

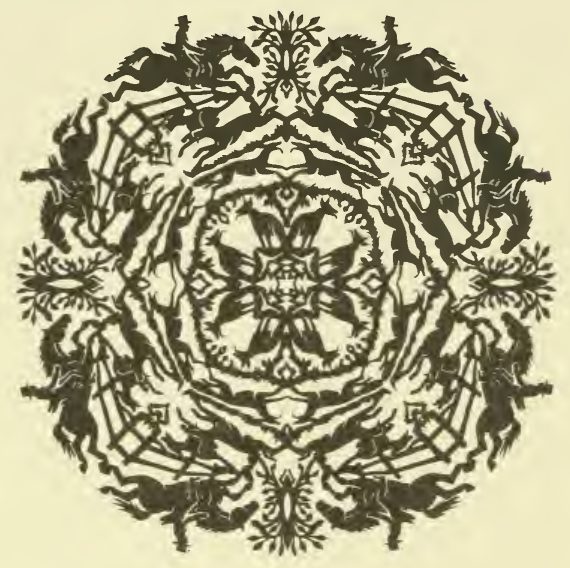

JOHN A.SEAVERNS 
TUFTS UNIVERSITY LIBRARIES

$\begin{array}{llll}39090 & 014 & 531 & 608\end{array}$ 
Webster Family Library of Veterinary Medicine Cummings School of Veterinary Medicine at Tuts University 200 Wectiono Road Narth 600 MA01536, 
- COMPLETE

\section{MODERN FARRIER}

A COMPENDIUM OF

\section{VETERINARY SCIENCE AND PRACTICE}

Showing the Best and most Successful Methods for the Prevention of all Diseases to which Farm Live-Stock are liable, and showing also the Best and most Successful Methods for the

Curative Treatment of these Diseases, the Subjects dealt with including

THE BREEDING, MANAGEMENT, AND VETERINARY TREATMENT OF HORSES FOR DRAUGHT, DRIVING, AND RACING

THE RULES OF THE JOCKEY CLUB

THE BREEDING, MANAGEMENT, AND VETERINARY TREATMENT OF DAIRY AND FEEDING CATTLE, WITH FULL INSTRUCTIONS AS TO THE PREVENTION OF TUBERCULOSIS, ABORTION, MILK-FEVER, AND OTHER BOVINE DISEASES

THE BREEDING, MANAGEMENT, AND VETERINARY TREATMENT OF SHEEP, DOGS, ETC.

\section{By THOMAS BROWN, M.P.S.}

LATE PRESIDENT OF THE ROYAL PHYSIOLOGICAL SOCIETY

WITH FOURTEEN FUI.L-PAGE COLOURED PLATES

\section{THIRTY-SECOND EDITION}

Thoroughly Revised, and in large part Re-written, so as to embody all the most Up-to-date Facts and Methods of Prevention and Treatment known to Veterinary Scientists

EDINBURGH: JOHN GRANT LONDON: SIMPKIN, MARSHALL \& CO., LTD. 



\section{P R EFACE}

ON account of the remarkable degree of public favour with which the former editions of this work has been received, this new edition has been thoroughly revised and brought fully up to date, so as to embody all the latest discoveries in Veterinary Science and Practice. In recent years great advances has been made in Veterinary Science, and the researches of Pasteur, Koch, and other eminent investigators in different fields of science have thrown a flood of light on the origin and nature of the diseases to which farm livestock are liable, and have also shown how in the case of many maladies the cause of the disease may be easily removed, so that in that way the disease may be prevented. The results of these researches made by eminent scientists, and confirmed by the practice of eminent veterinary surgeons, have been embodied in this new edition, which thus contains a great deal of valuable matter that was not to be found in the older editions. In the section relating to cattle, several new chapters have been added. The dire disease of tuberculosis-i.e., consumption-which is the burning question of the day to cattle-breeders and dairyfarmers, and which has been specially investigated 
by no fewer than three Royal Commissions within the last fifteen years, has been fully dealt with, and the means by which this scourge may be most effectively combated have been duly set forth in the following pages. Abortion, which is another source of disastrous loss to cattle-breeders, has also been dealt with, and full directions are given as to the best methods of preventing this plague. New chapters on Milk Fever, Diseases of the Udder, etc., have also been added. The sections relating to Horses, Sheep, Pigs, and Dogs have also been most carefully revised in the light of the most recent discoveries of Veterinary Science and Practice. The book has all along been designed to be a popular manual of Veterinary Practice, and is as free as possible from scientific technicalities, the author's expositions and instructions being expressed in language that can be easily understood by any intelligent stock-owner. 


\section{CONTENTS}

INTRODUCTION

\section{SECTION I}

\section{OF THE HORSE, ITS DISEASE.S AND STRUCTURE} CHAP.

I. Diseases of the Head, including the Brain, Ears, Mouth, Nostrils, Eyes, Etc. . . . .

Megrims-The Staggers, or Apoplexy-Mad Staggers-Rabies, or Madness-Tetanus, or Locked-Jaw-Epilepsy, or FitsPalsy-Glanders-Farcy-Nasal Gleet-Polypus-Lampas -Strangles-Canker and Wounds in the Mouth-Diseases of the Tongue; Tongue Bladders ; Bitten Tongue ; Vives, or Swellings in the Submaxillary Glands ; Barbs, or Paps; Gigs -Diseases of the Teeth-Diseases of the Lips-Diseases of the Eye; Common Inflammation of the Eye; Ophthalmia; The Haw ; Eruption on the Eyelids ; Warts ; Gutta Serena; General Blindness and Imperfect Vision-Fracture of the Skull.

II. Diseases of the Neck and Contiguous Parts • .

Poll-Evil-Roaring-Obstruction of the Gullet-Inflammation of the Jugular Vein.

III. Diseases of the Chest and its Contents . .

Inflammation of the Lungs-Pleurisy-Fever-Stomach-Staggers-Catarrh, or Common Cold-Bronchitis-Thick Wind -Broken Wind-Chronic Cough-Influenza, Epidemic Catarrh, Distemper-Fistulous Withers-Warbles, SaddleGalls, Etc.

IV. Diseases of the Abdomen and Intestines . • .

Inflammation of the Liver-The Jaundice, or Yellows-Hernia, or Rupture-Inflammation of the Kidneys-Inflammation of the Bladder-Stone in the Bladder-Diabetes-Staling of Blood-Inflammation of the Bowels; Inflammation of the Mucous Membrane; Spasmodic Colic; Entanglement of the Bowels; Stones in the Intestines; Intussusception of the Intestines-Intestinal Worms-The Bots.

V. Disorders of the External Parts of a Horse . .

The Fore Legs-Sprain of the Shoulder-Complaints and Injuries of the Arm; Fracture of the Elbow; Enlargement of the Elbow-Complaints and Injuries of the Knee; Broken Knees-Splent, or Splint-Speedy Cut-Knee-tied-Sprain of the Back Sinews-Wind-Galls-Rupture of the Suspensory Ligament-Grogginess-Sprain of the Fetlock-Cutting of the Fetlock-Sprain of the Coffin-Joint-RingboneOssification of the Back Sinew. 
CHAP.

Fracture of the Tuberosities of the Haunch-Sprain of the Round Bone-Sprain of the Stifle-Joint-ThoroughpinCapped Hocks-Mallenders and Sallenders-Enlargement of the Hock - Curb - String Halt - Bone-Spavin - BogSpavin-Of Swelled Legs generally; Swelling of the Cellular Substance; Swelling from Inactivity; Swellings after Grass-Grease.

VII. Diseases of the Foot .

Acute Founder-Chronic Founder-Contraction-Sand-Crack -The Navicular-Joint Disease-False Quarter-Tread, or Overreach-Quittor-Prick, or Wound in the Sole or Crust -Corns-Canker-Thrush-Ossification of the CartilagesWeakness of the Foot.

ViII. The Anatomical Structure of the Horse .

Different Bones of the Skeleton of the Horse-Bones of the Head-Side View of the Cranium-Section exhibiting the Internal Anatomy of the Head-Representation of the Palate and Teeth-External Anatomy of the Horse's HeadMuscles and Parts connected with the Eye-The Eye and its Visual Structure-The Teeth and their Development-Dental Indications of the Age of a Horse.

IX. Principal Muscles of the Limbs, Etc.

Muscles of the Outside of the Shoulder-Muscles on the Inside of the Shoulder-Muscles of the Outside of the ThighChief Muscles of the Inside of the Thigh-Structure of the Hock Joint-Bones of the Foot-Front View of the Pastern - Nerve, Vein, and Artery of the Pastern and Foot-Illustrations of Defects of the Fore-Leg-Attachments in Front of the Pastern Bones, etc.-Situation of Diseases of the Hind Leg-The Pastern and Foot, with their Bones and Integuments-External Parts of the Foot; The Crust or Wall of the Hoof-Cartilages of the Foot.

X. Sketch of the Internal Organisation of the Horse -

The Lungs-The Heart-The Pulse; Quick Pulse; Slow Pulse; Hard Pulse; Small Pulse; A Weak Pulse; Oppressed Pulse-The Abdomen and its Contents-The Peritoneum-The Diaphragm-The Stomach-The LiverThe Omentum, or Caul-The Spleen-The Pancreas-The Diaphragm, or Midriff-The Kidneys-The Bladder-The Intestines.

\section{Of THE Skin AND ITS Diseases}

The Cuticle-The Cutis-The Rete Mucosum-Pores of the Skin-Of the Hair-Clipping-Singeing-Colour of the Hair -Hide-bound-Surfeit-Mange-Warts.

XII. OPERATIONS

Bleeding-Blistering-Firing-Neurotomy, or Cutting the Nerve-Setons-Docking-Nicking.

X111. Of Breeding, Feeding, and Training Horses

Section I., Breeding. Section II., Feeding; Of Drink. Section IIl., Training. 


\section{CONTENTS}

CHAP.

XiV. Of Stabling, Exercise, Clothing, Etc

The Stable-Management of the Feet-Light-ExerciseGrooming-Clothing.

XV. Vices and Dangerous Habits of the Horse

Restiveness-Rearing-Backing, or Gibbing-Kicking-Biting Seizing the Cheek of the Bit-Running Away-ShyingRestiveness, and Resisting being Mounted-Vicious while Cleaning-Restiveness while Shoeing-Crib-Biting-WindSucking-Not lying down-Slipping the Collar-Pawing-Rolling-Weaving-Tripping.

XVI. Of ShoeIng

Preparation of the Foot for Shoeing-The Putting On of the Shoe-The Concave-seated Shoe-The Seated Shoe-The Expanding Shoe-The Hunting Shoe-The Racing ShoeThe Bar Shoe-Clips-The Hinder Shoe-Tips-Patent Safety Shoe-Water and Poultice Boots; Water-Boot-Felt, or Leather Soles.

XVII. How to Buy A HoRse . . . . . .

XVIII. General History of the Horse, with an ACcount of DIFFERENT BREEDS

Section I., Of British Horses: The English Race-Horse-The Hunter-The Hackney, or Roadster-The Charger-The Coach-Horse-The Cleveland Bays-The Draught-HorseThe Suffolk Punch-Horse. Section II., Of Asiatic Horses: The Arabian-The Persian Horse-The Tartar Horse-The Toorkoman Horse-The Turkish Horse. Section III., Of African Horses: The Barb-The Egyptian Horse-The Nubian and Dongola Horses. Section IV., American Horses.

XiX. The Ass and Mule

\section{SECTION II}

\section{DISEASES OF CATTLE}

INTRODUCTION

I. Diseases of the Head and Neck of Neat Cattle

Inflammation of the Brain-Choking-Locked-Jaw-Sore Throat-Snores.

II. Diseases of the Chest .

Inflammation of the Lungs (Pneumonia)-Contagious PleuroPneumonia-Inflammation of the Stomach-Inflammation of the Liver-Fog-Sickness, or Hoven-Catarrh, or ColdCough-Hoose, or Husk-Stomach, or Grass StaggersJaundice, or Yellows.

III. Diseases of the AbDomen

Inflammation of the Bowels-Inflammation of the Kidneys - Inflammation of the Bladder-Inflammation of the WombRed Water-Gripes-Diarrhœa, or Looseness-Dysentery -Fardel-Bound, or Excessive Costiveness-Mortification. 
CHAP.

IV. OF Febrile Diseases . • • • •

Splenic Apoplexy-Anthrax-Symptoms and Post-Mortem Appearances of Anthrax-Procedure-Disposal of CarcasesCleansing and Disinfection-" Black Leg," "Black Quarter," Parturient Apoplexy, or Milk Fever.

V. Diseases, Etc., of the External Parts of Cattle

Scurf; Scab-Cow-Pox-The Fouls-The Gad-Fly, Wurbles, or Wormals-Lice-Strains and Bruises-Wounds.

VI. Of Gestation, Parturition, Etc.

Gestation-Swelling of the Udder-Diseases of the Mammary Gland or Udder ; Physiological Congestion or Engorgement of the Mammæ; Mammitis, or Mastitis; Catarrhal Mammitis; Interstitial or Parenchymatous Mammitis-Agalactia-Ulcerated Teats; Chaps-Abortion-Falling Down of the Calf Bed-Parturition, or Calving-Rearing of Calves-Dentition of Cattle.

VII. Management and Feeding, Etc., of Cattle

Cow-Houses-The Feeding Cow-House.

ViII. The Various Breeds of Horned Cattle . . .

The Short-Horned, or Holstein Breed-The Kyloes, or Highland Cattle-The Long-Horned Cattle-The Galloway Breed -Milch Cows-Tuberculosis.

\section{SECTION III}

\section{DISEASES OF SHEEP}

INTRODUCTION

I. Diseases of the Head, Etc.

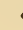

Staggers-Blindness-Catarrh.

II. Diseases of the Chest .

Fluke, or Liver Rot-Inflammation of the Lungs-Grass-IllMilk-Ill-Jaundice, or Yellows.

III. Diseases of the ABDomen

The Resp, or Red Water-The Red Water Blibes-The Braxy, or Sickness-Diarrhœa, or Looseness-Dysentery-ColicPinding.

IV. Diseases of the External Parts, Etc.

Scab, or Itch-Pelt-Rot-Erysipelas, or Wild-Fire-Cramp of the Legs, or Wood-Evil-Leg-Evil-Foot-Rot-VerminWounds-Fractures-Of Bleeding-On the Age of Sheep as indicated by the Teeth.

V. Gestation, Parturition, Etc. .

The Ram-Leaping-The Ewe-Lambing-Weaning LambsCastrating Lambs-Inflamed Udder, Etc. 
CIIAP.

VI. Management, Feeding, Etc., of Sheep

Shelter-Drifting Snow-Effects of Rain-Heat and FliesWashing-Shearing-Food-Fattening.

VII. Different BREEDS OF SheEP .

\section{SECTION IV}

\section{DISEASES OF SWINE}

\section{INTRODUCTION}

I. InTERnal Complaints

Inflammation of the Lungs-Quinsy-Blood-Striking.

II. Gargut, OR Distended UdDer • • • • . 585

ili. Breeding, Gestation, Parturition . • . . 586

\section{SECTION V}

\section{DISEASES OF DOGS}

\section{INTRODUCTION}

I. Diseases of the Head and Chest . . . .

Swelling in the Throat-Internal Ulceration of the EarCoughs-Inflammation of the Lungs-Asthma-The Distemper-Rabies, or Hydrophobia-Bites of Vipers-PoisonBlindness.

II. Diseases of the Abdomen, Etc.

Inflammation of the Bowels-Inflammation of the BladderWorms : Intestinal Parasites : Tape-Worms; Round-Worms; Sucking-Worms-Dropsy-Costiveness -Colic-Diarrhœa, or Looseness-Blend Water, or Bloody Urine-Piles-Fits.

III. Diseases of the External Parts, Etc. - •
Diseases of the Eye-External Ulcer of the Ear-Canker in the Ear-The Common Mange-The Red Mange-Foul Coats - Sore Feet - Wounds-Sprains and Bruises - ScaldsRickets-Thorns-Fleas and Ticks, etc.

IV. OPERATIONS

Blood-Letting-Method of giving a Bolus or Pill-WormingFractures-Cropping.

V. Gestation, Parturition, Etc.

On Breeding Generally-Parturition, or Pupping.

Vi. Feeding, Management, Training, Etc.

Greyhounds-Of Training Fox-Hounds-Training of Pointers and Setters - Vocabulary - Maxims - Retrievers - WaterDogs-Greyhounds-The Kennel. 
CHAP.

VII. Different Dogs used in Field Sports and for Domestic

$$
\text { Purposes, Watch-Dogs, Etc. }
$$

Section I. Dogs of the Chase. The Stag-Hound-The Fox-

Hound-The Harrier-The Beagle-The Terrier-The Otter-Hound - The Greyhound - The Scottish Highland Greyhound, or Wolf-Dog. Section II., Dogs used in Shooting. The English Pointer - The English Setter - The Springer-The Cocker-The Smaller Spaniel, or King Charles's Cocker-The Large Water-Spaniel-The Small Water-Spaniel, or Poodle. Section III., Watch-Dogs. The Mastiff-The Bull-Dog-The Bull-Terrier-The Newfoundland Dog-The Shepherd's Dog-The Coach-Dog, or Dalmatian.

APPENDIX-Rules of Racing

INDEX 


\section{LIST OF ILLUSTRATIONS}

RLATE

I. THE HORSE . . . . . . . Frontispiece

II. Bones of the SKeleton of A HoRse . • . . 16 I

III. External and Internal Anatomy of a Horse's Head - i68

IV. Teeth of the Horse from a Week to Six Years • - 192

V. $" \|, \quad$ SEVEn to FIFTEEN Years . 200

Vi. Muscles of the Fore Legs . . . . . 206

VII. " " HIND LEGS . . . . . 224

VIII. Bones of the Hock; the Cranium; the Stomach; the INTESTINES . . . . . . . . . 238

ix. Contents of the Chest; the Abdomen; Veins; Arteries AND NeRVES OF THE HiNd Legs • • • 257

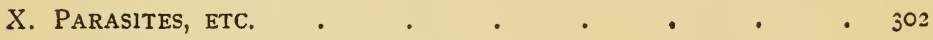

XI. Various Kinds of Shoes, Water-boots, etc. • • • 368

XII. " " $\quad$ Shoes, NAIls, KnIVES, ETC. • 374

Xili. Ear of a Horse; Stomach of a Ruminating Animal • 422

XIV. Head OF A Sheep • • • • • • • 544 



\section{MA N UAL}

OF

\section{MODERN FARRIERY.}

\section{INTRODUCTION.}

The term Farriery has been in use for a very long period, and embraced the Medical and Surgical treatment of the Horse, as well as Shoeing, and fitting him for taking the road and the field.

The persons employed in the manual labour of constructing and fitting the shoes of horses were originally termed Feveres, or Ferriers, from the Latin word ferrum, iron, and their practice ferriery, which has, in the course of time, been changed into the word Farrier, and now universally adopted.

In most country situations the blacksmith who shoes horses is called the farrier, because he is generally the only person, in many localities, who practises the art of horse and cattle doctor. Not only is this the case in Great Britain, but also in every country of Europe.

It is to be lamented that more attention is not devoted to the useful practice of the cure of domestic animals, more especially when we consider not only their great value, but also their commercial importance. As well may the medical care of man be intrusted to shoemakers and tailors, as the care of horses, cattle, swine, dogs, and other domestic animals be left to the tender mercies of an untutored blacksmith, who, although by practice he may be extremely competent 
to shoe or bleed a horse, is, nevertheless, most unlikely to be able to administer relief to animals whose physical constitutions are subject to as many and complicated diseases as humanity itself.

In France and Italy the importance of the cure of the diseases of horses and cattle was first manifested upwards of two centuries ago; and professorships established for the art of farriery as a medical and surgical science, and gradually the persons who professionally studied and practised this art assumed the names of Veterinary Surgeons. This term, although but recently adopted, is of great antiquity, having been in general use among the Latins.

It is, however, surprising that in Great Britain, where our domestic animals are of such vast importance, it is only very lately that schools and professorships for the study of the Veterinary art have been established; and not much more than half a century ago, the surgeons of our cavalry regiments administered medicine to horses as well as to the soldiers. But the study of Comparative Anatomy soon rendered it manifest that a totally different course of study became necessary for the treatment of animals whose internal organisation was so different from that of the human being; and now every horse regiment has its Veterinary Surgeon. All great towns, and many small ones also, possess medical and surgical practitioners, whose sole attention is devoted to the cure of domestic animals; these gentlemen having attended and acquired diplomas from the Veterinary Colleges for their knowledge and capability to practise the arts.

The intention of the following treatise is not to take the practice out of th: : hands of regular Veterinary Surgeons, but to serve is a ready manual to those living in the country or $\epsilon$ ven in towns, to give them 
a general idea of the diseases and remedies, so that they may be applied in time of need, and where a regular veterinary practitioner is not at hand.

Some of the diseases incidental to horses and cattle are so marked in their character, that a little experience will easily enable a person accustomed to be among those animals, to detect it with facility, and in such cases, medicine may be safely administered, according to the rules we have laid down; but there are other disorders, whose characters are of a more complicated form, which ought not to be treated by a person uneducated in the veterinary art. In such cases it will always be found safer and cheaper in the end to apply to regular practitioners.

Although in a certain sense there is some analogy between the diseases of man and animals, yet these are exceedingly different in their specific characters, and consequently in the remedies applied for their cures; the construction of the stomach, the length of the alimentary canal and small intestines, with other organic distinctions-all combining to render the specific quantity and character of the medicines to differ essentially.

Nevertheless, it is quite possible to acquire a considerable knowledge of all that is known in the veterinary art, by persons in private life; although considerable study, as may well be supposed, is necessary to attain this degree of knowledge.

The first thing to be studied is to acquire a knowledge of the skeleton, then of the muscles, and lastly of the internal organisation. The two first of these are pretty much alike in all our domestic animals, but a very great difference will be found in the internal structure. For the skeleton and muscles, works and good engravings will give a good idea to the be- 
ginner; and after acquiring the names of the different bones and muscles, the student must practise upon dead subjects, so that he may be enabled, by dissection, at once to determine what part of the body is affected by lameness or a wound. He must next acquire a thorough knowledge of the exact situation of the internal organs, and their comparative dimensions and structure. Attention must next be given to Chemistry, and the compounding of medicines, with the relative quantities to be administered to the different animals, which will depend upon, as above hinted at, the structure of the stomach and the length of the large and small intestines. Upon the character also of the nervous system will depend much of the treatment of animals, and the energy and immediate application of remedial means. Some diseases are slow in their progress, while others are so rapid that there is more art and utility in arresting them, than in a cure after they are fairly formed. But this can only be acquired by experience.

It is not pretended that the knowledge above specified is to be found within the limits of this work, which is chiefly intended as a popular digest of all that is truly useful to the country gentleman and practical farmer, and others possessing domestic animals. We shall, however, in the course of the work, give illustrations of the general structure of the Horse, with such other parts as should be generally known, and directions concerning their functions; so that the classes for whom it is intended may have such a knowledge of the subject, as will enable them to act for themselves, in ordinary cases, and in others to qualify them for detecting the impositions too frequently practised by ignorant quacks. 


\section{SECTION}

OF THE HORSE, ITS DISEASES AND STRUCTURE.

\section{CHAPTER I.}

Diseases of the Head, including the Brain, Ears, Mouth, Nostrils, Eyes, \&c.

THE diseases of the head in animals, as well as in the human being, are, for the most part, so complicated and obscure that few non-professional men are qualified to undertake their cure with success; but it will be our endeavour to treat the subject in as plain language as possible, so that those who have not regularly studied the veterinary art may at least acquire such a knowledge as will enable them to apply some judicious remedy, until the advice of a practitioner can be obtained.

\section{MEGRIMS.}

This malady is occasioned by a pressure on the brain, caused by an unusual flow of blood to it. The flow of blood through the brain is much greater in quantity than through any other portion of the body of equal bulk. To prevent as far as possible any unusual flow of blood to this organ, the arteries pursue an extremely winding and circuitous course, and enter the skull through small holes in the bony process. These holes are so small that they will admit but of little enlargement of the blood-vessels, 
and thus to a great extent the progress of inflammation is prevented. Yet, notwithstanding this beautiful provision in nature, the horse is liable to be afflicted with diseases in the brain, from violent and injudicious exercise, and hard driving or riding in warm weather, which forces the blood to the head, and distends the arteries of the brain more than the veins; and the consequence is, the small vessels which ramify in the substance of the brain get gorged with blood, and then its bulk is increased to such an extent that it will produce undue pressure upon the origin of the nerves, which is followed by loss of power and even consciousness, and consequences of a very serious nature frequently follow.

Symptoms.-These most commonly appear when a horse is over-driven. When attacked he will suddenly stop and shake his head, having been seized with giddiness and a slight degree of unconsciousness. If allowed to stand for a few minutes, this will go off, and he will be enabled to proceed on his journey. But it not unfrequently happens that the attack is of a more severe kind; and under such circumstances the horse will fall suddenly, or in other cases will run round several times and then fall. Sometimes he will lie quietly in a state of complete torpor; at other times he will struggle with great violence, and for a time be unable to rise. In either of these conditions he will continue for from five to ten minutes, when he will gradually resume sensibility, and then will be able to get on his feet, and may then proceed on the journey. But after these attacks he generally exhibits symptoms of dullness and exhaustion.

REMEDY.-Immediately after the first attack of this disease, if the animal is strong and robust, re- 
course should be had to bleeding. Three or four quarts of blood from the jugular vein generally has the effect of arresting the symptoms. Another method is to cut the palate, which will permit a sufficient flow of blood to have the desired effect, that is, from two to three quarts. This, of course, should only be resorted to if the driver happens not to possess a lancet at the time the horse is attacked.

The bleeding place of the palate is in a direct line between the middle and second cutting teeth, and situate a little more than an inch within the mouth. Here the vein and artery make a curve. A sharp penknife may be used, and cut down upon the spot where they intersect each other. The result will be a plentiful flow of blood, and which will stop of its own accord when two or three quarts have issued forth. In consequence of the artery being cut across, it will shrink and speedily cease to bleed, and the application of a sponge, or piece of rag and cold water, will stop the bleeding of the vein. In this operation the nerve is generally divided, but no bad effects will result from it.

If the cut is made a little too much on one side, and nearly opposite the second incisor tooth, it is possible the artery may be wounded longitudinally, but not divided, in which case there may be great difficulty in arresting the flow of blood. The most effectual method is to make a large and compact pledget of lint or tow, which should be rolled round a piece of twine, and then firmly tied round the front teeth, and its pressure on the surface of the bleeding part will generally stop it; but should this prove ineffectual, then a gag may be constructed so as to press upon the pledget, which is sure to stop the bleeding. 
It is only when a horse is on a journey that the above mode of bleeding, by cutting the bars of the palate, is to be resorted to, because there is no way of ascertaining the measure of the blood. Therefore it is only in cases of necessity that it is to be applied, as it may not only occasion much pain to the horse, but also a great deal of trouble to the operator. The ordinary mode of blood-letting is preferable in all cases. Immediately after the first attack, three or four quarts of blood should be taken from the vein of the neck. A short time afterwards, give a dose of physic, in the form of a ball, consisting of the following ingredients :-

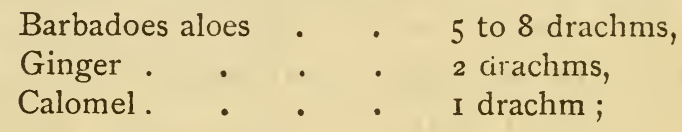

to be beat up in a mortar with a sufficient quantity of honey, so as to form them into a ball, which must be administered in the manner we direct under the head of "Medicines."

Megrims is a very dangerous disease, not only to the horse but also to the driver, as in many instances the horse falls down without the slightest previous indication of illness. If a horse has had one attack of this malady, he is liable to a return of it; and after a second attack, although proper means have been adopted to prevent a recurrence of it, the most prudent plan is to part with the animal, as he can never afterwards be depended upon.

THE STAGGERS, OR APOPLEXY.

Symitoms.-.The premonitory appearances are a low hanging of the head, and either supporting it on the manger, or extending it nearly to the ground. 
He moves to and fro while standing, and seems liable to fall at every movement. His sight and hearing are much impaired. He will remain in this condition from one to twelve hours; he then falls. His eyes are open and protruding, with a fixed, seemingly unconscious, stare, with the pupils much dilated; he grinds his teeth, the whole frame manifests twitchings, the veins of the neck are distended, and in attempting to swallow, the drink is returned by the nostrils and mouth, and he dungs involuntarily: strong convulsive twitchings follow, and these are the certain preludes to death.

In the first stage of the disease there is depression, sleepiness, and feebleness, which is distinctly indicated by dulness of the eye. As it increases, he presses his head against the wall or the rack, and when aroused from this position he seems alarmed.

Causes.-A deranged state of the digestive organs is the most ordinary cause, and this is the effect of over-feeding in many cases. Some persons are so foolish as to suppose that horses may have as much grain as they can eat, and that it will do them no harm. This is a serious mistake; because, even without the aid of water, the grain will swell in the stomach, and from being completely overloaded, indigestion follows, the stomach being too much distended to be able to perform its office. The head is affected, as in the animal economy a very intimate connection exists between the brain and stomach, each reciprocally influencing the other. The want of exercise with horses which are highly fed, also tends to derange the stomach, more especially during warm weather. The bracing influence of exercise being wanting to give energy to the actions of the intestinal canal, the food frequently lodges there. This is also 
caused by the food being indigestible. Another cause is irregularity in watering horses, as this element is peculiarly necessary to animals which live upon dry food. Every horse should be watered at least four times during the day, and in some cases while working hard, more frequently, and in smaller quantities.

REMEDY.-If the horse is robust or plethoric he may be bled in the jugular or neck vein, but if weak or debilitated from whatever cause, blood should not be abstracted. If the lower intestines, or rectum, is overloaded with dung, the hand should be passed up the rectum, and the bowels unloaded in this way. The following medicine should be administered, in the form of a ball :-

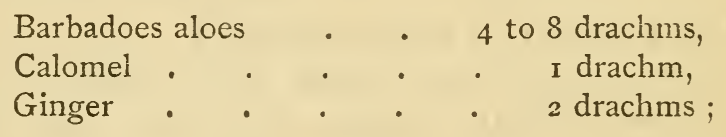

these to be mixed with honey or treacle, in sufficient quantity to form a ball.

Sometimes a clyster is useful, which may be formed of soft soap and hot water.

These clysters may be repeated twice or thrice with beneficial effect, as they facilitate the action of the purgative and unload the rectum.

Blisters behind the ears, Cayenne pepper blown up the nostrils, as well as bark and spices given internally, are perfectly useless. Indeed the two former are ridiculous.

After the purgative has acted, should the horse be able to take food, boiled barley, scalded bran or oatmeal, and lukewarm water, should be given to him for some days until he is able to masticate hay, which ought to be of the best quality. This must, 
however, be given in small quantities until his bowels have been thoroughly purged out.

To complete the cure, the following may be given :-

\begin{tabular}{l}
$\begin{array}{l}\text { Bicarb. of soda } \\
\text { Pulv. gentian } \\
\text { Pulv. ginger }\end{array} \quad \cdot \quad \cdot \quad \cdot \quad \cdot \quad \begin{array}{l}3 \text { drachms, } \\
\text { drachms, }\end{array}$ \\
\hline
\end{tabular}

these to be dissolved in a quart of hot ale; to be given the first thing in the morning in a tepid state of heat. This may be repeated daily for ten days.

It is hardly necessary to remark, that this disease is in general extremely rapid in its effects, and the utmost promptitude must be exercised in the remedies.

\section{MAD STAGGERS.}

Symptoms. - This disease proceeds from inflammation of the brain. In the earlier stages it is difficult to distinguish from the sleepy or stomach staggers. It soon, however, assumes a different character. The nostrils become distended, and he commences to heave at the flanks; his eyes assume a fixed, vacant, and wild stare, which is followed by complete delirium; he becomes furious, and dashes about in a violent manner from side to side, being quite unconscious of his actions.

The mad staggers are considerably alike in their symptoms to rabies. In the former of these maladies the horse retains his consciousness, and the violence of his actions will depend upon the peculiar character of the madness. In some instances a desire to be mischievous is manifested.

Causes.-This disease may be caused by the animal being too fat, too full of blood, or from a fracture of the bones of the skull, or disease of the 
blood-vessels of the brain, or its coverings. The fever produced thereby causes a determination of blood to the head, and thus terminating in what is generally denominated "brain fever."

Remedy.-The treatment in this malady is at all times exceedingly uncertain. If the pulse is full and strong, abstract blood from the jugular until its character is altered, thereafter give a dose of purgative medicine, and without delay call in the services of the veterinarian.

\section{RABIES, OR MADNESS.}

Symptoms. - The symptoms are nervousness, then excitability and violence. Determination to seize, bite, and kick any and all objects within reach of its mouth or heels. Sometimes the victim lacerates his own flesh with its teeth, and neighs frequently and loudly. Towards the close the animal experiences a difficulty in swallowing, and the respiration is loud and distressing.

The horse affected generally dies within thirtysix hours from the first commencement of marked visible symptoms.

Causes. - This incurable disorder is caused by the bite of a mad dog, or other rabid animal, the blood poison being contained in the saliva.

REMEDY.-When symptoms of rabies have manifested themselves, it is in vain to attempt a cure. But in cases where horses have been known to be bitten by a rabid animal, or, under doubtful circumstances, the wound should be well and deeply burned with lunar caustic. Rabies being a scheduled disease, its existence must be reported to the Local Authority under the Contagious Diseases of Animals Act. 
TETANUS, OR LOCKED-JAW.

Symptoms.-To the human being, as well as to the horse, this generally proves a fatal malady. This disease does not manifest itself of a sudden; but generally steals over the system by slow or insidious means. It first develops itself by the animal appearing heavy and unwell for a day or two; he feeds sparingly, frequently half chewing his food, and then drops it from his mouth. When he drinks, the water is gulped, in place of the ordinary mode of taking it. The action of the jaw becomes extremely imperfect, and the saliva trickles from the sides of his mouth. The mouth can at length be but imperfectly opened; and ultimately, the whole voluntary muscles of the neck, head, and upper portions of the body become immovably fixed. The earliest and most reliable symptom of tetanus is spasms of the retractor muscle of the eyeball, and this is in evidence the instant the animal elevates his head, the eye being drawn back and the haw (Membrana Nictitans) shot forward and upwards. In a short time nearly the whole muscles of the body are spasmodically affected.

Causes.-This disease is due to the introduction of a microbe (Bacillus Tetani) into the body of the affected horse through a wound. It sometimes comes on instantaneously, after the infliction of a wound, or sometimes a considerable time afterwards. Nicking and docking have frequently caused this affection of the nervous system. The usual way in which this disease comes on seldom leads to a suspicion of what it is, as few who have not previously watched its progress can trace its character. 
Hence it has assumed its climax before persons are aware of it.

Remedies. - If the cause of the disease has been from docking, the operation should be repeated higher up; and if from nicking, by making a deeper incision,-by these means the spasms have been in some instances removed.

If tetanus follows on a prick of a nail or puncture of the sole of the foot, the horn should be pared, the wound opened up, and carbolic acid poured in. As the Tetanus Bacillus is found in earth, the dust of streets and manure, wounds of the feet, and portions of the body on which the animal reclines, should be carefully protected by carbolic liniment, and tow and bandages, where these can be applied.

If the jaws are not fixed, administer an aloetic ball, and follow this up by the immediate hypodermic or subcutaneous injection of "Tetanus Antitoxin," but this and the after-treatment should be under the guidance of a veterinary surgeon.

In the treatment of this disease it is of the first importance that the horse be placed in a loose box, from which the light is excluded, and no one is allowed to enter, except the attendant on the animal, and he should be cautioned to make no noise or disturb the horse in any way. A pail of cold water, and one containing gruel (oatmeal and linseed) should be fixed up so that the animal may be able to drink without depressing his head. In some cases it is advisable to place the horse in slings, for should he fall down when asleep, he may be unable to rise.

EPILEPSY, OR FITS.

Symptons.-Epileptic fits in horses are, as in the 
human being, very sudden, of which no premonition is given. The horse suddenly stops, is seized with considerable trembling, looks around him with a vacant stare, and then suddenly falls. This is followed with convulsive struggles of a greater or lesser degree. The head and neck are considerably contorted. The convulsions seldom last more than a few minutes; he ceases to struggle, and on the recovery of consciousness, he generally springs to his feet. If in the stable, the horse will immediately commence feeding.

It is exceedingly unsafe to use a horse so afflicted, especially in riding. Indeed we would strongly recommend that after having one fit, he should never be again used for the saddle, as if once attacked, there is every probability of a return of the fits.

We shall not attempt to point out a remedy for epilepsy, as hitherto nothing has been discovered to prove a certain cure. Therefore, anything that may be attempted must be by a regular veterinary surgeon.

\section{PALSY.}

This is usually occasioned by blows, falls, or racks in pulling loads which are beyond the proper strength of the animal. It is, for the most part, situate in the hind-quarters. It is therefore evident that it proceeds from spinal irritation or organic disease of the spinal cord. It may affect the head and fore-limbs, and be associated with disease of the brain or the growth of tumours within the cranial cavity. Palsy is, for the most part, met with in draught-horses. Horses so afflicted seldom lie down either in the stable or field, as they have invariably considerable difficulty in getting up again. It seldom happens that this complaint is removed. Blisters, nerve stimulants, 
and friction are the most probable means to be applied.

\section{GLANDERS.}

Symptoms.-Of all the diseases incidental to the horse this is without doubt the most malignant, and most to be dreaded in a steed. The instant that there is any appearance of it, the horse should be immediately removed to a place by itself, as this malady is exceedingly contagious; and from want of due caution, when even a suspicion is entertained, the most disastrous consequences have been the result.

This disease is due to the introduction of a parasite, termed the "Bacillus Malleii," into the body of the horse, and is found in the discharge from the nostrils and in the "glanders nodules" in the lungs of affected horses.

There are various diseases which in their early symptoms have much the same appearance as glanders, and therefore it is necessary to watch these narrowly.

The very first visible symptom of glanders is a discharge from one nostril, clearer and of a lighter colour than in a common cold or catarrh, and more glutinous in its substance. If rubbed between the finger and thumb, it has a sticky feel.

A singular character of glanders is, that it generally attacks one nostril only, and that is the left one; only a few cases having occurred where it commenced in the right nostril. $\mathrm{Mr}$ Dupay, a celebrated veterinary surgeon, and director of this school of surgery at Toulouse, mentions, that out of eight hundred cases of glanders, which occurred during his practice, only one was affected in the right nostril. 
Shortly after the discharge from the nostril takes place, the horse is then affected in the glands of the lower jaw, which swell to a considerable extent, and ultimately atrophy and become attached to the bone. Some considerable time after the discharge has made its appearance, the gluey substance will be seen accompanying the mucous discharge. It is this pus mingling with the other gluey matter which, absorbed by the circulating vessels and carried to the gland, affects it. However, in common catarrh, the glands are sometimes swelled. But in glanders the swelling generally subsides considerably in a short time; and the glands are not in the centre of the channel, but adhere firmly to the jaw.

At this stage of the disorder, the mucous membrane of the nostril may become dark purple or of a livid colour; sometimes of a tone intermediate between these two shades. In some instances there is inflammation of those parts, which varies from the common appearance, being of a purple cast, instead of the high red which usually accompanies inflammation. This is followed by the formation of small circular nodules on the lining of the nostrils, and these in a short time ulcerate and discharge pus. When this has taken place, there can no longer exist a doubt that the horse is glandered. Care must be taken not to mistake the lachrymal or tear-duct for an ulcer. This duct opens by a small orifice or hole on the continuation of the common skin of the muzzle, which is situate a little way up the nostril; while the ulcerated tubercles are placed upon the mucous membrane above the duct, and well marked by a line of separation.

With the formation of "glanders nodules" the animal is certain to have become constitutionally 
affected. His coat will stare and fall off; he will lose flesh, and his belly will be tucked up; cough will follow; the appetite will be much affected, accompanied by a rapid diminution of strength; the nodules will multiply; discharge will be much more abundant, and will assume a purulent and bloody appearance, accompanied with a very fotid smell. The ulceration may extend down the windpipe, and the lungs will be in a very short time studded with glanders nodules. A test of the lungs having become affected, the breathing will be difficult, and a stifled, grating noise accompanies it, which is a certain prelude to death.

A common catarrh has often been mistaken for glanders; but a little attention will soon enable any one to perceive the distinction between those diseases. Catarrh is invariably accompanied by fever, sore throat, generally cough, loss of appetite, and a discharge from both nostrils, and, in most cases, very copious ; sometimes purulent; the glands are generally swollen in both sides of the throat, are movable and hot to the touch. The proper means being adopted, all the symptoms are abated. Strangles have also been mistaken for glanders. This disease usually affects young horses only. At first they resemble a common cold, with a severe cough and wheezing, and accompanied with a considerable thickening and swelling between the jawbones. The swellings become harder towards the middle, and a fluid can be felt in their centre, which ultimately breaks, and a discharge flows from it. The mucous membrane of the nostrils is of a vivid red colour ; and an ample discharge continues, which is mixed with pus from nearly the commencement.

CAUSE.-Ill-ventilated and not properly drained 
stables, we fear, are too frequently predisposing causes in the production of glanders. There the ammonia from the urine fills the whole atmosphere, and this being constantly inhaled, ultimately produces a poisonous effect upon the lungs.

We find that glanders almost invariably breaks out in ill-aired stables, and which are besides kept too hot. We find that in the lofty, well-aired stables of gentlemen this disease is almost unknown, and when it does show itself in such, it has in all probability been introduced by some addition to his stud of one or more horses previously affected. In such a case, all the other animals in the stable may catch the malady, as glanders is known to be highly contagious. In many of the crowded, ill-aired stables of London and other large cities, this disease is but too often an inmate, and frequently great havoc is made among the horses in consequence. Persons who are in the daily habit of riding to town, should bespeak a stall expressly for themselves in a livery-stable, because, by introducing a diseased horse, the contagium may be caught by their horses, in consequence of any slight wound about the muzzle coming in contact with the crib, on which the mucus of the glandered animal has been left, and is thus carried into the circulation. All public stables should have high divisions between the stalls, to prevent the muzzles of horses from coming in contact with each other, horses being very apt to smell at one another in a stable, as it is by this faculty alone they recognise their companions. Glanders may be propagated in three ways:-(I) By ingestion of food soiled with the glanders discharge from the nose. (2) By drinking water containing the discharge. (3) By inoculation of the virus into a wound, e.g., a glandered horse biting his neighbour. 
If doubt exists as to the animal being glandered, the "mallein test" should be applied by the veterinary attendant. Glanders is a scheduled disease, so that the existence must also be reported to the Local Authority, and the animal destroyed. Professor Nocard of Paris entertains the opinion from experiments which he has performed, that glanders in its early stages is curable by injections of mallein.

From an ill-judged piece of economy, many persons, after being aware of a horse being glandered, persist in keeping it in the same stable with others. Every hour is risking the health of all he possesses. It is the duty of every person, so soon as he is certain of his horse having caught this disease, to destroy it as speedily as possible. For, although a glandered horse may be able to work for a considerable length of time under the influence of this disorder, he will find ultimately that it is a bad piece of economy to keep him under such circumstances.

Many persons who have lost their horses by this disease have resorted to extremes to prevent a continuance of it. Some have even gone so far as to pull down their stables, and others to remove their racks, mangers, and partitions. It is quite sufficient if the mangers and other parts which the discharge from the nostrils have touched, is well washed with a scrubbing brush, with a strong solution of soda and water, and afterwards with chloride of lime, the proportion of which should be a pint-and-a-half to a pailful of water. The walls should be washed with lime and water, containing a pint of crude carbolic acid to the pailful of lime-wash, and all the halters, etc., destroyed, and the iron work painted.

CAUTION.-All purchasers of horses at fairs, or from dealers with whom they are not acquainted, 
should carefully examine horses as to their having this disease : because they, by infamous trickery, too frequently use means to deceive the purchaser. It is well known that if a horse is galloped pretty sharply, that the increased action in breathing will thoroughly drive the mucous substance out of his nostril. And to make it continue dry for a time, they force a pledget a considerable way up the diseased nostril ; after having blown powdered alum or white vitriol into it. But a little attention will enable any one to perceive that the animal is in pain, and will make ineffectual efforts to sneeze.

\section{FARCY.}

Syмpтомs.-This is the same disease as glanders, but confining its ravages to the skin and lymph vessels ramifying in its structure. The first symptoms manifested are the appearance of small tumours, popularly called farcy buds, or buttons, situate close to some of the lymphatics and following their course, connected by a sort of cord; and hence they are called corded veins by farriers. At first they are generally very small, and consequently may not be noticed for some weeks, until they have attained their full size; after which they usually increase more rapidly, become hot, and cause considerable pain, and at length ulceration ensues. They make their appearance about the face, neck, and throat; sometimes extending to the inside of the thigh, and produce lameness, and considerable swelling of the limb.

It sometimes happens that farcy is progressing in the constitution long before the buds make their appearance, or swelling along the course of the absorbents takes place. In some instances, the buds do not ulcerate, but assume a callous texture. At 
this period an apparent check to the disease takes place, and the horse seems to be quite recovered. This, however, is only a delusion; and although no symptoms of the complaint manifest themselves for a number of months, it is working in secret, and all at once breaks out in a most malignant form, and probably in a few days he expires under its influence.

Sometimes a considerable swelling of the head takes place, especially in the region of the muzzle, and from which an extremely fotid mucous fluid is discharged. Various portions of the body exhibit mangy eruptions; swellings in the limbs will follow, the heels will become cracked, exhibiting all the appearance of grease. The animal in most cases will become emaciated and weak.

Farcy assumes many different appearances in its various stages. It is no uncommon thing for one of the hind legs to swell suddenly to a very large size, frequently upwards of three times its natural dimensions, accompanied by abrupt projections and depressions, and which the poor animal will be unable to move. This is generally accompanied by a considerable degree of fever.

\section{NASAL GLEET.}

Symproms.-This is a constant discharge of a thickish fluid from one or both the nostrils; proceeding from the mucous membrane, which lines the internal cavity of the nose and sinuses of the head.

This disease is frequently brought on by the effects of a long-continued discharge from catarrh or cold. It is unattended with any feverish symptoms. The flow of this thick mucous gleet is often very considerable, and variable in colour. When the horse is living upon green food, the discharge is of a 
bright grass-green colour; but if his diet consists of dry food, and he is kept in the stable, then it assumes a very different hue; varying from creamwhite to brown, or straw-colour, and mixed with pus in some instances, and in others mingled with blood. The discharge is sometimes continuous, and at others it is only occasionally sneezed out. In the latter case it is generally thick, and when so the disease is on the wane. If, however, it is of long duration, it sometimes assumes a serious aspect, and may ultimately prove fatal to the horse.

Cure.-Give the following medicine twice a day :Sulphate of copper, or blue vitriol I drachm,

made into a ball with treacle and linseed meal.

Should this disease be attended with cough and fever, then the following draught must be prepared :-

\begin{tabular}{|c|}
\hline $\begin{array}{l}\text { Linseed } \\
\text { Treacle }\end{array}$ \\
\hline Vinegar \\
\hline
\end{tabular}

The linseed must be soaked or decocted in hot water for three or four hours, kept close to the fire, or on the hob of a grate. Let it be poured off, and the quantity of this tea which remains must be six pints. Then add the other ingredients.

Give the horse half-a-pint from four to six times during the twenty-four hours. The above tonic, consisting of sulphate of copper, should be continued along with the cough mixture. Should pus be found mixed with the nasal discharge, and the smell be disagreeable, then the following tonic medicine must be given :-

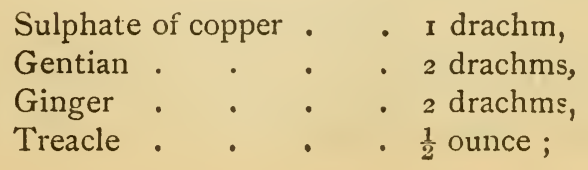


to be administered in a single dose, and repeated daily for a week or fortnight unless the bowels become costive.

\section{POLYPUS.}

This is the name given to a pedunculated tumour which grows from the mucous membrane which lines the nostrils, and although not painful, is attended with disagreeable consequences by obstructing the breathing, and often proves very annoying to the animal. We need not treat more of its cure or otherwise, as it can only be removed by an operation, which requires the aid of a skilful veterinary surgeon.

\section{LAMPAS.}

Symptoms. - This is the name given by farriers to a condition in which the bars on the upper palate are found red and swollen, and on a line with the cutting edges of the incision teeth. It is a condition more or less seen in all horses when growing their teeth.

Remedies.-In most instances the congestion and swelling will subside without medical aid. Slight purgatives and some mashes will facilitate its removal. The animal may also be relieved by a few slight cuts across the bars. This may be done with a penknife or lancet, only the operator must keep clear of the palatine artery ; the situation of which will be obvious by a reference to Plate III., fig. $2, e, e$. It is a common practice with farriers to burn the bars with a red-hot iron, a mode of treatment which cannot be too much deprecated.

STRANGLES.

Symptoms.-This is a disease incidental to young horses, and few colts escape it. Occasionally it 
attacks old horses, in which event it is more difficuit to cure. The usual period at which this disease shows itself is in the third and fourth years, although it is by no means uncommon to attack foals. The first symptom is cough, differing but little from that of a common cold, only that there is a more abundant discharge from the nostrils, which is of a yellowish colour, and unaccompanied by a disagreeable odour : it is also in most cases mixed with matter. There is, besides, a profuse discharge of slimy, stringy fluid from the mouth, the throat being sore. The membrane which lines the nose is intensely red. It will be found that considerable swelling has taken place under the jaws and accompanied by fever, which is distinguished by want of appetite, a quick pulse, and a hot mouth, a general weakness of the whole frame, producing a dejected appearance. To ascertain the amount of fever (or increase of combustion going on in the body), a graduated thermometer should be inserted in the rectum, and there allowed to remain for four minutes. The average normal temperature is $\mathrm{IOI}^{\circ}$, and all above this indicates the fever present, or in other words, the disturbance of the heat function and abnormal burning up of the tissues composing the animal's body. A temperature above $104^{\circ}$ is serious, and above $107^{\circ}$ generally fatal. There is likewise a quick motion of the flanks, and coldness in the ears and limbs. The swelling is in the form of a tumour between the jaws; increasing with various degrees of rapidity, occupying nearly the entire space, and gives pain to the horse when eating; he besides manifests a great disinclination to feed. This is accompanied by much thirst, but the swelling prevents him from indulging in water, and having swallowed a mouthful or two he desists. After which, and even after eating, 
he is frequently seized with a spasmodic cough, with suffocating symptoms.

Cause.-Strangles is an eruptive febrile disease dependent on a specific germ or microbe, and can be transmitted by cohabitation, so that if the dam has the disease she generally conveys it to her foal, and in this way all the foals and young horses running together at grass may become infected. It appears to be in some degree analogous to measles in the human being; and having passed through it, the constitution of the animal seems to have undergone purification and improvement. In some instances it has affected the animal in so mild a form, that it has passed through its various stages and gone off without much inconvenience to it, or any remedial means whatever having been employed. Every horse has this complaint once during his life, and once only.

ReMedies.-As the virus or blood-poison eliminates itself by the formation of an abscess in the lymph glands betwixt the jaws, or in the lymph glands in the parotid region, the first thing to be attended to is to bring the tumour to a suppuration. A sharp mustard blister is the first thing to be applied. This, administered in time, will facilitate the discharge a week or two earlier than it would have taken place, if allowed to come to a period naturally. The old practice of applying poultices and fomentations first were very ineffectual appliances, from the great thickness of the skin of the horse, but after one or two applications of the mustard, they materially assist in softening the inflammatory exudates and bringing the abscess to a point. The abscess from absorption and thinning of its walls and the tissues about it frequently bursts of its own accord; but if it should not, it must be laid open with a lancet. It will be found that 
where abscesses break spontaneously, the lips of the wound, from having uneven edges, will be more diffcult to heal.

If there is much fever, with difficult breathing, febrifuge medicine should be prescribed :-

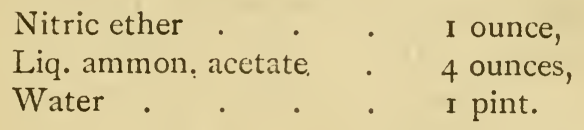

The above fever drink may be given morning and evening until the temperature becomes normal. If there is no fever, the animal will soon manifest a desire to eat. His food should principally be oatmeal gruel and bran-mashes, with a supply of green meat, consisting of cut grass, or tares.

If the complaint is followed by weakness, it will be necessary to have recourse to the following tonic medicine, which should be repeated daily until the horse recovers strength :-

Ginger $\quad$ : 2 drachms,
Sulphate of iron: $\quad 2$ drachms,
Gentian - $\quad 2$ drachms.

In bad cases of strangles the parotid gland may swell to a great size and become hard and indurated, and remain in this condition for a length of time. In all such cases an abscess deep in the structure of the glands forms. In such a case, it will require the aid of a regular veterinary surgeon, as an operation must be had recourse to, which no one but a regular and experienced practitioner will be able to perform.

\section{CANKER AND WOUNDS IN THE MOUTH.}

It is but a too common occurrence, that the sides of the mouth and other parts are wounded by the bit, which may be either too acute in its edges or may not 
fit. Frequently deep wounds are in consequence inflicted in the sides and inner parts, more especially between the grinders and the tushes, on which the bit rests. It is no uncommon occurrence for the entire flesh to be removed from between the tushes and grinders, and instances frequently occur of the bone likewise being so injured that portions of it have to be removed. It may be well conceived the very great pain this must occasion to the poor animal. Those who have had even a very slight inflammation of the gums will readily have an idea of its sufferings.

Every man of feeling will make it his first study to see that the bit fits the mouth of his horse properly, and that it in no way can injure either the sides of the mouth or palate. Even his own comfort ought to * dictate this; for no horse can perform his work pleasantly while he is suffering from an irritation in the mouth.

When the owner of a horse finds that the bone of the jaw is injured, he should immediately apply to a veterinary surgeon; but wounds and ulcers may be cured without the aid of a practitioner. The most simple remedies are tincture of myrrh, diluted in an equal proportion of water. The parts should be frequently washed with this; or, dissolve an ounce of alum in a quart of water, and use it as a wash. If the wound has become a settled ulcer, and looks foul in the edges, then it should be touched with lunar caustic, or with the liquid nitrate of silver, which will stimulate it and cause it to heal. At the same time the above wash should be applied to the parts. 


\section{DISEASES OF THE TONGUE.}

TONGUE BLADDERS.

Symptoms.-A careful groom will occasionally examine the interior of the mouth of horses under his charge. If he notices any swelling of the tongue, he must ascertain from whence it proceeds; or his attention may be directed to this organ by a discharge of ropy saliva from the sides of the mouth. This will be found to have its origin in inflammation, caused by one large or many small bladder-like swellings on the under sides of the tongue, frequently extending its whole length.

Renedy.-If these bladders are fully charged with matter, they should be opened with a lancet, which generally puts an end to the complaint, and the swelling will quickly subside. Should any degree of fever remain, an alterative powder should be given daily in mash, composed as follows :-

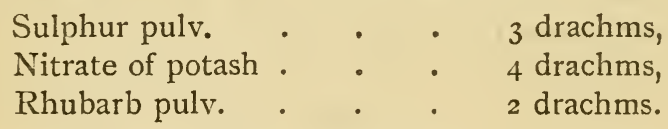

\section{BITTEN TONGUE.}

It often happens that horses bite their tongues. If the wound be slight, it will heal of its own accord; but when of large extent, veterinary aid must be called in, as it would be unsafe for a person not thoroughly acquainted with pathology to attempt a cure.

VIVES, OR SWELLINGS IN THE SUBMAXILLARY GLANDS.

During catarrh it is no uncommon occurrence for these glands to become enlarged, and also after strangles. In such cases, stimulating embrocations may be used, which generally relieve them; or they 
may, in most instances, subside, after the cause which has induced the enlargement has ceased. We would on no account allow operations to be performed for their removal.

These swellings are readily distinguished from those which accompany glanders, by their being, for the most part, larger, less distinct, and occupying the centre of the space between the jaws, and in never adhering to the jawbones, as in glanders.

BARBS, OR PAPS.

In inflammation of the mouth, produced by catarrh or any other cause, the ducts situated on both sides of the frænum, or bridle of the tongue, sometimes enlarge, and a redness is to be perceived under it. No operation is necessary in this disease; the only thing to be attended to is the removal of that which causes them. Unskilful and bigoted farriers often operate for the paps, but in this event the cure is worse than the disease, as abscesses are likely to follow operations, which years may not eradicate.

GIGS.

The sublingual glands are liable to inflammation during colds. They are situate under the tongue, or on its lower surface. They assume the appearance of small pimples when inflamed. If they ulcerate they should be washed with a solution of alum, or tincture of myrrh, which will always be found to heal them. Operations seldom fail to prove injurious.

\section{DISEASES OF THE TEETH.}

Caries and other diseases of the teeth in horses are common. From the constant use of the grinders, in chewing grain and straw, they are often worn down, 
and their edges occasionally present a sharp and rough appearance. This cuts the inside of the cheeks. To prevent this they should be filed down.

If from the above cause the cheek has been cut, and an ulcer produced, it must be frequently and carefully washed, either with a solution of myrrh, or alum and water; and if it prove obstinate, nitrate of silver must be had recourse to.

Sometimes the teeth grow irregularly in length, more especially the grinders. This proceeds generally from these teeth not being placed immediately opposite to each other. Instances are not wanting where such teeth have grown three-quarters of an inch, or sometimes more, above the general level of the grinders or molar teeth; and this pressing against the bars of the mouth irritates them, and generally ends in ulceration. The only remedy for this is to file or sheer off with an instrument the projecting tooth down to a level with the others. Unless the ulcer is very deep and spongy, it will heal of its own accord; but should it prove otherwise, its edges must be touched with lunar caustic or nitrate of silver, after it has been well washed out with a solution of alum and water. It often happens, from want of attention on the part of the groom in this case, that the horse cannot take his food, and will in consequence pine away, lose flesh, and become quite dispirited.

Teeth that have thus grown will always have a tendency to shoot out again, and must therefore be watched to prevent a recurrence of ulcers.

In all diseases of the mouth horses will half-chew their food and then clrop it, which is familiarly termed "quidding their food." 


\section{DISEASES OF THE LIPS.}

Few persons are aware of the very great importance of the lips of horses. They may justly be considered as the hands of that animal. Without their aid he could neither collect his food in the fields, nor even convey corn down his throat. To prove this, I shall give an account of an experiment which was tried with an ass, to ascertain the extent of the use of these important organs. The nerves which give feeling and sensation to the lips were divided, and instantly it was perceived that he was not aware when he touched food with them. They were entirely divested of motion, and he was in consequence unable to convey the oats, with which his manger was full, to his teeth. Compelled by hunger, he made a violent effort to lick up a few with his tongue, but they were nearly all rubbed off before they could be conveyed to his mouth.

The angles of the mouth are frequently lacerated, and become sore by the smallness of the bit, and from the unmerciful dragging of a heavy hand in either riding or driving him, and also from the shortness of the snaffle. The severe excoriation of those parts produces deep ulcers, which cannot be removed while the animal is worked. Washing with a solution of alum is one of the best curatives; and if the sore is callous, it must be burned slightly on the edges with nitrate of silver.

DISEASES OF THE EYE.

In the horse the diseases of the eye are not numerous; but they are of frequent occurrence, and often most difficult to cure.

COMMON INFLAMMATION OF THE EYE.

Srmptons. - This malady generally makes its ap- 
pearance unexpectedly, accompanied by considerable swelling of the eyelids, which has the effect of partially closing them, and causes a discharge of watery matter, or tears. The lid exhibits inflammation, and some of the vessels of the eyeball are gorged with blood. There will also be a dimness in the cornea.

CAuse.-This usually accompanies a catarrh. But it may also be caused by substances getting under the eyelid, such as a seed of hay; or from a blow. When inflammation occurs, the eye should be carefully examined, so that the cause may be discovered. This seldom affects the health of the horse, or prevents his feeding.

Remedies.-The eye should be bathed with the following lotion :-

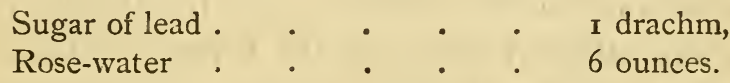

If this does not speedily abate the inflammation, then use the following :-

Tincture of opium, or laudanum, $\frac{1}{2}$ ounce,

Water. . . . . I pint.

Or the following will prove equally efficacious :-

Powdered leaves of digitalis . . I ounce,

Boiling water . . . . I quart.

His food should consist of mashes, with mild doses of physic. Three or four days should remove the disease.

OPHTHALMIA.

Symptoms. - This is manifested by great inflammation in the eyelids, as also the cornea and aqueous humour and iris, all of which assume a dim appearance, and lose their transparency. The animal can 
hardly open its eyelids from the pain produced by exposing the eye to the action of light. This disease is extremely difficult to combat; and after a month's constant treatment the eye will exhibit an alternation of remission and increase of the inflammation day after day. One day it will have all the appearance of being nearly well, and on the next exhibit more unfavourable symptoms than it has before assumed: the gorged appearance of the inner membrane of the eyelid will be much abated, and the inflammation on the white of the eyeball will have nearly quitted it; the hazy aspect of the cornea have assumed a certain degree of clearness, and to all appearance the malady has taken its departure.

It seldom happens, however, that it is so, for in six weeks or two months we too frequently find the eye again affected with all its former redness, often worse than on the former attack; or oftentimes both eyes affected. Indeed, from time to time a succession of these abatements and attacks will have succeeded one another, until a cloudy appearance and permanent opacity of the lens or capsule of the eye have taken place; and confirmed blindness in one or both eyes is the final result.

CAUSE.-The constant heated air of the stable may be considered the remote cause of this inveterate disease, to which all horses seem to be predisposed. The poisoned air is also a powerful agent in the propagation of ophthalmia. To these may be added the too frequent use of stables which are totally dark, so that when the animal is suddenly brought to the light, the abrupt transition produces congestion of blood in the vessels of the eyes. In some cases it is an hereditary affection, and animals with specific ophthalmia should not be bred from. 
Remedies. - When this disease first makes its appearance, the affected eye should have placed over it a thin sponge saturated with belladonna lotion. The horse should then be put upon a low diet, and gentle purgatives administered.

When the pain and inflammation have subsided, if opacity of the lens has taken place (cataract), we are not aware of any means of removing it and restoring vision, except by an operation, as in the human subject, and for obvious reasons it could not be a practical success.

THE HAW.

It is no unusual thing for a thickening of this part to take place, and it will then protrude itself on the fore part of the eyeball. In this disease the retractor muscle pulls back the eye to protect it from the irritating effect of the light, and this thickening of the haw pushing it forward, and the adjacent parts being also thickened, no retraction can take place.

The practice of cutting out this is exceedingly absurd, and ought never to be resorted to. It is intended for protecting the eye against dust or insects. In former times few farriers understood the use of the haw; and even yet it is surprising how few are aware of its value to a horse. In cases of inflammation of the eye, it sometimes itself becomes inflamed and increased in dimensions, and the contiguous parts likewise thickened. This either forces it out of its place, or it is voluntarily produced to protect the eye from the action of light. In some cases it does not return into its place, and has been mistaken for a tumour or extraneous excrescence, and has been cut out by ignorant persons, and the eye 
consequently left unguarded. Unless in those cases where there is a tumour growing in connection with the haw (Membrana Nictitans), it should not be interfered with, and where there is a growth requiring removal, the veterinary surgeon is the person to perform the operation.

\section{ERUPTION ON THE EYELIDS.}

Symptoms. - The edges of the eyelids are occasionally affected with scale-like eruptions. This is always attended with great itching, which causes the horse to rub his eyelid against any projecting part within his reach, in the performance of which it often happens that the animal injures the eye itself.

Remedies. - The edges of the eyelids should be annointed with the following:-

Nitrated ointment of mercury · $\quad \cdot \quad \frac{1}{2}$ ounce, Hogs' lard . . . . . $\frac{1}{2}$ ounce.

A few applications will generally produce the desired effect.

\section{WARTS.}

The eyelids are sometimes affected with warts, which are very irritating to the animal in consequence of his rubbing them on some prominent part: this causes them to bleed, and increases their number.

They should be cut off with a pair of sharp scissors, and the roots touched with nitrate of silver, lunar-caustic, or blue vitriol; but great care must be exercised in not making the cut through the skin large, or eversion or inversion of the eyelid may result.

GUTTA SERENA.

Sympтoms.-This exhibits itself by an extraor- 
dinary dilation of the pupil, which becomes immovable, and the eye has a bright glassy appearance. This is caused by a paralysis of the optic nerve, or its retinal expansion.

Cures in this disease are very few in the horse. Indeed, we doubt if ever the cure of a confirmed case of gutta serena, or glass eye, has been effected.

\section{GENERAL BLINDNESS AND IMPERFECT VISION.}

No subject is of greater importance than the state of a horse's organs of vision, and ought to occupy the first attention of a purchaser; for blindness, or a partial defect in the eyes, may lead to many unpleasant consequences to the proprietor, whether in riding or driving. It will require considerable knowledge of the anatomical construction of the eye to enable any one to judge correctly of its perfection or defects.

Independently of the beauty of a prominent eye, it is of much importance that the cornea should possess considerable convexity; but this must have a limit. If very prominent, the rays of light will be too convergent, which will cause indistinct vision; on the other hand, if the cornea be small and flat, the rays may not be sufficiently convergent, and consequently will render the vision imperfect. A horse is unsafe with either of these defects, both in riding and driving, as he is certain to start at objects which suddenly present themselves, or he may quickly bolt round, and in either case may over-balance the rider, or upset a vehicle.

The cornea should, therefore, be moderately convex, perfectly transparent, and totally free from all opacity or cloudiness over its entire surface. The best method of examining this organ is to place the 
cheek of the scrutiniser close to the cheek of the horse, both behind and under the eye, and the latter position is the most advantageous to see it thoroughly. The open air is not favourable for such an examination. The head of the horse should be a little within the doorway of a stable, and by looking outwards any defect is rendered more visible. If any faint whitish, milky-like streaks be noticed passing over the cornea, it is certain the animal has had inflammation in that portion of the eye. But, should the centre part of the cornea be perfectly transparent, and yet the margin of it, where it unites with the sclerotica, have a hazy ring, it may be concluded that this has been occasioned by recent inflammation, and consequently the eye will be predisposed to a return of it.

During the operation of thus inspecting the eye, no white or pale-coloured object should be near, as its form and great transparency are very likely to reflect these rays, and may deceive the person examining the organ.

Attention to the dilation and contraction of the pupil will materially aid a person in the detection of blindness or otherwise. When the cornea and crystalline lens are quite transparent, with the retina paralysed, and of course not liable to be affected by light, blindness in one or both eyes will be extremely difficult of detection. It generally happens, when a horse is totally blind, he has a constant and rapid motion in his ears. He also lifts his feet high, as if some obstacle presented itself, and he puts his feet to the ground with a cautious uncertainty. Particular attention should be directed to the pupils of both eyes, and to noticing whether they are both of a size while he is in the stable; and as he approaches the 
door, observe whether both pupils contract equally as they are subjected to the stronger light. If, however, the horse is examined in the open air and distant from a stable, place the hand over one eye, and then observe, after it has obscured it from the light for a little while, whether the pupil contracts. Repeat the same experiment with the other eye also.

\section{FRACTURE OF THE SKULL.}

The admirable construction of the skull of a horse, as will be seen in our anatomical description, renders a fracture a thing of rare occurrence. And such is the force required to produce such an effect, that it almost invariably proves fatal by injuring the brain. Horses that rear, and in that act fall, sometimes fracture their skull, and in some instances blows inflicted by their grooms with a heavy weapon may have the effect of fracturing the skull. There is little hope of a cure, except with the assistance of a regular veterinary surgeon. But in case one is not to be had immediately, the parts of the bones should be replaced and held together by the aid of adhesive plaster. Bleeding and low feeding must be resorted to, together with small doses of medicine. 


\section{CHAPTER II.}

Diseases of the Necks and Contiguous Parts.

\section{POLL-EVIL.}

Symptoms. - This disease consists of an inflammation of the muscle over the poll-bone and the first vertebra of the neck. It generally extends under the ligament of the neck which passes over the atlas-bone. This ligament is not attached to the bone, and the disease is consequently seated between it and the bone itself. It is being thus deeply seated which frequently renders it so difficult to cure. Before the swelling becomes very conspicuous, the part is very hot, and painful when touched, which can easily be noticed from the motion of the horse.

Cause.-The poll-evil is too frequently occasioned by a severe blow on the poll of the neck, given by illtempered and unmerciful riders or grooms; at other times it is brought on by the horse striking his head against the manger, or by the ligaments being too much stretched by severely tight reining. But, from whatever cause the malady proceeds, it frequently becomes exceedingly troublesome and tedious to cure.

Remedies. - Before suppuration takes place, every means should be adopted to suppress the inflammation, and, if possible, to disperse the swelling. Medicines of a laxative kind should be administered, and bleeding resorted to, and also cold lotions applied. This treatment will often have the effect of reducing the tumour. 
But if it is found that the swelling continues in spite of these, other means must be resorted to, and applications to facilitate the ripening of the abscess must be adopted. This will be best effected by poultices, warm fomentations, and stimulating embrocations. The progress of the disease must be narrowly watched, and when sufficiently advanced, which will be known by the softness of the swelling, it must be opened with a lancet, and afterwards kept open by means of a seton. The needle, with the cord attached to it, must penetrate the apex of the abscess, and be forced out a little way below the bottom, so that no matter can possibly collect in and lodge there. Gentle pressure should be used to squeeze the whole of the matter out. After this nothing more will be required except to keep it clean and occasionally fomented with warm water, and carbolic acid or Jey's fluid. If taken at an early stage of the disease, what we have recommended will generally effect a cure.

On the other hand, should the pus burrow deep and affect the ligaments of the vertebræ of the neck, the edges of the wound must be stimulated by nitrate of silver or caustic, to induce a healthy action into the muscular fibre, and excite it to granulate. When the interior of the abscess has assumed a callous appearance, it may be necessary to syringe it out with a very weak solution of nitrate of silver mixed with rose-water. Should this not have the effect of inducing a healthy condition, then it will be necessary to have the services of a regular veterinary surgeon. The cord used as a seton should be wet with the following mixture :-
Corrosive sublimate
$\frac{1}{2}$ drachm,
Spirit of wine .
2 ounces.

The cord should be cut off and united at both ends 
by wrapping a thread round it, and pulled round once daily during the twenty-four hours, taking care to wipe the part which has been in the abscess, and also to wet the portion which is to remain in it for a time, with the above solution.

When all the swelling has subsided, and the matter assumes a thick white appearance, the seton may be removed, and the wound kept clean with an antiseptic lotion.

\section{ROARING.}

Symptons. - This is a disease arising from an affection of the larynx and superior portion of the windpipe. When a horse so affected has been hard trotted or galloped, he may be heard at some distance to utter a whistling or roaring sound. Or when he is standing, if touched suddenly in the flank with a whip or stick, he will involuntarily grunt or groan. Dealers are frequently in the habit of practising this test ; because, if a horse is moderately exercised or at rest, this complaint never manifests itself.

Cause.-It is due to disease of the inferior laryngeal nerve, which leads to atrophy and fatty degeneration of the muscles which dilate the larynx, and as a consequence the mouth of the windpipe is narrowed and altered in its shape. After strangles this disease frequently follows. Tight reining is believed by some to be a cause of this evil, but cart-horses are more frequent roarers than any other, and they neither wear a "bearing rein," nor are driven with a tight rein. The practice of what is termed coughing a horse, to ascertain the state of his wind, is apt to produce roaring. This is performed by subjecting the larynx or trachea to violent pressure by squeezing with the fingers. A horse so afflicted is decidedly unsound. 
REMEdiEs. - In the early stage of the disease the application of a fly-blister over the larynx is to be recommended, and the administration of a sedative electuary, composed of belladonna, gum-camphor and treacle. When the disease has been of such a character as to threaten suffocation, the operation termed tracheotomy must be performed; but this is an operation that can only be successfully performed by a veterinary surgeon.

\section{OBSTRUCTION OF THE GULLET.}

Symptoms.-In this malady the gullet has become contracted, which compels the horse to swallow its food in small quantities, and that with much difficulty, giving the animal the appearance of choking when in the act of swallowing. Sometimes the stricture is near the upper portion of the gullet: in which case an experienced veterinary surgeon may devise some remedy; but if seated near the entrance into the stomach, no hopes can be entertained of a cure.

Horses eating too greedily sometimes swallow their food in too large masses, and before it is properly masticated, which has in many instances lodged in the gullet, producing alarming symptoms of suffocation.

Remedies.-The first thing to be tried is to force the ball of food which obstructs the passage downwards, by the aid of the elastic tube (probang) used for hove in cattle. Sometimes it cannot be dislodged even by means of this instrument. In that case the only other remedy is to cut open the gullet to prevent suffocation. But this operation need not be described, as it can only be successfully performed by a veterinary surgeon. 


\section{INFLAMMATION OF THE JUGULAR VEIN.}

In the horse the jugulars are single on both sides of the neck, while in horned cattle they are double. Inflammation is sometimes induced in them after the operation of bleeding, directions for which we shall give hereafter.

After bleeding has been performed, the practice is to bring the cut edges of the vein together, and to keep them in contact by inserting a pin through the skin above it, and twisting tow pretty tightly round it, so as to keep it from being removed. In three or four days the wound will have completely healed in most cases.

CAUSES.-A variety of causes may, however, operate to induce inflammation of the vein. Among these may be enumerated, striking too hard on the lancet with the fleam (an instrument used in bleeding), or using a blunt or rusty lancet. In other cases, by pulling the skin too far from the neck while drawing the wound together, and thus allowing space for the blood to insinuate itself into the cellular tissue; or working the horse immediately after the operation; or neglecting to tie up his head for a short while after bleeding, to prevent his removing the pressure of the pin from the wound by rubbing his head against the manger; and other causes.

Symptoms.-Swelling and separation of the cut edges of the wound; coagulation of blood within the channel of the vein; inflammation and thickening of the wall of the vessel, so that it feels like a cord under the finger. The obliteration of the jugular may extend from the point of operation to the base of the brain. This is generally followed by a series of small 
abscesses forming along the course of the inflamed vein.

Remedies.-Some veterinary surgeons, to stimulate the part, apply a hot iron to the margin of the orifice, which induces a union of its edges. Should this not prove effectual, and if the swelling is great and abscesses have made their appearance, the next remedies are to inject a weak solution of nitrate of silver or other caustic liquid into the abscesses, or to introduce setons into them. 


\section{CHAPTER III.}

\section{Diseases of the Chest and its Contents.}

\section{INFLAMMATION OF THE LUNGS.}

Syмpтомs.-Of all the diseases incidental to the horse, that of inflammation of the lungs is the most prevalent, and certainly the most fatal. Sometimes this malady is sudden in its appearance, but in the greater number of cases dullness and fever are premonitory symptoms. In its early stages the pulse is not very rapid, but indistinct and heavy in its action, sometimes feeling vibratory under the pressure of the finger. In other instances it is hardly to be perceived, so languid is its motion. This is followed by dilated nostrils, coldness in the extremities, and heaving at the flanks, which clearly indicate an oppression in the breathing; differing, however, from the laboured and irregular breathing in broken-winded horses, which appears as if it required two expirations for every inspiration of air into the lungs. The animal thrusts out his head, the mucous membrane of the nose becomes very red, especially in the inside angles of the nostrils, where it continues; although at times it extends further up, from which position it may disappear, but still maintaining its chief seat in the lower portion of the nostrils. The animal expresses great anxiety in his look, and turns his head frequently and hurriedly round towards his flanks, more especially to 
that side where the inflammation has settled. $\mathrm{He}$ stands in a stracidling manner, with his fore legs generally considerably apart. He seldom lies down, and when he does so it is but for a few minutes, remaining for days on his legs.

It does not, however, always happen that the premonitory symptoms are the same, for in many cases inflammation comes on slowly and in an insidious manner. Perhaps the horse may be off his feed and his coat will stare; his breathing may be but slightly accelerated and abbreviated, with the legs a little colder than usual. Sometimes inflammation of the lungs is preceded by symptoms which are attendant upon common fever, catarrh, or influenza. In such instances the true disease manifests itself in its full force when the groom or master of the horse least suspects it. The first manifestations are coldness in the limbs and ears, accompanied by the flurried pulsation and anxious look, with a seeming dread of lying down. This is soon followed by an irregularity and indistinctness in the pulse, and extreme coldness affects the legs and ears. The nostrils become livid-he hardly seems to breathe-he grinds his teeth-and these may be regarded as the too certain symptoms of dissolution; staggering ensues, and he finally sinks in his stall.

This last is a picture of that kind of inflammation of the lungs which has lurked in the constitution without exhibiting premonitory symptoms, and which in most cases proves fatal. There are, besides, cases in which the disease is so rapid that it will have undergone its entire stages in twenty-four hours. In this short time the entire mass of the lungs will have suffered complete destruction from engorgement with blood. Such a case has been satisfactorily proved not to 
proceed from long and deep-rooted inflammation, but assuredly from the very reverse.

There are bad cases, which are not so rapid in their termination, but which are nevertheless equally fatal. This happens when the lungs have become consolidated and the inflammatory products have formed pus. In such a case the breath of the horse will be extremely disagreeable, with a running at the nostrils, which is a sure indication that mortification has taken place in the substance of the lungs, and that death will soon follow.

Inflammation of the lungs may be distinguished from inflammation of the bowels, by the pulse in the latter case being small and wiry; the mucous membrane of the nose not being so red, and by pains in the belly, which are indicated by kicking, pulling, stamping, and lying down.

CAUSE.-This malady may be brought on by the numerous and sudden transitions from heat to cold, to which most horses are subjected. They are, under the careless and wanton folly of masters and grooms, often galloped, or otherwise worked and overheated, and then permitted to cool in the open air, or in the draught of a stable. The stable itself is also kept too hot, frequently from twenty-five to thirty degrees beyond that of the atmosphere; and its air is but too often of an impure kind, and which being breathed affects the membrane which lines the cells of the lungs. This is weakened, and hence rendered susceptible of irritation and inflammation from breathing an atmosphere which is impregnated with ammoniacal gas, generated by the manure and urine.

Remedies. - The treatment for inflammation of the lungs must be immediate and decisive. As the disease is rapid, so also must be the means employed. 
Although its first symptoms may exhibit a mild character, yet we have seen how rapid they become in their after-effects. To palliate it therefore would be absurd. In a previously healthy animal bleeding may be resorted to. During the flow of the blood the pulse should be felt all the while to mark its progress, and the slightest change in its character should be accepted as indicating that no more blood should be withdrawn. If the horse be costive, which is frequently the case in inflammation of the lungs, from four to six ounces of Epsom salts, mixed with a gruel, will be found useful, and we must next have recourse to blistering the chest with mustard.

Febrifuge medicines must now be resorted to, and the following doses may be given :-

$\begin{array}{llllll}\text { Nitre } & \cdot & \cdot & \cdot & \cdot & 3 \text { drachms, } \\ \text { Emetic tartar } & \cdot & \cdot & \cdot & \cdot & \mathrm{I} \frac{1}{2} \text { drachm, } \\ \text { Digitalis } & \cdot & \cdot & \cdot & \cdot & \mathrm{I} d r a c h m .\end{array}$

This medicine is to be given daily in a ball until the fever has subsided, as indicated by the thermometer standing at $10 \mathrm{I}^{\circ}$.

It is a great mistake to suppose that in this complaint the stable should be kept close and warm; for the very cause which in a great measure operated in remotely inducing the disorder is again brought into play. Rather let warm clothing be put upon the horse, which will have a tendency to keep up insensible perspiration.

After this the horse should be well rubbed down, and his legs in particular should have a smart application of the brush, in order to restore heat and an increase in the circulation, and then thickly rolled up with flannel to keep up the action. The rubbing should be repeated from time to time. The less he 
eats at a time the better. Corn must on no account be given. Green food and mashes may be set before him in small quantities, and as much drinking water allowed at all times as the horse desires.

If the oppression in the breathing now subside, heat be restored to the limbs, and the animal lie down, these are sure indications of the disease having abated. The strength and appetite will now gradually be restored. But much caution should be exercised in not allowing the animal to take too much food, which might have the effect of inducing a return of the malady : green meat, or, if that cannot be had, a gruel of linseed and oatmeal, mashes, and a little hay should form his diet. But to restore strength where much exhaustion prevails, tonics should be given.

$$
\begin{aligned}
& \text { Iron sulphate . . . . } 2 \text { drachms, } \\
& \text { Ginger . . . . . } 2 \text { drachms, } \\
& \text { Gentian . . . . . } 2 \text { grains. }
\end{aligned}
$$

Should the animal continue to improve, his diet may be extended, and probably in three or four days he may have a small quantity of corn, which may be increased as his strength is restored.

A cool and clean stable are the best means for preventing predisposition to diseases of the lungs, both of which should be scrupulously attended to. The heated air of a stable, and the poisonous gas arising from the dung, prove most injurious to the horse.

\section{PLEURISY.}

Symptoms. - This disease is entirely confined to inflammation of the pleura or membrane which envelops the lungs and lines the chest. The pulse is hard, but not oppressed. The extremities are cold, although not so much so as in ordinary inflammation, nor is the membrane of the nose so very red. 
If pressure on the sides is applied, the horse will feel pain, and express it by a quick and impatient grunt. The unwillingness of the horse to lie down will be manifested in this as well as in violent inflammation of the lungs.

CAUSE.-This disease has its origin from the same causes as inflammation of the lungs, viz., sudden transitions from heat to cold, etc.

REMEDIES.-Much the same treatment is required for pleurisy as for pneumonia. The two diseases are frequently conjoined, and a veterinary surgeon can alone decide this point.

If pleurisy is acute, it frequently induces dropsy in the chest, as in this complaint a fluid is thrown out from the vessels of the pleura, which, having no means of escaping, and being too large in quantity to be absorbed, lodges in the cavity of the chest. When this is the case little good is to be expected from the animal, and it seldom happens that a complete cure is effected. Sometimes the chest is punctured for it, which may carry off the fluid; but it too frequently proves an ineffectual remedy. Whenever it is suspected that water is formed to any great extent, puncturing should be had recourse to, the opening to be made by the instrument called the trochar. The locality where it is inserted is the intercostal membrane, between the seventh and eighth ribs, and as close to the cartilages as possible. Diuretic medicines, in combination with tonics, should be given. The following will be found the best :-

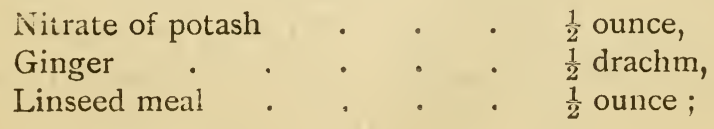
made into a ball with common syrup or treacle. 


\section{FEVER.}

Symptoms. - Fever commences with a cold and shivering fit; the animal manifests great dullness, with a desire to be inactive; his hair stands erect, or stares, and his legs and feet are cold; the pulse is quick, hard, and unequal, the temperature abnormal, ranging from $102^{\circ}$ to $107^{\circ}$, or higher; his mouth is hot, with a total loss of appetite, shivering, and a dejected appearance. This is followed by general warmth of the body; an unequal distribution of heat to the limbs, sometimes one being hot while the other is cold. He becomes very costive, with turbid urine; affected sometimes with colic pains; but there is no cough, pawing, or looking back at the loins. If these symptoms are allowed to proceed unchecked, the membrane that lines the eyelid becomes unnaturally red; the inflammation may then be considered as settled in some internal organ, and pure or symptomatic fever will have ensued. While this pure fever continues, the shivering fit returns daily, at nearly the same hour, and is followed by a warm one, and sometimes by a cold clammy sweat. This state continues for several days, and local inflammation ensues; or the fever gradually becomes abated.

Some veterinary surgeons have absurdly denied the possibility of fever in the horse; but those who have advanced such an opinion must have paid but little attention to the state of his pulse, and have never used a thermometer.

CAUSES. - General increased action in the heart and arteries is the cause of fever, produced by the sympathy of the system, induced by local inflammation; or it may exist without any perceptible local affection. It is too frequently induced by bad stable 
management, the sudden changes from heat to cold, and the lungs having breathed impure air from the filth and confined atmosphere of the stable. Inflammation of the lungs, feet, or any other part of the body is always accompanied by fever.

Remedies.-Gentle laxatives will be found beneficial, but strong ones exceedingly injurious. The following should be given morning and evening until the temperature becomes normal :-

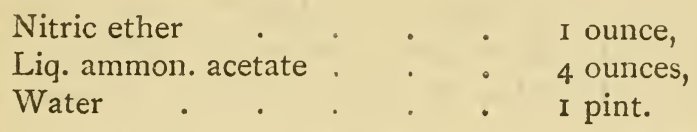

The above may be slightly augmented or diminished, according to circumstances.

Symptomatic fever should be treated in the same manner as simple fever, only that attention must be directed to the diseased part which caused the fever. If the inflammation can be subdued, then the fever will naturally abate, without other means being adopted.

\section{STOMACH-STAGGERS.}

Symptoms. - This disease is indicated by the dull, stupid, sleepy appearance of the horse, and he staggers about in his stall. He seems unconscious of what he is doing, and if roused from his lethargy will probably take a mouthful of hay, in a few seconds desist from chewing, and the hay will fall from his mouth. Many instances have occurred, when the disease has been allowed to acquire an ascendancy, that the horse would drop down and die while in the act of eating. In other cases the drowsiness goes off, and is succeeded by delirium; and after falling, rising, and staggering about, will die in convulsions. 
Cause.-Over-feeding is too often the cause of this sad disease, and especially if the food is of a bad quality. Careless servants will too often neglect a horse; and afterwards, by food being placed before him while ravenously hungry, he will swallow it rapidly and in too large quantity, without being properly masticated, consequently it swells in the stomach, and thus stretches it far beyond its natural capacity: its action is thereby impaired: the consequence is, the brain is unduly acted upon, and giddiness and drowsiness induced, which occasion staggering. Besides the horse being allowed to eat in this manner, the groom may neglect to give the animal water to assist it in converting it into a pulp and facilitating the operation of digestion, the natural juices of the stomach, from its gorged state, being inadequate to the performance of their office.

Remedies. - Before remedies are attempted it must first be ascertained what has caused the staggers; as the mad staggers present exactly the same symptoms in their early stage. In this disorder, as may well be supposed, medicine will have but little effect, from the gorged condition of the stomach. Some veterinary surgeons recommend bleeding; but we have never found this attended with any beneficial effects, as what must naturally debilitate the system can hardly be expected to aid the action of the stomach. Probably the safest plan is to allow nature to work its own cure, by abstaining from giving food. But as we know of no certain remedy for this disease, we should carefully guard against promoting it.

It is no uncommon occurrence for farmers and others keeping a number of horses to lose several of 
them within very short periods of each other with this malady, from which an opinion prevails with many that the staggers is contagious. Nothing can be more erroneous than this belief, as it is quite certain that the complaint is induced by bad stable management, or by feeding the horse with unwholesome food, or in the horse feeding too voraciously, as already mentioned. This disease is more common with old horses than others. We would strongly recommend the owners of horses to give some attention to the following:- Too much food given at one time after long fasting or hard work, and neglecting to give the animal water, is almost certain to produce the staggers. The hours of labour should be for limited spaces of time, with proper intervals of rest allowed, and the horse regularly fed during these intervals. Every man must have felt the effects of being without dinner for two or three hours beyond his accustomed time. From five to six hours are the intervals between the meals of a labouring man; and with a horse that is worked no longer time should be allowed to elapse without feeding and watering. When persons are occasionally so situated that they cannot unyoke their horses at stated times for food and rest, then they ought to carry hay and a nose-bag, and a supply of corn along with them. Indeed, it is surprising that if humanity does not influence many men to be kind to this valuable animal, self-interest ought to have the effect of inducing proper treatment.

Staggers often attacks horses while they are at grass. This, however, happens chiefly with such as have been previously stinted in their food, or where the pasture is very rich. In such cases, nature generally works its own cure; because, if the horse 
is kept from eating, the natural sap in green food will soon assist in promoting digestion.

\section{CATARRH, OR COMMON COLD.}

The sudden transitions from heat to cold to which horses are so frequently, and often so thoughtlessly, exposed, renders this a very common complaint with them. If masters and servants would bear in their remembrance the old and true adage, "that it is better and easier to keep well than to make well," their horses would not require half the medicines which are given to them.

A simple cold, if neglected, may end in an incurable disease, especially with horses of a delicate constitution. Catarrh commences with a slight degree of fever, with some little discharge from the nose and eyes, accompanied by cough, which is sometimes hard, frequent, and painful to the horse. If the cough is not severe, the complaint may generally be removed by simple treatment. The horse should be kept warm, and a few doses of the following mixture administered :-

$$
\begin{aligned}
& \text { Digitalis . : . } \frac{1}{2} \text { drachm, } \\
& \text { Nitre . . . . } 2 \text { drachms, } \\
& \text { Emetic tartar . . I drachm; }
\end{aligned}
$$

to be given in the form of a ball. Instead of corn, his food should consist of hay and mashes.

\section{BRONCHITIS.}

Syмртомs. - Inflammation of the mucous membrane of the bronchial tubes manifests itself by the breathing being considerably harder and quicker than in a common catarrh; and also by a wheezing sound accompanying the inspirations of air into the lungs, and which is temporarily removed when the horse 
coughs up the mucus secreted by the inflamed mem brane of the throat.

Cause. - Bronchitis is generally incluced by a severe cold, and is indicated by the cough being severe. The seat of the clisease is in the divisions of the trachea or windpipe, just before it enters the lungs. At this portion it branches out into a numerous series of vessels, and these are denominated the bronchial tubes, and hence the inflammation which attacks their lining has been called bronchitis. This disease is generally induced by catarrh having gained an ascendency, and the inflammation extending to the entrance of the lungs.

Remedies.-Blisters on the chest should be applied, and the following medicine given :-

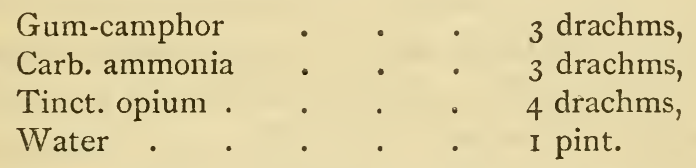

When bronchitis is neglected, it produces thick wind, which can never afterwards be removed.

\section{THICK WIND.}

Symptoms. - Thick wind is indicated by short, laborious, and frequent breathings when the horse is at rest, and becomes much more evident where he is employed in working, whether in a cart, carriage, or as a hunter or roadster. In the latter cases, the inspirations and expirations succeed each other in such rapid succession that lead us to suppose the animal is about to expire through suffocation, and his breathing can be heard at a considerable distance; his sides and loins exhibit marked symptoms of the difficulty with which he breathes. 
Causes. - This affection for the most part owes its origin to previous inflammation, and more especially inflammation and thickening of the mucous membrane which lines the bronchial tubes.

Remedies.-This disease does not come within the scope of medical treatment: all that can be done is to guard against overloading the stomach with food, and not to exercise the animal to any extent while his stomach is full. Abstain from subjecting him to hard labour, or trotting and galloping him violently. By these precautions the affection may gradually subside, and in time he may become almost sound.

\section{BROKEN WIND.}

Sympтомs.-A marked distinction prevails between this disease and thick wind. In the latter the inspirations and expirations are of uniform length and duration; while in broken wind, for one inspiration two expirations are required, which is plainly indicated by the motion of the flanks. This is caused by the rupture or union of several of the air-cells, which renders the cavities of the lungs more complicated, and hence requires that double action to expel the air. It may be easily conceived that when the lungs are expanded the air will rush in readily enough, and one exertion of the muscles of respiration is sufficient to expel the carbonised air; but when these cells have become united, the cavity of the lung is so irregular and many-cornered, that air is with difficulty expelled from them, and therefore two efforts are required to effect it.

Broken wind is generally accompanied by a hard, dry, husky cough, differing completely in its character from that of a common cold. 
Causes.-This disease may take place without any visible disordered condition in a horse. Voracious feeding may produce it, or food taken hastily and in too large quantities into the stomach, particularly coarse hay and straw, which, by distending the stomach beyond the natural limits, presses against the lungs and prevents their free action. Coarse and bulky food being frequently used, is extremely apt to produce broken wind, from the effect just mentioned. The constrained action of the lungs, from the frequently distended stomach, is apt to burst the air-cells, and thus lay the foundation of an incurable disease. Thick wind is usually the premonitor of the disorder.

Remedies.-A radical cure of broken wind, I believe, has never taken place; that is, after a rupture of a portion of the air-cells. All that can be effected is a palliation of the complaint. The food should consist as much as possible of that which affords the greatest nourishment in the smallest compass. Oats, and particularly beans and peas, as well as carrots, have this qualification. The bowels should be kept gently open, and for this purpose give an alterative powder in bran mash every second evening, viz. :-

$$
\begin{aligned}
& \text { Nitrate of potash } \quad \cdot \quad \cdot \quad 3 \text { drachms, } \\
& \text { Sulphur sub. }
\end{aligned}
$$

Avoid exercise or labour when the stomach is full. A broken-winded horse should never be pastured, as, by having his stomach constantly full and distended, the disease is increased. By strict attention to all these rules, and by gentle, well-timed, and progressive exercise, broken-winded horses may be rendered capable of performing a fair proportion of labour, and may 
even live a considerable number of years under the influence of this affection, by cautious treatment.

Experience must have taught many persons possessing a number of horses, that frequent instances have occurred of horses having been put into a strawyard perfectly sound, coming out broken-winded. The food affording but little nutriment, the animals require to keep their stomachs always full, which causes a constant pressure upon the lungs; and their action being thereby restricted, a violent effort becomes necessary to effect respiration, and hence rupture of the partitions of the air-cells take place.

\section{CHRONIC COUGH.}

Symptoms.-This is a constant, irritating cough, which in particular manifests itself after feeding and drinking, and on first being exposed to the open air in the morning, and more especially after any excitement, such as hard work, a sharp trot, or a gallop. In most instances the complaint is accompanied by a staring coat.

CAUSES.-Chronic cough has its origin in some previous inflammatory complaint. In becoming a fixed disease, it will proceed from inflammation of the air passages; or it may be from severe irritation of the lower portion of the windpipe. When the seat of the disorder is caused by inflammation in the substance of the lungs, it will manifest itself after eating, which is occasioned by the distended stomach pressing upon the diaphragm, and this organ in its turn pressing upon the lungs. This renders them less capable of transmitting the air through their passages; which occasions considerable excitement, from the great effort made to perform respiration, and hence the irritating cough is induced. 
Remedies.-The following dose to be given every night until the cough is modified :-

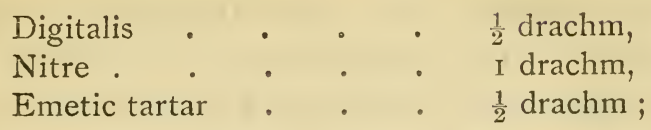

to be made up in the form of a ball, with tar. If this fails to lessen the irritation, a blister should be applied to the throat, extending from one ear to the other, and reaching six or eight inches down the windpipe, which will have the effect of lessening the irritation of the fauces or the larynx, if the inflammation exists in that situation. Sometimes a blister extending to the lower part of the windpipe, as far as the chest, has had a good effect.

The food should be of an opening nature, as dry feeding, such as straw and chaff, is always found to increase the complaint: grass and other green food will have a salutary effect in this and other similar diseases.

Horses may have chronic cough without their general health being affected by it; and should the above remedies prove ineffectual in removing it in a few weeks, there will be no use in persisting in attempts at a cure; and it is only when the complaint assumes a more than ordinary degree of coughing, that medical treatment should be resorted to. A great object is to avoid exposing the animal to sudden transitions from heat to cold; as a horse afflicted with chronic cough is more liable than others to be affected by changes of temperature.

INFLUENZA, EPIDEMIC CATARRH, DISTEMPER.

This is a febrile disease, depending upon a bloodpoison or specific microbe, and generally prevails as 
an epidemic in man, and an epizootic in horses. Like other microbic diseases it has a special affinity for certain organs, and the microbe of influenza selects the mucous membrane which lines the air passages (pulmonary influenza), the same membrane which lines the stomach and bowels (gastric influenza), and the ramifications of the same membrane in the liver (biliary influenza). It is a disease characterised by suddenness of attack, and from the first moment by marked debility and prostration. The temperature runs high, and this and the general debility leads to an accumulation or congestion of blood in the lungs, stomach, bowels, and liver.

Influenza is a highly infectious disease, and undoubtedly the microbes are wafted long distances in the atmosphere. The convalescent horse can carry the contagion and give it to others on arrival at his journey's end hundreds of miles distant from where he contracted it. Stallions convalescent, by the act of copulation, sometimes give influenza to almost every mare they cover. Experiments would point to the "semen" being contaminated.

Symptoms.-Loss of appetite, dullness, great and rapid prostration, weakness of the limbs, weakness of the heart's action and pulse, high temperature or fever, from $104^{\circ}$ to $107^{\circ}$. In forty-eight hours weakness so marked that the animal staggers on his limbs when made to walk. Breathing now becomes disturbed, congestion of the lungs having commenced. A bronchial cough is generally present. Constipation of the bowels may be present in the early stages, but in those cases where the bowels and liver are actively engaged in casting out the blood-poison, yellowness of the eyes and diarrhœea may be marked symptoms. A copious discharge from the nostrils may be present. 
or there may be no discharge. The legs generally swell and sometimes the floor of the abdomen.

Rheumatic complications sometimes exist from the onset of the malady, but rheumatism of the joints and tendons more frequently make their appearance at convalescence, and leave the animal more or less a cripple for life.

REMEDiEs.-Much skill is required in the treatment of this disorder, and it is necessary to study it with great attention before. remedial means are attempted, whether by the veterinary surgeon or the owner of the horse. The treatment generally adopted, until within the last thirty years, was bleeding and purging, and the mortality as attested was 50 per cent.; but since bleeding and purging have been abandoned, and the treatment we are about to prescribe has been substituted, 5 per cent. or less will cover the loss.

When the disease has found an entrance in a stud, the stableman should be instructed to look into the manger of each horse the first thing on opening the stable, and in every case where he finds the horse has not fed, on no account allow that horse to go to work.

Remove the horse to a well-ventilated loose box. Place on his body a warm rug and roll his limbs in flannel bandages. Then administer the following febrifuge drink :-

$\begin{array}{llc}\text { Nitrated ether . } & \text { - } & \text { I ounce, } \\ \text { Liqor. ammon. acetas . } & \text { - } & 4 \text { ounces, } \\ \text { Tinct. of aconite } & \text { - } & \text { 15 drops, } \\ \text { Water } & \text { - } & \text { - I pint. }\end{array}$

Let this drink be given morning and evening until the fever subsides. To regulate the bowels give an 
alterative powder in mash daily. The powder to contain the following ingredients, viz. :-

Nitrate of potash $: \quad \cdot \quad \cdot \quad 4$ drachms,
$\begin{aligned} & \text { Sulphur } \\ & \text { Rhubarb pulv. }\end{aligned}$

Should the animal commence to heave at the flanks and otherwise exhibit a difficulty in breathing, apply a mustard embrocation to the chest wall. Take a pound of the best mustard, place it in a small basin, add water till it is much of the same consistence as used at table. Rub the mustard well in with the hand, and allow it to remain on the chest wall for a couple of hours. If necessary, repeat the mustard embrocation or poultice. The food should be boiled barley, bran, linseed, oatmeal, grass if in season, carrots, etc. If the animal refuses the food, malt, porter, eggs, milk, port wine in gruels must be poured over his throat.

In those cases where the lungs or bowels are seriously involved in the disease, the aid of the veterinary surgeon is urgently demanded, and his services should at once be had in requisition.

\section{FISTULOUS WITHERS.}

Symptoms. - This consists of one or more hot and tender tumours formed upon the withers. If neglected it ends in a large and deep abscess, and will sometimes extend beneath the shoulder-blade, or scapula-and consequently become extremely difficult of cure.

Cause. - If the saddle does not fit exactly to the shape of the withers, these tumours may be formed by pressure.

Remedies.-When first detected it may possibly be removed by cold applications. The following 
to the elbow, and sometimes to the bones of the withers, and render them carious.

In severe cases, and where, generally from neglect, caries or ulcerative disease has attacked the spines of the dorsal vertebræ, a surgical operation ought at once to be performed, and the animal handed over to the veterinary surgeon.

WARBLES, SADDLE-GALLS, \&C.

CaUSES.-When saddles do not fit, various tumours are produced on the back of horses. The name "warbles" is applied to small tumours produced by pressure, which sometimes do not suppurate, but are nevertheless troublesome and painful to the animal. When they become indurated and callous they are termed "sitfasts," and may adhere so firmly as to require great force to remove, and frequently cannot be extracted without being cut out.

REMEDIES.-The first thing that must naturally occur to the mind of a humane man, is to have the stuffing of the saddle altered so as to make it fit, and thereby prevent undue pressure on any particular spot, and the animal should be allowed sufficient rest to permit the tumours to be taken up by absorption. To facilitate this, cold lotions should be frequently and plentifully applied, such as goulard, vinegar, or brine. If these fail, and "steadfasts" appear, then a gentle blister should be applied, which generally has the effect of removing them from their seats; after which the wound may be dressed with Friar's Balsam, or carbolic liniment.

Saddle-galls are generally cured with facility by an application of strong brine, with the addition of tincture of myrrh, in the proportion of a fourth part to three parts of brine. 
We have frequently been disgusted with the unfeeling cruelty of some thoughtless persons riding their horses day after day, with large sores under the saddle. Such individuals but ill deserve to be the possessors of horses. 
Should the above not remove the uneasy symptoms, it will be necessary. to have recourse to a veterinary surgeon.

\section{THE JAUNDICE, OR YELLOWS}

Symptoms. - The eyes are yellow, as well as the skin of the mouth, and also the other naked parts. The dung is voided in small, hard balls, and the urine of a high yellow colour; the appetite is blunted, and general weakness evinces itself.

CaUses. - This complaint is occasioned by some obstruction in the single duct-for the horse has but one-through which the bile is conducted from the liver to the intestines; or from an increased and undue quantity of bile. The horse being destitute of a gall-bladder, the bile flows immediately as it is formed.

Renedies.-Purgatives must be employed to restore the functions of the organs to their healthy condition. These must be administered with caution, and in small quantities, frequently repeated, until the bowels are freely opened. It would be dangerous to risk strong doses, as common inflammation of the intestines may cause the symptoms which apparently mark this disease. Let the following be given twice a day :-

$\begin{array}{llll}\text { Barbadoes aloes . } & \text {. } & \text {. } & 2 \text { drachms, } \\ \text { Calomel } & \text {. } & \text {. } & 1 \text { drachm. }\end{array}$

Thin, slightly warmed gruel should be given in abundance, together with carrots and green food, particularly tares. Let the horse be warmly clothed if the weather is severe; and the stable should be properly ventilated, but not allowed to be too cold. The following should afterwards be given :-Bran-mashes, with green meat, for four or five days; also drink of thin warm gruel. 
If purging should become pretty active after administering the above medicine, it should not be hastily checked unless inflammatory symptoms begin to appear, or if the horse exhibits weakness. In either of these cases then the following medicine may be given :-

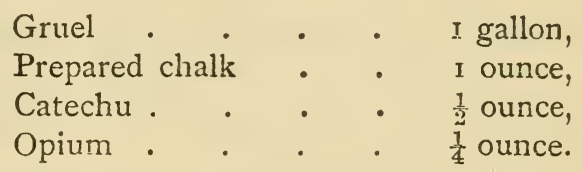

After this give the following tonic :-

Gentian . $\quad \cdot \quad \cdot \quad 2$ drachms,
Ginger . $\quad 2$ drachms;

formed into a ball, and repeated daily until the discharge is checked.

\section{HERNIA, OR RUPTURE.}

This consists of a portion of the intestine being protruded from the abdominal cavity, either through a natural opening or through one caused by disease. In many cases the intestine may be returned into the cavity, but it seldom is an effectual cure, in consequence of the impossibility of applying a truss to keep it up. There are other instances where the gut having passed through the inguinal ring and protruded into the scrotum of the entire horse, or in the groin of the gelding, which may in time become so narrow as not to admit of the gut being again returned, in consequence of becoming thickened and the protruded portion being filled with the dung. This is called strangulated hernia, and can only be successfully treated by a veterinary surgeon, and should on no account be attempted by any other, especially an uneducated person. 


\section{INFLAMMATION OF THE KIDNEYS.}

Symptoms. - This complaint is usually preceded by fever; and when completely formed, the horse stands with his hind legs wide apart, and has an awkward gait in his walk. He withdraws from the pressure of the hand on the loins, which also indicate an undue heat, considerably above that in the natural condition. When turning with moderate quickness, he feels pain, and looks wistfully back at his flanks; a suppression of the action of the urinary organs takes place, and is followed by a difficulty in voiding urine, which comes off in small quantities, and is generally high coloured, and not unfrequently mixed with blood. Strong efforts are made by the horse to discharge it in larger portions, but at length it is almost quite suppressed. Hence it will be manifest that there is an affection of the urinary organs; but whether in the kidneys or bladder it will be difficult at first to determine. At this stage of the disease the pulse is hard and accelerated; it soon afterwards becomes small, although retaining its character of hardness.

In order to ascertain the seat of the disease, the hand must be introduced into the rectum, and if the bladder (which is situated under the rectum) feels distended and hard, then it is certain that the neck or sphincture of the bladder is inflamed. If, on the other hand, it is soft and feeling empty, with a heat in the intestines over it, then there is inflammation of the bladder itself; but if there is no unnatural heat over it, then it may be certain that the affection is in the kidneys.

Cause.-Musty or mow-burnt oats, from their diuretic properties, are often the cause of inflammation in the kidneys. Indeed, very strong diuretics, 
frequently given, will bring on inflammation of the kidneys as well as weakness in the parts. A sprain in the loins often proves the remote cause of this disease; or a sudden check, throwing him back upon his haunches, and giving a sudden shock to the muscles of the loins, is another cause. Exposure to rain and being allowed to cool and dry without having been rubbed down, will cause inflammation in the kidneys.

Remedies.-Place the horse in a comfortable loose-box, and clothe his body with a warm rug and bandages. Give warm drinks of linseed and oatmeal gruel, and apply a counter-irritant over the region of the kidneys. If bowels are constipated, give one pint of linseed oil daily in boiled food, but if refused pour it over.

\section{INFLAMMATION OF THE BLADDER.}

Sympтoms. - When treating of inflammation of the kidneys, we described fully the symptoms of inflammation of the bladder. In this variety of the complaint the urine is voided in small quantities, and with considerable pain each time; and in extreme cases a total suppression takes place. When this is the case the bladder becomes exceedingly distended under the rectum, and may be easily felt by the means recommended in the former article.

CAUSES.-This is produced by some acrid substance, which has been generated in the system by the use of food of a heating nature, forming a chemical compound of an acrid quality in the urine. Stone in the bladder will also produce this disease. Some persons are so absurd as to administer the tincture of cantharides by way of hastening the season of horsing in the mare, which is almost certain to inflame the coating or sphincture of the bladder. 
Remedies. - Much the same line of treatment as has been prescribed for inflammation of the kidneys should be adopted for inflammation of the bladder. But should this not relieve the malady, then the following must be administered :-

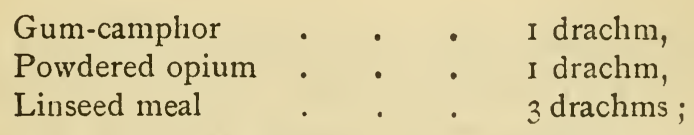

given as a ball every three hours. In cases where the mare is affected, the urine may be drawn off by means of a catheter. This will have a considerable influence in relieving the animal, and by taking off the tension will tend to abate the inflammatory symptoms. The same remedy is of difficult application in the horse, and should never be attempted by any one but a veterinary surgeon.

\section{STONE IN THE BLADDER.}

Symptoms.-Irregularity in the discharge of urine, with occasional suppression of it, and fits resembling spasmodic colic, are the symptoms which attend this disease. To ascertain if it is really stone which produces these effects, the horse should be thrown on his back and the hand introduced into the rectum, when the stone, if it exists, will be easily felt.

REMEDIES. - If the stone is of any size it must be extracted by an operation, which can only be performed by a regular veterinary surgeon. When it is small, or only appears in the form of gravel, diuretics are the only remedies which can do good; these, by inducing an increased flow of urine, may carry with it the smaller concretions. The following will be found a useful diuretic, given once a day in a drink :-

Purified nitre (nitrate of potash) 3 drachms,

Digitalis . . . . I drachm. 


\section{DIABETES.}

Symptons.-This disease consists in an excessive discharge of urine; and, fortunately, is not of such common occurrence among horses as in the human species.

CAUSES.-Bad food will produce irritation of the kidneys, as well as the improper and too frequent use of strong diuretics, which cause irritation and an increased action in the kidneys.

REMEDIES.-Order a complete change of food, and see that the hay and oats are of the best quality. Give boiled food once a day, and to it add the following powder:-

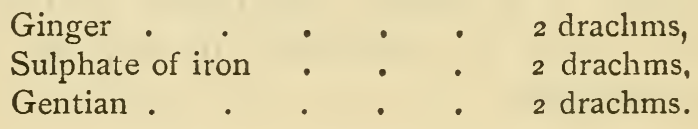

Whatever tends to lessen the undue action will be the best remedy. Green meat and carrots will be of much use during this disease.

\section{STALING OF BLOOD.}

Symptoms. - The symptoms of this complaint are nearly allied to those of inflammation in the kidneys; and it always manifests itself by the highly coloured urine, mingled with blood. If the bowels are not easy, let them be opened by the following cathartic :-

$\begin{array}{llll}\text { Barbadoes aloes } & \text { • } & \text { - } & 6 \text { drachms, } \\ \text { Ginger, powdered } & \text { - } \quad & \text { I drachm ; }\end{array}$

to be formed into a ball and administered. When it has operated, the following must be given once a day until the urine has assumed its natural colour:-

\begin{tabular}{|c|c|c|}
\hline an bark, po & dered & \\
\hline Nitre, in powder & 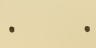 & \\
\hline Balsam of capiva & 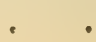 & \\
\hline
\end{tabular}


Let the above be well powdered together, and then given in three ounces of linseed, boiled in half-a-gallon of water reduced to three pints.

\section{INFLAMMATION OF THE BOWELS.}

The intestines are subject to two different kinds of inflammation, namely, that of the mucous membrane or internal lining of the gut. The second is in the external coating of the bowels. These are very different in their characters, and consequently in the mode in which they must be treated.

INFLAMMATION OF THE MUCOUS MEMBRANE.

Symptoms.-This is accompanied with violent purging, proceeding too often from over-doses of physic being administered to the horse, or from acid generated in the bowels by the food, or some other unknown cause. In addition to the purging, considerable pain attends this disease, which is indicated by the animal frequently looking round to his flanks, with a heaviness in his breathing, accompanied by a quick feeble pulse, with a hot mouth, ears, and legs.

CAUSES.-Sudden exposure from a warm to a cold atmosphere, and being allowed to drink plentifully of water.when over-heated, or having his belly and legs wetted with cold water when too warm, are all causes which induce this complaint, more especially in highfed horses.

REMEDIES. - When accompanied by excessive purging, with great pain, astringent medicines should not be administered. All food should be denied him, and in its stead give him gruel, a decoction of linseed, thin starch, or arrowroot. Clysters of warm gruel would also be proper. These should be administered with Reid's patent pump. If the irritation and indications 
of pain still continue, it will be necessary to give the following :-

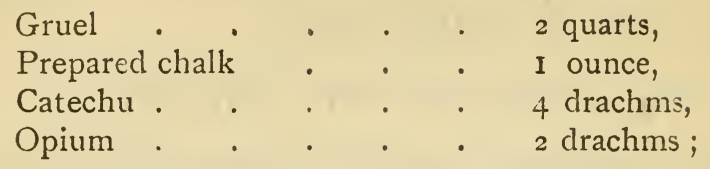

The above to be repeated every five or six hours until the purging and pain are allayed, after which the doses should be lessened in quantity and frequency.

The horse must be kept warm and well-clothed, with his legs bandaged after being rubbed thoroughly.

\section{SPASMODIC COLIC.}

Symptoms. - This disorder in general comes on very suddenly, without any premonitory signs. The horse becomes very restless, shifts his position, paws the ground, and looks round with anxiety at his flanks; sometimes raising his foot as high as his belly and striking it violently: he will also lie down and roll about on his back. In a few minutes the spasm subsides, and the animal after shaking himself will resume feeding. At longer or shorter intervals the attack is renewed, but with increased violence; he will throw himself with considerable force on the ground; will break into a copious perspiration, and heave greatly at the flanks. These spasms are renewed at intervals, and gradually become less frequent and less severe; or if, on the contrary, they are more frequent and acute, and at length manifest a nearly uninterrupted series, then it may be suspected that inflammation has taken place, and that death may speedily ensue.

CaUses.-The causes giving rise to spasmodic contraction of the muscular fibres of the bowels are 
very various. Stones and large earthy lumps in the intestines cause colic pains, but it cannot be known when these are present. Drinking cold water when the animal is over-heated is a frequent cause of these spasms and pains. Green food is also apt to induce these pains when taken in large quantities, and especially if too hard worked after it.

REMEDIES.-A combination of opium and ether are valuable specifics in this disorder, in the following quantities, and usually produce almost immediate relief :-

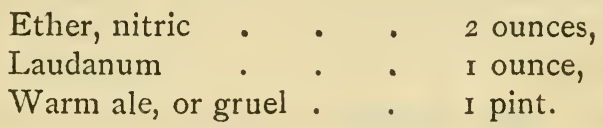

If in two hours after the above has been administered no visible mitigation of the complaint exhibits itself, it will be necessary to repeat the medicine and apply a mustard poultice to his abdomen; because a longcontinued succession of spasms is almost certain to induce inflammation. Injections of warm water, or gruel, mixed with a small quantity of aloes, will have an excellent effect in this complaint.

The following embrocation has produced good effects during the time the animal was suffering from spasms. It is made into a pretty thick paste and rubbed over a considerable portion of the belly :-

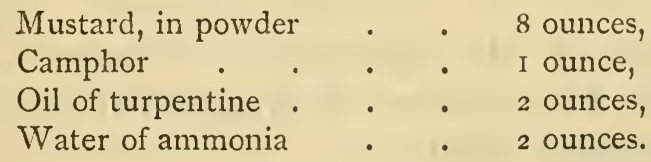

This disease and inflammation of the bowels are frequently mistaken for each other, which is caused by the general appearance being somewhat similar; but if strict attention be paid to both diseases, it will be found that there are symptoms connected with 
each disorder specifically different, which will at once be understood by the following tables:-

INFLAMMATION OF THE BOWELS.

I. Pulse considerably accelerated, but very indistinct.

2. Gradual in its approach, with previous febrile indications.

3. Lies down, seldom rolls on his back, starts to his legs suddenly.

4. Legs and ears generally cold.

5. Belly exceedingly tender, and when touched causing pain.

6. Constant pain.

7. Rapid prostration or strength.

8. Mucous membrane inside the nostrils very red.

9. Lining of the eyelids unusually red.

10. Peristaltic motion of the bowels excited, with the anus hot.
SPASMODIC COLIC.

I. PULSE natural, or lower than in its natural state, but accelerated and more full during the spasms.

2. Sudden in its attack, but destitute of febrile symptoms.

3. Lies down, and may roll on his back, which seems to give him relief.

4. Legs and ears of a natural heat.

5. Rubbing the belly gives relief to the animal.

6. Intervals of rest.

7. Strength hardly affected.

8. Mucous membrane of its ordinary colour.

9. Lining of the eyelids of its natural colour.

I0. Slight motion of the intestines, unless by purgative injections.

Pawing of the ground is common to both complaints, but in cases of entanglement of the gut he desists from pawing.

Both this and the preceding complaint are induced by various causes. But if symptoms similar to them are caused by eating green food in too large quantity, a different mode of treatment will be necessary. 
ENTANGLEMENT OF THE BOWELS.

This is caused by colic, in consequence of the animal throwing himself about while suffering under the pain of that complaint; portions of that intestine called the ileum become twisted and knotted, and drawn together with astonishing firmness. There is no remedy for this complaint.

\section{STONES IN THE INTESTINES.}

Horses that are subject to very frequent attaclss of colic pains have usually stony masses in the cæcum or colon. Sometimes they are some pounds weight. These, obstructing the passage of the gut, produce colic pains; and at other times, when exceedingly large, by pressing upon the mucous membrane, produce inflammation. But as yet no distinctlymarked symptoms have been detected by which their presence can be ascertained. Hitherto no certain mode of treatment has been discovered for their removal.

\section{INTUSSUSCEPTION OF THE INTESTINES.}

This is another evil arising from a long-continued spasmodic action of the ileum, which sometimes causes an inverted pressure from the cæcum towards the stomach, which overcomes the natural action, which forces this contracted portion of the intestine into a portion above it, which retains its natural calibre. The irritation thereby produced increases the upward action, and causes still more of the intestine to be forced inwards, until an obstruction of an insurmountable character is produced. Continued and unmitigated pain is the only symptom which may lead to a suspicion that this incurable malady has taken place. 


\section{INTESTINAL WORMS.}

A variety of worms inhabit the intestines of horses, and when they become numerous often prove injurious to the constitution of the animal. Although a respectable writer says they are not of much consequence unless they are numerous, we would recommend that the sooner they are expelled the better, however small the number may be, for this simple reason - that where there exists a single pair there will naturally be an increase of their species.

Symptoms. - Loss of appetite, griping pains, a rough coat, and tucked-up belly, are symptomatic of worms of the larger species, lumbricus teres, or long white round-worms very much resembling in form the common earth-worm, which is met with from five to ten inches in length; and itching of the rectum, evinced by a quick twitching of the tail and a small quantity of mucus, which hardens and assumes the appearance of a white powder at the anus, is indicative of ascarides-small, needle-formed worms, which lodge in the large intestines, and frequently find their way in great numbers to the cæcum. A third species sometimes, although of much rarer occurrence, inhabits various parts of the intestinal-canal from the stomach downwards. This is the tape-worm, which is known from its broad, flat, tape-like appearance, and consisting of many articulated joints. This species is the most formidable and the most difficult to be removed.

Remedies. - When the symptoms which we have pointed out are noticed, the groom should carefully watch whether worms are voided, to ascertain their existence. Still, however, they may be lodged in the intestines without being evacuated; and when there 
is a well-grounded suspicion that they inhabit the body, a dose of some vermifuge should be given.

For the expulsion of the round-worm, the following anthelmintic should be administered :-

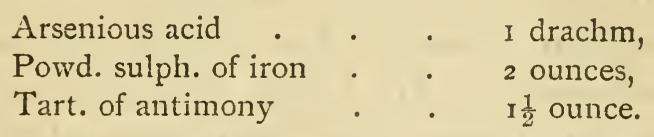

Mix and divide into twelve powders, and give one each morning; follow up on the fourteenth day by an aloetic ball.

If the existence of the tape-worm is apprehended, then the following will be the proper remedy:-

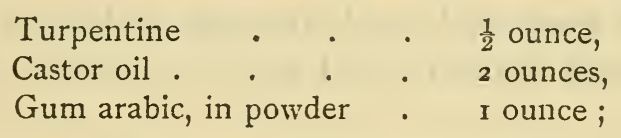

treacle to form it into a ball. Warm mashes to be given for a day afterwards.

It seldom, however, happens that these are removed by one dose; and unless it is quite certain that the entire animal has been discharged, the above medicine must be repeated. If even a link or two is broken off and left in the intestines, these will be regenerated into a perfect worm, as they have the property of reproducing the parts of which they have been deprived.

\section{THE BO'TS.}

Horses may be infested by bots without apparently suffering material injury from them. But we cannot agree with the opinion of Mr Bracy Clark, that they are destined by Nature to act upon the food in the stomach by trituration, or as pepper does in the human stomach. Like every other part of the animal body, this organ is so admirably constructed that in 
its healthy condition no artificial aid is necessary to enable it to perform its office. If the opinion of $\mathrm{Mr}$ Clark were correct, what would supply the place of those parasites during that portion of the year when the grub assumed its perfect condition?

Post-mortem dissection has proved that bots are not so inoffensive as $\mathrm{Mr}$ Clark supposed; as it has been found that horses which have been infested with these grubs had ulcers of considerable extent in the muscular coat of the stomach.

Symptoms and Habits. - The progress of the disease generally manifests itself gradually; the horse becomes hide-bound, his coat becomes rough and unhealthy, he loses flesh and strength, although he feeds with his usual appetite, and has frequently a tickling cough.

CAUSE. - The bots are a species of gad-fly called cestrus equi by naturalists, which may be observed in the month of July flying actively about the legs of horses in the fields. These flies are represented (Plate $\mathrm{x}$, fig. I), which is the common gad-fly. They may be seen flying rapidly towards the sides and knees of horses. These are the females depositing their eggs in the hair, to which they adhere by a glutinous fluid by which they are surrounded (figs. 2, 3). In a few days the eggs are hatched, and the minute grubs or caterpillars which they contain are set at liberty. This operation is performed by the horse, which, in licking himself, bursts the eggs, and the caterpillar adheres to his tongue, and in the operation of eating, the little animal is carried into the stomach along with the food. These caterpillars are provided with a small hook on each side of their mouths, and by means of which they cling pertinaceously to the cuticular portion of the lining of the stomach (see fig. 4); and so tena- 
ciously do they adhere, that the hook will break before they leave their hold. There these pests contrive to locate during the whole winter, and to the end of the following spring, feeding on the mucus; by which time they have grown to full size (fig. 5), and must now undergo a new transformation; they quit their hold of the coating of the stomach, are carried into the villous portion along with food, from thence pass into the intestinal canal along with the chyme, and at length are discharged with it. The caterpillars thus evacuated seek an appropriate place in the ground, where they assume the imago or chrysalis condition; and remaining in this state for some weeks, at length break out from their swathing and assume the form of the perfect insect. Immediately after this the males and females pair; the latter becoming impregnated, set about seeking an appropriate situation where to deposit their eggs, which, in imitation of the parent, by a peculiar instinct, they fix on the hairs of a horse, from whence they are licked off and swallowed in the larva state.

There are two species of gad-flies; the second is known by the name of the red bot, fig. 6 is its larva, and fig. 7 the perfect fly. Their natural history and habits are similar in all respects to the other. 


\section{CHAPTER V.}

Disorders of the External Parts of the Horse.

THE FORE I.EGS.

WE come now to treat of those portions of the frame of a horse most intimately connected with his action, and his essential value in the service of man. Unfortunately these are but too often liable to disease; and the animal afficted with maladies of those parts is not only rendered less useful to his possessor, but also less valuable in the market, as the extremities embrace the entire apparatus of progressive motion, and consequently that action in which mankind are chiefly interested in this useful animal.

To thoughtless and reckless masters, and careless, cruel, and unthinking servants, this noble animal owes many of his complaints, both internal and external. And what is revolting to every humane mind, is, after the poor animals are afflicted with a painful disorder, their unfeeling masters too often continue to subject them to hard work while they are labouring under acute suffering. Let any man who has had a severe sprained ankle fancy to himself that he was compelled to carry a heavy burden, or remove a quantity of stones from one part to another by means of a wheelbarrow, or suppose he were even forced to walk at all, and what would be his sufferings? The probability is, he would be utterly unable to perform one or the other task. But the poor horse has four legs, and probably 
three of these are sound, and he is thereby not only able to stand, but also to perform progressive motion ; but every time the lame limb is put to the ground and borne upon, his sufferings are equal to, if not worse than, what man himself would feel.

It is the interest of every man to have his lame horse rendered sound as speedily as possible; and this, in most cases, cannot be effected without allowing the animal a cessation from labour ; and it is monstrous cruelty to subject a horse to labour whilst under the influence of a painful malady. The finger of contempt should be pointed at all such unfeeling wretches.

In this portion of the work we shall treat of the diseases alone, and the anatomy of the various parts will be afterwards given, with complete references to the plates illustrative of those parts.

\section{SPRAIN OF THE SHOULDER.}

Many ignorant pretenders, who denominate themselves Farriers, frequently speak of what they call the "shoulder-lameness," without being able to state wherein this lameness consists. In innumerable instances they are wrong when they say or imagine that the lameness proceeds from the shoulder at all. We are decidedly of opinion that lameness of the shoulder is much less frequent than is supposed.

Symptoms. - To those who have attended to the anatomy of the shoulder of the horse, shoulder-lameness can be recognised with considerable celerity, as well as certainty. The horse generally suffers great pain in moving forward while under the influence of this complaint, which is indicated by dragging his toe along the ground instead of lifting the foot smartly up, which is the natural action in progressive motion; 
for it is this lifting of the foot which produces the pain, by giving motion to the muscles of the shoulder, some of which are inflamed and tender, in consequence of the sprain. But it must be obvious to any one acquainted with the anatomy of the shoulder, that the pain occasioned by lifting the foot must be both short and small in lameness of the shoulder, as the limb is allowed to bear the weight a much shorter time than in any other species of lameness. For example, in sprain of the back tendons, it is only when the horse is moving forwards that he suffers much pain, and this is most felt when the weight rests on the leg; consequently, there is a peculiar activity in moving up the limb in shoulder-lameness the instant the weight bears upon it. This is strongly manifested in moving down a hill, in which case additional pressure is given to the limb. And while the horse is in the stable, it will be found that his toe only is resting on the ground when afflicted with shoulder-lameness, while in a sound state he will have the foot flat on the ground. But one of its most prominent characters is, that when the foot is lifted and pressed considerably forward, the animal indicates a feeling of great pain, while the same action will give him no uneasiness if the lameness is seated in the leg or foot.

In diseases or injuries of the muscles, heat generally accompanies them; but from the muscles which are affected in this belonging to the under layer, of course the heat is not perceptible to the touch. Neither can the swelling be perceived for the same reason.

CAUSE. - This has its origin in some sudden and severe shock which the muscles have received. A slip of the foot or side-fall may also occasion this complaint.

REMEDIES. - In some cases of lameness a horse may 
be able to work, but not in shoulder-lameness. It takes away all chance of the animal being restored to soundness. At once have him put off work. Foment the shoulder with hot water for a few days, then apply a stimulating liniment all over the injured muscle. If lameness persists, blister the parts with oil of cantharidis, and if the animal is not thereby restored to soundness, call in the aid of the veterinary surgeon.

Numerous ridiculous operations, which only give pain to the horse labouring under this injury, have been resorted to, but all of them are as absurd as they are cruel. The following mild purgative ball may be administered, so as to keep the bowels open:-

$\begin{array}{lllll}\text { Barbadoes aloes } & \cdot & \cdot & \cdot & 5 \text { drachms, } \\ \text { Castile soap } & \cdot & \cdot & \cdot & 2 \text { drachms, } \\ \text { Oil of caraways } & \cdot & \cdot & \text {. } & \text { I } 2 \text { drops. }\end{array}$

COMPLAINTS AND INJURIES OF THE ARM.

FRACTURE OF THE ELBOW

sometimes occurs, and when this is the case, it would be exceedingly imprudent for any person to attempt a cure, except one skilled in the veterinary art, and even with the regular practitioner there is no certainty of a cure being effected.

\section{ENLARGEMENT OF THE ELBOW}

may be induced by a severe blow; or it may be caused by the heels of the shoes pressing against that part while the horse has his feet doubled under him.

REMEDY.-Let a seton be carried through the tumour if it is of small dimensions, and in most cases it will subside completely. But if large, an incision should be made in the skin along the centre of the 
prominent part, and the tumour removed by dissection. Some horses produce "Capped elbow" by resting too long on their elbows in the act of rising from their bed, and when this is the case, an "elbow pad" or "swab heel boot" should be worn during the night.

\section{COMPLAINTS AND INJURIES OF THE KNEE.}

\section{BROKEN KNEES.}

Much has been said, and a great variety of remedies have been recommended, for injuries received in the knees. It is a subject of very great importance, and therefore much patient attention should be bestowed upon it. Many fine horses have been blemished and disfigured for life by broken knees, and others have been so severely injured that it has rendered them unfit for future service, and in other cases has proved fatal to them.

This injury is generally sustained while the horse is going at a quick pace, consequently the extent of the wound will generally be in proportion to the rate of the speed at which the animal is progressing, and the weight behind, as well as the condition and texture of the road on which he falls. The horse in the action of falling naturally throws his knees forward, and consequently they receive all the weight of both horse and rider, and frequently are very severely lacerated.

Remedies. - The first precaution is to wash the wounds thoroughly with warm carbolised water and a sponge, so as to remove all dirt and gravel from the parts. The next thing to be done is to examine carefully whether the joint is opened, which is best ascertained by the application of a probe, but great care must be exercised, otherwise the joint may be 
opened by the point of the probe. But should a doubt remain as to its being open, then a poultice must be applied composed of linseed meal and carbolised water. This may be allowed to remain for about eight hours, which will have at least the effect of acting as a fomentation to the wound, and assist in allaying the inflammation. When the poultice is removed, if the capsular ligament of the joint has been injured, or the sheath of a tendon laid open, then it will exhibit the synovia, or joint-oil, which manifests a yellowish, glary, transparent appearance. If a doubt still remains, then a second poultice should be applied for the same length of time. Having ascertained the fact that the joint has been laid open by the flow of the oily matter, an attempt must be made to close it, and with this object tie up the horse's head and apply carbolic liniment to the injured parts. Tow saturated with carbolic liniment should also be applied to the knee and kept in position by bandage.

Prompt attention must be paid to closing the wound, an operation which is only fit for the skilful hand of a regular veterinary surgeon. It must be borne in mind that wounds from contusions of the kind mentioned are very different from a simple cut; as the edges are irregular, and there is a consequent loss of substance, which can only be restored by the tedious process of granulation. It must therefore be evident that the less action in the limbs the better, as if those tender, new-formed portions are either stretched or bruised, it protracts the cure.

If the cut in the joint be large, and the joint-oil continues to exude from it, and the horse exhibit symptoms of suffering. much pain, it may be considered that the wound has become associated with ulcerative disease of the bones, and under the circum- 
stances it will be but proper to destroy the animal, and relieve him from the torture which always accompanies aggravated cases of broken knees: for high fever is almost always induced, of which he is nearly certain to die; or if he survives it, the inflammation of the parts will result in a growth of bone and a stiff knee-joint.

To enable a person to judge of the exact situation of the wound in broken knees, he should have a thorough knowledge of the structure and situation of the bones of the knee-joint, which is the most complicated of all the bony structure of the horse. Between the lower termination of the bone of the arm and the superior portion of the leg-bones, there are interposed seven other bones, called the carpal bones. Six of these are arranged in two rows of three each, and the seventh is placed behind the others. Should the wound be situated opposite the bottom row, and if its dimensions are small, then there will be little difficulty in closing it; and even a pretty large one is frequently successfully cured, as there is but limited motion in that part. But if it is situate opposite the union of the two rows, its remedy is much more uncertain, in consequence of this being the seat of the chief motion of the joint, which has a tendency to disunite the lips of the wound, and also induces most irritating friction between the bones, which would become in close contact with each other, in consequence of the expenditure of the joint-oil.

When the skin has been broken, it is always visible afterwards, but the extent of the blemish will depend greatly upon the treatment of the wound in its early stages. Caustic applications should invariably be avoided, as they always leave behind them a greater blemish. But should the mark left be of 
some extent, even without using caustic remedies, then the best way to render it less visible is to apply a mild blister to the part, which will stimulate the hair to shoot out more abundantly round the scar. We do not think there is much faith to be placed in the application of ointments, except they may be stimulating, in consequence of being generally composed of turpentine or Spanish flies.

It is seldom a knee that has once been broken is so healed as not to be perceptible, although in some cases the hurt has been so slight that it can hardly be detected. But even in the least visible of these the hair is always slightly curled, and where this is noticed, the leg should be taken up and the knee bent, when the scar, however slight, will be at once perceived. But we would not have it supposed that a broken knee is invariably an indication of a stumbling animal, because the best of horses have come down, by being backed by a bad rider, or placing his foot on a rolling stone on a road when going at a quick trot, or other cause. However, a broken knee once detected will put the purchaser upon his guard to watch minutely the action and paces of the horse, as well as the form of his forequarters ; because, if his shoulder is thick and upright, and the legs placed far under him, then he is likely to have a bad action, and be apt not to lift his feet high enough to clear the ground properly. On the other hand, I knew one of the finest hunters that ever took the field with his knees broken, from his rider having forced him to a leap which it was impossible he could accomplish. If the shoulder is oblique and the withers high, with a strong, well-developed forearm, it may be fairly inferred that he has come down from some accidental cause. 
SPLENT, OR SPLINT.

A splent is an enlargement, or bony excrescence, of some part of the shank-bone, generally in the higher portion of it, and situate in the inside of the leg (Plate vI, fig. I). This is a complaint to which young horses are most liable; and it has been generally remarked, that as horses advance in years they gradually diminish, and not unfrequently disappear altogether. It seldom happens, unless the splent is tolerably large, and encroaches too much upon the knee-joint or the back sinew, that it is productive of lameness, unless the horse happen to strike it with the foot of the other leg. In other instances a splent not larger than a pea may be detected in consequence of being acutely sensitive, and produces such lameness as, without being experienced in this complaint, might lead to the belief that it was quite disproportionate to the cause.

Cause.-It is difficult to conceive how splent should appear on the outside of the small bones, except we suppose that the space between these bones is occupied by mechanism of an important character. It is much easier to account for their almost exclusive appearance on the inside of the limb. The inner splent-bone is situate nearer the central part of the body than the other; and from the nature of its connection with the knee, it is subject to a greater proportion of weight than the outer one, and hence is more liable to injury and inflammation, and consequently inducing this bony deposit, which has been termed splent. The inner bone supports the entire weight, which is transmitted to one of the small knee-bones. It is the only support of that bone, while but a portion of the 
weight is sustained by the outer splent-bone, and the pressure is divided between it and the shank-bone. Besides, many smiths who are imperfectly acquainted with their profession, most absurdly elevate the outer heel of the shoe to a great degree, which throws an additional quantity of the weight of the animal on the inner splent-bone. Severe blows sometimes occasion splents on the other portions of the shankbone.

During the formation of a splent, the horse is frequently lame, occasioned by the periosteum, or membrane which covers the bone, being inflamed and stretched to an unnatural degree, and causing great pain to the animal in consequence of the sensitive nature of that membrane. But when the inflammation has subsided and the membrane has been so stretched as to accommodate itself to the form of the growth causing the "splent"-unless it be in a situation which comes in contact with the tendon, or the animal from his peculiar action strikes the splent, the lameness will disappear.

"Splents" by a recent decision have been held as constituting a horse unsound, and as "splents" are always the result of a diseased action, viz., inflammation of the periosteum or covering of the bone, legally the decision appears a fair one, but as on an average 80 per cent. of horses have "splents," and as in all probability not over 5 per cent. of horses remain permanently lame from splents, but make a perfect recovery, it should only be held to constitute unsoundness under given conditions. A splent or splents on the limbs of well-formed horses, and whose action is straight, rarely lames except during the period of its growth, and is of no more practical consequence than a splent or knob of bone on his nose. On the con- 
trary, splents the result of striking in action should under all circumstances render the animal legally an unsound one.

Remedies.-When it is noticed that a splent is forming, which generally commences with a tumour, the hair should be shaved off all round it, and a small portion of biniodide of mercury ointment rubbed into it for two or three days, and after this a pretty strong blister should be applied. If these do not stop its progress, the cautery is sometimes used; although we would recommend that this should not be resorted to, except where the splent threatens to be large, and is making evident progress after the above remedies. It often happens that the effects of the mercurial ointment and blister do not manifest themselves at first; but after some little time the splent will begin to lessen, and eventually disappear altogether. In fact, most splents disappear by absorption when the horse begins to get old.

\section{SPEEDY CUT.}

This is an injury inflicted on the inside of the leg, just below the knee-joint, and extending to the head of the inner splent-bone. Horses of high action are liable to this injury, by severely striking this part with the edge of the shoe when they are trotting at a speedy rate. This, in some instances, occasions a bony enlargement, and in others considerable tenderness and great heat in the adjacent parts. Sometimes the pain inflicted is so great that the horse will suddenly drop as if life were extinct.

REMEDIES.-The only thing required is to prevent the shoe from extending beyond the hoof; and the shoe should have only one nail on the inside of the foot, and that placed near the toe. Let the shoe also 
be of equal thickness at the heel and toe, and formed so that the foot may bear equally on both sides.

\section{KNEE-TIED.}

In some instances, when the trapezium or hinder bone of the knee is not sufficiently prominent, the ligamentous ring by which the tendons are bound together, will confine the flexor tendons of the foot so tightly that the leg will be very deficient in depth under the knee. This is known by the phrase, that the horse is "tied below the knee." This defect has always been found to limit the speed of the animal as well as its endurance. If such a horse is ridden either fast or far, he is almost certain of being seized with a sprain of the back sinews. This is caused by the pressure of the ring producing such a degree of friction as will militate against the free action of the tendons, and thus requiring a greater degree of exertion to keep up progressive motion. This will be the case, even with the best horse, otherwise well formed, and who has a complete degree of muscular development, which by a continuance of the pressure and action must strain the tendons. But this is not all: when the back tendons are thus bound down, they are squeezed into a more oblique direction, consequently it requires a greater force to make the muscles act, and fatigue is induced in a shorter time, and it is almost always accompanied by a sprain. In short, it is one of the worst defects incidental to the horse.

\section{SPRAIN OF THE BACK SINEWS.}

The back tendons are enclosed in a sheath of thick cellular substance, which not only protects 
them from injury, but also keeps them in their proper situation. To prevent friction, a synovial fluid fills the space between the sheath and the tendon. When the horse has been over-exerted or over-worked, the tendon presses upon the delicate membrane with which the sheath is lined, and induces inflammation. Long-continued action will also sometimes rupture some of the fibres with which the tendons are bound. This is what has been termed a sprain of the back sinew, and when this takes place to a greater extent, the horse is said to have broken doron.

Symptoms. - It will be seen that during every movement of the limb the animal evinces great pain, in consequence of the excessive inflammation which accompanies this injury, and also the considerable local swelling and heat which accompany it. The horse will also stand with his foot off the ground, barely touching it with his toe. The first injury which is above pointed out, consists merely of inflammation of the sheath, or partial rupture of the fibres by which it is attached.

Remedies.-This is an injury so serious to the animal's future usefulness, as to necessitate his instant removal from work, because however slight the strain, if kept at work the animal will gradually "knuckle over" on his fetlock joint and become useless. To prevent constitutional irritation, administer a dose of purgative medicine made as follows :-

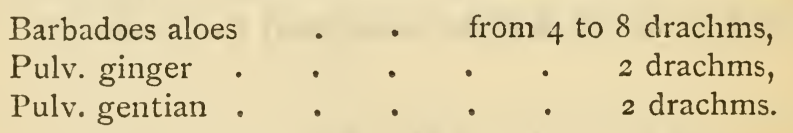

After this the limb should be well fomented with water three or four times a day, each time for about an hour; and in the intervals let large poultices of 
linseed meal be wrapped round the leg. A little of Goulard's extract, or vinegar, may be added to the poultice with advantage. A great deal depends, however, on the warmth and moisture of the poultice, as well as the fomentations. These appliances are intended to allay the inflammation. Stimulants are to be carefully avoided, as these are sure to increase the inflammation in the early stages of the injury.

When the horse begins to rest his foot upon the sole, and the heat has left the part, it is certain that the irritation will have considerably abated. But although this is the case, means must be used to prevent the recurrence of the inflammation, and also to reduce the swelling. Apply a thin flannel bandage to support the parts, which, by gentle pressure, will have a tendency to stimulate the absorbents and promote the absorption of the inflammatory exudate; and it should be applied tighter as the limb will endure increased pressure; and this appliance should be continued for at least fourteen days. It will be better to keep the bandage wet with the following lotion :-

$$
\begin{aligned}
& \text { Vinegar } \\
& \text { Spirit of wine } \quad: \quad: \quad \text { I pint, }
\end{aligned}
$$

This will have the effect of reducing the deep-seated inflammation.

If in a fortnight or three weeks all the symptoms have disappeared, the horse may be put to work, but of course with caution. But if after this time lameness still continues, then recourse must be had to a blister; and in this case it will be proper afterwards to turn out the horse for a month or two to allow proper time and opportunity for the reduction of the 
swelling and inflammation, otherwise an incurable lameness may be the result.

Severe sprains, as well as those which have been unskilfully treated, especially where from long-continued inflammation the structure of the part has been considerably altered; or if the swelling has become callous, and the skin thickened so as to prevent the free action of the limb, then it will be necessary to use the cautery, as the best means of rousing and stimulating to action the absorbents, so as to enable them to take up the coagulated cleposit, as well as the thickened skin and unusual increase of the cellular substance.

In firing, the cautery should be applied in straight lines so as to contract the skin, and by its natural elasticity to produce that pressure so necessary in this complaint.

It may well be imagined that it will require some time before this hardened fluid can be removed; not less than six months' cessation from work will suffice. A blister applied six weeks or two months after the use of the cautery is frequently attended with beneficial effects; but it should never be had recourse to except in cases where it becomes indispensable.

It is necessary to pay very strict attention to the appearance of the flexor tendons in the purchase of a horse; and if there is any thickening of the cellular substance, then it may be inferred that the horse has had one of those sprains, and that it has not been properly managed. The action of the horse is sure to be affected by it, and a return of the malady may occur; and although all appearance of lameness has left the animal, still he cannot under such circumstances be considered a perfectly sound horse. 
WIND-GALLS.

Symptons. - Wind-galls more frequently occur in the fore than in the hind legs. These are known by the puffy appearance so frequently noticad about the legs of horses which have been hard worked (see Plate vi, fig. 8, c). There is a beautiful provision in Nature, in placing little bags between the tendons and other parts where they are exposed to pressure or friction. These sacs contain a mucous fluid of a similar kind to the synovia, or joint-oil which oozes from the bags and lubricates the tendons of the muscles. In consequence of violent action and straining the tendons, these bags get injured, become inflamed, swollen, and hard, the tendons being generally inserted near the joints, where there is more pressure and motion, and consequently these bags are more liable to injury. They become inflamed, and during this state the horse is generally lame to a greater or less degree. However, unless these sacs attain a very large size, the horse cannot be reckoned unsound, as few are entirely free from wind-galls, - which appellation they acquired from the fancy that they contained wind. It was an old practice to cut or probe them, so that the air might escape. This has, in many instances, produced violent inflammation, and has been the death of many fine horses.

Remedies.-Unless wind-galls are large and interfere with the actions of the leg, we would recommend that no treatment should be attempted. The first appliance to a large wind-gall should be a bandage or roller of flannel, and under it, immediately over the swollen parts, a soft pad should be introduced, and firmly bound down. The bandage should be wetted with the same kind of lotion recommended in sprain of 
the back sinews, page 97 . This treatment in most instances will cause the wind-galls to disappear; but unfortunately they are liable to return, especially if the animal is hard worked. A blister is more likely to effect a cure, as it generally has the power of dispelling the swellings. Firing is still more effectual in large galls, as it immediately induces absorption of the fluid, and consequently the swelling disappears, by means of the contraction of the skin caused by the use of the cautery; and this contraction serves the purpose of a permanent bandage, and prevents a recurrence of the wind-gall.

RUPTURE OF THE SUSPENSORY LIGAMENT.

Extraordinary exertion will sometimes produce rupture in the suspensory ligament (see Plate vr, fig. Io, $f$ ). This rupture allows the sessamoid bones to fall down, and consequently the fetlock joint nearly touches the ground. It not unfrequently happens that this complaint is mistaken for rupture of the flexor tendons; but one thing will render it sufficiently evident that it is really the suspensory tendon which is at fault, namely, that the horse is able to bend his foot. No malady which affects a horse is more serious than this; for it is ten to one that the animal ever becomes effectually cured of it. In most instances he is ever afterwards lame.

REMEDY.-If a cure can at all be effected, it will be by keeping the animal quiet, and having the leg bandaged, and giving him a high-heeled shoe with stays.

\section{GROGGINESS.}

This consists of a singular knuckling over the fetlock joint, and general shaking over the entire fore 
leg. It is a complaint common to old, over-worked horses. Neither the fetlock or pastern joints are simply implicated in this complaint, although both are connected with it.

CAUSE.-Working the animal beyond his strength is usually the sole cause of this complaint. It is for the most part a want of power in the ligaments of this joint generally occasioned by frequent and severe sprains, brought on by cruel and oppressive treatment, in forcing the animal to pull more than his physical energies can accomplish without a desperate effort.

Remedies.-It seldom happens that an effectual remedy can be found for this complaint, because it is generally accompanied by inflammation of the synovial membrane and ulcerated disease of the bones within the joints.

\section{SPRAIN OF THE FETLOCK.}

The fetlock-joint is peculiarly liable to injuries, from its being the principal situation of action below the knee. In sprains of the back sinew, accompanied by inflammation, which is usually the case, inflammation is often induced in this also. And many of the sprains which are supposed to belong to higher portions of the limbs are, in fact, affections of this joint.

Symptoms.-It is no easy matter to distinguish affections of the fetlock from those situate in the superior portions of the limb. Pressure by the finger on the part, and heat, are the surest tests for finding out the seat of this complaint, but in some cases flexion and extension of the fetlock joint, with pressure of the thumb over the back tendons, will elicit more pain than by any other method.

Treatment.-Strong blisters applied with prompti- 
tude are the most effectual means of removing sprain of this joint; and afterwards plastic bandages should be used.

\section{CUTTING OF THE FETLOCK.}

The inside of the fetlock-joint is frequently bruised or cut by the shoe of the opposite foot. This depends sometimes upon natural defects in the form of the leg and foot; and in such a case it is seldom that a remedy can be devised; such as when the limbs are placed too near each other, or when the feet have an inward or outward inclination. Some horses are subject to this when much fatigued, arising, no doubt, from a relaxation of the joint ; and it is not unfrequent for colts to cut when they have not attained their full strength.

Remedies. - Many plans have been tried to remedy this defect, such as raising or lowering the inside and outside of the heel, as the case might require it. In some instances it has succeeded, and in others failed. In fact no principle can be laid down whereby to guide an operation for this defect. The most successful remedy hitherto discovered is to use a shoe of uniform thickness from heel to toe, so that the bearing may be perfectly level, and to put but one nail in, and that situated near the toe, inside of the shoe; this shoe to be applied to that foot which strikes the other. The greatest care must be exercised to prevent the shoe from extending beyond the hoof, besides having the crust a little rasped off the inside of the hoof. Great care must be taken that the shoe be equal in thickness on both sides, and also at the heel and toe, so as to equalise the bearing on both sides. Another method which sometimes succeeds when other methods fail is shoeing the animal with light plain shoes, having a 
small heel on the outside turned out thus, and no nails on the inside quarter.

When the defect arises from natural malformation of the setting on of the feet, many experiments have been tried to remedy this, as above stated; but

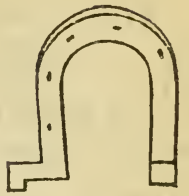
the most ingenious of these were instituted by Mr Moorcroft, by which he adopted a contrary practice to that above; he says, "If the inside of the foot be raised and the outside lowered, the supporting leg when in action will bring the body, and consequently the moving leg more to the side of the supporting leg, and hence more liable to be struck. On the other hand, if the outside of the foot be raised, the supporting leg will throw the body off that leg, consequently the moving leg will be further off the supporting leg, and hence less liable to strike." These arguments are good; but still there have been cases where the practice of these suggestions has failed to produce the desired effect.

But cutting does not always arise from natural defects, as it is often the consequence of unskilful shoeing. When this is the case, we usually find the upper edge, or that part which comes in contact with the crust, to be hammered inwards, leaving the lower edge on the ground side wider than the hoof, and projecting beyond it.

In purchasing a horse which manifests marks of cutting by callous thickening on the inside of the fetlock, it is safest to avoid the purchase, as it is most difficult to ascertain the true cause by inspection, unless the throwing outwards or inwards of the toes be considerable. Besides, as above stated, some horses will cut when tired, and others from a weakness of the fetlock. 


\section{SPRAIN OF THE COFFIN-JOINT.}

This disease is indicated by a sudden lameness; and a considerable heat exists round the coronet, as well as tenderness, which gives the animal pain when pressed upon. Sprain of the coffin-joint sometimes becomes a very bad complaint, in consequence of its being so often unobserved by grooms or even farriers, and it becomes deeply rooted before it is detected. Above all others, this complaint is frequently con. founded with lameness of the shoulder.

Remedies.-The first thing to be tried is hot fomentations: and afterwards a blister should be applied, and at the same time the animal should have occasional doses of physic. The following laxative may be given :-

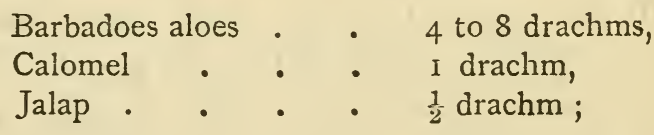

to be given in the form of a ball.

\section{RINGBONE.}

This is one of the worst species of lameness which is incidental to the horse. Whenever it exists, even in the slightest degree, it comes under the character of unsoundness, because it is so liable to increase; and when the bony deposit begins to enlarge, the lameness becomes incurable. This complaint commences in one of the pasterns, and usually near to the joint. It is liable to spread rapidly. When the first deposit is on and surrounds the lower pastern, emanating from a violent inflammation of the ligaments, a small enlargement or bony process is to be perceived immediately above the coronet (see Plate vi, fig. 8, 6). The hinr' 
feet are most liable to this complaint, as they are subjected to the greatest stress in the force required by them to propel the horse, and in this case the back part of the foot is most liable to be affected. But the disease is not of so serious a nature, being chiefly confined to the ligaments, and the bones have not been injured by concussion, to which the fore legs are peculiarly exposed; consequently, in them, diseases of the bones generally accompany ligamentary lameness.

REMEDies.-In the early stages of this complaint, when even a bony enlargement is visible on both sides of the pastern joint, or on one side only, and the enlargement is of but small extent, then there is a probability that it may be removed by the application of an active blister, or by the use of the cautery. But owing to the extreme action of these joints, the inflammation and the bony secretion rapidly spread, and then the joints become immovably fixed, or what is technically termed anchylosed.

\section{OSSIFICATION OF THE BACK SINEW.}

Ossification of the back sinew is very easily distinguished. When a healthy sinew is pressed upon with the thumb and two first fingers, from above downwards, it feels like a very tense cord, yielding slightly under the pressure, but springing back to its natural position the moment that pressure is removed. When the sinew is ossified, it is divested of that elasticity, so obvious to the touch of even the most inexperienced. It is true that in its incipient state it is not so readily discovered, as its bony texture has not been fully formed, although an expert veterinary surgeon will be able to detect it soon after it has begun to form. In the earlier stages of ossification of 
the back sinew, no lameness takes place, but it is certain to render the horse lame eventually, and that, too, for the remainder of his life. There is no remedy for this infirmity, but fortunately it is a rare termination of disease in a tendon. 


\section{CHAPTER VI.}

The Haunch and other Portions of the Hind Legs.

\section{FRACTURE OF THE TUBEROSITIES OF THE HAUNCH.}

THE portions of the haunch or pelvis which are most liable to injury or fracture are the tuberosities on the points of the various bones. It sometimes happens that those parts are fractured by a fall or heavy blow. When such is the case, the horse will walk lame, and swelling will take place in the contiguous parts, with a degree of heat, as inflammation is always an accompaniment of fracture. There are no mechanical means of bringing those parts together, to force a reunion and place them in their natural position; so that all we can do is to place the horse in slings and allow Nature to work her own cure. A large and strong adhesive plaster may be laid across the haunch, which will, in some measure, assist in keeping together and supporting the parts. It seldom, however, happens that the parts again adhere in their natural position, consequently deformity and lameness are entailed upon the animal for life.

\section{SPRAIN OF THE ROUND BONE.}

Symptoms. - The rounded termination of the femur or thigh-bone is in common language termed the round-bone. In a few isolated cases this part has been dislocated, and the rounded part which fits into 
the cavity of the hip-joint is fractured; but sprain of this part is more commonly the case. The indication of this is, that the horse drags his leg after him, with the toe only touching the ground as he walks. If the bone itself has been injured, heat and tenderness of the part will generally be manifested.

Remedies.-It seldom happens that strains of this joint are immediately relieved; and from the want of proper action the muscles of the limb waste away. A blister is the most likely thing to be of use, and the horse should not be subjected to any kind of work. Firing has sometimes relieved the complaint. Should this fail, the only thing that remains to be done is to put a charge over the joint, or insert a seton. Dress the seton with cantharides ointment. Change it weekly, and keep it in for three weeks or a month.

\section{SPRAIN OF THE STIFLE-JOINT.}

This joint is frequently sprained. When it does happen, heat and tenderness of the part will give an indication of it. Sometimes dislocation of the patella or small bone, answering to the knee-pan in man, occurs. This will be indicated by the horse dragging the limb after him, or by his resting it on the fetlockjoint, and being unable to flex the joints. The muscles of the inside of the thigh are sometimes sprained, which is known by the heat in all the contiguous parts.

REMEDIES. - For dislocation of the patella, the aid of a veterinary surgeon will be absolutely necessary. In case of muscles being sprained, fomentations must be used, accompanied by doses of laxative medicine, as recommended at page I 12. 


\section{THOROUGHPIN.}

In treating of wind-galls, we described certain little bags or sacs, containing a fluid for lubricating the joints. These, we mentioned, sometimes become inflamed and enlarged: similar sacs are situated contiguous to the hock-joint, and these also are liable to inflammation, and produce swellings of greater or lesser dimensions immediately under the strong tendon which unites with the cap of the hock. These swellings generally protrude on both sides, in the form of round swellings. Sometimes when the coverings of the tumour yield more readily in one direction than another, it will protrude principally in that part. The nature of the complaint is precisely the same as wind-galls, but from its situation has been termed thoroughpin (see Plate viI, fig. $3, e e$, and fig. $5, b, b$ ). These swellings generally produce stiffness of the joint, which sometimes wears off when exercise has induced absorption of the fluid which these bags contain. However, it is only when the sacs become large that they occasion any inconvenience to the action of the limb. Many persons think thoroughpin of but little consequence; but after a hard day's work, a horse will always manifest stiffness in the joint in which these swellings exist. And although a horse cannot strictly be called unsound which is afficted with thoroughpin, still a purchaser, when he notices these, should otherwise examine the horse with care, to discover, if possible, that they have not been induced by previous hard work, which will sooner or later tell on the animal.

Treatment.-The same remedies as recommended in wind-galls are applicable to this complaint. But 
there is no certain cure, and hard work is almost certain to make them reappear.

\section{CAPPED HOCKS.}

Capped hock consists of a swelling on the very point of the hock-joint, due to, in general, an infiltration of serum into the cellular tissue underneath the skin, but in some cases the synovial bursæ are also distended (see Plate vir, fig. 4, a). The tumour is soft and fluctuating. Lameness seldom accompanies capped hock, but nevertheless it is an awkward complaint, particularly in draught-horses, in consequence of this part being very liable to come in contact with the bar of a gig or carriage; and by causing pain to the animal, induces him to kick, and, if a high-tempered horse, often causes much mischief. It is generally produced by blows of some kind; but in many instances the injury is inflicted by the horse himself in kicking, and that when the animal is in harness, although some are in the habit of kicking while in the stall. It also occurs sometimes by the bedding of the horse being too thin, and the hock may be bruised in consequence.

Treatment.-If the swollen part be hot and tender apply the following :-

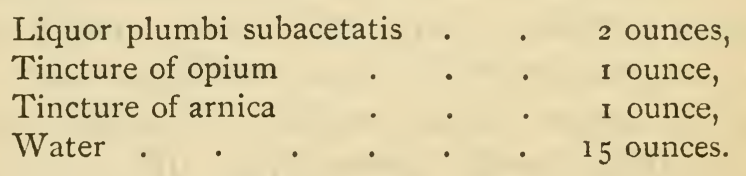

Apply in small quantity, and so as to keep the parts damp.

Sometimes the swelling disappears without any other means than rest being employed. But often capped hock becomes of a very large size, and callous in its structure, in which case the parts should be 
painted daily with tincture of iodine until tenderness is produced; or an ointment of biniodide of mercury may be rubbed in in small quantity.

If the horse caps his hocks by kicking at the stall, fix a large door-mat to each side of the trevis or partition, and when he kicks it and no sound follows, he frequently desists. A strap fixed round his pastern with a few links of a chain and wooden clog at the end of it, will frequently, by the clog striking the leg, cure him of his bad habit.

In purchasing a horse which exhibits capped hock, the whole of the part should be very carefully examined, in order to ascertain if there are any other bruises observable. His history should be obtained, if possible, and learn whether it has been the result of kicking. Horses that are given to kicking can seldom or never be broken from the habit.

\section{MALLENDERS AND SALLENDERS.}

These are scurfy eruptions, situate in the inside of the hock, a little way under the knee, sometimes even on it: the former appellation is given to those of the fore leg, and the latter to the hind leg. These seldom are accompanied with lameness, but if not attended to in time, they will ulcerate, when a thin watery humour will issue from them, which becomes difficult of cure.

Remedies.-The following ointment must be applied to the parts affected :-

$$
\begin{aligned}
& \text { Common tar . . . I ounce, } \\
& \text { Sugar of lead . . . } \frac{1}{2} \text { ounce, } \\
& \text { Lard . . . } 4 \text { ounces. }
\end{aligned}
$$

If the above does not stop the discharge in a week's application, then recourse must be had to the weak mercurial ointment. 
During the treatment of this complaint, the following laxative ball should be occasionally given :-

$\begin{array}{llll}\text { Barbadoes aloes } & \text {. } & \text {. } & 5 \text { drachms, } \\ \text { Castile soap } & \cdot & \cdot & 2 \text { drachms, } \\ \text { Oil of caraways } & \text { • } & \text {. } & \text { 10 drops. }\end{array}$

The cause of this complaint is in general owing to bad stable management and want of washing and brushing the skin.

\section{ENLARGEMENT OF THE HOCK.}

The origin of this is a sprain or inflammation, affecting generally the hock-joint, produced principally from some sudden or violent concussion; checking the horse abruptly when going at a rapid pace, or from the animal being compelled to draw a greater weight than his strength will enable him to do comfortably. Considerable lameness, with tenderness of the parts, always accompany this; but these are not so difficult to remove as in more limited inflammation. It will yield to rest and fomentation in general; but if not, it will be necessary to have recourse to firing, which generally proves effectual. However, it too frequently happens that the enlargement of the whole joint remains. But this will not incapacitate him for work; and if treated with ordinary caution, he may be worked during the remainder of his life without any increase of swelling or inflammation returning, although the parts around the hock-joint are altered in structure, and to a certain extent weakened. Although a horse may never afterwards exhibit lameness, still where permanent swelling of the hockjoint exists, he is, in point of fact, regarded as un sound; because, if he happen by necessity or accident to be subjected to extra hard work for a day or two, he is almost certain to become lame, and that too 
for life. Stiil, however, many a good and useful horse has enlargement of the hock.

\section{CURB.}

A curb is situated some inches below the point of the hock (see Plate vir, fig. $3, a$, and fig. $8, e$ ), and on a line with the head of the external small splint-bone (which head, if extra large, may be mistaken for a curb). It is caused by a sprain of the posterior straight ligament, or an injury (strain) of the flexor tendons at the same situation. When the limb is viewed from the side, a gradual puffing out of this part will be observable; and if it is at all large or inflamed, it is sure to be followed by lameness. If heat and tenderness of the part are perceived, and the horse is lame, it is certain that it has its seat in this spot.

The cause of curb, like other injuries of the hockjoint, proceeds from a sudden check, or over-exertion, and inducing extension of the ligaments, which bind down the tendons; or it may proceed, as stated, from an injury sustained by the sheaths of the tendons. This brings on inflammation, and frequently lameness ensues. It is most common to young horses, especially those which are cow-hocked-that is, with the structure of the hock and leg being like that of a cow, the hocks having an inward inclination, and the legs exhibiting a considerable outward angle. This formation of the limbs, it will be at once perceived, has the effect of inducing a continual stretch on the annular ligament, and thereby confining the action of the tendons.

When curb first appears it is generally accompanied by swelling and lameness : it is seldom, however, that the swelling is great, exhibiting generally a gradual 
curve, so little to be noticed that, when viewed from behind, it cannot be observed.

REMEDIES.-The first application should be cold, evaporating lotions, frequently used. These to consist of -

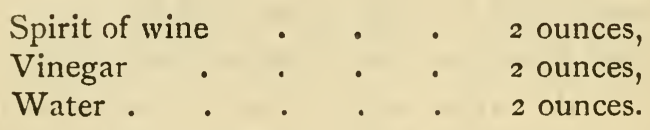

This to be often applied until the inflammation has subsided. Should the swelling and lameness continue, a blister of cantharides, or an ointment of biniodide of mercury should be applied. If these fail in restoring the animal to soundness, then the firing irons should be used, followed up by a blister; after which purgatives must be administered. The following should be made up in the form of a ball, and given :-

$\begin{array}{lllll}\text { Barbadoes aloes } & \cdot & \cdot & \cdot & 5 \text { drachms, } \\ \text { Castile soap } & \cdot & \cdot & \cdot & 3 \text { drachms, } \\ \text { Oil of caraways } & \text {. } & \text {. } & \text {. } & \mathbf{1 2} \text { drops. }\end{array}$

Long-continued rest is sometimes necessary in cases of curb, without which the lameness is liable to return upon the horse being worked.

Horses that have, or have had curb, are decidedly unsound.

\section{STRING HALT.}

This complaint consists of a convulsive or twitching action in one or more of the legs, and so evident is it in some subjects that it is perceptible to any one, however inexperienced he may be in the action of horses, but in other cases it is only observable when the animal is made to wheel or turn round quickly and set back. It is a complaint almost always confined to the hind legs, although in a few solitary instances it 
may be met with in the fore limbs. The animal lifts his leg high, suddenly elevating it towards his belly, and puts it to the ground with considerable force. Should both limbs be affected, then it gives the appearance as if the horse had very good and high action in his hind limbs, and many a purchaser has been deceived in consequence. This shows the utility of all possessors of horses having a general knowledge of the complaints incidental to this useful animal. String-halt may disappear after the horse has been heated a little, and apparently in some instances it is no detriment to him after he has been warmed; for we have seen and ridden some of the best of hunters which have had that affection, and which were first in at the death.

String-halt, pure and uncomplicated, is an affection of the sacro-sciatic nerve, and legally constitutes unsoundness, but practically it is, as a rule, a disease which neither impairs the animal's usefulness, nor limits his age. Owing to the peculiarity of the action of the limbs, it may be mistaken and confounded with the spinal and cranial disease, "shivering." Both are hereditary diseases, but the "shiverer" is always a bad subject, easily knocked up with work, always gets worse, and rarely lives above the adult period of life. String-halt requires no treatment.

\section{BONE-SPAVIN.}

A bone-spavin is an increased growth of bony consistence situated on the lower and most prominent part of the inside of the hock-joint (see Plate vil, fig. $9, a)$. A well-formed hock-joint gradually tapers down so as to unite almost imperceptibly with the soft or fleshy parts. In examining a horse, the hand should be passed over the inside of the hock in a downward 
direction, when horses having this injury will be found to have a little prominence near the lower point of the bone. This is an enlargement of the bone, and what is termed bone-spavin. It is a disease of the inner splint-bone, but in many cases ultimately it affects more or less all the small bones of the hock.

The true nature and causes which induce bonespavin cannot be properly understood without a thorough knowledge of the hock-joint, which we have represented on Plate vin, fig. I. In this figure the shank-bone is represented at $b$, and the two small bones behind at $g$ are the splint-bones; these support the lower layer of the bones of the hock. The cubebone, $f$, rest chiefly on the shank-bone, and in a slight measure on the outer splint-bone. The middle wedgebone, $e$, rests entirely upon the shank-bone, and the smaller wedge-bone rests in a slight degree on the shank-bone, but its chief support is on the inner splint-bone. From this arrangement the splint-bones support a very unequal degree of weight and concussion. The inner one is placed more under the body, and consequently nearer the centre of gravity, and besides nearly the entire weight and concussion is communicated to the little wedge-bone. Hence it is that during any violent action of this joint either in leaping, galloping, or under the pressure of a heavy draught, the inner splint-bone or its ligaments are injured. This is more especially the case with young horses before their joints have become firmly consolidated.

The shoeing blacksmith or farrier too often is the cause of inducing and increasing this complaint, by improper treatment of the feet. An erroneous notion has too long prevailed among smiths that cutting and wounds of the feet inflicted by the one foot treading 
upon the other can be prevented by adding a calkin on the outer heel of the shoe, which consists in the extremity of the shoe being bent, elevating the outer heel considerably above the ground, and thus the ligaments of the joints are subjected to an unequal strain, and especially those of the hock, which increases the tendency to spavin.

It is this which induces, in the first instance, inflammation of the cartilaginous union of the shank-bone, and this cartilage is absorbed, and a secretion of bony substance deposited in its stead; consequently the elastic action between them is terminated, and the splint inside the hind leg formed, and has the appearance of a tumour, when the head of the splint-bone is united with the shank, and always in front of that junction, as exhibited in Plate vir, fig. $3, c$.

Lameness generally accompanies spavin during its growth, but when the periosteum or membrane of the bone has been stretched, and taken the form of the bony enlargement, then the pain subsides, and with it the lameness. Many instances occur of horses with large spavins, and yet they are not much affected with lameness; sometimes having merely a stiffness of the joint, which is not apparent after the animal has had a little exercise. And, on the contrary, there are instances in which the bony deposit is very small, and yet the horse is very lame, so much so as to render him comparatively useless. In all such cases it will be found that the growth, large or small, or entirely awanting, is associated with ulcerative disease of at least two of the small bones of the hock, and removal of the cartilage which clothes and protects their gliding surfaces. The worst form of spavin (or so-called occult spavin) is characterised by an absence of bony growth. It must therefore be evident that we ought 
carefully to examine horses with suspected spavin before they have had exercise, and as soon as they have left the stable.

Sometimes spavin continues to enlarge considerably, and spreads over the lower wedge-bones (Plate vir, fig. $8, e$ ) in consequence of these being nearest the original seat of the enlargement. These bones are capable of a small degree of motion, and participate in every action of the joint, but their chief office is to prevent concussion. The principal motion of the joint is in the tibia, $b$, and the astragalus, $c$, and consequently stiffness more than lameness may accompany spavin, even when the small bones of the joint are affected. From which also it will be seen that there is a manifest advantage in each of these bones being provided with a separate ligament and membrane, and thus, as it were, constituting so many separate joints; so that any of them may sustain injury without its being communicated to the rest. It is not uncommon for the bony deposit continuing to enlarge, and embracing the second series of bones, enveloping the larger wedge-bones, $d$, and extending to the cube-bones on the other side; and even then the lameness may not be so great as to prove very injurious, for this reason, that the motion of these two joints, or rather parts of the joint, is small; but when it reaches to the union of the tibia, $b$, and the astragalus, $c$, when the joint in which the principal motion of the joint is affected, then the lameness is of a very serious kind, and the horse may be considered as no longer fit for use.

Although spavin unfits horses for active employment, yet for farm purposes they need not be rejected, especially by those who possess limited farms; for slow draught and other agricultural purposes they 
will be found quite useful, and from this sort of work, in which quick motion is not required, the horse may improve, and even the bony matter may be absorbed to a considerable extent.

One strong reason why spavined horses do not improve is that they seldom lie down, as they are conscious that they will suffer considerable pain in the act of rising again ; so that the continual pressure and weight of the body upon the limb keeps alive the exciting cause.

There is sometimes an enlargement of the heads of the bones of the leg, which may be mistaken for spavin.

REMEDIES. - Spavin can only be successfully treated, if at all, by blisters frequently repeated, which may induce an absorption of the bony deposit, or at least a diminution of the ligamentary inflammation. When this fails, then recourse may be had to the cautery, but no horse should be subjected to the suffering implied in blistering and firing until the seat of lameness and the character of the disease has been decided by an experienced veterinary surgeon.

\section{BOG-SPAVIN}

Is the name given to a distension of the capsule of the true hock-joint. This distension is of frequent occurrence in cart horses, young and old. It may be due to conformation, debility, want of exercise, and entire absence of disease of the joint, or it may be dependent on an injury, and associated with more or less congestion and inflammation (synovitis). Bogspavin constitutes a legal unsoundness, but in the majority of cases the animal is practically a sound animal, and in not a few the swelling of the hocks disappears. It may be a grave malady, or a condition 
of little importance, and the experienced veterinary surgeon is the individual who alone can decide the point.

REMEDIES.-In those cases where from conformation (straight hind limbs), the capsule of the joint appears conspicuous, no treatment should be attempted, as it is an impossibility to alter the shape. On the contrary, where the fullness of the hock or hocks is dependent on debility or want of exercise, tonics, good food, and regular exercise will be attended with benefit. An india-rubber bandage to give pressure is also of use. If the swelling persists, apply the following with a stiff brush, and repeat daily for some time, viz. :-

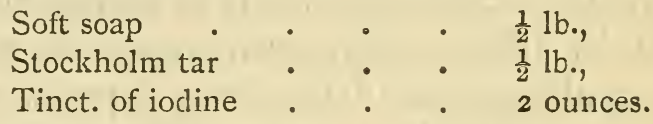

In those cases in which the distended capsules and increased synovial secretion is associated with pain and lameness, complete rest should be given, the hock-joints fomented, and thereafter rubbed with hot camphor and arnica lotion. After the inflammation has subsided stimulating embrocations and blisters may be employed, but their application should be under the guidance of the veterinary adviser.

\section{OF SWELLED LEGS GENERALLY.}

SWELliNg OF THE CELLULAR SUBSTANCE.

Both the fore and hind legs of horses are liable to considerable swelling, but the latter are most subject to be thus affected. Frequently, when a horse seems to be affected with no other disease, the hind legs will suddenly swell to a very great extent from the hock to the fetlock, and in some instances even from the stifle downwards. This is accompanied by heat and ex- 
treme tenderness of the skin, inducing lameness of a peculiar character. A quickened and hard pulse are usual concomitants of this seizure, with a considerable degree of fever. This complaint is acute inflammation of the cellular substance of the limbs, being sudden in its attack, very violent in its degree, is consequently attended with the secretion of a quantity of fluid on the cellular tissue. Young horses, and those which are over-fed, with little exercise, are most liable to be thus attacked, and without having had previous inflammation.

REMEDIES. - If accompanied by fever, the following diuretic should be given :-

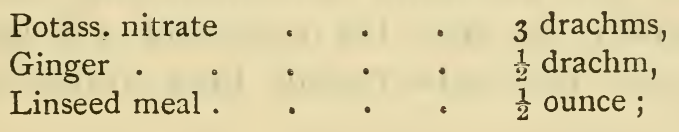

made into a ball with common syrup.

Twelve hours afterwards give the following purgative, in the form of a ball :-

$\begin{aligned} & \text { Barbadoes aloes } \\ & \text { Castile soap } \\ & \text { Oil of caraways }\end{aligned} \quad \vdots \quad: \quad \begin{aligned} & 5 \text { drachms, } \\ & 1 \frac{1}{2} \text { drachm, }\end{aligned}$

Also use fomentations, and in most instances the swelling will subside as rapidly as it appeared.

\section{SWEILING FROM INACTIVITY.}

The most troublesome, as well as the most frequent swelling in the limbs, is that caused by inactivity, from high feeding and want of due exercise. One kind is accompanied by actual or comparative debility, or loss of power in the part affected. Those horses which are over-fed, without exercise, are liable to swellings in the limbs from the parterial caillary 
vessels having sent forth an over-portion of fluid to the extremities, and in consequence of the want of muscular exertion and the perspiration naturally connected with it; and the fluids having accumulated in the extremities in consequence of the vessels not having sufficient power to return them. The heart is thus acting upon an additional quantity of fluid; while by the want of exercise, the limbs are deprived of that power by which the fluids are returned.

Remedies. - The above physic and diuretic should be administered. These will lessen the quantity of fluid; also exercise the horse freely, which will increase the perspiration externally, and diminish it internally, and the veins and absorbents will attain more activity, so that the complaint will gradually subside, and the limbs resume their wonted appearance.

\section{SWELLINGS AFTER GRASS.}

It is a common occurrence for horses just taken in from grass to be seized with swelling in the legs. This is occasioned by the difference of food, owing to its containing a greater proportion of nutriment, which naturally increases the quantity of the blood, and the want of that exercise which is necessary to carry it off by the skin. It will therefore be quite apparent that exercise and a little opening medicine will effect a cure.

Horses may also have swelled legs from general debility. The proper quantity or quality of food may induce this, or it may proceed from disease that has reduced the strength of the animal system. The limbs, being the most remote from the centre of circulation, first exhibit loss of power ; and this is mani- 
fested by swelling, in consequence of the accumulation of fluids in them.

The cure in this case would be to give such diet and tonic medicines as would tend to invigorate the system, as well as to administer mild diuretics.

Horses are liable to swellings in the limbs in the spring and fall of the year. This can be accounted for by the principal activity of the circulation being employed in preparing a fresh covering of hair at those periods, so that the vital influence in the extremities is somewhat diminished, and the same cause as above explained produces swellings in the legs. Administer diuretics to diminish the quantity of the circulating fluid, and give cordials to strengthen the system.

Swelling of the legs is also common to horses which are used for hunting and pleasure only. This, it will be seen, arises from irregularity in their habits, one day having a more than sufficient exercise, and probably standing for days or weeks in a stable, and only walked out or trotted for a short distance. In such cases the limbs should be well rubbed down every day, both morning and evening, so as to stimulate the vessels to activity. Pressure by means of bandages will also be found to have a powerful tendency to promote the circulation. It is too much the practice of grooms to give diuretics in swellings of the limbs, as these, by being frequently repeated, are sure to weaken the urinary organs and produce an incurable debility.

\section{GREASE.}

This is a disease of the skin of the heel, sometimes in the fore feet, but most commonly in the hind ones. The disease is too frequently the effect of washing the limbs with cold water while they are over-heated from 
exercise, and allowing them to dry of their own accord ; the consequent reaction after the application of cold being very great, produces inflammation. Another cause is taking a horse into a warm stable in winter, when the legs have been chilled by excessive cold from standing.

Grease is not contagious, yet it has been known to affect all the horses of a stable at the same time, after one has been seized with the complaint. This can only be accounted for by bad stable management. The skin of the heel is considerably different in its texture from that of other parts of the legs. The fetlock is subjected to a greater degree of motion and friction than any other joint, and it is provided with a soft unctuous matter to keep it from chapping or excoriation, which can be easily felt to be greasy to the touch. When inflammation ensues, this greasy exudation stops, and the heel exhibits a red, dry, and mealy appearance; and in consequence of the continual motion of this joint, cracks soon make their appearance; these increasing considerably, present an entire mass of sores, which ulcerate very much and assume a fungus-like appearance.

It is easy to account for the tendency of the skin of the heels to violent inflammatory attacks, which arises from its being so remote from the centre of the circulation, and also its constant exposure to extreme variations of temperature. In the first place, when standing in the stable the feet are subjected to a great degree of heat, from being at all times surrounded with straw, and then when the door is opened his heels, in many instances, being close to the door, are exposed to any cold draught which may rush in ; and then he may be taken from the stable to the open air and walked through soft and cold mud after being 
over-heated, and thereby chilled. It is easy then to see that they are constantly liable to inflammatory attacks by being thus exposed to sudden transitions from one temperature to another, and hence the difficulty of subduing those attacks of inflammation to which the horse is liable.

Farmers' and carriers' horses, and indeed those in general which are worked in carts, are not so liable to grease as riding and carriage-horses, being less exposed to those extreme transitions from heat to cold, or vice versâ. And the hair, which is usually allowed to grow plentifully at the heels, is a great protection against sudden changes.

REMEDIES. - The first thing to be done is to wash the heel well with soap and warm water, and remove as much of the white scurf as possible. When it has become perfectly dry, which should be accomplished by rubbing, then the following ointment should be applied until the parts are healed :-

$\begin{array}{ll}\text { Lard } & \text { I ounce, } \\ \text { Sugar of lead, well pounded } & \text { I drachm. }\end{array}$

In cases where cracks appear, the treatment will greatly depend upon their extent and depth: when they are small, the following lotion will be found beneficial :-

Blue vitriol (or sulphate of copper) 2 drachms,

Water . . . . I pint ;

or four drachms of alum may be substituted for the vitriol, with the same quantity of water. This will speedily dry them up and close them. When the cracks are large and deep, and discharge an ichorous matter, accompanied with considerable lameness, a poultice of linseed meal will be indispensable; but when the discharge is thin and accompanied by a 
fetid smell, it will be necessary to mix an ounce of finely powdered charcoal with the linseed meal, as it is a powerful antiseptic. The poultices to be applied until the inflammation has abated, and a thicker and more healthy matter flows from the cracks. After this apply the following ointment :-

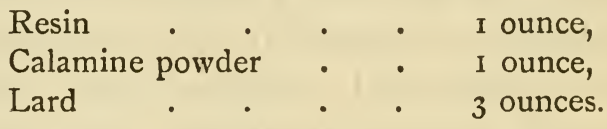

Let the resin and lard be melted together, and when sufficiently incorporated, allow the mixture to stand until nearly cold, then add the calamine, and stir them well together. This precaution is necessary, as the calamine would fall to the bottom if the mixture were thin. During the time of the above application, the cracks should be frequently washed with the solution, which will stimulate them to heal more speedily. Administer the following diuretic :-

$\begin{array}{lllll}\text { Turpentine } & \cdot & \text {. } & \text {. } & \frac{1}{2} \text { ounce, } \\ \text { Ginger } & \cdot & \text {. } & \text {. } & \frac{1}{2} \text { ounce, } \\ \text { Purified nitre } & \text {. } & \text {. } & \text {. } & \frac{1}{4} \text { ounce, } \\ \text { Linseed meal } & \text {. } & \text {. } & \text {. } & \frac{3}{4} \text { ounce. }\end{array}$

Three hours afterwards let the following laxative be given :-

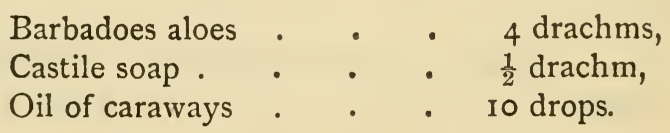

When the cracks have been healed up, the legs will sometimes continue swelled. In this case a bandage will be found of much advantage. But nothing is better than turning the animal out to grass, if the season of the year is favourable. Frequently the cracks spread over the whole heel and fetlock, or up the limb, as is sometimes the case; and while the 
leg is considerably swollen, accompanied by a discharge of a thin watery matter from the cracks, and makes its appearance also in other parts of the limb, accompanied by great heat, so much so that the heels smoke, the skin being so hot that the watery fluid partly evaporates as it oozes from the cracks or through the skin.

In the above state it would be attended with very bad consequences to stop the discharge suddenly. The first thing to be attended to is to allay the inflammation, and nothing is better calculated to do so than poultices, as already recommended, particularly those of carrots. When the heat, tenderness, and stiffness have abated, then the astringent, already recommended, should be used; but that made of alum, or a strong decoction of bark, are the best. Indeed, it would be preferable to use these lotions alternately, but not a mixture of the two. The ointment above named should be used for dressing the cracks, and as soon as the horse can bear the pressure without pain, a flannel bandage should be applied, extending from the coronet to some inches above the swelling.

From this period mild doses of medicine and diuretics should be given, accompanied by a third part of cordial mixture, which is composed of the following ingredients :-

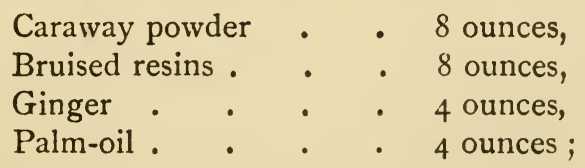

to be well beaten into a pulp.

If the horse is of a full habit of body, physic should always be given before diuretics, and in some instances it will be prudent to abstain entirely from 
giving the latter; but in cases where the animal is much debilitated, diuretics with the above pulp will be preferable. It will also be necessary to attend carefully to the feeding of horses at this time. Green meat will be found the best, and carrots are still better, and a very moderate quantity of corn, so that the tendency to fever may be kept down. The next essential is proper exercise, and care must be taken not to overdo it; and when the animal has been walked for some days, he may be afterwards exercised at a gentle trot.

There is a much worse kind of grease than that which we have just described and prescribed for, although it is not so common. In this species the ulceration spreads over the skin of the heel and the entire fetlock, and a highly sensitive fungus protrudes from both, mixed with scales: it is irritable in the extreme, and bleeds on the slightest touch. In a short time this fungus assumes a covering of a horny texture, projecting in the form of knobs and in congregated bunches, which, from their peculiar similarity to grapes, have acquired that name. From the entire surface of this callous substance issues a discharge of a peculiar fetid matter. In this complaint the horse suffers great pain and uneasiness, and quickly loses flesh. It would not be safe for any one but a veterinary surgeon to attempt a cure for this loathsome complaint. 


\section{CHAPTER VII.}

\section{Diseases of the Foot.}

The diseases of the feet in horses are more numerous, and of a more complex nature than the inexperienced can have any idea of. The structure of the foot is very complicated, and having to sustain the weight of so heavy an animal, besides being exposed to the chances of many injuries, a knowledge of these is of paramount importance, both as regards the safety of the proprietor and the intrinsic value of the animal. When, therefore, we arrive at the anatomical description of the horse, we shall dwell at some length upon the structure of the foot as well as of the pastern.

\section{ACUTE FOUNDER.}

Inflammation of the foot has been denominated "acute founder." It is the cause of many other diseases, and is more or less connected with them all.

Inflammation of the sensible lamellæ, or fleshy plates on the front and sides of the coffin-bone, is the cause of acute founder. These lamellæ are thickly intersected with blood-vessels, like every other vascular part; and, consequently, from their exposure to violent and long-continued action, they are elongated and strained, more especially when the hoofs have been subjected to a day's journey on a hard road. It is easy to imagine that if, after the feet 
have been thus heated, they should be washed and not immediately and thoroughly dried, or the animal allowed to stand, with his feet exposed to a cold draught of any kind, that inflammation is likely to ensue from this sudden change of temperature. This observation is equally applicable to the change from cold to heat.

Symptoms.-Inflammation in the feet is manifested by restlessness and fidgety action of the fore legs, frequently shifting the weight of resting from the one to the other, and the exhibition of great unwillingness to "set back" when made to do so. But there is no appearance of pawing, or of elevating the hind legs towards the belly, as in colic and other intestinal complaints. The pulse will rise to a pretty rapid state, the flanks will heave, and the inside of the nostrils will become very red, his countenance will exhibit an anxious expression, and that he is suffering pain will be indicated by moaning. $\mathrm{He}$ will then scrutinise his litter, as if indicating a wish to lie down, but will not do so immediately, from an apparent fear of drawing his limbs together. He still continues to shift the pressure of his weight from one foot to the other, until impelled by pain and fatigue he at length lies down. There is a marked distinction in respect to his lying down between this and inflammation of the lungs, for in the latter complaint the horse never lies down until he drops under the influence of complete exhaustion. When a horse which is affected with inflammation of the feet lies down, he invariably rests quietly, because the pain is so much relieved by the removal of the weight of his body; whereas in colic and inflammation of the bowels, he kicks and rolls about violently, and his constantly looking at the part affected as clearly shows the seat of the disease. A 
little attention to these will soon point out to the inexperienced where the malady exists.

After these symptoms have been manifested, the first thing to be done is to examine the foot, when it will be found very hot. Tap gently on the foot with a hammer, and the horse will express pain at the same time. The effects of inflammation in the foot are very rapid, and but a short time will elapse before the horse will be quite unable to get up, unless some means are adopted to check the progress of the complaint. Even when horses have been forced up while thus afflicted, they have been known to fall down immediately, from the intensity of pain which they suffered by resting on their feet.

REMEDIES. - If the animal attacked is robust, and the pulse hard and full, blood may be abstracted from the jugular vein. After this large poultices of linseed meal and bran should be applied, so as to cover the entire foot and pastern, and these ought to be frequently renewed, and every three hours have hot water poured over them. The shoe should be removed, and the sole pared as thin as possible. This operation should be done as gently as possible, as in founder the pain experienced from the excessive inflammation is very great. This having been accomplished, recourse must be had to very mild purgatives. The following may be given in the form of a ball :-

$$
\begin{aligned}
& \text { Barbadoes aloes . . . } 4 \text { drachms, } \\
& \text { Calomel . . . . . I I drachm. }
\end{aligned}
$$

Inflammation of the foot is always accompanied by intense fever, and there is a danger of the inflammation shifting from the feet to the bowels or lungs; as it is no uncommon occurrence for horses 
labouring under inflammation of the feet to have that suddenly transferred to the lungs, or vice versâ. In such a case it might, and indeed often proves fatal to the animal. It is therefore probably safer to have recourse to sedative medicines at first. The following febrifuge and sedative drink may be given :-

Liquor ammonia acetas

Nitric ether

Tincture of aconite

Water

$$
\begin{aligned}
& 4 \text { ounces, } \\
& 2 \text { ounces, } \\
& \text { I } 5 \text { drops, } \\
& \text { I pint. }
\end{aligned}
$$

Repeat this every eight hours until the temperature (fever) falls to normal, IO $^{\circ}$ Fahrenheit.

It should on no account be attempted to force a horse to rise, as the animal will not lie longer than is necessary for his recovery, and every symptom of lameness should be gone before walking exercise is attempted. A month or two's run at grass will be attended with much advantage after founder.

No disease is more to be dreaded than violent inflammation of the foot, as, even with the utmost attention to remedy the complaint, very bad consequences result from it. One of these, of frequent occurrence, is loss of the hoof. The first symptom of this is the appearance of a small separation between the coronet and the hoof. Great attention to this is necessary, as the horn thus separated will never reunite with the parts beneath, but the separation will continue to extend downwards, until entire disunion is effected and the hoof is ultimately lost. This is a most serious affair; for although a new hoof will be formed, the pedal bone having descended can never be replaced, and in consequence the sole of the hoof is convex instead of concave, 
can bear no weight, and the mode of progression is changed.

The treatment of founder is so difficult and its results so serious for the poor animal, that apart from pecuniary interests, it should only be entrusted to a veterinarian, if one can be found.

\section{CHRONIC FOUNDER.}

Chronic founder is merely a conventional term, adopted by farriers to express those changes which take place in the foot of the horse in disease brought on by bad shoeing or mismanagement in some way or other. In fact, it is a word too frequently used as a cloak by ignorant pretenders.

\section{CONTRACTION.}

To enable persons to judge of the perfect and healthy state of the foot of a horse, they should examine those feet of young horses in the natural condition which have not been shod or worked in any way. We have given a representation of the sole of a sound foot (Plate VI, fig. I2). It will be seen that it is very nearly circular, and is somewhat widest towards the quarters; the inner one is a very little wider than the outer. This form, however, seldom continues long; for the foot increases in length and gradually becomes narrow in the quarters, more especially at the heel, when the frog becomes contracted. The entire foot assumes a greater concavity, and the heels become higher. To those unacquainted with the different parts of the sole, we refer to the above figure for their form. The crust is represented at $a a$; the sole, $b$; the bars, $c c$; the frog, $d d$.

Contraction may exist without inducing lameness; 
nor does shoeing always promote this narrowing of the foot, although it is in many instances the source of contraction when unskilfully performed.

CaUse.-The cause of contraction, or rather alteration of the shape and size of the horse's hoof, is one which has given rise to much discussion. Some veterinarians deny that it ever is a cause of lameness, and at the worst it is but a symptom of a diseased condition. The feet of any and all breeds of horses, if shod with heels on their shoes, become contracted, because the frogs (whose function it is to keep the heels open) are removed from pressure, and coming in contact with the ground. As the frogs atrophy the heels "wire in," and the animal has undoubtedly in time a foot with small frogs and small heels, as compared to what they were before he first wore shoes with heels; but take off the shoes and turn the animal to grass, or shoe him with "Charlier shoes" (which have only a small web and no heels), and the heels and frogs will come back to their normal shape and size.

It is a grand mistake for persons allowing the shoes of horses to remain on longer than three weeks or a month. Whether the shoes are worn or not, they should be taken off, and the toes of the feet shortened, and the heels lowered with the rasp. Without this precaution the feet must become malformed. I have known instances where ridinghorses have been so seldom ridden or exercised, that their shoes have lasted three and even four months, and were not removed during this time, proceeding in some cases from the parsimony of the owners, and in others from their ignorance of the evil effects which would result therefrom. In some instances the shoes were made thicker than was 
necessary, consequently the animal subjected to carrying superfluous weight, besides being tightly fastened on and provided with an extra number of nails, and these too at the quarters. All of which increase the tendency to thickening. It is perfectly clear that without due attention to the above points, in proportion to the degree of neglect, so will the evil consequences increase, and the hoof must contract and close up the foot round the coronet. It is a notorious fact that low-heeled horses are seldom liable to contraction of the hoof.

Nothing can be more injudicious than to remove the bars, as they are a grand protection against contraction, their use being principally to prevent wiring $i n$, so that cutting them away is certain to facilitate and greatly increase the contraction after it has begun; but we must not have it supposed that the removal of the bars of themselves would produce this tendency.

It has been said that thrushes are often the cause of contraction, but they are more frequently, if not altogether, the consequence rather than the cause.

Many persons are disposed to have an undue objection to contraction, and at once reject a horse that exhibits the slightest degree of wiving in of the quarters. There can be little doubt but this is a malformation of the hoof; but one thing is certain, that its growth is very slow, the altered form extremely gradual, and the parts are progressively accommodated to the change of form.

\section{SAND-CRACK.}

This is a downward crack, or division of the hoof, as represented on Plate vi, fig. 8, a. It may occur either in the fore or hind feet. In the fore 
feet they are usually found in the inner quarter, although they sometimes exist in the outer quarter. The reason why the quarters are most liable to this is, that the chief stress of the foot is where it expands, and the inner quarter is weaker than the outer. Sand-crack almost always occurs in the front of the hoof in the hind feet, occasioned by the pressure applied by the toe in progressive motion, especially when the animal is dragging a heavy load behind him, and more particularly on an ascent.

Hoofs that crack in this manner are brittle in the crust, which is an extremely bad defect, and is caused by a want of that nutriment necessary to keep the crust supple. It also proceeds from disease in the foot. It may besides occur from a false step or over-exertion, or galloping on hard ground.

Sometimes the crack is only superficial, and does not extend through the hoof, in which case it occasions no lameness. It will, however, require attention, as, if neglected, it may reach the quick, and occasion much trouble.

Remedies. - When sand-crack is occasioned by brittleness of the hoof, let the following composition be rubbed into the crust twice a day until it becomes pliable :-

\section{Oil of tar}

Common fish-oil

2 ounces,

4 ounces.

In a superficial crack it should be pared and rasped entirely out; and if it has been of considerable depth, it ought to be strengthened by a coating of pitch, applied so thick as to replace all that has been rasped off, and formed so as to represent the natural shape of the foot. Indeed, rasping ought to be had recourse to with every crack, so as to 
ascertain its depth. Should it be found to penetrate through the crust without occasioning lameness, and is situated low down on the foot, recourse must be had to a red-hot firing iron, which should be drawn across the hoof, both above and below the crack, so as to prevent it from extending. The edges ought thereafter to be thinned, to prevent any pressure which might cause pain to the tender parts below; and it must be bound up, taking care to avoid pressure immediately beneath the crack. Neither must the shoe be allowed to press upon the part below.

Should lameness proceed from the crack having penetrated through the hoof, the cure is much more difficult. The first thing to be done is to examine the parts, to ascertain whether any sand or dirt has got in and has reached the quick below, and the crack thoroughly cleaned out. If proud-flesh is found in it, an application of chloride of antimony must be used to destroy it, and the edges of the crack must be considerably thinned. Some veterinary surgeons have used the cautery; but this has a tendency to thicken the edges of the horn and render it rough, which is liable to irritate the tender portions below. After the fungus has been destroyed, it will be necessary to apply the iron above and below, as recommended in a superficial crack. A pledget of tow should be put into the crack, and another over it, and then firmly tied down, and not examined until the third or fourth day after. If the proud-flesh has not been eaten away, it will be necessary to apply the caustic again. On the other hand, if the crack is quite dry and exhibits a hard horny crust, the pitch-plaster should be immediately applied. Bees'-wax is considered prefer- 
able by some, and the crack carefully filled with it. The wax must be applied warm; and to fill the crack properly, it is necessary to draw the heated cautery slightly over it. This gentle pressure will contribute to the healthy granulation of fresh horn, and at the same time prevent the access of sand or dirt, and also cold or wet, or the influence of the atmosphere from reaching the tender parts of the foot.

The worst case of crack is caused by tread, which divides the coronet; and where this is imperfect the horn or crust will grow down divided, because the growth of horn proceeds from the coronary ligament. The mode of cure which has been most successful in this crack has been to draw the heated cautery over the division of the coronet. This will be followed with some degree of inflammation; a scab will form on the part, which in a few days will fall off, when it will be found that no traces of the division will be visible, and fresh and sound horn will speedily grow downwards. When division of the horn at the coronet takes place, it requires five or six months to effect a cure. When the horn has grown downwards for an inch, the animal may be turned out, taking care that the part is well defended by a clambered pitch-plaster, which should be replaced by a new one as often as it separates from the hoof. At the same time a clambered shoe should be applied, but it must not press upon the hoof immediately under the crack. During the time the horse is subjected to surgical treatment for this complaint, he must have perfect rest, otherwise the cure will undoubtedly be protracted.

In purchasing a horse, it is absolutely necessary to examine with care the inner quarter of the feet, 
as it is very common for low, unprincipled dealers to cover over sand-cracks so neatly with pitch, and afterwards along the whole hoof, that it is so completely concealed, as not to be observable except by a narrow inspection.

\section{THE NAVICULAR-JOINT DISEASE.}

Behind and beneath the lower pastern-bone, and behind and above the heel of the coffin-bone, is placed a small bone, called the navicular bone. (See Plate vi, fig. Io, e.) The use of this bone is to support and strengthen the union between the lower pastern and the coffin-bone, and to assist the flexor tendon in its action as it passes over it, in order to be inserted into the bottom of the coffin-bone, and forms a sort of joint with that tendon. The navicular bone is subjected to much pressure, as is also this tendon; and besides, there is much play between them in the bending and extension of the pasterns.

CAUSE.-Like many other complaints of the horse, this is often induced by sudden and violent exercise after the animal has been allowed to stand in the stable inactive. The parts not being for some time adapted to overstrained action, there may be too much play between the bone and the tendon, and by concussion of the parts the synovial membrane which covers the tendon and forms the bursæ may be bruised; or the cartilage of the bone may be inflamed, and thus produce destruction of it, and cause a lameness of the most painful description. From the navicular bone being so obscurely situated, it is difficult to ascertain by inspection when it is diseased. And this has puzzled many to find out the cause of lameness 
emanating from it, and has too often been attributed to the shoulder. Indeed, the action of the horse with this lameness has all the appearance of being seated in the shoulder. Of late years the attention of veterinary colleges has been particularly directed to this point, and it has been found in numerous cases of dissection that this is the seat of this lameness, which has deceived and puzzled so many persons, both learned and unlearned. The membrane covering the bone has been found highly inflamed, and the cartilage itself completely ulcerated, and even the bones in a state of decay. Besides this, bony adhesions have often taken place between the navicular bone and the pastern, which consequently render this joint altogether unserviceable.

REMEdiEs.-Like some other diseases incidental to the horse, the cure of this is very uncertain. A poultice should be applied to allay the inflammation, and the bowels should be kept open. The following purgative should be given in the form of a ball, and repeated in a week:-

$\begin{array}{lllll}\text { Barbadoes aloes } & \cdot & \cdot & \cdot & 5 \text { drachms, } \\ \text { Castile soap } & \cdot & \cdot & & 2 \text { drachms, } \\ \text { Oil of caraways } & \cdot & \cdot & \cdot & 8 \text { drops. }\end{array}$

The ball should be formed with a little linseedmeal and treacle.

If this complaint is discovered at its commencement, and before ulceration of the articular cartilage has taken place, a cure may be effected; but, on the contrary, if the cartilage is ulcerated before remedies are applied, the difficulty in eradicating it will be exceedingly great, if not impossible. Caries of the bone is certain to take place, and 
that condition can never be changed. Blistering the coronet has been found beneficial in assisting a cure, by removing the inflammation. Setons passed through the part, as contiguous to the seat of the disease as possible, have either lessened the complaint or entirely removed it.

But this disease is altogether of so delicate a nature, that we would not advise its cure to be attempted by an unskilful person, as it is one of those which can only be successfully taken up by an experienced veterinary surgeon.

As in all other inflammatory diseases, there is heat in the seat of the navicular bone, and probably in the whole foot. In this complaint the animal suffers much pain, to alleviate which we would recommend neurotomy, or cutting out a portion of the nerve, which has in some instances very much reduced the lameness. But at all events it will lessen the sufferings of the horse. In cases of extreme lameness, either with or without contraction, then it would be injudicious to resort to this operation; because, if there was ulceration of the membrane or decay of the bone, the increased friction, in consequence of the freer action given to the parts, the feeling of pain being removed, would cause these to progress more rapidly, until complete disorganisation of the foot took place; or in all probability the tendon itself would be gradually worn through by rubbing against the roughened surface of the decayed bone.

\section{FALSE QUARTER.}

When the coronary substance is severely cut or divided, and has sloughed off, it is termed false quarter. In some instances it is destroyed by the 
application of caustic applied to other wounds and sores of the foot; when this is the case there will be a division of the horn as it grows down, having all the appearance of a sand-crack, or one portion of the horny substance will overlap the other. This is a very bad defect in the foot, and often occasions lameness, and there is very great difficulty in curing it. The coronary substance is that by which the horn of the hoof is secreted; consequently what must be first attempted is to restore it to the discharge of its healthy function.

Remedies. - Caustic has been used in many instances, and found effectual; but nevertheless it is a dangerous remedy, as it has often proved very injurious. Blisters have also been successfully tried, but they are too often found not to be sufficiently active. The remedy which has proved most successful is the heated cautery carefully applied to the injured part. The edges of the horn on both sides of the crack should be thinned down, and a thick plastering of pitch spread over the parts so as to hold them closely together, as well as to support the hoof. This plaster to be kept on undisturbed for at least fourteen days, and then the parts should be carefully examined, to ascertain the condition of the coronet, and whether union of the parts has taken place. Should adhesion not be begun, then it must again be covered up and not looked at for eight days, by which time it will have adhered. During this treatment it would be judicious to strengthen the hoof by the use of a bar-shoe, only great care must be taken that there is no bearing at, or immediately below, the separation of the horn. To secure this against such a result, if the crust be naturally thick, accompanied 
by strong quarters, then a little of the crust near the part should be pared off, to prevent it from resting on the shoe. On the other hand, if the hoof be weak, an inclentation should be made in the shoe itself opposite the part, which will prevent any stress, as well as the danger consequent upon a sudden or violent concussion, which might have the effect of again cracking the hoof before it had got firmly united.

In this complaint the horn sometimes grows down entire, but from an unhealthy action in the coronary substance, it secretes a narrow slip of horn, generally different in appearance from the other parts, usually of a lighter colour. Although this is the case, it may become perfectly strong, but it must always be considered as a defect, and clearly showing that the horse has had sand-crack, and of course predisposed to a return of it. The horse may be fit for all kinds of work, yet there generally will remain some degree of tenderness in the part, and may produce lameness when the shoe is allowed to press on it; or when the horse is subjected to hard work, lameness may return.

\section{TREAD, OR OVERREACH.}

This is nearly connected with false quarter, and comprehends wounds and bruises of the coronet, usually the effect of the horse either setting one foot on the other, which not unfrequently happens in the hind feet; or in the fore feet, by the hind foot overreaching the heel when in rapid action, and wounding it.

Remedies.-Although this is not in general a very serious injury, yet it should be immediately and carefully attended to. The first thing is to wash out 
any dirt or sand which may have got into the wound, and dry the part thoroughly with a cloth. After which a pledget of tow, wetted with Friar's balsam, should be firmly bound over the wound, which usually proves a speedy cure. If the wound is large or deep, then it may be a necessary precaution to poultice the part for one or two days before digestive ointment or Friar's balsam is applied.

It sometimes happens that a soft tumour will form on the part. This must be poulticed and brought to suppuration. When the whole of the matter has been discharged, the sides of the ulcer should be washed with a weak solution of blue vitriol (sulphate of copper), which will have the effect of expediting granulation of the parts; and a pledget of tow which has been dipped in Friar's balsam will soon effect a cure.

Ignorant farriers are frequently in the habit of applying caustic to wounds of the feet. This should on no account be permitted, as it is very apt to injure the coronary substance so as to render it incapable of afterwards secreting healthy horn.

\section{QUITTOR.}

A wound of the coronet, whether it proceeds from a tread, or otherwise, should be carefully and immediately attended to; because if sand or gravel get into the wound, it is likely to produce those deep-seated ulcerations that are termed pipes or simuses, which constitute the disease called quittor.

But it may also proceed from any wound of the foot, and in any portion of it. In wounds of the foot, there is much difficulty in the matter proceeding from inflammation and suppuration finding its way from under the hoof, which covers the foot with its various 
complicated parts. The consequence is, pus accumulates under the hoof until it has increased to such an extent that it must find escape in some way; and frequently forces itself out in all directions, separating the little fleshy plates from their connection with the horny ones of the crust; or disuniting the fleshy sole from the horny one; and in extreme cases eats its way deeply into the internal parts of the foot, forming pipes or sinuses, which run in all directions.

If quittor arises from a prick or wound in the lower portion of the foot, the matter which is collected in it issues from it, and induces a separation between the horny and fleshy sole; and having accumulated in considerable quantity, at length discharges itself at the coronet, generally close to the quarter. This, however, does not manifest itself to any extent, as both the aperture and quantity of matter which oozes out are apparently so insignificant, that they would lead an inexperienced person to suppose the discharge of little consequence. In this, however, they will be sadly mistaken; for most serious mischief lurks within, and the difficulty of removing it is extremely great. In this state of the disease, although the fistula is of very small dimensions, yet the effects of this confined matter will have extended over almost the entire quarter, and the horny sole may be separated from the foot.

REMEDIES.-It must be evident that this is a case which can only be successfully treated by a skilful veterinary surgeon. In most instances of such cases, it becomes necessary to remove the greater portion of the horny sole, and thereafter restore the healthy state of the tender surface beneath. When this has been effected, the horn will quickly be reproduced. But in cases where much of the 
sole has been removed, it will take at least six months to restore fully that which has been removed, so that the horse may again be subjected to labour.

Quittor often proceeds from neglecting bruises and injuries of the sole of the foot. When horses have flat feet, and are ridden quickly over a rough, uneven, stony road, the feet are very liable to be injured, and especially by getting a small stone between the shoe and the sole. Narrow webbed shoes are frequently the cause of bruises of the feet, by leaving too much of the sole unprotected. Another cause is, the smith paring out the sole too closely, or pricking the sole while in the act of shoeing, as also pressure of the shoe on the sole, and gravel getting insinuated between the sole and the shoe.

\section{PRICK, OR WOUND IN THE SOLE OR CRUST.}

The sole is peculiarly liable to be injured by sharppointed stones, flints, and pieces of glass which it may come in contact with on a road. In shoeing, too, a nail may be driven either into the sensitive part of the foot, or so near to it in the horn, that it will cause pressure and consequent irritation, probably inflammation, and this may end in suppuration. In all cases of lameness, particularly in those which are sudden, the legs should be examined with great care; and if no cause can be discovered to account for it, the probability is that its seat is in the foot. The shoe ought to be immediately removed, when very likely the cause will be ascertained. But if it is not at once apparent by the heat of some portion of the foot, then the crust should be rapped all round with a small hammer, and when the part which is affected is touched, the horse is sure to 
flinch from it; or pressure applied by pincers will answer the same purpose.

Should lameness occur within two or three days after the horse has been shod, the first suspicion should fall upon the foot. The best of smiths may prick the foot in shoeing, and he that acts honestly in such a case, by at once acknowledging it, or informing the owner, will not deserve blame. It is in concealing or denying the possibility of the thing that causes all the mischief. Because, whenever it is discovered, the shoe should be taken off, and that too with much care. Some foolish smiths are in the habit of wrenching off shoes, a practice which, under any circumstances, cannot be too severely condemned.

REMEDIEs.-As soon as the injured part is detected, the sole should be well thinned down around it, and at the punctured spot it should be pared to the quick. We are speaking of one of some days' standing, in which case matter will issue from it, and the sore will be thereby relieved. If the wound is quite fresh, then all that will be necessary after thinning the hoof is to apply a pledget with some Friar's balsam to it ; or fill the wound with carbolic liniment, and it will quickly heal if kept clean. If, however, it does not heal speedily, and it becomes hot, then inflammation will have taken place, and therefore it will be necessary to apply a poultice.

But in wounds of the foot much depends upon the particular part which has been injured. Although a pretty deep wound is inflicted towards the back part of the sole, and even extending into the frog, still it may not be attended with much danger or inconvenience to the animal, because there are no bones or tendons to be injured. Neither is much harm to 
be apprehended from a prick near the toe. But in the centre of the sole, where the flexor tendons pass over, especially where the tendon is inserted into the coffin-bone, a puncture may be attended with serious consequences, as well as a wound in the joint which unites the navicular-bone with the coffin-bone. If a wound inflicted in this situation is neglected, the animal may be rendered useless for life. In short, injuries of the foot require the utmost skill to examine their precise nature, as well as the manner in which they ought to be treated.

In searching for a wound, it is not uncommon for the smith to cut away an unnecessary portion of the horn from the bottom of the crust of the sole, and thus leave no hold for the nail of the shoe. In such cases it has frequently taken months before the horny substance was of sufficient substance to hold the nails.

When it becomes necessary to remove any portion of the horny sole in consequence of a wound, or if separation has taken place through suppuration, then the injured or wounded portion of the crust must be entirely removed, as the dead parts of the horn will never unite with that which is living. Then the fleshy sole which has been left exposed must be carefully touched with chloride of antimony, and some soft and dry tow or lint laid on it. But if there are inflammatory symptoms, it will be necessary to apply a poultice to the entire sole. So quickly is the horny substance secreted, that in a few days a thin pellicle will be found covering the whole, or part of the portion that has been stripped of the crust. If there are any symptoms of proud-flesh shooting through it, then it must be subjected to an application of chloride of antimony, and tow placed over it 
as before. In a few days the whole surface will be invested with a horny crust.

\section{CORNS.}

A corn is a bruise of the sensitive papilla of the sole, situated betwixt the bars and the wall at the heels.

CAUSE.-This disease has acquired a name which but ill expresses its nature. It bears but little affinity to corns of the human foot; although, like them, they are the effects of pressure. Instead of being hard as in the human subject, they are thin and very weak, and caused by pressure on the sole in the angle between the bars, as presented, Plate vi, fig. I 2, $c$ c. The horn becomes more spongy and softer than at other parts, and it is so sensitive, that upon the slightest pressure the horse indicates that he feels pain.

Nothing is more injurious than to allow a shoe to remain too long on, as it is sure to become embedded in the heel of the foot; consequently the crust grows down on the outside of it, and the bearing is thus thrown on the angular portion of the sole. Continued pressure on the sole is certain to induce inflammation and corns. The shoe being long on, wears and gets loosened at the heels, which admits of gravel between it and the crust, and having accumulated in the angle it naturally insinuates itself into the heel, and produces a sore.

As we have before said in another part of the work, nothing can be more injudicious than to allow shoes to remain too long on; even if they are not worn, they should be taken off every fourth week and readjusted, to free the feet from long-continued 
pressure on certain parts by the growing of the horny substance of the hoof. In shoeing, too, the bars are very often cut away, and this renders it necessary to be bevelled inward, so as to accommodate it to this injurious and ridiculous shaping of the foot; consequently an unnatural disposition to contraction is induced by this slanting inward direction of the heel of the shoe. From this the sole is subjected to double liability to injury; first, by being pressed upon by the shoe, and, second, by being squeezed between the outer crust and the external portion of the bar. This angle is less able to bear pressure than any other portion of the foot, being more exposed to accidental bruises and injuries of different kinds, in consequence of the shoe being made unnecessarily narrow at the heel.

There can be little doubt but corns are mainly owing to faults in shoeing, as well as the fact of shoeing itself, however well performed, preventing the due expansion of the horn when the sole is growing downwards, and thus confining and injuring this portion of the sole. And it is easy to imagine that if the shoe is badly constructed, or allowed to remain too long on, that corns must be induced. This effect will be rendered manifest by an attentive observation of the foot in Plate VI, fig. Io. When paring is not attended to in shoeing, or when it is injudiciously extended to the bars, the evil of corns is likely to arise. This unnatural pressure of the sole causes the blood to be thrown out, which enters into the pores of the soft and diseased horn which is then secreted; and by the colour and softness of the horn at this place, the existence and extent of the corn is judged.

Remedies. - The cure of corns is difficult and un- 
certain, because a diseased action is induced; and to check this is no easy matter after it has contracted the tendency to secrete diseased horn; for all shoeing produces pressure on the parts.

The first thing to be done is to ascertain the extent of the corns; and this can only be effected by paring out the angle between the crust and the bars; at the same time it will relieve, and to a great extent do away with, what has caused the complaint. This can be best accomplished by the use of a small drawing knife, with which the corn must be pared out to the bottom; but great care must be exercised not to wound the sole. After this operation, it will be seen whether there is any effusion of blood or matter underneath the corn. If there is reason to believe that such is the case, then an opening must be made through the horn, so that the matter may discharge itself and the separated horn be removed; and when the course of the sinuses have been ascertained, the same remedies must be employed as in quittor.

In bad cases of corns, where the inflammation has rendered the parts very sensitive, a bar-shoe will be of service, so chambered that there will be no pressure on the part affected.

If corns have once existed to a considerable extent, they are liable to return; therefore, before shoeing, the seat of the corn should be well pared out. The seated shoe (which we have described in our chapter on shoeing) should be used, with a web sufficiently thick to cover the situation of the corn, and extending as far back as possible without producing injury to the frog. When the horn has grown to some extent, if the horse is turned out to grass with a bar-shoe in the first instance, and afterwards with a 
shoe fastened on one side only, or with tips, it will frequently prove of much service.

In all cases where horses have low weak heels, they should seldom be pared with a knife, but merely rasped down to a level surface. This is peculiarly applicable to the inner heel. The hind feet are seldom affected with corns, because they are stronger than the fore feet, and less subjected to concussion; and even where corns do exist, they do not produce lameness.

\section{CANKER.}

Canker consists of a separation of the horny substance from the sensitive fleshy and bony portion of the foot, caused by the growth of fungus tissue shooting up and occupying parts of, or the entire sole of the foot and frog. It is induced either by a puncture, bruise, corn, quittor, or thrush, more commonly proceeding from the latter than any other cause. It seldom attacks high-bred animals, being almost always confined to the heavy breed of cart and dray horses, which class seem to have a constitutional predisposition to this complaint. Those which have previously had grease are peculiarly liable to canker, and especially those which have thick heels.

It is peculiarly necessary to give a watchful attention to all species of injuries in these heavy horses, as they are more likely than all others to assume a bad complexion. Their feet being clubbed, and their hoofs in general brittle, they are liable to be punctured while shoeing. Nine-tenths of the complaints of this kind arise from negligence either in the groom or master, and also from the carelessness or stupidity of the shoeing smith. It will only require a little 
attention to the subject to enable the master to detect where the mischief arises.

REMEDrEs.-Canker is a complaint which ought only to be undertaken by a veterinary surgeon, being exceedingly difficult and tedious in its progress towards a cure.

The first thing which is done in this disease is to cut out the fungus growth; and besides this it often becomes necessary to use both caustic and the cautery; and all that portion of the horn which has been separated from the fleshy and bony parts must be carefully removed. Should fresh fungus be generated, it must be got rid of, and means adopted to check its growth, and by proper applicants to induce the secretion of healthy horn. Unskilful veterinarians have been known to inflict severe and unnecessary torture on horses which had canker, by too deeply corroding the parts with caustic, and thus producing a sore. In this complaint gentle exercise is absolutely necessary where the disease is confined to the frog and sole of the foot, but moisture is carefully to be avoided. But it will be found that in effecting a cure the processes will be both tedious and painful, in consequence of the necessity of caustic applications, which, however slight, always cause considerable irritation in the parts.

In this complaint medicine is seldom had recourse to, as it is of little use, the disease being local. But it sometimes occurs that grease follows canker; in the event of which physic will become necessary, and these will be alteratives and diuretics.

\section{THRUSH.}

The disease thrush is characterised by a discharge which issues from the cleft of the frog and 
is more or less mixed with pus. From a healthy frog there is no discharge, but if the foot of the horse is allowed to soak in urine and dung for a time, inflammation is set up in the sensitive frog, and this ends in suppuration and separation of the horny frog from the sensitive frog from which it grows.

Thrush is a disease to which horses of all ages are liable, and in all situations. Even unshod colts are subject to this complaint. When the frog is in a healthy condition, the cleft sinks but a small way into it. Any complaint which affects the healthy condition and action of the frog, is almost sure to induce thrush. Differing from most diseases of the foot, thrush is generally more severe in the hind than in the fore feet. This can only be accounted for by bad stable management, and that the hind feet are subjected to being so much exposed to the baneful consequences of immersion in the dung and urine, producing irritation, and generating disease.

A horse may have thrush without being lame, and it often happens that no alteration whatever can be seen on the foot thus diseased, and it may require a close inspection to detect that it is affected. But it will always be manifested by the disagreeable and peculiar smell which invariably accompanies the complaint. In some cases no tenderness of the frog attends thrush, and therefore the horse is not reckoned legally unsound by many persons. This we, however, consider strange, as it is a complaint which may, and indeed is likely to assume a worse aspect, particularly if not remedied in time, and hence may lead to positive unsoundness. But it is only in cases where considerable alteration in the form of the hoof has taken place, that thrushes are likely to be of a severe kind; for we find that they may exist 
in an otherwise sound and well-formed foot, without being attended with immediate detriment, and may be easily remedied. Still, if the thrush be not removed, in the course of time it will alter the shape of the foot and also the action of the animal, and consequently becomes confirmed unsoundness. It is quite certain, if thrush is neglected, that however slow its progress may be, still it is sure ultimately to assume a serious and immovable complaint. The frog will contract, become rugged and tender, and will be followed by a copious and very fetid discharge ; the horn will gradually disappear, and leave the sensitive frog completely exposed and unprotected; it is so tender that the slightest touch gives exquisite pain to the horse. In a day or two fungus granulations shoot out, and if neglected may end in canker.

From this will be seen the extreme folly of neglecting thrush, however simple its aspect may be at first. We have known valuable horses having been lost to their owners from the erroneous belief entertained by themselves, or put into their heads by ignorant quacks, namely, that thrush would benefit the horse, as it was only Nature working its own cure by throwing off superabundant humours. Some ignorant country smiths, who call themselves farriers, have promulgated such a doctrine, and even recommended that the discharge should not be speedily dried up on this account; and some have even said that it should not be dried up at all. But, assuredly, in nine cases out of ten what we have pointed out will be the inevitable consequence of neglected thrush. Its operations are slow, but its effects are certain. Disease of all kinds should be instantly attacked, and speedily subdued if possible. 
Remedies. - Astringents generally are the best appliances to thrush; but those of a caustic nature are to be avoided. The foot should be carefully examined, and when the cause of the thrush has been ascertained, our first attention should be directed to the removal of that. As has been stated, the primary cause of thrush is allowing the animal's feet to soak in liquid and filth, hence it is all-important to place the animal where his feet will be kept clean and dry. Remove with the drawingknife all loose or under-run horn, then the following should be applied :-

$\begin{array}{llllll}\text { Honey } & \cdot & \cdot & \cdot & \cdot & 4 \text { ounces, } \\ \text { Verdigris } & \cdot & \cdot & \cdot & \cdot & \text { I ounce, } \\ \text { Vinegar } & \cdot & \cdot & \cdot & \cdot & \frac{1}{2} \text { pint. }\end{array}$

Let these be boiled for a few minutes, and the composition applied to the place from which the discharge emanates. This is, however, a mild application, and will be somewhat slow in effecting a cure. But the most speedy, yet safe, appliance is the follow ing :-

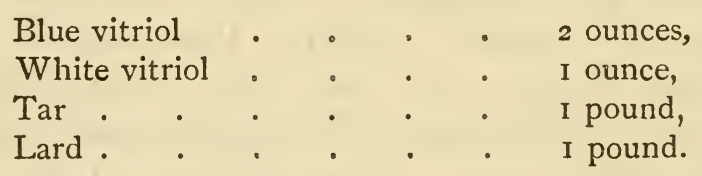

The vitriols to be finely powdered, and then mixed with the tar and lard.

A pledget of tow covered with the above should be inserted every evening as deeply as possible into the cleft of the frog, and renewed every day. Should the frog be much exposed, besides the pledget which is to be put into the crack, a larger piece of tow should be spread over with the above liniment, and applied over the whole exposed surface. Care 
must be taken not to force the pledget too hard into the cleft to press upon the tender part below, and during the treatment recommended it will be proper to keep the frog and foot as dry as possible.

In cases of thrush it is all-important that the feet should be kept dry; and, contrary to the practice of some who recommend turning out to grass with this complaint, we prefer keeping the animal in the stable until a cure is effected.

\section{OSSIFICATION OF THE CARTILAGES.}

These cartilages occupy a considerable portion of the back part and side of the foot, as represented on Plate vil, fig. $7, h$. Their use is to preserve the conformation and expansion of the upper portion of the foot. They are liable to inflammatory attacks, which in time leads to a conversion of their cartilaginous (gristle) tissue, into bone texture. This ossified condition of the cartilages is frequently connected with ring-bone, but it often exists without any affection of the pastern-joint. Side bones are almost entirely confined to cart horses of great weight, and apparently the weight of the body is the great cause. It is difficult to find an aged and extra weighty cart horse without side bones; and the converse holds good, viz., that it is difficult to find a light weight animal of any breed, from the pony to the thorough-bred horse, with "side bones."

Symptoms.-When the foot is in a healthy condition, the lateral cartilages will yield to the pressure of the thumb on the coronet, over the quarters. But when ossification commences, and the complaint progresses, then a commensurate degree of resistance manifests itself, and in process of time it will no longer yield to any pressure. It is then certain that complete 
ossification of the cartilages has taken place. During the transformation of the cartilage into bone, in horses with full-sized open feet no visible inflammation is observable in the foot, nor does much lameness appear, and, indeed, frequently lameness is not at all manifested, although a slight stiffness may be noticed; but in horses with small donkey-formed feet, there is generally marked lameness and a proneness for the lameness to become more or less permanent. These observations apply to cart-horses, or such as require only to be worked at a slow pace; but in horses that go at a rapid pace, it amounts to positive lameness. When this complaint is connected with ring-bone, very great lameness is the consequence.

Remedies. - If there is no lameness, have recourse to no treatment, as the conversion of cartilage to bone cannot be arrested. On the contrary, if the animal is lame and suffering much pain, a blister of cantharides may hasten on the ossific process, and thereby lessen the pain. The shape and size of the foot has much to do with the pain and lameness, and a horse with side bones and small feet is a much more risky purchase than one with good open feet.

\section{WEAKNESS OF THE FOOT.}

This is merely a conventional term in general use among horsemen and dealers, which might with more propriety be denominated "malformation of the foot," because, in point of fact, it is rather a bad formation than a disease. In many instances it is a natural infirmity, and likewise proceeds from some other disease. The natural slant or angle of the well-formed crust from the coronet to the toe is 
an angle of forty-five degrees; but in this formation the angle will not exceed from thirty-eight to forty degrees. This inclination is but ill adapted for resisting the effects of pressure ; and consequently, after the animal has been worked for one or two years, the line of inclination, instead of being straight, becomes hollowed half-way between the coronet and the toe- a defect that also occurs in pumiced feet. The surface presents an irregularly rough appearance, but is more frequently roughened in circles or rings; and the general structure of the crust assumes a conical form, with the lower portion, or sole, presenting an unnaturally wide aspect; and in most instances the foot is larger than it ought to be.

In this ill-formed foot the sole is always so flat that it will not stand paring when the horse is shod, and it will be found that the bars are very small in size, indeed, in many cases they can hardly be said to exist ; the heels are so low that the coronet appears almost to touch the ground, and the crust is so thin that it will hardly be capable of holding the nails of the shoe. Little good can be expected from horses with such feet. Besides, they are certain to be liable to corns; also to frequent bruises and convexity of the sole; to the crust being broken; to sprain and injury of the pastern, the fetlock, and flexor tendons; and to punctures from the nails in the operation of shoeing.

Feet of this construction are susceptible of little or no improvement. To those who have the misfortune to possess horses with these feet, we can only recommend that which we have condemned in the healthy state, namely, to shoe them as seldom as possible. The web of the shoe should be light and concave; the foot should be as sparingly pared as 
possible; hard work and rough roads are carefully to be avoided. With these precautions the animal may work for a considerable time; but an evil day must arrive, and the animal will turn out utterly worthless. 


$$
E
$$




\section{CHAPTER VIII.}

\section{The Anatomical Structure of the Horse.}

IT is not our intention to go deeply into the anatomy of the horse, as this would far exceed the limits of such a work as the present, and indeed would not be suitable for a popular treatise. We shall therefore confine ourselves to such an outline of his structure as will convey a pretty general idea of what is truly useful to possessors of horses in general.

We may briefly state, that the bones of the skeleton and the muscles which cover them are all double, if we except a very few bones which lock the two halves together; and that if an animal is divided correctly into two halves, these will be found exactly similar in the number of bones and the muscles with which they are covered. But this does not extend to the internal organisation in general, although "a few of its parts are also double.

\section{DIFFERENT BONES OF THE SKELETON OF THE HORSE.}

\section{PLATE II.}

Instead of giving the bare skeleton of the horse, we have considered it better to exhibit an outline of 
the external form of the animal, which will convey a more correct idea of the situation of the different bones as embodied in the muscles; and by comparing this view of the skeleton with our representation of the perfect horse in Plate I, the reader will have little difficulty in ascertaining the position which the various bones occupy under the superincumbent muscles.

FIG. I, I. The seven cervical vertebræ, or bones of the neck.

2. The sternum, fore part of the chest, or breast-bone.

3. The scapula, or shoulder-blade.

4. The humerus, or bone of the arm.

5. The radius, or bone of the fore-arm.

6. The ulna, or elbow.

7. The cartilages of the ribs.

$8,8,8$. The costæ or ribs, eight of which unite with, or are articulated to the sternum-these are called the true ribs; and ten are united together by cartilages, and are called the false ribs.

9. The carpus, or knee, consisting of seven bones.

10, 10. The metacarpal, or shank-bones: the larger metacarpal, or cannon, or shank-bone, in front, and the smaller metacarpal, or splint-bone, behind.

II. The upper pastern.

12. The lower pastern.

13. The coffin-bone.

14, I4. The eighteen dorsal vertebræ, or bones of the back.

15. The six lumbar vertebræ, or bones of the loins.

16, 16. The haunch, consisting of the ilium, the ischium, and the pubis.

17, 17. The femur, or thigh-bone.

i8, I8. The stifle-joint, with the patella, or knee-cap.

19, 19. The tibia, or proper leg-bone.

20. The fibula.

2I, 2I. The tarsus, or hock, composed of six bones. The prominent part behind is the os calcis, or point of the hock. 
22. The metatarsal bones of the hind legs.

23, 23. The pastern of the hind feet, including the upper and larger bone (fig. 23), the lower pastern (fig. 25), and the coffin-bone (fig. 24).

26,26 . The caudal vertebræ, or bones of the tail.

BONES OF THE HEAD.

PLATE VIII, Figs. 2 AND 3.

The head contains the brain and other important organs of sense. It is divided by anatomists into two parts, namely, the skull and the face. The skull, or cranium, is that portion in which the brain is situate; and the bones in which it is enveloped are destined for its protection. This division consists of nine bones: the two frontal bones, $a, a$; two parietal, $c, c$; two temporal, $d, d$; the occipital, $g$; the ethmoid; and sphenoid: the two latter are situate at the base of the skull, and are not visible in a front view, but their position will be seen in fig. 3 , Plate III, the ethmoid, or sievelike bone, immediately above $k$, and sphenoid, $l$. These nine bones are separated in the foal at an early period of its existence; but soon after birth they are firmly united together by the sutures, at which parts they are so strong that fracture seldom or never occurs there.

There is a beautiful evidence of design in this division of the head into so many bones. When the fotus of the foal in the womb first assumes a form, and may be said to be in life, this portion of the skull is merely a jelly-like consistence, which is gradually changed into a harder substance, called cartilage; and previous to the birth of the animal much of this cartilaginous substance is carried off 
by certain vessels emanating from the brain, called absorbents, and bone is deposited in its stead. In all the flat bones, such as those of the head, this deposit takes place from the centre, from which radiations, or rays of bone shoot forth in all directions. Therefore it is evident, that by having so many bones, there are so many more centres of radiation, and consequently the formation of bone is carried on so much more rapidly, and becomes perfected at the time when the necessities of the animal require it. But when the foal is born, this process is not completed, as the edges of the bones remain somewhat soft and pliant; so that in parturition they yield a little, and by overlapping each other render the birth more easy, and save the parent much pain, and contribute materially to the safety of the foal; and indeed without a change in the form of the head, from this compression and yielding of the bone of which it is composed, the animal could not be born.

a. The occipital bone, or bone of the hinder part of the head.

$b, b$. The parietal bones, or walls of the skull.

$c, c$. The temporal bones, or bones of the temple.

$d, d$. The temporal fossæ, or pits above the eye.

The age of a horse is pretty well manifested by the depth of these fossa. At the back part of the eye there is a cushion of fatty matter on which the eye rests, and revolves without friction. In aged horses this substance decreases, and consequently the eye sinks and the pit above it deepens. To deceive the unwary, dishonest dealers puncture the skin of the pit, and with a blow-pipe fill up what it has lost in substance with air. This puffed-up skin will continue for many hours. The 
name which is given to this by these unprincipled men is 'puffing the glims.'

$e, e$. The frontal bones, or bones of the forehead.

The frontal bones are articulated together by a curious and very intricate dove-tailed suture, which gives great strength, so as to defend the upper portion of the brain, which lies immediately below them, from injury. The frontal bones strongly manifest the breed or blood of a horse. Those which are high-bred have a broad angular forehead, with the face gradually tapering from the brow to the muzzle, as represented in this figure. The cranium of the dray or cart-horse is nearly as wide below as above. It is the full and largely developed forehead which gives to the blood-horse that fiery and intelligent expression.

$f, f$. The zygomatic arch.

$g, g$. The super-orbital foraminæ, or holes above the orbit for the passage of the nerves and blood-vessels which supply the forehead. The small hole beneath receives vessels which penetrate into and supply the bone. In some craniums there are several such holes.

$h, h$. The lachrymal, or tear-bones.

$i, i$. The orbits which contain and defend the eye.

$j, j$. The malar, or cheek-bones.

$k, k$. The nasal-bones, or bones of the nose.

$l, l$. The superior maxillary, or that portion of the upper jaw containing the molar teeth or grinders.

$m$, $m$. The infra-orbital foraminæ, or holes below the orbits, through which pass branches of nerves and bloodvessels to supply the lower portion of the face.

$n, n$. The openings into the nose, with the bones forming the roof of the palate.

$o, o$. The inferior maxillary, the lower portion of the jawbone, which is a separate bone in quadrupeds, contain- 
ing the incisors or cutting teeth and the upper tushes at the point of union between the superior and inferior maxillaries.

$p, p$. The upper incisors, or cutting teeth, or, as they are otherwise called, the nippers, a term which we limit to the two central ones above and below; the one next to these, on each side, are called the dividers, and the innermost ones on both sides are termed the corner incisors. There are in all twelve incisors in the head of a horse, viz., six in the upper, and six in the under jaw.

\section{SIDE VIEW OF THE CRANIUM.}

\section{PLATE VIII, Fig. 2.}

b. The parietal bone.

$e$. The frontal bone; the cavities or cells below which are called the frontal sinuses.

$f$. The zygomatic arch.

$g$. The super-orbital foramina.

$h$. The lachrymal bones.

$i$. The orbit which contains the eye.

$k$. The nasal bone.

l. The superior maxillary bone.

$m$. The infra-orbital foramina.

n. The opening into the nose.

o. The inferior maxillary.

$p$. The upper incisors or cutting teeth.

$q, q$. The molars, or grinders of the upper and under jaw.

$r$. The posterior maxillary or under jaw.

$t$. The lower portion of the under jaw.

u. The under incisors, or cutting teeth.

\section{SECTION EXHIBITING THE INTERNAL ANATOMY OF THE HEAD.}

PLATE III, FIG. 3 .

a. The occipital bone. 
$b$. The frontal bone, under which are cavities called the frontal sinuses, marked 16 , I6.

These frontal sinuses are cavities intervening between the frontal bone and a transverse plate of bone which grows within it. They communicate with the cavities of the nose, as also with those of the sphenoid, ethmoid, and upper jaw-bones. In consequence of this conformation, they increase the loudness and clearness of neighing. It sometimes happens that the larvæ of certain flies crawl up the nostrils and locate themselves in the frontal sinuses, occasioning great pain to the animal. Happily, this is not of very frequent occurrence with the horse, although sheep and horned cattle are more liable to such intrusion.

These sinuses are occasionally opened by trephining the bone, in order that the pus cells, which they frequently contain in large quantity, may be removed by direct injection of water and astringents.

In performing the operation we suppose a line to be drawn across the forehead from 2 and 3 , one foramen to the other, Plate vili, fig. $g, g, g$. On that line, and about half an inch from the suture, or line which separates the frontal bones, the sinuses or cavities are situated, and extend to an inch in depth, as represented in Plate III, fig. 3 , immediately under $b$, and marked 16, I6. If the position of $g, g$ in fig. 3, Plate vil and $b$ in fig. 3 , Plate III, are compared, a perfect idea of their seat in the forehead will be formed. At this part a small circular portion of bone is drilled out with a trephine, and into this warm water is injected by means of a common syringe, which will run out at the nostrils. 
c. The nasal bone, or bone of the nose.

d. The tentorium, or bony separation between the cerebrum and cerebellum.

c. The occipital bone.

$f$. The cerebrum, or brain.

$g$. The cerebellum, or little brain.

$h$. A portion of the medullary, or marrow-like substance of the brain; and the prolongation of it, which bears the name of the crus cerebri, or leg of the brain, and from which many of the nerves emanate.

$i$, and $m$. The ligament of the neck, or pack-wax, by which the head is chiefly supported.

$j$. The sphenoid, or wedge-like bone, with its cavities.

$k$. The cuneiform, or wedge-shaped process, or base of the occipital bone. Between it and the other portion of the occipital bone, $a$, lies the great foramen or aperture through which the prolongation of that portion of the brain called the spinal-marrow issues from the cranium, and is continued through the spine or back-bone.

l. The medulla oblongata, a prolongation of the brain after the marrow-like substance of the cerebrum and cerebellum have united, and forming the commencement of the spinal marrow. This portion has a ropy appearance.

n. The point of the atlas bone which sustains or carries the head.

o. The first bone of the neck.

p. The dentata, or tooth-shaped bone, the second bone of the vertebral column.

q. The cartilage covering the entrance into the eustachian tube, or communication between the mouth and internal part of the ear.

$r$. The spinal marrow, extending through a canal in the centre of the bones of the neck, back, and loins, to the extremities of the tail, and from which the nerves of feeling and of motion which supply every part of the frame arise.

$s$, s. The septım-nasi, or cartilaginous division between the nostrils. 


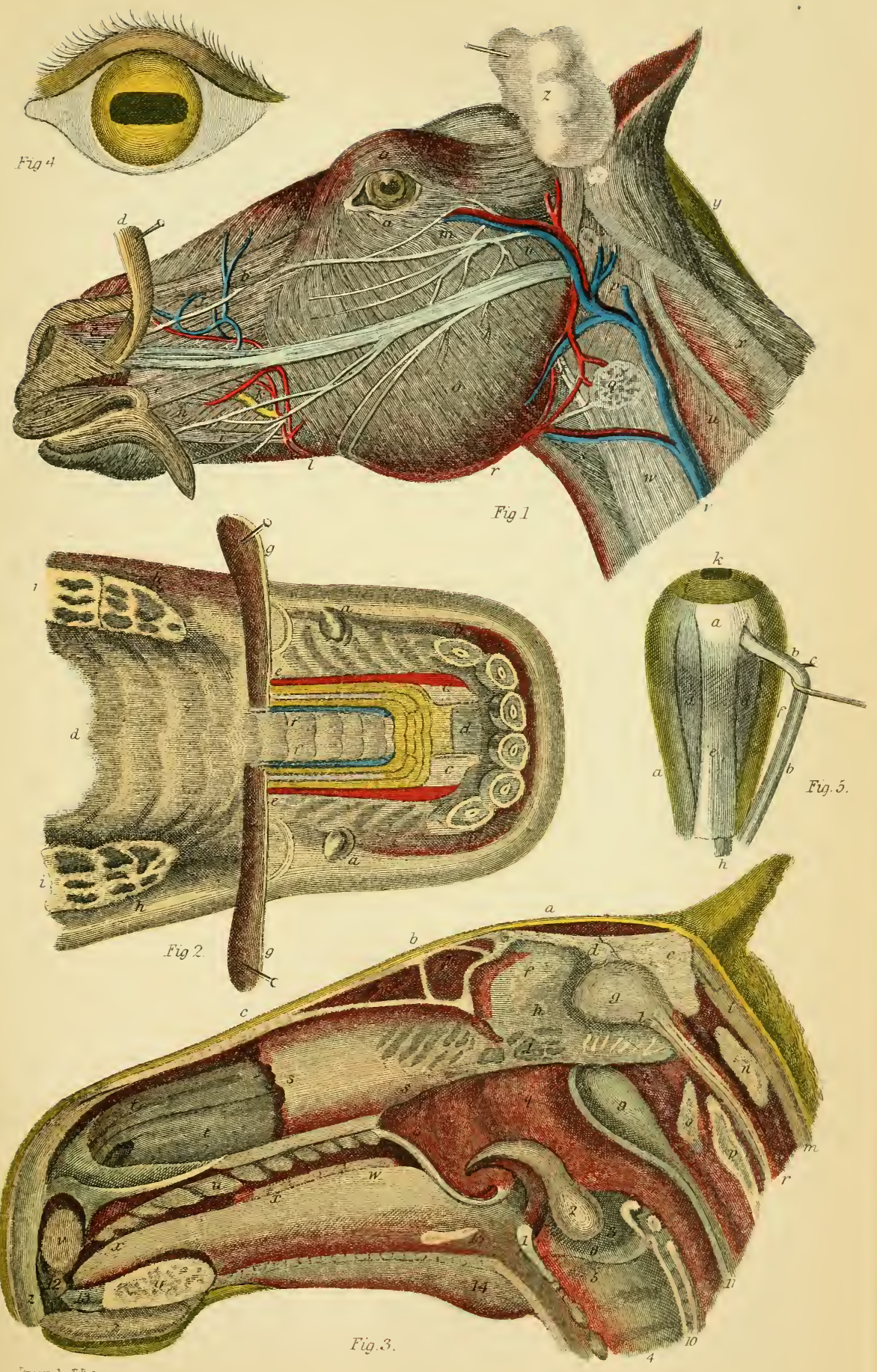

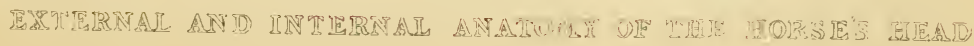



When the nostril is opened, the membrane by which the cartilage and whole cavity of the nose is lined is seen. By the colour of this membrane, and the lining of the eyelids, we are assisted in determining the amount of congestion of blood in the mucous membrane which lines the air passages and the bowels. This cavity on both sides is occupied by two bones, which, in consequence of being rolled up in the form of a turban, are called the turbinated bones.

$t, t$. The scptum nasi, cut off at the lower part to exhibit the spongy turbinated or turban-shaped bones, filling the cavity of the nostrils. Part of the cartilage is removed to display them. They are as thin as gauze, and, like it, perforated into a thousand holes. Between them are left sufficient passages for the air.

If these gauze-like membranes were unrolled, they would present a very considerable surface. On every part of them there is spread the delicate fibres of the olfactory, or first pair of nerves, which are the organs of smell. It is by this exquisite sense of smell that the horse selects such food as is best calculated for his nutriment, and is enabled to reject what is poisonous. By smell he judges of the quality of his food in a domestic state. The horse will recognise his master or favourite groom by the sense of smell, and frequently expresses such recognition by a neigh. These cavities intensify sounds issuing from the larynx-the organ of voice; the sound reverberates through them, and increases in loudness as through the windings of a French-horn. All the air which passes to and returns from the lungs must go through the nostrils, as he can breathe through 
the nose only. The nostrils ought therefore to be large and expanded. The skin also which covers them should be thin and elastic, that they may the more readily yield when the animal requires a greater supply of air while trotting hard or galloping. In the race-horse, the nostrils are wide and flexible, while in the cart-horse they are confined, and surrounded by a quantity of cellular tissue and thick skin.

There are, besides, four distinct cartilages attached to the nostrils, which are exceedingly elastic, and bring them back to their ordinary dimensions whenever the muscles cease to act. The bones also of the nose, $n$, Plate virl, fig. 2 , and $n n$, fig. 3 , are tapered off to a point, to give a wider range for the action of the muscles; while the cartilages are so constructed as not only to discharge the office above referred to, but also to prevent this tapering point of bone from injury.

u. The palate.

$v$. The inferior maxillary-bone, containing the incisor teeth, or nippers.

$w$. The molar teeth, or grinders.

$x, x$. The tongue.

$y$. The posterior maxillary, or jaw, with its incisors.

$z, z$. The lips.

I2. The upper incisory teeth.

13. The lower incisory teeth.

I4. The posterior maxillary, or jaw-bone.

.. The thyroid, or helmet-shaped cartilage, enclosing and protecting the contiguous parts.

2. The epiglottis, or covering of the glottis, or aperture of the windpipe.

3. The arytenoid, or funnel-shaped cartilages, having between them the aperture leading into the trachea, or windpipe. 
4. The trachea, or windpipe, with its rings.

5. One of the chorda vocales, or cords concerned in the voice.

6. The sacculus laryngis, or the sac or ventricle of the larynx, or throat, for the modulation of the voice.

7. The opening from the back part of the mouth into the nostril.

8. The soft palate at the back of the mouth, so constructed as nearly to prevent the possibility of vomiting by the mouth.

9. The muscle of the neck, covered by the membrane of the back part of the mouth.

Io. The cricoid, or ring-like cartilage below and behind the thyroid.

I I. The œsophagus, or gullet.

15. A portion of the os hyoides, or bone of the tongue.

\section{REPRESENTATION OF THE PALATE AND}

TEETH.

PLATE III, FIG. 2.

$a, a$. The tushes canines, or tusk-formed teeth.

$b, b$. The incisors, nippers, or cutting teeth.

$c, c$. The palatine nerve between the artery and the vein.

$d, d$. The palate, divided into ridges and bars.

$e, e$. The palatine artery.

$f, f$. The palatine vein.

$g, g$. A strip of the palate, dissected up, to exhibit the position of the vessels and nerve beneath.

$h, h$. The cheeks.

$i, i$. The molars, or grinding teeth.

\section{EXTERNAL ANATOMY OF THE HORSE'S HEAD.}

$$
\text { PLATE III, FIG I. }
$$

$a, a$. The orbicularis muscles, surrounding the eye, and destined for the purpose of closing the eyelids. 
b. The nasalis labii superioris takes its rise from a depression at the junction of the superior maxillary and malar bones, and extending to the angle of the nostril. Its use is to raise the lip, and dilate the nostrils.

c. Dilator magnus, or great dilator, which assists in the office of retracting the upper lip and in dilating the nostrils.

d. Dilator naris lateralis, or side dilator of the nostrils, reversed to exhibit the vessels and nerves which it covers, extending from the covering of the nasal and frontal bones to the angle of the mouth and side of the nostril. Its office is to retract the upper lip, and dilate the nostrils.

c. The zygomaticus, extending from the zygomatic arch and masseter to the corner of the mouth, for the purpose of drawing back the angle of the mouth.

$f, g$. The orbicularis oris, or circular muscle of the mouth. This muscle surrounds the mouth for the purpose of closing the lips and dilating the nostrils.

h. The buccinator, or trumpeter muscle, extending from the inside of the mouth and cheeks to the angle of the mouth, to draw it back.

i. Depressor labii inferioris, or puller down of the under lip, attached to the sides of the under lip to pull it down.

$j$. Branches of nerves, with small blood-vessels.

$k$. The parotid duct, penetrating the cheek to discharge the saliva into the mouth.

l. See letter $r$, and explanation.

$m$. The vein and attery passing under the zygomatic arch.

n. A branch of the fifth pair of nerves, the sensitive nerve of the face, emanating from under the parotid gland.

o. The masseter, or chewing muscle. This muscle occupies the entire cheek of the horse, and is exceedingly powerful, extending from the upper jaw-bone into the rough surface round the angle of the lower one ; which, in conjunction with the temporal muscle, is destined to chew the food and close the mouth.

$p$. The stylo-maxillaris, or styloid, pencil-shaped process of the occiput, extending to the angle of the jaw. Its office is to pull the jaw backwards and open it. 
q. The maxillary gland, or gland of the lower jaw, with its duct.

$r$. At this situation the submaxillary artery, a branch of the jugular artery and the parotid duct pass under and within the angle of the lower jaw, and reappear again at $l$; and, ascending the cheek, are distributed in a branching manner over it.

s. The subscapula hyoideus, emanating from under the shoulder-blade to the body of the os hyoides, to draw back that bone.

$t, w$. The stemo-maxillaris, or muscle belonging to the breastbone and upper jaw, from the cartilage in front of the chest to the angle of the lower jaw, for the purpose of bending the head; or, if one only act, to bend it on one side.

i. The levator humeri, or elevator of the shoulder, arising from the tubercle of the occiput, the mastoid, or nippleshaped process of the temporal bone; and the transverse processes of the four first bones of the neck and the ligament of the neck, and proceeding to the muscles of the shoulders and the upper bone of the arm, for the purpose of drawing forward the shoulder and arm, or to turn the head and neck; and, when the two levators act, to depress the head.

$v$. The jugular, or neck-vein. It is from this vein that blood is taken for all diseases in the head, neck, and contiguous parts.

$\therefore$ The tendon common to the complexo major, or larger complicated tendon; and the splenius, or splint-like tendon, and the mastoid process of the temporal, to hold up the head, or, the muscles on one side alone acting, to turn it.

$y$. The superior portion of the ligament of the neck.

$\boldsymbol{z}$. The superior portion of the parotid gland, or gland situate near the ear, reversed to exhibit the blood-vessels and nerves beneath it. 
MUSCLES AND PARTS CONNECTED WITH THE EYE.

PLATE III, FIGS. 4 AND 5.

The horse has a very extended field of vision. The eye is provided with seven muscles to move it in all directions; and that they may act with sufficient promptitude and power, no fewer than six nerves are directed to the eye generally, or to particular muscles; and that it may receive no injury from friction, it rests upon a mass of fatty matter, which also enables it to be turned without much exertion of the muscles. Four of the muscles, $a, e$, and $d$, are straight; these rise from the back of the orbit, and are inserted into the ball of the eye, immediately opposite, and at equal distances from each other. One of these, $f$, rises to the upper part of the eye, immediately behind the transparent and visible portion of it, the office of which is to raise the eye. When it contracts, the eye must necessarily be drawn upward. Another, $a$, is inserted immediately opposite, at the bottom of the eye, for the purpose of depressing the eye, or enabling the horse to look downwards. A third, $e$, is inserted at the outer corner, which turns the eye outward; and a fourth is inserted at the inner corner for turning the eye inwards. By means of all these the eye can rotate, or be turned in any direction at the will of the animal. Should the animal wish to look upward and outward, then the outer and upper muscles are called into action, and can be modified in any manner at the will of the horse. These muscles perform another duty, namely, keeping the eye in its place, for, while grazing, the principal weight of the eye rests upon them; and to aid them 
in this, another muscle, $d d$, called the retractor, is added ; it arises from the edge of the foramen, through which the optic-nerve enters the orbit. The use of this muscle is to support the eye generally, or when it is suddenly called into great action, and aided by the straight muscles, it draws the eye back out of the reach of danger, and in the act of drawing it back it forces the haw to protrude in the manner already described, as an additional defence. The cornea, $i$, is the only visible part of the eyeball of a horse, or at least it should be; and it is said that where much white is seen, that it is an indication of bad temper. The pupil, $k$, in the horse differs in form from that of all other animals. It is of an elongated, oblong, ovate form, and placed transversely, as represented in fig. 4, Plate III.

The eye is a very important organ in the horse. It ought to be large and rather prominent; the eyelid should be fine and thin. This thinness is for the purpose of preventing pressure, and at the same time to give more extensive and easy motion.

The horse is devoid of eyebrows, and the eyelashes are singularly arranged. The rows of hairs are longest and most numerous on the upper lid, and especially towards the outer or temporal corner, because the light falls on the eye from above; and as he stands, especially when he is grazing, as well as from the lateral situation of the eyes, the greater portion of the light, the attacks of insects, and the trickling down of moisture, is chiefly from the outside. Towards the inner corner of the eye there are hardly any eyelashes, because there is little or no danger from below, and as little light is thrown from below, the eyelashes are thin and short. While horses are grazing, insects may find their way to the eye towards 
the inner angle, the principal or only hair is found on the lower lid. All of which most beautifully proves design in the formation and adaptation of the animal. The eyelashes should on no account whatever be cut, as they have a most important office to fill, not only preventing the eye from being injured by dazzling light, but also protecting it from the intrusion of insects.

The horse is destitute of eyebrows, but in their stead he is provided with a number of scattered bristly hairs, as also a projecting fold of the upper eyelid, which answers the same purpose. Some persons are so foolish as to cut off these hairs, and have absurdly attributed them to weakness. The under eyelid also is furnished with projecting bristly hairs, which are so sensitive that they give immediate warning to the animal of the approach of any insect or other object which might injure the eye, and he naturally closes the eyelid instantaneously. Ignorant grooms sometimes denude horses of these admirable premonitory feelers. It will thus be seen that these muscles are admirable substitutes for the want of hands, in protecting the eyes against the intrusion of things that would injure them.

These muscles perform another important office in altering the focus of the eye to accommodate itself to the examination of distant or near objects. (See $c$ and $b$, fig. 5.) The straight and retractor muscles draw back the eye, and forces it upon the substance, and thus in a slight degree flattens it, brings it nearer the retina or mirror, and adapts the eye to the observation of distant objects.

But as these muscles are chiefly employed in supporting the weight of the eye, they might not have power to turn it so quickly and to such an 
extent as the animal might wish or require; therefore the eye is furnished with two other muscles, whose entire office is to turn it. They are placed obliquely, and in consequence are called the oblique muscles. The upper one, $a b$, is curiously constructed. It emanates from the back part of the orbit, and follows a direction upwards and towards the inner side, and there, immediately under the ridge of the orbit, it passes through a perfect mechanical pulley, and turning round takes a direction across the eye, and is inserted a little beyond the middle of the eye, and towards the outer side. Thus the globe of the eye is evidently directed inward and upward. This is not all which is accomplished by this remarkable mechanism. That the eye may be completely defended, it is sunk deep in the orbit, but it may be occasionally requisite to bring the eye forward and enlarge the field of vision. Under the influence of fear the eye is positively protruded, and it is not only forced more forward, but the lids are opened more widely. It may be asked, how can this be possibly accomplished? The remarkable pully-muscle, or trochlearis, at $b \quad c$, readily effects this, while the straight muscles at the same time do not oppose it, or only regulate the direction of the eye, it is really brought forward. The lower oblique muscle has its insertion just within the lachrymal-bone, $n$, and proceeding across the eye is fixed into part of the sclerotica, opposite to the other oblique muscle, and turns the eye in an opposite direction, and also assisting the upper oblique muscle in bringing the eye forward from its socket.

There is another beautiful provision preventing impurities or insects from being carried to and lodging in the corner of the eye, which would, if allowed to 
accumulate at the inner angle of the eye, be carried down the duct, which would irritate and obstruct it. No sooner do any of these annoyances enter the eye but they are carried off by the haw, which lies concealed within the inner corner of the eye. This haw is a black or pied cartilaginous substance of a triangular form, concave within, and made exactly to suit the globe of the eye; while it is convex externally, accurately fitting the membrane lining the lid, while the base of it is reduced to a thin or almost sharp edge. The horse has the voluntary power of suddenly protruding this from its concealment, and passing it rapidly over the eyeball, clears off every nuisance mixed with the tears, and then being quickly drawn back, the dust or insect is wiped off as the cartilage again passes under the corner of the eye.

In treating of disease of the haw, page 35 , we mentioned the barbarous practice of cutting out this valuable appendage to the eye. This member is destined by nature as a substitute for the want of hands for wiping the eye and cleansing it from offensive matter. This being removed, subjects the poor animal to pain in its eye for life, and lays it open to the constant chance of inflammation from dust or small pieces of gravel being blown into it. The pain they thus inflict on the horse may be easily conceived by any one having dust or other extraneous matter lodged between the eyelid and eye, and being without hands to wipe it out.

THE EYE AND ITS VISUAL STRUCTURE.

PLATE X, Fig. 8.

The eye is of a spherical figure, yet not perfectly globular; or it may rather be considered as com- 
posed of the parts of two globes, the half of the one, A, Plate $\mathrm{x}$, fig. 8, smaller, and transparent in front; and of the other, B, which is larger, with an opaque coat behind.

It is an established law in optics, that all objects become visible from the rays of light which flow from these objects into the eye. These rays pass through the pupil and fall upon the retina, which is a fine expansion of the optic nerve, interwoven like net-work in the back part or bottom of the eye, and there the rays form a picture of the object, whose apparent bulk depends upon the size of such picture so formed upon the retina. We shall suppose the animal looking at an arrow with the barb of it downwards, C, D. From every part of the arrow rays of light will be sent forth in straight lines, and in passing through the pupil, Plate III, fig. $5, k$, it is clear that those which flow from the under portion of the object, C, must flow upwards, while those above, D, must pass downwards; and pursuing this principle, all the intermediate rays, $f$, will intervene, consequently a reversed picture of the object will be formed upon the retina, as seen at $g, h$.

Paley makes the following interesting observation on this subject: he says, "In considering vision as achieved by means of an image formed at the bottom of the eye, we can never reflect without wonder on the smallness, yet correctness, of the picture, the subtlety of the touch, and the fineness of the lines. A landscape of five or six square leagues is brought into a space of half-an-inch in diameter; yet the magnitude of objects which it contains are all preserved, are all discriminated in their magnitudes, positions, figures, and colours. A stage-coach passing at its ordinary speed for several minutes, passes 
in the eye only over one-twelfth of an inch, yet is the change of place in the image distinctly perceived throughout its whole progress."

$i, i$. The points where the rays, having passed the cornea, converge by the refracting power of the lens.

$j$. The cornea, or horny and transparent portion, which is covered by the conjunctiva uniting different parts together.

The cornea fills up the vacuity which is left by the sclerotica; but although it is closely united with it, may be easily separated and will drop out. A prominent eye adds greatly to the beauty of a horse, and this will depend upon the projection of the cornea. But if too prominent, the rays of light may be rendered too convergent, which will produce indistinct vision. If it be small and flat, the rays may not be sufficiently convergent, which will produce imperfect vision. An eye that is moderately convex will be found best; because, when either too prominent or too flat, the horse is liable to starting and shying upon a road. The cornea is quite transparent in the healthy eye, and when cloudiness is observable, then it is an indication of disease.

In the purchase of a horse, the utmost attention should be given to the condition of the cornea. Perfect transparency over the whole surface is indispensable. The eye should be carefully examined in the manner pointed out at page 37 ; first in front, and afterwards through its substance.

$k, k$. The crystalline, or glassy lens, situated behind the pupil and in front of the vitreous humour, which is so named from a supposed resemblance to melted glass; it is a clear gelatinous fluid, very much resembling the white of an egg.

l. The sclerotica, a hard, firm coat, covering the whole of 
the eye, except that portion occupied by the cornea. The choroides, or choroid coat, covered with a black secretion, or black or dark brown paint, called the pigmentum nigrum.

This delicate membrane extends over the whole internal part of the eye, from the optic-nerve to the cornea. This is intended to absorb the stray rays of light which might dazzle and confuse the animal, and is not found on any portion which may be called the field of vision; but in its stead a bright green substance is spread, which extends more over the upper than the lower portion, because the objects which are most necessary for him to see are below the level of the head. This in some way, yet undiscovered, enables the animal to see even when it is comparatively dark. In the dusk this beautiful sea-green colour may be seen in the eye of a horse.

Cream-coloured, or perfectly white horses, have not this dark pigment; so that the ordinary appearance of the pupil is red instead of black. In looking into their eyes we do not see the covering, but the choroid coat itself.

$m, m$. The iris, or rainbow-coloured circular membrane, situated under the cornea in front of the eye, and on which the colour of the eye depends. The duplicature behind is the uvea, so called from its colour resembling a grape. See also Plate III, fig. $5, i$. The iris acts as a curtain, and floats in the aqueous humour.

In horses the colour of the iris is subject to little variation, but for the most part has some analogy with that of the hide, varying in different degrees from hazel to dark brown.

$n$. The pupil is placed in the centre of the cornea, and through which all the rays of objects pass to the retina or mirror of the eye. See also Plate III, fig. $5, k$. 
In the horse it is of an oblong form (see Plate III, fig. 4). It is variable in size, depending upon the intensity of the light in which it is viewed. In the open air it will be much contracted, so as to prevent too large a quantity of light being thrown upon the retina, as it is painful and injurious to that object to receive too great a portion of it, as well as prejudicial to vision; while in a dark stable it expands, in consequence of a deficient portion of light reaching the retina.

This contraction and expansion of the pupil is of much service in enabling purchasers to judge of the state of the horse's sight. There is a description of blindness, in which the crystalline lens and cornea continue quite transparent, but the retina of the eye is palsied, and consequently not affected by light; so that the pupils are hardly if at all altered by a change from light to darkness, or vice versâ. In purchasing a horse, the size of both pupils should be strictly observed, to see that they both expand and contract to the same extent by the change of light. The hand ought to be held over the eyes for a short time, and then notice if both pupils expand alike, when removed.

Suspended from the upper edge of the pupil of the eye two or three black bodies may be discovered on close inspection, the size of millet-seeds. When the horse is suddenly brought into an intense light, the pupil suddenly contracts; these little globes are pressed out from between the edges of the iris, and an equal number, but of much smaller size, are attached to the lower edge of the iris. Their use, probably, is to intercept portions of light which would be injurious to the eye. But their chief function is called into action while grazing, and perform the 
duties which are attributed to the eyelashes, namely, to obstruct the rays of light in those directions in which it would come with the greatest force both from above and below; while at the same time the field of view is quite open, so far as respects the pasture on which the horse is grazing.

$o, o$. The ciliary, or hair-like processes.

$p$. The vitreous, or glass-like humour, which fills the whole of the cavity of the eye behind the lens. It is a clear gelatinous fluid, resembling the white of an egg. It fills about three-fourths of the globe of the eye, and extends from the posterior part as far forward as the ciliary ligament.

$q$. The aqueous, or water-like humour which fills the space between the cornea and the crystalline lens.

It is by means of this humour that the cornea is preserved in its rounded form. A small portion of it is behind the iris.

$r$. The retina, or fine net-like expansion of the optic-nerve, which is spread over the whole of the choroides, as far as the lens.

s. The optic-nerve, or nerve of sight.

$t$. The conjunctiva, or that membrane which covers the fore part of the eye, and which lines the lids, and even extends to the transparent portion of it. It is transparent, and transmits colour to the parts beneath. It is very liable to inflammation, during which the vessels of the lining of the lids will become engorged with blood, and present an intensely red appearance, which extends itself to those vessels in the white of the eye, which will also become completely congested with blood, and will ultimately render the cornea clouded and opaque.

A defect of sight in the horse is more dangerous than total blindness, for this reason, that one with a defect of sight will start and shy at every object 
which he meets with upon a road, and the rider may be thrown off when he least expects it; while a horse that is quite blind will generally resign himself to the guidance of his rider or driver.

\section{THE TEETH AND THEIR DEVELOPMENT.}

Mastication in the horse is performed in two ways, namely, by a champing motion, and also a grinding motion.

At $o$, Plate III, fig. I, is the masseter-muscle, which is of great strength, and constituting the cheek; it has its origin at the superior maxillary bone, under the ridge continued from the zygomaticarch, Plate viII, fig. $3, f, f$, and inserted into the lower jaw. This acts in conjunction with the temporal muscle in closing the jaw, and in producing its direct cutting, or champing motion.

Inside of the lower jaw, on each side, and occupying the entire of the hollowed portion, and opposite the masseter, or cheek-muscles, are the pterygoidmuscles, which proceed from the jaw-bones to those more in the centre of the channel, also shutting the mouth, and likewise by their alternate action giving that grinding motion so necessary in preparing the food of the horse.

The channel which occupies that portion of the lower jaw between the branches, is of considerable importance in the conformation of the head of the horse. If this part is too wide, it always gives a clumsy, heavy appearance to the face; and when too narrow, it prevents the animal from bending his head with freedom and grace. Horses with such heads are unpleasant in the hand either of the rider or driver, as they constantly keep pulling and 
stretching their head, which also prevents them from being well reined in.

The changes which successively take place in the incisor teeth throughout the whole period of the life, form the most certain test of the age of the horse.

The teeth of a horse are forty in number, and in the mare thirty-six, being destitute of the four canines or tushes. The incisor, or cutting teeth, are temporary, and are replaced by others from two and a half years to four or five. The marks on them which distinguish the age become obliterated at eight years.

Teeth are composed of three distinct structures, viz., dentine, enamel, and crusta petrosa. The dentine surrounds the "pulp cavity" in all teeth. The enamel forms the white shell, and the crusta petrosa or cement joins the three structures together. The incisor tooth of the horse is known from other incisor teeth, not only by its shape, but by having a cavity in the crown, termed the "infundibulum" or pit, which descends about a third way down the body of the tooth and ends in a blind pouch.

In the early stages of their formation, the incisor teeth of the horse resemble a cellular body whose sides are soft and membranous, and quickly become hard and thickened, and are then reflected at the side of the table. From this primary dental production emanate two cavities that have no communication with each other, and which are essentially different, the largest being situated next the root. (See Plate v, fig. I I.) This contains the pulpy substance and the blood-vessels and nerves of the tooth. The other cavity, or so-called 
"infundibulum," is open at the side next the table, and forms a reflected funnel.

Through wear, the enamel of the incisors is divided into two portions, the exterior or casing enamel, and the other, the interior, which envelopes the infundibulum. The enamel being harder, and offering more resistance than the dentine and crusta petrosa that surrounds it on all sides, the central enamel presents a slight prominence, and takes on different forms in proportion as the funnel becomes destroyed and contracted.

As we have stated, the horse has forty teeth, namely,-six incisor, or cutting teeth, in both jaws, (see Plate III, fig. 2, b, b); two tushes, or canines, in each jaw, $a, a$; and six molars, or grinders, in both jaws, $h, h$. There is a considerable vacant space between the incisors and tushes, as also between the tushes and grinders, as will be seen by the figure referred to. These teeth are inserted into sockets, consisting of a spongy bony substance, called the alveolar process, which forms the edge of the maxillary bones. All the teeth are first germinated in the interior of the maxillary bones; and having acquired a certain size, and the exterior table of their sockets having been removed by absorption, they push out above the gums. Those which appear shortly after birth are called sucking or temporary teeth; these consist of all the incisors and the three first molars. The formation and appearance of the others are later, and are called the permanent teeth; and those which succeed the temporary are called the replacers, or horse-teeth. 


\section{DENTAL INDICATIONS OF THE AGE OF A HORSE.}

Nothing can more clearly indicate a proof of design than the beautiful contrivance in the formation of the molars of the horse for the purposes required. They are, like the incisor teeth, covered on the sides with enamel, but not on the top, though several portions of enamel enter into their substance in their internal structure. They are subjected to much more friction than the cutting teeth in grinding down the harder portions of their food, and nature has made ample provision for their strength and endurance.

We have given a representation of the section of a grinder (Plate v, fig. I2) to show its interior structure. The teeth are prepared and formed in cavities within the jaw-bones. In the unborn animal a delicate membranous bag, containing a jelly-like substance, is situate in the small cell within the jaw-bone. By degrees this substance assumes the form of a tooth, and then the jelly within the membrane begins to change to a bony consistence; then a hard crystallisation is formed on the outside of this membranous covering. This is the enamel of the tooth. In the formation of each grinder of the horse in the upper jaw, there are originally five of these membranous bags filled with jelly, and four in the lower jaw. This jelly is gradually superseded by bony matter, which is deposited by little vessels penetrating into it. These vessels are represented by the black streaks in the carker central portions of the figure, around each of which the crystallisation of enamel can be distinctly traced, so that there would be five 
distinct bones or teeth. The white spaces in the figure represent a very powerful cement, which unites all these distinct bones into one compact body, thus making one tooth of the five as they originally appeared to be; this being accomplished, an outer coating of enamel invests the whole of the sides, but not the top, which completes the tooth. It will thus be manifest that this is a beautiful and perfect contrivance to prevent the wearing down of the grinders by the constant friction to which they are subjected by chewing the harder portions of their food. Thus it will be seen there are columns of enamel penetrating through the entire substance of the tooth; this, together with the bony matter and cement by which the different layers are united, and which fill the spaces between the columns, soon begin to wear away, while the enamel remains, thus occasioning the uneven surface presented by them, and which is the very best structure for them to possess in order to grind down the food.

The grinders in the lower jaw are smaller and narrower, and more regular in their form than the upper ones. They are placed horizontally in both jaws; but in the lower one the higher side is within, and gradually shelving outwards, while in the upper jaw the higher side is without, and shelving inwards; by which beautiful arrangement the triturating or grinding motion is most advantageously performed. Each of the grinders is different in appearance, and can be respectively recognised with the situation of, and the jaws to which they belonged.

When the foal is born, it has the first and second temporary molars, which are large compared with the size of those by which they are afterwards 
replaced. In six or eight days after birth, the two central incisors make their appearance. These are large in comparison to the size of the jaw, and occupy its whole front, as represented in Plate IV, fig I. In three weeks or a month the third temporary molars will have made their appearance, and within six weeks an additional incisor (middle) on each side of the two first, both above and below, will be visible, and soon after completed, and the jaw will have assumed the form exhibited in fig. 2. In from nine months to twelve months the corner temporary incisors appear. The two molars that the foal has at birth remain until the animal is three years of age, at which time they fall out of their sockets by the protrusion of the second set, or permanent molars. But from this time there is no use in consulting the grinders for the age of the horse, as the best tests and easiest come at are the incisors. The permanent molars appear in the following order: the first permanent (but the fourth by position in the jaw) at one year, the fifth by position at two years; the first and second by position at three years; the third and sixth by position at four years.

The incisor teeth are continually undergoing some change, in consequence of the friction produced by the action of their rubbing against each other. The anterior edge being considerably more elevated and sharper than the posterior, the wear first commences there, and in a short time it is level with the posterior, then both wear together; the longitudinal cavity, or infundibulum, becomes narrower, and afterwards triangular, and, finally, at a certain period disappears, and is replaced by the small end of the funnel next the root; it is 
this regular wear which is termed "losing the mark" (see fig. 4). The obliteration of the mark has frequently taken place by the time the corner teeth are beginning to appear. It must be distinctly understood, that in speaking of the marks in the tooth we constantly refer to the incisors of the under jaw, except when otherwise expressed; and the ages of all horses, except thoroughbreds, are reckoned from May.

When an incisor tooth has commenced wearing, and its two edges are parallel, the table exhibits two bands of enamel, the one exterior, that surrounds the tooth, which is termed the casing enamel; the other internal, only surrounding the cavity, and this is called the central enamel (see Plate Iv, figs. 3 and 5). The incisor teeth of the lower jaw always wear more quickly than those of the upper, and uniformly more regular. The reason of this has never been satisfactorily accounted for, but it is certainly very remarkable. From this fact it is obvious that it is more difficult to judge of the period of obliteration of the mark in the upper jaw. In the lower jaw the marks of the central incisors are always obliterated at about ten months, in the dividers or second teeth at one year, and from the corner or inner incisors varying from fifteen months to two years.

By this time the marks of the upper nippers have become almost entirely obliterated, so that at two years old the cavities in all the temporary incisor teeth have disappeared.

At this period the crowns of the incisors become insensibly smaller, and with their base necked. They also assume a yellowish-brown aspect, soon after which they loosen and almost entirely lose their 
attachment in the gums, and finally fall out. It is at this time the second period in the age of a horse commences.

The mark in the tooth is occasioned by the food blackening the infundibulum or hollow pit which is formed on their surface by the bending in of the enamel, which passes over the surface of the teeth; and by the gradual wearing down of the enamel from friction, and the consequent disappearance of it, we are enabled for several years to judge of the age of the animal.

In the third year, the tushes sometimes begin to make their appearance, although there are instances of their not being developed until the fifth or sixth year. But the most general time for their appearing is in the fourth year. Little dependence can, therefore, be placed on them in determining the age of the horse. We have given a representation of a three-year-old mouth (fig. 5), in which it will be seen the central teeth are larger than the others, and are provided with two grooves in the outer convex surface. The mark is long, narrow, deep, and black; and these teeth, not having attained their full growth, are somewhat lower than the others. In the two next teeth the mark is nearly worn out, and in the corner teeth it is slowly disappearing.

At this period it is not unusual for dishonest dealers to deceive the unskilful with regard to the age of a horse. If they happen to possess one that has been foaled a little earlier than usual, probably in January, and which may have acquired bulk and strength, they punch or draw out the central nippers, and the others appear some months earlier than they otherwise would. This will allow the 
teeth to rise much quicker than they would do naturally, and three or four months may be thereby gained in their reproduction; consequently dealers who wish to take advantage of this may sell a colt for a year older than he actually is.

But we must look to the general form of the animal, and when young it will be manifested by the small development of the forehead. The second pair of incisors will still have the mark, and it will also be much stronger on the corner ones, and probably with an enlargement or irregularity about the gums, in consequence of the teeth having been violently forced out; the first and fifth grinders will be small, and the sixth will not have yet appeared. As it is the teeth of the lower jaw which are usually consulted as to his age, dealers who wish thus to impose seldom extract the teeth of the upper jaw, therefore it would be well to examine it also, when all the signs will remain. Attention to these points will enable anyone to detect the true age of the colt.

At two years to two-and-a-half the central temporary incisors are cast, and at three years replaced by the central permanent. At three years to three-and-a-half the middle temporary incisors are cast, and at four years replaced by the middle permanent. At four years to four-and-a-half the corner temporary incisors are cast, and at five years replaced by the corner permanent.

A horse at three years of age will have the central permanent nippers growing with their edges sharp in comparison to the others.

The tushes are now fully half-an-inch above the gums, with an external rounded prominence, and a groove on each side. $U p$ to the period of the reproduction of the corner incisor the animal is 

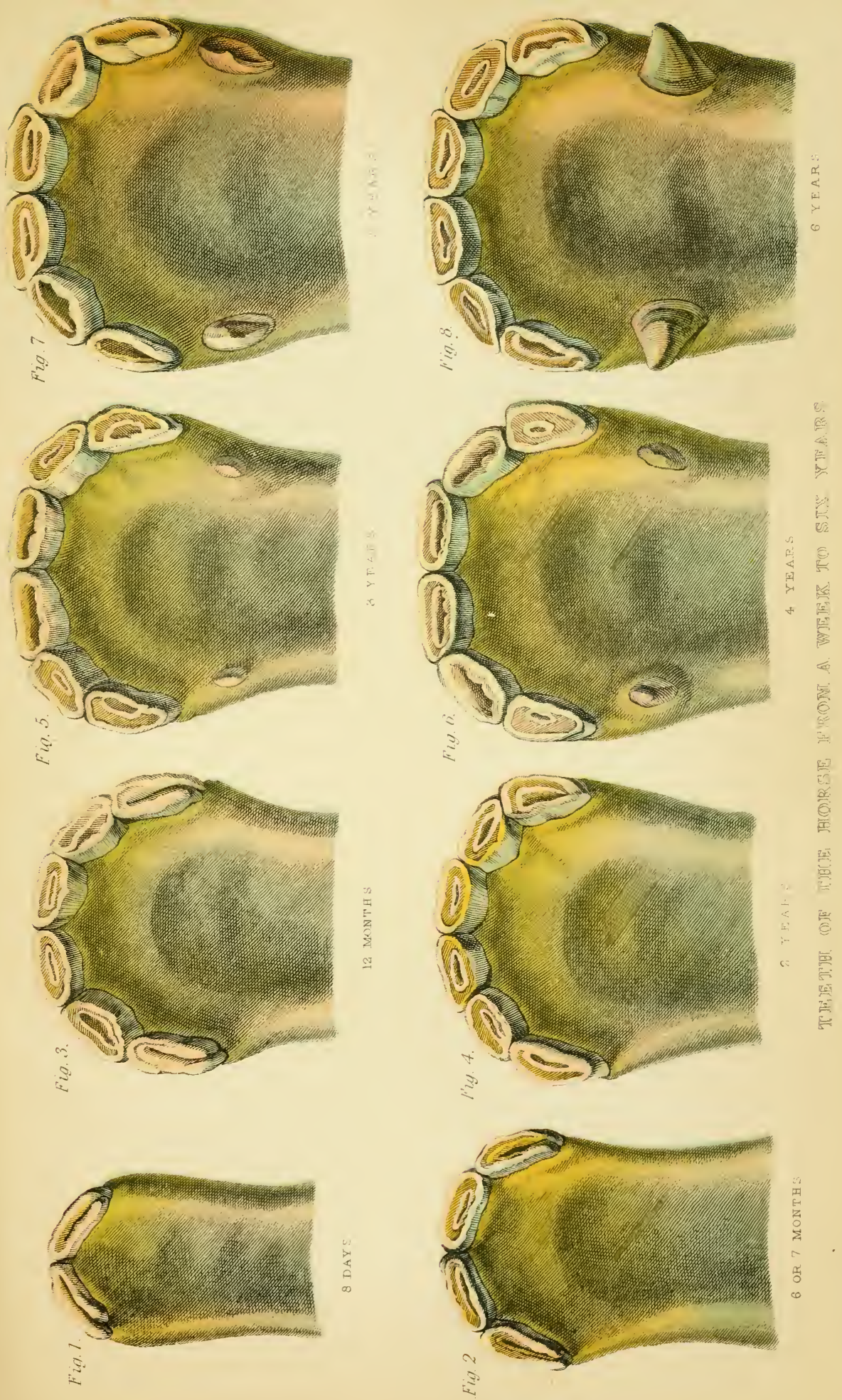

designated a colt, but it now assumes the name of horse; the female, which up to this time has been denominated filly, is now called mare.

The mouth of the horse is nearly perfect at the age of five years. The corner incisors are fully developed, with the mark on the inside long and irregular. (See Plate IV, fig. 7.) The other nippers will exhibit considerable indications of wearing, the tushes be much grown, and the grooves on the sides will have nearly or quite disappeared, presenting an almost regular convex surface. They are still, however, as concave within, and with their edges nearly as sharp as they were six months previously. The third and sixth molar teeth will have attained their complete height. The three last grinders and tushes are never shed.

When the animal has attained its sixth year, the mark on the central incisors, or nippers, will be completely worn off, leaving, however, a little difference of colour in the centre of the teeth. The cement which fills the hole produced by the dipping in of the enamel will be somewhat browner than that of other portions of the tooth, and will exhibit evident proofs of the edge being surrounded by enamel. This condition has perplexed the inexperienced, as many expect to find the surface of the tooth plain, and of a uniform colour, whereas they are both irregular and discoloured, as we have above described. (See Plate Iv, fig. 8).

In the second incisors the mark is shorter, broader, and more faint, with the enamel of the edges of the corner teeth more regular, while the surface gives sufficient evidence of wear. The tushes will have attained their full size, being fully an inch long, convex outwardly, and a little concave 
inwardly, accuminated towards the point, and slightly incurved at the apex.

During the long period which the horse is subjected to teething, it is surprising how little the animal has suffered from it, and hardly a day of his valuable services will have been lost. In some instances heat and swelling of the gums take place, but this can easily be removed by scarification. Here, then, we have an example of the wisdom and perfection of the works of creation; were the horse to suffer as much as the human being, he would not be able to chew his food for weeks, and must consequently fall off in flesh. Dogs are liable to convulsions while cutting their teeth, and many even die during this process.

At seven years the marks in the four centre incisors are worn out, and are speedily disappearing in the corner ones. The tushes are now undergoing an alteration of form; they are rounded at the point and edges, but still inflated outwardly, and beginning to round inwardly; the inner edge of the corner teeth is on a level with the outer, in consequence of wear. Sometimes a notch is observable in the upper corner teeth. (See Plate v, fig. I.)

At eight years old all the marks on the teeth of the lower jaw will be obliterated, and will be on a level at the surface; their form is changed, they assume an oval shape, and the cavity is altered into an elongated transverse protrusion of enamel, which is the termination of the central enamel, or funnel next the root. (Plate v, fig. 2.) After this period it is the practice to examine the nippers of the upper jaw, and to draw deductions as to the age of the horse; because, for years after the marks 
have become obliterated in the lower jaw, traces of them exist in the upper jaw. This is in consequence of the enamel which occupies the pit in the centre of the tooth not being elevated to a level with the general surface, so that there is a greater depth to be worn down in order to rub it off; and besides, the upper incisors are less liable to friction and wear than those of the under jaw, in consequence of the lower jaw alone being moved in the act of chewing, and the upper jaw being fixed and without motion: its office is only to resist the pressure of the under jaw in eating.

The tushes are of no use whatever in enabling us to determine the age of a horse, because the change of their form is very uncertain. They do not rub against each other like the teeth, and are consequently less liable to be worn down.

At nine years the infundibulum is gone in the inferior incisors, and the inside and outside walls of the upper corner teeth are level.

At ten years the upper central incisors loose mark; at eleven years the middle, and at twelve years the corner. To tell the age of the animal beyond this period is difficult and uncertain, but the shape of the teeth and other conditions facilitate the undertaking. Young permanent teeth are about twice as broad as thick, whereas the old are the reverse, and this difference graduates itself yearly, but not by growth and change in the teeth themselves. This change is by wear of the crown and body of the teeth, and absorption of the alveoli or sockets. In the young the teeth describe a semicircle, and stand perpendicular, and the alveoli or sockets are deep. In the old they shoot almost straight out, like the point of scissors, the upper 
incisors overlapping the under, and the alveoli are shallow from absorption, just as is seen in old people when the chin and nose meet.

At ten years a longitudinal groove makes its appearance in the middle of the body of the corner upper incisor teeth, immediately under the line of the gum. This groove or longitudinal line takes eleven years to come down from top to bottom, so that when it reaches the bottom of these teeth the horse is twenty-one years of age. To return, at thirteen years the groove is a quarter way down; at fifteen to sixteen years, half-way down; at eighteen years, three-quarters ; and at twenty-one years, all the way. After twenty-one years, the top of the corner teeth next the gum commences to get round in shape and yellow in colour, and the groove ceases. The longer the round portion of the tooth above the groove, the older the horse. At twenty-five or twenty-six years the round portion is a quarter way down, at thirty years it is round half-way down.

In consequence of the continual growing up of the teeth of the bone from the side next the root, and the socket not being sufficiently long, the pressure of the new portion of the root gives the tooth an outward inclination. Besides, these new portions of the teeth being always narrower, the sockets must necessarily contract, in order to secure the teeth. At this time the sides of the superior maxillaries become flattened, and the head assumes a lengthened and pointed form, which gives to the animal an appearance which is very indicative of age. The horizontal direction, owing to the same cause, is always a mark of advanced life, but this direction is much developed in some horses, and 
not at all in others, the cause of which remains still a question.

The horizontal direction of the teeth, which increases with the years of a horse, will be easily understood by a reference to Plate v, fig. I8. This figure represents the under incisor teeth of a horse known by the name of "Old Billy," which attained his SEventy-sixth year, and was perhaps the oldest horse that ever lived. The cranium, with the muscles preserved, is deposited in the Museum of the Manchester Natural History Society, from which I made the drawing. The whole of the incisors are much elongated horizontally, but not perpendicularly elevated more than those of a horse at six years of age. The upper surface of the nippers and dividers are of a quadrangular form, the inner margins being a little rounded, while the corner teeth are oblong oval, nearly the form of an egg, with the most acute end outwards. The tushes are conical, a little blunted on the crown, and turned backward, with an elongated, shallow, curved groove on their inner sides. It will be seen that the outer edge of all the cutting teeth is nearly parallel, the corner teeth being only a ittle less produced than the others. It does not appear that the jawbones themselves have been lengthened, the teeth alone having shot forward ; consequently, their surface is lying obliquely, and hence their elongated form from front to back, as more particularly developed in the corner teeth. The tushes are considerably larger in proportion than in a horse under twenty years of age.

The portion of the tooth which is buried in the gums is called "the root"; the nippers are placed parallel to the axis of the jaw, that is, perpendicular to it; the dividers are somervhat oblique, and the 
corner teeth still more so, with their roots turning inwards; the semicircle formed by the roots of these teeth is considerably narrower, and occupies less space than that of the free portion.

The entire length of the temporary incisors varies from an inch and a quarter to an inch and threequarters; and the permanent incisors from two inches and a half to three and a quarter. Their form is not the same throughout. Thus, next to the surface of wear, they become flattened from front to rear, they narrow towards the edge of the socket, are at first oval and then rounded, and of a triangular form towards the base of the root, with the extremity flattened from side to side. This variation is much more remarkable in the nippers and dividers than in the corner teeth, which is rendered more obvious by making several sections of an incisor tooth at somewhat about a quarter of an inch apart. We have given five transverse sections of the tooth of a young horse to exhibit this: Plate v, figs. I 3, I 4, I 5, I6, and I 7 , have the funnel, and the sections, I 6 and I7, show the septum of the root of the tooth.

The incisors of the upper jaw are stronger, broader, and more developed than those of the lower; consequently the outer edge of the lower corner teeth rests against the centre of the upper corners, and wears them in such a manner as to produce in some jaws a triangular nick, which leads in a certain degree to the determination of the age of a horse. This nick never appears until the horse has attained its seventh year. It becomes obliterated in time, but sooner in proportion as the jaw assumes a horizontal direction.

We have given representations of six teeth, show- 
ing the exterior of the incisor teeth, together with their internal organisation.

The tooth of a foal viewed from its posterior or inner surface. Plate $\mathrm{v}$, fig. $8, a$, the mouth of the cavity of funnel of the table.

Another foal's tooth, viewed anteriorly, fig. $7: a$, exhibits the body of the tooth; $b$, the neck; and $c$, the root.

The tooth of a very young foal, in which the casing or outer enamel is cut through its whole length, and exhibiting the central enamel, fig. Io, $a$.

The molars, or grinding teeth of "Old Billy," mentioned at page 197, are worn in a singular manner. The first grinder of the lower jaw, on both sides, is worn down to about half-an-inch above the gum ; the second, on a level with the gums, forming a hiatus into which the second grinder of the upper jaw fits, which is fully a quarter of an inch longer than the first grinder, and very uneven and unequal on the surface. About half-an-inch of the outer portion of the fourth grinder is parallel with the third, but the remainder is worn level with the jaw; the fifth and sixth molars are almost entirely worn down, except a small portion of the inner part of the sixth. The third upper one is more than half worn down, and the fourth level with the socket; part of the fifth and sixth are worn obliquely down, the cavity formed by which is occupied by the lower opposite teeth, and the worn down fifth and sixth of the lower jaw are occupied by the upper ones. In short, it is curious how mastication could be performed.

It seems probable that the natural age of the horse is from thirty-five to forty. It would be most erroneous to estimate his life according to the age 
at which he is worn out in a state of servitude. Few of these valuable animals live to anything like what they would do in a state of freedom, most of them being unserviceable or destroyed by excessive labour. Mr Blaine informs us of one gentleman who had three horses which attained a considerable age: one at thirty-five, another at thirty-seven, and the third at thirty-nine. $\mathrm{Mr}$ Cully mentions one which received a ball in his neck at the battle of Preston, in 1715 , and which was extracted when he died in $175^{8}$, so that he must have been forty-seven or forty-eight years of age. Albertus mentions that in his time there was a charger, proving serviceable at the advanced age of sixty, and Augustus Nephus says there was a horse in the stable of Ferdinand the First that had attained the age of seventy years. This is the oldest horse mentioned by any author as far as I know; so that in all probability "Old Billy" is the Parr of horses.

The incisor tooth of a horse, divided into two parts its whole length, fig. I 2 , and which exhibits the external cavity, $a$, and the internal cavity, $b$.

The incisory tooth of a young horse, with an opening at the middle of the anterior surface, leaving exposed the lower portion of the funnel, fig. I I, $a$.

The incisory tooth of a young horse, divided throughout, fig. 9, $a$, shows the exterior cavity, and $b$, the extremity of the funnel.

Independently of the teeth, there are other signs by which an aged horse is known: first, the temporal fossæ or pits above the eyes become much sunk, grey hairs are also seen above the eyes and about the muzzle, the lips being thin and hanging, the back sinks, the withers become sharp, the quarters lengthened, spavin, windgalls, and all kinds of 

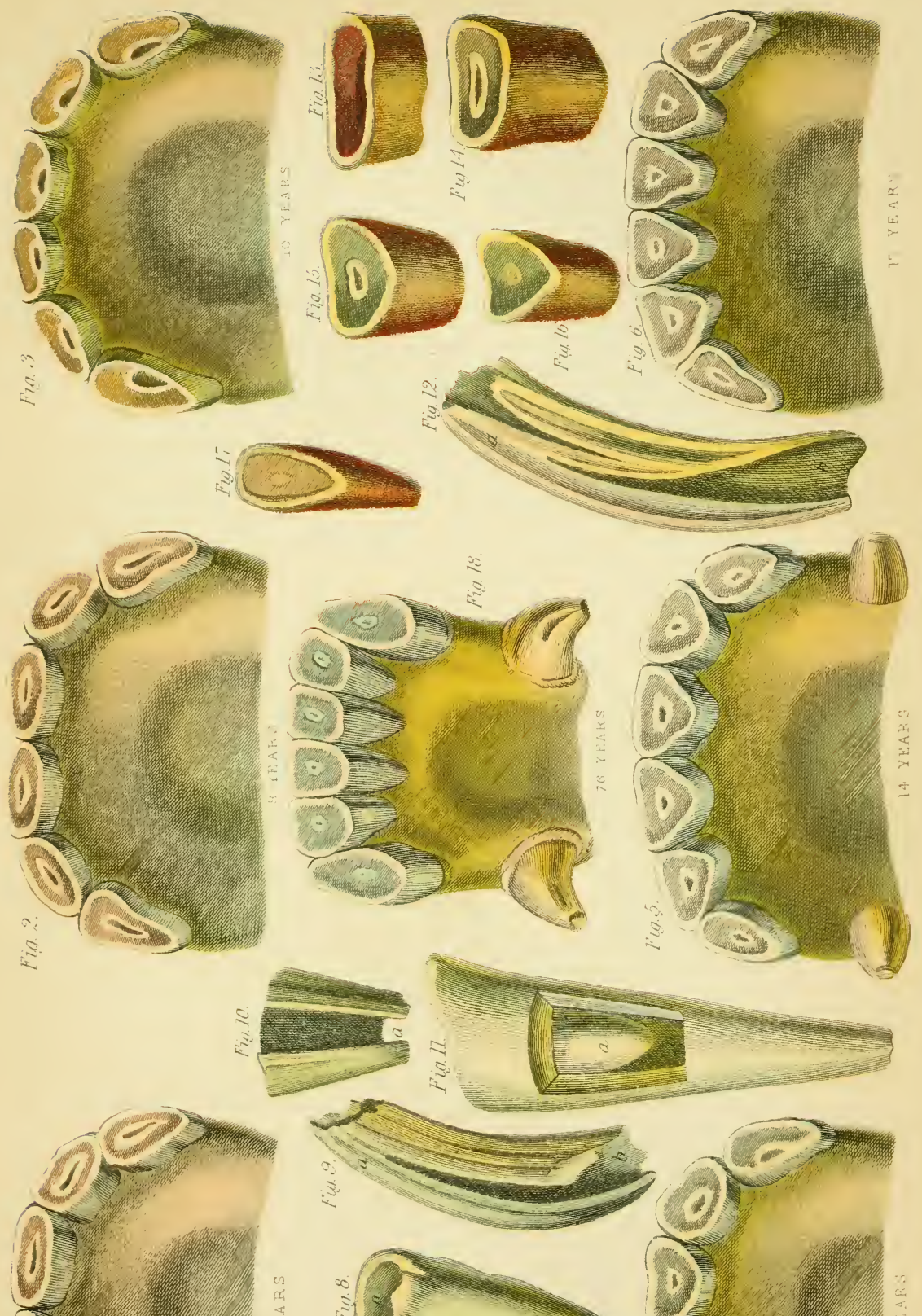

(1)
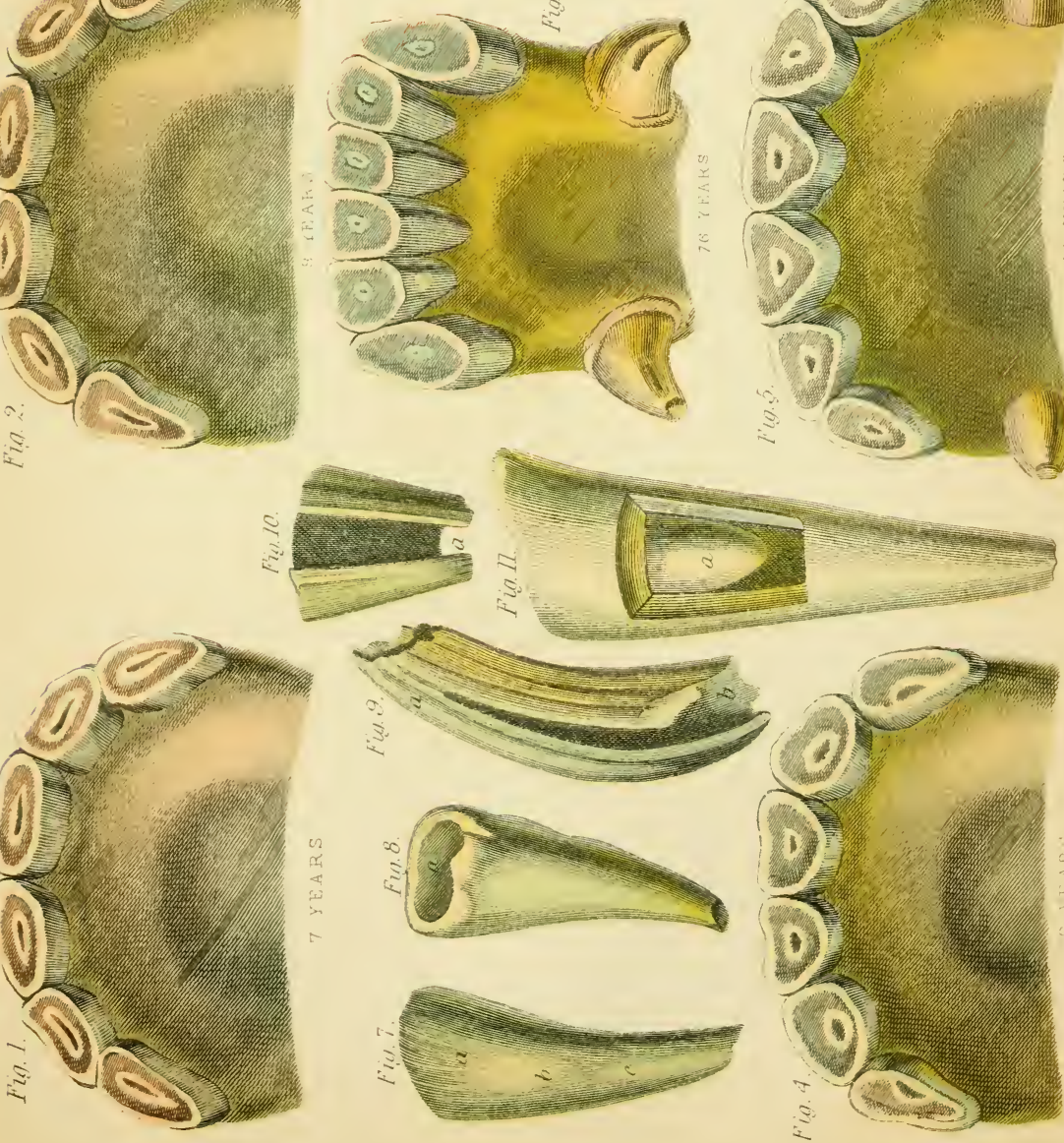

$$
\text { 돈 }
$$



tumours disappear, and for the most part the animal has a dull and heavy appearance, with hanging of the head.

Various are the tricks resorted to by dealers to impose upon the uninitiated. They endeavour by fraudulent means to make them appear most near the age at which they are of the greatest value, and when they can of course obtain the highest price for them. If too young, they endeavour to make them seem older, and on the contrary, if too old, they try to give them a youthful appearance. Besides what we have mentioned at page I9I, there is a practice amongst dishonest dealers to prolong the youthful marks of the teeth of a horse by what has been designated Bishoping, after the infamous inventor of this fraud. This trick is to imitate the appearance of the mark. A horse of eight or nine years of age is taken, and, by the aid of an engraving tool, a hole is dug in the surface of the corner teeth to imitate that in a horse of seven years of age. The hole is then burned with heated iron, until it leaves a permanent black stain. This is sometimes extended to the dividers, in a slight degree. But upon a narrow inspection this trick will be detected. The irregular appearance of the cavity, the absence of the ring of enamel around the infundibulum, the diffusion of the black stain around the tushes, the sharpened edges and concave inner surface can never be successfully imitated so as to deceive the experienced. Besides, if the incisors of the upper jaw be examined, they will aid the most ignorant in detecting the imposition. 


\section{CHAPTER IX.}

\section{Principal Muscles of the Limbs, etc.}

WE do not consider it necessary for the general reader to be acquainted with the entire muscles of the horse, although a knowledge of such as are more immediately called into active operation will aid him in discovering the seat of sprains and bruises, and to administer for their relief, in the absence of a regular practitioner.

\section{MUSCLES OF THE OUTSIDE OF THE SHOULDER.}

\section{PLATE VI, FIG. I.}

a. A portion of the sterno-maxillaris, or muscle which is common to, and connected with, the lower jaw, as well as the fore part of the chest. This is one of the muscles employed in lowering the head. It lies immediately under the skin, and arises from the cartilage projecting from, or constituting the breast-bone, Plate II, fig. 2. It then traverses the neck in an upward direction, and is neither of great dimensions nor strength. About threefourths of its length upwards, it changes to a flat tendon, and is exhibited, Plate III, fig. I, $w$, above which it insinuates itself between the parotid and submaxillary glands, that it may be inserted into the angle of the lower jaw. It is exerted in bending the head towards the chest.

$b, i$, Is a muscle which is the raiser of the shoulder, and takes its rise from the nipple-shaped process of the temporal 
bone, $c, c$, Plate III, figs. 2 and 3, and extending down the fore part of the neck, is inserted into the middle part of the lower bone of the shoulder, and thence continued down to the arm. The use of this muscle is to bend the head; or, the head and neck being fixed, to elevate and bring forward the arm. When the horse is running at full speed, with the head thrown forward, this muscle is in powerful action.

$c, d$. The serratus major, or great tooth-shaped muscle of the shoulder, which constitutes a large proportion of the lower part of the neck. It is deeply seated, arising from the five last bones of the neck and the two first ribs. Its lower portion springs from all the true ribs. The whole of its fibres have a downward tendency, and are inserted into the inner surface of the shoulder; by means of which the shoulder is attached to the chest, and the weight of the body thereby supported.

When the horse is in a standing position, this muscle occasionally performs another important office. The shoulders and legs, by means of the weight of the body, are fixed and immovable; consequently, having no longer any power over the motion of the limbs, it now exercises its power in enlarging the cavity of the chest, and thus materially contributes to give freedom to the action of the chest in breathing.

In treating of inflammation of the lungs, page 47 , we mentioned that horses labouring under this disease would stand night and day. The cause of this is, that the breathing is laborious and painful, and the animal in standing obtains the aid of this muscle in giving ease to the respiration. Therefore, when he does lie down, it is a pretty strong proof that the violence of the disease has abated, and consequently he no longer requires the assistance of this muscle.

$e, f$. These parts represent portions of the trapezius muscle, which is of a quadrangular form, and rises from the 
sides of the withers. Its base is turned upwards, and its right side forwards in a parallel line with the spine of the scapula or shoulder-blade. It occupies the space between the withers and upper part of the scapula, and is large and strong in proportion to the height of the withers, and the oblique direction or slanting of the shoulder. Its use is to elevate the scapula, as well as to draw it backward. This muscle is one of the most important as connected with the action of the horse, and prominently illustrative of the advantage of high withers and a slanting shoulder. We have represented a portion of it turned back to exhibit the muscles beneath.

$g$. Is the little pectoral or breast-muscle; it is connected with both the breast and shoulder-blade. It takes its rise from the breast-bone, and extends to the covering of the shoulder-joint and to the muscle of the shoulder. It lies below the greater breast-muscle. Inwardly it is attached to the anterior half of the breast-bone, and to the cartilages of the first four ribs. Its action is common with that of the larger breast-muscle (pectoralis major), and to assist it in drawing back the top of the lower bone of the shoulder and the lower part of the scapula, as well as to keep the latter bone more upright in its position.

h. The antea spinatis muscle, or muscle before the spine, is placed on the external part of the shoulder, before the ridge of the shoulder-blade, and fills the whole of that space. It proceeds downwards, dividing into two parts, each of which is attached to the summits of the greater and lesser tubercles of the humerus, or bone of the arm, and also to the capsular ligament of the shoulder-joint. It is a muscle of great strength, and extends the arm or humerus upon the scapula, and moves it forward. See the description at $b$.

$k$. The postea spinatus niuscle, situated behind the spine or ridge, and occupying the space called the fossa postea spinata. It extends to the lower bone of the shoulder, and is inserted into the upper head of that bone. It is of a triangular form, and is flattened, and broacier, but not so thick in substance as the antea spinatus muscle. It assists the flection of the humerus, and at the same time rolls it outwards. This is the tendon of the long 
extensor of the arm, which reaches from the upper angle and the posterior border of the scapula, to the point of the elbow and the inside of the arm. This muscle is a very important one.

$0, q, t$, Are the three divisions of another muscle concerned in the same office with $p$, arising from the shoulder-blade and the lower bone of the shoulder, and likewise attached to the point of the elbow by a very powerful tendon.

t. This is the middle flexor, which is one of the numerous and powerful muscles which bend the leg. It is also represented at $e$, Plate VI, fig. 2 , and is the muscle which bends the shank-bone, because it is situated precisely in the middle of the back part of the arm. It has its origin from the inner head of the lower bone of the shoulder, and is inserted into one of the bones on the inner side of the knee. The other is seen at 2, fig. I, and is called the external flexor of the leg, in consequence of lying on the outer side of the arm, towards the back. It is inserted on the outer head of the lower bone of the shoulder; advancing towards the knee, it is tendinous; and the tendon divides into two portions, one of which is inserted into the same bone of the knee, and the other into the outer small bone of the leg. The internal flexor is exhibited at $g$, Plate vi, fig. 2 .

$u$. This is one of the muscles of the lower bone of the shoulder. It is the external one, whose office is to bend the arm. It arises from the inner and back part of the neck and body of the lower bone of the shoulder, and turns obliquely round it, and is inserted into the inner and upper part of the bone of the arm.

$w$. This is the extensor of the leg; it is the principal one of the fore arm, is of considerable bulk, and occupies the front part of the arm. It takes its rise from the lower portion of the body of the lower bone of the shoulder, and from its outer head. As it descends down the arm, it becomes tendinous. This tendon passes under one of the ligaments of the knee; it then spreads out, and is inserted into the fore and superior portion of the shankbone. It is also seen at $e$, fig. 2 .

$x$. The middle flexor, or bending muscle of the shank-bone. This is situated immediately on the middle of the back part of the arm. It arises from the inner termination of 
the lower bone of the shoulder, and is continued and inserted into one of the bones on the inner side of the knee. It is also seen at $e$, fig. 2 .

2. The external flexor of the leg, and which is situated on the outer side of the arm, towards the back. It has its origin in the outer head of the lower bone of the shoulder, stretching towards the knee. It is of a tendinous character, and divides into two portions, one of which is inserted into the same bone of the knee, and the other into the outer small bone of the leg.

3. And extending down to 7,4 , and 5 , are the principal artery, nerve, and vein of the leg.

7. This is the perforated flexor muscle. It arises from the lower and back portion of the inner head of the lower bone of the shoulder, and is intimately intermixed with the perforating flexor muscle. As it descends along the bone of the arm, it becomes tendinous, and extending to the knee, it is bound down by ligamentary bands to prevent it from starting in sudden or violent motion. From the knee it widens and partly laps round the tendon of the perforating muscle; they then descend together in contact, but are not adherent, sliding over each other freely and safely by the aid of the lubricating fluid. They are both encircled in a thick sheath of cellular substance which is attached to them by numerous small fibres. Reaching near to the fetlock, the tendon expands still more, forming a complete ring round the tendon of the perforating muscle. This is shown at $k$, Plate vi, fig. 7 . The perforated tendon soon divides, and is inserted into the larger and smaller pastern-bones, its office being to bend them.

8. The subcutaneous vein of the side of the chest.

\section{MUSCLES ON THE INSIDE OF THE SHOULDER.}

\section{PLATE VI, FIG. 2.}

a. The flexor of the arm, which is one of the most powerful of the flexor muscles. It has its origin in the extremity of the ridge of the shoulder-blade, in the form of a large and round tendon, which extends between two 


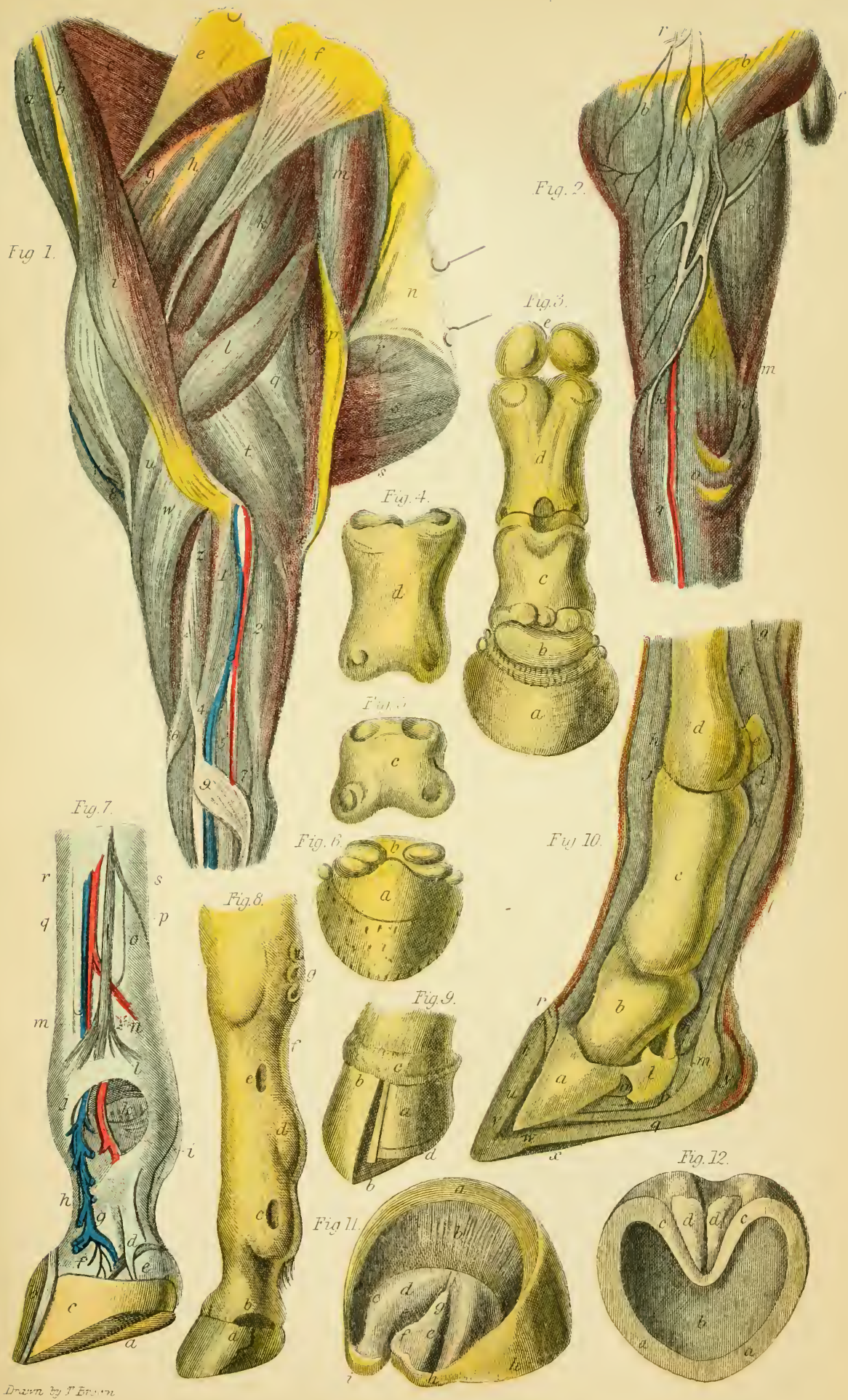



prominences in the upper portion of the front of the lower bone of the scapula. This groove, or pulley, is as perfect as it is possible to suppose it, and is lined with smooth cartilage, between which and the tendon there is interposed an oily fluid, which enables the tendon to move in this pulley without the danger of being injured by friction. Passing on from this pulley, and extending beyond the head of the lower bone of the shoulder, the cord spreads out into a round fleshy substance, containing numerous tendinous fibres. It is deeply seated, and gives that fine fullness to the front of the arm. It is inserted into the head and neck of the bone of the arm, as also into the capsular ligament of the elbow-joint. It is the chief muscle, by which nearly the entire of the leg below the arm is bent.

$b, b$. This muscle is called the pectoralis transversus, or the muscle which crosses the breast. It takes its rise from the first four bones of the chest, and, stretching across to the inner part of the arm, is inserted into the tendinous substance which covers the muscles of the fore-arm, and extending a considerable way down the arm. Its use is to bind the arm to the side of the horse, and it also keeps the legs straight before the horse when it is at speed, so that the weight of the body may be received on them in a direction most easy and safe to the horse as well as the rider, and most advantageous for the full action of all the muscles connected with progression.

c. This represents the disease called capped elbow, or an enlargement of the joint of the elbow, as described at page 87 .

g. The internal flexor. It takes its rise from the inner head of the lower bone of the shoulder, and is inserted into the head of the inner splint-bone. Its office is to bend the leg, and to turn it very slightly.

$r$. The principal veins, nerves, and arteries of the shoulder and arm. 


\section{MUSCLES OF THE OUTSIDE OF THE THIGH.}

\section{PLATE VII, FIG. I.}

a. The great glutæus muscle, or gluteus maximus. It occupies the anterior, middle, and external parts of the haunch. It arises from the spinous and transverse processes of several of the bones of the loins, from the sacrum, and from the different edges of the ilium, and is inserted into the protuberance of the upper bone of the thigh, behind and a little above the joint that unites the thigh to the haunch-bone. It is this muscle which gives that fullness and roundness to the haunch so much admired. It is one of the chief muscles of progression.

b. The outer glutæus or buttocl-muscle, which can only be considered as a fleshy slip attached to the great glutæus. Its origin is as high as the spine, and it runs along the back part of the thigh in the form of a ridge, and is inserted into the smaller outer prominence of the upper bone of the thigh.

o. This muscle arises high up from the bones of the spine, from others at the root of the tail, from the protuberance of the ischium, and from other bones of the pelvis. It is, in fact, a sort of triple muscle, as it has three heads; it is called the triceps femoris, or threeheaded muscle of the thigh. It is inserted into the upper part of the lower bone of the thigh, and its office is to draw back the thigh when placed under the trunk, and so to throw forward the trunk of the body.

d. This muscle descends from the sacrum, and from the first bones of the tail runs down posterior to the triceps femoris, and constitutes the hinder border of the haunch. It is inserted into the lower bone of the thigh, and assists in performing the same kind of motion. The whole of these muscles are very much developed in the thoroughbred horse, and hence his strength and speed, which is besides another very important point in the horse. To perform their full action, these muscles should be so prominent that the 
horse, when the observer stands behind him, should be perceptibly wider at the thighs than at the loins and haunch.

$e$. The root of the tail, with its muscles.

f. This is the tendinous expansion which binds and strengthens the above muscles.

$g$. Is the flexor metatarsi, or muscle used to bend the hocks, or bender of the leg. It arises from the lower part of the upper bone of the thigh, and is inserted into the upper portion of the shank bone, and also the inner splint bone. It is a muscle of considerable porver.

h. The extensor pedis. It is situated on the anterior part of the thigh, and is attached to a roughened depression upon the antero-inferior part of the external condyle of the femoral bone, and below to the coronal process of the os pedis, and to the upper edge of the bone in the interval between the lateral cartilages. Its office is the extension of the foot, as well as the fetlock and pastern joints.

$i$ This letter is placed at the situation occupied by the principal nerves before they pass under the muscle. They take a direction nearly in a line with the letter $c$.

$l, p$. The poplitaus or femoro-tibialis obliquus, a short, thick, triangular muscle, extending from the upper to the lower thigh bones, bending the stifle, and turning the limb inward.

$k$. The use of this muscle is to extend the hock. It is an exceedingly powerful muscle, having its origin in the head of the upper bone of the thigh, and, midway down the lower bone of the thigh, ending in a flat tendon, which is inserted into the joint of the hock. It is advantageously placed for powerful exertion, for it acts nearly at right angles.

$m, n$. The peronculs, which is another of the extensor muscles, and so called from a name given to the fibula. It arises from the whole course of the fibula, and also becomes tendinous before reaching the hock. About half-way down the shank, it occupies the same sheath as the principal extensor muscle, and is inserted along with it into the coffin bone. The 
province of the extensor muscles is to raise the foot from the ground, and to bring it forward under the body.

o. The flexor pedis, one of the chief bending or flexor muscles of the foot, which has its origin in the upper part of the tibia. As it approaches the hock, it is distinguished by its large round tendon, which is seen to enter into a groove at the back of the hock. It is continued down the back of the leg in the same manner as a similar muscle in the fore leg. It is the performing flexor muscle of the hind leg, and assists in binding the pastern and coffin joints.

\section{CHIEF MUSCLES OF THE INSIDE OF THE THIGH}

\section{PLATE VII, Frg. 2.}

$b$. The blood-vessels belonging to the groin.

$d, x$. These represent the course of the principal anterior arteries and veins, which extend to $x$, and include in their range $d$ and $k$.

$c$. The gracilis, or slender muscle, which is very broad, and occupies the greater portion of the surface of the inner part of the thigh, and especially the prominent part of it. It has its origin in the lower part of the haunch bone, and in its downward passage unites with the sartorius, and is inserted with it into the lower bone of the thigh. Its province is also to turn the leg.

$j$. A portion of the muscle described at $k$, fig. I.

$k$. The peronaus. This muscle is attached above to the head of the fibula, continuing its attachment for the whole length of that bone, and below to the coronal process of the os pedis.

l. The poplitanus, a short muscle.

$m$. The flexor metatarsi, or bender of the leg, which arises from the lower part of the upper bone of the thigh, and is inserted into the upper part of the shank bone, as well as to the inner small splint bone. This muscle has considerable power. 
n. The extensor pedis, described at $h$, fig. $\mathrm{r}$.

o. An inside view of the perforating muscle of the foot.

$p$. The veins of the posterior part of the leg.

$v$. The ligamentous bands, which are of such importance in confining the tendons at the bending of the hock.

w. Immediately behind the bend of the hock, at $w$, from the anterior superior part of the metatarsal bone, issues a thin layer of fleshy fibres, enveloped in cellular substance, and concealed in part of the tendon of the extensor pedis, with which (about one-fourth of the cannon downward) they form a union, and make some addition to its substance. In action, these supplementary fibres will brace the tendon, and are probably furnished to prevent it from being coinpressed by the flexion of the hock.

$y, y$. The large cutaneous vein, or vein immediately under the skin.

8. The inguinal vein, which is one of rather large size, coming from the groin, which owes its formation to a considerable branch emanating from the muscles of the thigh, and the above superficial or cutaneous abdominal vein. The femoral vein is the continuation of the external iliac trunk below the brim of the peivis, and becoming the main channel into which the deep-seated veins of the hind extremity pour their blood.

\section{STRUCTURE OF THE HOCK JOINT.}

PLATE VIII, FIG. I.

This is one of the most important points of the animal, and which ought to be thoroughly known by all who possess a horse. We are convinced that this is much more frequently the seat of disease and lameness than is suspected. Besides, upon the proper formation of this joint, the value and excellence of the horse chiefly depends.

As the knee answers to the wrist of man, and is 
therefore analogically regarded as the carpus, so in like manner the hock becomes the correspondent part to the instep, and is consequently considered under the technical appellation of tarsus. It consists of six small bones.

a. The astragalus, or knuckle bone. Its form is like that of a pulley. Its surface consists of two bold semicircular prominences, with a deep capacious groove between them; these are admirably adapted to the two grooves, parted by their middle projection in the lower extremity of the tibia, and these opposite prominences and grooves are received, and as it were morticed into each other. At the posterior part its convex surface is received into a concavity near the base of another bone, and with which it is united by very strong ligaments to the os calcis, $c$, or bone of the heel, and it projects upwards, flattened at the sides, and receives into it the tendons of powerful muscles, which are strongly implanted into it. The lower surface is smaller than either of the others, and is irregularly flattened, and almost wholly articulatory; it is embraced by the superior part of the large cuneiform bone. The two bones above described rest on two others, the os cuboides, or cubeshaped bone, $e$, behind, and the larger wedge-shaped bone, $d$, in front. This larger wedge-shaped bone is supported by two small ones, $f$, and these two smaller ones and the cube-shaped bone by the upper heads of the shank bone, $h$, and the splint bone, $g$. The cube bone is placed on the external splint bone and the cannon bone; the small wedge bone chiefly rests on the inner splint bone, which cannot be seen in our figure, and the middle wedge bone rests on the shank bone, $h$, alone. All these bones are connected together by very strong ligaments, which prevent dislocation, but are sufficiently flexible to allow a slight degree of motion among them, and the surfaces which are opposed to and move upon each other are thickly covered by elastic cartilage.

b. The inferior end or base of the tibia. This bone reaches 
from the stifle to the hock; it is connected with the round bone above, and the os calcis below.

c. The os calcis, which forms the posterior projecting part, called the point of the hock.

d. The os cuneiforms magnum, large wedge-shaped or cuneiform bone, situated immediately under the astragalus.

$c$. The os cuboides, or cuboid bone, situate on the outer part of the hock.

$f$. The os cuneiforme, or middle cuneiform bone, situated immediately underneath the large cuneiform bone, and upon the hind cannon bone.

g. The splint bone.

h. Upper head of the shank bone, or metatarsi magnum.

This joint is subjected to very great stress and weight, and consequently peculiarly liable to injury both in the draught and during rapid motion. However, nature has constructed it with wonderful skill; and it is, by the beautiful adjustment of its parts, rendered less liable to disease than might be expected from the important and heavy duties it has to perform. The provisions made for this end are, that the pulley-like heads of the astragalus and tibia fit deeply into each other, and are strongly confined in their position by extremely powerful ligaments, which are so contrived that they admit of the necessary freedom of the hinge-like motion of the joint, but completely prevent that lateral or side motion to which the joint is exposed in rapid movements, or passing over uneven surfaces. It will be seen, by inspecting the figure referred to, that the weight of the hind-quarters is principally thrown upon the tibia, $b$, and that it rests almost entirely on the astragalus, $a$; but it will be observed that this weight does not press perpendicularly, but in an oblique direction, so that much of the concussion which 
would otherwise take place is avoided, by the springy action this slanting pressure produces among the several bones which compose the joint. This will be more readily understood by a reference to the skeleton, Plate II, fig. 21. As we have already shown, this joint consists of six bones, all of which are covered with articular cartilage, and each admitting of a certain degree of motion, which diminishes concussion by the weight, pressure, and action being diffused among them all, and thus the concussion is neutralised and rendered harmless. Besides the cartilaginous covering, each of these bones has a membranous covering, which secretes the synovia or oily fluid, which we have already mentioned at page 89 and elsewhere. In fact these bones may be considered as so many distinct joints, all separated from each other and protected from injury, yet united by different ligaments, binding them so firmly together as to prevent the possibility of dislocation, yet permitting sufficient motion for the important office they have to fulfil. Beautiful and wonderfully powerful as this joint is, it is sometimes injured,-too frequently, we lament to say, - by the brutal cruelty of those to whose care horses are entrusted. In ordinary action this joint has heavy work to perform, but often more severe exertion is exacted from it than even its admirable construction can possibly perform. Much of the lameness of the hind-quarters will be found, upon careful examination, to be seated in the hock. If the exact locality of lameness cannot be detected elsewhere, we may almost consider that it is somewhere connected with the complicated structure of the hock joint, and we may particularly refer to the various diseases of this joint described towards the beginning of Chapter VI, page 107. 


\section{BONES OF THE FOOT.}

\section{PLATE VI, FIG. 3.}

This figure represents a back view of the bones of the pastern joint and of the foot, and those connected with it. The coffin bone constitutes its osseous fabric, to which the navicular bone may be regarded as an appendage. The anatomy of this part should be well understood, as it is of much importance in the action of a horse, as also very liable to disease.

a. The coffin bone, with its horny laminæ. It is situated within the hoof, which it nearly resembles in form, being in its outline crescent-shaped. Its form, however, varies with the natural make and morbid changes in the form of the hoof. The coffin bone is of a soft, spongy texture.

b. The navicular bone, or shuttle bone, which is situated at the back of the coffin joint, into whose composition it enters. It is crescent-shaped. One of its extremities is directed outward, and the other inward ; their points are obtuse, and are fixed by lateral ligaments to the coffin bone.

c. The lower pastern, or coronet bone. Its situation is between the pastern and the foot, and answers to the second phalanx of the human foot. Its form is nearly square.

d. The upper pastern is situated below the cannon bone, with which, from taking an oblique direction, it forms an obtuse angle. It is connected with the cannon and coronet bones, and with the two sesamoids.

e. The sesamoid bones, or os sesamoidea. These are situated at the back of the articulation formed by the pastern and cannon bones. They are articulated only with the large metacarpal bone, and are connected both with that and the pastern bone.

\section{FRONT VIEW OF THE PASTERN.}

PLATE VI, FIGS. 4, 5, AND 6.

We have described some of the injuries to which the fetlock is liable, page ror. 
a. The coffin bone, or os pedis. The outline of its form is semi-lunar, convex before and above, concave below and behind.

b. The navicular bone, or os naviculare.

$c$. The lower pastern, or os coronce.

d. The upper pastern, or os suffraginis.

NERVE, VEIN, AND ARTERY OF THE PASTERN AND FOOT.

PLATE VI, FIG. 7 .

This gives a representation of the nerve on the inside of the foot as it approaches the fetlock and passes over the pastern. It will be noticed that branches are given off above the fetlock, which proceed to the fore part of the foot and give it feeling. The continuation of the nerve under the fetlock principally supplies the quarters and hinder parts of the foot.

This figure is intended to show the parts and situation of the vein, artery, and nerve, which has been operated upon during diseases of the foot, to alleviate the pain the animal suffers under some of these affections. This is termed neurotomy, or cutting of the nerve.

a. The sole of the foot.

b. The horny crust.

c. The fleshy, or sensible laminæ, covering the coffin bone, the horny crust being removed.

d. The posterior lateral ligaments.

$\iota$. The internal, or sensible frog.

$f$. The branch of the nerve which supplies the fore part of the foot with feeling.

$g$. The lower part of the vein before the artery.

$h$. The same vein spreading over the pastern.

$i$. The continuation of the nerve, $s$, and proceeding downward to supply the back portion of the foot with feeling. 
$j$. The extensors of the foot.

$k$. The deeper flexor tendon continued downward, called the perforans, or perforating, and contained within the other.

$l, m$. The division of the nerve on the fetlock joint.

n. The tendinous band in which the flexors work.

o. One of the flexor tendons.

$p$. The deeper flexor tendon.

$q$. The artery between the vein and nerve.

$r$. The vein before the artery.

$s$. The nerve on the inside of the off leg, at the edge of the shank bone, and behind the vein and artery.

\section{ILLUSTRATIONS OF DEFECTS OF THE FORE LEG.}

PLATE VI, FIG. 8.

a. The situation of sand-crack in the foot of the fore leg, as described at page $\mathrm{I} 35$.

$b$. Representation of ringbone when it first appears on the side of the pastern. See page 104.

c. The situation of wind-gall. See page 99 .

$d$. The situation and appearance of the enlargement which accompanies sprain of the back sinews. See page 95 .

$e$. The ordinary position in which splint occurs on the side of the shank bone, which, however, does not produce lameness after its first formation, in consequence of its not interfering with the motion of the knee, nor does it injure the suspensory ligament. See page 92.

$f$. The tying in of the leg below the knee.

$h$. The situation of the disease called mallenders. See page I I I.

\section{ATTACHMENTS IN FRONT OF THE PASTERN} BONES, ETC.

PLATE VI, FIG. 7 .

a. The coffin bone.

$b, c$. Branches of the suspensory ligaments, proceeding to unite with the extensor tendon.

$d$. The back of the upper pastern. 
$e$. The back part of the lower pastern.

$f$. Back of one of the sesamoid bones.

$g$. The lower part of the shank bone.

h. The lateral cartilages of the foot.

$i$. The ligaments connecting the two pastern bones together.

\section{SITUATION OF DISEASES OF THE HIND LEG.}

\section{PLATE VII, FIG. 3 .}

a. The situation of grease of the foot. See page 123 .

b. Represents a wind-gall. See page 99 .

c. Capped hock. See page i Io.

d. Curb. See page iriz.

$c, c$ The position of thoroughpin. See page Iog.

We have given a series of different representations of these, as follow, on Plate vir :-

a. Fig. 4, Capped hocks. See page i ro.

$b, b$. Fig. 5, Thoroughpin. See page Iog.

e. Fig. 6, Bog and blood-spavin. See page Iry.

e. Fig. 8, Curb. See page II3.

d. Fig. 9, Bone-spavin. See page Ir5.

THE PASTERN AND FOOT, WITH THEIR BONES AND INTEGUMENTS.

\section{PLATE VI, Fig. Io.}

At the interior portion of the shank bone, immediately below the knee, and occupying the space between the two splint bones, two remarkable and important ligaments are situated. They are not only elastic, but also particularly well adapted to obviate concussion. They have their origin from the head of the shank bone, and likewise from the heads of the splint bones. They descend down the leg, and fill the groove between the splint bones, although they are not attached to either. A little 
lower down they expand on both sides, and as they approach the pasterns, separate, and are inserted into two small sesamoid bones, situate at the back of the upper pastern. These form a joint, both with the lower termination of the shank bone and the upper pastern bone, to both of which they are united by ligaments, $f, i$, and $k$, but more firmly united with the pastern than the shank. Between them pass the flexor tendons, through a synovial bag, which prevents the friction to which they would otherwise be exposed in passing through so confined a situation. This ligament is continued over the sesamoid bones, and afterwards is directed obliquely forward over the pastern, where it unites with the long extensor tendon, and downward to the perforated tendon, which it surrounds, and fixes in its position, and likewise to the smaller pastern bone.

a. The coffin bone. This bone is fitted to, and occupies the fore part of, the hoof, filling about half of it. It nearly resembles the hoof in form, being half-moon shaped; it is convex above and in front, and concave behind and beneath. It varies, however, with the natural form of the hoof, and also adapts itself to such changes in the hoof as are induced by disease. Its structure is light and spongy, and perforated with numerous holes. These are adapted for the passage of the blood-vessels of the foot, without which the circulation could not be so safely and conveniently kept up, and affords another striking example of those beautiful provisions of nature for effecting certain ends. But for this, these vessels would be frequently subjected to great pressure, which would occasionally obstruct its free passage. The upper surface, it will be seen, is concave, for the reception of the rounded end of the lower pastern, $b$; at the back, $o$, is a depression for the perforating tendon, $g$. We have described the diseases connected with this joint at page 104.

b. The lower or smaller pastern bone. 
c. The upper or larger pastern bone.

$d$. The shank bone.

$c$. The sesamoid bone.

$f$. The suspensory ligament. See rupture of this described, page 100.

g. The tendon of the perforating flexor, inserted into the coffin bone after having passed over the navicular bone.

$h$. A long ligament, reaching from the pastern bone to the knee.

$i$. The small inelastic ligament, which fastens down the sesamoid bone to the larger pastern.

$j$. The extensor tendon, inserted into both the pasterns and the coffin bone.

$k$. A continuation of the suspensory ligament, inserted into the smaller pastern bone.

l. The navicular, or shuttle bone. One of the chief uses of this bone is to take off a portion of the weight from the coffin bone; and from the navicular bone it is thrown on the tendon, which rests on the elastic frog beneath. See diseases of this bone, page 139 .

$m$. The inner, or sensitive frog. This is a wedge-shaped body projecting from the bottom of the foot, together with the substance continued from it, and occupying the interval between the cartilages.

n. A ligament which unites the navicular bone to the smaller pastern.

$o$. A ligament uniting the navicular bone to the coffin bone.

$p$. This is the seat of lameness of the navicular joint.

$q$. The sensitive sole between the coffin bone and horny sole. It is placed between the coffin bone and the sole; and, from its yielding nature, assists in preventing concussion, and also forms a supply for the horn of the sole. It is furnished with nervous fibres, and is highly sensitive. The lameness which is caused by the pressure of a stone or of the shoe on the sole is occasioned by inflammation of the sensitive sole. From the same cause, corns between the crust and the sole result.

$r$. The coronary ring of the crust.

$s$. The covering of the coronary ligament, from which the crust is secreted. 
$t$. The sensible laminæ, to which the crust is attached. $u, v$. The crust, or wall of the foot.

$w$. The place of bleeding at the toe.

$x$. The horny sole.

$y$. The cleft of the horny frog.

It is obvious, from the situation which the suspensory ligament occupies, that splints formed backward on the leg are more liable to produce lameness than those which are formed on the side of the leg; because they interfere with the motion of this ligament, and if large may press upon and injure it. The chief action of the suspensory ligament is to suspend the sesamoid bone in its place.

It will be seen by the figure above described, that the pasterns are united to the shank in an oblique direction, differing in degree of obliquity with the various breeds of horses. A portion of the weight which falls upon the pasterns must be communicated to the sesamoid bones. The yielding of the pasterns, taken in connection with their oblique position, is a beautiful contrivance to prevent jarring in the action of the horse; and this is materially assisted by the sesamoid being suspended, and no bone being under it; so that the suspensory ligament gradually yields, lengthens, and contracts in proportion to the weight or pressure applied to it, thus rendering all severe concussion impossible, because it yields to the force it has to sustain, and lengthens; but as soon as the foot is raised from the ground and pressure is removed, its elastic power is again exerted, and it contracts to its natural length, and the sesamoid bone springs back into its place, and by this quick and rapid return assists in raising the limb. This action is thus clearly described by Mr Percivall:- "Furthermore, it seems to us that 
these elastic parts assist in the elevations of the feet from the ground in those places in which they are called into sudden and forcible action. The suspensory ligament, by its reaction instantaneously after extension, aids the flexor muscles in bending the pastern joints. The astonishing activity and expedition displayed in the movements of the racehorse at speed, seem to be referable, in part, to the promptitude with which the suspensory ligament can act before the flexor muscles are duly prepared; the latter, we should say, catch, as it were, and then direct the limb first snatched from the ground by the powers of elasticity."

The spring and elasticity in the action of a horse depends, in a great measure, upon the length and obliquity of the pasterns. It should be long in the race-horse, less so in the hunter, still shorter in the hackney, and considerably less so in the cart and dray horse. In the latter the concussion is exceedingly little, because their movements are slow; and the short and upright pasterns enable him to sustain and drag the heavy loads which he is destined to support and move forward. But in a horse that is to be used for the saddle, the short and upright pastern is not only a great defect, but is also very unsafe, as he is exceedingly liable to come down when trotting. Besides, such joints soon begin to knuckle over, even with ordinary work, which is the precursor of ossification of the cartilages, ringbone, and contracted feet. 


\section{EXTERNAL PARTS OF THE FOOT.}

THE CRUST OR IVALL OF THE HOOF.

The formation of the foot of the horse fits him, as well as the ass, above all other animals, for the service of man. In short, had the hoof of the horse been cleft, he would have been incapacitated for many of the useful departments of his employment; and a correct knowledge of the structure of every part of the foot is indispensably necessary to render us scientific overseers of the farrier's art.

The crust is that portion which reaches from the termination of the hair to the ground. Its depth is greatest in front, and is denominated the toe; it is more shallow at the sides, which are called the quarters, and still less behind, which is termed the heel. When the sole is placed on the ground, the front exhibits an angle of about forty-five degrees, differing, however, considerably in many horses to the extent of the angle. But a healthy and well-formed hoof very nearly approaches what we have stated, that is, a fourth part of a semicircle. With a greater degree of obliquity, it is said the crust has "fallen in," and when the sole is too flat, and is said to be pumiced, or convex; and if the front be more upright than the above angle, it is the proof of a contracted foot with the sole too concave. When the crust is deep at the heel, it is a foot liable to contraction, thrush, sand-crack, and inflammation. The pastern will be found too upright, and the horse will have a bad and unpleasant action. If, on the other hand, the crust diminishes too rapidly from front to back, and the heels are low, this is always accompanied by too great ob- 
liquity of the pastern, producing a weakness in the joint, and liability to sprain of the back sinew, described at page 95. The foot itself will be weak, and have a general tendency to that hidden lameness called "the navicular joint disease," particularised at page I 39 .

The general thickness of the crust in front is somewhat more than half-an-inch, becoming gradually thinner towards the quarters and heels. This will show the necessity for shoeing-smiths being adepts in driving the nails, seeing the small space for that purpose, and more especially behind. The crust is thinner and a little higher in the inner than the outer quarter. This is another beautiful provision, because, being placed under the inner splint bone, more of the weight rests on the inside than the outside, consequently it is enabled to expand more, and thus by its elasticity. assists in lessening concussion. When, therefore, expansion is prevented by the inner quarter being nailed firmly to the shoe, corns, contraction, and sandcrack are induced. The crust is not liable to much variation in thickness, as will be seen by a reference to Plate vi, fig. Io, $u$ and $v$, and $c$ in fig. 9, until near the top, at the coronet, or where the horn of the hoof unites with the skin of the pasterns, where it becomes abruptly thin, as will be seen on a reference to $s$, in fig. 10. Here it appears as if scooped out, and here also its colour and consistence are changed, and it appears like a continuation of the skin. This thin portion is called the coronary ring, $r$, fig. Io, which covers a thickened prolongation of the skin called the coronary ligament, $c$, in fig. 9. This extension of the skin is supplied with numerous densely set blood-vessels, connected to- 


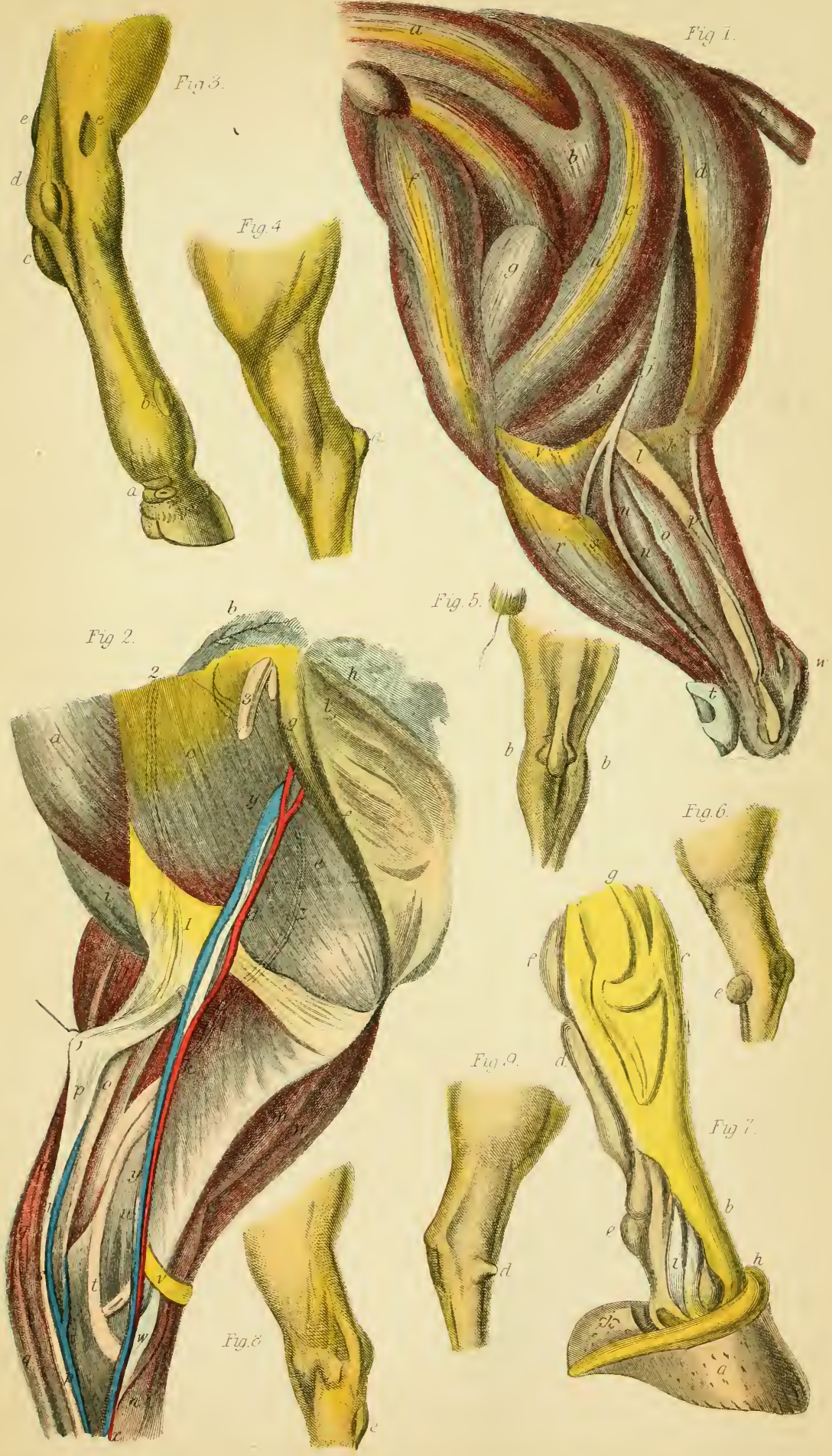



gether by a fibrous texture, many of which have the property of secreting the horny substance which forms the crust. The sensible laminæ, $a$, fig. 9, have the power of secreting some horn, which furnishes an immediate defence against injury in cases where the crust is either purposely removed, or has sustained injury. This is sufficient proof in cases of quittor or sand-crack, when it becomes absolutely necessary to remove a portion of the crust. The exposed portion is soon covered by a film of a hard horny texture. The crust, however, is chiefly formed by the coronary ligament; and hence in quittor and sand-crack its growth is slow and downwards, and is only replaced with the natural lengthening of the crust, in the same manner as in injury to the human nail. And there is situate below the coronary ligament a strip of horny matter, emanating from the frog, which seems intended for a similar purpose to that which surrounds the root of the human nail-namely, to strengthen the union of the part where it rests, and to bind together the various substances which meet there.

The crust is composed of numerous fibres, which proceed directly from the coronet to the ground, but which follow an oblique course from the heel forwards. These fibres are kept together by a glutinous substance.

In a sound condition the crust of the foot is smooth and hard, and when there are rings or other thickenings, it is a sure indication of disease in the foot, and that, too, to a considerable extent, as may be well supposed, to induce this irregularity of growth. When the front is depressed, it may be inferred that a sinking of the coffin bone has taken place, and that the sole will be flat and pumiced. 
When hollow at the quarter, it is a clear indication of contraction to a considerable extent.

The perspective representation which we have given, Plate vI, fig. II, will convey a clear idea of the structure of the crust and its various component parts.

a. The coronary ring, which is a circular, attenuated, concavo-convex part, entering into the composition of the coronet. Its extent is marked exteriorly by the whitish aspect which it assumes, and likewise by a partial separation and eversion of the outer flakes of horn around its junction with the wall, or crust below.

$b$. The small horny plates which line the crust. These consist of numerous narrow laminæ, or processes, arranged with the nicest order and mathematical precision upon the internal surface of the wall. They extend in uniform parallels in a perpendicular direction from the lower edge of the superior border to the line of junction of the wall with the sole, and are so thickly set, that no part of the superficies remains unoccupied by them. They are also continued upon the surfaces of the bars. They are soft, yielding, and elastic; but from exposure they become dry and rigid. Every plate exhibits two edges and two surfaces. By one edge it grows to the wall, and the other, which is somewhat thinned, hangs loose and floating within the cavity of the hoof. These are two smooth lateral surfaces, and, considering the magnitude of the lamella itself, of enormous extent; so much so, that it may be said almost to be constituted entirely of superficies. Looking at this, we are naturally led to the contemplation of the great and magnificent designs which Nature evidently had in view in their formation and beautiful adaptation, viz., the production of ample surface within a small space, an end that has been obtained through the means of multiplication. A mathernatical calculation was made by the late Thomas Evans, LL.D., of what the united superficies of these lamellæ amounted to, and it was found that they afforded an increase of actual surface more than the single internal area of the 
hoof would give, of about twelve times, or about two hundred and twelve square inches, or nearly one square foot and a half.

c. These above described laminæ are continued over the bars in this situation.

d. The bars are processes of the wall, inflected from its heels obliquely across the bottom of the foot. These are also seen in figure $12, c, c$. They extend from the base of the heel into the centre of the foot, between the sole and the frog ; behind, they are continuous in substance with the wall or crust, with which they form acute angles; anteriorly they stretch as far as the point of the frog, constituting two inner walls between that body and the sole. They seem formed for the purpose of offering resistance to the contractions of the heels.

$e, e$. Two concave surfaces of the inside of the horny frog.

$f$. That portion which externally is the cleft of the frog, the inferior surface of which exhibits a remarkable cavity, broad and deep, and of a triangular form, bounded on the sides by sloping prominences, which diverge from the convexity forming the toe of the frog, and terminate at the heels. This cavity is called the cleft of the frog.

$g$. The frog, as also exhibited fig. I $2, d, d$. In its superior surface it is continuous, uniform, and porous, being the counterpart in form of the inferior surface, presenting only reverses, where the one is hollow, and the other swelling. Opposite to the cleft is the frog-stay, which is elevated and bounded on its sides by two deep channels, and a hollow of shallower dimensions in the front. This bold, horny elevation is admirably calculated to form that dove-tailed connection with the sensitive foot, which greatly augments their surfaces of opposition, and establishes their union beyond all risk or possibility of dislocation.

$h, h$. The external crust, or wall ; as also seen fig. $12, a, a$, and fully described above. At the first $h$ and $i$ is the rounded portion of the heels belonging to the frog.

1. Fig. 12, is the external surface of the sole, or the arched plate entering into the formation of the bottom of the hoof, and covering the whole inferior surface of the foot, excepting the trog. No individual part requires 
such undivided attention as the sole, as regards shoeing; since the success of this mechanical operation chiefly depends upon the paring and defence of this arched horny plate. Viewed from below, the sole commonly presents an arch of more or less concavity. It is subject to vast variety in the degree of the arc; in some feet it is of surprising depth, and in others the arch is converted into a flattened surface, and yet both seem to perform equally well. In the hind feet the sole is generally more arched than in the fore, and approaches in figure more to the oval than the circle. Its thickness is about one-sixth of an inch. That portion most elevated from the ground-that which forms a union with the bars-is nearly double the thickness of the central or circumferent parts, and next to this in substance comes the heel. This is situate at the back part of the foot, at which point the crust of the hoof, instead of being continued round and forming a complete circle, is abruptly bent in, as will be seen by a reference to fig. 12 , and at $h$, fig. II.

\section{CARTILAGES OF THE FOOT.}

The cartilages are two broad, scabrous, concavoconvex cartilaginous plates which surmount the sides and wings of the coffin bone. There is a groove extending along the upper part of the coffin bone on each side, except at the protuberance, which receives the extensor tendon, and which extends to the very posterior portion of the foot, rising about the quarters fully half-an-inch above the hoof, and diminishing in height backward and forward. These cartilages occupy a greater portion of the foot than does the coffin bone, as will be observed in fig. 7, Plate viI, $i$, where it will be seen they extend far behind the coffin bone. They are fixed into two grooves, excavated in the superior lateral borders of the coffin bone, and are thus perfectly secured.

Between these cartilages is the sensible frog, occu- 
pying the whole of the space, and answering several important purposes, it being an elastic bed on which the navicular bone and the tendon can play with security, and without concussion. This will be understood by referring to Plate vi, fig. Io, $l$. Thus all concussion to the cartilages of the foot is prevented, and these cartilages kept asunder, and the expansion of the upper part of the foot preserved. This mechanism is both beautiful and important. The yielding and elastic substance of the frog is pressed upon by the navicular bone as well as the tendon and the pastern, and being incapable of condensing into less compass, is forced out on each side of them, and expands the lateral cartilages; and these again, by their inherent elasticity, revert to their former situation, when they are no longer pressed outward by the frog. It thus appears that by a different mechanism, but both equally admirable and referable to the same principle, namely, that of elasticity, the expansion of the upper and lower portions of the hoof are effected, the one by the descent of the sole, and the other by the compression and rising of the frog. The preservation and usefulness of the limbs of the horse are chiefly maintained by this upward expansion, when the destructive methods which are adopted in shoeing are calculated to destroy the expansion beneath. From the long-continued and violent pressure on the frog in draught-horses, and conveyed from the frog to the cartilage, inflammation is frequently produced, and too often terminates in the cartilages being turned into bony matter. 


\section{CHAPTER X.}

Sketch of the Internal Organisation of the Horse.

THE LUNGS.

PLATE IX, FIG. I, ETC.

THE lungs are two spongy bodies formed for the purpose of breathing. They are contained in the lateral regions on each side of the chest, $a, a, a$, separated from each other by the mediastinum and heart, which occupy the middle region. The lungs are two in number, - the right and the left, partitioned from each other by the mediastinum. They are further divided into lobes, - that on the right side, which is the larger of the two, consists of three lobes and the left has only two. These lobes are merely partial divisions, of variable extent, which serves to adapt them more accurately to the cavities of the chest, and at the same time render them fitter for the purposes of expansion and contraction. When the windpipe enters the chest, it is divided into two branches, one extending to each lung; and when these enter the substance of the lungs, they separate into numerous branches, each terminating in a little bag or cell. These bear a considerable resemblance to minute bunches of grapes. Around these cells are spread innumerable blood-vessels, being the extreme ramifications of those which conveyed the blood from the right side of the heart to the lungs, and the com- 
mencement of those which conduct it back from the lungs to the left side of the heart. These cells and blood-vessels are connected together by an intervening substance of a fibrous and cellular texture.

The blood circulating through the capillaries of the body contribute to the nourishment of the animal system, and furnishing all the secretions, becomes changed, and is no longer capable of supporting life: it becomes of a poisonous quality, in consequence of having in it a too large portion of carbon. This must be expelled before the blood can again be rendered subservient to the purposes of life. That portion of the atmospheric air called oxygen having a strong attraction for carbon, unites with it whenever they come in contact. The chest enlarges by means of the diaphragm and the muscles between the ribs, called the intercostal muscles, and others, and the lungs expand with the chest in order to fill up the vacuum which would otherwise exist between them and the sides of the chest. These cells enlarge, and a sort of vacuum is formed in each of them, as the air rushes down and fills them; and being divided from the venous and poisoned blood by these membranes alone, it is enabled to act upon the blood and abstract from it the carbon, and by this means purifies it, and the arterial blood is fitted for the purposes of life. This purification being performed, the chest contracts, and the lungs are compressed into smaller compass, and a portion of air, holding in it a quantity of carbon, and rendered poisonous in its turn, is squeezed out. Immediately afterwards the chest expands again, and the lungs expand with it, and pure atmospheric air is drawn into them, which is immediately thrown out again by the compression of the lungs, which, like the preceding expiration, is 
poisoned by the carbon of the blood. These alternate contractions of the chest and lungs constitute what is termed breathing.

When the horse is subjected to powerful exertion, it is obvious that a more ample supply of uncontaminated blood will be required to sustain the energies of life, and violent action of the muscles forces the blood more rapidly through the veins, and hence the quick and deep breathing of the animal when running at speed. Therefore, the more capacious the chest, the greater will be the supply of pure blood, as the lungs will have more room to expand and perform the functions which we have above described. Besides, a capacious chest will fit him for a longer duration of speed.

Those who are accustomed to hunting or horseracing, must have frequently witnessed the wonderful relief which loosening the girths have afforded to a horse after a severe gallop. The tightening being removed, permits the chest to expand and contract to a greater extent, and consequently yields a larger portion of purified blood. A very short rest will sufficiently manifest how much the exhausted energies of those organs will recover when the greater expenditure is not necessary.

Even for animals which are not required to possess speed, such as cart, waggon, dray, and farmers' horses, a capacious chest is equally necessary ; for this reason, that in these there is generally a great accumulation of both flesh and fat, which require a large portion of the blood to supply his growth; consequently, large and ample chests are requisite so as to afford room for the necessary provision of a rapid purification of the contaminated blood.

Diseases of the lungs are among the worst to 
which horses are liable, and most frequent of occurrence, and hence the most likely to impair his usefulness.

It is not to be wondered at, that so many horses are afflicted with lung complaints, most of them resulting from carelessness. The poor animals are too often over-heated, and afterwards put into cold stables in a state of profuse perspiration, and allowed to dry. This should never be the case. The animal ought to be instantly rubbed down, until his coat is quite dry. If this is not attended to, inflammation of the lungs and a host of other complaints may be the consequence.

\section{THE HEART.}

The heart is enclosed within a membrane or bag, called the pericardium, Plate Ix, fig. I, $c$, and both together occupy the middle space of the cavity of the chest. The pericardium contains within it a small quantity of serous fluid, which serves to lubricate the contiguous surfaces of the sac, and to preserve them against any ill consequences arising from friction.

The use of the pericardium is to confine the heart in its situation, to sustain it in its reciprocal action with the lungs, and guarding it from any undue collision, and to serve as a guard to the heart. When the pericardium or the heart becomes inflamed, an undue secretion of this fluid is induced, sometimes to such an extent as to obstruct the beating of the heart. When such is the case, the animal is said to have dropsy of the heart.

The heart itself, fig. I, $b$, is the organ by which the blood is circulated through the body. It is of a conoid form, with the base turned uppermost, and is opposed to the fourth, fifth, and sixth vertebræ 
of the back, from which it is suspended in its situation in the middle of the cavity of the chest, by the attachment of the venous and arterial trunks immediately connected with it. Its apex hangs loose within the cavity of the pericardium, pointing downwards and backwards, and rather inclined towards the left side. It is composed of four cavities : the two uppermost are called auricles, from their form being somewhat like the ear of a dog; and two ventricles, or belly-shaped cavities, which occupy the substance of the heart. Although the heart is chiefly composed of fleshy fibres, still a tendinous substance is found in the middle, which seems to be the common medium of attachment between its auricles, ventricles, and vessels, one to another.

The heart is supplied with blood by two coronary arteries; the first branches are given off from the aorta, or great artery. Its veins pour their blood into the coronary vein, by which it is returned into the right auricle.

There are two orders of blood-vessels, arteries and veins; the former conduct the flood from the heart to all parts of the body, nourishes it, and returns to the heart through the veins. It enters the auricle on the right side, where it is accumulated as a reservoir, until there is sufficient to fill the ventricle below. The auricle then contracts, and forces the blood into the ventricle, which in its turn contracts, and drives the blood through an aperture that leads to the lungs. It cannot be drawn again into the auricle, because there is a complete valve, like that of a sucker of a pump, to prevent this. The blood which has thus been forced into the lungs traverses every portion of them, by the minutely ramified blood-vessels, and entering all the little cells, there to undergo the 
important change of being subjected to the action of the atmospheric air which the lungs have inhaled, and be purified by the oxygen contained in the air, and from which substance it owes its beautiful red colour. It is now carried to the left auricle, and from thence it descends to the left ventricle, and by the powerful closing of the ventricle is propelled into the arteries. These vessels, in all their numerous ramifications, emanate originally from two main trunks, the pulmonary artery and the aorta; the branches of the former penetrate the lungs, and the latter are spread over every part of the body.

The aorta, with its numerous branches, when taking them as a whole, may be compared to a short but straggling and very branching shrub or dwarf tree, of luxuriant but extremely irregular growth. It takes its rise from the left ventricle, and the blood by the force communicated to it by the sudden contraction of the ventricle, and aided by the elastic power of the arteries, keeps them open and free from obstruction, and likewise, by the pressure of the muscular and elastic coats, endeavouring to return to their former dimensions, flows in a continuous stream through every portion of the frame.

The pulmonary artery is a vessel of larger dimensions and calibre than the aorta. It has its origin in the posterior upper part of the right ventricle of the heart, and winding upwards to the root of the left lung, there divides into what are termed the right and left pulmonary arteries. These divisions immediately enter the substance of their correspondent lungs, and therein ramify in all directions, like the minutest threads, the branches regulating their course and division by the ramification of the bronchial tubes.

The heart is liable to disease, as it is sympatheti- 
cally affected by almost all the complaints incidental to the animal frame, no matter how distant that malady may be from it. An injury of the foot will in a very short time cause the heart to beat or pulsate with double its ordinary quickness. This is not a complaint of very frequent occurrence, but extremeiy dangerous, and such is the strength and rapidity of the heart's action, that its pulsations may be distinctly seen at the animal's side, and even heard at some yards distance. In this complaint the animal exhibits great energy of expression of countenance, with a quick and restless motion.

\section{THE PULSE.}

As the pulse is so intimately connected with the action of the heart, we may with propriety treat of it in this place. As in the human subject the pulse is a useful key to the health of the system, it is likewise of the same value to those practising the veterinary art.

Although there are several parts in a horse where the pulse may be felt, yet the most convenient is at the lower jaw, a little behind the part where the submaxillary artery and vein, and the parotid-duct, pass under the jaw. (See Plate III, fig. I, $r$.) At this spot the pulsations may be not only distinctly counted, but also the character of the action of the pulse accurately ascertained, which is of much importance, because its hardness and softness indicate certain conditions of disease. Many persons place the hand on the side to ascertain the state of the pulse, but this will only give the number of its beats in a minute, without its condition.

In a healthy state, the pulsations in the heart of a farmer's horse range from thirty-five to forty in a 
minute; in the thoroughbred horse from forty to fortyfive. These are considered the standard healthy pulses; but even in health these are subject to some variation either above or below these numbers of beats. It must be understood that this is the condition of the pulse when the animal is at rest, or at least when he is not in the act of using exertion; because, even in pulling a heavy load or during trotting, and for a short time after both of these, the pulse will be higher, according to the degree of exertion to which the horse has been subjected. Besides, exercise, a warm stable, and fear will materially augment the action of the heart.

When the animal is at rest, and the pulse reaches fifty or fifty-five beats in a minute, then it may be suspected that there is a degree of fever, and its cause must be investigated. When it is from seventy to seventy-five, a high state of fever may be the consequence, and active means must be resorted to, but the only reliable test for the existence of fever and its intensity is that indicated by the thermometer.

When horses are labouring under disease, it is necessary to approach them with caution, because either abruptness or speaking harshly to them will have a tendency to raise the pulse ten or fifteen degrees. The animal should be patted and spoken to gently, and the pulse felt a second time before its real state can be determined. The conditions of the pulse may be arranged under the following heads :-

\section{QUICK PULSE.}

This is always an indication that the animal is under some excitement. This will vary in degree according to the force of the exciting cause. The business of the veterinarian is to ascertain and remove 
this as speedily as the circumstances will permit. We have above stated the number of beats in a healthy and diseased state. The heart may be excited to more frequent and also to more violent action. It may contract more powerfully upon the blood-vessels, and consequently drive the fluid with greater force through the arteries, and the expansion of the coating of the arteries will be greater and more abrupt. The quickened pulse indicates a tendency to fever and irritation.

\section{SLOW PULSE.}

This is an indication of an oppressed condition of the heart's action, and accompanies diseases of an opposite kind from those which are the concomitants of a quick pulse. It proves that the malady with which it is connected results from a deficiency of nervous energy. It is always a concomitant of sleepy staggers.

\section{HARD PULSE.}

This is indicated by a firm and jerking feeling under the pressure of the finger, and at the same time accompanied by a fullness in the flow of blood through the vessels.

\section{SMALL PULSE}

Is indicated by feebleness in the beat and a feeling of languidness in the circulation, very easily discerned under pressure of the finger. It, however, sometimes happens that small pulse may be accompanied by hardness and jerking, but still the remarkable smallness in the circulating fluid will be perceptible. 


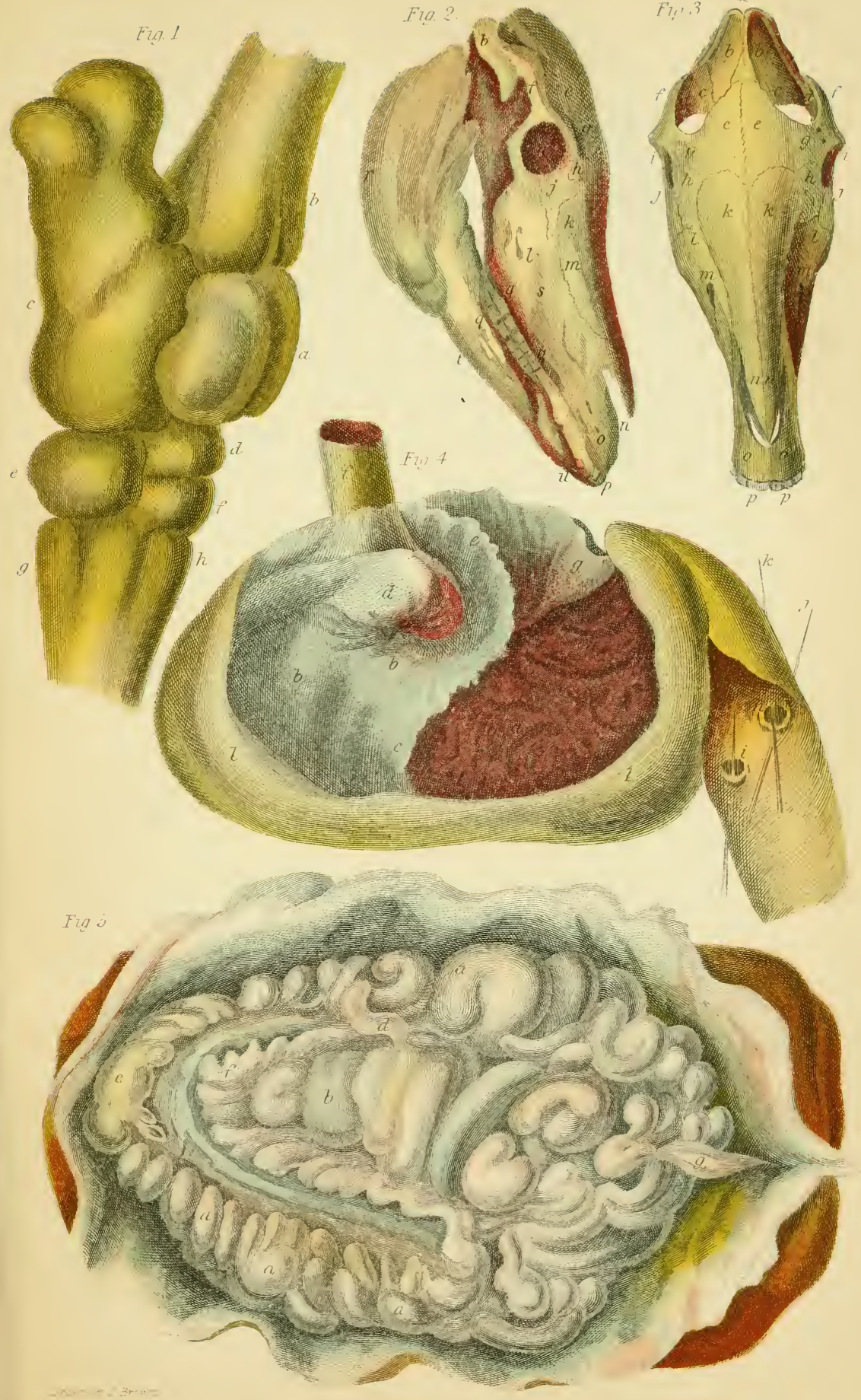





\section{A WEAK PULSE.}

This is caused by a feeble action of the heart, as the stream of arterial blood is flowing slowly, and hence is hardly to be felt. It indicates debility. Stimulants are generally necessary in this condition of pulse.

\section{OPPRESSED PULSE.}

When the arteries are fully distended with blood, and the pressure upon them is greater than their calibre will convey with ease, owing to some obstruction in their interior, and the action of the heart is unable to press forward the current, and in consequence the pulsation feels irregular and unequal, the pulse is said to be oppressed. In sudden inflammation of the lungs this condition is common.

The pulse is subject to various modifications besides those above enumerated, which it would be very difficult to explain, and which are well understood by those who are practised in feeling the pulse.

\section{THE ABDOMEN AND ITS CONTENTS.}

PLATE VIII, Fig. 5, and PLATE IX, Figs. I ANd 2.

Having given a brief account of the contents of the chest, or rather those parts most essential to the general reader, we now descend to the abdominal viscera. The heart and lungs may be considered the moving powers of the animal system, which, however, require the materials to keep up and supply that motion. The organ which prepares and distributes that stimulus is the stomach, and is lodged in the higher region of the belly, while the intestines which carry off the waste are situated in 
the middle and lower portion of the abdominal cavity.

The abdomen, or belly, is formed chiefly of soft parts, which principally consist of the four pair of abdominal muscles which mainly constitute its broad superficies below and laterally. In its interior part, the most important viscera are situated, viz., the stomach and liver. It is bounded by the false ribs, and in front by the diaphragm; its posterior compartment is bounded by the pelvis, and above by the dorsal and lumbar vertebræ and muscles belonging to the loins.

The abdominal viscera of the horse differ from those of man chiefly in the shape and comparative size of the stomach, and the intestine called the colon; their general relative situation is much the same in both.

\section{THE PERITONEUM.}

The peritoneum is the membrane that lines the cavity of the belly, and is reflected upon the contained viscera. It presents a shining secreting surface, of a whitish aspect, and considerable transparency. Its internal surface is smooth and humid. The use of this organ is to secrete a serous fluid, which is intended for lubricating every part of the membrane, in consequence of which those viscera that are continually moving within the belly glide over one another, not only without friction, but without exciting the least consciousness of their motions on the part of the animal himself. In addition to this, the peritoneum furnishes most of the viscera with a complete external tunic, and thereby adds strength and firmness to their several textures. It attaches, supports, and confines those 
viscera (within certain limits) in their respective places; and it strengthens the abdominal cavity altogether by its uninterrupted extension everywhere through and around it.

\section{THE DIAPHRAGM.}

PLATE IX, FIg. I, $h$.

This forms a fleshy and tendinous partition, dividing the cavity of the chest from that of the abdomen. It is of a broad circular form flattened from before backwards; its front surface is convex, and concave behind; divided or forked above, and having two elongations or appendices extending backwards, with pointed extremities. On that side next the chest it is invested by the membrane which covers the lungs, and towards the belly by that which covers the intestines. It adheres to the spine, the ribs, and the breastbone, by strong muscular fibres. Its structure is fleshy and tendinous. The fleshy parts are those which form the circumferent portions of the large muscle, and the principal part of the crura or appendices. The tendinous parts consist of a thin circular expansion, occupying the middle of the larger muscle, and uniting that with the lesser. Through the muscle are seen three remarkable openings, an upper one in the interspace between the crura for the passage of the aorta; one a little lower, formed by the decussation of the crura for the œsophagus; and the third, or lower one, perforating the cordiform, or heart-shaped tendon, for the reception of the posterior vena cava.

The diaphragm is the chief agent in inspiration; it acts in opposition to the abdominal muscles, which are the chief expiratory powers. By the contraction 
of its radiated fibres, with the assistance of that of the crura, the cordiform tendon is transformed to a plane surface, and the dimensions of the chest from front to back thereby considerably augmented. When this muscle acts, in consequence of the shortening of its fibres, it loses its convexity, as above stated, and the chest being thereby enlarged as well as the lungs, the air rushes in, and inspiration is performed. This muscle also assists in the natural constant motion of the bowels, and lends its powerful aid in expelling the fæces and urine; and in females, facilitates the birth of the young animal.

The membranes by which the diaphragm is covered-viz., the pleura in front and the peritoneum behind-are very liable to inflammatory attacks. In many cases of disease of the lungs and bowels, the diaphragm is inflamed; and this is sometimes the cause of the breathing of the horse being so much affected during inflammation of the chest and abdomen. It is likewise concerned in coughing, yawning, and sighing. Sometimes it is ruptured, occasioned by any violent exertion. We are, however, unable to give distinct indications of this condition. In cases of small rupture some portion of the intestines insinuates itself into it, and there becomes entangled, so that an incurable obstruction is the consequence. In the event of a large aperture, the intestines protrude through it, and by pressing upon the heart totally suppress respiration.

The gullet passes through the diaphragm into the stomach, and in which it terminates. See Plate viII, fig. 4. $f$. 


\section{THE STOMACH.}

PLATE VIII, Fig. 4, ANd PLATE IX, Fig. 1.

The stomach is situated in the left side of the belly, resting upon the large intestines. Its anterior or convex part lies upon the diaphragm and the false ribs of the left side; its posterior or concave part is concealed by the intestines, and its lower surface is invested by the omentum; attached to its left extremity is the spleen, and its right end is in contact with the left and middle lobes of the liver.

The stomach may be compared to a pouch or bag, formed for the reception of the food after it passes through the œsophagus. Perhaps no animal, in proportion to its size, has so small a stomach as the horse. The stomach of a middle-sized man, of about twelve stone weight, will contain somewhat more than three quarts of water; whereas that of an ordinary-sized horse, whose bulk and weight exceeds that of the man by eight times, will only contain three gallons, or four times the quantity of the man's. However, we must bear in mind that the stomach, like other hollow muscles, has the property of accommodating itself to the bulk of the matter which it contains.

This organ is of vast importance in the animal economy; in short, it is indispensable to their being; no animal is without one. This is not the case with the brain, and much less with the heart, as we know that animal life is sustained in some species without either. That great anatomist, John Hunter, in his physiological disquisitions, showed that the existence of a stomach was the chief characteristic between animals and vegetables. The stomach has been 
truly said to be the organ of digestion, because within it the aliment transmitted by the œesophagus in a crude state, undergoes its primary and principal change in a process, the object of which is to convert it into material for the support of the body, and the distribution and transmission of those fluids which sustain life and motion in its different parts.

It must be obvious, from the situation of the stomach, that it is not only attended with great inconvenience and pain, but also danger, to work a horse hard after a full meal. Indeed, many have sustained irreparable injury from this cause. By the action of the diaphragm, the stomach must be displaced and forced back in the belly by every contraction of the diaphragm or act of inspiration; then in proportion to the fullness of the stomach will be the weight to be overcome in breathing, and hence the increased labour of the diaphragm, and consequently the exhaustion of the animal. Besides, if the stomach is very full, and consequently distended, its weight may prevent it from being forced sufficiently far back to allow ample room for the necessary volume of air which the animal requires during a state of exertion. Hence the short, frequent, and oppressed breathing during rapid action, and which too often destroys the animal. On a journey, a horse should therefore be fed moderately, and more frequently than in a state of rest, and care should be taken not to allow him too much water, which ought also to be given in small and frequent quantities.

It would seem that Nature had wisely foreseen. that as the horse was destined to be the servant of man, and to render him more valuable and fitted for the labour that would be required of him, it 
became necessary to diminish the inconvenience and danger from pressure which would necessarily accompany a large stomach, that the animal should have one proportioned to the situation he was destined to fill in creation. The great bulk and consequent expenditure of his frame require a large quantity of food to be consumed to afford nutriment. Yet the stomach is wisely formed small, to prevent pressure as much as possible; and in addition it has the power of rapidly digesting the food, which speedily descends to a portion of the intestine remote from the diaphragm, where the pressure of the food cannot inconvenience him. Indeed, the whole of his digestive system is quick, and consequently his food passes rapidly through him; otherwise life never could be sustained, considering the small proportional nutriment contained in the ordinary food of the horse.

We shall now proceed to describe the several parts of the stomach. The situation which the stomach occupies in the abdomen will be seen by a reference to Plate $\mathrm{Ix}$, fig. $2, b$; and its general form and several parts are represented in Plate viII, fig. 4 .

$a, a$. The mucous or villous portion of the stomach in which the food is chiefly digested, or converted into a soft and pulpy substance. It extends over that portion of the stomach left unoccupied by the cuticular part. It is of a yellowish cast, inclining to red in some places.

$b, b$. Is that portion of the stomach which is covered by cuticle or insensible skin. This cuticular substance is of the same nature as the lining of the œsophagus, with which, indeed, at the cardia it is continuous. Numerous small openings are visible upon its inner surface, through which issues a mucous fluid, the product of follicular glands underneath, which is useful in the process of 
digestion, and where it may be said properly to commence. This is called the gastric juice, which mixes with the food already softened, and converts it into that fluid substance called chyme.

$c, c$. The margin which separates the cuticular from the villous portions.

d. The entrance from the gullet into the stomach. The circular layers of muscles which invest this part are very strong and thick. By their powerful contractions they assist in rendering it difficult for the food to be returned or even vomited. This orifice is called the cardiac orifice, in consequence of its contiguity to the heart. It is constantly closed by strong muscular fibres, except when the food is passing through it into the stomach.

$f$. The œesophagus, or gullet, through which the food is conducted from the pharynx into the stomach. It has its commencement in the pharynx, and is there placed at the upper and back part of the larynx, the first part of its course being behind the trachea, between it and the cervical vertebræ. After proceeding a short way down, it inclines to the left, and soon after makes its appearance altogether on the left side of the trachea, and continues so on its passage down the neck. This will explain what has puzzled many, why we look for the bolus during the act of swallowing on the left, and not on the right side of the animal. Accompanying the trachea, the œsophagus enters the chest between the first two ribs, at which part, running above that tube, it diverges from the trachea, and in connection with the superior mediastinum, and traversing that cavity a little way below and to the right of the aorta.

$g$. The communication between the stomach and the first intestine.

i. A small orifice through which a portion of the secretion of the pancreas enters the intestines. Its direction will be seen by the probe which is passed through it. The pancreas, otherwise called the sweetbread, is a glandular body lying across the spine in the epigastric region, underneath the crura of the diaphragm, immediately 
behind and a little above the small curvature of the stomach.

$j, k$. Two probes passed through the common orifice through which the bile and the pancreatic secretion pass into the first intestine. The part where the two probes intersect each other mark the spot where these tubes unite.

\section{THE LIVER.}

\section{PLATE IX, FIG. 2, a.}

This organ is situated between the stomach and the diaphragm. Its right is in contact with the duodenum and the right kidney, and the micldle and left divisions with the stomach. It is confined in its place by means of what have been termed its ligaments, which, with the exception of one, are nothing more than elongations proceeding from the peritoneum. The one attaching the right lobe to the diaphragm is called the right ligament; a similar one connecting the left lobe to it, the left ligament; between the diaphragm and its middle lobe, is the suspensory ligament; and immediately above that, surrounding the posterior vena cava, is the coronary ligament; and that within the folds of the suspensory ligament are the remains of the umbilical vein.

In our description of the heart, at page 234, we mentioned that the blood which is conducted to the different parts of the body by the arteries, is returned to the heart by the veins. But that portion of the blood which is returned from the stomach, intestines, pancreas, spleen, and mesentery, instead of taking a direct course to the heart, passes first through the liver. Two large vessels conduct it thither, and as soon as they have entered its substance, they spread out into innumerable minute branches, traversing 
through every part of the liver. During the passage of the blood through the liver, a fluid is separated from it, which is called the bile. This is carried by the gall-duct, $l$, into the duodenum, $m$, in such quantities as are required for aiding digestion. The horse has no gall-bladder, and, consequently, the bile flows into the duodenum, or first intestine, $m$, immediately after it is separated from the blood. As we have already mentioned, the stomach of the horse is small in proportion to the quantity of food which he must consume, and consequently must be much oftener emptied; and hence the necessity for uninterrupted flow of bile to aid the process of digestion.

The diseases connected with the liver are inflammation and jaundice, treated of at page 69.

\section{THE OMENTUM, OR CAUL.}

This is a doubling of the peritoneum, investing the lower portion of the stomach, to the great curvature of which, and to that portion of the colon which crosses the spine to form the sigmoid flexure, or last turn, it is attached. The omentum is small in the horse, and seldom contains much adipose matter. It is situated between the intestines and walls of the abdomen, to prevent concussion and injury during rapid movements of the horse. See fig. $2, c$.

\section{THE SPLEEN.}

This is situated on the left side of the stomach, fig. 2, $e$, and between it and the short ribs, with the hinder cartilages of which its margin beneath corresponds, so that if the belly were pierced from the left side posteriorly to the last rib, this organ would 
escape injury. It is attached to the left half of the great curvature of the stomach, but the chief portion of it lies behind and rather above the stomach. Its anterior end comes in contact with the left lobe of the liver; its posterior is connected to the left kidney, and concealed by the convolutions of the colon. The particular use of the spleen has never been yet satisfactorily ascertained.

Great enlargement and also rupture of the spleen has been noticed after the death of horses; but little is known of the causes nor the symptoms which indicate disease of this organ.

\section{THE PANCREAS.}

This organ is situated between the stomach and left kidney. It lies across the spine, within the epigastric region, underneath the crura of the diaphragm, immediately behind and a little above the small curvature of the stomach. Its structure bears a strong resemblance to that of the salivary glands, contiguous to the mouth, and secretes a fluid very much resembling common saliva. This fluid is conducted into the intestines by a duct, which enters at the same aperture with that which proceeds from the liver. There is every reason to believe that this Huid aids digestion, but in what way has not been ascertained.

\section{THE DIAPHRAGM, OR MIDRIFF.}

The cavity of the chest consists of two compartments, and the division or separating wall between which is called the diaphragm, as more fully described, page 24I. See fig. 2, $i, i$, and fig. $1, h$. 


\section{THE KIDNEYS.}

These are two somewhat oval, reddish-coloured bodies, which occupy the upper part of the abdomen, and are situated under the loins. Their form is like that of a kidney bean. The right kidney lies most forward, and is placed under the liver; the left one lies more backward, rather behind the stomach and spleen. They lie above and are concealed by the intestines, consequently upon dissection they are not visible until the intestines are removed, as will be seen by Plate Ix, fig. I. In fig. 2 these are removed, and the kidneys, $d, d$, are exposed, as well as other organs which occupy the chest and abdomen. The chief function of the kidneys is the secretion of the urine, and carrying off an ingredient which enters into its composition, called the urea, a substance which, if allowed to circulate with the fluids, would prove poisonous. To each of the kidneys a large artery runs, which conducts nearly a sixth part of the blood of the body to them. These branch off into innumerable minute vessels, which ramify every portion of the substance of the kidney, and by this means, and the uriniferous secreting structures, the watery portions are separated from the blood, and by the ureters conducted to the bladder. The urine varies more in its quantity and quality in the horse than in any other animal with which we are acquainted, and hence the necessity of attending to its appearance and composition during disease; because attention to this enables the veterinarian to detect the disease, and also to judge the quantity of medicine which may prove beneficial to the animal. In the application of these much good or much evil may be the result. Ignorant ostlers, seeing the effects of nitre in promoting staling and also in purifying the 
urine, are too prone to use it with indiscretion. These diuretic medicines, if used in too great quantities, stimulate the kidneys to separate more of the watery fluid than they do in a natural and healthy condition, and the effect is to lessen the quantity of blood. Although this property in nitre is advantageous in many disorders where the heart is burdened by the pressure required to force the blood forward through the arteries in inflammatory complaints, it is easy to see that taken in too great quantities, or too frequently, may not only render it too thin, but also deficient in quantity.

Diuretic medicines are of much value in many diseases. For example, in swellings of the legs the use of diuretics have the effect of carrying off-as we have above shown-a greater than ordinary quantity of the watery portion of the blood. Nature has always a tendency to keep in equilibrium all her machinery and laws; consequently, the absorbent vessels are stimulated to greater action in order to compensate for this waste, and take up and convey into the circulation that portion of the fluid which had affected the limbs. In short, many horses are so predisposed to swelling of the limbs, that it is impossible to render them fine without the use of diuretics. We shall treat this part more fully in our chapter on medicines; but we may in the meantime offer the following precautions in the use of diuretics. First, let the horse have as much drink as he will take, as this will promote the urinary evacuations. Secondly, the stable should be kept cool, and the clothing thin; because, if this is not attended to, the medicine given to stimulate the kidneys will pass off by perspiration, and the effect intended will be thus frustrated. It is a law of the animal economy that when the skin 
gives off perspiration, the action of the kidneys is reduced.

\section{THE BLADDER. \\ PLATE IX, FIG. $2, k$.}

The bladder occupies the middle and lower portion of the pelvis. In its undistended condition it is wholly confined to the cavity of the pelvis; but when full, its fundus advances before the pubes into the abdomen, the advancement being in ratio with the degree of distention. It is pretty nearly pear-shaped.

We have described how the urine is separated from the blood, when treating of the kidneys. The urine which is separated is discharged by these minute vessels into larger ones, which terminate in the kidney, which is termed its pelvis, and from thence led into the ureter duct, $n, n$, to the bladder, $k$, which we have represented distended with urine. The urine is constantly secreted and flows continually from the kidneys through the ureter ducts. It is this elastic property of the bladder which enables animals to retain the urine beyond the time which it is filled to its unstretched capacity, and prevents that constant flow which would be the result but for this beautiful provision.

The bladder is provided with three coats. The outer one covers the greater portion of it, and is a part of the peritoneum; the muscular coat consists of two layers of fibres; the external running longitudinally, and the inner circularly, which enables it to yield to the pressure of the urine as the cavity fills, and again contract to a small size when emptied. This contractile property also assists in expelling the urine from the bladder. The inner or mucous coat is 
white, soft in its texture, and highly organised. It possesses numerous follicles or little glands, from whose excretory pores issue a plentiful mucous secretion, to defend it from the acrimony of the saline and other matters contained in the urine; this mucous matter being perpetually washed off from the surface of the inner coat by the urine, is kept constantly renewed, and it is sometimes voided in considerable quantities. When this is the case, it may be apprehended that the urine is unusually acrid, or that calculi or other irritable matter is within the bladder. About an inch before the cervix or neck of the bladder, in the sides of the bag; the orifices of the ureters are placed, which cnter the bladder in an oblique direction, and prevent any reflux of the urine at the time the bag is contracting, and which gives them the property of valves. The bladder terminates in a small neck, round which is a powerful muscle, which keeps the passage closed and retains the urine until the animal wishes to expel it; or when the bladder contains a certain quantity of fluid, the muscular coat contracts, and, the lungs being filled with air, the diaphragm is rendered convex towards the intestines, and they are by that means pressed upon the bladder, and by their united powers the fluid is forced through the sphincter muscle at the neck of the bladder, and escapes. We have described the disorders to which the bladder is liable at page 72 .

\section{THE INTESTINES.}

The intestines are cylindrical tubes of very unequal dimensions, forming one continued but convoluted canal from the lower orifice of the stomach to the anus, in which the process of digestion, begun in 
the stomach, is completed, and ultimately expelled in the form of fæces.

The abdominal viscera, taken collectively, cannot be said to occupy any particular region of the belly, for they are spread chiefly over the lower portions of it-immediately supported by the abdominal muscles, and one or more of them are found in every portion of the cavity of the belly, and collectively fill nearly its whole space.

The length of the intestines of the horse of full dimensions is ninety feet, or between eight and nine times the length of his body. Those of man are about sixty feet long, or six times the length of the body.

The food having been partially digested in the stomach, and converted into a substance called chyme, passes through the pyloric orifices into the intestines. The length of the intestines in animals bears a proportion according to the nature of the food. The nutritive portion of vegetables is extracted with much more difficulty than with animal substances, and hence the necessity of the alimentary canal being much longer and more complicated in the horse and other animals which feed upon vegetables. This viscera is divided into the small and large intestines, from the latter exceeding in volume the former. Each of these is subdivided into three parts, all of which are composed of three coats: the first, or external one, is called the peritoneal; the second, or middle, the muscular; and the third, or internal, the villous or mucous coat.

The peritoneal coat is a covering continued from the peritoneum itself, which includes the mesenteric vessels and nerves in its course to the intestines, and connects them to the spine, to one another, and to 
other viscera. It intimately adheres by fine cellular tissue to the muscular coat underneath. It serves to strengthen the tubes, and to furnish a lubricating serous perspiration, which renders the membrane smooth and moist, and serves to prevent all friction and concussion. By the aid of this coat the bowels are confined in their proper situations. If the intestines were allowed to float loosely in the abdomen, they would be liable to constant entanglement and injury by jolting during the rapid and violent motions which the horse is subjected to.

The middle, or muscular coat, like that of the stomach, is composed of two kinds of fibres, one of which is longitudinal, just under the peritoneum, and stronger circular fibres are situate more inwardly. In consequence of these combined actions the intestine may be contracted in all directions. It is by the action of the circular fibres that the aliment is gradually forced through the intestines.

The villous, or mucous coat-so named from its surface being studded with small giands which give out a mucous fluid to lubricate the coating, and sheaths and defends it from acrimonious or mechanical irritation of the aliment, and also to facilitate its passage through the intestine. It is also crowded with innumerable small apertures, which are the mouths of minute vessels, by which the nutritial portion of the food is absorbed, and which is ultimately conveyed into the circulation and distributed through every part of the body.

Having given an account of the structure and appearance of the intestines generally, we shall now enter into their peculiarities. 


\section{PLATE VIII, FIG. 5 .}

This gives a general view of the appearance of the belly with the intestines, as they are presented without any of them being removed, the skin of the abdomen only cut open and thrown back so as to expose the viscera.

$a, a, a, a$. The colon, which in the horse is a gut of enormous size, being the longest and most capacious of the large intestines. Such is its capacity, that it will contain about twelve gallons of water. The course and figure of this intestine are peculiar. It begins at the head of the colon, and expands into a cavity larger in dimensions than the stomach itself; it then begins to contract, and continues to do so gradually until it has completed its second convolution round the cæcum, or blind gut, where its dimensions are not more than one of the small intestines.

b. The cæcum, or blind gut, which is the first subdivision of the large intestines, originating in a large capacious receptacle, called the coccum caput coli, or blind head of the colon, from which it extends downward and terminates in a blind extremity. The cæcum differs from all the other intestines in having but one opening into it, so that all the substances which enter into it must reascend into the caput coli, in order to be carried through the intestine. The exterior parts are braced by three longitudinal bands, and puckered by them into three sets of cells internally, which will be better understood by a reference to Plate Ix, fig. I.

c. A portion of the mesentery. It is a duplicature of the peritoneum, which bears this appeliation. The colon is attached in like manner to the bone by a production of the same membrane, called the mesocolon, and the rectum is kept in its place by a similar reflection, called the mesorectum.

$d, e$. Are portions of the small intestine.

$j$. The beginning of the colon. 



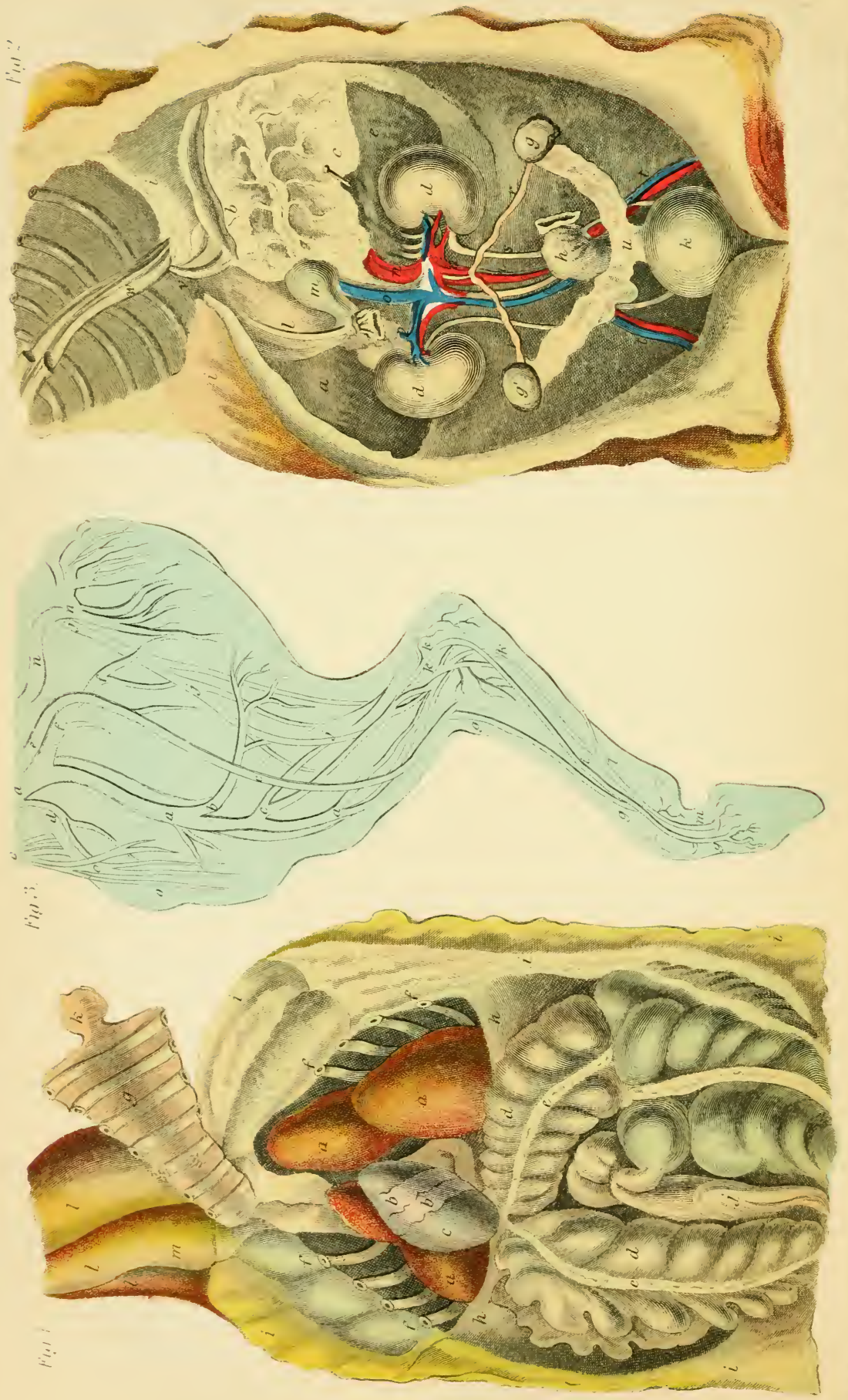


g. The rectum. As soon as the colon has reached the basis of the sacrum, it ends, and it then assumes the name of rectum, being to a certain extent straight ; however, it is not perfectly so, as it follows the curve of the bones. It terminates by a large extremity, called the anus. The rectum will hold about three gallons of water or fluid matter. The outer extremity is furnished with a circular muscle, called the sphincter ani, the use of which is to keep the anus closed, to retain the feculent matter until so much of it be accumulated in the rectum as to excite a desire to discharge it.

\section{PLATE IX, FIG. I.}

This figure is intended to display the relative situations of the principal organs, with only part of the intestinal canal. These lie exteriorly to other important viscera.

$a, a, a$. The different lobes of the lungs, already described, page 230. Its vessels are seen upon its surface.

b. The pericardium, or bag which surrounds the heart.

c. The heart.

$a, a, d$. The colon, already described in Plate vin, fig. 5, $a, a, a, a$.

$e$. The ligamentous bands of the colon, which pucker it into folds.

$f, f, f, f$. The ribs.

$g$. The sternum or breast-bone, removed from its junction with the ribs, and thrown back to exhibit the contents of the chest beneath.

h. The diaphragm, already described, page $24 \mathrm{I}$.

$i, i, i, i$. The skin thrown back, to show the contents of the chest.

$j$. One of the small intestines.

$k$. The ensiform, or sabre-shaped cartilage.

$l, l, l$. The neck.

m. The situation of the trachea, or windpipe. 
PLATE IX, FIG. 2.

The chief organs represented in this figure are principally hidden by those described in the last figure.

a. Lobe of the liver.

b. The stomach, described at page 243 .

c. The omentum, or caul, described at page 248 .

$d, d$. The kidneys, described at page 250 .

$e$. The spleen, described at page 248 .

$f$. The uterus, or womb, which is a hollow membranous organ, united to the anterior part of the vagina, and in the mare is of a striking and peculiar form. Its body spreads out anteriorly into two horn-like processes. The vagina resembles a bottle, and the uterial portion is like a head and neck. This is in the female which has never been fecundated. But during the period of gestation the womb is almost incredibly augmented in size, and never afterwards resumes either its identical form or virgin state of contraction.

$g, g$. The ovaries. These are two egg-shaped bodies, situated a little further forward than the Fallopian tubes, within the cavity of the abdomen. They are the female testicles, and are about the size of walnuts.

$h$. Part of the rectum.

$i, i$. The diaphragm.

$k$. The bladder, distended with urine to show its dimensions and form.

l. The gall-duct.

$m$. The duodenum takes its rise from the right extremity of the stomach-being the first of the small intestinesand soon after forms a curvature around the head of the pancreas, having the liver above and the great arch of the colon below it. When it reaches the concave part of the liver, it makes a sudden turn backward, and becomes attached to the right kidney, then crosses the spine, between the roots of the mesentery and mesocolon and left side, where it assumes the name of jejunum. The duodenum receives 
the food whicl has been converted into chyme by the digestive power of the stomach, and in this gut is converted into chyle. It is mixed with the bile and the secretion from the pancreas, which enter into this intestine about five inches down from its origin. The bile appears to be the principal agent in this change, for no sooner does it enter into combination with the chyme, than the fluid begins to separate into two distinct ingredients, namely, the thick white liquid termed chyle-which is the nutritive portion of the food-and a yellow pulpy substance, which becomes excrement. A more perfect separation of these substances takes place further on in the intestines, the chyle is sucked up by the mouths of the numerous small vessels called the lacteals, leaving the excrement alone.

The next part of the small intestine which follows the duodenum is the jejunum, through which the food passes with great rapidity, for it is generally found quite empty in the dead subject. It is paler in colour and less in calibre, and also much longer than the duodenum.

The next intestine is the ileum, which is the longest of all the intestines, and forms the greater part of the convoluted tube, which lies chiefly in the umbilical region. The small intestines altogether will contain about eleven gallons of fluid.

That part of the food which has not been taken up by the lacteals and absorbents in its course through the small intestines, passes through the valvular opening of the ileum, the fluid portion of it finds its way into the colon, and the rest enters into the cæcum. Here it seems to remain a considerable time, in order that the nutriment may be extracted from it. This blind pouch is plentifully supplied with blood-vessels and absorbents, which perform their office of carrying off the nutritive portion. 
Horses will frequently drink more at a time than the stomach is capable of containing. It does not, however, remain in it, but passes on to the cæcum, which acts as a reservoir in time of need. This organ will contain four gallons of fluid.

$n, n, n$. The aorta descends in both cavities.

$o$. The vena cava descends.

$p, p$. The emulgent veins, which empty their blood into the vella cava.

$q, q$. The emulgent arteries, emanating from the aorta.

$r, r$. The spermatic arteries and veins, which are united by a cellular substance, soon after their origin is distributed to the ovaria.

$s, s$. The ureters, which have their origin in the kidneys, and are inserted into the upper part of the bladder.

$t, t$. The iliac vessels, which are bifurcations of the aorta and vena cava. 


\section{CHAPTER XI.}

\section{Of the Skin and its Diseases.}

THE skin of the horse consists of three parts, differing in appearance from each other, namely, the cutis, or true skin; the cuticle, epidermis, or scarf-skin; and the rete-mucosum.

\section{THE CUTICLE}

Is placed externally; it is very thin and somewhat transparent and tough. In the living animal this may be proved by application of a blister : serum is effused from the exhalents of the cutis, and the cuticle becomes elevated by it in the form of small pellucid bladders. This membrane is continually growing, and the scales which are brushed out in grooming are scaly portions of the cuticle.

The cuticle seems to be composed of very thin flexible scales, somewhat resembling the scales of a fish, and similar to them in arrangement. The cuticle is produced by the true skin, and is perforated by both its exhalent and absorbent pores. In almost all parts of the body the cuticle is thickly clothed with hair, but that of the nose, the lips, and the interior of the ear, the borders of the eyelids, and the inside of the superior portion of the thighs, is naked; and in all those places is thinner in substance than on the other parts, which are invested with hair. The colour of the cuticle is the same in all 
horses, whatever be their hue. But it is a known fact that the skin of the silver grey Arabian horse is of a bluish black; but whether this colour is in the cuticle or in the rete-mucosum I have not yet been enabled to discover.

The epidermis is everywhere perforated by minute holes, corresponding in situation, size, and number to those of the cutis. First, there are the pores for the hairs; secondly, the perspiratory, or exhalent pores; thirdly, the absorbent, or inhalent pores ; and lastly, larger-sized pores, through which unctuous secretions in various parts are emitted. The cuticle is destitute of both nerves and vessels, and consequently devoid of sensibility.

\section{THE CU'TIS.}

This is the true skin, or that portion which is converted into leather, and lies immediately under the scarf-skin. It reaches over every part of the animal. It is attached to the under parts by the cellular membrane, and in some places fits so tensely that it is incapable of motion of any kind. In other situations it is more loose and can be pulled into folds; about the forehead, the back, and near the hock and pasterns, it is so tightly braced that it is hardly possible to pinch up a part of it between the finger and thumb; upon the sides of the face, neck, ribs, along the flanks, front of the chest, and upon the arms and thighs, it is more easily folded. In the blood-horse it is thin and highly sensitive; while in the cart-horse, it is much thicker, and far less sensible; and is liable to considerable variation in different breeds. The texture of the hair seems to depend in a great measure upon that of the cutis, for we find that of the thick-skinned black horse much coarser than in the 
racer and other high-bred varieties. It is a rare thing to meet with a black thoroughbred horse.

The skin is beautifully adapted for giving strength as well as a protection to the muscles; for we find in those parts that require to be firmly bound together, such as the bones of the knees, the pasterns and tendons of the limbs, it adheres with such tenacity that it cannot be raised from those places; thus acting the part of a powerful ligature to the parts which are subjected to the greatest degree of stress; and in those situations where being tight would interfere with the action of the horse, it is loose.

The cutis is of a strong fibrous texture, very tough, yet supple, elastic, very vascular, and sensitive. Its fibres are curiously interwoven in almost every direction, and so interlaced as to give great strength to its texture, making it almost impenetrable by a knife in the living animal, and possessing extreme elasticity. It is this quality which adapts it so closely to the animal, whether he is plump and muscular, or reduced to skin and bone. In man, and most other animals, where from disease a great reduction of the muscular fibre has taken place, the skin becomes loose and shrivelled. It owes this great elasticity to the innumerable larger and smaller glands which penetrate its entire substance, and furnish that unctuous matter, preserving the skin soft and pliable, and maintains that greasy moisture which its surface ever possesses, giving that beautiful sleek appearance to the hair. When the animal gets out of condition, and the skin is diseased, then the coat assumes a rough appearance, the hairs refuse to lie down, and it is then said that the coat stares.

The skin at the bend of the knee and hock is 
bountifully supplied with this mucous matter to give them suppleness, and to preserve from friction those parts which are subjected to such constant and active movements. Sometimes this secretion exceeds the quantity necessary for the due action of the parts, and from want of attention and cleanliness becomes inspissated, and collects about those parts: and, if this hardness is permitted to remain, it will become a watery sore, which will terminate in lameness, stiffness, and pain in the joint when the animal bends it. When this is situated in the bend of the knee, it is termed mallenders, and when it is seated in front of the hock joint, it is called sallenders, complaints which we have described at page III. If these complaints are attended to in their early stage, nothing more will be required than to cleanse the part from the scurf or scab which it produces by soaking it in hot water, and carefully washing it every day with a sponge and some astringent mixed with the water, such as a small portion of vinegar.

The skin of the heel has numerous glandular pores, through which the unctuous secretion oozes; and sometimes these are unduly excited, and this matter becomes altered in its substance and odour, and produces that disease called grease, described at page 123 , and which in some instances proves somewhat difficult to cure.

The cutis, when destroyed by any means, does not regenerate quickly. Great care should therefore be taken not to allow any portion of it to be broken. Many think that it is of little consequence for horses to have the skin of their back rubbed off by friction from a saddle. Such parts as have lost their cutis have it but slowly reproduced; and even when it has been restored, its vital power is much weaker 
than it originally was; for, although it appears at first to be very vascular, its vessels after a time either shrink in calibre, or some of them become altogether obliterated. It invariably happens that when horses have had fistulæ or saddle-galls, they are always more disposed to subsequent injury in those parts.

\section{THE RETE MUCOSUM.}

The third part of the skin consists of a membrane which bears this name; it is a fine, delicate, laminated tissue, interposed between the cutis and the cuticle. The skin takes its colour from this membrane. It is from this membrane being black in the negro that he takes his dark colour; for dissection has proved that his cuticle when separated from this membrane is as white as that of a European. Its use appears to afford protection to the delicate vessels of the nerves from outward injuries.

\section{PORES OF THE SKIN.}

The skin exhibits an infinity of pores over its entire surface, and probably the whole of these transmit hairs. There are also numerous others, smaller and less perceptible, which are called perspiratory pores, from their emitting an imperceptible vapour, denominated the insensible perspiration, the sensible perspiration being what is ordinarily called sweat. The situation of these pores is rendered manifest by the condensation and collection of this exhalation. But these pores can be rendered visible by maceration, or putrefaction in the dead animal. Besides these there is another set of pores, of larger dimensions, more discernible than others in some places, which are the mouths of follicles. These are of large 
size in the nose, for the secretion or mucus; and the tubes of the ears have many of them for the passage of the waxy matter; and all the portions of the skin which are liable to friction are numerously provided with them, which preserves it in a soft and pliable condition, and produces that greasiness of feel which is constantly kept up in the skin.

This process of perspiration in the horse cannot be so controlled by the use of medicine as in the human being. The visible perspiration can only be increased in a limited degree in the horse, although we can in some measure lessen profuse perspirations which accompany the moult, disease, or want of condition.

The visible perspiration, as far as we know, cannot be produced by any kind of medicine. Antimony and sulphur, however, have considerable effect in opening the pores, and in a certain degree exciting the vessels to action.

There can be little doubt but the skin is furnished with absorbent vessels, which take in any substance in a fluid state and conduct it into the circulation. We have strong proofs of this in the horse being more easily salivated than man himself, as we have known a horse to be salivated by rubbing a splint with mercurial ointment.

\section{OF THE HAIR.}

The hair of the horse is of two qualities: the one covering the entire surface of the skin, called its coat, and which is of a fine soft material; and the other, which invests the ridge of the neck, crown of the head, and forms the tail, of a coarser texture, and much lengthened. The foretop appears only formed as an ornament; while the mane is a protection to the neck 
during combat; and the tail acts the double purpose of an ornament, and as a switch to drive off insects from its rear. The tufts of hair springing from the fetlocks defend them from contusion, and also protects the heels. The coat is of a uniform thickness all over the body, excepting upon the inner parts of the thighs, under the arms, etc. The coat varies in quality, colour, and length in different breeds. The thoroughbred racer, the Arabian, Barbs, and Turkish horses are remarkable for the shortness and sleekness of their coat ; while the cart-horse, the Shetland pony, and horses of all northern climates, are distinguished by the length, roughness, and coarseness of their hair. The lighter the shade of colour, the finer the hair; and it has been found that in the chestnut and light bay horses there are many more hairs in a square inch than in black and other dark-coloured horses.

The coat is shed twice a year, in spring and autumn. In a state of nature this commences with great regularity as to time; but in a state of domestication, this process is much influenced by the temperature and stable management. The hair of the mane and tail is never shed. Regeneration of the hair is attended with a considerable expenditure of nervous energy, and consequently the strength of the animal during this process is affected, as well as his general health. The horse at this time feels a general languor, and consequently should not be subjected to violent exercise or hard work. This weakness will invariably be manifested by profuse perspiration when at work. Hard labour at these periods frequently induces various complaints.

To facilitate the process of moulting, or changing the coat, spices and other stimulants have been given to horses, which no doubt has the effect of hastening 
the change; but we would prefer allowing nature to pursue its own course, as there can be little doubt but these stimulants incluce a higher degree of fever at the time, and may bring on a regular febrile attack. Gentle friction will be beneficial; but care should be taken not to abuse this. The currycomb should not be used at this time; the brush only should be had recourse to, and that applied with caution. Moderate exercise and warmer clothing ought to be adopted during the biannual moult.

The slightest attention to the subject will easily convince any one of the altered state of an animal during shedding the hair. It will be found that at this time there is always an increased pulse, redness of the nose, and heat of the mouth, unusual in the healthy condition. Hence it is evident that everything exciting should be avoided. Many grooms are in the habit of giving the animal cordials and stimulants, mistaking febrile excitement for debility. The following alterative will be found beneficial :-

$\begin{array}{llllll}\text { Digitalis } & \cdot & \cdot & \cdot & \cdot & \text { I drachm, } \\ \text { Nitre } & \cdot & \cdot & \cdot & \cdot & 2 \frac{1}{2} \text { drachms, } \\ \text { Emetic tartar } & \cdot & \cdot & \cdot & \cdot & \text { I drachm, } \\ \text { Aloes } & \cdot & \cdot & \cdot & \cdot & \text { I } \frac{1}{2} \text { drachm. }\end{array}$

The stable-clothing should be warmer, the usual quantity of food should be diminished, and branmashes given instead of hard food.

\section{CLIPPING.}

It is an utter absurdity to denude the animal of its natural quantity of clothing. It has been a practice to clip hunters, so that the coat of the animal may sooner dry after a long run; but there is less danger to be apprehended from the longer coat, although it 
does not thoroughly dry, than when the short hair of the clipped animal exposes the overheated skin to the chilling effects of a frosty atmosphere, and thus during pauses from exercise the horse must suffer severely from cold, and besides is liable to be attacked by inflammation.

I am aware that many persons of much experience will differ with me in what I have said upon this subject. Waiving my objections, it must be admitted that the skin of the animal will dry much sooner after hard labour when he has a thin coat, and undoubtedly much labour will be saved to the groom, which is of material consequence. It has been said that horses which have short hair feed much sooner after a hard run than those which have a long and rough coat; and then when once it is dried, there is less chance of its afterwards breaking out into a sweat.

\section{SINGEING.}

Many approve and recommend this operation, and by a little practice it can be singed nearly as close as in clipping. The instrument used is a piece of iron, about four inches wide at the extremity, made in the form of a Dutch-hoe, and inserted into a handle six or eight inches long. Some wick-cotton must be rolled round the bar at the bottom of the instrument as evenly as possible. It must then be dipped in naphtha and lighted, and drawn gently over the coat of the horse, taking care not to go too deep at once, but repeating it frequently until the hair be burnt as close to the skin as possible. During this operation a blunt table-knife must be held in the left hand, with which to scrape out the flame from time to time, to prevent it burning too deep in any part, as well as to preserve the skin from being blistered. The singed hair must 
always be scraped carefully off before the flame be re-applied. The same part should not be singed several times in succession without allowing it to cool thoroughly, otherwise great irritation and fever may be induced. In singeing the neck, the mane must be turned to the opposite side to that which it is intended to burn, and a wet brush should be passed over the roots of it, to prevent the flame running over it. We would recommend that the operation of singeing should not be done all at one time, but by degrees on several successive days.

\section{COLOUR OF THE HAIR.}

Fashion too frequently regulates the prevailing colour of horses, because, as we have already observed, the colour of the coat is dependent upon chemical action, and consequently the constitution of the animal is connected with it. It will be found that there is also a sympathy between the colour of the hair and that of the eyes. The three primitive colours in the horse are white, red, and black; and all the intermediate shades are modifications of these. Milk-white horses have very often wall-eyes, while blacks have dark hazel, and chestnut brown, varying in intensity with the depth of colour.

Black horses have in general excellent constitutions. In Lincolnshire much attention has been paid to the purity of their large and stately blacks. They are peculiarly well adapted for waggons and other heavy machines, but are defective in point of activity. In the above and adjoining counties considerable attention has been devoted to a smaller breed of blacks, for the purpose of cavalry horses. They are remarkable for their high action, a quality which, however valuable in a draught-horse, is objectionable in a roadster, their 
paces being disagreeable. Few racers or hunters of character have been known of this colour.

Of all the colours, bays have been the best in this country. They are liable to many modifications of shade. Those which have no white about them, and whose limbs are black from the knees and the hocks to the feet, are the most desirable to possess, having in general good constitutions, and also well-formed feet. When they verge into the bay-brown, they are not so showy, nor is their action so good; but they will be found to be hardy, durable, and useful. They possess more substance than the lighter coloured bays, and have a greater depth of leg. If they happen to be high-bred, they will generally turn out good.

There are three varieties of chestnuts. The lightest red, which is denominated sorrel, have too frequently white about either their feet or face. In general they are found to be light in the carcass, and possessing delicate constitutions, and for the most part are hot-tempered. Many of them, although light in the figure and tolerably well formed, are nevertheless totally devoid of good breeding, and certainly incapable of endurance. If we have a predilection for chestnut, which is undoubtedly a showy colour, we must look to the lighter coloured horses with less of the red, and tending more to the bay or brown. The action of these horses is generally pleasant, but sometimes possessing irritable tempers. They are, however, considered rather constitutionally weak. The dark chestnut possesses a finely-moulded form, with well-rounded quarters, although the legs have a tendency to be rather fine. This horse is capable of great endurance, and possesses a healthy constitution. His temper will be found fiery and high. Small feet 
are frequently met with accompanying this colour, and which are generally disposed to contraction.

Snow-white horses are now seldom to be met with, although at one time they were very common; I mean, those which are white in their earliest years; as light grey animals soon become white, especially those which have dark-coloured joints.

The silver-grey is now not common, but they are in general high-bred, and of undoubted descent from the Arabian or Barb. Their height seldom exceeds fourteen and a half hands (which is the ordinary size of their progenitors), with a well-rounded body, firmly knit, light legs, oblique pasterns, and high shoulders. They are active and fleet, fitted for hard work, and possessing excellent health. Their action and appearance peculiarly fit them for the use of the ladies.

The iron-grey is a larger horse than the above, generally higher in the withers, with a thinner body, flatter chest, and more angular in all his proportions, with his legs often too long. They are showy horses, and well adapted for a carriage. In some instances they make good hunters, and are useful as roadsters, being capable of greater endurance than would be expected from the flatness of their chests. They are, however, found often to have a tendency to contraction of the feet, and hence lameness.

The dappled grey is usually a well-formed and active horse, and divested of all the angular points which characterise the iron-grey. They look remarkably well in harness, and are showy as hackneys. When these horses are high-bred, which is mostly the case, they are not only handsome, but also active. If they are dark-coloured when young, they seldom or never become white, but retain their dappling and 
fine rusty colour for life. Formerly, this breed were heavy animals, but their form has been materially improved by the admixture of foreign blood.

The dun is a hardy kind, and especially if it is of the smaller or Galloway size. Some of the best hackneys which I have known were of this colour. If of the paler dun, they are particularly well adapted for the phaeton of a lady. I have seen duns with a good deal of blood possessing very great activity. It is, however, curious that the larger horses of this colour, unless high-bred, are conspicuous for their want of action.

Roans, although frequently showy animals, are not remarkable for activity nor for constitution. Their delicate habits seem to give them an elasticity of joint which renders their paces easy, and they are therefore well adapted for ladies. This variety is very apt to have white legs and feet, and they are too frequently weak in consequence.

The cream-coloured steeds are a breed originally imported from Hanover, where they have for a very long period been bred as horses for the royal stud. There is a marked peculiarity in this breed: possessing a large yet perfectly well-formed carcass, with a beautifully arched crest; the action is graceful and dignified, which peculiarly fits them for the stagecarriage of a monarch. The pupil of these horses is red, and the eyeball or iris white, so that they are true albinos. Their constitution is delicate, and they are incapable of great exertion and endurance.

The pied horse is in many instances an attractive animal, although seldom used as a riding-horse. If more than half-bred and well matched, they are showy in a carriage. They are, however, liable to have 
white legs and feet, points not desirable in any horse.

\section{HIDE-BOUND.}

Symptons. - This complaint consists of an alteration in the substance of the skin, proceeding from a hardness and unyieldingness of texture, in consequence of the want of the ordinary oily secretion on its surface, as well as in its whole parts. The skin to the touch has a dry and rigid feel, which proceeds from a want of energy in the vessels of the skin, rendering them unfit to perform their functions, and indeed in the vessels of the stomach and bowels, which must affect those of the whole system.

This rigidity of the cuticle naturally causes a dryness in the scales which cover it; and these being separated in all directions, turn the hairs in that irregular manner, and produce the rough, uneven coat, which is termed staring, always indicating a want of condition in the animal. This complaint may rather be considered as the concomitant of disease, than the ailment itself; for the root of the evil is seated in the stomach and bowels. This being the case, we must apply the remedy to the seat of the disease, as well as to the skin itself.

Cause.-This state of the skin accompanies various complaints, consequently the first thing we must look to is to discover its cause; and so soon as the cause is removed the skin will be restored to its natural healthy condition and appearance.

Remedies.-If the complaint is connected with any of the maladies to which we have referred, then the medical treatment already recommended in these must be adopted; but if we are unable to detect the cause, which is frequently the case, it may then 
be suspected that it is connected with the suspension of some important secretion, and with the alimentary canal generally. Then we should administer some mild purgatives, which may consist of the following prescription :-

$$
\begin{aligned}
& \text { Aloes . . . . } \mathrm{I} \frac{1}{2} \mathrm{drachm} \text {, } \\
& \text { Nitre . . . . } 2 \text { drachms; }
\end{aligned}
$$

made into a ball with treacle.

Let the above be twice repeated, and on the third day the following should be given :-

$$
\begin{array}{lllll}
\text { Calomel } & \text {. } & \text {. } & \text {. } & \text { I drachm, } \\
\text { Aloes } & \text {. } & \text {. } & \text {. } & 2 \text { drachms. }
\end{array}
$$

The horse should be kept from dry food, and in its stead give him bran and other mashes. This treatment must be pursued until a regular and healthy action of the alimentary canal is re-established, which will be indicated by the appearance of his coat. Some veterinarians recommend the following purgative in hide-bound cases:-

Levigated antimony $\quad . \quad 2$ drachms,
Nitre $: \quad . \quad: \quad 3$ drachms,
Sulphur $: \quad . \quad$ drachms;

made into a ball.

This is repeated every night, and accompanied by mashes until a healthy condition is established. Perhaps it is better to vary these different medicines. Sulphur and antimony combined produce a peculiar effect upon the skin, while the sulphur acts upon the bowels, and the urinary organs are cleared out by the nitre.

If the horse is off his feed, and no symptoms of fever, it may be apprehended, as above hinted, that a want of energy of the vessels is the cause of the 
complaint. In that case it will be necessary to add a tonic to the above prescription. Let that be---

$\begin{array}{llll}\text { Gentian : } & \cdot & \cdot & \text { I drachm, } \\ \text { Ground ginger } & \cdot & \cdot & \frac{1}{2} \text { drachm. }\end{array}$

Besides what we have above recommended, it will be found that friction may be advantageously employed, as it arouses the dormant energies of the secreting vessels. Additional clothing may also be resorted to with great advantage. We must, however, draw a marked distinction between heat thus employed from that of keeping the stable warm. This is too frequently resorted to by injudicious grooms, who thus contaminate the atmosphere of the stable, and keep up what is very often the cause of the disease. Nothing is of so much consequence as a well-aired stable; and if warmth is necessary, let it be applied in the shape of clothing.

\section{SURFEIT.}

Symptons. - In the spring large pimples or lumps suddenly make their appearance on the skin of the horse. They differ materially in their effects, as some are attended with much itching, and others seem to give the animal no uneasiness whatever. Instances occur in which they disappear as suddenly as they came. When they do remain, in a few days the epidermis peels off, leaving a small scale-like spot, which is rarely a sore. In some instances these lumps are confined to the neck; but more frequently they extend over the back, loins, and quarters.

Cause.-The true cause of this cutaneous disease is not known, although it has been called surfeit, from a supposed resemblance to those eruptions in 
the skin of the human being which follow unwholesome or indigestible food. This complaint, however, most frequently exists after or during the time the horse is shedding his hair, and at which period there exists a stronger tendency to stomach-affections, and consequently indigestion has been supposed to affect the skin in the manner described. Poisonous substances taken into the stomach, such as herbs and mow-burnt hay-the chemical qualities, in the latter case, having changed its properties-have been known to cause this eruption. Drinking cold water in immoderate quantity, as well as improper exposure to cold air when the animal was over-heated, have been known to induce affections of this kind. There can be little doubt but it is caused by some obstruction of the pores of the skin, which produces swellings around them, and depending upon a primary affection of the cuticle, or from a sympathy with the digestive organs.

Remedies.-The following alterative must be administered :-

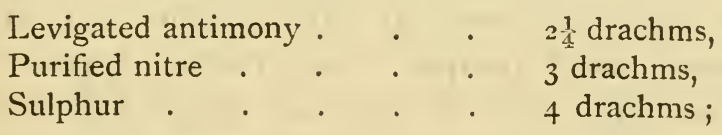

given at night, either in a mash, or formed into a ball with the ordinary substances; to be repeated for several nights in succession, with the use of warm clothing, which, together with heat of the stable, will cause the sulphur and antimony to act with more effect upon the skin. His drink should be slightly heated, and a moderate quantity of green meat should be given him, if it can be procured at the time. In the mornings he should be walked out for half-an-hour, but his clothing must be warmer than usual. His food must consist of mashes. 


\section{MANGE.}

Mange is a parasitic disease, similar to "Scab" in sheep, and the "Itch" in the human subject, but the parasites are distinct. There are three kinds of mange parasites found on the body of the horse, but all of them are so small that they cannot be recognised with the naked eye, and therefore a microscope has to be used in order to determine their presence. Certain chronic non-infectious skin affections lead to changes in the skin, very much similar to those changes produced by the burrowing and breeding of the mange parasites, and therefore it is imperative in all suspicious cases to examine the scales and hairs with the microscope.

Sумpтомs.- The symptoms are intense pruritus or itching. Touch the skin and the horse shows his sense of pleasure by stretching his neck, curling his lips, and clenching his teeth. He rubs his body against the stall or any object to which he can gain access, and this rubbing and biting and burrowing of the parasites sets up inflammation in the skin. Numerous small pimples and vesicles appear on the skin, the hairs fall off and are glued together by a liquid. The epidermis thickens, and fissures are formed in it. Owing to the continued irritation to which the animal is subjected, he falls off in flesh and condition, and if he is allowed to come in contact with other horses, or the harness, or the clothing, or the brushes which have been in contact with his body, the so-called mange disease appears on them. Grooms should be very careful not to use the same currycomb to a diseased and to a healthy horse indiscriminately, neither should the same brush be used.

Remedies. - In the early stage, when the parasites 
are not numerous, "mange" in horses, like "scab" in sheep, is not difficult to eradicate, but when the parasites are numerous and have burrowed into the skin and formed galleries therein, considerable time and frequent application of agents destructive to insect life are required.

Commence the treatment by washing the skin thoroughly with hot water containing carbolic acid and soap. Then clip the hairs off, soften the scabs with carbolised black soap, a thick coating, and leave it on the skin for a day and a night. Wash off, then apply :-

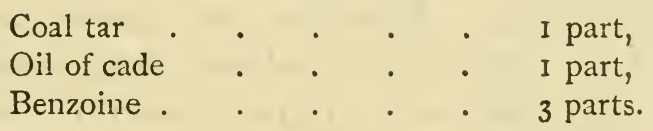

Mix the tar and cade and then add the benzoine. During the application of the liniment, an alterative ball should be daily administered, such as we have recommended at page 277 .

When the above treatment has been continued for some time, then the following ointment may be prepared, and the parts daily anointed with it:-

$\begin{array}{llllll}\text { Sulphur } & \cdot & \cdot & \cdot & \cdot & \frac{1}{2} \text { pound, } \\ \text { Oil of } \operatorname{tar} & \cdot & \cdot & \cdot & \cdot & \text { I pint, } \\ \text { Palm oil } & \cdot & \cdot & \cdot & \cdot & \text { I pint. }\end{array}$

Every fifth or sixth day the ointment should be washed off with spirit of turpentine, and afterwards well cleansed with soap and water, which will enable the groom to see what progress has been effected towards a cure. But should the disease still continue, the application must be still proceeded with. Mange often proves extremely obstinate, and weeks of trouble have been required to restore the skin to a healthy condition. In the various washings the soap and 
water should be pretty warm, as this will have the effect of opening the pores, which is of material consequence while the animal is undergoing anointment.

While the animal is subjected to this treatment, his food should be plentiful and nourishing, but not of stimulating quality. If during warm summer weather, he should be turned out to graze; but if the weather is cold, he should be plentifully supplied with green food.

Sometimes this complaint is deceptive in its appearance, and seems better than it really is after the above treatment. Therefore, to guard against a return of it, the rubbing should be continued for three or four days after it is apparently well. The alterative medicine should also be continued for ten or twelve days after a cure has been effected.

It is hardly necessary to say that the clothing which the animal wore during the use of those remedial means must be thoroughly cleansed. To render contagion impossible, they should be first soaked in water, with the addition of a thirty-fifth or fortieth part of the saturated solution of chloride of lime, and afterwards well washed with soap and water. In short, every part of the harness, rack, manger, and partitions should be completely scrubbed with a strong solution of soda and water; and when dry, with a solution of chloride of lime, in the proportion of a pint to three gallons of water. The currycomb should also be well cleaned, and the brush thrown away.

We would particularly caution all persons possessing horses to be watchful of this disease; and whenever they observe indications of itching in the head or neck of the animal, to examine carefully if any 
symptoms of this disagreeable disease exist, and to attack it instantly.

WARTS.

These are tumours of various sizes which spring from the cuticle, and afterwards are based in the true skin. They grow on various parts of the body, and sometimes are found in the prepuce and eyelids, or inside of the ears. These can only be removed by an operation, and that safely and effectually done by a practical veterinary surgeon. If the roots be very small, which is sometimes the case, they may be cut out close to the skin by a pair of small sharp-pointed scissors, and the part afterwards touched with caustic. If the stem of the wart be thick, it will not be prudent to cut it off, but a thread of fine waxed silk may be tied round it, and tightened every day until it drops off. Sometimes warts are in considerable clusters. When this is the case, it will be necessary to cut them off close to the skin, and afterwards sear the place with a red-hot iron, which will prevent them from growing again. If the horse is of a spirited nature, it will be necessary to cast him, and operate under chloroform. 


\section{CHAPTER XII.}

\section{Operations.}

Although operations are more the province of the veterinary surgeon than of private individuals, yet it would not be proper to pass over these without partially touching upon the subject. It is well for the untaught at least to know how the most simple of these are performed, and to them only shall we draw attention.

It is easy to imagine that in several operations the animals must suffer considerable pain, and under these are frequently very restive. It therefore becomes necessary to have certain instruments constructed to restrain the animals, and prevent them from injuring themselves, as well as for the safety of the operator. These we shall describe in the first instance.

The Travis.-This machine, considered by Continental horse-shoeing blacksmiths as indispensable accompaniments to their forge, is comparatively rare in this country. On the Continent, even the quietest horses are shod in the travis. It consists of very strong bars of wood, between which the horse is confined and slung. Although this is well calculated to prevent danger to the blacksmith, yet many fine horses have been rendered useless from their violent resistance to such confinement.

The Barnacles. - These are the handles of the 
pincers, which are placed over and enclose the muzzle. These are compressed by the assistant in operations. They give great pain, but are indispensable with some horses to enforce obedience. Sometimes this can be effected by blindfolding, but cannot be depended upon. Sometimes the use of the barnacles are ineffectual, which renders the side-line and twitch inclispensable.

The Side-Line. - This is found useful in the operations of nicking, docking, and slight firing. It consists of the long line of the hobbles, or common cart-rope, with a noose at one end. This is fixed to one of the pasterns of a hind leg. The rope is then carried over the neck, and afterwards round the withers, and this is tied to that portion which comes from the leg. By drawing this leg forward, it renders him incapable of kicking with the other; and should he attempt to use his fore leg, the other may be seized and held up, which renders any exertion on his part nugatory. The above will be found effectual in all the more simple operations; and that even with restive horses, if the assistants are determined and know how to restrain the animals. Sometimes the side-line is made so as to include both limbs, but in the struggles of the animal he is apt to throw himself, and may be injured in his fall.

For the more severe operations of firing and castration, improved hobbles are used. These permit of any single leg to be set at liberty and returned at pleasure; and when the operation is finished, all the legs may be freed from restraint in an instant. We need not describe the methods employed to throw a horse, because these are well known to the practical veterinarian, to whom alone operations which require this degree of restraint should be entrusted, and therefore we shall not touch upon them. 


\section{BLEEDING.}

It is a too frequent practice for gentlemen who have given a little attention to the veterinary art, as well as with some professional surgeons, to order a certain quantity of blood to be taken away, without themselves attending to the operation. Although we have pointed out the probable quantity under particular diseases, yet it must be distinctly understood that much depends upon the strength, size, and condition of the animal at the time; and although we may suppose, from what we have seen effected by practice, that the quantity which we have ordered will suffice, yet in this we may be greatly mistaken. No regular surgeon, however great his practice may have been, can predict this to a certainty. We have already pointed out, at page 236 , the nature of the pulse and its different indications. Before bleeding, we are supposed to have ascertained the state of the pulse, and our object is to reduce it as nearly as possible to its natural or healthy condition, or at least until a change has been effected in its action; therefore, the operator or his assistant should keep his finger on the artery while the blood is flowing, and, without strictly adhering to the quantity which he had previously supposed, continue to take blood until a marked alteration in the pulse has taken place.

The operation of bleeding is performed with a fleam, or lancet. We would recommend the former to be used by private individuals, or at least by such as have not had sufficient practice with the lancet. A piece of hard wood, loaded at one end with lead, called a blood-stick, is used for striking the fleam into the vein. Care should be taken not to strike too hard, as by doing so it is likely to wound the opposite side of 
the coating of the vein, which may produce severe cases of inflammation. If the fleam is sharp, which it always should be, a blow with the doubled fist will answer the purpose of a blood-stick.

The jugular vein is the part usually selected for general bleeding. It is necessary to blindfold the horse, or to turn away his head from that side from which blood is to be taken. The hair is smoothed along the course of the vein with the finger, which has been previously moistened; then with the third and little fingers of the left hand, in which the fleam must be held, sufficient pressure is applied to the vein so as to bring it completely into view, taking care, however, not to distend it too much, as the too rounded surface is apt to roll or slip when the blow is given to the fleam. The particular part from which the blood is taken lies about two inches below the union of the two branches of the jugular vein at the angle of the jaw. (See Plate III, fig. I, $p$.) The fleam must be placed in a direct line with the course of the vein, and over the precise centre of the vein, with its point as close as possible to the skin, without touching the vein. A sharp but not heavy rap from the blood-stick or fist on the back of the fleam, directly over the blade, will cut through the coating of the vein, and the blood will immediately flow after the fleam is withdrawn. A large-bladed fleam should always be preferred, which will make a greater opening, and thus facilitate the operation; besides, what is of greater importance, blood drawn speedily has far more effect on the system than double the quantity taken slowly ; and the wound, although larger, will heal as fast as a smaller one. A slight pressure on the neck with the pail, or other vessel used while blood is taken, will be enough to cause the blood to flow sufficiently fast. Some persons 
introduce the finger into the mouth between the tushes and the grinders, which by gently moving it about induces motion in the jaws, and facilitates the rapidity of the stream by the action of the muscles in connection with the vein.

In the operation of bleeding the blood should be received into a vessel the dimensions of which are exactly known, so that the operator may be able to judge from time to time of the quantity that has been taken. When it is necessary to repeat the bleeding, it will be better to make a fresh incision lower down than to open the old wound. The blood coagulates soon after it is drawn. That portion of it which is coagulable is composed of two substances - that which gives colour to the blood, and the thinner part in which the red corpuscles float. By degrees these separate, and the red corpuscles sink to the bottom. If coagulation takes place slowly, the red corpuscles have more time to sink through the fluid portion, and there appears on the top a thick, adhesive, pale, yellowish orange substance, called the buffy coat. In proportion to the slowness of coagulation and the thickness of this coat, the degree of inflammation used to be determined. In the healthy condition, coagulation is more rapid, and consequently the red particles have not time to sink, and the buffy coat is thin. When the horse is much exhausted, and there is a general decay of his constitution, coagulation is imperfect.

When the necessary quantity of blood has been taken, the edges of the wound must be brought close together and kept in their place by a small pin being passed through the contiguous skin, with a little tow wrapped round the extremities of the pin so as to cover the entire wound. In bringing the edges of the wound 
together. care should be taken not to draw the skin too much from the neck, otherwise blood will insinuate itself between the skin and the muscles, and cause a swelling (thrombus), which sometimes proves troublesome. The edges of the wound will heal in fortyeight hours, but the pin should be allowed to remain in the skin for about seven days.

The jugular being the largest superficial vein, and most readily got at, is generally selected to bleed from. In local inflammation, blood may be taken from any of the nearest superficial veins. For example: in affections of the shoulder, fore leg, or foot, the plate-vein may be used, which lies along the inside of the arm, and runs up immediately in front of it, towards the jugular vein. The disposition of this vein will be seen at Plate vi, fig. $2, r$, and the branches of the other veins of this portion of the limb are exhibited in the same figure. Where the hinder extremity is affected, blood is sometimes taken from the saphæna, or thigh-vein, which runs across the thigh. The course of the different veins of this part will be seen on referring to Plate vir, fig. 2. Where the foot is at fault, blood may be taken from the coronet; or, what is better, from the very point of the toe, by cutting down by means of a searcher at the union between the crust and the sole until the blood flows. Should it not do so freely, the discharge may be increased by dipping the foot in warm water. Any quantity of blood may be taken from this situation, and its flow is easily stopped by putting a little tow in the cut, and then placing the shoe slightly over it, to prevent it from getting out.

In inflammatory cases, the finger of the veterinarian should never be off the artery during blood-letting, as this will be a better indication of the quantity neces- 
sary to be drawn than any preconceived opinion, as nothing can be more absurd than drawing blood indiscriminately. The prudent man will bleed until the oppressed pulse becomes more distinct and fallen, or the strong pulse of high fever softer.

We would recommend a sharp-pointed, broadshouldered lancet, but especially the spring lancet invented by $\mathrm{Mr}$ Weiss of London, as the most safe and certain one in use, not only for bleeding from the larger, but also the smaller veins, etc. We especially recommend great care in keeping the lancet clean, and after every operation it should be wiped very carefully. Private individuals should occasionally look at and rub their lancets, because they are apt to rust even when not in use.

\section{BLISTERING.}

We have already mentioned blistering in treating of the various diseases to which it is applicable. Blisters act on the principle that two intense states of inflammation cannot exist in contiguous parts of the system at one time; therefore we apply some irritant substance to the skin to induce external inflammation, to draw away that existing in some deeper contiguous parts. Upon this principle we apply a blister to the skin of the side in cases of inflammation of the lungs; for inflammation of the bowels, we blister the belly; the legs are blistered for that of the cellular substance surrounding the sheaths of the tendons; and for inflammation of the navicular joint, we make the coronet or heel the medium of operations. Besides their use in inflammation, blisters are useful in increasing the activity of the vessels contiguous to the places where they are applied. In cases of strangles, for cxample, a blister is used to induce earlier suppuration. 
Such is the power of blisters, that they will excite the absorbents to greater energy, and by this means they will carry away tumours, and callous, and even bony substances.

In the application of blisters there is some nicety required to determine when a stronger or weaker one must be applied; whether by long-continued, or sudden and violent action, the desired effect is most likely to be produced. In inflammatory cases, strong blisters will be found best; but in old and longseated tumours and swellings, milder means must be adopted.

It is necessary to prepare the skin of the horse for a blister, by clipping off or shaving closely all the hair of the part where it is to be applied, and also washing the skin. The best composition for a blister is Spanish flies, lard, and resin, in the following proportions, viz. :-

\section{Powdered Spanish flies}

Powdered resin . . Lard .
I ounce,

I ounce,

4 ounces.

Mix the lard and resin together and then add the Spanish flies. This should be thoroughly rubbed in, otherwise it will not rise. After the application of a blister the head of the horse must be tied up for at least three days, to prevent him from disfiguring the part with his teeth, as well as injuring his muzzle. If, however, the sides are blistered, the clothes may be so fixed that he will be unable to reach the part. When the blistering ointment has remained on for three days, the part may be rubbed with a little olive oil, which will assist in alleviating the pain, as well as in softening the skin and preventing it from cracking, which it is liable to do, and these become difficult to heal. The oil must be applied morning and evening 
to the part, until the scab falls off. When the scabs show symptoms of peeling, their removal may be facilitated by the application of a lotion of soap and water, applied with a sponge or piece of flannel; but they must not be forced off, as by doing so a blemish will be left.

Some persons recommend the removal of all the litter and straw from the stall of the animal, if blistered on the belly or sides; but we think this unnecessary, as it would be better to put additional clothing, or cover the part with something soft, well secured against being irritated by the straw. It would be as well to keep the horse standing for two days, taking care that he cannot reach the blistered part to blemish his skin, which will be best effected by what is called a cradle. This consists of round strips of wood attached together, extending from the lower jaw to the chest.

For a sweating blister the best application is an infusion of Spanish flies in turpentine, and that should be reduced to the proper strength with neats'-foot oil, according to the degree of excitement required.

If the blister has not the effect of removing the disease, it may be repeated after the scab has been healed and removed from the old part.

It sometimes occurs that absorption of a portion of the flies takes place, which produces strangury, or spasm of the neck of the bladder, and this, upon the principle of counter-irritation, will assist in allaying the more dangerous one. However, this must not be allowed to continue long, as it is of itself a most painful complaint. It will be necessary to supply the horse plentifully with linseed-tea, made in the following proportions, until the complaint is abated, which will be known by the animal staling less frequently; as in inflammation of the bladder or its sphincter, the 
animal stales very often, and that is voided in small quantities :-

Take a pound of linseed a little bruised, and pour on it two gallons of boiling water, and allow it to stand until nearly cold; then pour off the clear mucilaginous fluid. This should be given to the animal in portions of a quart at a time every three hours. Half-a-pound of Epsom salts should be dissolved in a quart of water, and administered. Every six hours the following ball should be given :-

$$
\begin{aligned}
& \text { Opium } \\
& \text { Camphor }
\end{aligned} \cdot \quad \cdot \quad \cdot \quad \cdot \quad \begin{aligned}
& \text { I scruple, } \\
& \text { I drachm; }
\end{aligned}
$$

made into a ball with linseed-meal and treacle.

Mustard blisters have frequently been applied to horses with beneficial results, and produce even a greater degree of swelling than cantharides. Those are made by pouring water on a pound of good mustard powder, according to the dimensions required to be excited. It should be of the consistence of a thick paste. It has been found to relieve inflammation of the kidneys more rapidly and effectually than cantharides. Tincture of croton makes an active liquid blister; and for milder applications hartshorn has been used.

In treating of sprain of the back sinews, page 95, we recommended a blister in certain cases; however, this must never be resorted to while any heat or tenderness remains about the places affected, as this would only increase the superficial inflammation, without allaying that which is more deeply seated, and might produce enlargements of the limb, and finally obstinate ulcerations, and thereby render the horse unfit for work. It is a mistake to suppose that blistering is beneficial in grease, as it rather increases than 
diminishes the complaint. Blistering in cold wintry weather must be done with great caution, because if the part should be exposed to cold it is very likely to end in grease. Blistering all round the limbs is not only a cruel but a dangerous practice, and ought never to be resorted to.

\section{FIRING.}

We cannot contemplate the use of firing without associating with it a degree of cruelty; but in many cases it must be had recourse to as the only method of removing the complaint. The principle on which firing is adopted is by exciting some superficial parts, and thereby, by counter-irritation, relieving deeplyseated inflammatory action. This strong remedy has also the effect of exciting the absorbents to carry off bony matter which is apt to be deposited in the limbs. In short, it is the most powerful stimulating agent with which we are acquainted; but should never be had recourse to, except when there is no chance of milder remedies being effectual.

When it becomes necessary to fire a part, the hair is clipped as close as possible, and afterwards shaved, so that the iron may come into immediate and close contact with the part, as also to prevent the smoke arising from singeing the hair, obscuring from sight the part requiring to be operated upon. In some cases it is absolutely necessary to throw the horse, not only for his own safety, but also for that of the operator. And to prevent the animal from using violent exertions, the hobbles, mentioned at page 283 , must be used.

As this is an operation which ought never to be attempted but by a veterinary surgeon, we shall not descend to the details of how it is performed. The 
operation of firing requires both tact and skill; and even with the best operators, acciclents will occur to the horse, in consequence of his violent struggles, which it is impossible in some instances to prevent, and the skin will be unavoidably cut through with the iron; but the accident cannot be fairly attributed to the surgeon. The skin thus partially divided is sure to separate in two or three days after the operation; but the ulceration which follows will be slight and easily cured, when compared with the skin being actually burnt through with the iron, as this is sure to be followed by violent inflammation, ulceration, and sloughing, producing effects very difficult to cure, besides leaving unsightly blemishes.

It is the practice of some veterinary surgeons to blister after firing. This is a most unjustifiable procedure, unless in cases of large bony tumours, spavin with considerable lameness, and ring-bone investing the entire coronet, and in old and obstinate affections of the round-bone; but in less serious complaints it is a piece of great cruelty.

The day after the operation of firing, the part should be rubbed with lard, or, what is still better, neats'-foot oil. This will give a pliability to the skin, and render it less liable to separate or ulcerate; but if cracks or ulceration take place, then calamine ointment must be applied to it. The composition of this ointment will be found amongst the medicines in the following chapter. A bandage must not be used, as it would prove injurious by irritating the part.

There can be little doubt of the superiority of firing over blistering. The skin being partially destroyed by the iron, is restored, and the surface lessened by its being drawn together, which acts as a kind of bandage, and consequently tightens and binds the whole parts; 
and by adding pressure is of much advantage in reducing hard and callous substances. After firing, it will be found of much advantage to turn out the animal to grass for three or four months.

Firing should always be either in parallel or longitudinal lines, particularly on the coronet, fetlock, and back sinews, for the reason that the skin will contract, so as to form the greatest uniformity of pressure.

\section{NEUROTOMY, OR CUTTING THE NERVE.}

The valuable services of the horse would be in a great degree circumscribed, but for the artificial protection of his hoofs by an iron shoe. Without this his feet would soon be battered to pieces going over our hard roads. But while shoes protect his flexible horny hoofs from injury, they at the same time cramp and confine them, from the inflexible nature of the iron and tight nailing, which, without great care, lays the foundation of many diseases, and those too of a very painful description.

To alleviate to a certain extent the severity of those painful complaints, veterinary surgeons have adopted the practice of cutting the nerve which goes to the foot. This nerve has its origin in the union of several of the spinal nerves, and consequently is a nerve which gives both motion and feeling to the foot. The fibres, however, which are connected with motion are carried only to those parts which are concerned in producing motion, and these are muscles. The influence of the nerves acting upon the muscles cause them to contract, and consequently the limbs are moved. The bones, blood-vessels, and other parts are merely passive agents. The muscles of the leg do not extend below the knee, and the fibres of the nerves concerned in motion are distributed above that joint, 
so that no part concerned in the production of motion extends below the knee; and when the nerve is divided either above the fetlock or on the pastern, not a fibre is touched concerned with motion, but those of feeling alone, and those are continued to the point of the toe. It will be seen therefore that this operation does not at all interfere with motion; but the sensibility or feeling of the foot is taken away, and the poor animal relieved from the torture which diseases of the foot generally cause. By this means the irritation of the foot is relieved, and this in most cases produces an abatement of the inflammation, and the horse will be able to perform work, and have the free use of his foot.

When horses have inflammatory diseases of the foot, they generally keep beating it on the ground, which not only keeps up the inflammation, but even increases it, while they frequently destroy the hoof by this constant battering.

Many persons have supposed that by cutting the nerve, the horse must lose its foot, but it is seldom that sloughing of the hoof results, unless suppuration has been set up in the hoof by a prick with a nail in the act of shoeing the animal. This nerve is connected with sensation alone; those on which the nutrition of the foot depends are the ganglial nerves, which wind round the veins and arteries, even to their most minute branches, and enable them to perform their functions. These cannot be touched in the operation of destroying the nerve of sensation, nor will it in the slightest degree militate against the functions of nutrition.

I have given a representation of the parts connected with the operations of neurotomy, Plate vi, fig. 7 , and described these parts at page $2 \mathrm{I} 6$. It will be 
seen that the nerve is on the inside of the foot, as it approaches the fetlock and passes over the pastern, where it branches off above the fetlock. The prolongation of the nerve below the fetlock is extended principally to the quarters and hinder part of the foot, for the purpose of supplying it with feeling. Before operating for neurotomy, the seat of the disease which causes pain must be ascertained. The artery, vein, and nerve lie close together, and run in the same direction; the vein is next to the fore part of the leg, then the artery, and the nerve behind. Before commencing the operation, the horse is cast and properly secured. Then remove the limb to be operated on from the hobbles. The hair must be shaved from the part. The skin is then cut through with great caution for an inch and a half in length, which will expose the vessels above referred to, and the nerve will be distinguished as occupying the position above pointed out, as well as from its whiteness. Pass a crooked needle with a silk thread under it, raise it a small degree, and then dissect it from the cellular tissue beneath : and when fairly felt, cut out about a quarter of an inch of it, making the first incision above, and the second cut will not be felt by the animal at all. The same must be performed on either side of the foot, as the nerves proceed down both sides. The sides of the wound are now brought together and secured by a bandage. The head must then be tied up for a day or two. In less than a fortnight the horse will be fit for work.

The operation of cutting the nerve may also prove of service for ring-bone, where the side cartilages become ossified, with partial stiffness of the pastern and coffin joints. They will be more liable to recover their usual action in consequence of the animal using 
the foot freely, as he will not feel pain while in motion. Neurotomy may also prove of much advantage in long-continued lameness, where no unusual heat is discoverable, nor contraction or alteration in its form can be perceived; neither will the extremely acute pain caused by the pressure of the horny crust on the sensible parts be longer felt, and the animal will be able to place his foot firmly and fully upon the ground ; all appearance of lameness will be removed, and the ultimate result will be a partial restoration at least of the form and elasticity of the foot.

The above are some of the benefits which will be derived from neurotomy; but there are other cases where cutting the nerve would be attended with unfavourable results. When the pastern or coffin-joints are quite stiff and unyielding, if this operation were performed, the animal would naturally bring his foot to the ground with force, and the joints being divested of their natural elastic play, the bone would be still more injured. In inflammation or ulceration of the cartilages and ligaments, it would be injudicious to cut the nerve, as the animal feeling no pain, the too active use of the foot would hasten the progress of disease by bruising the parts. Neither would it be proper to have recourse to neurotomy in pumiced and convex soles of the foot, because it would be speedily worn out and destroyed by the animal now pressing on it ; whereas before the operation the pain of pressure naturally induced him to bear lightly on it.

From what we have said, we hope all the prejudices against this operation will be removed, and by resorting to it many suffering cart and waggon horses will be freed from suffering, and prove more serviceable to their owners. But by all means let the nerve be cut by an experienced veterinary surgeon. 


\section{SETONS.}

In abscesses, ulcers, and tumours, the use of setons will prove of great service. A seton is a piece of cord or tape passed through the base of such ulcers as have deep sinuses, or between the skin and muscles. This is effected by means of an instrument resembling a needle, and the seton is kept in its place by means of a knot at each end, or tying the ends together. The tape should be moved daily, and wetted with turpentine, spirit of wine, or some acrid liquid, in order to keep up the inflammation and promote the discharge of matter, which is the purpose for which setons are used.

Setons are often useful in increasing a discharge in the places contiguous to inflammation. For example: In inflammation of the eye, a seton in the cheek will act with much advantage, on the principle of counterirritation. In deep-seated inflammations many favourable results have been experienced in the use of setons. For example: In inflammation of the navicular-joint, great advantage has been derived from a seton, although the reverse is the case with frog-setons for all diseases of the foot. But where there is inflammation in the larger organs, we must not expect to reap great advantage, as their power of action is too limited.

Where there are abscesses or tumours in the withers or poll, the seton should be passed entirely through from the bottom to the top, by which the fluid will be discharged and the accumulation of more prevented. They are especially valuable in deep fistulous sores, by giving an outlet to the matter secreted in them, which, if not discharged, would burrow deeper into the parts. 


\section{DOCKING.}

It has been a long-established custom to dock the tails of horses-convenience justifies it and fashion guides it. The length of the stump varies with the taste of the times, or the fancy of the owner. A medium length is undoubtedly the best, and a very short one is not only unseemly, but also a very great injury to the animal, as he is thus deprived of that which nature intended as a switch, and as a substitute for hands to drive off insects.

The operation of docking is performed at different ages of the animal; some consider it best to do it at two years, while others think the earlier the better. From what we have ourselves noticed, we coincide with the latter opinion. At a fortnight it may be done with perfect safety, and even sooner; and it unquestionably affects the foal less at this period than at the age of two years.

The manner of performing the operation is to fix upon a part of the tail, and having determined on that, take the one next joint to it; let the hair be turned up and fastened with tape for an inch or two above the joint, while that which grows upon the vertebra itself must be cut off. The horse is then restrained by the side line, and the surgeon now applies his docking machine, and cuts it through at the division between the vertebræ at one stroke. It is not uncommon for farmers and other breeders to perform the operation with a sharp knife, resting the tail on a board, and striking the back of the knife with a mallet or hammer. Although considerable bleeding is caused by this operation, there is no danger to be apprehended from it. To stop this in the speediest manner is to sere the stump with a red-hot iron, with a hole 
in its centre to prevent it from touching the bone, which would cause exfoliation; or, if severely injured, it would fall off at the joint above, and thus shorten the desired length of the tail. The bleeding vessels are all on the outside of the bone. The iron must not be too hot, nor much pressure applied to it, neither should it be long continued. No kind of dressing is required after this operation, but it should be remembered that while docking is a simple and generally safe operation, many horses have fallen victims to the parasitic disease "Tetanus," immediately or shortly after being docked.

\section{NICKING.}

It is to be lamented that fashion has long held a sway in promoting and maintaining this cruel operation, even in its simplest form. However, of late, public taste has materially improved in this respect, for the fashion at the present day is to have horses with long switch tails, possessing much of that beauty which nature wisely intended by the formation of that organ ; as also, like that of other quadrupeds, to assist him in turning when going at a rapid speed, as well as to defend him from the attacks of numerous insects with which he is constantly annoyed when in the field.

We wish we could pass over the subject of this barbarous operation without noticing how it is performed, but as there are still persons so devoid of taste and feeling as to approve of it, the work would be necessarily imperfect without a description of the operation.

We have given a figure of the skeleton of the horse, Plate II, and referred to it at page I6I. It will be seen by a reference to these that the horse 
has eighteen dorsal vertebræ, or bones of the back or spine, extending from 'figs. I4 to 14 ; besides five lumbar in some subjects, and in others six, fig. I5, there is a continuation of these called the sacrum, which consist of five bones, which are separate in the colt, but which become consolidated in the adult animal. To the sacrum the bones of the upper and side portion of the haunch, or pelvis, are strongly articulated, so much so that they resemble a solid mass rather than a joint. From the lower or outer extremity of the sacrum, the bones of the tail emanate; these are fifteen in number, extending from figs. I6 to 16 , and gradually diminishing in size towards the point. The spinal-marrow extends to the extreme tip, as well as the continuation of the arteries of the sacrum. The setting on of the tail and its particular character and curvature from the loins is much looked to by those who are judges of horses, as from this they discover the purity or impurity of the breed. The line from the setting on of the tail ought to be almost straight, or with a slight inclination downwards, as there is no surer test of a high-bred horse than this character of tail.

The tail has three sets of muscles, the one for raising it, Plate VII, fig. I, $e$, and another immediately below for depressing it, and a third for giving it lateral motion in every direction. When the animal is at rest, the power of the lateral set of muscles seems to predominate, as the tail is constantly inclined downwards, resting upon the buttocks; but when energised the tail is carried higher, which gives that spirited character to him when excited. It was to convey this expression constantly that the operation of nicking was devised; therefore the depressor and part of the lateral muscles are nicked through to a 
greater or lesser degree, as the wish of the operator may suggest to him, as to the height at which he wishes the animal to carry his tail.

The operation is performed in the following manner. The horse is cast, and the hair at the point of the tail firmly tied together, so that a weight may be afterwards attached to it. The tail is then held firmly in the hand and lifted up, and the exact central spot of one of the bones is ascertained, and the muscle is divided deep with a very sharp knife or scalpel from the edge of the tail to the centre, and continuing the incision across the bone of the tail, it is cut as deep as on the other side. All this may be done with one rapid and steady incision without lifting the scalpel. In a high-bred horse this will be sufficient for the purpose; for a hunter two incisions are usually made, the second being about two inches below the first, which should also be as near as possible to the centre of one of the vertebræ. In the hackney it has been the practice to make three cuts, so as to make him carry his tail still higher. Two cuts only are made in the tail of a mare, and the second one is seldom deep. Any of the fibres which may reach across the incision must be cut off with a pair of curved scissors. Each incision must now be filled with pledgets of tow, and inserted deeply into the wounds; and it must be distinctly ascertained whether the muscles are cut uniformly on each side, otherwise the animal will carry its tail awry. They must be secured by a bandage, but it must not be made too tight, as bad consequences are likely to follow where this is done. Indeed, if not loosened in the course of two or three hours, intense inflammation and swelling will ensue, and in all probability death may follow. In about 

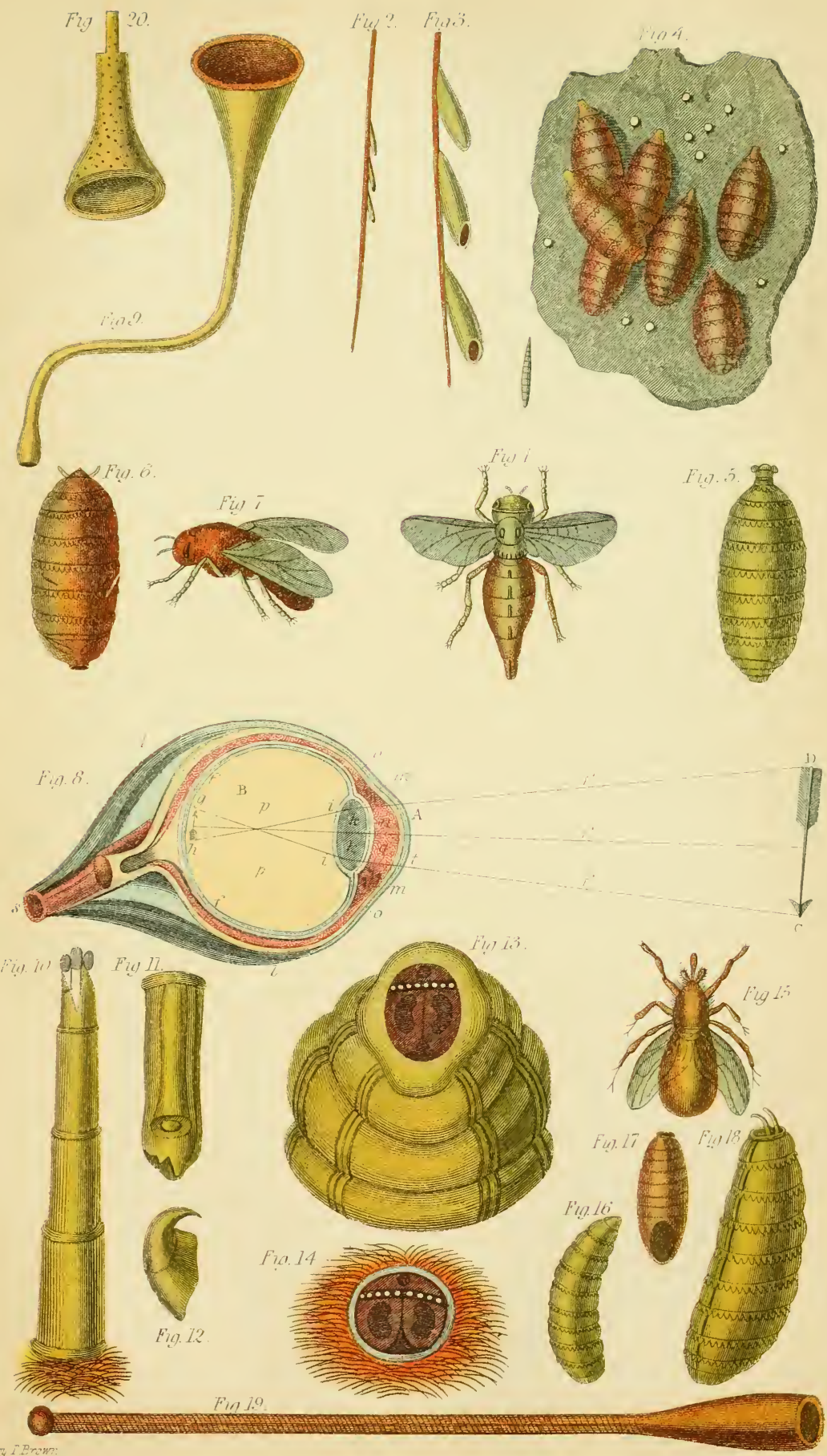

twenty-four hours after the operation, the bandage may be thrown aside and the wounds kept clean, which is all that will be required.

The tail must be afterwards kept bent back for some weeks until the wounds are healed; because if allowed to hang down, the edges of the cuts would come in contact and soon reunite again. This is accomplished by means of a cord from one to two feet in length, which is attached to the hair at the point of the tail, and this is affixed to another divided cord, each division passing over a pulley on either side of the back of the stall. To each extremity a weight is suspended, sufficient to keep the cuts of the tail open to the extent required; but it is an act of cruelty to hang too heavy weights to these, as the smallest quantity beyond what is necessary inflicts great pain on the animal. Some persons do not use pulleys at all, but by keeping a slight girth on the horse, attach a cord to the tail and pull it backwards. However, whether the pulleys or simple cord are made use of, the tail must be relieved from the weight once or twice a day, and the horse exercised. Ten days or a fortnight will be required to complete the operation, as if too soon removed, the desired elevation of the tail may not be effected.

It sometimes happens that great irritation and inflammation take place in nicking, and even lockedjaw has been brought on by it. When the first of these ensue, the weight must be removed from the tail, and the parts carefully fomented with warm water, and gentle purgatives administered. When locked-jaw has taken place, the joints of the tail should be amputated at the first joint above the highest nick. 


\section{CHAP'TER XIII.}

Of Breeding, Feeding, and Training Horses.

\section{SECTION I.-BREEDING.}

THE utmost attention should be paid in the selection of brood-mares, because the progeny depends more upon the dam than the horse, and the size and strength of the foal will bear a considerable similitude to hers. As a proof of this, we have found that those horses that have been the produce of an Arabian stallion and a mare, if she were large and well-formed, have not resembled the horse in their stature. Up to the year I 829, only one Arabian horse had been brought to Scotland, which was in the reign of Alexander I., who, in the year II3I, presented to the Church of $\mathrm{St}$ Andrew's an Arabian horse, furnished with costly trappings ; this is the first that was brought to Great Britain. In I829, Capt. Horne, of the Madras Artillery, introduced a beautiful silver-grey horse, called the Humdanieh Arabian. His height was fourteen hands and a half - a size which the Edinburgh breeders thought too small to be a good breeding stallion, and refused to use him as such. Sir Robert Keith Dick, Bart., however, who had been long in India, was well aware of the fallacy of size being an objection. He offered to keep him at Prestonfield and breed from him. The first colt of his produce turned 
out the largest that Sir Robert had ever bred. It is an established axiom, that in general the produce partakes of the qualities, or the mingled qualities of both parents. Above all things, avoid breeding from either a horse or mare which has any nervous disease, as it is almost certain that the progeny will inherit it ; and like diseases in the human race, if it does not show itself in the first generation, it is more than probable it will break out in the second; so that breeders should always know the history of both sire and dam. And we again repeat, that however perfect the form of the horse, these may be neutralised by a defective mare. Wherever bad points appear in either the male or female of animals the breeder is anxious to avoid, or, on the other hand, keep up, from possessing some good qualities and points, attention should be paid to put an animal of the opposite sex to the other having those points in a more perfect degree; and it cannot be too strongly impressed upon the mind that excellence on the part of the mare is as essential as that of the horse: for it very rarely happens that a good foal is produced by a sorry mare.

We see what has been effected in Yorkshire and other midland counties in breeding the larger-sized cart-horses, by a strict attention to have both sire and dam as perfect as possible in their form. Both parents should be in full possession of their health and strength ; and it is a great mistake to suppose that a mare can be fit for breeding when incapacitated by disease for work. Many suppose that because a mare is rendered lame by accident, that she is nevertheless useful as a brood-mare. This, however, is sometimes found to be a mistake.

Breeding in and in, as it is termed,-that is, forming a constant union of the same blood, is always 
attended with bad results. However good both sire and dam may be, if too closely allied, the progeny will in all probability turn out weak, and become liable to all those diseases to which delicate constitutions are subject; but where parties have a predilection to a particular stock, these evil consequences may be obviated by occasionally introducing fresh blood, either by crossing with the sire or dam.

A good brood-mare should be selected with a rather long carcase, so as to give sufficient room for the growth of the foetus; at the same time she should be compact in the make, and rather short than otherwise in the leg; while the stallion should be somewhat opposite in his form, which ought invariably to be short, with as many of the good points which we have before enumerated contained within a small compass. The inclination of the shoulder is also an essential point to be attended to. A large stallion with upright shoulders never got a good hunter or hackney; but for a draught-horse, this form to a limited extent is desirable.

To secure a good and healthy progeny, youth on the side of both sexes is very essential. Both ought to be in the prime of life, and in full health. From three to four years of age is a proper time to commence breeding from a mare, but to begin sooner (unless the animal is large and well fed), before their form has assumed its full strength and vigour, cannot fail to limit the growth of the mother and her progeny. Mares that are little worked may be safely bred from until they attain the age of eighteen or twenty, and instances have occurred where they produced splendid foals at twenty-seven years. But, on the contrary, if hard worked, there is little chance of the progeny being good. 
The mare comes into heat early in the spring, and generally goes with foal for eleven months, although it not unfrequently happens that they vary, even so much as from a month to six weeks, either earlier or later. From the time which the mare is covered until she foals, she may be moderately worked with safety, and even with advantage to both parent and colt; but we would recommend for a week or two before foaling that she should not be worked, but subjected to regular daily and moderate exercise. The time can be pretty nearly guessed from the time she was covered. About a day before foaling an adhesive matter issues from the teats of the mare, and after this she should on no account be subjected to any sort of work, but allowed to go at large, and constantly watched by some careful person.

After the mare is about half advanced in pregnancy, she should be well fed, and from one to two feeds of oats given to her daily, as a greater quantity of nourishment after this period is required for the support of the fœtus. If the system is allowed to become debilitated from want of a supply of food to meet the increased demand, then there is a probability that her weakness may lead to her proving abortive, to which mares are more liable at this period than either before or afterwards. There can be little doubt that good feeding and moderate exercise will be most conducive to the prevention of dropping a fotus; but after this time hard work must be avoided, and also galloping or hard trotting. Should a mare once abort it is extremely likely to happen afterwards. It is a fact that where two or three mares in foal are on the same pasture, if one aborts, the rest are very liable to the same occurrence. It is therefore evident that as soon as such a circumstance occurs, other mares that may 
be in the same field should be removed to another pasture.

If mares are in good health while in foal, there is little danger of any mischance during parturition; but should any such occur, either as regards a difficulty in parting with the foal or a wrong presentation, recourse must instantly be had to a regular experienced veterinary surgeon, who, from practice, is most likely to afford the necessary relief, and will save the suffering animal pain, and frequently save her life. Many fine mares have been lost, or rendered useless, by unskilful persons attempting to afford relief.

It would be absurd in a limited treatise to attempt laying down rules for enabling persons to perform those duties successfully, which can only be acquired by a lengthened course of study; and which, consequently, would far exceed our limits. Mares generally come in heat about the tenth day after foaling, and thereafter every third week.

Whenever parturition has been accomplished, the mare with her foal should be turned out into a pasture, which ought always to have a shed to retire to for shelter. If this happens to be early in the spring, that is, in April, before the grass has become sufficiently plentiful, then the mare should be supplied with three feeds of oats daily; otherwise she will be unable to supply the foal with a sufficiency of milk, a lack of which is sure to lay the foundation of weakness, and in all probability arrest his growth, as this is the most important time in the life of a foal. In a week or two the foal will be able to take crushed corn along with the mother. Bran and shielings are also very fit food for both mother and foal at this period; but after grass grows more plentifuliy, both kinds of food may be discontinued. 
The foal may be weaned in five or six months, as may be determined from its strength. This is done by gradually withdrawing it from the use of milk, and after lessening the quantity for a week or two, it should be turned into a rick yard, where it can have benefit of both food and shelter during bad weather, and occasional draughts of meal and water given to it. The mare, in the meantime, should be put upon dryer food, so as to lessen the secretion of milk, and a little harder work will have a tendency to give a different action to the fluids, and thus diminish the supply. Should it, however, prove troublesome, a physic ball and one or two diuretic balls will be found beneficial in that case.

After the foal has been separated from the dam, he should be well fed until he has attained his full growth. During the earlier stages, bruised oats, bruised beans and pease, and also bran should form a part of his daily food. This applies to all kinds of colts, whether racers, hunters, draught, or saddle kinds. During the time foals are sucking, the greatest caution is necessary not to overheat the dams, as this is sure to affect the milk, and proves most injurious to the colt.

To save the heavy expense of corn-feeding in young horses, clover, lucerne, and tares, are substituted ; carrots have also been successfully used. But no species of food is so nourishing as pease, half a feed of which is about equal to a feed of oats. Young horses should invariably be plentifully fed; and a want of attention to this has been the cause of many of them acquiring the practice of biting their racks, and thence called crib-biters. To prevent this practice, a little hay or straw should be constantly kept in the rack. 


\section{SECTION II.-FEEDING.}

Hay and oats being the chief food of horses, it is highly necessary that the owner should be, at least, a tolerable judge of their quality. The best kind of hay for horses is that which grows on upland meadows. It should be bright in its appearance, of a greenish cast, possessing a fragrant smell, and not too dry; for when it is so, and crackles when squeezed in the hand, it is a sure sign that its nutritive qualities have been dissipated by too long exposure to the sun and air after it has been cut. It would, however, be proper occasionally to vary the quality of hay by giving white clover and also rye-grass in limited quantities. Care must be taken that it is not too new, as in that state it is apt to produce acidity and flatulence. Twelve pounds of hay is sufficient for any ordinary-sized horse per day, with eight pounds of oats and two of beans. The cart or agricultural horse will require about twelve pounds of oats, with two of beans, added to eighteen pounds of chaff or hay, but where the hours are long and the loads heavy, a larger quantity of grain will be required to keep the animal in fair condition. The horse having consumed the above quantity of food, requires none during the night, and it would be proper to keep his rack without hay, but where the animal has long hours of work during the day he must have a quantity of long hay to eat during the night.

Some horses which are greedy feeders swallow their pease and oats without being properly chewed, and much of both pass through the stomach and intestines without undergoing much change; indeed, this is the case to a certain extent with all horses; 
the consequence is, that the animal is deprived of their nutritive qualities. Horses which do not chew their food, can easily be detected by examining their dung, when it will be found to contain much grain in its perfect condition. When this is the case, the grain and pulse should be bruised, and also mixed with a portion of chaff, or cut hay and straw, which he cannot swallow without chewing. Besides deriving all the benefit of the nutritive qualities of the food, the animal is prevented from bolting his food too quickly and overloading his stomach, and rendering him unfit for being used immediately after feeding, as we have already explained when treating of the stomach, page 243. Slow feeding is of much importance, because in the lengthened process a greater portion of saliva is carried into the stomach with the food, which materially assists in the process of digestion.

Machines have been constructed for cutting hay into chaff. Meadow hay, clover, wheat, barley, and oat straw are cut into pieces of a little more than half an inch in length, and the whole well incorporated, and the proportional quantity of bruised oats and beans added, and measured out at meal-times to the animal. If the chaff is slightly wetted immediately before feeding; the horse is enabled easier to chew it. With some horses the bruised grain produces scouring; when this is the case, it must of course be given whole, but this very rarely happens with bruised grain when mixed with chaff, as we have above recommended. Horses that are driven rapidly in harness are more liable to be purged with bruised grain than those of slow draught; and it has been found that diminishing the proportion of straw-chaff, and increasing the quantity of hay in the proportion 
of two trusses of hay to one of straw, has had the effect of obviating this. For horses which are used as hunters, the quantity of oats must be materially increased, as affording a substantial meal of considerably less bulk, and consequently does not distend the stomach so as to press upon the lungs, and thereby impede the process of breathing, as it will be seen by an examination of our figures, I, 2, and 3 of Plate IX, that these organs lie close to each other. It must be understood that the same applies to the race-horse as well as the hunter.

Horses which are accustomed to bruised grain and chaff, become so fond of it that they prefer it to the oats or beans alone. We cannot too strongly impress upon the owners of horses the danger of feeding them on either grain or hay which has become musty, as either are almost certain to induce disease; and although no particular complaint is manifested, the horse is sure to fall off in condition. One great advantage in manger-feeding is that the horse when returning from a fatiguing journey or hard gallop, is sooner enabled to fill his stomach and lie down. If the rack is used, it takes from two to three hours to eat his fill, whereas a little more than an hour will suffice with bruised grain and chaff, which gives him so much more additional time to rest, which is of vast importance to such horses as are used in mail and stage-coaches.

When small farmers or others unfortunately possess musty grain, the smell will to a considerable extent be removed by kiln-drying it; but in this process great care must be taken not to overheat it, which renders it unpalatable to the horse, and more heating in its nature, producing an increased discharge of urine as well as diseases of the skin, and is apt to induce 
inflammation of the eyelids and of the ball of the eye.

Horses that have been fed all winter on dry food will receive much benefit by being turned out in the spring, when the grass has become luxuriant ; or when this is inconvenient, cut grass may be given to him in the stable. The use of green tares will also be found very beneficial. Green food is certain to purge the animal, and that in a moderate degree, which carries off all the evil effects of constant feeding on hard meat.

Wheat is too expensive to be used as food for horses in Great Britain, and it is only when damaged that farmers think of applying it for this purpose. Although wheat contains a large portion of nutrition, it is not by any means desirable as food for a horse, as it has a tendency to form obstructions in the bowels by becoming caked ; and besides, it is difficult of digestion. This has been found to be especially the case when they are watered soon after feeding; for the water, flowing quickly through the stomach and small intestines in its passage to the cæcum, or blind-gut, carries off with it all the gelatinous substance of the grain, and leaves the more indissoluble mass behind, and the masses thereby formed have often caused the death of the horse. A small portion of hay should be given along with wheat.

Beans are an excellent and nutritious food, but should never be given whole when dried, as their skin being so very hard the horse swallows many of them whole, or drops others.

Pease are still more nutritive than beans; but should never be given unbruised, being difficult to grind by the horse, in consequence of their round form. They are frequently swallowed quite whole by the 
horse, and when an undue quantity has been given, the most injurious consequences have happened to the animals, especially if much water is given soon after being fed with them.

On the Continent, barley is a common food of the horse. It contains more nourishment than oats; yet it does not seem so well adapted for the food of the horse as oats, except where the animals are hard worked. It has been found that horses fed on barley are more liable to be affected with complaints of an inflammatory character, and likewise to surfeit and other skin diseases. In Britain, farmers have been known to feed their horses on unsound and unsaleable barley, which in many instances has produced serious maladies. In the form of mashes, barley is found to be advantageous, from its stimulating properties; but we certainly do not approve of it as a general food.

Tares are valuable in their green state in the early part of summer, as they possess medicinal properties, as well as being very nutritive. In the event of horses having surfeit-lumps, green tares to the extent of ten or twelve pounds daily, cut up and mixed with the chaff, will be found to remove them very quickly.

It has been satisfactorily proved that tares have not the effect of producing a rough coat, although this opinion still exists with many persons.

Carrots form an excellent food for the horse when mixed with the chaff in the proportion of half-a-bushel a day, and excluding the oats and beans. With this quantity, or not exceeding three quarters of a bushel, horses will stand any quantity of work.

Swedish turnips are a tolerably nutritive food, and very easy of digestion. Twenty pounds of sliced turnip, to twelve pounds of bruised oats and six pounds of straw, forms an excellent meal for a horse. 
Nothing contributes more to the health of a horse than feeding regularly, and at fixed intervals. As his digestion is very rapid, he should be regularly fed. When kept long without food, the horse is apt to take his meal too rapidly, and by distending the stomach produce tympanitis and rupture.

That the food may be well ground down to prepare it for digestion, it must be previously moistened. The horse is fed in a stable on dry food. In the vicinity of the mouth are placed the parotid and submaxillary salivary glands, destined to secrete abundantly a limpid fluid of a saltish taste. This fluid (estimated to be secreted at the rate of eighty-four pounds in twenty-four hours) is conducted into the mouth by various ducts while the animal is chewing his food; and being mixed with it, assists in making it more easily ground and conducted into the stomach, and better prepared for digestion.

Sometimes horses are afflicted with inflammation of the stomach from having eaten poisonous plants, although this is of rare occurrence.

The deleterious plants of this country which affect the horse are but few. The common hemlock, and water-hemlock, which prove a deadly poison to many other animals, are eaten with impunity by the horse.

Water-parsley, if not eaten in too large quantities, will not act as a poison ; but if much is consumed, will produce palsy.

Water-dropwort is poisonous, but is generally rejected by horses; although instances have occurred of mares in foal having died from eating it.

But of all the vegetable poisons, yew is the most to be guarded against, as horses are very apt to eat its leaves. When eaten it produces great sleepiness, from which it is hardly possible to rouse the animal; 
and that is only for short intervals, for he soon relapses into a torpid state, and dies without manifesting pain. The remedy to be tried is the following:-Give ten grains of the farina of the croton-nut whenever it is known the horse has eaten yew-leaves; then a drink of half-a-pint of vinegar, mixed with a pint of gruel. Repeat the croton every six hours until it operates.

\section{OF DRINK.}

Nothing is of greater importance than the quantity of water which is given to a horse as drink. Accustomed for the most part to dry food, water is indispensable to nourishment and health. Its quality, too, is of vast importance. Mr Lawrence, in his treatise entitled "The Horse," makes the following remarks, which convey all we can say upon this point-

"It has frequently been observed, and not easily accounted for, that horses do not thrive on changing from one part of the country to another, although their treatment in every respect be the same, difference of water excepted. This perhaps may, in a great measure, be owing to the quality of the water they drink, and which may be possessed of different chemical properties from that to which they had before been accustomed.

"This is particularly observed in those places where the stable-yards are supplied from pit-wells, some of which are very deep, and the water very hard, which occasions that chilliness, trembling, and shaking which is frequently observed in horses when they drink it immediately after it is new pumped, and which causes their coats to stare and stand on end for a considerable time, and sometimes they are griped, and much out of order. Spring-water is liable to partake of all the metallic or mineral properties of the strata through which it passes; hence it becomes noxious or salutary 
according to the nature of those substances with which it has been in contact. River-water has, likewise, its different qualities, from the various soils through which it travels; but, in general, it is much softer than water that runs underground. Pond-water (under which head may be included all stagnant water, which generally proceeds from rain), if lying on a clear and clayey bottom, and fresh, answers well for cattle of all kinds; but in warm weather it is apt to corrupt and ferment, which renders it unwholesome and unfit for use."

To correct the hardness of pit-water, and render it more salutary for horses to drink, it should be pumped into a large trough, and exposed to the open air for some time before it is used; or if a cart-load or two of clay or chalk be thrown into the well, it will greatly improve the water. It has likewise been found that breaking down a piece of clay, about the size of an apple, in a pailful of hard water, before it is given to a horse to drink, morning and evening, has produced a considerable change in their coats.

Indeed, it will be found where horses are obliged to drink hard water, they are for the most part roughhaired, and at the same time have a great deal of dusty matter at the roots of their coats, even though they are well curried and brushed every day; from which we infer this is occasioned by the bad quality of the water they drink.

In cases where stagnant water can only be procured in summer, unslacked lime will materially improve it; but ground charcoal will render even the most impure water sweet and wholesome.

When horses are warm, they should never be allowed more than a few mouthfuls of water; neither should they be permitted to drink too freely while on 
a journey, or while they are subjected to any active employment. But when they have cooled, two or three quarts may be given to them, and after that their feed. Before finishing their corn, two or three quarts more may be offered them.

If horses refuse their food after travelling, it is a bad sign of them, as a healthy and vigorous animal will always feed well after he is properly cooled down and has had a drink; and when horses do refuse their food on a journey, they ought not to be again made to travel that day, or at least for some considerable time afterwards, and not until they have taken their feed.

Horses will invariably, if left to themselves, prefer soft to hard water, and when cool may be allowed to drink their fill, and no evil will result therefrom. Instinct or experience has taught them this; they will leave crystalline hard water, and resort to soft, however turbid it may be.

Horses kept in the stable should be watered in summer at least three times a day; and if this salutary advice be attended to, many of the diseases to which they are liable will be prevented. Horses subjected to hard labour require a great deal of drink to supply that moisture which is thrown off by perspiration, and the poor animals but too frequently suffer much from a want of due attention to this. Let any one observe how eagerly a horse plunges his muzzle into a pail of water, and with what difficulty he can be removed from it while a drop remains, and he will be able to judge of the thirst which he must have suffered. If they are allowed a moderate quantity of water while warm, they must not be permitted to stand still for some time afterwards, otherwise very bad consequences may follow; and nothing is so likely irreparably to 
injure the wind of a horse, as to gallop him immediately after drinking; but a litt'e, taken at intervals, will not harm him. If horses are allowed to drink freely when overheated, and remain quiet, violent spasms, inflanmation, and sudden death is likely to ensue.

\section{SECTION III.-TRAINING.}

Training should be commenced as soon as the colt is taken from the mare, and, as before hinted at, he should be placed under the care of a man of a mild and gentle disposition. Kind treatment and caresses are the only sure methods to obtain obedience, attachment, and confidence in man. This maxim should be applied to horses even of the most stubborn temper, for assuredly if gentle measures will not render them obedient, harsh treatment never will. In short, most of the vices in horses may be traced to their being early entrusted to the care of persons of brutal dispositions, who destroy their temper by cruelty and injudicious severity. Others again are taught all manner of tricks for the gratification of idle folly. Tractability, steadiness, and good temper, are the qualities for which a horse is chiefly valuable to man; so that the utmost attention should be paid by breeders to points on which depend so much of the safety and comfort of those who may become their owners. Indeed, gentle treatment has been more effectual in taming wild animals than any other.

Much difference of opinion prevails as to the time which horses ought to be worked. My unalterable belief is, that at three years is the earliest period at which a horse should be used either for the turf, hunting, the road, or harness ; consequently breakingin should not be completed until after the second 
winter, although, of course, it ought to be gone about gradually from his weaning. A bit should be selected with a plain snaffle, that will not hurt his mouth, and it ought to be of a small size, in the centre of which should be attached a stabbering-bit, which, resting gently upon the tongue, excites the horse to move his jaws, and prevents him from pressing too heavily upon it, as this would deaden and harden the mouth, which is one of the worst faults a horse can have. $\mathrm{He}$ should be accustomed to the use of it in his mouth for some days before the hand of the trainer is applied to it. He ought then to be led out, and gently checked by it when he is getting too playful, until by degrees he will bear its control without manifesting irritation.

After the colt has thus been partially broken-in, the next thing to teach him is implicit obedience to his instructor. This should be effected by steadiness and firmness, while severity should be carefully avoided. He should be spoken to in a soothing, rather than an angry tone of voice. He must be taught to know the effect of the whip and spur, but their uses must be administered with much caution; and only showing him that we have the power of enforcing submission.

If a young horse refuses to allow the bit to be placed in his mouth, it must not be attempted to accomplish it by force, because this will only redouble the resistance. Coaxing and gentle trials day after day will be the quickest means of accomplishing the object. When it has been effected, then kindness should be shown him, and caresses used. A headstall is now put on him, and a cavesson is then affixed to it, with long reins. The cavesson is an apparatus intended to confine and pinch the nose; but this being a powerful and severe instrument, it should be used gently. Instances have been known of the bones and 
gristle of the nose becoming diseased from harshly pulling the cavesson. He must first be taught to obey the action of the rein, and after he has become obedient to it, he must next be led round a ring on soft ground. To the cavesson is attached a small rope or cord, which is held by the breaker, and the colt is first led round the circle at a walk. When he has acquired his paces, and become obedient to this action, he should then be trotted round the circle, but at an easy rate, and only for a short time at once. When stopped, he should be caressed. He should be accustomed to go both to the right and left. If any circumstance occurs which may frighten a young horse, and he refuses to proceed in consequence, another horse ought to be led on before him, and he is almost certain to follow. At first the circles should be large, and gradually diminished; because small ones are apt to produce giddiness and too much fatigue at first. In performing those revolutions, he should be frequently stopped by the trainer, and pulled up to him gently, to show him that no injury is intended, and he ought to be caressed at the same time. The cord should be long and loose; his paces all regular and correct, and if he gets false in these, he should be at once stopped, and recommenced. Should he become restive or frolicsome, let the person who holds the whip crack it to show him he is there, but upon no account should he touch the horse with it; or if he stands still and plunges or rears, the whip should be cracked, and only ought to be applied gently to him, when he absolutely refuses to proceed. When the trainer changes the direction of the colt, he should invariably be stopped, and each time be enticed to approach, which will have the effect of accustoming him to have confidence in his attendant or groom, always 
caressing him when obedient; and whatever gait he is performing, on no account allow him to depart from it, as it is only by strict attention to the action wanted that he will learn to be correct in his paces. If he happens to hold his head too low, shake the cavesson, to remind him to raise it; but be sure always to adopt one mode of directing his attention to any particular thing required. All his lessons should be short, the pace should be kept distinct and perfect in each, and he should be rewarded for attention and obedience by handfuls of corn and caresses. When the colt becomes tractable and obedient in all his lessons, crupper-straps, or something similar, should be attached to his clothing to accustom him to it, that he may not be afterwards tickled and become restive by the rider's coat-tails. A few days will suffice to make him endure this patiently, because when he finds that he suffers no harm from them, he soon becomes reconciled to them.

The regular riding-bit should now be applied to his mouth. It ought to be large and smooth, to which should be attached the reins, buckled to a ring on either side of the pad. The reins ought to be flat and allowed to be slack, and gradually tightened. The trainer should occasionally stand in front of the animal, and take hold of each side-rein near to the mouth, gently press upon it, and thus begin to teach him to back and stop by the pressure of the rein, always rewarding obedience, but gently punishing him by a slight jerk when obstinate.

The colt should now be taken to the street, or road, and led about, to accustom him to meet carts and other objects without starting and shying; but if he does start or shy, he should not be allowed to pass on, but ought to be quietly led up to the object of his 
fear, and shown that it will not harm him. But on no account should he be beaten on such occasions. And should he be still shy, let him be taken past the object of his fear, first at a greater distance, and then nearer, until he may be quietly led close to it. It is only by patience on the part of the breaker that these difficulties are got the better of; whereas if the animal is forcibly and suddenly taken up to the object before the fear has subsided, a habit may be established, which will never afterwards be eradicated. Nothing can be more absurd than to beat even an adult and thoroughtrained horse for shying. This is certain to establish rather than remove the fault. The same system should be adopted with an aged horse, as recommended for the colt, and this I have found effectual in every case. The best horse is liable occasionally to shy at a white post, or other object which he may suddenly come upon, especially in the evening or in the dark; but on no account should the rider proceed on his journey without quietly and deliberately leading up his horse to the object, and allowing him to see what it is.

When the colt has been inured to walking on a road or street, and will pass any object that may be presented without shying, the breaker should then walk by his side, throw his right arm over his back, while he holds the reins in his left. The breaker must invariably walk by the left side of the animal, so that he may be thoroughly accustomed to permit him to approach on that side, which is the one universally adopted for mounting. The pace should now be occasionally quickened, and at the same time the colt should be gently tapped on the right side with the whip, which ought always to be held in the right hand. This being repeated at each time the pace is quickened, will 
familiarise the animal to it, and will associate in his mind the increased action required by such a signal. Horses in general have excellent memories, and seldom forget what they are taught. If, however, the colt does not attend to the gentle tap, a sharper one may be applied, and the feeling of pain exerted as a monitor to increased action. These lessons must be repeated until the animal is reduced to perfect obedience.

The next thing to be attended to is to apply the saddle, which should be put on his back with great caution. The breaker should place himself at the head of the colt, and by caresses and patting divert his attention. Let one assistant on the off side put the saddle gently on his back, while another on the near side gets hold of the girths, and slowly tightens them. They ought to be but loosely drawn at first, only to such an extent as to prevent the saddle from turning round. If a crupper is used, it ought to be sufficiently long to prevent it from galling the tail. He should then be led about with the saddle on his back for at least a couple of days, the trainer occasionally leaning his arm as heavily as possible. The girths during this time must be gradually tightened, until that firmness is acquired which is necessary when a man is mounted on his back. If the previous process of breaking has been effectually accomplished, he will generally submit to all this quietly, if done with caution. On or about the third day the trainer must then attempt to mount. At first two assistants will be absolutely necessary. His first business will be to remain at the head of the animal, patting and caressing him, while the person who intends to mount must first pull the left or near stirrup pretty heavily with both hands, while the man 
on the off side presses equally on the other stirrup; and after having repeated this several times, the person on the near side must put his left foot into the stirrup, and gradually apply pressure to it, the man on the off side pressing on the other stirrupleather as before, until the colt will endure the whole weight of the rider, mounted, and leaning his hands upon the saddle; and if the animal proves very refractory, no further attempt must be made at that time. If this is in the morning, the same course may be pursued in the evening, and by repeating twice a day, it may be fairly mounted in about two days. During this operation, a handful of corn should be occasionally given to the colt.

After the colt has been fairly accustomed to the rider balancing himself in the stirrup, and has become docile under it, the rider may gently throw his right leg over the saddle, and quietly seat himself, taking care that he has a firm hold of the reins in case the horse should plunge and attempt to throw him off. But if he submits to it with little resistance, the breaker will then gently and slowly lead him round the ring, while he is followed by a man with a whip, as in the early part of his training-the rider sits quite still. $\mathrm{He}$ will then endeavour to direct the horse round the ring by means of the reins, which must be done by as gentle pressure as possible, while he frequently pats the animal on the neck and encourages him to proceed. When he intends to dismount, it must be done slowly and with much caution, and the colt should be given some corn or green meat to encourage him to obedience. Mounting and dismounting should now be frequently practised for a day or two, but he ought not to be much exercised during this operation. When 
he has become quite reconciled to this, the rider must now apply pressure with his legs, and also a gentle touch of the heels when he desires to quicken his pace, which will finish the process of training.

All this accomplished, rewards must be gradually withdrawn, and obedience instilled by gentle and kind treatment, which in most instances is all that is required. But should the colt at any time become obstinate and resist the commands of his rider, the whip and the spur must be applied to enforce obedience. These means, however, should be very sparingly used, for whenever he finds that he must yield to the power of his rider, he will seldom attempt to disobey him; but on a recurrence of restiveness, soothing should first be attempted to restrain him, which in most cases will have the effect; firmness and gentleness are more likely to prove effectual than cruelty and harshness. Few horses are naturally vicious.

The above is applicable to a horse that is to be used for riding, we come now to those means best adapted for fitting him to endure harness and the draught. At first, portions of the harness should only be placed upon him, and then blind-winkers, and in a few days the whole trappings. He should then be put into the shafts of an empty cart or waggon; and the better to teach him to draw, another horse may be placed before, and there is little danger but he will soon be taught to follow, gentle patting alone being used to encourage him. He should then be tried alone, and if he works, then a little weight may be added, and increased by degrees, until he will draw a full load. The horses used in agriculture will frequently be required for 
riding, and if they have not been regularly brokenin, as we have directed, before putting them in harness, their feeder should be put on their backs while they are in the team, and it is seldom they will resist his continuing to ride.

We would caution all those who ride horses occasionally used in harness, to keep a firm bridlehand, that is, to feel the mouth constantly; because they are accustomed to depend for support on the wheel-carriage, and thus have a tendency to lean forward, and hence are very apt to stumble and come down upon the road.

We shall not attempt to give directions for training the higher bred blood-horses for carriages and the turf. This can only be effectually accomplished by persons whose entire profession it is. To those unaccustomed to it, the attempt is at best a hazardous undertaking. 


\section{CHAPTER XV.}

\section{Of Stabling, Exercise, Clothing, Etc.}

\section{THE STABLE.}

A PROPERLy constructed and well-regulated stable is of the utmost importance. All proprietors of horses ought to give much attention to this subject, as for want of a thorough acquaintance with, and care as regards it, many of the diseases incidental to horses have their origin. A stable ought to be built in a dry situation, roomy, high in the roof, and well aired, without, however, having a great draught passing through it; but when the horses are out, the stable. doors and windows should be thrown wide open, and allowed to remain so as long as they are abroad. But nothing is worse than to allow the wind to blow directly upon the horse, or a cross-draught of any kind. Grooms, however, are too prone to go to the other extreme, and in general allow the stable to become too warm, and block up with the utmost care every place where air is admitted. Some practice this from an erroneous opinion that they should be kept very warm, while too many do so that the animals may have a fine shining coat. By this injudicious practice, the air becomes contaminated with the unwholesome vapour generated from the litter and urine, which produces a strong exhalation of ammoniacal gas. This being breathed for a length 
of time, has a pernicious effect upon the lungs of the horses; digestion is impaired, and all the vital functions injured. Inflammation of the eyes, chronic cough, with a host of concomitant ailments, are the consequences of inhaling this deteriorated and semipoisonous vapour. Let any person enter a stable in the morning which is not properly ventilated, and he will be sensibly alive to the pungent smell, and even pain in the eyes, produced by the vitiated atmosphere. The chemical action of the urine commences soon after it is voided. It is from this cause that horses are but too frequently attacked with distempers in the spring of the year, or in autumn. This is, however, seldom the case in small well-regulated stables. In short, I have gone into many stables where their heat induced copious perspiration in a few minutes, This must have a strong effect upon the skin of the horse, and especially if his clothing is on; and when strapped and suddenly taken to the open air thus overheated, induces coughs and other diseases in the mucous membrane, as well as inflammation in the kidneys and lungs; and should the weather be cold, rheumatism and stiffness in the joints, because horses are frequently allowed to stand for some time before they are put in motion, and are in consequence chilled. It also too frequently happens that carriage or cart-horses are allowed to stand an hour or more in harness after they have been overheated by severe exercise or labour. This is the reason why such horses seldom live to be aged, and too frequently die in the prime of life. The horses of stage and hackney coaches seldom live beyond the age of eight or nine years, and this is chiefly owing to the carelessness of their drivers and grooms; whereas horses have been known to acquire 
a very great age when they are looked after with that care which so valuable and useful an animal requires. A remarkable example of this occurred in Warrington, where a horse attained the extraordinary age of seventy-six years, and was well known by the name of Old Billy. As far as I have been able to learn, this was the oldest horse which ever lived, and may hence be considered the Parr among horses. He belonged to the Mersey and Irwell Navigation Company, and more than half his life has been spent in towing boats. The Company, for many of his last years, on account of his great age, kept him without working. In summer he grazed on the luxuriant pasture on the banks of the Mersey, and in winter was taken into stable and fed on mashes and soft food. When he died, the Company had his head preserved, the skin stuffed, and the cranium cleaned, and presented it to the Museum of the Manchester Natural History Society, where it is still to be seen.

Few people are aware that after a horse has been worked hard or galloped, that his return to a hot stable is nearly as dangerous as subjecting him to a cold atmosphere from a warm stable. Many a horse has been seized with inflammation and fever after having been worked and returned to a hot stable, filled with the noxious gas above alluded to, and more especially if he was cold at the time. Nothing is worse than the sudden change from one temperature to another. From this thousands of horses yearly meet their death.

Stables should never be built longer than with accommodation for five or six horses, as repose after working is of vital importance; and where there are many together, it is more than probable that some 
will be awake while the others are asleep, and disturb them.

The dimensions of a stable, in proportion to the number of horses, is a most important point. A stable for six horses should be from thirty-eight to forty feet in length, from thirteen to fifteen feet wide, and about twelve feet in height. It is always of consequence to have the roof of a stable plastered, whether there is a hay loft above it or not. This will prevent currents of air from passing through the floor There should always be a few central tiles to allow the hot air to escape and give place to that which is pure and cold. These tiles should be furnished with protecting ledges, to prevent the rain from entering; or, what is perhaps better, large tubes should be carried through the roof, with caps a little way above them, to prevent the rain from beating in. A third plan is to have gratings placed high in the walls. These last ought to be as near the roof as possible, and shut and opened by a cover, as occasion requires.

In summer and autumn the stable ought never to be more than a few degrees warmer than the atmosphere. In winter not more than fifteen degrees, because the hair is thicker at this season than in summer.

If the hay is kept in a loft over the stable, there should be no holes over the racks for throwing down the hay, as by these openings foul air will be carried up to the hay, and render it unwholesome. And besides, it not unfrequently happens that seeds fall down into the eyes of the horse, and occasion serious inflammation in them.

While the floor of the stalls should be so constructed that the urine will be speedily carried off, yet a little reflection will show that the practice of making 
them gradually slope from the stall to the outside is very prejudicial to the horse, and too frequently the cause of lameness by straining the back sinews. $\mathrm{Mr}$ Lawrence justly remarks, that "If the reader will stand for a few minutes with his toes higher than his heels, the pain he will feel in the calves of his legs will soon convince him of the truth of this remark. Hence, when a horse is not eating, he always endeavours to find his level, either by standing across the stall, or else as far back as his halter will permit, so that his hind legs may meet the ascent of the other side of the channel."

This sloping direction of the floor of the stall is also a frequent cause of contraction of the heels, by throwing too great a proportion of the weight upon the toes of the foot, and removing that pressure which tends most to keep the heels open. It must therefore be evident that the floor should slant no more than is absolutely necessary to drain off the urine sufficiently quick to prevent chemical action taking place. This is the kind of stall most suitable for mares, but for horses it should be constructed with a grating in the centre, and an inclination of the floor on every side towards the middle. This should be carried off to the outside by means of a small drain and lodged in a reservoir, as urine is a valuable acquisition to the farmer. To prevent an offensive smell or current of air passing through the drains, cheap traps have been invented to stop the grating. I have lately seen stables constructed with the patent wooden pavement, which answers many good purposes; it is much warmer for the feet, and at the same time softer, and entirely free from noise.

Some persons imagine that the horse should not stand on litter during the day, because the heat which 
it produces may prove injurious to the hoof of the horse. I imagine that little injury will result from standing all day on litter, providing it be dry and not so deep as entirely to cover the hoof. It is quite certain that standing on litter must be much more comfortable to the horse than on cold, hard stones, and we therefore recommend its adoption.

Farmers are in the practice of using the haum of peas, beans, and potatoes, as well as heath. But we would have them to recollect that they must be much oftener changed than straw, as they soon begin to ferment, and consequently the gas emanating from them is noxious; and besides, the heated litter proves injurious to the feet. We cannot too strongly impress upon farmers and others the great impropriety of allowing an accumulation of litter in their stalls and stables. Some are in the practice of nightly heaping fresh straw or other materials over that of the preceding day, instead of having it removed. It is sure to be hurtful to the animal from the reasons we have above stated.

Before closing this subject, we must state our entire disapprobation of double-headed stables, that is, having a range of stalls along each wall, with the rear of the horses standing towards each other. It too frequently happens in inns that from want of room these stables are so narrow that the hind legs of horses are too near each other, and serious injury is often done by kicking. Many fine horses have been rendered lame for life, and even have had their legs broken, from the kicks of quarrelsome horses. If it is absolutely necessary that such a construction must be adopted, then there ought to be at least a free passage of about eight feet. Every stall should be at least six feet wide, and ought always to be divided by 
a boarded partition as high as the back of the horse.

\section{MANAGEMENT OF THE FEET.}

The feet of a horse should be examined with great care every morning, for the purpose of ascertaining, in the first place, if the shoes are all firm, and that none of the clenches are raised, which might wound the limbs. A worn shoe is also liable to press on the sole or heel, and prove injurious.

One of the first things to be attended to after a horse has come off a journey, or has ceased from his day's labour, is that his heels should be thoroughly brushed out. If an agricultural or cart-horse, it will be better to apply the hand than washing, as the long hair with which the heels are generally invested will take a considerable time to dry, especially during winter, which may occasion grease. The feet should then be stopped, after the picker has been used to remove all stones or clay between the hoofs and shoes. With other horses, the feet may be washed with a brush. The shoes should be removed at least once a month.

\section{LIGHT.}

It is surprising that in many parts of the country, farmers' stables, and indeed those of other individuals, have no other light than probably what is admitted by a few panes of glass over the door; or some have an open window closed by a shutter, which is only occasionally opened. To this cause may be attributed many of the diseases to which the eyes of horses are liable, and ultimately blindness itself. It is easy to imagine what the animal must feel, and the consequences which are likely to be the result, from our own 
painful and giddy sensations on issuing from a dark place to the full blaze of the noonday sun, or even when a candle is introduced, after sitting for some time in a dark room. A repetition of this several times during the day would inevitably be attended with most injurious consequences, from the sudden shock it gives to the optic nerve, and the vessels of the retina. Besides, it may produce inflammation in the coating of the eye, which may end in the total destruction of the organ. There can be little doubt but horses that are liable to start at objects, frequently owe this dangerous quality to the cause above alluded to.

A stable ought to be as well lighted as a house, so that this source of injury may be avoided, and also on account of all parts of it being properly seen, so that masters may be enabled to detect a want of due attention to cleanliness, a subject which we cannot too often impress upon masters and servants.

In stables which have a due quantity of light, the shutters may be partially closed when the animals have fed properly, and lie down to sleep. Many horses stand too long upon their limbs, and therefore this subdued light is more apt to produce drowsiness, and thus incline him to repose, during which time the food is well-known to have the most salutary effect in increasing the muscular fibre, and the cellular and adipose substances.

While we have shown the utility of a well-lighted stable, we would guard our readers against allowing that part of the wall next the head of the horse being too light; because the refraction of the rays of light constantly beaming into the eye will stimulate the nerve too much, and is apt to produce exhaustion of energy in the optic nerve and retina. If the stable is 
well provided with windows, the walls should be painted of some subdued tone of colour; and when otherwise, white should be used.

\section{EXERCISE.}

This with the horse, as with man himself, is of paramount importance for the preservation of health. A horse kept in a stable ought to be exercised for two hours regularly each day, without which he will never be fit for work, as he will suffer more from absolute idleness than hard labour. The quantity of exercise should be regulated according to the age of the horse. A young horse requires more than an old one; but violent exercise must be carefully guarded against, especially with young horses, which, although prone to activity, must not be indulged too freely. The beginning and termination should be moderate, and in the middle he may be trotted smartly, or galloped for a short distance. When horses are of full habit, the exercise should be of medium quantity, and increased a little daily; but those who fatigue or even drive a horse hard when in full condition, may find it followed by inflammation.

In training the race-horse and the hunter, the utmost regularity in exercising them is quite indispensable, otherwise they never can perform the task required of them, and grievous disappointment is certain to be the result. The one will be knocked up with half a day's work, while the other is certain to be winded before he performs a circuit of the course.

I have particularly to caution the inexperienced against working a newly-purchased horse too hard, as these are invariably made up by the dealer, by feeding and idleness, so as to please the eye. To take a horse to the hunting-field under such circumstances, is run- 
ning a great hazard. Let him be carefully and regularly worked for some days before hunting him, and there is little danger of bad consequences following, if gradually cooled and well groomed at the end of his work. We would recommend the proprietors of horses to attend themselves to this salutary and necessary operation, as grooms but too frequently neglect it, or, in many instances, injudiciously perform it.

The above remarks are applicable to the horses of the gentleman and tradesman, but those of the agriculturist need but little attention, as they are generally worked with moderation and regularity, and hence are not predisposed to those diseases where a different mode of treatment exists.

\section{GROOMING.}

Horses kept constantly in a stable should be subjected to constant grooming. It is of the utmost consequence to their health and appearance. The free use of the currycomb and brush should never be neglected, as the scurf which accumulates at the roots of the hair, and stops the pores of the skin, will otherwise prove injurious to the health of horses, by retarding free perspiration. Horses which are turned out in a field do not require grooming, as Nature provides a means of removing the scurf. Besides, without grooming, the coat of a horse will never have that sleek appearance which so much heightens the beauty of this fine animal. Lazy and careless grooms prefer giving the coat that smooth texture, by keeping the stable above that temperature which is safe for the health of the horse. The use of the currycomb and brush gives an increased action to the surface of the skin, and accelerates the circulation in the external vessels, which stimulates the animal and rouses all his 
energies. Indeed, any one may perceive the salutary effects of grooming on the spirits of the horse after this operation, which should always be performed in the open air when the weather is favourable, which braces the skin and conduces to health. It, however, frequently happens that grooms in using the currycomb give too much pressure, and thereby often irritate the cuticle and give pain instead of pleasure to the animal, especially to those which have thin skins. It ought therefore to be used with gentleness, and a longer time bestowed upon it. We also disapprove of a very hard brush, and especially one which has irregular hairs on the surface. A soft brush well applied will be equally effective, and produce less irritation to the horse.

After violent exercise, or a long journey, the legs of horses should be well rubbed down both with the hand and brush. This will prevent swelling, and even allay it if it has actually taken place.

\section{CLOTHING.}

If stables are kept dry and all cross-draughts are avoided, then the use of horse-cloths will be unnecessary. Nature has given the animal a covering which perfectly fits it, to prevent the necessity of artificial clothing while under a comfortable roof.

If a horse has been overheated, the true method to prevent his taking cold when put in the stable is to rub him well down with straw or hay until his skin is dry ; but if it should so happen that the groom cannot possibly spare time at that moment to attend to it, a cloth may be thrown across his loins, until he is fairly cooled down, when it should be immediately removed.

Nothing can be more absurd than to clothe coach and post-horses in the stable, because when out of 
doors this cannot be done, and the consequence is, it renders them extremely susceptible to colds and inflammation. When horses are overheated and have occasion to stand any length of time in the street, it will be a very proper precaution to throw a cloth over their loins, but this will be only necessary in very cold weather. In summer it would be rather injurious than otherwise.

But with horses that are used for riding, the greatest attention must be paid to prevent them being subjected to any sudden transition from heat to cold; and, as above noticed, good grooming is the best and surest preventive.

We would recommend that all stables should be provided with a thermometer, so as to enable the groom to preserve, as far as possible, a uniform temperature. 


\section{CHAPTER XV.}

\section{Vices and Dangerous Habits of the Horse.}

The horse is an animal of a noble and generous disposition, and naturally possessed of few vices, although he is occasionally met with having a bad and even furious temper, and, as may be expected, manifests great variety of natural habit. His vices, however, are too often attributable to the effects of improper training, and to tricks which he is taught by the bad treatment and folly of his groom or keeper.

The first breaking-in of the horse should only be entrusted to persons of mild dispositions, as it is by kind and patient treatment alone that we can hope to succeed in rendering this valuable animal truly useful and docile. I have no doubt but in nine cases out of ten, where horses exhibit furious or stubborn tempers, that these have been produced from the cruelty and ignorance of their first trainers.

\section{RESTIVENESS.}

The most unpleasant and dangerous of all vices possessed by the horse is that of restiveness. Sometimes this proceeds from a naturally bad temper, and at others from faultiness in education. This term includes plunging, rearing, kicking, bolting, and general impatience while mounting. A horse with any of the above faults can never be depended upon, for, 
although we may use means to counteract a particular vice, whether by compulsion or gentle measures, he may exhibit that vice when we are off our guard and are the least expecting it. Force may bring him to obedience, and he may succumb to him who has had the determination to subjugate him; but when mounted by another he is extremely likely to break out again. A horse that kicks in harness may be driven with safety by a cautious and experienced driver or coachman, but still there is no certainty of his not exhibiting the same trick years afterwards; indeed, most horses which have been kickers return to it again.

However high the temper which the horse may exhibit, we would recommend that he should be broken from his vices by kind and soothing means, and these exercised with patience for a considerable length of time; and force should only be resorted to when all other means have failed.

There have been several striking instances of persons who possessed the power of taming vicious horses by gentle measures; the most remarkable is recorded in the Rev. Mr Townsend's "Statistical Survey of the County of Cork," who remarks, that although the following circumstances appear almost incredible, yet they are nevertheless true, as he was an eye-witness to them :- "James Sullivan was a native of the county of Cork, and an awkward, ignorant rustic of the lowest class, generally known by the appellation of the whisperer; and his profession was horse-breaking. The credulity of the vulgar bestowed that epithet upon him from an opinion that he communicated his wishes to the animal by means of a whisper, and the singularity of his method gave some colour to the superstitious belief. As far as the sphere of his control extended, the boast of veni, vidi, vici, 
was more justly claimed by James Sullivan than by Cæsar, or even Buonaparte himself. How his art was acquired, or in what it consisted, is likely to remain for ever unknown, as he has lately left the world without divulging it. His son, who follows the same occupation, possesses but a small portion of the art, having either never learned the true secret, or being incapable of putting it in practice. The wonder of his skill consisted in the short time requisite to accomplish his design, which was performed in private, and without any apparent means of coercion. Every description of horse, or even mule, whether previously broke or unhandled, whatever their peculiar vices or ill habits might have been, submitted without show of resistance to the magical influence of his art, and, in the short space of half-an-hour, became gentle and tractable. The effect, although instantaneously produced, was generally durable. Though more submissive to him than to others, yet they seem to have acquired a docility unknown before. When sent for to tame a vicious horse, he directed the stable in which he and the object of his experiment were placed, to be shut, with orders not to open the door until a signal was given. After a tête-a-tête between him and the horse for about half-an-hour, during which little or no bustle was heard, the signal was made; and after opening the door, the horse was seen lying down, and the man by his side, playing familiarly with him like a child with a puppy-dog. From that time he was found perfectly willing to submit to discipline, however repugnant to his nature before. Some saw his skill tried on a horse which could never before be brought to stand for a smith to shoe him. The day after Sullivan's half-hour lecture, I went, not without some incredulity, to the smith's shop, with many other 
curious spectators, where we were eye-witnesses of the complete success of his art. This too had been a troop-horse, and it was supposed, not without reason, that after regimental discipline had failed, no other would be found availing. I observed that the animal seemed afraid whenever Sullivan spoke or looked at him. How that extraordinary ascendancy could have been obtained, it is difficult to conjecture. In common cases, this mysterious preparation was unnecessary. He seemed to possess an instinctive power of inspiring awe, the result, perhaps, of natural intrepidity, in which I believe a great part of his art consisted, though the circumstance of thetête-a-tête shows that upon particular occasions something more must have been added to it. A faculty like this would, in other hands, have made a fortune, and great offers have been made to him for the exercise of his art abroad ; but hunting, and attachment to his native soil, were his ruling passions. $\mathrm{He}$ lived at home in the style most agreeable to his disposition, and nothing could induce him to quit Dunhallow and the fox-hounds." Among the many strikingperformances in this way, none was more remarkable than his taming the celebrated racer King Pippin, one of the most ferocious horses that ever lived. Such was his furious temper, that to saddle and bridle him was almost impracticable, even by his ordinary attendants. His particular propensity was flying at and worrying any person who came within his reach; and he has been known to turn round and tear the leg of his rider with his teeth, and drag him from his back. On one occasion, when he had bid defiance to all, the "whisperer" was sent for, who was shut up with him the whole night, and in the morning, so completely subdued was this furious animal, that he followed Sullivan round the course like a dog-lying down at 
his command-permitting his mouth to be opened, and any person's hand to be introduced into it-in short, he was as quiet as a lamb.

"At the same meeting, on the Curragh of Kildare, he won a race, and continued his docility for three years; but again broke out, and having killed a man in one of his furious fits, he was ordered to be destroyed."

As I have before said, there is little chance of reclaiming a bad-tempered horse by harsh treatment, as I believe it will always be found to have an opposite tendency. An ill-tempered groom should never be allowed to enter a stable, however fit he may be for his business in other respects. For a surly, bullying fellow is sure to frighten horses so much that as soon as he enters the stable they will jump from side to side at his approach. Many a scar has been inflicted by such a man, by using his pitchfork instead of soothing the animal for a fault; and if asked how the horse came by the blemish, he invents a falsehood to account for it.

\section{REARING.}

Rearing is one of the worst vices in a horse, and is practised with the intent to throw the rider off. Sometimes it is the result of playfulness, but even then it is a dangerous and unpleasant fault. The use of a deep curb and sharp bit will, in some instances, cause even a quiet horse to rear, and when this is the case, immediate recourse must be had to the snaffle bridle.

As in kicking, however, this is seldom or never cured. Horse-breakers have attempted it by absurd and dangerous means, namely, that of pulling the horse backward on a soft piece of ground. This has 
ruined many horses, some having had their necks broken, or their spine so severely injured as to render them ever afterwards useless. If rearing proceeds from determinedly vicious habits, it is a hopeless case, as the animal seldom abandons it. Sometimes horses rear from playfulness, which is, however, very different from that which proceeds from passion.

\section{BACKING OR GIBBING.}

It is not an unfrequent occurrence for horses in harness to back instead of drawing when first started, and some add to this considerable viciousness, combined with obstinacy. In this case soothing and persuasive coaxing should be tried, and some patience exercised; and it is not until these have failed that the whip should be applied, and this must be exercised with moderation. In nine cases out of ten, if severely punished with the whip, the animal becomes obstinately determined not to move, or he may proceed a short way on his journey, and probably he will again stop at the first hill he comes to on the road.

In breaking, great care should be taken not to start the horse uphill when using the break, because he feels the entire weight of the machine at once, whereas if he were put in motion on a level road, the heavy pull would be less perceptible. Some trainers are so foolish as to teach the horse backing by placing his head uphill, and making the animal draw a little. He feels the weight of the break, and then by halting and pulling him backwards the weight is removed, and the animal finds it much easier to back down hill than to pull; and hence a habit of backing is acquired, which is both troublesome and dangerous.

With horses which have this habit at starting, 
the best method to break them off it is to place a large heavy stone behind the wheel; and the horse, feeling he is unable to back, will generally proceed forward, finding it more easy to do so; and by carefully continuing this practice, the horse will gradually be broken off the bad habit. Another plan, nearly as good, is to start the horse, if it can possibly be managed, with the back of the machine placed towards a rising ground; and as it is more difficult at all times to force it backward than forward, besides the hill being against him, he will prefer going forward to backward. Sometimes it will be necessary to lead the horse for a short distance, and when the groom has quitted the reins, a gentle touch with the whip will make him proceed. If, however, he is determinedly obstinate, there will be little chance of succeeding by forcible means; and if the driver is resolved to use compulsion, we would recommend that it should not be attempted unless there is a wide space, where by tight reining the driver may back him in the particular direction which he wishes, and it would be very desirable to do so uphill if the ground inclines in the neighbourhood. But still there is considerable danger in the attempt.

Gibbing and backing are frequently produced by the pain inflicted on a horse where the collar is tight or does not fit. Some horses have also a great dislike to a cold collar, and when this is the case it ought to be lined with cloth instead of leather, or a false collar or strip of cloth may be worn round the shoulders. Many horses, not otherwise gibbers, will not start if their shoulders have been chafed with the collar and has left a rawness, as the coldness of the collar gives considerable pain; but after the collar becomes of the same temperature as the animal, then 
he will go on. To prevent unpleasantness of this kind it would be well to warm the collar at a fire before putting it on. Some horses, which were inveterate gibbers, have been cured of this vice by constantly wearing a false collar; while others have been reformed by keeping the ordinary collar on night and day. This is, however, not to be recommended, as it interferes with the animal's rest.

Many horses are such determined gibbers that they will never cure. When this is the case, they should be sold to the owners of a stage-coach, in which fourin-hand are driven, and if placed as the near wheeler, they will be forced to do their work. Some have also been worked in a team by farmers; but nobody would think of keeping an animal which can only occasionally be rendered serviceable.

\section{KICKING.}

The vice of kicking is too often caused by horses being teased, tickled, and pinched by grooms, from wanton mischief or thoughtless folly. The habit becomes habitual with the animals, and what was at first only done in play, is exercised in anger, and often too when one least expects it. In short, it is a dangerous and incurable vice.

Horses with an irritable or fidgety disposition kick the stall or bail, and especially during the night. This is a great annoyance to other horses in the stable, and breaks their rest. Besides, the animal is liable to injure himself seriously, and bring on swelled hocks or other malady. Mares are more given to this than horses; and in either it is difficult to eradicate if once confirmed. As soon as it is discovered that a horse has this vice, a furze or thornbranch should be fixed to the partition or post; and 
few horses will continue to kick when they are pricked at every attempt. Many cures have been effected by this simple means, although cases have occurred where it was not a remedy. The next remedy is to have recourse to the log. This consists of a heavy piece of wood attached to a chain, and buckled a little way above the hock, so as to reach half way down the leg. As often as the horse kicks, he receives a severe blow from the log; and he soon learns to desist, finding the pain which it inflicts. However, not unfrequently considerable injury is done to the limbs by the bruises and severe swellings which have followed.

Kicking is a dangerous vice, especially with horses used in harness; bad with a chaise, but much more so with a gig behind them. The slightest touch on their quarters, even by the reins touching, will set them to kicking; and in many instances the bottom of the chaise will be driven in, or a gig may be battered to pieces, and the horse frequently coming off with a broken limb, or the driver may sustain serious injury. With kicking horses, the greatest care should be taken not to allow the reins to pass under the tail, as the moment they feel it, the tail is pressed suddenly and tightly down, so much so, that it is impossible to extricate the reins; and the more the driver pulls, the more the animal kicks and plunges. When the driver finds that the reins are so entangled, he should on no account attempt to extricate them by pulling, but quietly dismount, and relieve them by lifting the tail gently.

This vice is seldom eradicated. Where persons cannot afford to part with such horses, as they must be sold at a great loss, a strong kicking strap may be used, which circumscribes the use of the hind 
limbs, and prevents the horse from raising them to kick. But even this is no security, as by violent efforts on the part of the animal the strap may break, and no one can tell what may be the consequence. I had a particularly handsome and powerful mare which possessed this vice, and although I adopted every means to break her off it, I found it impossible. I sold her, and the person who bought her was sure he could effect a cure; but he was mistaken, and he parted with her. Her fine form soon found a ready purchaser, and in six months she was in the hands of eight different persons. Never trust a kicker.

\section{BITING.}

This trick often proceeds from play, and is taught by the folly of grooms or stable-boys teasing the animals. But what they have thus acquired as sport, becomes a fixed habit; and when thwarted in any manner, they will sometimes bite with great severity. Like other vices, this is difficult of cure, and it is but seldom they can be really broken off it. Teasing a horse should be strictly forbidden, and the groom or stable-boy severely punished when detected in the act. Biting, like other vices, should never be taught the animal, as it is easier to avoid it than to effect a cure. Gentle treatment is the best suited for all our domestic animals, and the surest way to command their affections. Bad-tempered and tricky grooms ought to be scouted by every one; and any master giving such a person a character, is highly culpable, and ought to be held up to public scorn.

SEIZING THE CHEEK OF THE BIT.

Some horses are so cunning as to get the cheek of the bit into their mouth, which gives them a great 
command over their rider or driver. There is no cure for this; and the only thing that can be done is to fasten a round piece of leather, or use some other mechanical contrivance, so that the animal cannot possibly get the cheek of the bit into his mouth. Neither soothing nor beating will remedy this trick, as the horse who has once been guilty of it is sure to seize the first opportunity to repeat it, when anything vexes him. Many very serious accidents have happened to persons from horses running away with the cheek-bit between their teeth.

\section{RUNNING AWAY.}

The only thing that can be done in this case is to use a strong curb with a sharp bit, and at the same time always keeping a firm bridle-hand. But if in spite of these precautions he does run away, if there is plenty of open space, or a ploughed field at hand, turn him into it, and apply the curb, spur, and whip, as vigorously as possible, and make him run until he is heartily tired of it. If anything can effect a cure, this will. Some horses only bolt off when they hear the cry of the hounds, and will not be restrained while the chase continues; other horses seem to be well aware when they are mounted by unskilful riders and endeavour by bolting to throw them off; while some vicious, headstrong animals bolt even with the best of riders.

\section{SHYING.}

Of all the vices incidental to the horse, shying is one of the worst, and more accidents have happened from it than any other of the vices or defects of a horse. Shying proceeds from various causes, but one of the principal is defective vision; timidity 
stands next; and it often proceeds from a disposition to be playful. This vice is far less common among high-bred horses than with those which are halfbred, although we have met with it in some of our first racers.

When we have a horse given to shying, our first attention should be directed to the cause; that is, whether it proceeds from friskyness, timidity, or defective vision.

When shying proceeds from playfulness, it is difficult to judge what mode of cure is best to be adopted, because if corrected for it, he will associate with any object that diverts his attention the infliction of punishment, which will tempt him to run away, under the dread of a flogging; and if caressed for the fault, it is liable to induce him to repeat it. But, of two evils, gentle correction must be adopted, and rather to pass by the object than to take him up to it. He should also be spoken to sharply.

If shying proceeds from fear of new objects, the true way to correct him of this is not to force him up to them, but to pat him and soothe him, but avoid beating; and take care to pass the objects of his fear again and again, always going nearer to them every time you pass. This will familiarise him to them. Seeing that these are harmless, he will soon learn to pass by unnoticed any novel object which he may meet with upon a road.

When an animal is given to shying from defective sight, the only method to effect a cure is to take him up to it, and in the act of doing so he must be coaxed to approach it, and on no account must he be beaten; and although it sometimes happens that the horse will manifest great reluctance to do so, he should be persevered with, and not allowed to proceed 
until he has seen closely the object of his fear. After he has been a few times thus treated, he will soon learn to pass with indifference any object which he may meet.

We cannot better define the folly of beating a horse for shying than by the following, which we extract from "The Veterinarian" :- "We will suppose a case, an every-day one. A man is riding a young horse upon the high-road in the country, and meets a stage-coach. What with the noise, the bustle, the imposing appearance altogether, and the slashing of the coachman's whip, the animal at the approach erects his head and crest, pricks his ears, looks affrighted, and no sooner comes alongside of the machine than he suddenly starts out of the road. $\mathrm{His}$ rider, annoyed by this, instantly commences a round of castigations with whip, spur, and curb, in which he persists until the horse, as well as himself, has lost his temper, and then one whips, spurs, and pulls, and the other jumps, plunges, and frets, and throws up his head, until both, pretty well exhausted by the conflict, grow tranquil again, and proceed on their journey, though not for some time afterwards, in their former mutual confidence and satisfaction. Should they on their road, or even on a distant day, meet with another coach, what is the consequence? That the horse is not only more alarmed than before, but now, the moment he has started, being conscious of his fault, and expecting chastisement, he jumps about in fearful agitation, making plunges to strike into a gallop, and attempting to run away. So that by this correction, instead of rendering his horse tranquil during the passage of a coach, the rider adds to the evil of shying that of subsequently plunging, and perhaps running away." 
If a horse is aged and prone to shying, and will not be broken off it by gentle treatment, then severe measures must be resorted to. Many years ago, I rode a remarkably spirited and active hackney, which was much given to shying, and particularly in the evening or at night, although he had no defect in his sight. About a mile from my residence there was a salt-work. The first time I had occasion to pass this at night, the flames were issuing from the top of the furnace. My horse came to a stand when within about fifty yards from it, and neither coaxing nor force would induce him to proceed. I turned his head homewards, and applied both whip and spur smartly, and galloped him at full speed to my gate; I then drew up, and turning round, returned at a sharp trot, and on coming up to the salt-work, he passed it without manifesting any signs of fear, and never afterwards showed the slightest reluctance to proceed when he came to it.

Some horses have a trick of shying on coming out of the stable. This is acquired by having received some injury while entering the door, or striking his head against the top of the doorway if too low. This is incurable, so far as I know; for both kind treatment and severity have been tried without success.

\section{RESTIVENESS, AND RESISTING BEING MOUNTED.}

Some horses which possess a lively or impatient temper, manifest a desire to start off before the rider can get seated in the saddle. Indeed some will attempt to set off whenever the foot is put in the stirrup. This is a troublesome and dangerous fault, even with the most expert horseman, and especially so to those who are inexperienced or infirm. Horses 
sometimes become so cunning that they know a good from a bad horseman, and finding they are either to be, or actually mounted, by a timid or indifferent rider, will endeavour to throw him off, or bolt away before he is properly seated.

The horse mentioned in the preceding article on shying, when I first had him, exhibited the utmost reluctance to be mounted; so much so, that it was impossible to succeed without some one holding his head; and no sooner did the servant quit the reins, than he plunged and leaped about in all directions. However, I refrained from flogging or spurring him, but patted him on the neck until he became quiet. I overcame this by making the groom hold his head, while I mounted and dismounted a dozen or more times successively. After repeating this once or twice a day for upwards of a week, I then led him out to a broad garden walk, and commenced by patting him on the neck and shoulder, and then putting my foot in the stirrup, quickly mounted him. When seated, I gently walked him about, soothing and speaking kindly to him all the while; and then dismounted, and led him about for a few minutes, and again mounted many times, which he became quite reconciled to. This I repeated several times daily for about a week, but never rode him out of the grounds during this time. At last he became so tractable by kind treatment that he never afterwards was troublesome to mount. I sometimes rewarded him with a handful of oats while pursuing this treatment. When a horse has such a propensity, he should be mounted quickly and without fear, and not allowed to proceed on his journey until he is perfectly quiet. Instances are not few where horses that are generally pleasant and easy to mount, become restive when too high fed 
and having too little work. The remedy for this is obvious. But when animals continue to manifest this vice, they should be sold.

\section{VICIOUS WHILE CLEANING.}

Very great difference exists in the temper exhibited by horses under the operation of cleaning. Some that are steady and quiet on the road and in the field, cannot be cleaned without great hazard to their grooms, as well as the danger of laming themselves. This often proceeds from a very sensitive skin, and at other times from their grooms having inflicted severe chastisement on some former occasion when cleaning. Besides, ill-disposed grooms, by teasing the animals, or currying them with a brokentoothed comb or uneven-surfaced brush, teach them this bad habit, and have even a delight in seeing the animals show their teeth; and this is continued until it becomes a fixed vice. If a change of groom takes place, what was done partly in play is then manifested in anger, and serious injuries have been inflicted upon the unsuspecting stranger. It therefore behoves grooms to be cautious how they handle a strange horse.

There is much variety in the sensibility of the skin of horses, some being so tender that moderate rubbing gives them uneasiness, while others are so much the reverse that the whip hardly excites it.

It will not be difficult to overcome this vicious habit. When the groom discovers it, the best plan is to use a gentle hand while cleaning, and lean lightly on those parts which seem most sensitive; and avoid punishing the animal for exhibiting restiveness, and he will soon lose all recollection of the 
former ill-treatment which he had received from his groom, and become quiet and steady.

\section{RESTIVENESS WHILE SHOEING.}

When a young horse is first shod, great caution should be used, and gentle means adopted to induce the animal to submit to this novel operation; and it would be much better to pay the smith a small gratuity for his loss of time in coaxing the horse to submit to it, than to use the gag hurriedly. It must naturally be expected that a young animal will exhibit uneasiness for the first few times he is taken to the smithy. He should on each occasion be led thither by the person who feeds him; and above all things the smith must not strike him for a fault; as in most instances horses which are vicious under the operation of shoeing, are rendered so by severe treatment either by the smith or groom. Patience at first shoeing will be well rewarded, and when the animal finds he receives no injury, he will soon become obedient under this necessary operation; but if severe chastisement has been resorted to, he is certain to be troublesome every time he is shod, having in remembrance his former treatment, and the pain he suffered under the twitch and the gag. The business of the smith is to be mild and yet firm. Shoeing a quiet horse in the presence of a young one has had the effect of teaching him to submit to it. For his own sake, the smith should avoid teaching this evil, as he is in constant danger during the operation; and the horse is liable to be pricked and lanced in his struggles. Some horses are so vicious that they never can be conquered, and it becomes necessary to cast them every time they are shod, and confined in the trevis. When this is 
the case, it may be expected that sooner or later the animal will meet with an accident which will render him useless.

\section{CRIB-BITING.}

Crib-biting is one of the worst habits which a horse can acquire, and is seldom or ever cured. The horse seizes the manger with his teeth while he stretches his neck forward, and after some spasmodic action of the throat, a slight grunting sound is uttered, which appears to be accompanied by a drawing in of air. The cause of this trick is not yet well understood; and whether it proceeds from a bad habit, or a defect in the formation of the soft palate and back part of the mouth, still remains undiscovered; and therefore we shall not indulge in any speculative opinions as to its origin.

One serious effect of this trick is the wearing down of the teeth; and instances have occurred where they have been broken. It has likewise been found that crib-biters are more liable to colic than those without this vice.

It has been found that crib-biting is acquired by horses being in the stable with one which has the trick. Among the expedients which have been resorted to for the cure of crib-biting, the edge of the manger has been lined with iron; also with sheep-skin besmeared with aloes, tar, and other disagreeable substances, but all with no effect. The only thing in this case is to resort to a preventive, and that will be found in the use of a strap buckled tightly round the neck, which has the effect of compressing the windpipe, and rendering it impossible to resort to it; but no sooner is the strap removed, than the horse recommences his old habit, so that it must be constantly 
worn to be of use. But the continual use of it is apt to produce irritation in the trachea, and this will terminate in the affection termed roaring, which we have particularly described at page 42. A five or six months' run in a field has also been tried without proving a remedy. Crib-biters are generally in low condition.

A muzzle barred across the bottom will prevent crib-biting. This must be made only of sufficient width to allow full action to the lips, so that the animal may pull his hay from the rack and eat his corn, but so close as not to admit of him grasping the edge of the manger. Crib-biting is legally considered unsoundness.

\section{WIND-SUCKING.}

This is so intimately connected with crib-biting that it may properly be considered a modification of it ; as it is accompanied by a want of condition, and the same bending of the neck, with the head drawn inward, is manifested, and the horse alternately opens and closes his lips, and a sound is produced similar to sucking air. The remedies attempted have been tying up the head of the horse, except when feeding ; and the application of a muzzle with sharp spikes bending towards the neck, which will prick him when drawing in his head.

\section{NOT LYING DOWN.}

Horses are sometimes prone to standing constantly ; and some only lie down once in a fortnight, or even a longer period. When this is the case, they are generally liable to swellings in the limbs, and seldom able to go through much work. Such horses should, if possible, be put into a stable by themselves and left 
at liberty, and a well-made bed will sometimes tempt them to lie down. No means can be adopted to force the animal to take rest by lying down. When it is not possible to place him in a stable alone, an empty box should be constructed so that he may be left for the night unhaltered in it. I had a remarkably fine draught-horse that never was known to lie down, and yet he kept in good condition, and was not troubled with swelling in the limbs: but this is a rare occurrence. He sometimes fell down on his knees while asleep, but the groom always found him on his legs before he could reach the stable, although his house was next door.

\section{SLIPPING THE COLLAR.}

Some horses are very expert at getting out of their collar, and range at large in the stable during the night, which subjects them to the liability of being kicked by their neighbours, besides keeping all the others awake. To prevent this, the web of the halter should be accurately fitted to the neck, and made so as to slip only one way; to this a strap must be attached, so as to buckle round the neck, taking care that it is not too tight.

\section{PAWING.}

This is a disagreeable and very bad habit, and proceeds from an irritable temper. Bruised feet and sprained legs too often proceed from it. The shoes are quickly worn down in front, and the litter considerably wasted. The only remedy for this is the use of shackles, to which should be attached a chain sufficiently long to allow the horse to shift his posture and move about in his stall. These to be used only 
in the daytime, as if kept on at night the horse will not lie down.

\section{ROLLING.}

Horses that roll in the stable are apt to be injured from want of sufficient room, and also to get entangled in the halter ; and, strange as it may appear, although he may get severely hurt and be nearly choked by the halter, he will repeat it night after night. The only thing which will prevent him from rolling, is to give him just enough of collar to enable him to lie down, but so short that his head will not touch the ground, because it is impossible he can roll over without resting his head upon the ground. If a horse is in a field, rolling is a harmless and even healthful amusement.

\section{WEAVING.}

Animals of an impatient, irritable temper, that dislike confinement in a stable, will sometimes keep moving their head, neck, and body to and fro, like the motion of a weaver's shuttle: these have been called weavers. Such horses seldom or never carry much flesh, from their fretful temper and incessant movement. The only preventive is to tie the head close up, except when feeding.

\section{TRIPPING.}

Innumerable attempts have been made to cure this dangerous quality in a horse, but few indeed have been the cures. It is only a waste of time to enumerate these. The true remedy is to get quit of the horse. 


\section{CHAPTER XVI.}

\section{Of Shoeing.}

A FINE horse without sound feet may be compared to an elegant building without a firm foundationboth are in constant danger of falling.

It is not known when the practice of shoeing horses with iron was first practised, although it is certain that William the Conqueror introduced it into Britain.

We shall not dwell upon the methods which were practised to preserve the hoofs of horses before the invention of iron shoes, but proceed to consider the best methods now adopted. The construction of our roads and streets render shoeing indispensable; at the same time it must be obvious that shoeing with iron, and nailing them to the hoofs, is productive of very serious mischief, and is the cause of various diseases, most of which we have already noticed in the preceding part of the work.

Much attention has been devoted by eminent veterinarians to the best form of shoe for the prevention of contraction, and the consequent destruction of the expansion of the hoof; but no means have yet been devised to obviate this completely.

There cannot be a greater error than the possessors of horses exercising parsimonious economy in having their horses shod by those smiths who will per- 
form the office cheapest. The first object with every one should be to get the work well done, because it is being "penny wise and pound foolish" to have the shoes of a horse ill fitted, for the saving of a few pence.

\section{PREPARATION OF THE FOOT FOR SHOEING.}

The first thing to be done by the smith is to remove the old shoe, and it is of importance to see that this is performed in a careful and proper manner. Some smiths are very careless and rash in removing the shoe, and instead of turning up the points of the nails tear off the shoe by force. The shoe is fixed to the foot by nails which penetrate from the sole to the upper surface of the hoof; these are broken off, allowing sufficient length remaining to turn down and be clenched into the horny substance. These clenches should be carefully raised and rendered as straight as possible, so that the nails may be pulled without injuring the hoof or increasing the dimensions of the nail-holes. By thus wrenching off the shoe, there is danger that some of the stubs may be left in the crust and cause future lameness, or portions of the crust itself may be torn off. In most cases, where such violent treatment is practised, the horse exhibits by his flinching that he suffers pain from it, and is the too frequent cause of animals being troublesome to shoe.

When the shoe has been removed, the crust must be rasped down in the edges; and although a little roughness may be exercised in this, yet there is little danger of injury to the hoof, only that too much must not be removed, so as to render it too thin.

It sometimes happens that the horn of the sole 
becomes so hard and thick that it is removed with very great difficulty, in which case it becomes necessary to soften it by heat. This is effected by means of a flat iron heated, and drawn over the sole, and even kept close to it for a little time. If the sole is thick, no injury will be sustained from it, and, on the contrary, it will render the paring more easy and less disagreeable to the horse; but if the desquamating portions of the sole has been regularly pared out during shoeing, this must not be permitted. The quantity of paring necessarily varies, according to the formation and condition of the foot, but as a rule little horn requires to be removed by the drawing knife from the soles of perfectly healthy feet, for as the horn of the hoof grows in lairs it is more or less shed. The foot which is pumiced should only have the ragged parts cut away; when the foot is flat, no paring is needed. Care must always be taken that the crust is not reduced to a level with the sole, as this would permit the sole to press upon the edge of the seating, and thereby be bruised and injured. The entire circumference of the crust should be perfectly level, but projecting a little beyond the sole.

We must now direct particular attention to the heels. More stress is thrown on the inner heel than on the outer, and, from natural weakness of the quarter there, it generally wears quicker than the outer one. This being the case, less horn must be pared from it than from the outer, as taking the same quantity of horn from it would leave it lower than the other, whereas they should be perfectly on a level.

Almost all smiths have a fancy for opening the heels, from the idea that it does good by rendering the foot neater, which is a complete fallacy, as they ought seldom or never to be touched; for, as we have 
already shown in our description of the anatomy of the foot, the bars are the only check in preventing contraction. Nothing, therefore, should be removed but the ragged and detached portions.

It is intended that the heel of the shoe should rest partly on the bar, and partly on the heel of the foot, consequently the bar should be allowed to remain nearly in its original condition, from its first inflection, and extending down the side of the frog. It is only when the frog becomes level with the crust that it should be pared at all. We have already shown, when treating of contraction, page 133 , that when the bars have been removed we have destroyed the props which are the main preventions of that defect.

The quantity to be pared from the frog depends chiefly upon its prominence and the shape of the foot, but here again as in the case of the healthy sole, so also in that of the healthy frog, the less horn removed by the knife so much the better, as the larger and stronger the hoof, the more fitted is it to bear pressure and protect the delicate structures underneath.

From what we have shown it will be sufficiently evident that skill is necessary in the preparation of the foot for the reception of the shoe; and it is equally incumbent on the groom and his master, as well as the smith, to possess this knowledge; and it is well for some one always to be in attendance during the operation of shoeing.

\section{THE PUTTING ON OF THE SHOE.}

The foot having been prepared, the smith selects a shoe from among those already made, which he thinks may fit as nearly as possible, and what alterations are necessary are made on the shoe. This frequently 
requires both the use of the hammer and the file, and the conscientious workman will not nail it on until it is so formed that it rests firmly on the sole, and its external shape precisely corresponds with that of the crust. On the other hand, the careless and inconsiderate mechanic will pick a shoe, and suit the hoof to the shoe, frequently paring the sole and crust to the quick; and often cutting it so thin that it will not hold the nails firmly, besides rendering the unprotected part of the foot liable to be punctured, and exposing the sole to pressure which may occasion lameness, and that too of a permanent character.

A properly constructed shoe should have the web of equal thickness from toe to heel. When thinner at the heel than the toe, it permits the heel to sink too much, which has a tendency to induce sprain of the flexor tendon, and when the shoe is thicker at the back than the front, it elevates the frog too much, is sure to promote disease, and will impair its function, and is certain to bruise the toe, which from its naturally exposed position is more liable to injury than other parts.

Every possessor of a horse should be well acquainted with the different kinds of shoes now in general use, and have such a knowledge of the varied form of hoofs as to enable him to judge the particular kind best adapted to the shape of the foot of his own horse. Country smiths pay too little attention to this point, and with a predilection for one form, apply it to whatever shaped hoof they may meet with.

In a state of nature the hoof of a horse is admirably adapted to give security to its steps; but when the foot is shod, the weight and bearings of the whole limb, or, more strictly speaking, of the entire animal, are changed. It therefore becomes a matter of much 
importance to investigate what form of shoe is best calculated for this altered condition, and which will produce the least mischief to the feet; for the best of shoeing must ever continue to be a source of diseases and inconvenience to the horse. And any one who strictly investigates the structure of the foot, with its numerous modifications of shape, will soon perceive that no universal form will be suitable for all feet.

Some parsimonious individuals contract with their smiths to supply shoes at a certain sum per annum. From what we have said in this, as well as other parts of the work, it will easily be seen how absurd such a practice is; because the smith will, in nine cases out of ten, put heavy shoes on the horse to save the labour of repeated shoeing; nor will he think of removing the shoes at stated intervals, as we have already recommended.

\section{THE CONCAVE-SEATED SHOE.}

Considerable difficulty has been experienced in having enough of room to pass a picker between the foot-surface of the patent-safety shoe and the sole of the foot, more especially where soles are flat, with an inclination to convexity. The consequence has been, the soles of such feet in some cases have been pinched by the pressure of the shoe, although this shoe is not more difficult to fit than any other. To obviate this supposed difficulty, and to meet the views of those who think it desirable, a concave-seated shoe has been invented, concave to the ground, and seated on the foot surface.

This shoe presents a perfectly level surface to the ground, so as to give as many points of bearing as possible. There is a groove round the outer edge, in 
which the nail-holes are punched; when the shoe is on, the nails project but a little way beyond the general surface of the shoe, but are soon worn level with the face of the shoe.

The web of this shoe is of equal thickness throughout, and parallel from toe to heel, deviating in width according to the form of the foot to which it is to be applied. The foot-surface of the shoe is sufficiently wide to protect the sole from bruises, and as wide at the heel as the frog will permit, in order effectually to cover the situation where a corn grows.

It is seated on the foot side, and the outer portion made as accurately flat as possible, and of the exact width of the crust, which it is designed alone to support, so that the entire weight of that union which exists between the numerous little plates which are arranged upon the internal surface of the wall of the foot (which are more particularly described at page 226), and this portion, supports the whole weight of the horse. This flattened portion of the shoe is wider towards the heel, and occupies the entire breadth of the web, to support the heel of the crust and its reflected portion, the bar; so that while it protects the horn included within the angle from injury, it promotes that equal pressure upon the bar and crust, which is most likely to prevent contraction as well as the growth of the corn.

We have given a representation of this shoe, Plate xI, fig. I. It is secured to the foot by nine nails, five on the outside, and four on the inner side of the shoe. These are not placed parallel to each other, but the outside ones extend a little further towards the heel than on the other side, because the outside heel has more nail-hold, and is thicker and stronger than the inside. When the feet are of moderate size, and not 
a great deal of work required of the animal, three nails on the inside and four on the outside will be sufficient, and the last nail being distant from the heel, will permit of expansion in that part. Besides, it is always desirable that as few nails as possible should be used, so that they are sufficient to secure the adhesion of the shoe. That there may be no pressure on the sole, the inside of the web of the shoe is bevelled off, or rendered concave. As we have already explained, the foot of the horse is exceedingly susceptible, and easily bruised if the sole comes in contact with hard substances. Although it is so far protected by the iron shoe, the sole descends slightly when the foot of the animal is placed on the ground, and is unable to bear constant or even temporary pressure for any length of time; and if it bears upon the shoe, the sensible sole between the coffin-bone and horny external sole would be so much bruised as to occasion lameness, and if long continued it would be of a very serious character. Working horses too early has a strong tendency to flatten the natural concavity of the sole, and may induce a disposition to continued descent. If the feet are pumiced, the shoe must have an extra degree of bevelling to protect them.

When shoes are flat, gravel and dirt constantly insinuate themselves and lodge there, and are certain upon a journey to bruise and injure the foot; but in bevelled shoes, it is hardly possible for either to remain between the sole and foot, as they would naturally be shaken out every time the foot comes in contact with the ground.

Another advantage in this shoe is that the web is of that proper thickness, that when the crust is properly pared the prominent part of the frog will lie im- 


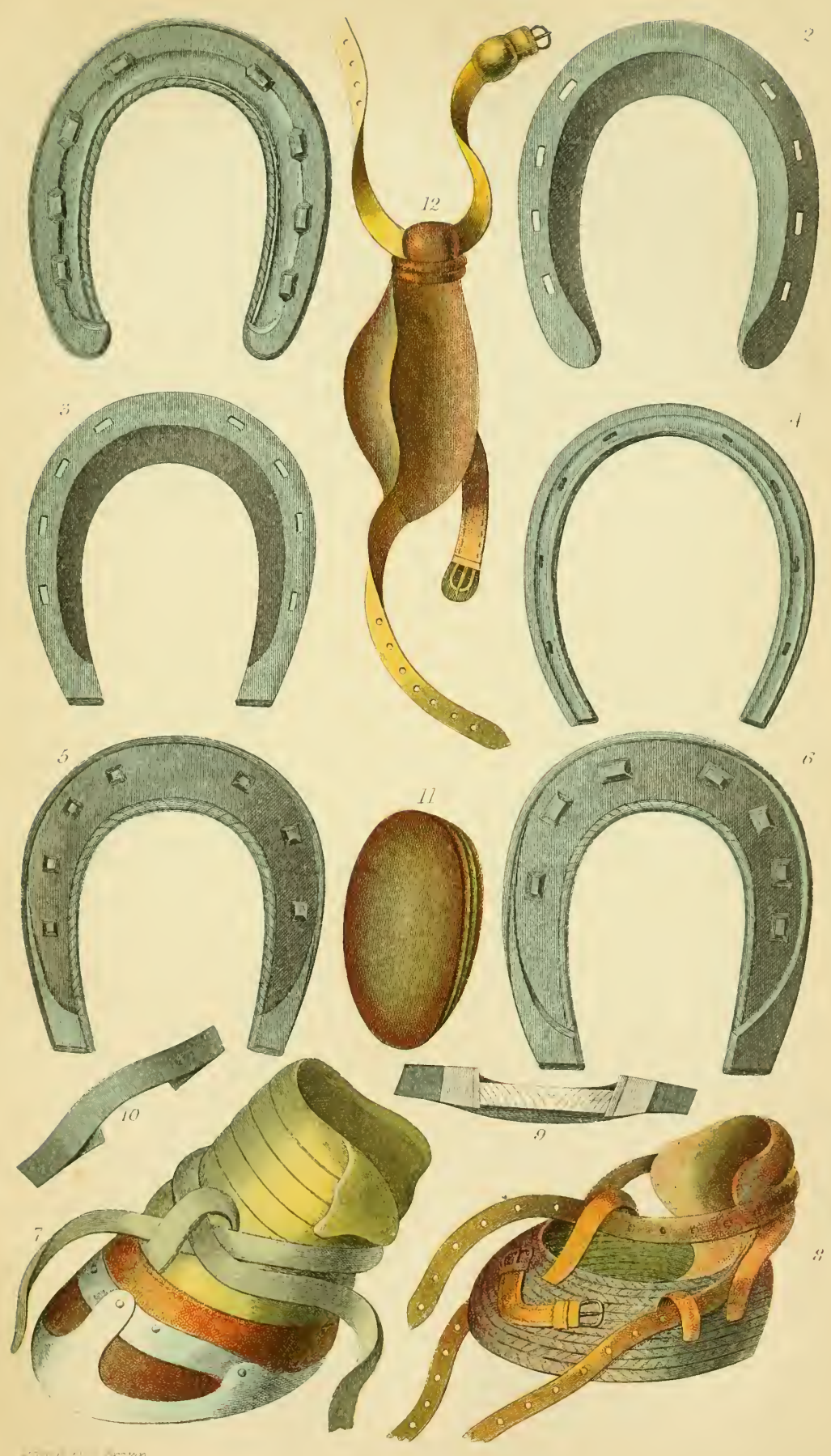



mediately within and above its ground surface, permitting the frog to rest sufficiently on the ground, so as to act as a wedge, and produce a tendency to expansion in the quarters; while at the same time it is protected from the injury it would sustain if it reached the ground with the full and first shock of the weight. In the common shoe the ground surface is a little convex, and its inward rim first comes in contact with the ground; so that the weight, instead of resting fairly on the crust, is sustained by the clenches and nails, which cannot fail to be prejudicial to the crust, and must often tear and splinter it.

The nail-holes must be situated as near the outer edge of the seating as the strength and security of the web will permit. The nails will consequently have a natural tendency to take an inward direction, and therefore will have a firmer hold, and be divested of that strain to which they are subjected in the common shoe.

We have given a representation of the undersurface of this shoe, Plate XI, fig. 2.

\section{THE SEATED SHOE.}

\section{PLATE XI, FIG. 3 .}

The seated shoe is of an equal thickness, perfectly flat, and parallel from the toe to the heel; only varying in width according to the form of the hoof to which it is to be applied, and similar to the ordinary shoe in the fullering, nailing, etc. The foot-surface, however, differs in having a narrow plain rim, about the same width as the thickness of the crust, extending round the edge of the shoe, except at the heel, where it presents a flat surface, to the extent of an inch; the other part of the shoe is hollowed out, bevelling from 
the inner edge of the seat, making it thin except at the heel, which is of the same thickness throughout. The intention is that the crust should bear upon and be supported by the seat of the shoe. The nail-holes are situated in the inner part of the seat. but the nailing is the same in principle as the common shoe.

The advantage of this shoe over the ordinary one is that of the crust resting on a flat surface instead of an inclined plane; and as it bears on the edge, it is less liable to induce contraction. But with that advantage, yet there are several objections to it. The shoe being flat without the proper degree of curvature, and the mode of fastening by pitching the nails inward, is equally destructive to the crust, although there is no strain upon the nails and clenches, as in the common shoe; and it has been found that its use has not the effect of diminishing the number of corn cases, which arises from the quarters being removed to fit the flat-shoe, and the weight is then sustained more by the heels than the quarters. Besides, it is a difficult shoe to make.

\section{THE EXPANDING SHOE.}

This consists of a shoe of the common English form, with a joint at the toe, which the inventor, $\mathrm{Mr}$ Bracy Clark, intended to relieve the feet from the restraint of the shoe and nails, by admitting the natural expansion of the hoof, and thus avoiding all the evils arising from shoes made on the common principle. This, however, has not been realised.

\section{THE HUNTING SHOE.}

PLATE XI, FIG. 4.

The hunting shoe differs from that of a horse used 
upon the road, both in weight and form. It is not so much bevelled off as the common concave-seated shoe, and only enough of space left between the shoe and sole for the introduction of the picker; as, going over heavy ground, the clay would insinuate itself; and by its tenacity would have a tendency to loosen the shoe, or indeed, what has been the case, tear it off altogether. It is also made shorter in the heels, so that they may not be torn off by the toe of the hind feet when galloping.

THE RACING-SHOE.

\section{PLATE XI, FIG. 5 .}

The racing-shoe, or plate, should be constructed of the best Swedish iron, and made of sufficient thickness to prevent breaking or bending when used. A flat surface on the foot side is generally used; and the same form of plate as in the shoes intended to be put on after the removal of the plate. Three or four nails on each side according to the size of the foot will suffice. The heels of the plate should not be longer than the horn of the heels, rather a trifle shorter, to prevent them from being torn off by the toe of the hind foot when at great speed.

\section{THE BAR-SHOE.}

This is a useful invention to remove the pressure from any tender part of the foot, and throw it on the frog which is quite sound. It consists of the common shoe continued round the heels and across the frog. Its chief use is in cases of corns and weak heels, and it quite covers their seat. It elevates the sole in pumiced feet, and thus secures them from pressure. It is also useful in sand-crack, as by it we can remove the 
pressure from the slit, and throw it on each side of it. In short, in all the diseases of the foot the bar-shoe will be found very serviceable if worn during these diseases; but it must be discontinued as soon as a cure has been effected. When the bar-shoe is used for sand-crack or corn, the crust and frog ought to be perfectly parallel, and the bar should be the widest part of the shoe, so that an extended bearing may be placed upon the frog. This shoe, however, is by no means safe during frost.

\section{CLIPS.}

These consist of portions of the upper edge of the shoe, hammered out, and turned up so as to embrace the lower surface of the crust, which must be a little pared out so as to receive the clip. The chief use of the clip is to give greater security in attaching the shoe to the foot, and lessening the stress upon the nails, which might prove injurious. In horses subjected to heavy draught, clips are indispensable, and are useful to all employed in draught of any kind. They will be found a useful preventive in securing the shoes from being torn off, when the strain is great on the feet while drawing. Clips are also beneficial when horses are given to stamping and pawing; as either of these tricks are likely to loosen the simple shoe. But clips should only be used in such horses as we have named, because they press upon the crust as it grows down, and are therefore objectionable with horses which are employed in light draught or hackneys.

THE HINDER SHOE.

As the hinder limbs are the chief instruments of propulsion in the animal, except while walking, the 
whole stress of the frame rests upon them. In consequence of this, the shoes of the hind feet are always made broader than those of the fore feet, and the toe is widened still more by rasping. When there is the slightest tendency to over-reaching, the toes of the hind feet should be shortened as much as possible, by sloping in the surface, and rendering the shoe somewhat less projecting than the horn at the toe. The hinder differs a little from the fore foot, in being straighter in the quarters. The nails in the hinder shoe should be situated nearer to the heel than in the fore shoe.

\section{TIPS.}

Tips are short shoes which reach only half way round the foot, and are worn by the horse while at grass, as a protection to the crust, to prevent it being injured by any hard parts in the ground. They are especially necessary when horses have a practice of pawing. The reason why these shoes are made short is to permit those feet which have a tendency to contraction to return to their natural condition by expansion.

\section{PATENT SAFETY SHOE.}

PLATE XI, Fig. 6.

This shoe is constructed of malleable cast-iron. This is accomplished by a chemical process called annealing, which renders the cast-iron less liable to break; as ordinary cast-iron is nearly as brittle as glass. The figure referred to exhibits the ground side of this shoe. The letters $a, a, a, a$, are a raised border, intended to strengthen the inward edge of the shoe, as well as to nrevent the intrusion of small 
stones, flint, and dirt, between the sole and shoe, which it is likely would take place but for this protection.

\section{WATER AND POULTICE BOOTS.}

\section{WATER-BOOT.-PLATE XI, Fig. 7.}

Considerable difficulty has been experienced in keeping diseased feet effectually wet by the application of a poultice. The usual method has been to put the poultice in a piece of old rag or sacking, and set the foot into it, and to tie the edges of the cloth round the fetlock. It must be obvious that the pressure of the foot will soon displace that portion under the sole, where probably it may be most required. Besides this, the cloth is liable to be cut through by the crust, and consequently the greater portion of the application escapes.

The poultice and water-boot is constructed with a leather bottom, defended by iron plating of different sizes. When required for the application of a poultice, a linen drill top is only necessary; but when required for a continued application of cold or warm water, it is necessary to have two or three thicknesses of bathcoating sewed together, and cut of a proper form for the top. In order that the whole foot may be subjected to moisture, a bottom consisting of two or three pieces of this cloth are sewed together, of the exact form and size required. The top must be soaked in water, and this will naturally descend to the bottom of the boot, where it is absorbed by the doubles of cloth. To keep the boot duly moist, it would be proper to dip the foot with the boot on into a pail of water occasionally through the day, and immediately before shutting the stable for the night. In this way the cooling moisture may be kept up for any length of time. 

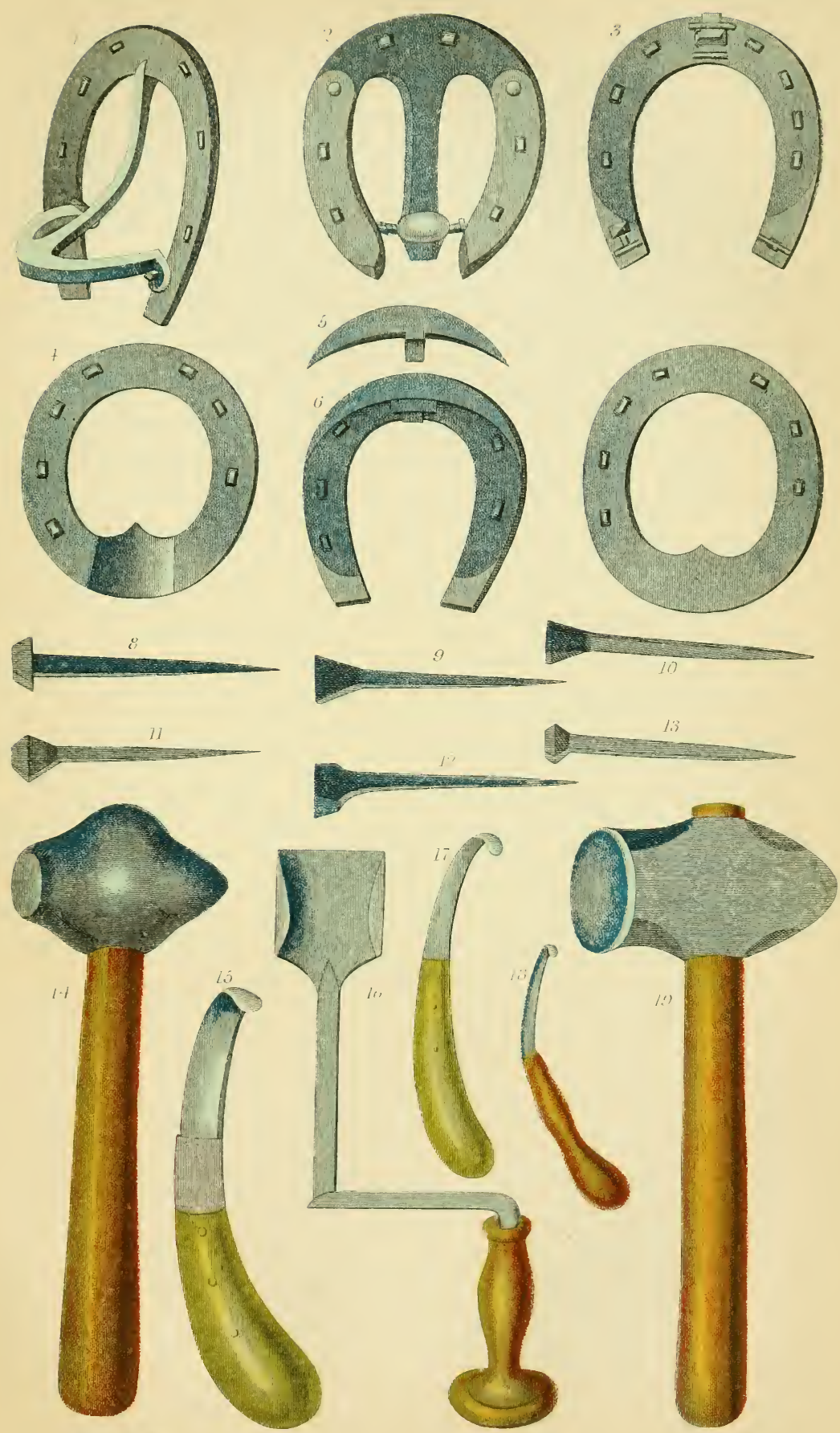

However, long-continued moisture has a tendency to destroy the texture of the frog, as well as the tough elastic property of the hoofs. To obviate this, two days in each week should be appropriated to keeping the boots off, and an unguent of tar applied to the surface of the foot, which should be well rubbed in with the hand. This will completely saturate the horn, and prevent that crumbly tendency which manifests itself when the foot is long kept moist. During this time the horse should have a well-supplied bed of litter to prevent injury to the hoof.

If during the two days on which the boots have been removed the animal paws with his foot, it is very liable to sustain injury; to prevent this, the use of a rope-boot will be necessary. See Plate xI, fig. 8 .

\section{FELT, OR LEATHER SOLES.}

In cases of bruised or inflamed feet it has been found of much benefit to insert a strip of leather or felt between the shoe and the crust. The intention of this is to lessen the vibration or shock which is given to the sensible portion of the foot, in consequence of the want of elasticity of the iron shoe. This is useful where disease exists, but must by no means be adopted where the feet are sound; for one reason-the nails can never be so firmly driven in when any substance is interposed between the hoof and the shoe, and by its alternate contraction and expansion with dry or hot weather, it is sure to lessen the security of the shoe; causing too much play upon the nails, and consequently enlarging the perforations in the crust, and rendering a portion of it liable to be torn away. 


\section{CHAPTER XVII.}

\section{How to Buy a Horse.}

Litrle do novices in horse-flesh think how many tricks are resorted to by dishonest dealers to conceal the defects of a horse, and take in the uninitiated. To exhibit a few of these will be the aim of this chapter, as well as to throw out several hints which may be useful in assisting the unexperienced in the purchase of a horse.

The first thing to be attended to is the form of the animal; and this differs materially in the various breeds, and its good points will depend upon their adaptation to particular kinds of work. The head in all the breeds should be fine, broad between the eyes, and tapering towards the nose; the jaws ought to be clean, and not possessing too much flesh; the eye full, sparkling, and lively; the nostrils rather large, open, and of a clear red; the space underneath between the jaws should be roomy, and free from glandular swellings or lumps; the ears should be well set into the head and pointed forwards, but not large; the neck should be well curved,' lightly formed rather than muscular, and considerably arched beneath at its union with the jaws; the shoulder should be high and sloping; the withers should be of medium breadth, and not too high, as it will be found that highwithered horses are generally narrow in the chest, 
which is always a bad point, as not allowing sufficient scope to the lungs, and never so pleasing to the eye as a broad expanded front. Still, some horses have proved both hardy and good in point of action with narrow chests ; but these have had depth to compensate for the want of breadth. However, there is a medium in the width of the chest, because great width is invariably accompanied with want of action ; and such horses are better adapted for cart or farm purposes.

The back should be short and somewhat arched across the loins; the chest deep, and the ribs expanding, especially between the last rib and the hucklebone, or hip, so as not to permit of a hollow betwixt them, which is always unsightly to the eye, if it is not a physical defect. No feeding will fill up a hollow in that quarter; nor can a horse be pleasing to the eye which has not well-formed hind-quarters. These should be rounded and full, and the muscles. of the hips well developed. A low rump is a characteristic feature of the Irish horse. This is termed among dealers goose-rumped. Many blood-horses have this characteristic. Horses long in the quarter are seldom serviceable; if a horse is required for field sports, always choose him short in the quarters, as this is a character possessed by all good leapers. The thighs must be muscular, and extending to the hock, from which to the hoof he should be clean, flat, and sinewy. The back part of the thigh ought to have a considerable bend, as a straight-legged animal seldom possesses good action, although there are exceptions to this rule. Avoid those which are cat-hammed, that is, with their hocks nearly touching each other. See that the fore-legs are strong and muscular down to the knee, and otherwise formed as we have described 
the fore-legs. Let the feet be nearly circular, gradually increasing as they descend towards the sole. Their inclination outwards should not be so great as that of the pastern; the chances are that feet which slope too much forwards are diseased, or liable to it; besides, this obliquity throws the animal too much on his heels, which produces tenderness of the part, and straining of the back sinew.

The position of the legs and feet, or what may be termed their setting on, is a most important point. Viewing the horse in front, his legs should be as nearly straight as possible, and his feet neither inclining to the right or left; as feet turned outwards are very liable to cut and trip, and the action of the horse is seldom good or agreeable to the eye, having an outward stride, and loses ground thereby at every step. Horses with an inward inclination are said to be pintoed, or pigeon-toed. These generally throw the foot outwards, exhibiting the sole of the foot while in action to those who are standing on one side of him. Such horses have usually a laboured action, which fatigues the animal, and is equal to a third, less or more, of the distance he has actually travelled. The fore-legs should be set well under the fore part of the shoulder, affording ample support to it; such as have their legs placed forward possess neither power nor action. When the legs are viewed sideways, or in profile, they should be nearly straight, as in our representation of the horse (Plate I), but when horses have what are called calf-knees, that is, with a slight inclination inwards, and with the shank sloping forwards, it is a certain sign of weakness, and such horses will always more easily knock up with hard work than those which have straight or even prominent knees. 
The hind legs should either be straight from the hock downwards, or having a slight inclination uncler the beily. Horses so formed are, for the most part, low in the rump, and will throw out their legs well under them when in action. On the contrary, horses which throw their legs outward are always disagreeable to ride, and seldom work well. Horses that stand with their hind legs much under them, and at the same time which droop in the hind-quarters, may be suspected to be diseased in the spine or the kidneys, and should be carefully examined on those points; and while doing so, on no account permit a dealer's servant to hold up the horse by the bridle, or to stand on rising ground. This should be most especially attended to while examining the legs. It is the invariable practice of dealers' grooms when exhibiting a horse to throw the snaffle and curb reins over the head, and to hold him back with the latter, while he touches him up behind with a long whip, which has the effect of making him elevate his head, and brings him to his mettle, and therefore he hardly knows where he places his feet, consequently he will both bend his knees and throw out his feet much more than he does in his ordinary style of going. These wily servants take care always to bring horses to a stand, with his fore-quarters on rising ground, which makes him advance his fore-legs, so as to conceal any knuckling of the knees, or pasterns, and will give a groggy animal all the appearance of sounclness. Therefore let the horse that is intended to be purchased, be examined thoroughly on level ground, with his head at liberty.

The first point to be attended to is the crown of the head; to ascertain if he has the disease called poll-evil (see page 40), examine his nostrils, and if 
there is a fetid discharge, he may be glandered (I6) or have nasal gleet (22). To ascertain this, the nostrils should be pinched together for about a minute, to prevent him from breathing, and on removing the hand he is sure to snort, which will blow out any matter if he is diseased. The tongue should also be particularly looked at. Examine the eye for gutta-serena and blindness ( 36 and 37 ); see that the withers are not fistulous $(64)$; carefully scrutinise the knees (88), because a horse that has had broken knees must be suspected of stumbling; what he has once done, he may do again; see that there is no appearance of splent below the knee (93); nor grogginess in the region of the fetlock (100); nor ringbone of the pasterns (IO4); nor thorough-pin of the hock-joints (I09); attend particularly to the hocks, in case they are capped (IIO); and notice that there is curb a little way below these points; examine narrowly the inside of the hock-joint, in case bone-spavin or enlargement of the bony substance exist there (I I5); descend to the feet, and examine if there are symptoms of grease (123); see that there does not exist sandcrack in the horny substance of the hoof (I 35$)$; nor canker separating the horny substance from the sensible and fleshy part of the foot (I39). These are a few of the external maladies which the purchaser must particularly attend to, all or any of which dealers will be at no loss to account for. Disbelieve all their eloquent excuses; err on the safe side by rejecting the purchase. Sweeping as this condemnation may be thought, it is unfortunately too true that this class of men are not to be depended upon; and considering the risks which they themselves are liable to, it is not to be wondered at. If the legs exhibit any sign of having been bandaged, a well- 
grounded suspicion may be entertained that all is not right.

Besides the points to which we have directed attention in the examination of the mouth, are the teeth, whereby the age of the horse is determined. Take care that he has not been bishoped (2OI); or had a tooth extracted (I9I). The different changes of these we have very fully described at p. I 84 . But besides the dental indications, the physical signs of age must also be looked to ; because a young horse may have been early and hard worked, and to that extent that he is to all intents and purposes aged in strength and action. When heated by being trotted or galloped, all his infirmities will disappear; but these will re-appear whenever he is again cooled down.

A horse with an upright shoulder is more fitted for harness than riding; and a sloping one is best adapted for riding, from having generally better action and less of his own weight to sustain on his fore-legs. A long-necked horse is generally admired; but we consider this a fault, as such are generally weak, and are predisposed to roaring. Short-necked horses are for the most part clear in the wind; but one of medium length should be preferred. When the head joins the neck at too sharp an angle, it is always disagreeable to the eye. Horses whose limbs have marks of having been fired, should never be purchased but at a low price, for it is a proof of disease: although many horses will work well after being cauterised; we have known animals of high reputation as hunters and racers, which have been subjected to this operation.

When the cornea of the eye is of a yellowish tinge, it is indicative of liver complaint. This being observed, turn up the lips and notice their internal structure, and 
if they are of a similar hue, avoid the purchase of the animal.

If the coat of a horse stares, it is certain he is not in good health. It will be noticed that their dung is either unusually fetid and slimy, or it is soft and washy, like that of a cow. When not disturbed, such animals are languid and sleepy, but dealers take care in showing them off temporarily to rouse them from their lethargic condition, by inserting ginger into their anus, salt into their mouth, and giving them a smart touch or two with the whip; and no sooner does the master enter the stable, than they commence champing their bits, cocking their tails, and exhibiting for a short time all the appearance of perfect health and spirits. In the meantime, the dealer exerts his clap-trap eloquence to induce the novice to believe the animals are the best and most active horses in Britain; and they too often succeed in inducing such a belief. The hand should be drawn over the ribs, and the finger pressed firmly between them. If the skin appear tight and unyielding, it may be inferred that he is hide-bound, and consequently labouring under some internal disease. I would particularly direct the attention of the purchaser to the mark of a cut which may be some inches in length, and situated parallel with the shank-bone, immediately below the pastern-joint. If such a mark is found and the animal shows no signs of feeling, when his limb above the hoof is struck with the point of your boot, it is certain that the nerve operation has been performed, which we particularly noticed at page 218 . Such a horse should instantly be rejected.

In examining the foot, if the front and sides of the hoofs are marked with circular depressions of any depth running parallel to the coronet, it is certain that severe inflammation has existed at one time in the sensible 
portion of the foot, and is consequently liable to return again. See that both fore-feet, as well as the hind ones, are of equal size, and ascertain that they are quite cool, and equally so. Attend to what we have already said respecting the examination of the sole, and its proper form. Thrush may be detected by the fetid smell of the foot, besides the other symptoms which we have described, but it is not of much consequence.

As the feet of horses are of such importance, we would especially recommend to all to acquire a thorough knowledge of them; and we know no better plan than to attend daily at a well-employed shoeingforge, and for a small gratuity the smith will allow the various feet to be examined; and if he has had experience, he will be able to point out readily the indications of disease. He will also see why differently formed hoofs require the shape of the shoe to be modified, and adapted to the peculiarity of structure, or probably existing disease.

The next important point to be attended to is the wind and breathing of the animal. Study all we have said on the different complaints connected with the lungs; namely, roaring, broken wind, whistling, etc. The wind may be tested by pinching the wind-pipe immediately behind the jaw. If the horse give a long sharp cough, it is an indication that he is sound in that respect; but if the cough be short and hollow, it may be inferred that he has unsound lungs: let gentle pressure on the wind-pipe be repeated in order fully to test this. Be sure to perform this test with your own hand, as dealers know that by compressing the windpipe firmly with one hand and the fore-finger of the other, the horse is forced to cough while they do so, which produces that shrill sound considered a test of 
good wind, by the air rushing through the limited aperture. Having satisfied yourself by this first experiment that the horse is sound in the wind, proceed next to watch the flanks in breathing. If the belly of the animal swells out, and the inspirations and expirations are regular, it may be reasonably inferred that his lungs are sound; but if these are irregular, and the inspiration stops before it is completed, with a considerable drawing in of the flanks, with the ribs very apparent, then it is certain his lungs are unsound. The third test of broken wind is to trot the horse pretty sharply, and watch the motion of his flanks, and observe if he utters a noise in breathing, with considerable blowing. If this is the case, reject him.

To examine whether a horse is a roarer, piper, or whistler, place him with his side against a wall or the side of the stall; take hold of the bridle near the mouth, and hold his head high, and give him a smart blow on the ribs with your doubled fist, or touch him smartly on the belly with a stick; and if he utters a grunt at each blow, he is a roarer; and if he dances about in consequence of the blows, sobbing, and drawing his breath quickly, this will be found an indication of his being a whistler, or piper. But for testing all diseases connected with the lungs, nothing is better than a good gallop or hard trot. Consider no time wasted which is spent in thoroughly investigating all points connected with the health of a horse.

Having satisfied yourself on the above points, stand in the rear of the animal, and scrutinise carefully the prominences of the hip-bones, and see whether or not they are on a level, and especially mark the round bones, which are situated a little posterior to the prominences of the hip. This is liable to lameness from strains, blows, and other 
causes. If there is any fault here, there generally will be a wasting of the muscles, and the defect will be more readily detected when he is in motion. If any symptoms of lameness are observable, pass the hand over the spot, and heat will be found to exist in it, and it may be probably verified by the smell of some liniment, which may have been applied.

When examining a horse, never permit the dealer's man to hold his head high, nor to place his fore-feet on rising ground; because, while a horse stands in this position, the defects (if he has any) of his fore-legs will not be apparent; whereas if he stands with his feet upon level ground, if the limbs have been shaken from hard work, they will exhibit a tremulous appearance, and the knees will be more or less bent, and the heels will not rest firmly on the ground, as they ought to do. Horses that have been severely worked, have the fetlocks of the hind-legs bent and relaxed, and the natural elasticity of the tendons and ligaments will have departed. The horse that is groggy, when standing in a quiescent state, will be found in a posture leaning over the fore-legs, the feet of which will be further under the belly than the upper part of the leg, and the entire limb forming a flat semi-circle with the knee at the extreme point of the curve.

In looking at the action of a horse, see that his fore-feet are lifted high, and that he completely clears the ground and throws his legs out freely and lightly. This is especially necessary in a saddle-horse. Horses with a short, confined step, can never have good action, and are always disagreeable to ride. In walking, the knee ought to be moderately bent, but only sufficiently so that he may fairly clear stones and other objects which he may meet with on a road; and 
when the foot is set down, the sole should fall flat, so that the toe does not first touch the ground. The "legs should be thrown straight out; that is, the toes should neither be turned outwards nor inwards, nor should the sole be seen by a person standing on either side. In a larger-sized horse, the step must be lengthy and regular; in a smaller, compact horse, it ought to be sharp, active, and springy; and in either the marks produced on the ground by the fore-feet should be stepped on by those of the hind-feet; if, however, the animal is wide hipped, the hind-feet will rest on the outside of the marks left by the fore-feet. The head should be carried high.

In trotting, see that the horse does not lift his feet too high, and that he places them flatly and firmly on the ground, for if the toe first touches the ground, he is liable to trip. If the shoe is examined, it will at once indicate the part which comes soonest in contact with the ground, by being most worn down. Bloodhorses never raise their feet so well as those of more inferior breeding, and are in consequence not so safe to ride upon the road. Their action in the trot will, however, be found much more pleasant and easy to the rider. Blind horses are almost invariably high steppers, and therefore whenever you find a horse lifting his legs very high, it would be prudent to examine his eyes carefully.

Ladies generally prefer horses which go at a canter instead of a trot. Indeed, it is a much safer action for them, in consequence of their peculiar seat. In the choice of a lady's horse, take care that he has been accustomed to lead with both legs; because he is capable of longer duration, and the wear of his feet and shoes will be more equal. In the canter the hindlegs should be thrown well under the body, because it 
is a minor species of gallop. It will be found that horses with an oblique shoulder will perform both the canter and gallop better than those which are more upright.

If a horse is required for speed, do not choose one which holds his head high, as such is incompatible with a great stretch; consequently the style and bearing of a horse intended for show and park use is very different from those intended for rapid action; and these latter always carry their heads low. It is the habit of blood-horses always being trained to go over a smooth and level surface, which is the cause of their not lifting, being unaccustomed to meet with obstruction in their way. The hunter, on the contrary, being subjected to all kinds of ground, soon acquires the habit of lifting his feet sufficiently high to enable him to surmount all the difficulties which he must constantly encounter. The style of their gallop must also be essentially different: that of the racer, a lengthened stretch; and of the hunter, a rounded gallop. 


\section{CHAPTER XVIII.}

General History of the Horse, with an Account of Different Breeds.

Plutarch says a good man will take care of his horses and dogs, not only while they are useful to him, but also after age renders them unfit for service. A beautiful illustration of this benevolent maxim is recorded of the Athenians, who, when they had completed the building of the Hecatompedon, set at liberty the animals employed in its erection. It is related that one of these at the head of his fellowlabourers some time after the completion of the temple, led the way to the citadel, which so highly pleased the people that a decree was made by the senate, enacting that these faithful and willing servants should be kept the remainder of their lives at the public expense.

Near the tomb of Cimon were placed the graves of the mares who bore him, on three several occasions, victorious at the Olympic games.

Every humane mind must feel sensibly alive and indignant at the brutal treatment to which that noble and generous animal, the horse, is but too frequently exposed in Europe. The ass, also an animal of great sagacity and gentleness, is almost invariably treated with savage barbarity. Let these unprincipled and unfeeling wretches look to the mutual love which 
subsists between the Arab and his steed, and the kindness manifested by the people of eastern countries to their asses and mules, and the benefit they derive from such a mode of treatment. If no other principle will awaken their kindly feelings towards those most useful animals, surely that of self-interest should stimulate them to adopt gentler measures.

The first breaking and training of the horse should only be entrusted to persons of mild dispositions, as it is by kind and patient treatment alone that we can hope to succeed in rendering this valuable animal truly useful and docile; for although force may produce obedience, it will be found, as with man himself, that as soon as fear has subsided and the animal has discovered its own strength, revenge will generally follow. I have no doubt that in nine cases out of ten where horses betray furious or stubborn tempers, that these have been produced from the cruelty or ignorance of their first trainers. The horse is an animal of great intelligence; but everything addressed to his perceptions should be clear, short, and distinct, for he is incapable of following a train of spoken language. Few words, delivered with precision, accompanied by caresses and gentle treatment, will be found more effectual than any other course.*

The domestication of the horse may be regarded as one of the most important acquisitions made by man from the animal kingdom. Without this useful quadruped, civilisation must have made comparatively but little progress, and we should have been later by many centuries in emerging from barbarism. The horse contributes largely to our luxuries, pleasures,

* Wo ! used by carters to horses, is derived from the NormanFrench, and signifies, "attend, stop, listen." Gee! is derived from the German verb gehen, "to go." 
and service; he facilitates and lessens the labours of the field; he transports burdens, and man himself, to the most distant parts, with certainty, celerity, and ease; he is ever the faithful and obedient servant of his master. His form, sagacity, and temper, have been most admirably and wisely adapted for our use ; he is fitted in an eminent degree to fill a most important part in the scale of being.

The horse is framed with such a pliability of physical structure and constitution, that man may mould him to the form or bulk best fitted for the particular service in which he is to be employed. Whether we contemplate the powerful and symmetrical structure of his frame, the elegance of his limbs, evincing strength and speed in their movements; the delicacy and glossy sleekness of his skin; his large and sparkling eyes, which either beam with mild intelligence, or flash with energetic fire; or the docility and tractability of his disposition, we cannot fail to regard him as one of the noblest of animated beings. In addition to these qualities, he possesses the most intrepid courage; he has been from most remote times the bearer of man in the field of carnage, where he fearlessly meets every danger ; the most appalling discharges of musketry and the thunders of a cannonading, he faces with a fortitude as dauntless as that of his rider, and seems even to enter into the spirit of the attack. This has been his character from the earliest ages; for he is spoken of in Job, one of the oldest books in the world, and, few will deny, one of the best ever written, in the following powerful language, which is amended from the common translation by my late learned friend Dr Scot, Professor of Hebrew in the College of St Andrews :-

"Hast thou given spirit to the horse? Hast thou 
clothed his neck with a mane? Canst thou make him bound as a locust? The majesty of his snorting is terrible. He paweth in the valleys and exulteth; he goeth on to meet the armed men. He mocketh at fear, and trembleth not ; nor turneth he back from the sword. Against him rattleth the quiver, the glittering spear, and shield. He devours the ground with fierceness and rage, and is impatient when the trumpet soundeth. He uttereth among the trumpets, Ha! $\mathrm{Ha}$ ! He smelleth the battle afar off, the thunder of the captains, and the shouting."

The time at which the horse was first domesticated is now lost in the cloud of antiquity. He is mentioned by the earliest writers, and in all probability his subjugation has been nearly coeval with the earliest state of society. From the Scriptures we learn that seventeen hundred and two years before the Christian era, horses were used; for in the 47 th chapter of Genesis we are told that Joseph gave the Egyptians bread in exchange for horses. It seems probable, from the earlier chapters of Genesis, that horses were unknown to the Hebrews and Egyptians; as we find from the I th chapter of that book that Abraham " had sheep and oxen, and men-servants, and maidservants, and she-asses and camels," but no mention is made of horses: this was 1920 years before the birth of Christ.

But after this time they seem to have propagated and greatly increased in Canaan; as it is said in the eleventh chapter of Joshua and fourth verse, of certain kings opposed to Joshua, that there were "much people, even as the sand that is upon the seashore in multitude, with horses and chariots very many."

From many other parts of holy writ we find that 
horses were numerous in most of the kingdoms of the East, but no mention of the country from whence they were originally derived. It is a generally received, although erroneous opinion, that Arabia was the native country of the horse. We find that even so late as the seventh century of the Christian era, when the prophet Mahomet attacked the Koreish, not far from Mecca, he had only two horses in his train; and although, in the plunder of this horrible campaign, he carried with him in his retreat twentyfour thousand camels, forty thousand sheep, and twenty-four thousand ounces of silver, there is no mention of horses being part of the booty.

Solomon's stables seem to have been magnificent. He kept horses both for pomp and gain. His stud, even in our own times, is unequalled. He is said to have had four thousand stalls for horses and chariots, and twelve thousand horsemen! The price of a horse in those days was fifty shekels of silver, which amounts to about seventeen pounds, two shillings sterling; a very large sum at that remote period.*

It seems certain that Egypt was the first country in which the breed of horses was first improved, and from whence most of the early Eastern monarchs procured their studs. Solomon obtained many from thence; and although it abounded in horses, still I

* Dr Scot wrote me upon this subject:- "We allow that there is some controversy among scholars about the exact number of the stalls, and we dare not say that no mistake is introduced into the text. The probability, indeed, is very great that the most ancient and authentic copies of the text are corrupted. We should conceive that four thousand was a large number. This indeed is stated to be the number in 2 Chronicles, chapter ix, verse $25^{\text {th }}$; and even the parallel passage in I Kings, chapter iv, verse 26 th, commonly translated forty thousand, will bear to be so interpreted." 
think it more than probable that the Egyptians obtained their best steeds from the south, as the local situation of that country was but ill adapted to preserve them in perfection. This monarch, as well as several of his successors, obtained coursers from Togarmah, now understood to be the modern Cappadocia, or the lands which border the Euxine Sea, which was in early times the seat of several flourishing and powerful kingdoms.

To trace the history of the progress of the horse from that period to the present time would require a larger space than our limits will admit, and we must now turn to that part of his history which refers to the place of his nativity.

Left only to conjecture, we can only suppose, from a combination of circumstances, that Asia was the original country of the horse; for there he is found to the present day roving in unrestrained freedom, and we are without any historical record of his having been introduced by man into those extensive wilds. One thing is quite certain, that he was not found either in America or New Holland on the original discovery of these continents. The great tract of desert country around the Sea of Aral, as well as those of the Caspian Sea, have been supposed to be the native residence of the horse; but if this conjecture be correct, he must have widely extended his geographical range, for he is found in a wild state in Asia, as far north as the sixtieth degree, and to the utmost southern extremes of that vast continent, and also in many parts of Africa.

On each side of the river Don horses are found in a wild state; but these are supposed to be the offspring of Russian horses which were used at the siege of Azof, in the year 1697, as many were turned 
loose upon that occasion for want of forage. In South America, on these immense plains extending from the shores of La Plata to Patagonia, immense troops of horses are found, sometimes to the extent of ten thousand individuals. These are the offspring of emancipated horses which were taken to that continent by the Spaniards : for it is quite certain that the horse was unknown in America when that continent was first discovered. Indeed, the natives considered the horseman and horse as one animal. There great troops do not always feed in company, but are dispersed into smaller herds, and only congregate when they are alarmed. These animals are impelled by a natural instinct, which looks remarkably like reason, for they are invariably preceded by a leader in cases of alarm, and are sensible that their safety consists in united force, and a principle of subordination - the first things to be attended to, even by man himself.

In a domestic condition the horse is found in every country, such being the pliability of his physical constitution, that he thrives in very opposite extremes of temperature, except within the limits of the Artic circle itself. But in Great Britian he seems to have acquired the highest degree of symmetrical proportions and powers of speed; as our race-horses are universally admitted to be the finest and fastest gallopers in the world.

The celebrated race-horse, known by the name of Flying Childers, was the fleetest horse that ever ran, having repeatedly accomplished nearly a mile in a minute; and, carrying nine stone two pounds, has been known to perform the course at Newmarketwhich is three miles and three hundred and sixty yards-in six minutes and forty seconds, or at the 
rate of eighty-two feet and a half in a second of time. Eclipse was next to him in fame, and nearly his equal in speed, but considerably his superior in conformation, and from whom spring most of the finest horses which this country has produced.

Long ages of domestication, as well as the variety of climate to which horses have been subjected, has produced the great varieties in size and general conformation in the horse. Every country possesses horses with a peculiarity of character belonging to itself; and in each country there is great diversity of shape and size.

\section{SECTION I.-OF BRITISH HORSES.}

THE ENGLISH RACE-HORSE.

The form of the head, in this horse in particular, is like that of the Arabian. His beautifully arched neck is finely set on, and his shoulders are oblique and lengthened; his hind legs are well bent, his quarters are ample and muscular, his whole legs are flat, and rather short from the knee downwards, although not always so deep as it ought to be, and his pasterns are long and elastic.

The thoroughbred horse is by no means a safe one to ride on a road, as he seldom lifts his fore-feet high enough to enable him with certainty to clear the inequalities of the road. His action, in consequence, is much more pleasant; and he possesses another quality of much importance, namely, that he seldom or never will shy at any object on the road; two things of valuable consideration to the rider.

Thoroughbred is a term employed in Britain to indicate the descent of a horse from a South-Eastern 
courser. The English racer has therefore been the progressively improved breed, from a commixture of our own horses with those of Asia. The horses of the first blood, or such as are the nearest possible to the Eastern stock, are those immediately produced from the Arabian, or Barb; any stallion with an English mare, which has been already crossed with a Barb or Arabian steed, in the first degree; or that which has sprung from two crossings in the same degree. In its action the English race-horse is somewhat like that of the Arabian, but differs from the Spanish horse in carrying the whole of his frame forward with an energetic power, while the motions of the latter are measured with more of a graceful motion and shorter step.

In breeding, a mare should be chosen with as great a proportion of the blood of King Herod as possible. She should be deep in the girth, long and full in the fore-arm and thigh, short in the leg, standing clean and even upon the feet, and wide and spreading in the hind-quarters. It is a curious fact that the produce of our first-rate mares and an Asiatic horse seldom or never are good racers; and they must be one remove at least from the foreign stock before they can be depended upon.

\section{THE HUNTER}

Is a combination of the thoroughbred race-horse and half-bred horses of greater strength, and less lengthy in their carcase. He should be from fifteen to sixteen hands in height. The points most likely to discover a horse of good proportions as a hunter, are a sanguine and healthy colour, with a lofty forehand, a head and neck as light as possible, clear wide jaws and nostrils, 
large and thin shoulders, strong and muscular thighs, deep chest, and short back. Above all, his joints should be strong, firm, and closely knit, his legs and pasterns rather short; for I believe there never was yet a long hinder-legged horse that was able to gallop down steep hills and take bold leaps with a weight upon his back, without sinking or foundering. And, lastly, his feet should be moderately large and sound.

\section{THE HACKNEY, OR ROADSTER,}

Should be a hunter of a small size; his height not exceeding fifteen hands and an inch; rather below than above that size. His make should be more compact than that of the hunter, with considerably more substance according to his height, so as to fit him for the fatigues of everyday work. His forehand should be high, but rather light; his head small, and placed on the neck in a gradually tapering manner, with a clear, full, and sprightly eye; his shoulder should be deep and large; his back straight, and his loins strong; his withers well raised, his fillets wide; and his croop gradually descending, but must not drop too abruptly, nor must his tail be too low set. The forearm and thighs should be strong and muscular; and the legs rather short than otherwise, straight, and rather near set, but the touching of the hoofs to be carefully avoided. An essential point is, that the shank-bone should be solid and flat. It is of great consequence that the bones beneath the knee should be deep and flat, and the tendon not too much tightened. His feet ought to point straight forward, with the heels wide and open; the fore-legs closely set, and as straight as possible; for a horse with bent 
knees is very likely to stumble and fall when his feet come in contact with the smallest obstacle or inequality of the road. In his action he ought to lift his forelegs high, and have a well-bent knee; his hind-legs should be placed considerably behind him, and widely set.

We must, however, remark, that he ought only to lift his fore-legs moderately high. Some are of opinion that the higher he lifts them the better, and conceive that while possessed of this quality he never will come down. This is a mistake, and it will be found that a horse that raises his feet too high in trotting, produces a disagreeable action, and shakes and fatigues even the best of riders; besides, he batters his hoofs to pieces in a few years. The principal thing to be attended to is the manner in which the hackney puts his feet to the ground; for if his toes first touch the road, he is sure to be a stumbler; the foot should come flat down on the whole sole at once, otherwise the horse is not to be depended upon in his trotting. But every rider in passing along a road should be constantly on his guard, as the best horse may come down by a rolling or loose stone getting under his feet, and therefore his mouth should always be felt by the rider.

Some persons prefer hollow-backed horses on account of their paces being generally easy; but it is an ascertained fact that they will never stand much work: the back should be straight and rather short than otherwise. Many suppose that if the fore-legs of a horse are close, the feet must necessarily cut the pasterns; but this is by no means the case, as it is only when the feet are twisted or irregularly set in one way or other that they cut. Indeed a saddle-horse can hardly be too close before, or too wide behind. 
Another most essential point in a hackney is, that his sight is good, otherwise he is sure to shy at every object which suddenly meets his vision; and consequently by starting to one side is very apt to throw his rider.

\section{THE CHARGER.}

Much diversity of opinion prevails in this, as well as in other countries, respecting the kind of horse best suited for a charger. At one period, the whole British cavalry were mounted on strong, heavy horses, which were bred from the large Flander's horses, crossed by those of Britain. But in the Peninsular campaigns, it was found that lighter horses, with a considerable proportion of blood, were the most useful, as they got over wet, marshy tracts of country better than heavier horses. The qualities of a charger, or troop-horse, are much the same as those of a hunter. His action should be great, as well as his spirit; the neck deep and arched, with a large swelling breast; the ribs full and finely bent; the chine broad and straight; and the rear round and full; the legs broad and flat, and the pasterns short. In action, the charger enters into the spirit of the attack, as the hunter does that of the chase. In the words of the poet-

"The fiery courser, when he hears afar

The sprightly trumpets and the shouts of war, Pricks up his ears, and, trembling with delight,

Shifts place, and paws, and hopes the promised fight.

On his right shoulder his thick mane reclined, Ruffles at speed, and dances in the wind:

His horny hoofs are jetty black and round.

His chine is double, starting with a bound, He turns the turf, and shakes the solid ground; Fire from his eyes, clouds from his nostrils flow, He bears his rider headlong on the foe." 
The charger which has been in many battle-fields retains as long as he lives a remembrance of his past services, which is thus beautifully expressed in "The Pleasures of Memory," one of the finest didactic poems in our language :-

"And when the drum beats briskly in the gale, The war-worn courser charges at the sound, And with young vigour wheels the pasture ground."

THE COACH-HORSE.

The better kind of coach-horses owe their origin to the Cleveland bays; the greatest attention being paid to breeding them in Yorkshire, Durham, and Northumberland. Some fine horses of this kind have also been bred in Lincolnshire. The most useful are those which are propagated by a cross of the Cleveland mare, with a three-fourth or thoroughbred horse of sufficient substance and height. They have a fine knee action, lift their feet high, which gives grandeur to their figure and paces; the head is generally well carried, and with a beautifully elevated crest.

\section{THE CLEVELAND BAYS.}

This fine breed emanated from Cleveland, in Yorkshire, but are now bred in Northumberland and Durham. They are of a superior size and of a good form, with a strength and activity surpassing most other horses. They are chiefly distinguished by their bay colour. Mares of this breed, with full-blood stallions, produce excellent hunters and roadsters ; and with half-bred stallions, an offspring very suitable for farm purposes, particularly that of ploughing. 


\section{THE DRAUGHT-HORSE.}

Much variety of opinion has prevailed respecting horses for the purposes of draught, and consequently these are found to vary throughout the kingdom; but one principal character now prevails, that of weight, to give them more physical force. For ordinary purposes they should not be above sixteen hands high, with a light, well-shaped head and neck, short pricked ears, and brisk, sparkling eyes; the nostrils large and wide, to allow freedom in breathing; their chests should be full and deep, with large, strong, muscular shoulders, but rather lower in front than otherwise; that is, with a large and round rump, which should be higher than the forehand; the tail firm, strong, and well furnished with hair; the back straight and tolerably long; but not too much so, as that is found to impair the general strength of the animal ; the legs should be rather long, flat, and broad ; the fillets large and swelling, the joints closely knit ; they should stand wide on all their legs, the hind-quarters being wider than the fore.

Large horses are better adapted for waggons, and have frequently been bred seventeen hands high, and even more, with elevated forehands, and deep counters. The great object in the breeding of draught-horses is to increase strength, activity, and power; to remove weight as much as possible, and procure them of the height of sixteen hands for general utility. Indeed it has been proved that horses of this size have performed feats of strength of greater magnitude than those of elephantine proportions. I remember to have seen a black cart-horse, of sixteen hands, draw thirty-six hundred weight of baggage from Glasgow to Stirling, a distance of twenty-seven miles, in about eleven hours. Instances have been known where a single horse has 
drawn a weight of three tons for a short distance. In former times, when burdens were removed from one locality to another by horses without carts, the packhorses of Yorkshire were accustomed to carry the weight of four hundred and twenty pounds over the old roads, which usually traversed high and precipitous hills.

\section{THE SUFFOLK PUNCH-HORSE.}

This hardy and active breed has now become nearly extinct. They are rather under sixteen hands in height, and their colour chestnut or sorrel. Their heads are rather large and coarse; their ears being too long and placed too distant from each other for modern taste. The body is deep, capacious, and compact; the shoulders wide and thick at top, and somewhat low, with the rump more elevated than the shoulder, which it is supposed enables them to throw much of their weight into the collar. They are large and strong in the quarters, full in the flanks, flat and short in the legs, with short pasterns.

In the "Sportsman's Repository" we are told that "they were the only race of horses which would collectively draw repeated dead pulls, namely, draw pull after pull, and down upon their knees, against a tree, or any body which they felt could not be moved, to the time of Jup, Ji!! and the crack of the whip (once familiar, but abominable sounds, which even now vibrate on our auditory nerves) as long as nature supplied the power, and would renew the same exertions to the end of the chapter."

The hideous yelling of most carters and farm servants, which is still prevalent when driving horses, not only in this country, but also on the Continent, is a barbarous custom ; for I have known many instances 
where gentlemen subdued this practice in their servants, and the most gentle and temperate accents were found to succeed better than the frightful and thundering exclamations in general use. Every possible means should be used by those who have either influence or power over that class of men, to abolish this noisy and useless practice, which not only stuns the poor, willing animal, but is also a great nuisance while passing through the streets of a town.

The old Suffolk breed of horses brought very high prices, but of late a larger breed has become more fashionable in that country and neighbouring districts, which for largeness and beauty certainly excel the old breed. They have been produced from a cross with the Yorkshire half- and three-part bred horses of the coach kind, and are particularly beautiful and lofty in the forehand. In the year I8I3, at a sale in Suffolk of the stock of a celebrated breeder, which was, in consequence, numerously attended by persons of rank and opulence, the horses brought considerable prices. The following were a few of them:-A mare, with a foal at her feet, $£ \mathrm{I} 24,4 \mathrm{~s}$; a three-year-old filly, $£ 85$, Is ; a mare, which had lost the sight of one eye, but of a beautiful form and powerful make, $£ 98$, i 4 s. The whole of his stud consisted of fifty mares, geldings, and foals, and brought the large sum of $£ 226_{3}$, I 3 s 6d.

\section{SECTION II.-OF ASIATIC HORSES.}

THE ARABIAN.

Arabia being sufficiently above the level of the sea, and having a surface composed of sand, mixed with a portion of vegetable mould (a circumstance favourable to pasture-ground), and the plains of Persia, 
situated still higher above the ocean, and consisting of a deposit of alluvial soil, resting on granite, are naturally dry, and by means of their heat attract moisture from the horse. On the other hand, the aromatic vegetation, which is there strong and succulent, drives from him those humours, the exudation of which is favoured by the imperceptible, but continual, perspiration incidental to a warm climate. The consequence is, that the horses of those and other countries with similar pasturage, are completely free from the strangles and other glandular diseases, which are so frequent and fatal to those of Europe.

The pure Arabian is considerably smaller than our racehorse, seldom exceeding fourteen hands two inches in height. His head is very beautiful, clean, and wide between the jaws; the forehead broad and square; the face flat; the muzzle short and fine; the nostrils large and open; the eyes prominent and brilliant; the ears small and handsome; the skin of the head thin, through which may be distinctly traced the whole of the veins; the neck rather short than otherwise. The body, as a whole, may be considered too light, and the breast rather narrow; but behind the fore-legs, the chest generally swells out greatly, and with much depth of ribs, leaving ample room for the lungs to play; the shoulder is superior to that of any other breed; the scapula, or shoulder-blade, inclines backward, nearly at an angle of forty-five degrees; the withers are high and arched; the neck beautifully curved; the mane and tail long, thin, and flowing; the legs fine, flat, and wiry, with the flanks placed somewhat oblique, which has led some to suppose that their strength was thereby diminished; but this is by no means the case: the bone is of uncommon density, and the prominent muscles of the 
forearms and thighs prove that the Arabian horse is fully equal to all that has been said of his powers. The Arabian is never known in a tropical climate to be a roarer, or to have curbs; the shape from the point of the hock to the fetlock being very perfect. It is a remarkable fact that the skin of all the lightcoloured Arabians is either pure black or bluish black, which gives to white horses that beautiful silvery grey colour so prevalent among the coursers of noble blood. If an Arabian horse exceeds fourteen and a half hands in height, the purity of the blood is always doubted in India. Three of the swiftest horses which were known in our own times at Madras, were under fourteen hands.

Above all others, the Kohlan horse of Arabia is distinguished for his superior qualities and the beauty of his form. He possesses an uncommon mildness of temper, an unalterable attachment to his master, a courage and intrepidity as astonishing as they are innate in his noble breast, an unfailing remembrance of the places where he has been, of the treatment he has received; not to be led, not to be touched but by his master; in the midst of carnage in battle he is cool and collected; he never forgets the place he came from, and though mortally wounded, if he can gather up sufficient strength, he carries back his desponding rider to his defeated tribe. His intelligence is wonderful, and he seems to know when he is sold. When the proprietor and purchaser meet for that purpose in the stable, the Kohlan soon guesses what is going on, becomes restless, gives from his beautiful eye a sideglance at the interlocutors, scrapes the ground with his foot, and plainly shows his discontent.

The action of the Arabian in his native plains is very beautiful. He carries his head high, which gives 
him a dignified aspect; his tail is turned up in the air, and forms a most graceful curve, which our English clealers have vainly attempted to imitate by the cruel and absurd practice of nicking the vertebræ.

In Arabia the horse is treated with the utmost gentleness, kindness, and affection. He inhabits the same tent with his master and family. His wife and children, with the mare and her foal, associate together in indiscriminate friendship, occupying the same bed, where the children may be seen prattling with and climbing over the bodies, and hanging round the necks of the docile creatures, who in their turn will frequently repose with their heads inclining on some one of the family. Whipping, by an Arab, is considered the greatest cruelty to horses, and it is by gentle measures alone that he secures the willing service and affection of his steed. Their friendship is mutual; for if the rider falls, although in the most rapid career, the horse instantly turns round, and halts till remounted by his master.

The Arab will never sell a mare on any consideration whatever. The genealogies are always recorded from the dams. In the pedigree of their steeds they are more particular than any other people on earth; it is an undoubted fact that they have pedigrees among them of not less than five hundred years. In this respect they look upon it as of more importance than that of their chiefs. Among the great dealers, they pride themselves upon being rigidly strict, and are more to be depended on than many of those of Europe in the pedigree of the horses they offer for sale. Weston, in his "Fragments of Oriental Literature," gives the following pedigree, which was hung about the neck of an Arabian, purchased by Colonel Ainslie 
during the campaign of Egypt :- "In the name of God, the merciful and compassionate, and of Seed Mohammed, agent of the high God, and of the companions of Mohammed, and of Jerusalem! Praised be the Lord, the Omnipotent Creator! This is a highbred horse, and its colt's tooth is here in a bag about his neck, with his pedigree, and of undoubted authority, such as no infidel can refuse to believe. $\mathrm{He}$ is the son of Rabbamy, out of the dam Lahadha, and equal in power to his sire - of the tribe of Zazhalah. $\mathrm{He}$ is finely moulded, and made for running like an ostrich, and great in his stroke and his cover. In the honours of relationship, Zaluah reckons Lalaack, sire of Mahat, sire of Kallack, and the unique Alket, sire of Manasseth, sire of Alsheh, father of the race down to the famous horse, the sire of Lahalala;---and to him be ever abundance of green meat and corn, and water of life, as a reward from the tribe of Zazhalah, for the fire of his cover; and many a thousand branches shade his carcass, from the hyæna of the tomb, from the howling wolf of the desert ; and let the tribe of Zazhalah present him with a festival within an enclosure of walls, and let thousands assemble at the rising of the sun, in troops, hastily, where the tribe holds up under a canopy of celestial signs within the walls; the saddle, with the name and family of the possessors. Then let them strike the hands with a loud noise incessantly and pray to God for immunity for the tribe of Zoab, the inspired tribe."

The Arabs have a breed of horses which they pretend is descended from the stud of King Solomon; but this, of course, is not the case; at all events, exceedingly unlikely.

The Persian, Tartar, and Turkish horses are all 
somewhat similar to the Arabian, but not so perfect in their symmetry.

THE PERSIAN HORSE.

From the most remote ages Persia has been famous for its breed of horses, which even now are only excelled by the Arabian breed. The former, however, were noted for their excellence long before we have any account of the latter. The Persian horse is considerably larger than the Arabian. The neck is beautifully arched, with a finely-formed crupper, and the whole frame more developed. We are informed by historians that Alexander the Great considered a Persian horse as a gift of the highest value; it was one which he only bestowed on potentates and favourites of the first class.

The Parthian kings used to sacrifice Persian horses to their divinities; this they considered the most costly offering they could make.

THE TARTAR HORSE.

In the widely extended plains of independent Tartary, there are various breeds of wild horses, which differ considerably in their external conformation. Those in general are ill-made, clumsy animals, but are said to possess great speed. They are hunted by the natives on account of their flesh, which is considered a great delicacy, more especially that of the foals, which is prized above that of all other food. The better formed animals are tamed and domesticated.

The largest of these wild coursers are a little larger than the smaller Russian horses, with large heads in proportion to the size of the body, and some 
of them have ears nearly as long as those of asses; their mane is very short and curled, their tails more or less covered with hair, but always shorter than that of a common horse, and certainly far from being a graceful ornament. They are for the most part of a mouse colour, and a few are of an ash-grey; their coat is very long, and extremely thick.

\section{THE TOORKOMAN HORSE.}

Turkistan is situate to the north of the Caspian Sea, or South Tartary, and has been long celebrated for a breed of horses possessing great physical powers : these are called Toorkomans. Some travellers affirm that they are greatly superior to the Persian race for enduring long-continued exertion.

In their form, however, they are by no means attractive, being too small in the barrel, with too long legs, with lank necks, and heads disproportionably large to the body. In size, they are from fifteen to sixteen hands high, and of excellent temper.

In that portion of central Asia peopled by the Tartars and Calmucks, are many herds, or tabunes, of wild horses. These herds may be seen grazing together on the same plain, but never intermingle, nor do any individuals of the respective herds stray from them. Their forms are so different that horses of one herd can at once be distinguished from those of the other.

\section{THE TURKISH HORSE.}

The horses of Turkey are chiefly descendants from those of Arabia, Persia, and Barbary. Their foreheads are slender, and they carry their heads higher than the Arabian breed; their bodies are longer, and 
their crupper more elevated. They are possessed of a great deal of fire and spirit, extremely active, and peculiarly adapted to the Turkish mode of performing military evolutions. They are said to evince great affection for their masters.

\section{SECTION III.-OF AFRICAN HORSES.}

The horses of Africa are next to the Arabian in point of lineage and excellence, and are probably sprung from them. They generally possess a fine form and that mildness of disposition peculiar to the Eastern horses.

\section{THE BARB.}

The present horses of Morocco are a race nearly allied to the Arabian, and have been produced by a cross of those of Algiers, which are supposed to have had their origin in a south European breed, crossed with the Arabian, but somewhat larger than the latter in size, with fine heads and crests, and in general well formed about the shoulder, straight backed, but droop considerably towards the haunches. They are exceedingly swift; and as none of them are geldings they possess great spirit, and are naturally fiery in their dispositions.

The forehead of the Barb is generally long and slender, and his mane rather scanty; his ears are small, beautifully shaped, and so placed on the forehead as to give him great expression; his shoulders are tight, flat, and sloping backwards; withers fine, and standing high; loins short and straight; flanks and ribs round and full, without producing in him too large a belly; his haunches are strong and elastic; the croup is sometimes long to a fault; the tail is placed 
high ; thighs well turned and rounded; legs clean and beautifully formed, with the hair thin, short, and silky; the tendons are detached from the bone, but the pasterns are frequently too long, and bending; the feet are rather small, but sound for the most part.

\section{THE EGYPTIAN HORSE.}

These horses are not at all to be compared with those of Persia and Arabia. They have neither the fire nor shape of these breeds, and are said to be thick in the breathing. These bad qualities, in all probability, arise from the humid atmosphere, and the low alluvial flats on which they are pastured.

\section{THE NUBIAN AND DONGOLA HORSES.}

Bruce says, "The Nubian horses are beautiful and symmetrical in their parts, of large size, great strength, and most active, agile, nervous, and elastic in their movements, capable of great endurance of fatigue, docility of temper, and seeming attachment to man, beyond any other domestic animal ; and if these faculties can promise anything for a stallion, the Nubian is, above all comparison, the most eligible in the world. They are all kept monstrously fat." They are black or white, but a vast proportion of the former to the latter; and a few bright bays, or inclining to sorrel.

The horses of Dongola, like those of the district of Nubia, are of large size, standing full sixteen hands high; but the length of the body, from the shoulders to the quarters, is considerably less, so that their form is quite opposed to that of the Arabian or English thoroughbred horses, which are longer than they are high by some inches. Their necks are long and 
slender, and their crests very fine; the withers sharp and high, producing a beautiful forehand. They are, however, faulty in the breast, being too narrow, and the quarters and flanks too flat, with the back somewhat bent. Bosman thinks them the most beautiful in the world. One of these horses was sold in Grand Cairo in 1816 for a sum equivalent to one thousand pounds sterling.

Several of these steeds have been imported into Europe, and some into England; but they did not turn out so well for breeding from as was expected. This failure might possibly arise from not breeding them with the kind of animal to which their qualities are likely to be the most useful. It is very probable that they might improve our cavalry horses by crossing them with three-part bred mares.

\section{SECTION IV.-AMERICAN HORSES.}

Horses are found in vast numbers in a wild state in the immense plains of South America, extending from the shores of La Plata to Patagonia. They are an emancipated race, emanating from those which were carried thither by the Spaniards, after their discovery of the new continent; and have increased with such astonishing rapidity, that they are to be seen in troops of many thousands. Azara affirms that they sometimes congregate in squadrons of not less than ten thousand individuals. They are invariably preceded by a leader, by whose movements they are governed; and all they do seems to be conducted in a systematic style.

These immense troops do not always feed together, but are dispersed into smaller herds; though when disturbed they congregate, and continue so until the 
cause of alarm has passed away. In form they bear a strong resemblance to the horses of Barbary and Turkey. Their colours are chestnut, bay, sorrel, or black; the latter, however, is not very common, chestnut being the prevailing colour, from which some authors suppose that this must have been the original colour of the horse; but we do not find it to be the prevailing colour of the Asiatic wild breeds, bay and dun being the most common amongst these.

When the Spaniards first entered Mexico, their horses were objects of the greatest astonishment to all the people of New Spain. At first they imagined the horse and his rider, like the centaur of the ancients, to be some monstrous animal of a terrible form; and supposing that their food was the same as that of man, brought flesh and bread to nourish them.

In South America mares are never ridden. An Englishman, who once attempted to ride a mare, was so hooted and pelted by the natives, that he had a narrow escape, and thought himself fortunate to get off without serious injury.

Wild horses are captured in South America by the native inhabitants of the plains, who are called Gauchos. They are taken by these men with much dexterity, with a halter called a lasso; which is thus described by Miers, in his Travels in Chili :- "The lasso is a missile weapon used by every native of the provinces of Chili. It is a very strong, plaited thong, of equal thickness, half an inch in diameter, and forty feet long, made of strips of green hide plaited like a whip-thong, and rendered supple by grease. It has at one end an iron ring, about one inch and a half in diameter, through which the thong is passed and forms a running noose.

"The Gaucho, or native Peon, is generally mounted 
on horseback when he uses the lasso. One end of the thong being affixed to his saddle-girth, the remainder he coils carefully in his left hand, leaving about twelve feet, belonging to the noose end, in a coil, half of which he holds in his right hand. He then swings this loose noose horizontally round his head, the weight of the iron ring at the end of the noose assisting in giving to it, by a continued circular motion, a sufficient force to project it the whole length of the line."

The Gaucho takes a wild horse by first mounting an animal which has been accustomed to the sport, and gallops over the plain in the direction where the herd of wild horses are, and, circling round, by degrees gets near to one of them; and as soon as he has approached sufficiently near, "the lasso is thrown round the two hind-legs, and as the Gaucho rides round a little on one side, the jerk pulls the entangled horse's feet laterally, so as to throw him on his side, without endangering his knees or face. Before the horse can recover the shock, the rider dismounts, and snatching his cloak from his shoulders, wraps it round the prostrate animal's head. $\mathrm{He}$ then forces into his mouth one of the powerful bridles of the country, straps a saddle on his back, and bestriding him, removes the cloak; upon which the astonished horse springs on his legs, and endeavours by a thousand vain efforts to disencumber himself of his new master, who sits quite composedly on his back and by a discipline which never fails, reduces the horse to such complete obedience, that he is soon trained to lend his whole speed and strength to the capture of his companions."

There is a remarkable difference in the dispositions of the Asiatic and South American wild horses: those of the former country can never be properly tamed, unless taken and trained very young; if 
captured when adults, they frequently break out in fits of rage in after life, exhibiting every mark of natural wildness; whereas those of America can be brought to perfect obedience, and even rendered somewhat docile within a few weeks. 


\section{CHA P T E R XIX.}

\section{The Ass and Mule.}

\section{THE ASS.}

THIS animal belongs to the same natural genus as the horse, and has been under the dominion of man from the earliest ages of which we have any account. Indeed, he seems to have been sooner domesticated than the horse; for we find asses mentioned in the twelfth chapter of Genesis, as domesticated, I920 years before the Christian era, although nothing is said of the horse.

In early times, the ass was not, as is now the case with us, considered a despicable animal; for we find that he was rode by the rich and noble in preference to the horse, as will appear from the following instances, which we select from many that are recorded in the sacred writings:-When Abraham went to offer his son Isaac, he rode upon an ass: Joseph's brethren rode on asses when they went down to Egypt to purchase corn; and we are told that when Moses left Jethro, his father-in-law, he took his wife and his sons, and set them upon asses, and returned to Egypt. In the enumeration of Job's property, which appears to have been very great, we find that he had five hundred she-asses ; and in his prosperity he is said to have had a thousand. It is probable that the preference given to she-asses arose from the 
circumstance, that as the ass can subsist on a scanty and coarse fare, the female would not only bear the riders and their goods through the desert, but also with her milk contribute to the support of her master and his followers.

The ass is by no means that stupid animal which he is generally supposed to be. He possesses all the senses in a very high degree, and his perceptions are clear and precise; and it may be affirmed that he has more solid good qualities than the horse. His disposition is naturally timid; hence the caution of his movements, which the thoughtless have improperly attributed to stupidity and obstinacy.

The ass is robust in constitution, and is liable to few diseases. His temperance is very great, being able to subsist on a scanty meal, of the coarsest herbage. He is more sure-footed than the horse, and superior to him in comparative strength. The Hebrew word athor is a term for the ass, from a root which signifies strong or firm; and he is less easily startled than the horse, a failing in this quadruped by which many lose their lives. Those very qualities which unthinking men ought to appreciate, have, on the contrary, the effect of bringing upon the unfortunate and patient animal an overwhelming load of unmerited hardships, barbarous chastisement, and contempt. His services are despised by the rich; and he is destined only to share the labours of the poor and needy, whose hearts are hardened by poverty, with scarcely a kindly feeling to bestow on the humble and patient animal, who exerts all his energies in their service, and whose scanty meal is often so limited as to be hardly sufficient to sustain life, and on whom, when weak and debilitated, the abject wretch but too frequently wreaks his chagrined feelings. Is it then 
to be wondered at, that the poor animal should exhibit a character of stupidity and dullness? It is chiefly in Britain that this valuable animal is treated with barbarity, and is a disgrace to our national character. Cruel treatment to any animal should be punished with the utmost severity by the legislature; for although the Almighty has given man dominion over the beasts of the field, yet the All-wise could never intend man to tyrannise over the poor, helpless creatures which have feelings equal to his own, and gratitude far surpassing the lords of the creation, when properly treated.

In Eastern and mountainous countries, the ass is held in high estimation, and is treated with that care which he so justly merits. In mountain tracts his services are invaluable in transporting travellers and their baggage where the horse is of no use. In Aleppo there is a large and handsome breed, and in Persia also, where they sell for seventy-five ducats to one hundred crowns.

\section{THE MULE.}

It is a matter of surprise that mules are not more bred in this country, as their action is much more agreeable than that of the horse; and they are also more hardy in their nature. It is not known when mules were first bred. The first mention of them in the sacred writings is I 740 years before the Christian era. In the book of Genesis it is said, "This was that same Anah that found mules in the wilderness as he fed the asses of Zibeon his father." It is a disputed point whether he was the first breeder of them : Aristotle and Pliny are of opinion that he was. However this may be, mules do not appear to have become 
common in India until the reign of David, which was about 300 years after the death of Anah. We think it very improbable that wild mules were found, as hybrids are only known to be generated under the influence of domestication. Or, if the manner of engendering mules were known to the Israelites, that people probably desisted from breeding them in consequence of the law of God against their propagation ; for it is said, "Ye shall keep my statutes. Thou shalt not let thy cattle gender with diverse kinds." It is, therefore, likely that the mules which David and his nobles rode were imported from other countries, where they appear to have been common long before his reign. In Greece and Cappadocia they abounded in early times, for the latter country paid an annual tribute of mules to Persia.

The mule, like its progenitor, the ass, is much more useful as a beast of burden in mountainous countries than the horse, being very sure-footed. Their long and sweeping pace on a plain makes their motion extremely easy and agreeable to the rider. Fifty or sixty pounds sterling is no uncommon price for a fine mule in Spain. 


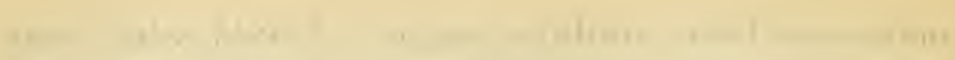

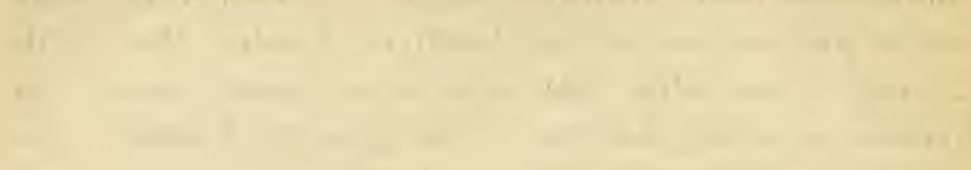

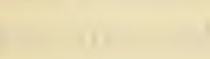

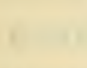

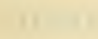

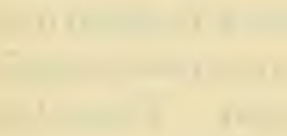

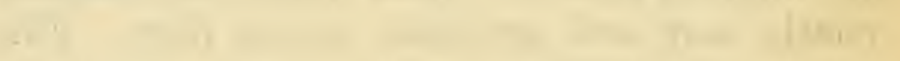

and 


\section{SECTION II.}

\section{DISEASES OF CATTLE.}

\section{INTRODUCTION.}

THE chief attention of the veterinary art has been devoted to diseases of the horse. This has proceeded in a great measure from selfishness on the part of those practising it, being better paid for the treatment of horses than cattle. This department has been hitherto almost entirely practised by farmers, and uneducated country blacksmiths. However, $\mathrm{Mr}$ Dick, of Edinburgh, Professor of Veterinary Surgery to the Highland Society, has done much towards increasing our knowledge in this most important branch : indeed, none is more so, when we consider so much of the comfort and health of the human race depends upon the quality of that food so much used in almost every country.

The following judicious remarks are made by $\mathrm{Mr}$ Lawrence in his excellent work:- "It should be considered that animals, living in a state of nature, regulated by the reason and experience of man, would be almost wholly exempt from disease; that their appetites, like our own, may be held under a constant control; that their diseases result purely from negligence or erroneous treatment of their owners. They are either too much exposed to the 
rigour and changes of the weather, or they are gorged with food, denied a sufficient quantity, or supplied with such as is unwholesome. Here we learn the chief cause of their maladies. Learn to prevent them, instead of undertaking the tedious, unsuitable, and hopeless task of learning to cure them. Of all things let the proprietors of cattle renounce for ever the insane folly of offering premiums for curing incurable diseases, and the hope of providing medicines which, by a sort of miraculous operation, will enable men to continue in the habit of exposing their animals to the constant risk of such diseases. I have no infallible recipes to offer. On the contrary, I wish to impress my readers strongly with the idea that all infallible recipes are infallible nonsense."

We agree with $\mathrm{Mr}$ White, that, "almost all the diseases of cattle arise either from exposure to wet and cold weather, from their food being of a bad quality, or deficient in quantity, or from being changed too suddenly from poor, unwholesome keep to richer pasture. It is necessary to observe also that the animal is more liable to be injured by exposure to wet and cold, when previously enfeebled by bad keep, old age, or any other cause, and particularly when brought from milder and more sheltered situations. I have scarcely met with a disease that is not attributed by those who have the care of cattle, to a chill; and under this impression the most stimulating medicines are usually employed : among which we generally find grains of paradise, ginger, long pepper, and mustard, in large doses. It unfortunately happens that the disorders arising from a chill are often of an inflammatory nature, and require a very different treatment. It must be granted, however, that cattle more frequently require stimulating medicines than horses; 


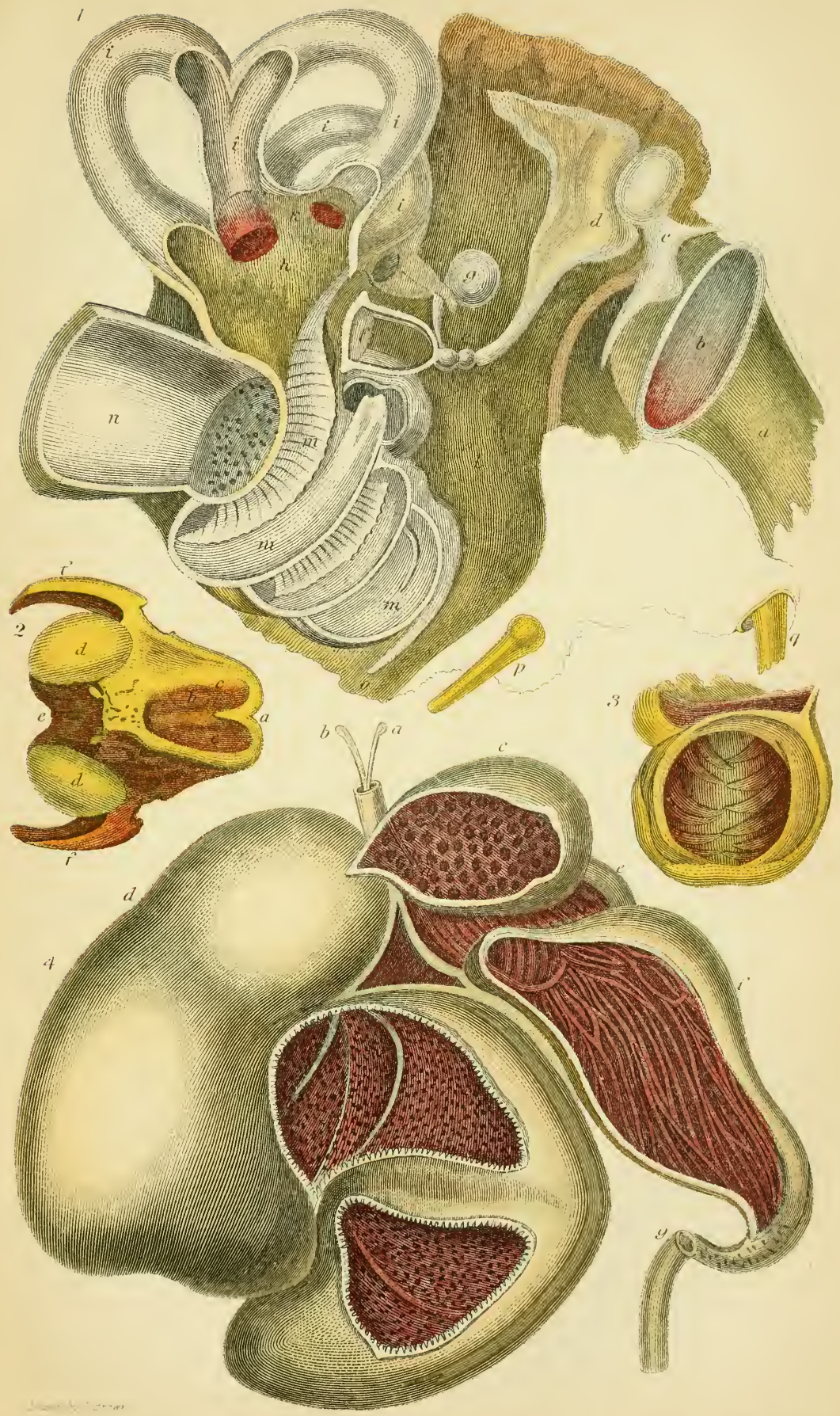


and that bleeding is not so frequently necessary, nor is it carried to such an extent in the former as in horses, particularly in milch-cows. Many of the medicines of which their drinks or drenches are composed are quite inert, some are nearly so, and others are very nasty."

Black cattle, sheep, and goats are included in a distinct order called ruminants, or those animals which chew the cud. They have three kinds of teeth; and are destitute of the incisory or cutting teeth in the upper jaw, but are furnished with eight in the lower one, which are opposed to a dense callous structure in the upper gums. There are twelve grinders in each jaw, marked with two double crescents of enamel on their crowns, of which the convexity is outwards in the lower, and internal in the upper jaw. They have four stomachs, calculated for ruminating, or the faculty of masticating their food a second time, by bringing it back to the mouth after a deglutition, a faculty depending upon the structure of their stomachs. The three first stomachs are so disposed that the food may enter into either of them, the œsophagus terminating at the point of communication.

The first, and greatly the largest, is called the paunch or "rumen," and occupies a considerable portion of the abdominal cavity. In this bag the food is macerated after very slight mastication ; it is divided externally into two saccular portions. It is in this cavity that all these morbid concretions are formed, called hairy balls, etc. (see Plate xIII, fig. 4, a). The second stomach is called the honeycomb-bag or "reticulum," in consequence of its parieties being laminated like a honeycomb. It is much smaller than the first, and of a globular form. Its office is to seize, moisten, and compress the food into little pellets, which afterwards 
successively ascend to the mouth to be re-chewed. The animal remains at rest during this operation, which lasts until all the food first taken into the paunch has been submitted to it. The aliment thus re-masticated descends directly through the œsophagus into the third stomach. Now, as this latter tube communicates with three of the stomachs, the contents of the mouth may be sent into any of the three by the will of the animal. This stomach is the smallest of the three, and resembles a rolled-up hedgehog; its external coat has broad duplicatures, like the leaves of a book, and is called the manyplies or "omasum" (see $3^{a}$ ). There the food only remains a short time, and undergoes some change which fits it for being received into the fourth stomach, which is called the abomasum, the sides of which are wrinkled, and which is the true organ of digestion, corresponding with the same organ in man and other mammiferous animals. The internal coating furnishes plentifully the ordinary gastric secretions for facilitating digestion. There is a beautiful provision in Nature, that while the young ruminants are still feeding on milk, this stomach is the largest of the whole. The paunch is only developed by receiving increased quantities of grass, which finally gives it an enormous volume. The intestinal canal is very long, though there are but few enlargements in the great intestines. The cæcum is likewise long and tolerably smooth.

Of all animals, the ruminants are the most useful to man. He can eat all parts of the animal ; and it is from that he procures most of the flesh which constitutes his aliment.

The passage of the food through these various stomachs will be easily understood by reference to fig. 4, Plate xiII. Its course is indicated by the 
direction of the probes $a, b$. The osophagus communicates on one hand with the paunch, I, and on the other, with the cavities, 2, 3, 4; and by the aid of a muscular fold formed by the walls of the second cavity, a passage may be formed which leads directly into the third stomach, without communicating with the second. It would therefore seem that the process of rumination is effected in the following manner:-The herbage when first swallowed in an unmasticated form passes into the paunch, I, where it accumulates, and undergoes a kind of preliminary mastication. When this paunch is filled, the animal desists from grazing, and the food is again regurgitated into the mouth to undergo that more complete mastication. It then passes into the second stomach, or reticulum, and is there formed into a smooth, lubricated bolus, which being expelled into the œsophagus, is immediately seized by the spiral muscles surrounding that canal, and forced forward into the mouth. After undergoing a thorough trituration, the aliment is again swallowed, and then enters the third stomach. Here it is spread out over the extensive surface formed by the laminated walls of the manyplies, and is prepared for admission into the last or true stomach. 


\section{CHAPTER I.}

\section{Diseases of the Head and Neck of Neat Cattle.}

\section{INFLAMMATION OF THE BRAIN.}

Symptoms. - This fatal disease but seldom occurs in neat cattle. The animal afflicted with the disorder exhibits a frightful appearance; he is unusually watchful, starts frequently, and groans loudly, as if labouring under severe and sudden pain; his respiration is slow, although he will sometimes make long inspirations, and has all the appearance of his breathing being entirely suspended. The animal becomes exceedingly restless, lying down, starting and rising up suddenly and frequently, with signs of delirium, and groans piteously. When this becomes great, the eyes have a red and furious appearance; and at other times he manifests symptoms of stupefaction and listlessness, and shows evident signs of constant fear, avoiding every object which he approaches. Sometimes he is quite ungovernable, and never lies down to repose. If the disease has been allowed to gain an ascendency, or has not been abated by the ordinary treatment, then the animal sinks into a state of stupefaction and total want of energy or tact. In some cases, the animal urinates frequently, and it is of a high colour; at other times it is paler than usual, but in both cases he stales frequently. 
When strong lethargic symptoms ensue, in consequence of sudden abatement of the frenzy and irritation, the pulse becomes feeble, and the strength quickly diminishes. This is strongly symptomatic of approaching dissolution. But on the other hand, if the fever and redness of the eyes gradually subside, without the pulse suddenly sinking, or great and quick debility ensuing, it is pretty certain that the animal is in the way of recovery.

When cattle have died of this complaint, their heads have been opened, and it has been found that the membranes have exhibited strong inflammatory symptoms, and in many cases in the substance itself, and even effusion of lymph, serum, and blood, where the animals have been greatly frenzied.

Causes. - Great heat, in exposed situations, where there is no shade to screen them from the mid-day sun, an excess of exercise, a sudden change from poor to rich pasture, and an inadequate supply of water, poisonous plants; with other causes which induce congestion and inflammatory febrile complaints.

REMEDy.-Whenever the symptoms which we have above described are noticed, prompt decisive measures must be adopted. Either the jugular vein or temporal artery should be opened, and blood taken. When the animal labours under a very high degree of excitement, the more rapid the discharge of blood the better. When bleeding has been effected, a strong stimulating blister should be applied to the crown of the head, over the brain, and the sides of the neck should be rubbed with a mixture of cantharides and oil of turpentine, and every possible means adopted to induce external inflammation, so as to determine the blood from the head. The bowels should be kept 
open by means of an active purgative, according to the following recipe:-

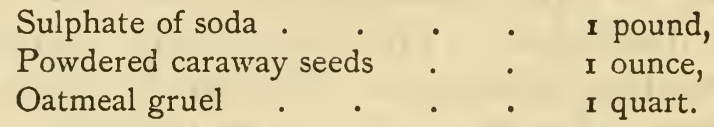

\section{CHOKING}

Is the name given to a mechanical obstruction to the passage of food from the mouth to the stomach. This obstruction may take place in the pharynx or entrance to the œsophagus or gullet, or about halfway down the œsophagus, or where the œsophagus ends in the stomach. The cause given rise to the arrest is generally the shape and not the size of the article swallowed. A turnip sliced, rarely chocks a cow, but if the animal be allowed to eat it with her teeth, the last portion of it swallowed is prone to stick, it being now more or less pointed and irregular in its outline.

Choking never results in the passage of the food from the rumen to the stomach, as the bolus or food is preceded by a quantity of fluid which lubricates, and at same time distends the walls of the œsophagus. There has been invented by $\mathrm{Mr}$ Alexander, a farmer of Tweeddale, a useful instrument, called a probang, for pushing down the food when it sticks in the throat, which can be had of all instrument-makers who deal in implements for the use of farmers. But where farmers or others do not possess this instrument, a substitute may be made in the following manner.

Three small canes of about five and a half or six feet long, are firmly bound together by waxed twine, rolled closely round its whole length. To the extremity of the canes is attached an elongated knob, 
made of shammy leather, stuffed with tow, and having a cup-shaped extremity. This knob must be firmly attached, by having long thongs proceeding from the edges of the leather, and firmly welted down by the enveloping twine. See Plate x, fig. I9. The purpose of the cup-shaped extremity is to make certain of pushing forward the obstructing food, which but for this termination might slip past it. Great care must be taken that the knob is properly attached, as, should it come off, it must ever remain in the stomach, an undigestible incumbrance, which is certain to create disease. The cup-shaped termination should be formed of tin, with a number of holes in it to permit of the leather being sown to it. See Plate x, fig. 20. Whalebone, if sufficiently long, is preferable to cane, as being much more elastic. Great care must be exercised on using the probang, or otherwise the œsophagus may be ruptured.

\section{LOCKED-JAW.}

Symptoms. - Tetanus or locked-jaw is at once distinguished by the animal being incapable of moving his jaw or of opening his mouth for the reception of food. It consists of a spasmodic contraction of the muscles by which the jaw is moved. If means cannot be devised to remove the spasm, the animal attacked must necessarily die for want of food.

CAUSE.-The causes giving rise to lock-jaw and other forms of tetanus, is the same in all animals, viz., the entrance, through a wound, of the "Bacillus Tetani" into the tissues of the affected animal, and as these have been already considered in dealing with the disease in the horse, the reader is referred for further information to page 13 of this work. 


\section{SORE THROAT.}

Congestion and inflammation of the mucous membrane which lines the pharynx, or anterior portion of the gullet, is not an uncommon occurrence in cattle, and when associated with the formation of abscess in the "Velum Pendulum Paleti," or soft palate, gives rise to great distress in breathing. Tumours frequently grow from the mucous membrane of the pharynx, and according to their situation interfere with the breathing.

Symptoms of sore throat are stretching of the head and neck, pointing of the nose, heaving of the flanks, disinclination to eat or drink, difficulty in swallowing, and a sound heard in inspiration.

Remedies.-Apply a mustard embrocation to the throat, or cantharides blister. Feed the animal on oatmeal and linseed gruels, and if bowels are constipated, administer a dose of purgative medicine. Unless the bowels are markedly constipated, do not administer any medicine by pouring it over, as there is a liability for the liquid passing into the larynx and thus entering the lungs instead of the stomach.

If an abscess forms in the soft palate, the distress in breathing will be great, and necessitate the performance of tracheotomy. The abscess when ripe will also require to be opened, and neither of these operations should be undertaken except by an experienced veterinarian.

\section{SNORES.}

Sympтомs. - The snores or snivels are known by a collection of thick, coagulated matter, inside the nostrils; and when the accumulation becomes great, it impedes the respiration through the nostrils, and 
produces a snivelling sound, as the air is expired and inspired. This complaint is sometimes mistaken for a disease in the throat, where it is imagined it causes obstruction. However, an inspection of the nostrils will readily show whether it is the snivels or not. The inflammation causes swelling in the nostrils, and it is not until suppuration has taken place, that the animal is relieved.

CAUSE.-The cause of this complaint is exposure to cold, producing inflammation of the mucous membrane of the nose.

REMEDY.-To effect a speedy cure, every means should be adopted to facilitate suppuration; and fomentations or heating liniments will prove the most effectual. It has been found that oil of bays injected up the nostrils frequently has the effect of inducing speedy suppuration. Steam of hot water has also been successfully employed; or holding a thick cloth steeped in warm water to the nostrils will produce the same effect. Putting a warm mash of bran into a thick canvas bag, and attaching it to the nose, in the same manner as the nose-bag of a horse, has also been attended with speedy results. While the animal is labouring under this disease, he should be kept warm, and in a house, and supplied with nutritious food. 


\section{CHAPTER II. \\ Diseases of the Chest.}

INFLAMMATION OF THE LUNGS (PNEUMONIA).

Symptoms.-The premonitory symptoms of this complaint are loss of appetite, with depression and an appearance of anxiety; rapidity of breathing, and consequent increased motion of the flanks, accompanied by a hard, dry, short cough. The pulse at first is full and oppressed, thereafter quick and small, and difficult to be felt. The mucous membrane lining the eyelids is considerably congested, and sometimes approaching to a deep orange colour. Milch-cows soon become dry, and the horns, ears, and legs are generally cold.

CAUSE.-This complaint is caused by sudden changes of temperature, or by removing the animals from close, sheltered situations, to exposed, high, and cold localities. Cattle feeding in low and damp situations, exposed to dense fogs and moist atmospheric changes are liable to be affected with inflammation of the lungs. Suddenly checking the perspiration by drinking a large quantity of cold water, when the animal is subjected to a high temperature, is another frequent cause. From this it will be manifest that sheltered sheds will be most beneficial in the feeding of cattle, either to retire into when exposed to the meridian of a summer sun, or during rains. It will be seen that in either case cattle will invariably avail 
themselves of shelter when it is within their reach. And, during variable seasons, movable sheds, however temporary, would be found of much value in preventing diseases of this description.

Remedies.-As soon as the symptoms which we have described are noticed, recourse may be had to bleeding. It is a mistake to bleed too profusely, as such weakness is often induced, that the animal never again rallies, and death follows. To act as a sedative on the heart, and lessen the frequency of its pulsations and force, Fleming's tincture of aconite may be given in fifteen drop doses every third hour, but as it is a deadly poison, care must be exercised in its use, and not more than ten doses given except under the guidance of the veterinary surgeon.

Tartarised antimony : $: \quad \frac{1}{2}$ ounce,
Saltpetre $: \quad 3$ drachms,

dissolved in a quart of linseed gruel, and the dose repeated every twelve hours, until the symptoms have disappeared. Counter - irritation by mustard and turpentine to the chest should be employed and repeated until the chest wall becomes tender to the touch of the fingers.

Instances have frequently occurred where cattle died in twelve or fourteen hours after being attacked with inflammation of the lungs.

Some veterinarians recommend a large seton in the dewlap, and to be stimulated with an embrocation of ammonia, spirit of turpentine, and mustard; but the connection of the dewlap with the lungs, is too remote to act in the manner intended, namely, by counterirritation. 


\section{CONTAGIOUS PLEURO-PNEUMONIA.}

Cattle, like all other creatures which have lungs, are liable to attacks of congestion and inflammation of these organs from the causes which produce inflammation in other animals, but they are also liable to be attacked by a specific form of inflammation of the lungs, which as yet has been found in no other but the Bovine race.

This contagious and specific form of inflammation of the lungs and its covering (Pleura) was unknown in this country until the year I84I. According to the literature on this subject, and on the authority of Bourgelat (Founder of the French Veterinary College), this disease existed in France in 1769 , and appeared in Prussia and Germany in 1802 ; in Russia in 1824 ; Belgium, I827; Holland, I833; Sweden, I847; Denmark, I848; Cape of Good Hope, I 854; United States of America, I 843; Melbourne, I 858; New Zealand, I864; and in all these countries named, with the single exception of Great Britain, it still exists.

The name by which the disease is known, viz., "Contagious Pleuro-Pneumonia," is a misnomer and very misleading. It is a parasitic disease, and the lung lesions or structural changes in the lung and its covering are the results or consequences of the specific organisms breeding and burrowing in the lung tissue. It is a highly infectious disease, the organisms being given off in the breath of the affected animal.

The late Professor Dick did not believe in its infection, and as a consequence the bulk of veterinarians held the same opinion, and no measures having been taken to prevent the spread of the malady by infected animals, within a few years of its first appearance in 
this country, it had become widespread, and the deaths very numerous.

In all probability another reason for its infectiousness having been overlooked may be found in the fact that the lung disease does not manifest itself in recognisable symptoms, for at least six weeks after the animal has become infected.

Instances have been cited tending to show a period of incubation or hatching for twelve months, but undoubtedly the average period of incubation in a primary and uncomplicated case of epizootic pleuropneumonia is six weeks.

Symptoms.-The first and earliest symptom is a cough of a peculiar short, sharp, and dry character, increase of temperature, pulse full and quick, breathing quickened, nostrils dilated, bowels constipated, a slight grunt at expiration, and marked tenderness when spine is pressed. On recourse to auscultation and percussion, certain sounds are heard which indicate the existence of bronchial disturbance and lung disease. As the disease advances the temperature increases, the grunt becomes louder, and the breathing distinctly abdominal.

Causes. - The cause of this, like all other specific diseases, is the entrance of the living specific organism into the lungs. Unlike many other specific or germ diseases, it cannot be propagated by inoculation of the diseased exudates of the lungs, neither can it be propagated by ingestion, that is by the introduction of the virus into the stomach in food or drink. It can only be propagated in one way, and that is by Inhalation, inhaling the breath of the living affected animal, and the moment the animal dies the infection or power to infect ceases. 
Remedies.-Like other germ diseases, once the germs have gained an entrance to the lungs, the diseased conditions or structural changes will progress until the last germ has ceased to exist. We know of no agent which will destroy these germs and not destroy the lung tissue. But we know of a method and an agent whereby cattle whose lungs are sound can be rendered proof to the attacks of the organisms, and that method consists in inoculating the lymph found in the early stages of the disease in the lung of the affected animal into the tail of the sound animal.

Inoculation of the virus has been looked upon by some as producing a milder form of the disease, and some have gone the length of asserting that it is a curative agent, but in our opinion it is neither the one nor the other. The result of inoculation in our hands has never demonstrated the existence during life of a lung affection, nor has it demonstrated, on postmortem, diseased lungs, and therefore we hold that it does not produce a milder form of the disease or any form at all. Moreover, an inoculated animal, if its lungs were sound, that is free of the pleuro germs at the time of inoculation, has never been known to give the disease to other cattle. Then as regards the curative power of the inoculated lymph, numerous experiments have satisfied us that it is neither a diagnostic nor curative agent, the symptoms exhibited and the series of changes produced at the point of inoculation, being as pronounced in the affected as the non-affected animal.

A sound animal successfully inoculated with the lymph found in the lungs of an affected animal, is beyond all doubt immune to an attack of infectious pleuro-pneumonia by the natural method.

This disease, as has already been stated, is non- 
existent in this country, and not being an indigenous disease, will not appear again until re-imported, but as the importation of cattle, except for immediate slaughter at the port of debarkation, is prohibited, there is little chance of it again ravishing our herds.

In stamping out the disease (which many asserted would never be achieved), two methods were advocated. The first, by slaughter of all the cattle on the farm or premises where the disease was found to exist; and second, the slaughter of all affected animals, and the inoculation of all the other cattle in the country.

The last method being the more humane, was the most popular, but it was not supported by the members of the veterinary profession who advised the Board of Agriculture, and for various reasons, some of which we will now refer to :-

I. Cattle are subject to two forms of inflammation of their lungs, one of which is non-infectious and curable, the other infectious and generally fatal.

II. In the living animal the two diseases cannot be differentiated by veterinary skill, or by inoculation or any other method known to the veterinary profession.

III. The undoubted existence of infectious pleuropneumonia, as distinguished from simple pleuropneumonia, can only be affirmed after a post-mortem examination of the lungs has been made.

IV. Granting that the existence of contagious pleuro-pneumonia has been correctly diagnosed in one or more members of a herd, and all have been slaughtered which exhibited the slightest symptoms of a lung affection, and all the remaining animals have been most carefully and apparently successfully inoculated, where is the guarantee that not one of the inoculated animals was labouring under pleuropneumonia at the time of inoculation? 
The prepared vaccine of tuberculosis, or as it is termed, "Tuberculin," when inoculated into the body of a sound ox produces no sudden and marked rise of temperature, but it does so act on the animal affected with tuberculosis, and in this way the veterinarian can with almost absolute certainty separate the affected from the non-affected, but we repeat the vaccine of contagious pleuro-pneumonia reacts on both alike, and while it protects the sound animal from an attack of the disease, it in no way protects the affected animal, or assists in its recognition or detection, and consequently it (Inoculation) has failed to stamp out the disease in any and all countries where it has been employed for that purpose.

If the vaccine of contagious pleuro-pneumonia had been a detective as well as a protective agent, then the stamping out of the disease by the cruel and brutal method of slaughter of affected herds, would never have been advocated by the members of the veterinary profession, nor adopted by the Government of the country.

\section{INFLAMMATION OF THE STOMACH.}

Symptoms. - The symptoms of inflammation of the stomach are not well marked; loathing of food is the most prominent, together with foul breath, with a tightness of the hide about the shoulders.

CaUses.-This is brought on by some acrid substance taken into the stomach while feeding, or from the animal feeding too greedily when removed from a poor to a rich pasture; it sometimes proceeds from debility of the system, and the food in consequence remaining in the paunch until it has undergone a certain degree of fermentation, which produces heat, 
and inflammation, and obstructs the circulation, inducing great distention of the parts.

REMEdies.-The medical treatment of this complaint must be something like that in inflammation of the lungs. Bleeding as a primary remedy may be resorted to, depending upon the strength of the animal and character of the pulse, and followed by cooling aperient medicines, to carry off the indigestible matter lodged in the stomach. We do not know a better medicine than the following:-

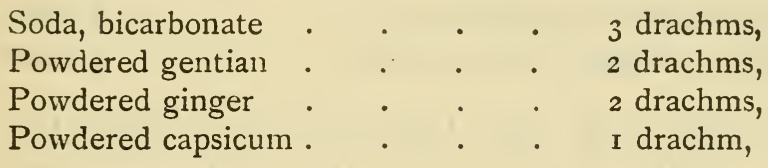

these to be given with a pint of linseed gruel. Let it be repeated every twelve hours for two or three days.

\section{INFLAMMATION OF THE LIVER.}

Symptoms. - The diagnosis of this disease is difficult, but we may state generally, that it is accompanied by a difficulty of breathing, accompanied with fever, high temperature, a certain amount of tenderness over the last ribs on the right side, hot clammy mouth, furred tongue, and mucous membranes of the eyes yellowish in colour. The bowels irregular, and forces bad smell, the breath has also a bad smell. The animal has usually no inclination to eat, but drinks large quantities of water if permitted to do so. Emaciation rapidly results with marked nervous prostration.

Causes.-One of the most common causes is feeding animals upon food too rich in albumen and fat constituents and a want of sufficient daily exercise. As exciting causes may be named, sudden changes of 
temperature, sudden chills as a result of warm days and cold nights, sleeping on damp pastures, cold dewy nights, fast driving after idleness, intense heat. It may also proceed from blows or injuries of various kinds.

Remedies.-Bleeding is seldom beneficial, and more benefit will be derived by producing a drain upon the portal circulation by changing the diet and administering saline purgatives and counter-irritants. With these objects in view, administer the following :-

Soda sulphate (glauber salts) . . . . I pound,

Sodium chloride (common salt) . . . $\frac{1}{2}$ pound,

follow this up by the application of a blister over the region of the liver, and if the animal continues indisposed send for the veterinary surgeon.

\section{FOG-SICKNESS, OR HOVEN.}

CAUSE.-This unpleasant, and frequently troublesome malady, is sometimes caused by cattle being removed from house or yard-feeding to rich pastures of meadow-grass or young clover, on which they feed so voraciousiy, that the stomach being overloaded with succulent food, fermentation takes place, gases are generated, which descending into the bowels, produce a general swelling of the belly. The cause is the animal taking in too great a quantity at once, without periorming the necessary act of chewing the cud, by which the food is reduced into a more liquid consistence, and prepared for its passage from the paunch into the other stomachs. It not unfrequently happens that the stomach is so distended with food and gas that unless immediate relief be given, the animal will die from paralysis of the nerves of respiration and circulation. It must be obvious that 
it is better to guard against the direness of this malady, than to remedy the evil. Care should be taken not to turn cattle into rich pastures when they are hungry; but if it is absolutely necessary that they should be turned out, they ought only to be allowed a limited time for feeding, and then return to their former situation, to chew the cud; and thus, by little caution, the evil may be avoided. This should be repeated daily, until the animals are habituated to the change. . The sudden gorging of the paunch, and the evolution of air, creates such a distention in it, that the function of chewing the cud is entirely prevented, and consequently it is seldom that Nature works its own cure, as is the case with other complaints.

Tympany or hoven may not be due so much to the condition of the food, as to general debility and impaired digestion. Foreign bodies, e.g., hair balls, portions of an old leather shoe, parasites, etc., etc., may and generally set up indigestion and evolution of gases. Hoven is a marked symptom in most of those cases of traumatic heart disease, due to a hairpin or sharp body having become fixed in the heart. Dairymaids should be careful in preparing the food of cattle, and if they must wear hairpins, see that they do not fall into the animal's food, for if they do, they will find their way through the walls of the stomach and become fixed in the heart.

REMEDIES.-Where there is very great swelling, the most speedy way of affording relief, in the absence of a trochar and cannula, is to make an incision with a sharp penknife, a scalpel, or other instrument, beneath the short ribs, and insert a quill so as to allow the air to escape. The exact point to puncture is midway betwixt the last rib and the prominent 
point of the pelvis or hook-bone. Cut the skin, then plunge the instrument (trochar or knife) into the wound with the cutting edge looking downwards. The trochar being withdrawn, the cannula may be tied round the body and left there so long as gases are formed and escape.

This surgical remedy is, however, not very often required, as medicines given internally, if promptly administered, generally have the effect of relieving the animal. The medicines are various. The following has been found effectual :-

Gin or whisky . . . . . . I pint, Water . . . . . . I pint.

It has been found that the following medicines afford relief :-

Aromatic spirits of ammonia . . . . 3 ounces,

Sulphate of soda . . . . . . I ounce,

Ether . . . . . . . 2 ounces,

Carbolic acid. . . . . . . 2 drachms.

In using these and other agents in solution or suspension in water, a good plan is to taste the liquid, and thus ascertain their strength, and if too pungent, add more water.

\section{CATARRH, OR COLD.}

Symptoms.-Colds are two fold-one is of a simple nature, and the other an Enzootic known by the name of Influenza. Cold, raw, and wet weather frequently induce common colds, and neglect of these often ends in serious evils, and are too frequently the remote cause of consumption and death. The influenza cold 
will frequently attack a whole straw-yard of cattle, even in the course of a single night. The first symptoms are a dull and languid appearance, with watery eyes, which are partially closed, and a mucous discharge from the nostrils. These are commonly accompanied by cough and loss of appetite. If neglected, the animal gradually loses flesh, and becomes hide-bound, with a rough, staring coat.

Causes. - Many causes induce colds ; the generality of these are too well known to require pointing out; but we may generally remark, that cold springs, which follow mild winters are very conducive to colds.

Remedies.-Prompt attention to these complaints, as in most others, is most likely to effect a speedy cure, and even to prevent the more serious consequences attendant upon neglected colds.

In the earlier stages let the animal be placed in a warm situation, and supplied plentifully with warm fluid drinks of gruel, infusion of malt, or linseed. But when the animal manifests chillness and shivering, with coldness of breath, warm stimulating draughts should be given to restore the action of the stomach and intestines. The following draught should be given every eight hours :-

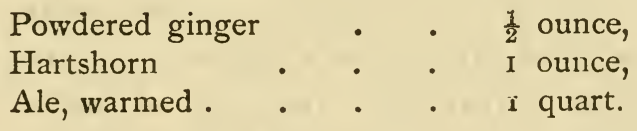

For drink, the water must always be given a little warmed. If feverish symptoms appear, an ounce of nitre, dissolved in a pint of warm water should be given.

Sometimes the throat is affected, and the animal experiences a difficulty in swallowing. When this is 
the case, the following liniment should be applied to the throat:-

Oil of turpentine . . $\mathrm{I}_{2}^{\frac{1}{2}}$ ounces,

Liquid ammonia, or hartshorn $\frac{1}{2}$ ounce, Rape-oil . . . . 2 ounces.

This is applied for the purpose of inducing counterirritation.

When influenza has been allowed to get hold of the constitution, extreme weakness generally ensues. When this is the case, warm, nourishing, and stimulating diet should be given, together with some tonic medicine twice or thrice a day.

Should the animal be costive, it will be necessary to administer the following laxative, which will be sufficient for two doses :-

$\begin{array}{lll}\text { Sulphate of soda } & \text {. } & \text { I pound, } \\ \text { Powdered caraway-seeds } & \text {. } & \text { I ounce, } \\ \text { Oatmeal gruel } & \text {. } & \text { I quart. }\end{array}$

In all cases of influenza the animal should be kept in a cow-house or shed, and a moderate quantity of grass or tares given daily, if they can be had at the season.

Whenever influenza appears to be epidemical, the animals should be immediately removed to a warm situation; and if in the winter or cold spring weather, a house or shed should be chosen to remove them to, as it is easier to prevent than cure a disease: indeed the old adage, "It is easier to keep well than to make well," applies to most complaints incidental to man and animals.

COUGH.

Syмртомs.-Cows after calving are frequently seized with a tickling, short, irritating cough, in conse- 
quence of being removed from a cow-house to some exposed situation. It may also proceed from sudden transitions from heat to cold. It is also occasioned by some extraneous matter adhering to the interior of the throat or trachea.

REMEDIES.-Some recommend warmth, with a constant but moderate supply of succulent food. Colds may also be relieved by a pint of the following mixture, to be administered twice or thrice a day :-

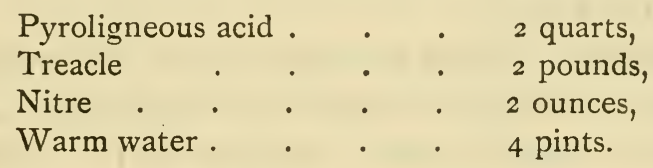

Besides the above, the animal should be drenched twice or thrice every day with the following dose of medicine :-

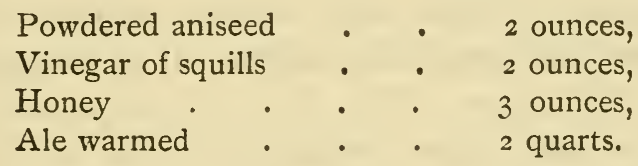

HOOSE, OR HUSK.

This is the name commonly given to an affection characterised by a spasmodic husky cough, and dependent on the presence of parasites in the airpassages. Calves and yearlings on certain farms are prone to be affected with a fatal form of bronchitis, and the deaths annually from this disease are numerous. In other farms "Verminous Bronchitis" is unknown. The parasite belongs to the family classed strongylus, and is named "Strongylus Micrurus." A similar parasite attacks foals. A somewhat similar, but a different parasite (Strongylus Filaria) is found in the bronchial tubes and windpipe of lambs and ewes. Low-lying, damp pastures 
are the favourite breeding-grounds of these parasites, and first and second growths of clover, especially where water occasionally rests in pools. Lambs following sheep pasturing, if the ewes do host to the parasite, rapidly become affected. The embryos are swallowed in grass, or drank in water, or entering the bodies of snails, in the water or food, thus gain the stomach of the calf or lamb. On being hatched, it bores its way into the blood or lymph vessels and finally it reaches the lungs.

Symptoms.-Husky cough, with not much nasal discharge, rubbing the nose on the ground, or against a hurdle; mouth open; tongue out; hurried and difficult breathing; rapid loss of flesh and strength. If the parasites infest the bowels there is profuse diarrhœea.

Remedies. - While the embryo worms are in the bowels, and the cough but slight, oil of turpentine and milk will kill them. The dose of turpentine is two drachms morning and evening in milk or linseed oil gruel. If the lungs are infested to any extent, chlorine evolved from bleaching powder (chloride of lime), or from common salt, black oxide of manganese, and sulphuric acid, or sulphuric acid gas, obtained by burning stick sulphur on a few live coals. Injections of oil of turpentine, carbolic acid, and opium into the windpipe is also a certain remedy, but it is a method of treatment which requires veterinary skill and supervision. When possible, change the pasture, and keep up the strength of the herd or flock by liberal use of hand-feeding.

STOMACH, OR GRASS STAGGERS.

Sympтомs. - This disease is indicated by a staggering, unequal walk, as if the animal were intoxicated, 
accompanied by a dull, heavy eye, with a hard, and oppressed pulse, and great drowsiness. In some cases, owing to disturbance in the cerebral circulation, the animal is greatly excited and bellows loudly and frequently, paws the ground, foams at the mouth, and almost presents as violent symptoms as seen in cases of rabies. In other cases the animal is blind, does not hear, and stands as if rivetted to the spot ; violent spasms of the voluntary muscles is sometimes a pronounced symptom.

CaUSE.-The cause of this stomach disorder is generally due to the grass upon which the animal is fed. Rye-grass, unless eaten down " shoots," and in this state from the large quantity of woody fibre present in it, is difficult to digest. On light sandy soils, rye-grass shoots readily, and if the season is a dry one and the animals have little water to drink, grass staggers is not only a widespread affection but a deadly one.

Remedies.-Crop the grass and thus prevent the rye-grass running to woody fibre. Give a pound or two of oil cake daily to each animal on the grass and thus prevent constipation of the bowels, and see that there is at all times plenty of water to drink.

Affected animals should be removed to the farmstead, and have a dose of purgative medicine administered. If brain symptoms are exhibited, employ the services of the veterinary surgeon.

\section{JAUNDICE, OR YELLOWS.}

Symptoms.-Cattle are very liable to this disease. Its seat is in the liver. It prevails in the spring and autumn. It is known by yellowness of the eyes and inside of the mouth; a dull and languid aspect, 
accompanied by debility and loss of appetite. It is generally attended by costiveness, and the animal is disinclined to feed.

Cause. - The cause of the disease may be associated with inflammation of the liver, or congestion of the organ, or disease and obstruction to the flow of bile from the liver to the gall bladder, or from the gall bladder to the bowel. Sometimes "Jaundice" is a symptom of verminous disease of the liver, e.g., fluke disease in cattle and sheep.

REMEDIEs.-If the jaundice is due to functional disturbance of the liver, a dose of laxative medicine and a less nutritious and stimulating diet, may be all that is required. If due to organic disease of the liver, with or without the presence of parasites in the bile ducts, the treatment must be entrusted to an experienced veterinary surgeon.

When the disease continues for some considerable time, it may be inferred that the liver is in a very disordered condition, and in that case a cure is hopeless. 


\section{CHAPTER III.}

\section{Diseases of the Abdomen.}

\section{INFLAMMATION OF THE BOWELS.}

CatTlE seldom are attacked with inflammation of the bowels, whereas horses are remarkably prone to the disease. This immunity in all probability is due to the prolonged and very thorough cooking process to which the food is subjected in the stomachs of ruminating animals.

Symptoms. - The chief symptom by which this disease is characterised, is great restlessness, accompanied by a violent, griping pain, and the animal will frequently turn his head towards his belly, and even endeavour to strike it with his hind-foot. The pulse is rapid, and the breathing oppressed; and it is not unusual for the animal, while suffering severe pain, to break out into profuse perspiration. The most prompt measures must be adopted, or the disease will end in mortification and death.

CAUSE.-This malady may proceed from drinking cold water too copiously after being overheated by being overdriven, or being exposed to the noon-day summer sun, or placed in a sheltered locality where there is no free circulation of air. It may also have its origin in costiveness.

REMEDIES.-The treatment to be adopted will 
depend upon the condition and extent of the evil. If the pulse is unusually quick, the eyelids unnaturally red, and the breathing most oppressive and hard, bleeding may be resorted to. If the bowels are constipated, then the following drench must be given :-

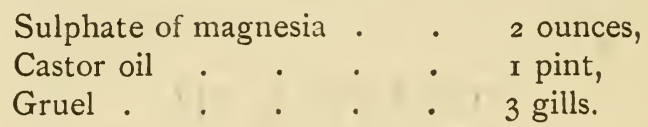

The salts should first be dissolved in the warm gruel, and the oil added afterwards.

If this drink does not operate quickly, clysters should be given to facilitate the evacuation. They should consist of -

$$
\begin{aligned}
& \text { Hog's lard . . . . } \frac{1}{4} \text { pound, } \\
& \text { Gruel, warm . . . } \quad \text { r pint. }
\end{aligned}
$$

If there is no redness in the under surface of the eyelid, the complaint will not be so severe as to render bleeding necessary; and particularly if the pulse should not be very quick and hard, and the bowels open. With a moderate attack, when the animal is in low condition, anodyne drinks may be substituted for bleeding. The following will be a sufficient dose, which should be repeated every six or eight hours, according to circumstances :-

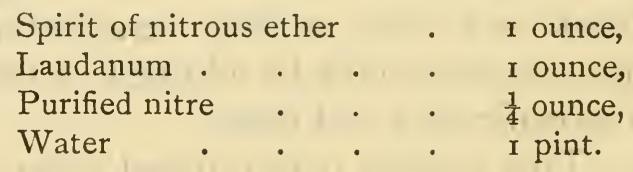

If the complaint proceeds from costiveness, after having been fed upon dry fodder (which will be manifested by a difficulty in dunging, and what he voids will be more solid than usual, differing in colour and offensive in smell), then the laxative drink above recommended must be given. Counter irritation to 
the abdomen is also recommended, and with this object in view apply I lb. of mustard and 2 ounces of spirits of turpentine. Rub them well into the skin. Rugs dipped in boiling water and tightly wrung out, may also be applied to the body of the animal.

\section{INFLAMMATION OF THE KIDNEYS.}

Symproms.-This complaint may be distinguished by a quick pulse, loss of appetite, and a frequent inclination to stale; and only a small quantity of urine being voided at a time, of a dark red colour, and accompanied by much suffering to the animal, indicated by every motion expressive of pain. There is also much stiffness in the hind-quarters in walking. This complaint requires immediate attention, otherwise it may terminate fatally. It differs from the malady called red water.

CAUSES.-External injuries or irritating substances taken into the stomach, and passing through the kidneys with the urine, will produce inflammation. It may also be induced by the injudicious administration of strong diuretic medicines and exposure to cold.

Remedies.-Keep the animal in the cow-shed, apply warm rugs, give plenty of water to drink to wash out the kidneys, and administer a dose of purgative medicine. If a difficulty in making urine or a small quantity is only passed, digitalis in $\frac{1}{2}$-drachm doses may be given in linseed gruel or mucilaginous drinks. A fresh sheep skin, fleshy side in, may be applied on back over the region of the kidneys. Copious injections of warm water into the rectum has a soothing effect, and tends to hasten the action of the purgative. 


\section{INFLAMMATION OF THE BLADDER.}

Sумттомs.-While cows are in calf, especially during the latter portion of their gestation, they have sometimes a frequent desire to void urine. This proceeds from an irritable condition in the sphincter of the bladder.

CAUSE.-This in most instances is caused by a continued pressure upon the region of the bladder.

Remedies.-The first thing is to draw off the urine by means of a catheter, and then to follow up the treatment recommended for inflammation of the kidneys. If a catheter is not at hand, the finger introduced as far as the neck of the bladder will permit the urine to flow freely.

\section{INFLAMMATION OF THE WOMB.}

A loss of appetite, accompanied by a rapid pulse, a languid action, and loss of milk are the ordinary symptoms of this complaint. As the disease progresses, a fotid discharge from the vagina takes place, accompanied by straining arching of the back and a constant endeavour to void urine, which comes off in small quantities at a time. Sometimes a complete retention of the urine takes place, which can only be relieved by the introduction of the catheter. The cow frequently becomes so weak as hardly to be able to stand, and moves about with much difficulty.

Causes. - This complaint may have its origin in keeping the cow on too full and rich a diet during the latter period of gestation, but it much more frequently follows after parturition, and due to exposure to cold or injuries sustained as a result of violence in assisting the delivery of the calf. 
CURE.-When retention of urine takes place, the catheter must be used as above mentioned. If the after-birth has been retained, it should be carefully separated and removed by hand. An injection of carbolised tepid water should be thrown into the womb and repeated, and the following laxative drink may be given :-

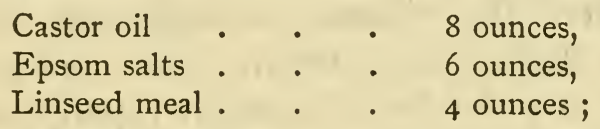

the whole to be dissolved in half-a-gallon of thin, warm gruel. Clysters of warm gruel and oil will be found beneficial, and then give the following anodyne :-

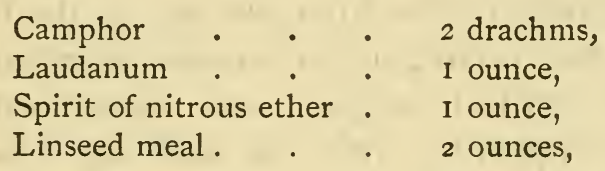

dissolved in a quart of gruel. If the animal does not exhibit symptoms of relief in ten or twelve hours, the same dose may be repeated. And if the straining and painful symptoms of frequent urinating continue, then an anodyne clyster may be given, composed of the following :-

$\begin{array}{lllll}\text { Laudanum } & \cdot & . & \cdot & 2 \text { ounces, } \\ \text { Thin gruel } & \cdot & \cdot & . & \text { I quart. }\end{array}$

RED WATER.

This is the name given to a disease in which the affected animal passes urine the colour of blood. A microscopic examination of the urine reveals the presence of broken down blood cells, and imperfectly formed blood cells. An analysis of the urine proves the existence of albumen and the colouring matter and 
iron which exists only in the red corpuscles of healthy blood. The disease is a blood disorder, and due to the food upon which the animal is feeding.

Symptoms. - At the commencement, this complaint is manifested by frequent, but unavailing attempts to emit urine; but when the disease has acquired a more advanced condition, the discharge is in large quantities. When the disease is neglected, the urine assumes a darker hue, and in time looks like foul coffee, and now he loses strength rapidly, and sinks into a lethargic condition; and unless speedy remedies are had recourse to, the animal will seldom exist under the complaint for more than ten or twelve days. Great pain is felt in the urinary passage, and the animal generally retires from the rest of the herd, and extends its tail, raising up its back in an arcuated form.

In the earliest stages, sunk eyes, restlessness, and want of appetite, with hot and dry nose, and a staring coat are the ordinary concomitants of this disease.

Red water is usually accompanied with considerable costiveness; and although this may not be the case at first, it generally shows itself in the more advanced stages of the complaint. Dry feeding is certain to induce costiveness in this malady, and more especially if strong estringents are given; and in this case such a degree of constipation may be induced, that the animal will die in consequence, as no medicine will give relief. So prone to costiveness are animals labouring under this complaint, that it will take place even when feeding on grass. Relief may sometimes be given by drawing off the dung with the hand. This complaint differs in a material degree from inflammation of the kidneys, as the urine is not voided in small quantities, as in that disorder. 
CAUSES.-The geological formation of the locality has much to do with the prevalence of this disease. Before drainage was introduced, red water was a common disease, but now it only exists on farms undrained and where the soil is wet, and the grass coarse and rank. Turnips grown on such soils may cause red water. Sometimes the disease manifests itself as an enzootic in cattle and sheep, and may be due to the animal eating the leaves of resinous trees-coniferæ-as they fall in early autumn, especially if the pasture is bare.

REMEDIEs.-At the commencement, laxatives have been generally found efficacious. The following formula has been recommended as the best:-

$\begin{array}{lllll}\text { Epsom salts } & \cdot & \cdot & \cdot & 4 \text { ounces, } \\ \text { Purified nitre } & \cdot & \cdot & \cdot & \text { I ounce, } \\ \text { Castor oil } \cdot & \cdot & \cdot & \cdot & 4 \text { ounces, } \\ \text { Thin gruel. } & \cdot & \cdot & \text {. } & \text { I quart. }\end{array}$

After the purgative has acted, the food should be highly nutritious, boiled barley and linseed. If the animal is weak and evidently bloodless, decoction of linseed, milk, eggs, and porter, should be poured over, and diffusible stimulants and iron tonics prescribed.

\section{GRIPES.}

Symptoms.-Young cattle are extremely liable to colic, which is manifested by great restlessness, frequently lying down and rising again groaning, and striking against the belly with the hind-feet, and sometimes even with the horns. It is seldom that any change of the pulse takes place. The body is, however, liable to swell, particularly on the left side; and unless remedies are speedily applied, the pain generally increases greatly, accompanied with inflam- 
mation, and a quick, hard, and unequal pulse, while the feet, ears, and horns are very cold. When the last symptoms appear, they are generally the forerunners of death.

CaUses.-Retention of food in the third stomach, with costiveness, will produce gripes. Even when the bowels are lax, or in a natural condition, cattle have been known to be griped. Dry food, such as grain and hay, will produce it in cows. It also occurs from the animal taking large meals of rank grass, and drinking cold water when much heated: in this case, the disease is sudden in its attack; but when caused by costiveness, its effects are more gradual, but more difficult to remove.

REMEDIES. - When costiveness is the cause of this disease, recourse must be had to purgatives, taken in combination with aromatics, or stimulants. The following prescription general proves effectual :-

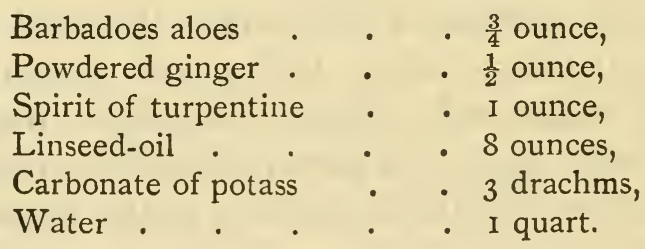

In country situations where all of the above medicines cannot be readily procured, the following formula may be used as a substitute :-

$$
\begin{aligned}
& \text { Common salt . . . . } 8 \text { ounces, } \\
& \text { Rape-oil, or melted hogs' lard . . } 8 \text { ounces, } \\
& \text { Mustard . . . . . . I ounce, } \\
& \text { Rum, or whisky . . . . } 2 \text { ounces. }
\end{aligned}
$$

When the animal is in high condition, with redness on the inner surface of the eyelid, bleeding may be resorted to. But when accompanied with looseness of the bowels, or in an ordinary condition and a tendency 
to weakness, blood must not be taken, and the following carminative medicine given :-

\begin{tabular}{|c|c|}
\hline Spirit of turpentine & I ounce, \\
\hline Laudanum & 6 drachms, \\
\hline Spirit of nitrous ether & 2 ounces, \\
\hline Linseed-tea & . I $\quad \frac{1}{2}$ pint. \\
\hline
\end{tabular}

When it is necessary to have recourse to a purgative mixture, its operation will be facilitated by clysters.

If colic is induced from the animal gorging itselt with dry and hard grain or hay, great caution must be exercised in feeding for some days after the complaint has been removed. At the same time it will be proper to give the animal the following stomachic, as a restorative, once or twice during the twenty-four hours :-

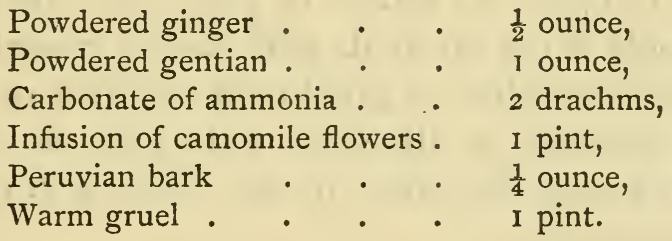

If, however, costiveness is the proximate cause of the colic, an alterative must be the remedy administered, and the purgative above recommended must be given. Sometimes a change from dry to green food will produce the desired effect without having recourse to medicine. Salt mixed with the food has been known to relieve the pain, and also to restore a healthy condition in the animal. Or, if he refuse the dry salt, a solution of four ounces, daily, in a quart of water will give a desire to drink, which, if water is given plentifully, will be of much service in obviating the complaint. 


\section{DIARRHEA, OR LOOSENESS.}

Sympтомs. - This complaint is manifested by general weakness and loss of flesh, increasing paleness of the eyes, frequent discharge of dung mixed with the slimy substance which lines the intestines, which has a most fotid smell, accompanied by inflammatory fever and griping pains, with irregular pulsation.

CAUSES.-Diarrhœa is induced by a variety of causes; the most frequent, however, is when cattle are either turned out from a straw-yard or poor pasture to a luxuriant field of grass-which is always preferred by cattle-where they gorge themselves to such an extent, that they bring on fog-sickness, and inflammation, as well as this complaint.

Remedies. - If the malady is taken at first, it has been found that putting the animal into a house, and feeding it upon dry fodder or grain will effect a cure. But should it not do so, its food should consist of maltmashes, linseed-tea, or gruel made of flour or oatmeal. If the scouring is attended with pain, an ounce of laudanum may be given in the food, or in a drink of a pint of gruel.

In cases where the discharge is very great, half-apint of castor oil may be given in a drink. If in five or six hours there is no abatement of the disease, let the following medicine be given every six hours, until the scouring is less violent, and the dung resumes its natural colour :-

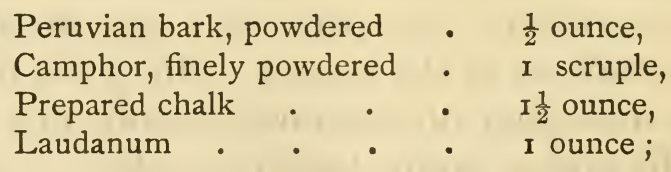

to be administered in a quart of gruel, made of flour. 
In diarrhœa, it is not the object of the veterinarian to stop the disease suddenly; and he that knows his profession will not prescribe strong astringents, and these in large doses, as this mode of treatment may produce worse effects than the disease itself.

If the discharge becomes intermittent, and the dung occasionally hard and difficult to pass, then it will be necessary to have recourse to laxatives, to clear out the exciting cause of this irregularity. The first and most simple to try is a dose of half-a-pint of castor oil, or half-an-ounce of the powder of bitter-apple, either of which may be given in a pint of flour-gruel.

Great attention is required to the different conditions in diarrhœa, otherwise the disease may assume that character which is termed rottenness or scouringrot, a disease which too often terminates fatally. The system should not be allowed to become too low; to prevent which, nourishing mashes of oatmeal and malt should be frequently given, and in small quantities at a time; for during the progress of this malady, it is found, that so long as the animal is permitted to indulge in a full meal, so long will the complaint continue.

It will be found that cattle, when left to themselves will seldom eat more than is proper to enable them to perform the necessary function of chewing the cud. It is only when they have been previously starved, or removed from a poor to a rich pasturage, that they exceed in this respect: so that, in those cases, they ought to be allowed to remain only a short time at first in such situations.

A low condition of the system will induce diarrhœa, and when it proceeds from this cause, there is more danger to be apprehended, and the animal is, consequently, less able to withstand any sudden transitions from poor to rich living, or from heat to cold, or from 
cold to heat. When, either from a dearness of food, or scarcity, the animal is kept on short allowance, it is a wise plan, when the luxuriance of spring arrives, to continue feeding the animal in the straw-yard, or house, upon a mixture of chopped hay and succulent grass, for a short time previous to turning him out to the field.

It is not uncommon for calves at the time of weaning to be affected with diarrhœea, which at this tender age should be stopped by

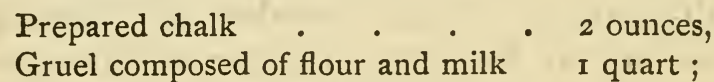

to be given every six hours, until the symptoms are arrested. But if in two days evident signs of a cure are not visible, to the above must be added a drachm of powdered ginger, and one drachm of laudanum.

\section{DYSENTERY.}

Sympтомs.--This is a dangerous malady, and sometimes is very deadly. Long-continued purging induces loss of flesh and general weakness; and so reduced is the muscular fibre, in many instances, that the skin may be seen hanging like a loose garment about their bodies. In other instances the animal is hide-bound; the hair changes its colour to sandy or grey; the eyes grow pale ; they eject a thin and slimy matter ; and their pulse is weak and irregular: the excrements often change colour, more particularly in the earlier stages of the complaint. In the more advanced stages of the malady, the fæces appear like food half-masticated, as it passes through the stomach and intestines without undergoing the ordinary digestive process. If the animal is pressed on either side of the spine, immediately behind the shoulders, after a long continuance of 
scouring-rot, it will manifest symptoms of suffering much pain. When this is the case, it may be inferred that the animal has become unsound, or tainted, and the chances against his recovery are exceedingly small. The excrements in the advanced stage are of a dark colour, with a frothy appearance, and the smell exceedingly offensive.

Causes.-Exposure to cold and long-continued rain, especially if the animal has been previously overheated and overdriven; also drinking cold water when warm. Want of a nutritive diet in milch-cows frequently induces this disease, which is one of the most difficult to cure.

REMEDIES.-A great variety of prescriptions have been given for the cure of this complaint, and some have been found very efficacious in some cases, which have no effect whatever in others. One of the points to be chiefly attended to, is to take the affected animal home to the straw-yard or shed, and subject it to dry feeding, which in some cases effects a cure without medical treatment. The following prescription has been found of much utility ; it is one of Mr Blaine's :-

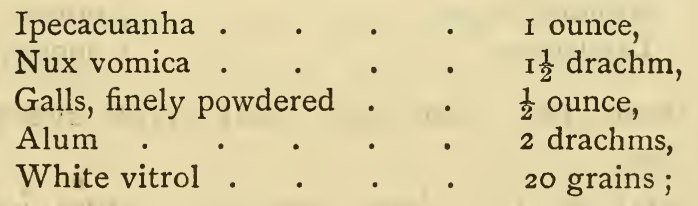

to be well incorporated in a quart of boiling water. The following recipe has been highly recommended:-

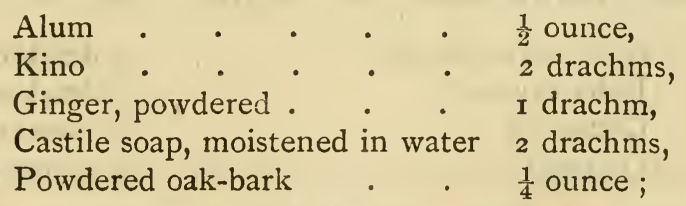

to be made into a ball, with a sufficient quantity of treacle. 
When scouring has continued long, considerable irritation in the membrane which lines the intestines and loss of its mucus must be the consequence. To mitigate this, mucilaginous drinks should be freely given. For this purpose, let a pound of linseed meal be boiled to a jelly in two gallons of water, and add to it an ounce of nitre. This will be sufficient to form six draughts, of a pint and a half each, to be diluted in half-a-gallon of warm water. Some practitioners recommend a quarter of a pound of starch to be added to the above quantity of linseed. Gruel made of milk and flour may be given with advantage; and if the dung assumes a dark colour with a disagreeable odour, then about a gill and a half of castor oil must be administered, and this may be assisted by clysters of gruel, butter, and laudanum.

When the disease becomes inveterate, some veterinarians have recommended strong tonics, which, in many instances have proved effectual. The following recipe will frequently prevail in bad cases :-

$\begin{array}{lllll}\text { Prepared chalk } & \text {. } & \text {. } & \text {. } & \frac{1}{2} \text { pound, } \\ \text { Ground cassia . } & \text {. } & \text {. } & \text {. } & \mathrm{I} \frac{1}{2} \text { ounce, } \\ \text { Ground ginger . } & \text {. } & \text {. } & \text {. } & \frac{1}{2} \text { ounce, } \\ \text { Laudanum } & \text {. } & \text {. } & \text {. } & \text { I ounce; }\end{array}$

to be made into four balls, and given every four or five hours.

Should all the above remedies prove ineffectual, the disease may then be treated as an affection of the liver, and the following ball administered :-

\begin{tabular}{|c|c|c|}
\hline Blue mercurial pi & & \\
\hline India rhubarb. & & \\
\hline Castor oil & & - \\
\hline Oatmeal & & . \\
\hline
\end{tabular}

which should be repeated for three or four days in succession. This may, however, produce sickness 
and gripes, accompanied with increased scouring; and in that event it will be necessary to administer astringents, and warm gruel should form the chief drink of the animal while he is under the above treatment. Let the following be given:-

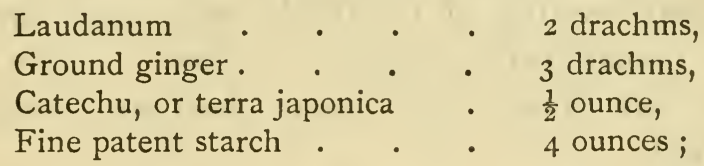

the whole to be diluted in two quarts of warm water, and given as a drink.

If the animal has received benefit from the above, and a progressive improvement becomes apparent, the animal should be allowed green pasturage for a few hours daily; but the situation must not be low or damp, otherwise a relapse will be the consequence, which will very likely prove fatal. Cows that are constantly milked and poorly fed are frequently attacked with scouring-rot. The remedies which we have above pointed out, will be also applicable to this case. But, while medicine is administered, drenches of oatmeal and flour, mixed, should be given at the same time, but not in great quantities at once, as too sudden changes would be injurious to the animal. After he is able to take a greater portion of food with safety, bean and pea-meal, mixed, should be given as a drench, which contains a greater portion of nutriment than any other vegetable substance. Bran-mashes may be given as a change from the above, until the animal is sufficiently recovered to be turned out entirely.

FARDEL-BOUND, OR EXCESSIVE COSTIVENESS.

Symptoms. - This disease manifests itself by extraordinary costiveness, and alternating with looseness 
at intervals; the fæces consisting of a profuse slimy discharge, with portions of hard dung in the form of balls, of different dimensions. Sometimes the complaint is accompanied by considerable swelling of the body, terminating in inflammation which is manifested by a short heaving of the flanks with hard breathing, and a considerable degree of fever. The animal loathes his food, and soon becomes weak and emaciated.

Causes.-This disease is generally due to the animal being fed on too large quantities of grains or meals, such as bean-meal, pea-meal, undecorticated cotton cake, and more especially if these are coarsely ground and not steamed or boiled.

Sometimes constipation in oxen is due to the cut spermatic cord twisting round a portion of bowel and thus mechanically preventing the contents of the incarcerated bowel passing downwards and being voided. Fardel-bound is generally a constipation of the third stomach.

Remedies.-Give the animal a pint of castor oil in half-a-gallon of warm oatmeal-gruel, with four ounces of Epsom salts and one ounce of carbonate of ammonia. If in twelve hours this purgative does not restore the bowels to their proper condition, repeat the dose and allow the animal to drink as much cold water as it desires.

Veterinary surgeons frequently treat that form of constipation known as "Gut-tie" by operation. The animal is first thrown on the ground, and an incision made in the right flank sufficient to admit the hand; when the operator will insert his hand to feel for the ligament by which the intestine is bound, and having discovered it, he separates the ligament with the nail of his thumb, which enables the intestine to assume 
its natural position. The part is then sewed up, and it will soon heal if the animal is in a good condition of body.

\section{MORTIFICATION.}

Symptoms. - This complaint shows itself by swellings behind the kidneys, and sometimes in the flanks, shoulders, side, or legs, accompanied with a desire to remain in a lying posture undisturbed. The premonitors to dissolution are a most fotid odour proceeding from the animal. It is seldom that he lives long after the first appearance of the malady: from five to twenty-four hours generally puts a period to his existence. Animals which have died of this complaint have been skinned, and it has been found that the swollen parts contain a gelatinous matter, mixed with very dark-coloured blood, nearly approaching to black. The animal dies without any symptoms of pain.

CAUSES. - This is a complaint which has only been known to exist in Connecticut, America, where it has proved very fatal to cattle. It is attributed to a change of pasture, or from bad to good fodder, which causes plethora, or fullness, and gorging of the vessels of the body. Cattle in the highest condition are most liable to be affected.

REMEDIES.-Little can be effected where this malady is not attacked at the outset, and the medicines must be those of a preventive quality. These must be, first, not to make too sudden a change from a bad to a good pasturage, nor too abrupt a transition from a poor to a rich diet. The following aperient should be first administered in the form of a drink :-

$\begin{array}{llll}\text { Calomel } & \cdot & \frac{1}{2} \text { drachm, } \\ \text { Tartarised antimony } & \cdot & \cdot & 2 \text { drachms, } \\ \text { Cream of tartar } & : & \cdot & \frac{1}{2} \text { ounce, } \\ \text { Camphor } & \cdot & \text {. } & 2 \text { scruples, }\end{array}$


to be given in a pint of gruel of wheat-flour. And when it has operated, give the following medicine every day until the symptoms disappear:-

$\begin{array}{llll}\text { Peruvian bark } & \cdot & \cdot & \frac{1}{2} \text { ounce, } \\ \text { Nitre, powdered } & \cdot & \cdot & \text { 2 drachms, } \\ \text { Salt of steel } & \cdot & \cdot & \text { I drachm, } \\ \text { Camphor, porvdered } & \cdot & \cdot & \text { I scruple, }\end{array}$

to be administered in a quart of warm oatmeal gruel. 


\section{CHAPTER IV.}

\section{Of Febrile Diseases.}

\section{SPIENIC APOPLEXY-ANTHRAX.}

Anthrax is a disease which not only attacks cattle and sheep but also pigs and horses, and by inoculation of the blood of an affected animal, it may be conveyed to almost all warm-blooded animals, including mankind. The manner in which cattle, sheep, and horses are infected is difficult to trace, but there is but one way that the disease can be propagated, and that is the introduction into the body of the animal of the spores or seeds of a rod-shaped organism, termed the "Bacillus Anthracis."

Anthrax being a scheduled disease, stockowners are required to report to the local authority its existence, and treatment of the affected animal is not permitted. The Board of Agriculture, having published a leaflet on Anthrax, and expressly requested that the information which it contained might be brought under the notice of agriculturists and stock-owners throughout the country. We gladly comply with that request :-

I. Anthrax is due to the existence in the blood on a minute rod (Bacillus Anthracis), which is one of a large family of fungi, and grows from spores or seeds.

2. Any substance which is brought on to a farm may act as a carrier of the infecting agent : fodder, 
litter, manure, whether from home or foreign sources, may contain the spores. A water-course may carry the poison. In fact, the channels through which the infecting agent may be conveyed to the susceptible animal are beyond calculation.

3. Diseased animals do not transmit the infection to others in the ordinary way by association. The organism on which the disease depends must be introduced into the blood through a wound however small; or an abrasion however slight, before the affection can be communicated, and it may be said without exaggeration that the carcase of an animal, dead of anthrax, is more dangerous than a living diseased animal.

\section{SYMPTOMS AND POST-MORTEM APPEARANCES OF ANTHRAX.}

4. In most cases, the first sign of an outbreak of anthrax or splenic fever is the discovery of a dead animal in the pasture or byre. Probably the animal was left a few hours before in apparent health; at least there was nothing to attract attention, or give any warning of the approaching catastrophe. Occasionally, and in the case of sheep not uncommonly, there are certain premonitory symptoms of an attack of anthrax which can be recognised by an expert. The affected animal is dull and disinclined to move. If one of a herd or flock is attacked, the fact is indicated by the separation of the sick animal from the rest. Close observation will enable the observer to detect an occasional shiver, which seems to pass rapidly over the body, and then ceases. Sometimes a little blood is discharged from the nose and also with the fæces, and from time to time the animal 
will cease to feed, and stand with the head bent towards the ground. On closer inspection, especially in the case of swine, it will often be found that there is a good deal of swelling under the throat, extending down the neck; and the swollen part will at first be tender to the touch, and hot, but as the disease goes on it becomes insensitive, cold, and clammy. The shivering fits now become more frequent, and perhaps, while these signs are being noted, the animal will suddenly roll over on its side, and, after a few violent struggles, expire.

5. According to the severity and suddenness of the attack, the post-mortem appearances will vary in degree, but they are tolerably uniform in kind. Under the skin there are usually patches of effused blood, and a considerable quantity of viscid serous fluid will be seen in those parts which were swollen during life. If the cavities of the chest and abdomen are examined, some red serous fluid generally escapes. The spleen is enlarged to three or four times its proper size, and is of a deep purple or black colour, soft and easily broken down. Effused blood is also found in masses under the kidneys, and red patches are seen in various parts of the serous membranes. The lining membrane of the intestines is often congested, and the contents are generally mixed with blood; sometimes, indeed, the intestinal canal is almost filled with that fluid.

6. The symptoms and post-mortem appearances which have been described may, as a rule, be accepted as evidence of the existence of anthrax. But it is very desirable to avoid opening the carcase of an animal which has died of anthrax, and the bacillus may be detected by putting a drop of blood from the ear or foot on a glass slide, covering it with a piece of thin glass, and examining it with a magnifying power 
of, at least, 400 diameters. The thin rods will appear like short pieces of fine thread crossing each other in every direction, and enclosing the blood corpuscles. This examination may be conducted in the shed or pasture, but in the laboratory staining processes are employed.

\section{PROCEDURE.}

7. The healthy animals on the pasture or other place where the outbreak occurred should be moved under proper restrictions to a convenient place for isolation, and should be examined by a veterinary surgeon every day for a week. If a rise of temperature is discovered in any of the isolated animals they should, as far as practicable, be removed from the rest of the herd until they have recovered.

8. Slaughter, by a Local Authority, of healthy animals in contact is only justifiable under special circumstances, i.e., where the animals are fat and fit for the butcher, or where the animals are few in number and of little value, or in outbreaks where the disease is spreading rapidly and there are no means of proper isolation.

9. Antiseptics, such as hyposulphite of soda, have been administered to the in-contact animals with apparent advantage. Medical treatment of animals should, however, only be carried out under the advice and direction of a veterinary surgeon, and with regard to this and other preventive measures it may be said that it is impossible to determine the degree of efficacy which they possess, owing to the fact that anthrax frequently ceases after the loss of a single animal.

IO. Inoculation, on the system recommended by M. Pasteur, could not be adopted except by an expert accustomed to operate, but the results of the operation 
in this country and elsewhere have not been of such a nature as would warrant the Board in recommending it to stock-owners as a means of dealing with outbreaks of anthrax.

\section{DISPOSAL OF CARCASES.}

II. Carcases of animals that have died or been slaughtered, affected with or suspected of anthrax, should be dealt with by the Local Authority in accordance with the following provisions :-

Either the Local Authority should cause the carcase to be buried as soon as possible in its skin in some convenient or suitable place removed from any dwelling-house, and at such a distance from any well or water-course as will preclude any risk of the contamination of the water therein, and at a depth of not less than six feet below the surface of the earth, having a layer of lime not less than one foot deep beneath, and a similar layer of lime above the carcase.

Or the Local Authority may, if authorised by licence of the Board, cause the carcase to be destroyed, under the inspection of the Local Authority, in the mode following: The carcase should be disinfected, and should then be taken, in charge of an officer of the Local Authority, to a horse-slaughterer's or knacker's yard approved for the purpose by the Board, or other place so approved, and should be there destroyed by exposure to a high temperature, or by chemical agents.

Before a carcase is removed for burial or destruction as above suggested, it should be covered with quicklime. In no case should the skin of 
the carcase be cut, nor should anything be done to cause the effusion of blood.

It is most important that the carcases should be left uncut, as the bacillus of anthrax disappears when kept from contact with air.

Any method, therefore, of destroying or disposing of the carcases which involves skinning or cutting up should be avoided. The disease is likely to be spread thereby, and, in addition, people engaged in such work run a very serious risk of being inoculated with the disease.

\section{CLEANSING AND DISINFECTION.}

The following are the provisions of the Anthrax Order of 1895 as regards the cleansing and disinfection of premises in which cases of anthrax have occurred :

The Local Authority shall, at their own expense, cause to be cleansed and disinfected in the mode provided by this Article-

All those parts of any shed, stable, building, or other place in which a diseased or suspected animal has been kept or has died or been slaughtered;

Every utensil, pen, hurdle, or other thing used for or about any diseased or suspected animal ;

Every van, cart, or other vehicle used for carrying any diseased or suspected animal on land, otherwise than on a railway.

The mode of the cleansing and disinfection of such shed, stable, building, or other place, or the part thereof, shall be as follows :

All those parts aforesaid of the shed, stable, 
building, or other place, shall be swept out, and all litter, dung, or other thing that has been in contact with, or used about, any diseased or suspected animal, shall be effectually removed therefrom: then

The floor and all other parts of the shed, stable, building, or other place with which the diseased or suspected animal or its droppings or any discharge from the mouth or nostrils of the animal has come in contact, shall be, as far as practicable, thoroughly washed or scrubbed or scoured with water: then

The same parts of the shed, stable, building, or other place shall be washed over with limewash made of freshly burnt lime and water, and containing in each gallon of limewash four ounces of chloride of lime or half a pint of commercial carbolic acid, the limewash being prepared immediately before use ;

Except that where any place as aforesaid is not capable of being so cleansed and disinfected, it shall be sufficient if such place be cleansed and disinfected as far as practicable.

The mode of the cleansing and disinfection of such utensil, pen, hurdle, or other thing, and such van, cart, or other vehicle aforesaid, shall be as follows :

Each utensil, pen, hurdle, or other thing, van, cart, or other vehicle, shall be thoroughly scraped, and all litter, dung, sawdust, or other thing shall be effectually removed therefrom : then 
It shall be thoroughly washed or scrubbed or scoured with water: then

It shall be washed over with limewash made of freshly burnt lime and water, and containing in each gallon of limewash four ounces of chloride of lime or half a pint of commercial carbolic acid, the limewash being prepared immediately before use.

All litter, dung, or other thing that has been removed from any such shed, stable, building, place, van, cart, or vehicle as aforesaid, shall be forthwith burnt or otherwise destroyed or disinfected to the satisfaction of an inspector of the Local Authority.

In regard to the disinfection of fields and like places in which animals affected with anthrax have died or been slaughtered, there are serious difficulties which cannot be completely removed. A top-dressing of lime is the most simple method which can be employed, and after a month or six weeks the lands may be re-stocked. But it must be understood that a certain degree of risk is incurred even after a much longer interval, and occasionally it has been found impossible to render contaminated land safe. In the majority of cases, however, the plan suggested will be effectual.

"BLACK LEG," "BLACK QUARTER."

Stock proprietors generally look upon Black Leg and Anthrax as one and the same disease, or if not very closely related, but they are perfectly distinct affections, although both are dependant on specific organisms.

The specific organism of Black Leg is smaller and 
thicker than the bacillus of Anthrax. It is motile (seen to move under the microscope), rounded at the end, and generally contains a bright oval spore. The blood and the spleen contains the anthrax bacilli in large numbers. The bacilli of black leg do not invade the spleen nor the blood stream, but they are found in large numbers in the exudate in the muscles and joints of the locally affected parts, e.g., loins, hip, knee, hock, shoulder, etc.

Anthrax may affect any animal and at any age. Black Quarter always attacks the young, and is confined to cattle and sheep.

Dogs and cats lapping the blood or eating the flesh of an anthrax subject, contract the disease and die, but we have never known death to result from lapping the blood and eating the flesh of a black leg subject.

Symptoms.-In the early stage of the disease, there is stiffness and soon marked lameness, and the inexperienced are apt to connect these with an injury; but in the course of a few hours the locally affected part becomes swollen and crepitates (crackles) when pressed with the fingers. This is a valuable symptom and shows that mortification has set in. The other symptoms are a quick small pulse, grinding of the teeth, standing in the field by itself, dullness and unwillingness to move from the spot.

Treatment-Preventative. Draining the land and the application of gas lime. Rock salt to lick. Feeding the animals so as to maintain at all times fair condition. Animals meagrely fed during winter, and then by a rush of grass in spring, put on rapid condition, are found in largest numbers to be the victims of this disease. As a preventative some proprietors have great faith in the introduction of a seton into the 
dewlap inserted a week before the cattle are turned out to graze in spring. Sulphite of sodium in ounce doses daily is a valuable preventative agent.

\section{PARTURIENT APOPLEXY, OR MILK FEVER.}

This form of parturient disease not unfrequently commences twelve or from that to thirty-six hours after calving. It sometimes occurs before calving, but it is seldom, and even then it may be confounded with parturient paralysis.

Syмpтомs.-Uneasiness, moving off the one hind $\operatorname{limb}$ on to the other, swinging to and fro of the body, staggering, ultimately falling down. Eyes at first bright, clear, expressive of cerebral excitement. Eyelids and ears spasmodically moving. By-and-bye, dullness, grinding of the teeth, head tossed from side to side, coma, deep breathing, puffing of the cheeks, and falling down of the lower jaw. The cow now lies in a deep sleep and is perfectly unconscious.

Causes. - The causes of this disease hang together as a narrative. First, the breeding is allimportant; Ayrshire and shorthorns are more prone than any other, but even these, unless deep milkers, escape. Second, the age of the animal is a predisposing cause. Rarely is any cow attacked earlier than at the third calving. Third, the feeding and condition are powerful links in the chain of causation. Plethora (rich in blood) inducing the disease.

Treatment. - The curative treatment of this disease is frequently disappointing, whereas the preventative is highly satisfactory. We will first describe the preventative. Bring all cows to the calving in fair condition and none in a state of plethora. To 
do this, if the animals are calving on the grass, have the pastures rather bare than otherwise, so that the animals will require to take a deal of exercise in procuring their food. When the cow is due to calf indoors, see that she has food to keep her in fair condition but nothing more, and as much as possible allow her to be out of doors daily for exercise and grazing.

Be careful to prevent constipation of the bowels from whatever cause, and by bran-mash and treacle, with a few boiled turnips and chaff, bring her to the day of calving with open and rather empty stomach and bowels. As soon as the labour pains commence, administer the following :-

\begin{tabular}{|c|c|}
\hline Epsom salts & I pound, \\
\hline Common salt & I pound, \\
\hline Croton beans & 20 (crushed and sound), \\
\hline Ginger powdered & I ounce ; \\
\hline
\end{tabular}

dissolve in four pints of water and pour over.

If the cow has much milk on her, do not hesitate to milk her daily before calving, and thus prevent congestion of the udder. After calving, milk her thrice daily or more frequently, doing it slowly, rubbing the gland and leaving a quantity of the milk undrawn. The diet for a week after calving should be only sufficient to keep the animal in health, and neither cakes nor meals of any kind, nor draff must be allowed. If grass is procurable, a sufficiency of it and plenty of water is all that is required. If grass is not in season, good hay and a few boiled turnips or cabbages will suffice. In eight days after calving, if all goes well, now the forcing feeding (meal, cakes, draff), may commence, but not earlier. We have practised this preventative treatment for years, and the cases of milk 
fever which occur under it are few indeed, and seldom prove fatal.

The curative treatment after the animal is seized, is too serious and complicated to be undertaken by any other person than the experienced veterinarian. 


\section{CHAPTER V.}

Diseases, etc., of the External Parts of Cattle.

SCURF, SCAB.

Symptoms.-This is a disease of the skin, and is manifested by its stiffness, and sticking fast to every part of the body, as if it were too small for the carcase, and first becomes visible about the head and jaws, the skin exhibiting a pale, dry, scurfy appearance, and then extends down the shoulders and back. The animal rubs itself violently against every object which it comes near; and unless remedies are applied, it will tear its skin, and cause bleeding, and thereby produce scabs, which retard the efficacy of the ointment, and allow the disorder to become more deeply rooted.

CaUses.-Poverty of the blood is an immediate cause of this complaint.

Remedies. - The best specific is mercury; but the animal is so apt to lick its skin, that it is a dangerous remedy. The following ointment has been found effectual in removing the complaint :-

Sulphur vivum, powdered

To be mixed with hogs' lard . . 6 ounces.

Let the whole parts affected be rubbed with this ointment, and repeated in the course of three or four 
days, if the itching does not abate. Some think that the following lotion is as certain as the above:-

Corrosive sublimate, finely powdered . . $\frac{1}{2}$ ounce, Muriatic acid . . . . . . . I ounce, River, or rain-water . . . . . 2 quarts,

to be applied with a sponge daily to the parts affected.

\section{COW-POX}

The following is the description of this disease, as communicated by Dr Jenner, the discoverer of it, and who first applied the lymph contained in the cow-pox vesicles to the human being, and which has proved an inestimable blessing to mankind as a preventative of small-pox. Dr Jenner was a medical practioner at Berkeley, in Gloucestershire, and often saw the complaint in various dairies in that neighbourhood. $\mathrm{He}$ says, "In this dairy country a great number of cows are kept, and the office of milking is performed indiscriminately by men and maid-servants. One of the former having been appointed to apply dressings to the heels of a horse affected with the grease, and, not paying due attention to cleanliness, incautiously bears his part in milking the cows, with some particles of the infectious matter adhering to his fingers. When this is the case, it commonly happens that a disease is communicated to the cows, and from the cows to the dairy-maids, which spreads through the farm, until most of the cattle and domestics feel its unpleasant consequences. This disease has obtained the name of cow-pox. It appears on the nipples of the cows, in the form of irregular pustules with depressed or umbilicated centres. At their first appearance they are of a palish blue, or rather of a colour somewhat 
approaching to livid, and are surrounded by erysipelatous inflammation. These pustules, unless a timely remedy be applied, frequently degenerate into phagedenic ulcers, which prove extremely troublesome. The animals become indisposed, and the secretion of milk is much lessened.

Frequently another kind of eruption appears on the udder of the cow, which, when not carefully examined, may be mistaken for the cow-pox. It manifests itself by the appearance of a number of white blisters on the nipples, filled with a whitish serous fluid. They are distinguished from the cowpox pustules by not having the bluish colour of the latter, as well as their never eating into the fleshy parts, being entirely confined to the skin, and terminating in scabs. This eruption is infectious, but not so highly so as the true cow-pox.

Dr Jenner was of opinion that this spurious eruption had its origin in the transition of the cow in the spring from a poor to a rich diet, at which period the udder becomes more than usually vascular from the supply of milk. In the West of England dairies there is still a third kind of inflammation, accompanied by pustules, which is not uncommon. When a cow with a naturally small udder is intended for sale, she is neither milked by the hand or by a calf for a day or two previously to her disposal. Consequently, an unusual quantity of milk is accumulated, and the udder is stretched out to an unnatural extent, which also has the effect of distending the nipples, and frequently produces inflammation, accompanied by pustular eruption.

REMEDY.-It is not necessary to apply any remedy for this complaint; all that is required is to keep the teats clean, and handle them gently during milking, 
and in a few days the eruption disappears. If the sores on the teats are painful, a few applications of glycerine and olive oil will assist the healing process.

\section{THE FOULS.}

Symptoms. - This disease is nearly allied to the disorder called canker in the horse, and is manifested by the discharge of fotid matter from the cleft of the hoof, or, in some cases from one of the claws. Veterinarians have divided this complaint into two kinds, the soft fouls and the horny fouls, which are differently treated by them. In the soft fouls, the discharge issues from the cleft of the hoof, and also from the heels, and in both cases the animal becomes very lame.

CaUSES.-Hard driving and long journeys often produce the fouls, more particularly if the roads are bad, and dirt lodges and dries in the cleft, or behind the heels. It affects cattle of all ages.

Remedies.-For the soft fouls, when cracks take place, these must be well washed with soft-soap and water, and then anointed with tar-ointment, and the feet kept as dry as possible. When a distention of the horny substance takes place, and soft, spongy protrusions appear, accompanied with the discharge of matter of a most offensive smell, these soft parts must be cut, or scraped off, and a caustic liquid applied to the parts. The following will prove effectual if well applied :-

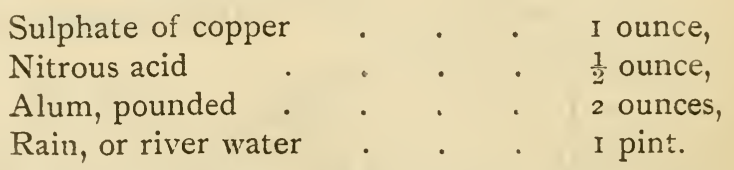

When the parts are properly washed with the above, 
and it has become dry, then a pledget covered with tar-ointment should be applied to the parts.

For the horny fouls, if the hoof feels very warm, and the animal manifests pain on pressure being applied to the parts, and they feel hard underneath, it will then be discovered that a horny substance is penetrating into the softer parts of the foot, either between the two halves of the hoof, or at the heels; these horny parts must be eradicated by cutting. In performing this operation, it will be necessary to throw the animal on some soft ground, or upon straw, the more easily to effect the cutting. After this is done, let the parts be rubbed with the above lotion, and the whole foot wrapped round with a cloth moistened in it. The animal may then be turned out to a soft pasturage. Should, however, the inflammation not subside in a couple of days, bleeding from the veins of the foot will be necessary.

\section{THE GAD-FLY, WURBLES, OR WORMALS.}

The gad-fly is exceedingly troublesome to cattle during the summer months, more especially in very hot seasons. This has been minutely investigated by Mr Bracey Clarke, and is scientifically called by him cestris bovis, or ox-fly. It has otherwise been called the breeze-fly. Their bite is very painful, and always creates a small swelling in that part of the skin penetrated by its tongue. But that evil of which we are about to treat has been successively denominated wurbles, or wormals, probably a corruption of wormholes, which are frequently found on the backs of cattle. The female gad-fly, which produces these humours, we have represented in Plate $\mathrm{x}$, fig. I5. From the observations of the celebrated naturalist and 
physiologist, Reaumur, it appears that the female is furnished with a singularly constructed ovipositor, which lengthens by a series of sliding tubes (Plate $\mathrm{x}$, fig. Io), precisely like that of an opera-glass. There are four of these tubes, as may be seen by pressing the belly of the fly till they 'come into view. Like other ovipositors of the same sort, they are composed of a horny substance. This instrument is furnished with five points, three of which are longer than the other two, and at first sight not unlike a fleur-de-lis, though, upon narrower inspection, they may be discovered to terminate in curved points (Plate $\mathrm{x}$, fig. I 2), somewhat like the claw of a cat. The two shorter pieces are also pointed, but not curved; and by the union of the five, a tube is composed for the passage of the eggs. (See Plate x, fig. I I.) It would be necessary, Reaumur confesses, to see the fly employ this instrument to ascertain in what manner it acts, though he is disposed to consider it fit for boring through the hides of cattle.

$\mathrm{Mr}$ Bracey Clarke, taking another view of the subject, is decidedly of opinion that the fly does not pierce the skin of cattle with its ovipositor at all, but merely glues its eggs to the hairs, while the grubs, when hatched, eat their way under the skin. If this be the fact, as is not improbable, the three curved pieces of the ovipositor, instead of acting, as Reaumur imagined, like a centre-bit, will only serve to prevent the eggs from falling till they are firmly glued to the hair, the opening formed by the two shorter points permitting this to be effected.

The extraordinary effects produced upon cattle, on the appearance of one of these flies, would certainly lead us to conclude that the pain inflicted by the ovipositor, is excruciating. Most of our readers may 
recollect to have seen, in the summer months, a whole herd of cattle start off across a field, at full gallop, as if they were racing, their movements indescribably awkward-their tails being poked out strait and stiff behind them, and their necks stretched forward to their utmost length. All this consternation has been known from the earliest times to be caused by the appearance of the gad-fly.

If $\mathrm{Mr}$ Clarke's views be correct, when the egg of the ox-fly is hatched, the grub (Plate $x$, fig. I7) immediately burrows under the skin, where it feeds upon the fatty matter, and remains until it has reached maturity. As the grub continues to grow, the bumps, or wurbles, increase in dimensions, in proportion to the size of the grub (Plate $\mathrm{x}$, fig. $d$ ). It is not until the middle of May that these bumps can be seen full grown; but, owing to particular circumstances, they do not attain an equal size. The largest of these are sixteen or seventeen lines in diameter by the base, and about one inch high, but they are scarcely perceptible before the beginning, or during the course of the winter.

Young cattle are most commonly attacked by these insects, and the most healthy are fixed upon for the deposition of their eggs; a wonderful proof of the instinct of these flies. Cattle-dealers and farmers have remarked this fact, and the latter always find the hides the best that these flies prefer. The number of bumps, or wurbles, which are found upon a beast, is equal to the number of eggs which have been deposited in its flesh; or, to speak more correctly, the number of eggs which have become matured under the skin, as those which have been fertile. Sometimes there will be found a single wurble on a cow, while in others upwards of thirty have been counted. 
The ordinary size of the full-grown grub is represented on Plate $\mathrm{x}$, fig. 16 ; and 18 is a magnified figure. Fig. I 3 is a greatly magnified view of the tail of the grub.

Remedies.-As soon as it is detected that the skin of the cow is penetrated by this insect, the part may be squeezed between the two thumbs, and the grub will be forced through the aperture in the skin. If the person have not sufficient strength to dislodge it, this can be easily accomplished by the aid of a pair of shoemaker's pincers. This may be done at any time while the grub remains. Any other mode of cure is of no avail. When the grub has been ejected, let a drop of turpentine be put into the cavity, and in a short time it will heal up. A dressing of carbolic soap or sheep dip tends to prevent the fly depositing its eggs.

\section{LICE.}

When young cattle have been turned out in cold weather they are very subject to lice; more especially if they are in a low habit of body, and have not been kept clean. Parasites are not only disagreeable to the animal which they infest, but are also apt to be communicated to others. The speediest means should be adopted to destroy them. The following recipe will generally have the effect:-

\begin{tabular}{l} 
Common tobacco \\
$\begin{array}{lll}\text { White hellebore-root, ground } & \cdot & \text { I ounce, } \\
\text { Vinegar } & \cdot & 2 \text { ounces, }\end{array}$ \\
\hline
\end{tabular}

these to be boiled together for half-an-hour, and the warm decoction to be applied with a soft brush or 
sponge. A second application seldom fails to destroy the vermin.

Carbolic acid and warm water destroy the parasites.

\section{STRAINS AND BRUISES.}

Cattle are not so liable to strains and bruises as horses; and as they are seldom necessitated to active exertion after an accident has occurred to them, there is less difficulty in curing them. Whether the affection is in the muscular or tendinous parts, we would recommend similar treatment as that employed for the horse under such circumstances.

Fomentation should first be tried, and when the inflammation has been subdued, the following liniment must be well rubbed on the part affected, two or three times daily, until all the symptoms have disappeared :-

$\begin{array}{ll}\text { Linseed oil . . . . } & 5 \text { ounces, } \\ \text { Spirit of turpentine, } & \text { I ounce, } \\ \text { Hartshorn, or liquid ammonia . } & \text { I ounce. }\end{array}$

When any portion of the limbs has been sprained, so as to occasion lameness, and has not been removed by applying the above liniment, it will be necessary to have recourse to a blister.

\section{WOUNDS.}

In treating of this part of our subject, we shall confine our observations chiefly to wounds of a simple nature, for this reason, that when cattle are injured in the internal parts, which requires a long and tedious attention, it will be better at once to kill the animal, because the trouble and expense will counter-balance the profit of a tedious recovery. The flesh of cattle 
which have received severe wounds, if the animal be immediately destroyed, is perfectly wholesome. Besides, in the attempt to cure severe wounds, there is always a risk of the animal's dying, under the best devised treatment.

In the cure of wounds much depends on their situation, and the form of the instrument by which they were inflicted. A cut from a sharp instrument, provided it be clean, is very easily healed; all that is required being to bring the edges of the wound together, and keep them in contact by means of a slip of adhesive plaster. But if they are in situations where the plaster is not likely to remain on, in consequence of the action of the muscles or otherwise, a few stitches with strong thread or fine twine will have the same effect; and when it is found that adhesion of the parts has taken place, the string must be cut away, and the needleholes will quickly fill up. Or the wound may be kept together by means of a pin or two, with thread twisted crossways round them, which can be drawn out after the part has healed. Care must be taken that the wounds are rendered perfectly clean before their edges are brought together. But in fresh wounds, the use of stimulants as a wash, is to be carefully avoided, all that is necessary being to wash the parts with warm water. Ignorant quacks often apply salves and other greasy substances to fresh wounds, which, instead of healing them, produce suppuration and sores; and stimulants rather retard the progress than facilitate the natural healing process.

Where wounds are inflicted by a round substance, or an unequal-sided, or triangular instrument, they are more difficult and more tedious to heal. And if the wound is deep, sewing or pinning the parts must not be had recourse to. In such cases, deep-rooted 
suppuration is almost certain to attend them; and although the external surface may be brought together and healed, ulceration will be lurking beneath, and the matter there collected must sooner or later find vent. Whenever it is suspected that ulceration is going on below, it will be better to keep the surface open, and in about a week probe the wound to the bottom, and thus make a free opening for the escape of the accumulated matter, which should be pressed out, and a warm poultice applied to the part to draw out all that remains. As soon as the inflammation completely subsides, granulation of the sides of the wound will take place, and it will ultimately heal up.

In cases where valuable breeding cattle have received deep and severe wounds, and the proprietor is anxious to preserve the animals, then every means must be adopted which are known in the veterinary art to effect a cure. One of the first things to be attended to is to keep down inflammation. In cases of the animal being gored, or deeply wounded in the belly, by leaping over a fence or other object, it often happens that the incision is of such an extent as to permit the bowels to protrude. 'The first thing to be done in such cases, is to see that the intestines are clean, and free from dirt or hair ; and if they are not so, let them be carefully washed with a sponge and carbolised warm water, and then return them into the abdominal cavity, and stitch up the wound carefully with a crooked needle and strong well-waxed linen thread, or metallic wire, and afterwards apply a broad roller round the carcase of the animal, to prevent the internal pressure from tearing the sides of the wound asunder. The animal should then be placed in a situation where it can remain undisturbed. Its diet for some time must be limited, and consist of such food 
as will prevent it from becoming costive, such as mashes of bran, and linseed or thick oatmeal-gruel, and occasionally fresh grass. But should costiveness come on, then let a pint of castor oil be given in a quart, or half-a-gallon of thin warm gruel. It is a most important point to keep the bowels open in all such cases.

In protrusion of the bowels it often happens that a quantity of air collects in the part which has been forced out, and so enlarges it that the orifice of the wound will not admit of its being returned. It will therefore be necessary to enlarge the opening with a sharp knife, but very great caution is necessary not to cut any vital part. This is most effectually prevented by guiding the knife with the fore-finger.

When, however, such dangerous wounds have been inflicted, it will be safer to have them treated by a skilful veterinary surgeon; and it is only for the instruction of those who are far from such aid when an accident occurs that we have pointed out the best mode of treating it.

When parts which are greatly inflamed become gangrenous, instead of suppurating, exciting stimulants must be applied. Let the following simple application be first tried :-

$\begin{array}{lllr}\text { Tincture of myrrh } & \cdot & \cdot & 2 \text { ounces, } \\ \text { Corrosive sublimate } & \cdot & \cdot & \text { I } 2 \text { grains, } \\ \text { Pure water } & \text {. } & \text {. } & 4 \text { ounces. }\end{array}$

Some practitioners recommend a solution of the sulphate of copper, in the proportion of half-an-ounce to four ounces of water.

The following has also been advantageously used:-

$\begin{array}{llll}\text { Hog's lard } & \cdot & \cdot & 6 \text { ounces, } \\ \text { Bees' wax } & \cdot & \cdot & \text { I ounce, } \\ \text { Spirit of turpentine } & \cdot & \cdot & 6 \text { ounces, } \\ \text { Powdered verdigris } & \cdot & \cdot & \text { I ounce. }\end{array}$


Let the first three ingredients be melted together, and the verdigris then added. The mixture must be stirred until cold, to prevent the verdigris from falling to the bottom.

All other wounds must be treated by practical veterinarians, or dealt with according to the judgment of the possessor of the animal: to particularise all such as may occur would require more space than our limits will admit of. 


\section{CHAPTER VII.}

Of Gestation, Parturition, etc.

GESTATION.

THE period of gestation in the cow is about nine months, and she rarely produces more than one at a time, although instances have occurred of two, and even three being brought forth; but the latter case is exceedingly rare. The time at which the cow has taken the bull should be carefully noted, that she may be dried off in proper time before calving. She should also be allowed as much rest as she will take for some time previous to calving. Her food should be of good quality, and rather nutritious than otherwise. By pressing the closed hand on the left side of the belly in an early stage of pregnancy, the calf can be felt.

It may be well for the information of those who only keep one or two milch cows, to know that by proper attention to their food, the animals may be continued in milk, without detriment to the cow or her calf, till within two months of the time of calving.

In cold and severe weather, in the winter, when cows are in calf, they must be taken into houses during the night, and even in day-time when stormy. This is more especially to be attended to for a couple of weeks before calving, as, should the cow drop the calf in a cold, wet field, there is a possibility that both 
may perish. Even in the finest weather it will be prudent to take the cow and calf into the house at night, for a week or two.

During the time of gestation, cows are liable to several complaints, such as costiveness and strangury, or a difficulty in voiding urine. These must be carefully attended to, otherwise they may cause the cow to slip her calf.

STRANGURY will be known by the animal making frequent painful attempts to stale, and by her voiding only a small quantity of urine at a time, and frequently none at all. The following must be administered until the complaint is removed :-

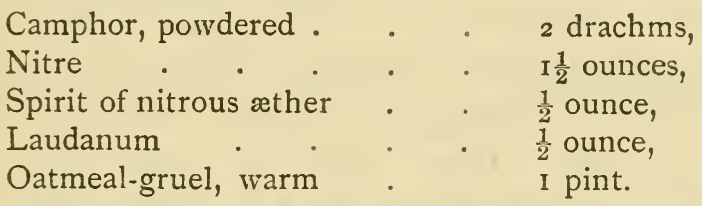

When strangury is accompanied by costiveness, which is frequently the case, immediate recourse should be had to laxatives. A pound of Epsom salts, dissolved in half-a-gallon of warm gruel, may be given; and the discharge assisted by administering clysters at the same time, consisting of four ounces of linseed-oil, and a quart of thin warm gruel, with half-an-ounce of salt of tartar to make them incorporate. If an evacuation does not speedily take place, the clyster must be repeated in half-an-hour afterwards, and continue every twenty minutes, until it has the desired effect.

It will be injudicious to feed a cow too much, previous to calving, as in that event they may be attacked with what is termed the milk-fever. If it is found that cows have too great a tendency to fatten previous to calving, they must be removed to a less nutritious pasture, or stinted in their diet, which is 
much more safe than reducing them by the aid of medicine.

\section{SWELLING OF THE UDDER.}

This is a complaint to which cows are liable at the time of calving, and is generally an indication that the animal will milk well. The swelling is due to congestions of blood in the vessels of the skin, with effusion of a watery liquid into the cell tissue underneath.

Symptoms.-Distention of the udder, pain and tenderness to pressure of the hand.

Remedies.-As a rule the swelling gradually subsides by absorption of the liquid within a few days after calving, and nothing is required but gentle rubbing with lanoline or vaseline ointment. The swelling of the udder may be associated with mammitis or inflammation of the udder, and when so, the treatment to be adopted will be found under that heading.

\section{DISEASES OF THE MAMMARY GLAND OR} UDDER.

In the cow the mammory gland is divided into four quarters, and there are four teats and four orifices; but in the mare there are only two quarters and two teats, with four orifices. The gland is connected to the abdomen by a sheet of connective tissue. It is further bound and supported to the abdomen by tendinous structure running betwixt the two halves of the gland, connected on the one hand to the muscles of the abdomen, and on the other by delicate fibres to the lactiferous (milk) ducts. It is in virtue of this arrangement that 
the cow can "keep up her milk" for a time, and only give it freely to those she likes when being milked.

A healthy udder is recognised by the two halves being equal in size, and the four teats being equal in size and length. If, as is sometimes the case, one teat is markedly smaller than the other three, it indicates disease, and on examination with the hand, the teat may be found blind and the quarter diseased and secreting no milk.

When the cow comes to the calving the udder is generally swollen, tender to pressure, and more or less hot. This is a natural state, and constitutes what is generally termed

PHYSIOLOGICAL CONGESTION OR ENGORGEMENT OF THE MAMME.

If the animal is come of a race of good or deep milkers, and possesses this qualification, then this physiological congestion is well marked, but on the other hand, if the animal neither inherits nor possesses the power of secreting a large quantity of milk, the congestion or "swam" is small.

The physiological engorgement referred to may lead to undue dilatation of the blood-vessels, and by pressure the arrest of the secretion of milk, or by "hefting" to engorgement of the milk tubes, and in this way to pathological (diseased) congestion and inflammation termed

\section{MAMMITIS, OR MASTITIS.}

Inflammation of this gland is much too common, especially in dairy cows, and probably in many cases it is due to a want of attention. It may appear before parturition, immediately after, or at 
any period during lactation. Sometimes it appears in dry cows (in calf or barren), put to grass, and is most troublesome to the grazier, as they cannot give the animals the attention required. If the cow is destined for the butcher, the teat is amputated at the root, and the pus formed in this way allowed to escape.

The type of inflammation may be acute or chronic. It, the inflammation, may be confined to the lactiferous (milk) tubes, and the delicate mucous membrane which lines their interior, and when this is the case the disease is termed

CATARRHAL MAMMITIS.

In other instances the inflammation primarily affects the secreting gland and connective tissue structures, and then it is termed

INTERSTITIAL OR PERENCHYMATOUS MAMMITIS.

If the udder prior to the attack was healthy, and the inflammation catarrhal, the symptoms are generally as follows, and confined to one quarter.

Pain (not acute at first nor for some time) on stripping the teat, the feeling of little hard lumps distinct, and only felt here and there. No uniform thickening and hardening of the gland and its connective tissue, because they are not involved in the inflammation. On withdrawing milk it is seen to be changed. It may be tinted more or less red, or watery, or curdled, and found to contain mucous, pus, and blood-cells. Very little milk may be able to be withdrawn, the teat sinuses or reservoirs being blocked with coagulated milk and pus. Here, if relief be not given, an abscess or abscesses form, 
and the gland and connective tissue may now become the seat of inflammation also. If the case is seen early and proper treatment adopted the inflammation may subside, but if not it is apt to become a chronic affection like a chronic catarrh of the mucous membrane of the nose, and when this is so there is neither thickening of tissue to be felt nor pain on pressure exhibited. The milk secreted remains more or less watery, purulent, and bloody.

Causes. - The causes are various, exposure to cold, or cold drafts of air coming in under the door, and blowing direct on the udder. Hefting, that is allowing two or more meals of milk to remain in the udder, a practice followed by many persons when exhibiting a cow in milk, in a fair for sale or show purposes. Another cause is carelessness in milking the animal and not removing by the hand all the contents of the gland. Blind teat resulting from an injury, and gradually closing up, and the introduction of teat syphons which are not scrupulously clean, may also lead to and terminate in inflammation of the udder.

Remedies. - Keep the animal within doors and free from drafts. Limit the food to that only sufficient to support the strength and not form milk. Administer a dose of salts sufficient to purge the animal freely, and keep up the purgative action for a few days. Gently strip the teats to remove the milk, and do this frequently. Anoint the udder with an ointment of palm oil and camphor, and rub it gently in with the hand, taking plenty of time so as to assist in softening the lumps. If necessary pass up a teat syphon, but see that it is clean and disinfected. Should the inflammation end in suppuration and the formation of an abscess, then the sooner the veterin 
arian is in attendance on the animal, it will be to the advantage of all concerned.

\section{INTERSTITIAL, OR PARENCHYMATOUS MAMMITIS.}

When it originates as a primary affection, it generally does so suddenly. It may be an animal milked in the evening and all right; next morning no milk of consequence, and the udder tender, painful, and swollen. She has no desire for food, is stiff and unwilling to move, and disinclined to allow you to manipulate the gland.

The inflammation once set up proceeds rapidly, and is prone to invade at least one-half of the udder, and frequently it involves the whole of the organ. Fever is a marked symptom, the thermometer standing at 106 or more. The pulse is full, the breathing disturbed, and the animal seldom ruminates.

By early and appropriate treatment, the inflammation may subside, and resolution by absorption of the exudate take place, but if so, the area of the inflammation must be limited. If the inflammation spreads rapidly, the udder in a day or two is enormousiy enlarged and becomes as hard as a stone, and in this indurated condition it may remain for an indefinite period, the lacteal secretion having ceased. If the animal lives, the hard masses of inflammatory exudates may commence to break up, softening and suppuration following each other.

The purulent matter may discharge itself by the teats, by sinuses through the body of the gland, and in places it may be retained in cysts or abscesses, so that we have circumscribed and diffused suppuration as in the lung tissue in pleuro-pneumonia. 
If the inflammation be acute and the induration extensive, the animal may die before that softening and suppuration has set in, the pressure of the inflammatory exudates on the blood-vessels of the udder being so great as to stop the circulation and lead to mortification of the udder.

If the suppuration be diffused, death may also result by blood-poisoning (septicæmia pyæmia) and general prostration, the animal partaking of no food to sustain life.

CaUses.--The causes are various : rough handling and bad milking. Blows from the head of the calf or lamb in the act of sucking. Lying on a damp or cold surface, chills from drafts of cold air from doors and windows, and sometimes contagion, as in abortion, from organisms gaining an entrance to the udder by the teat.

Remedies.-Have the cow or ewe housed and made comfortable. If cold weather, cover the body with a rug. Administer a full dose of saline purgative. Restrict the diet to one that will only support life, and not form milk.

Support the udder with a bandage and anoint the udder with camphorated mercurial ointment or belladona ointment. Apply the ointment three times daily with gentle friction and manipulation, but do not irritate the skin; at same time strip the teats and remove the secreted milk and pus. Fomentations and poultices may also be required, and for contagious or septic inflammation of the udder, injections of boracic acid, and other agents should be employed, but as the operation is a delicate one and in some cases has led to serious and fatal consequences, we would not advise any one but an experienced veterinarian to attempt it. The prevention of the spread of contagious mammitis 
to other animals in the herd or flock, is one requiring the guidance of a veterinary expert, and will well repay the stock proprietor for the outlay.

\section{AGALACTIA.}

Agalactia or arrest of the milk secretion, sometimes takes place within a day or two, and sometimes it affects a number of cows at the same time. When a number are affected, and they are all being depastured in the same field, it may be taken for granted that it is due to some herb in the grass, which at this stage in its growth, has this power of affecting the circulation of blood in the mammory gland, and the secretion of milk.

REMEDIES.-Change the pasture and give thrice daily warm drinks of bean and oatmeal with a little linseed, if bowels are constipated. In a fortnight or so, the cows may in all probability be returned to the same pasture and fed thereon without bad effects.

\section{ULCERATED TEATS-CHAPS.}

Cows that are cat-hammed, or go close behind, are liable to be affected with chapped udders, in consequence of their thighs rubbing against them. The skin of the thighs may also be inflamed. These may be readily cured by the parts being regularly washed with carbolic soap and warm water, and afterwards sponged over with a lotion, composed of-

Extract of goulard

Camphorated spirits of wine

2 ounces, 4 ounces.

When the teats are chapped, which frequently proceeds from a want of cleanliness on the part of the 
milker, great care must be exercised, otherwise deep fissures and ulcers may form, and the same affection may appear on the teats of all the other animals milked by the same unclean person. Mammitis may also result from the animal suffering so much pain on milking that she refuses to give up her milk.

Remedies. - Chapped and other diseased conditions of the teats are largely preventable. With this object in view, the hands of the milker should be washed before and after milking a cow, and the farmer or dairyman should have clean water, towel, and soap so placed as to allow of this being carried into practice without inconvenience and trouble to the milker.

Before (if necessary), and always after, milking, the teats should be sponged and dried, and if the skin is tender (as it generally is in all cows for some time after calving, but especially in cows subjected to milking after their first calf), anointed with camphorated olive oil and glycerine. If this treatment is adopted from the first, the tender skin having now been used to the friction and heat in milking, will not become inflamed and tender, and when this stage has been reached, the ointment may be discontinued, and all that is now required is to keep the teats clean.

\section{ABORTION.}

This is the term in general use to signify a premature expulsion of the fotus from the womb at any period before, when born, it is capable of a separate existence. A calf seldom lives if born at seven months, and even at the end of eight months it is small and weakly. Abortion may take place as early 
as the sixth week, but in cows it is more frequently the sixth or seventh month.

The causes giving rise to abortion may be ranked under two heads : first, Accidental or Non-Contagious ; and, second, Contagious.

Under the head of accidental causes, may be enumerated (I) exposure to cold and wet, (2) fright, (3) over-exertion, (4) injuries of various kinds and particularly over the region of the abdomen, (5) food indigestible, ergotised grasses, frozen turnips, cabbages, grasses, etc., (6) water impure, or at a low temperature, (7) bad smells, e.g., cows hanging their placental membranes, or after-birth, etc., etc.

As a rule the cases of abortion due to accidental causes are not numerous even in large herds and flocks, but when abortion is due to contagion, or the entrance of micro-organisms (via the vagina), betwixt the placental membranes and the womb, then the cases in general are numerous and prone to become continuous.

Symptoms. - The symptoms of abortion depend upon the stage of development of the young at the time of its expulsion from the womb. If prior to the fourth month, no premonitory symptoms may be exhibited. The embryo lamb or calf may be found in the field and close examination required to single out the unfortunate mother. If after the fourth month, the cow about to abort, generally has a bloody discharge coming from the vulva, the ligaments of the pelvis relaxed, and the udder more or less congested. Shifting of the feet, arching of the back, and straining, are also symptoms.

Remedies. - If the animal about to abort is detected, early abortion in some cases may be prevented. In all cases it is of the utmost importance 
to remove the animal and place her in a loose-box away from all other. A sedative drink should be administered, composed of the following :-

Linseed oil

Tincture of opium . . . . . 2 ounces,

Tincture of ginger . . . . 2 ounces.

This drink may be repeated in twelve hours. The food should be restricted to small bran mashes and plenty of tepid water to drink.

If the animal aborts and the placental membranes are not cast off, as is generally the case, they must be removed by the hand without delay, and the womb washed out with Jays' fluid and tepid water, but the removal of the membranes should be entrusted to the veterinary surgeon.

As preventative treatment various agents have been administered, and by some carbolic acid is looked upon as a specific. Pure carbolic acid in from one to two drachm doses may be given daily in drinking water or in the food.

In contagious abortion it is all-important not only to administer carbolic acid internally, but also to sponge out the vagina and wash the vulva and tail with carbolised soap and water, and to do this daily to all the animals in the herd until the malady disappears.

The strength of carbolic acid for disinfection and sponging purposes varies, but in the proportion of I oz. of carbolic acid to $50 \mathrm{Oz}$. of water, it is safe. A solution of sulphate of copper is useful, and another inexpensive and effective lotion is:-

Corrosive sublimate

Muriatic acid.

Soft water
$2 \frac{1}{2}$ drachms,

$2 \frac{1}{2}$ ounces,

2 gallons. 
In tracing the original cause of the outbreak of contagious abortion, and its continuance in a herd, it is all-important to remember that the contagion may be carried by the bull as well as the cow, and, all things considered, it is advisable to keep two bulls for service in a herd where contagious abortion has obtained a footing. It is also advisable to divide the herd into two portions, and to graze and house all the cows who have aborted by themselves, and to serve them by the one bull. The sound cows and the other bull should congregate together, and, if possible, have separate attendants.

\section{FALLING DOWN OF THE CALF BED.}

The extent to which this takes place varies. In some cases it is only a prolapsus of the walls of the vagina, and is only seen to take place when the cow lies down. In other cases not only the vaginal wall descends, but also the whole body of the womb or calf bed.

REMEDIES. - Where the prolapsus is restricted to the vagina, and only takes place in the recumbent position, all that is required in general, is to lower the Ann: in finnt, or raise the floor behind, so that when the animal is lying down, her body behind will be raised up, and the calf in the womb will gravitate forwards, instead of downwards and backwards.

When the womb has become inverted the first thing to be done is to remove the placental membranes, support and protect the womb with a clean cloth underneath, and have it returned at once, and this as a rule can only be accomplished by a veterinary expert, so that we need not describe the operation in detail. 


\section{PARTURITION, OR CALVING.}

In most instances animals do not require the aid of man in bringing forth their young, as nature works her own ends. The principal thing to be attended to, is to have the animal placed in a dry and warm situation. But, as it is possible, that a wrong presentation or an accident may occur, it will be proper to have a person watching the animal when parturition is near at hand.

The natural presentation of the calf is with its head and fore-feet, the muzzle resting between the feet, with the back of the animal upwards. $\mathrm{Mr}$ Downing, a veterinary surgeon, who had much experience, enumerates seven different preternatural presentations ; namely :-

Ist. Reverse, or with the tail and hind-quarters first.-This position being favourable for extraction, if the calf be a small one, let nature take its course, but assist the animal in a speedy delivery, to prevent the possibility of suffocation. Let the haunches be pressed back with the palm of the hand, and seize the bend of the hough of one leg, and pull it until the foot is reached, which will facilitate the extraction of both feet.

2nd. The fore-feet first, the head not being visible.-Get hold of the nose or face-bone, and place the head in its proper position, between the fore-legs. This requires the arm of the operator to be stretched its full length, being careful that the fingers are firmly fixed, so that advantage may be taken of every throe, as it occurs.

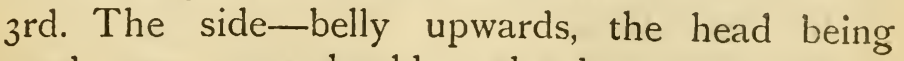
reversed over one shoulder, the legs appearing.- 
Let the calf be moved backwards by gentle pressure, and bring the head forward to the legs.

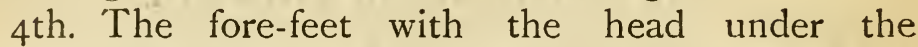
brisket.-Let the calf be pushed back until the head is felt, then take hold of the nose and pull at it, when it is certain that the head is in a proper position; but force must on no account be used until it is certain that it is so.

5th. The head alone, or with only one fore-leg accompanying it.-Push the calf back against the shoulders and brisket, and the feet, or one of them, will be found folded under the belly; pull the feet forward, one at a time, while the hand is gently placed on the bend of the knee. It not unfrequently happens, that the head becomes so much swollen and bruised, that it cannot be returned; when this is the case, the only thing which can be done to save the cow, is to amputate the head of the calf, after having first skinned it. Cut through the skin of the forehead in a straight line, from the nose to the poll, and force it back over the first joint of the neck, at which place let the head be divided from the body, and push it back into the womb so as to enable you to lay hold of the knees, and after wrapping the loose skin over the end of the neckbone, while an assistant keeps hold of the skin, in order to guide it clear of the haunch-bones of the cow; let the calf be extracted gently; but should it not come fairly forward, let the calf be pushed back immediately, and properly adjusted before again pulling it out.

The same treatment is applicable to the 6 th, when there is a presentation of the head and a single leg, or the head alone.

7 th. The calf lying on its back, with its four 
legs folded nearly together, and close up to the cow's back; the head appearing, or doubled back, even with the ribs, on one side or other; the hindleg perhaps appearing.-If the latter is the case, let it be put back, as it is impossible to extract the calf with a hind and fore-leg together, and the difference between the knee and the hough will be at once discovered. The head in this case being doubled back, must of course be returned to its proper position, as mentioned above. As this is a case where time is required, the cow often becomes very impatient and restless; but if quiet, the operation may be performed easily and deliberately. But if it is found that extraction cannot be accomplished without the use of the hook, then the operator must take hold either in the sockets of the eyes, cavity of the ears, or under the jaws. Sometimes the calf is unnaturally swollen, in consequence of dropsy, and thus the cow is unable to give birth to it. When this is the case, it will be necessary to pierce the belly of the calf with a knife, to allow the water to escape.

A popular author says, "When every other plan has failed for turning the calf, so as to put him into a favourable position for delivery, the following has often succeeded:- Let the cow be thrown down in a proper position, and placed on her back; then, by means of ropes and a pulley attached to a beam above, let the hind parts be raised up, so as to be considerably higher than the fore parts; in this position, the calf may be easily put back towards the bottom of the uterus, so as to admit of being turned, or his head and fore-legs brought forward without difficulty." 
$\mathrm{Mr}$ Lawrence, an experienced veterinarian, gives the following rules, which merit attention:-

" Ist. Employ timely assistance before the animal is exhausted.

" 2nd. Extraction never to be attempted in an improper position.

" 3 rd. Supple the hand and arm with warm water and fresh lard.

" 4 th. Examination best made, the cow standing, and in the interval of pains.

" 5 th. In pulling at the feet, enclose the claws, or hoofs, in the hand, that the horn may not bruise the cow.

" 6 th. The navel-string bursting, and the usual flux of blood of no consequence.

" 7 th. Instruments to be used only as the last resort, and by experienced and steady persons only."

Should it be necessary to assist the cow, the position of the calf may be ascertained after the waters have been seen. A cord should always be at hand to attach to the fore-legs of the calf, in order that each natural exertion, or throe, may be assisted. Always keep the head free from obstruction.

It sometimes happens that the passage to the uterus is so contracted and sinewy as to obstruct the passage of even the smallest hand; and, indeed, even the finger, in some cases. This is called horning (scirrhus) of the lye, or calf-bed. This occurs even at the last stage of gestation; and many cows cannot give birth to their calves in consequence, and not a few have fallen victims to it. When the period of parturition has arrived, and the animal manifests difficulty in parting with her calf, 
a small hand, which has been anointed with hogs' lard, should be introduced into the vagina, so that it may easily be effected, and it may then be ascertained whether this horning of the lye has taken place; and if it should be found to be the case, recourse must be had to the following operation, which, however, we would recommend to be performed by a veterinary surgeon, if one can be had; but if not, there will be no alternative but to proceed with the operation:-Procure a small, very sharp knife, with a blade of about an inch and a half in length, and with a hollow on the back part, near the point, on which the extremity of the fore-finger must rest, to guide the knife in cutting, and let the point and edge of the knife be covered as much as possible, to prevent it cutting the vagina. It must have a short handle, so that the fore-finger of the operator may always be kept forward, as far as the extremity of the blade, to prevent any danger from its edge cutting the sides of the vagina. With the point of the knife cut the lower side of the passage to the womb, and when the horny obstruction is completely divided, the animal will feel immediate relief, and the natural pains will be resumed, and with a little assistance the calf may easily be extracted, and in many instances by the efforts of the cow alone.

When this horny obstruction exists, it will be observed that those necessary alterations preparatory to calving do not take place, and when this is noticed, particular attention must be paid in examining into the cause, why due preparation has not been made by nature for the change about to take place.

As a rule in the cow, the placental membranes are expelled by the contractions of the muscular fibres of 
the womb within twelve hours, but should they not be expelled within three days, they ought to be removed by the hand of an expert. Much force should not, however, be used, as this might cause hæmorrhage, and endanger the life of the animal. If allowed to remain long after the birth of the calf, it is apt to become putrid, and mortification in the womb may follow. Some cows, after they have dropped their calf, and the after-birth has come away, have a propensity to eat the latter, which should never be permitted, if possible.

After a cow has calved, she should be kept in the house for two days, and not allowed to drink water which is quite cold; it will also be of benefit to mix a little oatmeal with the tepid water.

\section{REARING OF CALVES.}

Some cows will disown their progeny soon after birth; to prevent this, let a little salt be thrown over the back of the calf, and the mother will speedily take to licking it, and afterwards allow it to suck her. If the cow inclines to horn the calf, chain her up, or if she kicks it violently, tie her two hind legs together with a small soft rope or strap, above the hocks.

About an hour after birth, we would recommend that the calf be allowed to suck its mother. And when it is designed to wean calves, for the sake of the cow's milk, the operation is commenced when they are about a fortnight old, in which cases the best substitute for the milk is that recommended by the late Duke of Northumberland, who was a zealous agriculturist. The following is the mode of preparing it:-Take one gallon of skimmed milk, and to about a pint of it add a tablespoonful of treacle or golden 
syrup, and after mixing them well, take an ounce of linseed oil cake, finely beaten down, and strew it slowly into the milk, stirring it all the while with a spoon, until it is thoroughly incorporated; and when it is so, add to it the other portion of milk, and place it on the fire until it is the warmth of new milk, when it may be given to the calf. Sometimes water is substituted for milk; when that is the case, let four ounces of linseed meal be well boiled in a gallon of water, and afterwards add half a pound of treacle, which must be added when the linseed meal has been slowly and thoroughly boiled.

To secure health and strength to the calves, the surest plan is to allow them to continue with their dam, and be suckled by her, until they have acquired sufficient energy to provide for themselves. Therefore they should be permitted to run about with her for twelve months. Calves, however, are apt to bruise the udder and teats, by butting against them with their head while sucking. To obviate this, they are prevented from sucking by means of a small piece of leather, with little sharp iron spikes upon its outer surface, and attached to the snout of the calf in such a manner as to permit it to feed upon grass, while it will prick the udder of its dam, and she will therefore not permit the calf to suck her. The cow is only milked twice a day, and it is at those times that the calf is permitted to suck the teats of one side, while the milkmaid strips those of the opposite side; and when she has completed the milking, the muzzle is again replaced.

In the great Yorkshire dairies, calves are hardly ever permitted to suck; they generally drink the new warm milk from a pail. This is continued for two or three weeks, and for the next three weeks they are 
fed upon half new and half skimmed milk; after this, upon skim milk alone, and even sometimes mixed with water, and ultimately with the addition of a little oatmeal porridge, until they are able to feed entirely upon grass.

The practice in Cheshire is to allow the calf to suck its dam for the first three weeks, and it is afterwards fed upon warm whey or butter milk, to which water is sometimes added, with the addition of oatmeal or flour. To every forty or fifty quarts of liquid, one quart of meal or flour is considered sufficient. On this they are fed night and morning for some weeks, and then it is only given to them once a day until they are three or four months old, when they are considered able to live entirely upon vegetable food.

In Gloucestershire, calves are only permitted to suck about two or three days, after which they are fed with'skimmed milk warmed. When they are able to masticate a little, they have given to them chopped hay, split beans, or oatmeal and water; and sometimes oats, which latter is an expensive food.

In Norfolk, calves are permitted to suck their dams twice a day for a fortnight, and for the next fortnight to be fed with the pail; then only once a day for a month or two, and accompanied by cut hay, turnips, or carrots.

A different mode of treatment takes place in Sussex, where calves are permitted to suck the cows for two months to twelve weeks ; after which they are fed with skimmed milk, with a mixture of oatmeal and water. Sometimes they are weaned at the end of this period, and fed upon cut hay, turnips, etc.

It has been found that linseed boiled to a jelly, in the proportion of one quart of seed to six quarts of water, forms an excellent substitute for milk in rearing 
calves. A pint of this to be given three times a day in a quart of water slightly warmed. Calves fed in this way thrive as well as if fed upon milk.

In America calves are fed with gruel, made of onethird barley and two-thirds oats, ground very fine. To each quart of flour add twelve of water, and boil it for half-an-hour. Let it stand until it has become of the heat of milk from the cow. Each calf to be given a quart of this morning and evening. When calves are ten days old, a bundle of soft hay is tied up in the middle of the cow-house, which they will come to eat by degrees. The quantity of gruel must be gradually increased until they are two months old, by which time they are generally enabled to provide for themselves. Three bushels of the above compound will raise six calves.

One of the most important objects to be attended to in rearing calves, as well as in feeding all animals, is to preserve the utmost regularity in the hours of feeding, and not to give too much at a meal. It has been found better to feed thrice a day instead of twice.

When calves are not intended for breeding from, it is the practice to castrate the males and spay the females. These operations should be performed when the animal is fifteen or twenty days old. After the operation great care is necessary to prevent the wounded parts from being exposed to the action of the air. They must be kept quiet and warm for the first three or four days afterwards, and not allowed much drink.

The time when cows should be put to the bull is not until they are from two to three years old, although it is not unusual for farmers to commence breeding from them at two years. But this latter has a tendency to weaken the system, as cows can hardly be 
expected to have attained their full strength at two years.

There are many other methods adopted in feeding and fattening calves and cattle, but these come more within the scope of a work on agriculture than of one on farriery.

\section{DENTITION OF CATTLE.}

The period at which the teeth appear in the mouth varies according to the breed and early maturity of the animal, and without doubt the feeding of the animal affects the growth of the teeth. Wellbred and well-fed animals have the teeth earlier in the jaws than under-bred and under-fed cattle. As an example, the mouth of a well-bred shorthorn at three and a half years is as advanced as that of a West Highland at four and a half years, so that to judge correctly we require to calculate the shorthorn by the table of early dentition and the West Highland by that of late dentition. As a guide to early dentition the following table may be used :-

At birth, or within one month

At birth, or within one month

At six months

At fifteen months
2 central temporary incisors. $\{2$ middle temporary incisors.

2 lateral temporary incisors.

2 corner temporary incisors. $\int$ Ist, 2nd, and 3 rd temporary $\{$ molars on each side, and ( above, and below. $4^{\text {th }}$ permanent molar. 5 th below permanent molar.

At one year and ten months to two years, the central temporary incisors are thrown off, and replaced by the two central permanents, and about the same time, the 6th molar makes its appearance in the jaw.

At two years and six months, the middles are cast and replaced by the two permanent middle, and at this time, also, the Ist and 2 nd temporary molars are replaced by the Ist and 2 nd permanent molars.

At two years and nine months to three years, the lateral tem- 
porary incisors are cast and replaced by the lateral permanent incisors, and a little earlier or at the same time, the 3 rd temporary molar disappears, and its place is taken by the $3^{\text {rd }}$ permanent molar. Lastly, at three years and three months, or three years and six months, the two corner temporary incisor teeth fall out and are replaced by the corner permanent incisors.

The perfect mouth consists of eight cutting or incisory teeth in the under jaw, and none in the upper, with six grinders or molar teeth on each side, both in the upper and lower jaw, making a total of thirty-two teeth. At three years, the horns are furnished with a wrinkle at their base, and every succeeding year they acquire an additional wrinkle; so that after the animal has reached its third year, its age may at once be ascertained by counting the wrinkles or circles at the base of the horns. In some cattle these wrinkles are not so apparent as in others; and many unprincipled dealers file down some of the wrinkles when they wish to pass off old cattle for young ones, and thus deceive those who are unsuspicious of the imposition. But experienced persons may detect the trick by a close examination of the horns, as it is hardly possible to perform this unworthy trick in so nice a manner as to give the part which has been rasped down the natural appearance of the horn. 


\section{CHAPTER VII.}

Management and Feeding, etc., of Cattle.

\section{COW-HOUSES.}

Nothing is more conducive to the health, and consequently to the feeding of the animal, than well-aired and ample cow-houses. The dimensions of these will depend upon the quantity of stock which the farmer may desire to bring up for immediate and marketable purposes. Many different plans have been adopted in the construction of such buildings; some have been well and usefully conceived, while others have been erected with more than necessary accommodation and elegance. In the latter respect none was, perhaps, more absurdly so, than that one erected by a jointstock company at Edinburgh, about fifty years ago, which was ironically designated "The Cow-Palace.' The speculation did not succeed, and this building stood long, a monument of extravagance and folly, not being easily convertible into any other purpose.

Perhaps the most useful and economical construction for a cow-house is one with a central wall, which should be about fourteen feet high, with a roof resting on it, sloping downwards to an outer wall of seven feet in height. The total width on each side should be about twelve feet. Each stall should be four feet two inches in width. At the heels of the cattle must be placed a gangway about three feet and a half broad. 
Urine runs should be conducted from the ends of the stalls, towards the outer wall of the cow-house, where there ought to be a receptacle for the urine. There should be a dung-pit, constructed of stone, or brickwork, for the reception of the dung. At about five feet apart should be a series of windows, covered with flap-boards, for giving light and air, when required. There should be a door at each end of the building, for admitting of a free current of air, as the occasion requires.

Some feeders prefer a circular building, on the score of economy; the animals standing with their tails towards the wall, which would offer facility in throwing out the dung, and the area within would answer for the purpose of feeding, and attendance upon the cattle.

The strictest attention should be paid to ventilation, and attendants should watch that the upper timbers of the house are dry, as they are liable to become damp from the condensed perspiration, and vapour arising from the respiration of the cattle.

Some experienced feeders affirm, that it is better to leave a gangway of sufficient breadth for the attendants to pass along, at the heads of the cattle, to feed them. And, if possible, troughs well supplied with running water. Where this can be obtained, there can be little doubt of its great utility.

\section{THE FEEDING COW-HOUSE.}

It has long been ascertained that the cleaner and more comfortable cattle are kept the better; and the utmost diligence should be employed in preserving the food as clean as possible. Upon these depend, in a great measure, their healthy condition; and consequently the sooner they will become fat and market- 
able. To attain these objects, $\mathrm{Mr}$ Hunter, of Blackness, near Dundee, a gentleman who paid much attention to agricultural pursuits, had cow-houses constructed in so complete a style, as to answer most effectually these desirable ends. His plan was to erect a house of two apartments, an inner one for feeding the cattle, and an outer one for containing the turnips and fodder.

In wintering young cattle, it is of much importance to keep them sheltered from the wet, and in a warm situation, as they feed much better. They should be tied up in sheds or houses constructed for the purpose ; and, if possible, these should be in a yard, surrounded by a wall. The cattle should be tied up in stalls; and it will be found that they feed faster than when running loose in a yard, and with much less consumption of straw and fodder.

A principal object with Mr Hunter was to obtain as much manure as possible, and at the same time to save the urine. After the turnips are fully ripened, they are gathered together in large quantities, and the roots are separated from the leaves, with large knives, made of old scythes, or knives purposely constructed, and as much of the earth removed from them as possible, and then carried into a barn, where they are deposited for use. The cattle are first fed with the leaves, until they are exhausted, which they eat with avidity when not withered. The turnips should be as closely piled up as possible, and covered with straw, to preserve them from frost. It must be observed that all the turnips are not removed from the ground at once, but taken up from time to time, as the leaves may be required for food. When turnips are permitted to remain in the ground during the whole winter, the green parts are rendered useless by the alternations of 
frost and thaw, and the turnips themselves much injured.

The feeding-house should be at right angles with the barn; and, at about three feet and a half distance from the principal wall, there should be a series of troughs from which the cattle feed. These should be constructed of fire-clay, which is preferable to wood. The floor should be an inclined plane, on which the troughs rest, and in the ends of each there ought to be an arched aperture for the passage of water from one trough to the other, by which means they are easily kept clean, by throwing a few buckets of water into the higher one, and the last or lower one empties itself by a spout led through the wall. By means of this arrangement, the food can be kept clean without much trouble. The cattle should be chained to stakes, situate at a proper distance from each other, and these are attached to a horizontal beam, running from one end of the house to the other, and attached to the roof by an upright support. The three and a half feet space between the beam and the wall, is intended as a walk for the men who feed the cattle, and from which they deposit the cut turnips into the troughs. The cow-house should have a window at both ends, to enable the men to see that no accident befalls the animals.

Should any of the cattle be seen choking, the attendant must immediately apply the instrument described, with the mode of using it, at page 429 , and represented on Plate $\mathrm{x}$, fig. 19, which ought constantly to be at hand.

In large establishments, the cattle-feeders should be on the spot night and day, and ought to have a sleeping apartment at the end of the cow-house, with a window looking into it, so that he may hear and see anything which may require his attention. 
At about a foot distant from the hind-quarters of the cattle, there should be a hewn stone dung-groop and urine-gutter, so that the latter may run off as soon as it is discharged by the animal, and be conducted into the urinarium in the yard, which should be enclosed for the purpose.

There should be several vents constructed for allowing the breath of the cattle and ammoniacal gas to escape, as the inhaling of the latter is very detrimental to the health of cattle; and these also prevent too great an accumulation of heated air. 


\section{CHAPTER VIII.}

\section{The Various Breeds of Horned Cattle.}

THE SHORT-HORNED, OR HOLSTEIN BREED.

IT is from this breed that we derived the best of our English cattle, which now, in most parts of the kingdom, far exceed the parent stock. They differ much from all the older British cattle in the shortness of their horns. They are wider and deeper in their form, and feed to a much greater weight than most other breeds ; they yield a large quantity of tallow, and their hides are greatly finer in texture, thinner, more compact in fibre, and with a thin coating of hair.

It is not the province of a work of this kind to enter into an elaborate detail of all the methods pursued by breeders for improving their stock, which would exceed the limits of a treatise of this kind; but we shall quote the words of $\mathrm{Mr}$ Beilby, who, in speaking of the improved Holstein breed, says, "We shall, however, give the general principles which have been laid down, and steadily adhered to, in the improvements of several breeds of cattle, and which have been so successfully brought into practice. The first, and most obvious, is beauty of form, a principle which has been, in common, applied to every species of domestic cattle, and, with great seeming propriety, was supposed to form the basis of every kind of improvement, under an idea that beauty of form and utility were inseparable. 
But at present a distinction is made by men who have long been conversant in practice, between a useful sort and a sort that is merely handsome. Utility of form is, therefore, the next general principle, and may be considered as arising from a larger proportion of those parts which are deemed offal, or which bear an inferior price, should be small in proportion to the better parts. A third principle of improvement laid down by breeders consists in the fineness of the muscular parts, or what is termed flesh. But the great object which engrosses the attention of breeders at present, is the fattening quality, or a natural propensity in cattle to arrive at a state of fatness at an early age, and in a short space of time; and it appears, from observation, that beauty and utility of form, the quality of flesh, and its propensity to fatness, are principles consistent with each other, are frequently found in the same individual, and hereditary in particular lines or families of cattle. In regard to the means of improvement, it has long been an established maxim, that, to improve the breed, it is necessary to cross it with others of an alien stock under an opinion, that continuing to breed from the same line, weakens the stock. This idea, however rooted it may have been in the minds of former practitioners, is now entirely set aside by the modern practice of breeding, not from the same line only, but from the same family: the sire and the daughter, the son and the mother, the brother and sister, are now permitted to improve their own kind; and in this way the improvement of the several breeds has advanced rapidly to a height unknown before in any age or nation."

This we may admit has been the case to a certain extent; but a limit must be put to it, otherwise degeneracy and weakness are sure to follow, and also a train of diseases, which will become hereditary. 
Crossing the different breeds throughout the United Kingdom has been so much practised within the last thirty years, that it is now difficult to say what is the origin of the cattle of any part of the country. Bulls and cows of improved breeds have been transported from one district to another to such an extent, that an almost uniform improved stock prevails throughout Britain and Ireland. These, however, are varied to a certain extent by the opinions and practice of various farmers. We must, however, peculiarise.

\section{THE KYLOES, OR HIGHLAND CATTLE.}

This hardy and small breed is peculiar to the Highlands of Scotland and its Western Islands. They are mostly black or light brown in colour, their horns white, with long and rather shaggy hair. These cattle feed to a good size in proportion to the dimensions of their bodies. The flesh is well flavoured, and the muscular fibre of a fine texture. They are much sought after by gentlemen in England, to be turned out in their parks, from the gentleness of their tempers, and the ease with which they fatten.

\section{THE LONG-HORNED CATTLE.}

This breed was formerly called the Lancashire breed, and prevailed over that county, as well as Cumberland and Westmoreland. Of late this breed, which was a favourite one, is so altered by the mixture of other kinds, that it is now nearly extinct.

\section{THE GALLOWAY BREED.}

The cattle of this and the neighbouring counties have long been the polled kind. They are of medium 
size, generally weighing from forty to sixty stone, although some have been known to weigh seventy stone. Their form is shorter than the Lancashire breed, and otherwise resemble them, except in their want of horns. They fatten on the most valuable parts, and their beef is finely marbled or mixed.

\section{MILCH COWS.}

Great differences are known to exist in the quantity of mills which cows yield, as well as the quality of the milk itself.

The short-horned and Ayrshire breeds give most milk; more cream is thrown up on its surface, and it produces a greater proportion of cheese. Some persons think the Alderney breed the best suited for dairies, as yielding a great proportional quantity of milk and cream, and also for consuming a smaller quantity of food. The Suffolk duns are also remarkable for the quantity and excellence of their milk.

\section{TUBERCULOSIS.}

The bodies of all animals are open to invasions of a growth generally termed "tubercle," and no organ is exempt. The human subject and cattle are especially liable to this invasion, and it is calculated that tuberculosis proves fatal to 25 per cent. of the human race, and invades from 25 to 50 per cent. of milch cows.

If we examine these tubercles as we find them in the lung of the human subject, they are seen to be little, round, hard, grey-coloured bodies, about the size of a millet seed, and yielding no fluid when crushed (miliary tubercle). In a more advanced stage it is of a yellowish colour, and of the consistency of putty or cheese (yellow tubercle). 
In cattle the miliary tubercles are larger than in man, and form bunches sometimes as large as grapes. They adhere or grow out from the membrane which lines the chest (pleura), and the membrane which lines the abdomen (peritoneum). They also invade the lung tissue, the lymph glands, mammary glands, mesenteric glands, the liver, kidneys, bowels, womb, testicles, brain, etc., etc.

If we now have recourse to the microscope, and make an examination of a recent tubercular growth in an acute case of generalised tuberculosis, we find it is made up of a number of cells like those found in the blood (leucocytes), like those found in a wound (granulation cells), like those found in the skin (epitheloid cells), and, although last not least, one or more micro-organisms belonging to the class termed "Bacillus Tuberculosis."

This bacillus has been singled out and grown on blood serum, glycerine-broth, glycerine-milk, glycerineagar, agar, potatoes, etc. If the cultivation material be injected into the subcutaneous tissue, or into the blood stream, or into the chest, or into the abdomen, or into the anterior chamber of the eye of rabbits or guinea-pigs, in about three weeks they exhibit symptoms of tuberculosis, and on post-mortem examination tubercles are found in the various organs of their bodies.

CAUSE.-It is therefore certain that the cause and spread of tuberculosis is the introduction of the living parasite, the "Bacillus Tuberculosis," into the body of the individual or animal, and without its introduction and multiplication within the body, the disease tuberculosis, or consumption, could not make its appearance in either the human subject or the lower animal. Regarding the experimental transmission of the 
disease to the human subject from the lower animal, for reasons which are self-apparent, the field of operation is a shut one, but light is indirectly thrown upon this point by experiments, having for their object the transmission of the disease from the human subject to the lower animals. A considerable number of experiments in this direction have been made, and all with the same result, but time and space will only permit of one example being given in detail.

The experiment to which I now refer was conducted by Professor Edgar M. Crookshank, King's College, London, and reads as follows :- "I obtained sputum containing numerous bacilli from an advanced case of phthisis. The sputum was shaken up with sterilised salt solution and injected into the peritoneal cavity. A few weeks afterwards the calf showed signs of illness. The animal looked dull, did not feed well, had a slight cough, and showed less inclination to move about than usual.

These symptoms gradually increased, and death occurred forty-two days after inoculation. Extensive lesions were discovered at the post-mortem examination. The mesentery was adherent to the abdominal wall at the seat of the inoculation, and to the rumen; the liver was adherent to the diaphragm. There was extensive tubercular deposit at the seat of inoculation, and an abscess the size of a walnut. Extending over the mesentery from this point, there were hundreds of wart-like, fleshy new growths, some quite irregular in form, others spherical or button-shaped.

There were similar deposits on the under surface of the liver, on the spleen, in the gastro-splenic omentum, and on the peritoneal surface of the diaphragm. On microscopical examination of sections, extremely minute tubercles were found to be dis- 
seminated throughout the whole of the substance of the lungs and liver, and tubercle bacilli were found in these and in the peritoneal deposits. The calf died of pyæmia, but sufficient time had elapsed for marked local infection leading to generalised miliary tuberculosis."

We thus see that human and bovine tuberculosis are one and the same disease, and that no difficulty is experienced in transmitting the malady from the human subject to the calf or cow, and can there be a doubt as to the transmissability of the malady from the calf or cow to the human subject?

The natural methods by which tuberculosis is propagated from the human subject to the lower animals are (I) inhalation of the dried expectoration of the consumptive person, or contamination by the lungs. (2) Ingestion of the expectorated sputum, or contamination by the stomach, and cases can be cited proving its transmission by these methods in dogs, and poultry which readily pick and swallow the sputum.

We do not incline to the opinion that many of the lower animals receive the infection from the human subject, nor are we inclined to believe that a large percentage of the cases of human tuberculosis are traceable to contamination from eating the flesh or drinking the milk of tubercular cattle.

Tubercular deposits varely are found in the flesh.Its favourite seats are the investing membranes of the chest and abdomen, and the glands, and these are removed by the butcher in dressing the carcase. Further, the heat to which the flesh is subjected in preparing it for human consumption, is sufficient to destroy the bacilli, and tuberculous beef without living bacilli cannot produce tuberculosis in those who eat it. 
But while we believe that few cases of tuberculosis in the human subject are traceable to the eating of the flesh of tuberculous animals, we entertain a different opinion as to the propagation of tuberculosis by drinking the milk of tuberculous cows, because it is partaken of as drawn from the animal, and rarely subjected to heat or cooking of any kind.

But even here again, the cases of tuberculosis which owe their origin to drinking the milk of tuberculous cows, are not so numerous as at first sight would appear, because numerous experiments in the feeding of calves on the milk of tuberculous cows, tend to prove that if the mammary gland be free from tubercular deposits (which in the majority of cases it is), the milk does not produce tuberculosis in the calves fed on it.

Regarding the hereditary transmission of this disease, until lately it was the unanimous opinion that consumption, or tuberculosis, descended from parent to offspring, and was propagated in no other way, but as it is a parasitic disease, or a disease produced by a specific parasite (bacillus tuberculosis), and without the parasite there can be no such disease, then it cannot be a parental inheritance, or disease flowing from the parents of the affected animal, but a disease associated with, and entirely dependent on, the presence and existence of the bacillus tuberculosis in the body of the affected animal.

We do not, however, incline to the opinion that all individuals and all animals are alike exempt from, or prone to be attacked by, this parasite. On the contrary, we hold the decided opinion that certain families in the higher and lower animals inherit a condition of body which predisposes or lays them open to be attacked by this parasite. 
Symptons. - The symptoms of tuberculosis depend upon the stage of the disease. The earliest symptom is a short, dry cough frequently repeated, especially in the morning, and while feeding. By-and-bye, according to the activity of the disease, there is wasting, enlargement of the cervical glands, hidebound, and a dirty pale colour of skin. The breath is generally bad, the eyes dull and sunken in their orbits, the appetite is frequently good, and the secretion of milk considerable; in other cases, fastidious appetite and almost no secretion of milk. Abnormal sounds are heard on auscultation, and dullness on percussion.

If the udder is invaded, nodules are felt, which are hard and painful to pressure, but must be distinguished from the indurations produced by simple inflammation in cases of mammitis.

In doubtful cases, or in those cases where it is desirable to ascertain if there are any of the herd affected with tuberculosis, injections of "tuberculine" should be made; but as this is an operation which should be conducted by a member of the veterinary profession, we shall not enter into details.

REMEDIES.-Where tuberculosis exists in a herd of valuable breeding animals, in order to get rid of the disease, it is not imperative to slaughter all of them. Those markedly affected should be slaughtered, and thereafter the remaining animals (young and old alike) should be subjected to the tuberculine test. Having by this test divided the herd into affected and non-affected animals, then house and graze the two sections apart. At stated intervals re-apply the tuberculine test, and add any re-acting animals to the affected section. The calves of the whole herd to be removed from their mothers as soon as born, and hand. 
fed with milk from the sound cows. The calves, in time, also to be subjected to the tuberculine test, by adopting these and other repressive measures which the experienced veterinarian will suggest; and by excluding all additions to the herd which re-act to the tuberculine test, the stock proprietor will, in a few years, find himself in the possession of a herd of cattle in which tuberculosis does not exist. 


\section{SECTION III.}

\section{DISEASES OF SHEEP.}

\section{INTRODUCTION.}

ThE duties incumbent on the shepherd are of a most arduous kind; and he that performs them rigidly, even with a moderate range of pasturage, will have little leisure time on his hand. Sheep are accustomed to enjoy an ample district of country for feeding, and being widely scattered, the shepherd has little opportunity of watching the condition of their individual health. Some will naturally be seized with maladies, unobserved by the shepherd, and in many instances die without being seen in some sheltered corner, where they retire for quiet, when overtaken by disease.

It is of great importance to have shepherds well versed in the different complaints to which sheep are liable, and to be able to distinguish the disease immediately on its earliest appearance. The diseases are comparatively few, and these in general well marked.

The sheep is not that stupid and defenceless animal which many suppose him. In extensive mountain ranges, where they but seldom see mankind, and where they are not dependent on the protection of the shepherd, sheep will be found to 
assume a very different character. Rams and wedders have been known to attack a single dog, and in general are found more than a match for one of ordinary strength. But when danger of an imminent kind presents itself, the whole flock assume an attitude of defence,-forming themselves into a compact body, with the females and young in the centre, the males place themselves in the front, and, keeping close to each other, boldly wait the attack; and when their enemy has approached within a few yards, the rams dart upon him with such impetuosity, as to lay him dead, unless he takes to flight, and thus eludes their vengeance. Dogs and foxes have no chance of breaking their ranks when thus formed. 


\section{CHAPTER I.}

Diseases of the Head, etc.

STAGGERS.

Symptoms. - This malady has a variety of names applied to it, as indicating various diseases, namely, sturdy, goggles, fernsick, and hydrocephalus, or water in the head; but they are in fact only one.

The staggers is a disease which more particularly attacks young sheep or hogs, from nine to eighteen months old. Sheep that have died of this disorder, on their skull being opened, a collection of water has been found between the skull and brain, and even sometimes in the ventricles of the brain.

The first symptom which manifests itself, is the sheep lagging behind its companions in the pasture ground, and assuming a dull, sluggish appearance. Then the animal will be noticed to go round, as if giddy; its eyes look as if they were blind; and ultimately he dies. But all these symptoms may be apparent for a considerable time before death ensues. Sheep labouring under this complaint sometimes feed well to the last, and die in good condition.

CaUses.-One form of this disease proceeds from an over-abundant supply of that fluid which moistens the ventricles of the brain. This is considered in- 
curable. The more common state proceeds from a species of worms, called hydatids. These parasites are found in little bags or cysts, filled with a fluid. They do not inhabit the substance of the brain, but lodge between it and the skull. It is by the pressure of those cysts disturbing the functions of the brain that this malady is produced, and which generally terminates fatally.

The hydatid is the cystic form of the tapeworm of the dog, and the sheep becomes the victim by swallowing, in the grass, the ova or eggs of the tapeworm extruded in the faces of the dog. If the dogs herding the sheep have no tapeworms in their bowels, the sheep will have no hydatids in their brains.

Remedies. - The celebrated poet, Hogg, better known by the name of the Ettrick Shepherd, discovered one mode of curing the species of disease which is caused by hydatids. His plan was to thrust a knitting-needle up the nostril of the animal and force it through the skull, into the cyst, which destroyed the hydatid, and the sheep then recovered its usual health.

In cases where a portion of the skull becomes soft, it is penetrated by a common awl, which permits the water and hydatids to escape, and a cure is effected. Care must be taken not to pierce the substance of the brain by forcing the awl too deep. Trepanning has been recommended, and successfully performed by veterinary surgeons. It is, however, too delicate an operation to be attempted by unskilful hands, and should therefore be entrusted to no one but a regular veterinarian. The animal must be properly secured, and placed in such a position that the part to be operated on should lie convenient to the hand. An incision of an inch 
and a half in length is made through the skin, which is crossed by another at right angles to it. The portion of bone above the cyst, and which in general is felt to be more or less soft to pressure, is removed by a circular saw termed a "trephine," or a hole is made by a "borer." The cyst is punctured by a "trochar," and the fluid withdrawn by a "syringe" applied to the "canula." Care must be exercised in the removal of the cyst. When this has been effected, the parts of the skull must be brought together, and crossed by slips of adhesive plaster. Remove these in three days, and wash the part with carbolic soap, if any matter appears, and dress it in the same manner. Two or three dressings generally is all that is required.

$\mathrm{Mr}$ Rhind, Surgeon, Edinburgh, describes, in the first volume of the Edinburgh Journal of Natural and Geographical Science, a species of worm, which was found in the frontal sinus of a sheep; but is unable to account for the animal being found in such a cavity. It belongs to that genus of worms called Pentastoma, and may be specifically termed ovis. We have given a representation of this worm on Plate xIv; fig. 9, the worm the size of nature; fig. II, the head magnified; fig. Io, the point of the tail; fig. 8, a cross-section of the body, showing the intestines.

\section{BLINDNESS.}

It may naturally be supposed that this malady subjects the animal to the risk of accidents, and consequently such as become blind should be fed in an enclosure where there is no inequality of ground. 
Cause.-Blindness is frequently induced by removal from warm and sheltered localities to such as are exposed to cold winds and storms. Moist situations are also apt to produce blindness. Exteme heat has also the same effect, especially in the dogdays. Poor feeding is likewise instrumental in producing blindness; and that peculiar condition of the atmosphere, generally denominated blight, produces an affection similar to what is called sty upon the human eye. When the cases are numerous in the flock, and at the same period in summer, it is caused by the dust of the grasses at the flowering season.

Remedies. - The eye being an extremely delicate organ, cures should only be attempted by a veterinary surgeon. The ointment made from the nitric oxide of mercury, drawn across the eye-ball by a camelhair pencil, or a feather, will frequently effect a cure; and the common mercurial ointment has been found beneficial.

\section{CATARRH.}

Symptoms. - The discharge of a purulent fetid matter from one or both nostrils, the membrane excoriated, accompanied by dullness, and the wool parting easily from the skin, together with want of appetite, are symptomatic of this malady.

Cause.-Subjection to wet or boggy layers in cold, wet weather, or during wind, as also exposure to damp situations during snow, are apt to produce catarrh. There can be little doubt but the disease is sometimes contagious, as it has been frequently observed, that when one animal has been seized with the complaint, others soon become infected. 
Remedies. - The following preparation has been found an effectual cure if taken in time:-
Gum-guaiacum .
$\frac{1}{4}$ ounce,
Camphor, finely powdered . $\frac{1}{2}$ drachm, Tartarised antimony . . $\frac{1}{4}$ ounce,
Fennel-seeds, powdered . . $\frac{1}{2}$ ounce,
Mithridate. . . . . $\frac{1}{2}$ ounce;

to be made into a ball with treacle. 


\section{CHAPTER II.}

\section{Diseases of the Chest.}

\section{FLUKE, OR LIVER ROT.}

Symptoms. - The name rot has been given to many diseases, which are, however, widely different in their character. But this complaint is a distinct malady, exhibiting characters peculiar to itself. The lungs, liver, and whole system are affected, and water is effused into the abdominal cavity. The chief seat of the malady seems to be the liver, which is always found in a diseased state; and a species of worm, called the fluke-worm, is found lodged in the bile ducts. The sheep invariably falls off in flesh, which also becomes flabby; the eyes are heavy, dull, and yellow in colour, and the animal appears stupid, and weak. The gums and tongue are of a livid hue; and the breast loses its fine rosy colour; the breath is fetid, and the teeth become loose. The wool parts from the skin easily, and in some instances the animal scours.

CAUSES. - Sheep that are fed upon dry, and especially mountain land, are seldom subject to this malady. On the contrary, it has been ascertained that those fed on low, wet pastures are liable to be attacked by this complaint. It has been especially remarked to affect sheep brought from hill-pastures to those which are damp and luxuriant, for the sake of speedily 
fattening them. The immediate cause of the disease is the introduction into and the presence within the liver of two fluke or leaf-like parasites, termed the Distomum Lanceolatum and Fasciola Hepatica, and their ova, which are present in thousands, disorganising the liver, preventing the secretion and excretion of bile, and draining the body of its blood until it becomes anæmic and generally dropsical. The ova, or eggs, of these parasites are not hatched within the body of the affected sheep, but pass out with the dung, and unless moisture and heat are afforded they come to nothing, but if they gain a pool of fresh water, they are hatched, and, floating about, are on the out-look for molluscs, the bodies of which they pierce, and then become encysted and form brood capsules, and within these many young embryos may be formed. If the molluscs are swallowed by the sheep in the grass or water, then the embryos develop into mature flukes, inhabiting the bile ducts and reproducing themselves only by eggs. One fact, however, is certain, namely, that where sheep are fed on salt-marshes, the complaint never occurs; and in Lincolnshire sheep pastured in such localities are uniformly well fed, and in most excellent condition, and bring high prices. It will now be apparent to the reader why high lying and dry pasture lands are almost exempt from fluke disease, and low, undrained soils, where fresh water rests in pools, is infested; and, also, why salt marsh lands are exempt from the malady and may lead to recovery of the affected sheep, the salt being a poison to the parasites.

Remedies.-As preventative, drain the pasture lands and get rid of the pools of surface water; if this cannot be accomplished dress the pastures with 
salt-no breeding stock should be reared on fluke lands, but a flying stock kept, and liberal handfeeding allowed so as to push the sheep forward for the butcher. Up to a certain point the sheep thrive well, and the moment they are seen to be standing still or going back in condition, they should be consigned to the butcher. Rock-salt and sulphate of iron assist in the destruction of the parasites and strengthen the constitution of the sheep.

\section{INFLAMMATION OF THE LUNGS.}

This complaint is most common in lambs, and occasions a short, hard cough. Whenever this is observed, give the following specific, every day, until the cough is subdued :-

Tartarised antimony . . . I drachm,

Linseed-tea . . . . 4 ounces.

\section{GRASS-ILL.}

Grass-ill is a disease which affects lambs from three weeks to a month old, and is caused by eating grass before the stomach is properly habituated to it, and no doubt from indigestion. It is most rapid in its progress, and speedily terminates in death, if not promptly attended to. The following should be given whenever the animal exhibits a languid, dejected state :-

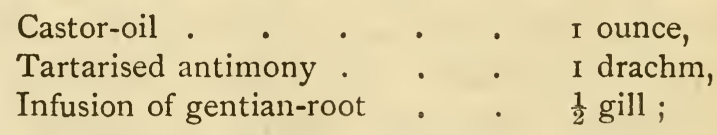

to be administered with the flexible tube funnel.

MILK-ILL.

Symptoms.-The animal exhibits a dull appear- 
ance, with the ears spread and hanging on either side of the head, instead of standing up in the ordinary manner.

Cause and Cure.-This complaint is one affecting lambs, and follows weaning, and is probably owing to indigestion, and acids generating in the stomach. It is nearly akin to grass-ill, and we are not aware of any other remedy than the one recommended for that disease. When they are affected with scouring, from twenty to thirty drops of laudanum may be given with effect.

\section{JAUNDICE, OR YELLOWS.}

This complaint is not a common one amongst sheep. It manifests itself by the white of the eyes assuming a greenish-yellow cast, and their skin being of a yellow tinge all over. This disease, as in other animals, is caused by a diffusion of the bile over the different parts of the body. Give the following :-

$\begin{array}{lll}\text { Salt of tartar }: \quad & \text { I drachm, } \\ \text { Epsom salts }: & : & \frac{1}{2} \text { ounce, } \\ \text { Bitter infusion } & : & \text { I gill. }\end{array}$

The following has also been found effectual in removing the complaint :-

Calomel . . 3 grains,

Jalap . . . I drachm. 


\section{CHAPTER III.}

\section{Diseases of the Abdomen.}

THE RESP, OR RED WATER.

Symproms. - The beginning or end of winter are the times when this complaint makes its appearance. It is less common in higher mountain ranges, but pretty frequent in lower situations when hoar-frost prevails. Where sheep are put under cover during the night, they always escape this malady, which manifests itself by an inflammation of the skin, and induces blisters, filled with a thin watery fluid, of a reddish colour. These first appear about the breast and belly, which is generally distended. After a short time they break, and the matter flows from the blisters, which, when fully discharged, are soon covered by a blackish scab. It is a disagreeable disease, but seldom proves fatal. Sheep affected with red water become melancholy, and are usually seen lagging behind their fellows while feeding, and may be observed poking out their heads in an awkward manner, and soon afterwards a discharge of saliva takes place from the mouth, and accompanied by swelling of the belly.

CAUSES.-Cold and wet are the chief sources of this complaint, as well as many others in sheep. The skin being fretted by the wet, watery blisters rise on it. Sometimes, however, the animal is affected with internal cold, which produces a slight febrile attack, 
and causes these watery vesicles to appear on the skin, and are similar to the eruptions which are produced about the mouth and face of many individuals when they have caught a severe cold. This affection has but little influence on the blood, although a small portion of it finds its way into the vesicles under the skin, and produces that red tinge to the thin glary matter of these little blisters, from which the name of the complaint is derived.

Remedies. - The first thing to be done when the disease is violent, is to remove the animal to a place by itself, and then the vesicles should be laid open by means of a small knife, scalpel, or lancet; after which let each of them be well washed out with tepid water and carbolic soap. Afterwards administer the following for three or four successive mornings :-

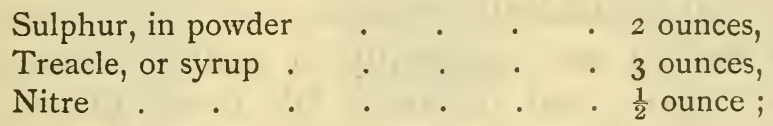

to be made into six doses, and given them in the form of a ball, or in half a pint of water, a little warmed. On the seventh morning give the animal an ounce of Epsom salts; and on the following or next day, wash the whole parts affected with lime water, and the sheep may then be considered as cured.

\section{THE RED WATER BLIBES.}

Symptoms. - This disease is so similar to the preceding, that there is no visible distinction in their appearance; but they differ entirely in their origin, the latter being caused by the animal feeding on succulent grasses or on turnips, which induce an inflammatory state of the system. This complaint attacks sheep which are quite healthy and in high 
condition, and not unfrequently carries them off in a day; for besides the external appearance, the whole internal organisation is affected.

REMEDIES.-An ounce to an ounce and a half of Epsom salts should be given to clean out the bowels effectually. Then give on the two following days a cooling draught, viz:

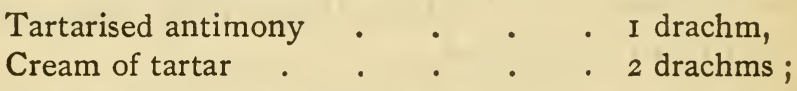

to be made up in a ball with treacle.

\section{THE BRAXY, OR SICKNESS.}

Symptoms.-This is above all others the most insidious and rapidly fatal malady to which sheep are liable. Before any symptoms manifest themselves, the animal is suddenly overtaken with the complaint, while feeding and apparently in perfect health, when he will start, and instantly fall down lifeless. If opened immediately after death, it will be found that the stench emanating from the carcase is quite intolerable, caused by the putridity which has affected the entire internal parts.

In some instances there are premonitory symptoms, by which it may be guessed that this complaint is making progress in the animal's system, as he seems uneasy and restless, lying down and rising up frequently, and sometimes standing with his head down and his back raised; and when forced to run, he manifests pain by his awkward action, and gradually ceases to feed with his usual regularity, and drinks frequently. The eyes have a watery appearance, and the eyelids are half closed, with considerable inflammation of the eyeballs. The mouth, tongue, nose, and skin become dry and parched; the pulse becomes strong and quick, 

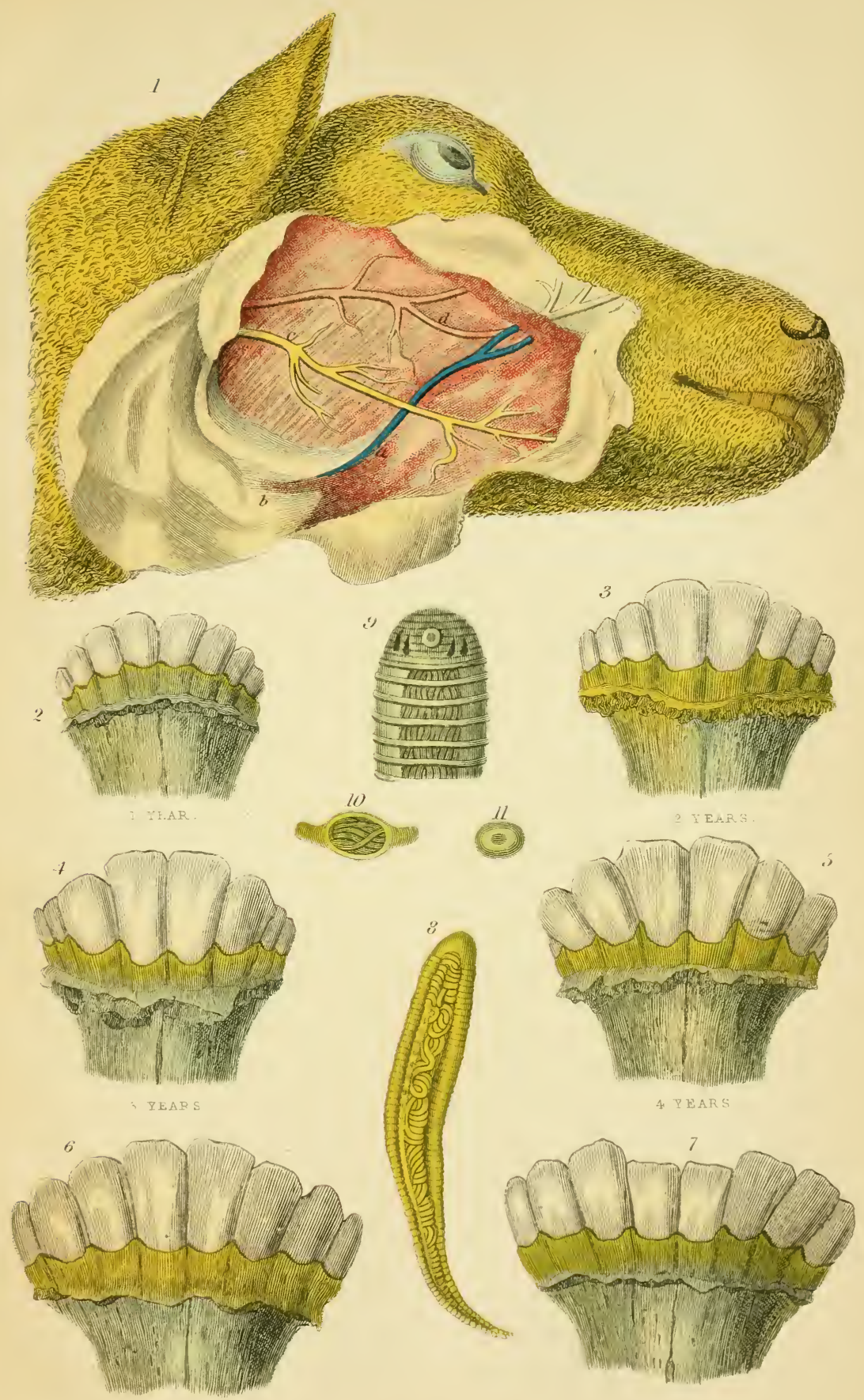

5 YEARS

YEARS

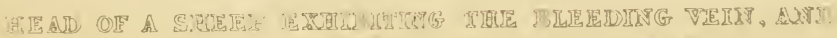

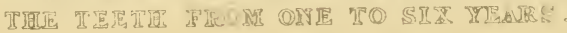



and the breathing rapid and difficult. Inflammation is going on, and considerable pain must be felt, terminating in mortification, after which, as is usual under such circumstances, the pain subsides, and the animal will frequently commence feeding with his usual avidity; and thus persons who have not attentively watched the progress and symptoms are deceived, and the animal dies when they little expect it.

CAUSE.-Cold, wet weather will have the effect of producing inflammation of the bowels, and thus lay the foundation of the disease. Costiveness is also supposed to occasion it ; or it may arise from drinking cold water, when the animal is much overheated; from being washed in cold water when very warm, or any other sudden change of temperature.

REMEDiEs. - Bleeding is the first thing which must be had recourse to after it is suspected this disease is coming on, which of itself will frequently have the effect of checking it. From half-a-pint to a pint may be taken. This should be followed by an ounce of Epsom salts, dissolved in a pint and a half or a quart of cold water, and given in two doses. The lower intestines being generally constipated on the approach of this disease, it will be found beneficial to assist the purgative by a clyster of warm broth or gruel, with an ounce of butter or lard melted in it. Give the following three times a day, until symptoms of recovery are apparent :-

Nitre, ground into a fine powder, I drachm ;

made into a ball with treacle and linseed meal. It will be found of much importance to sprinkle the animal's food with salt. The food should consist of cut grass or turnips; and if the complaint attacks the animal in the winter, green food should be occasionally given, if possible. 


\section{DIARRHCEA, OR LOOSENESS.}

Sympтомs.-This disease and dysentery have frequently been confounded, although they are sufficiently different in their characters. Diarrhœe consists in an unusual discharge from the mucous membrane of the intestines, and is always accompanied by pain, gripings, and frequent stools, but unaccompanied by fever; nor is there any blood or slime: it almost always occurs in the spring; weak sheep and hogs being most liable to its attacks. In this disease there is only a temporary or partial wasting of the flesh, while in dysentery a rapid diminution of the muscular fibre takes place.

Cause. - Sudden changes from poor to rich pasturage; and bad food will induce it.

REMEDIES.-It is not prudent to stop this disease too rapidly; but it is equally wrong to allow it to continue too long, as, if the discharge is either too long continued, or the purging very copious, it brings on great debility. If caused by change from dry to moist food, then it will be proper to resume the dry feeding for a time, which will generally effect a cure within a few days.

If a change of food does not prove effectual, give the animal half an ounce of prepared chalk in a pint of cow's milk, slightly warmed. If the purging has not been subdued by this, it may be repeated on the second day. If the discharge is very great, and accompanied by symptoms of pain and straining, two drachms of rhubarb may be given as a first dose, and chalk as a second. If this has not the effect of relieving the animal, then from twenty to thirty drops of laudanum may be given with advantage, and afterwards the chalk, as above recommended. 


\section{DYSENTERY.}

Symptoms. - This complaint is distinguished from the last disease by being always accompanied with fever, and other symptoms of an inflammatory condition of the intestines, whereas diarrhœa is generally a state of simple irritation or weakness of the bowels. In dysentery there is great pain, frequent gripings, especially when at stool; the matter discharged is in hard balls, accompanied by mucous blood, and often purulent matter from the bowels. Sheep affected with dysentery have generally the wool clapped, with a languid expression of eye, the mouth dry, and rough skin, with a greatly accelerated pulse. When the disease is in an advanced state, the fæces are very black and fetid. The animal eats very little, and is rendered incapable of ruminating. This complaint frequently ends fatally by ulceration of the bowels.

REMEdiEs. - The treatment of dysentery should be commenced with a change of food, e.g., crushed oats, bran and rye grass and clover hay. If costive, the bowels to be moved with castor oil, three ounces; and laudanum, thirty drops. In twenty-four hours follow this up by the daily administration of one drachm of pulv. rhubarb with half an ounce of prepared chalk, mixed in a pint of warm milk. The food should be sprinkled with salt. Hay and a slice of Swedish turnip may be given to him during the continuance of the malady.

\section{COLIC.}

It not unfrequently happens after sheep-shearing, that they are affected with flatulent colic, when the weather is cold and damp. They roll much about 
when seized with the complaint, and are considerably swelled.

Remedy.-Two table-spoonfuls of the following mixture, given twice or three times at intervals of an hour, will generally prove effectual :-

Compound tincture of senna, 6 drachms, Spirit of nitre . . . I ounce, Tincture of rhubarb . . 6 drachms, Laudanum . . . . 6 drachms, Water. . . . I quart.

\section{PINDING.}

This is a complaint with which lambs are afflicted, generally when very young. The frcial discharge is of a thin, glutinous consistence, which has the effect of making the tail adhere to the anus, effectually interrupting the passage of the dung, and proves most injurious to the lamb, sometimes causing its death. This is, however, completely prevented by docking the tail a day or two after birth.

This disease, which consists of pretty copious purging, will be alleviated by the following prescription :-

$\begin{array}{lll}\text { Laudanum } & \cdot & 20 \text { drops, } \\ \text { Ground ginger } & \cdot & \frac{1}{2} \text { drachm ; }\end{array}$

made into a small ball with linseed meal and treacle, and forced down the animal's throat. 


\section{CHAPTER IV.}

Diseases of the External Parts, etc.

SCAB, OR ITCH.

Symptoms. - This troublesome, infectious, and destructive malady is analogous to the itch in the human being, and the mange in horses and dogs, being due to the presence of parasitic acari lodging on, feeding from, and breeding in the skin of the affected sheep. As soon as a sheep has caught the complaint, it rubs itself against every object which it meets. It will even tear off its wool with its teeth. The skin emits a peculiarly sickly smell, and presents a red, fretted appearance, which in a short time hardens into scabs all over the body. This disease is highly contagious, so that when one is discovered to be affected, the whole flock should be carefully examined, and those in which the slightest indications are manifested should be separated from the rest, otherwise the entire flock will catch the complaint. But even here the assiduity of the shepherd must not terminate, as it will be necessary to go carefully over the flock for three or four days successively, as some of them may have caught the malady, although it has not become apparent at first.

REMEDIES.-A variety of means have been adopted for the cure of this disease, and it has been remarked that sheep which have been smeared seldom are 
attacked by it. The following ointment has been found an excellent remedy:-

$\begin{array}{lll}\text { Strong mercurial ointment } & \text {. } & 6 \text { pounds, } \\ \text { Spirit of turpentine : } & : & 3 \text { gills, } \\ \text { Hogs' lard, tallow, or butter } & : & 6 \text { pounds. }\end{array}$

The hogs lard, or butter, must be first melted, and poured over into a vessel; then add the mercurial ointment, stirring them well together until properly incorporated, and all cloudiness disappears, when the mixture will be of a uniform pale grey colour. The turpentine must then be added, and the whole stirred together until the ointment is thoroughly mixed, and then pour it into a large flat dish, that it may cool rapidly, so that the mercury may not sink to the bottom, to prevent which the mixture should be stirred until cold.

The following preparation has also been found effectual, and is considerably cheaper than the preceding :-

Corrosive sublimate, finely powdered 8 ounces, Train oil

6 gallons, Resin . . . . . 2 pounds, Tallow . . . . . 2 pounds.

Let the corrosive sublimate be put into a vessel with a pound of the tallow, and when warm, let them be well stirred together, and then the resin added, which, when melted, will keep the corrosive sublimate suspended. When these are well incorporated by means of stirring, the remainder of the tallow should be added; and when thoroughly mixed, put in the whole of the oil, and after being well mixed let the salve be poured into a flat vessel to cool; or it may be allowed to remain in the vessel in which it was melted: in either case keep stirring the mixture until cold. 
If the weather is warm, the preparation may be found too thin; to obviate this, add an additional quantity of tallow and resin. This ointment is applied in the same manner as the smearing preparation, commencing with a line along the back, and one on each side, one down all the legs, and on the centre of the belly, as well as the inside of the thighs, and both sides of the neck. Unless the weather is cold, the wool should be shorn previous to the use of any of the above appliances, and the whole skin well washed with soda and water, or strong soap and water, with a soft shoe-brush, and after the animal is perfectly dry, the ointment may be then applied over the whole body.

After the ordinary process of shearing, if the sheep were anointed with a very weak preparation of the above ointments, it will have the effect of keeping off the scab and other cutaneous diseases.

It will require great caution in the application of these ointments, taking care that too great a quantity is not used, because it may bring on salivation, and consequently endanger the life of the animals ; as many have died out of a flock which have been anointed with these mercurial preparations. Instances have occurred where nearly the half of flocks have been salivated, while the other portion exhibited no such symptoms. This may be accounted for in two ways: first, by the shepherd having been too liberal in the application with those which are so affected; or the salve may not have been properly prepared, from want of attention to the stirring of the ointment until cold, and consequently a greater proportion of the mercury falling to the bottom, and thus rendering it unequally charged with this substance.

When the animals are salivated-which will be seen by saliva issuing from the sides of the mouth 
and the teeth becoming loose, then the following prescription must be given, which will counteract the destructive effects of salivation; two table-spoonfuls to be given every twelve hours, until a change takes place :-

Sulphuret of potass . . $\quad \mathrm{r}_{2}^{\frac{1}{2}}$ ounce,

Wine steel . . . . 6 ounces.

In cases where the disease has not gained an ascendancy, let the animals be shorn and well washed with a strong solution of soda, applied by a soft shoebrush; and when dry, rub off the scabs and scurf with a dry shoe-brush. Then anoint them with the following preparation, which is less hazardous than mercurial preparations :-

$\begin{array}{llll}\text { Hogs' lard } \quad \cdot \quad & \cdot & \text { I pound, } \\ \text { Flower of sulphur } & \cdot & \cdot & 6 \text { ounces. } \\ \text { Spirit of turpentine } & \cdot & \cdot & 4 \text { ounces. }\end{array}$

Melt the lard over a slow fire, and then add the sulphur, which should be well incorporated by stirring: and when removed from the fire let the turpentine be mixed with it, and continue stirring it until cold.

After numerous and repeated trials of various agents, we have found the following in our hands to be the most efficient, viz. :-

FOR POURING 20 SHEEP.

Tobacco juice (Fleming's patent), I gallon, Ground hellebore . . . I pound, Black soap . . . . 2 pounds.

Directions. - The hellebore to be boiled fifteen minutes with water and soap, and then add the tobacco juice and as much more hot water as will make the wash in all five gallons.

In pouring or dipping sheep affected with scab, it 
must be remembered that the dip can only destroy the parasites but not their eggs, and therefore the affected sheep require several applications before all the parasites, young and old, are destroyed.

\section{PELT-ROT.}

This is a complaint caused by the animals which are in low condition lying in wet commons, woods, or copses, with their fleeces soaked with rain, and the debilitated condition of the sheep producing low spirits and inactivity, so that it does not shake itself. This produces a relaxation of the action of the vessels of the skin; and consequently the wool falls off, leaving large patches of the hide bare. Scanty and poor pasturage will also induce this complaint; and it is sometimes caused by a sudden transition from poor to rich pasturage.

When this disease is noticed, the animal should be removed to a warm straw-yard, and fed upon diet of a nourishing kind, such as mashes of malt and oatmeal, mixed with pea-meal; and also give him cordial draughts.

ERYSIPELAS, OR WILD-FIRE.

Symptoms.-This is an affection of the skin, and is extremely contagious; and if not attended to will quickly spread over the whole flock. The skin becomes very red, and considerable inflammation prevails, but differs from red water in never blistering. It is caused by some peculiar condition of the atmosphere, and generally appears in August and September. It seldom, however, continues more than eight days at a time, although sheep which have been affected with it are liable to a relapse. In some seasons the disease proves very fatal to sheep. 
Remedies.-Cooling medicines, such as antimonial preparations, are useful in this complaint. Give a dose of from one ounce to one ounce and a half of Epsom salts for three days successively, after half-apint of blood has been taken from the animal. Then let half-an-ounce of purified nitre be given for two days thereafter, when generally a cure will be effected. Sir George Mackenzie recommends the salts and nitre to be given in a cold state, which he says will prove more powerful, and more beneficial. The parts which are most inflamed should be washed with goulardwater, to cool them.

\section{CRAMP OF THE LEGS, OR WOOD-EVIL.}

Cold is the cause of this complaint, which suddenly seizes sheep, and renders them incapable of walking, by partially paralysing the action of the nerves of the limbs.

REmedies.-A teaspoonful of mustard given night and morning for some days has been found to restore power to the limbs, while they are rubbed twice or thrice a day with strong camphorated spirit of wine, mixed with about a fourth of that quantity of spirit of turpentine. This is applied with a flannel rubber. Let the animal be kept very warm, and the following prescription given twice a clay:-

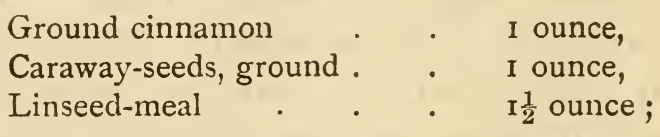

to be made into six balls.

\section{LEG-EVIL.}

This is a complaint in which the legs are liable to considerable swelling, which sometimes ends in 
mortification and death. Sheep which are attacked by this complaint should be immediately taken into the house, and their legs well washed with soda and water; and after they are quite dry, let them be properly anointed with citric or nitric ointment. When the swelling suppurates, it should be dressed with tar-ointment, and a rag rolled round it, and the anointing to be repeated every second day.

\section{FOOT-ROT.}

Symptoms. - The earliest symptom of foot-rot is lameness, after which a fetid discharge issues from between the claws, and soon afterwards proud-flesh makes its appearance; and this is followed by a gangrenous or cancerous aspect of the fleshy portions of the foot.

CAUSES.-Wet, cold pasturage, is one of the chief causes of this complaint; and if the sheep are removed to a distance when the horn is still soft, the effects of travelling on a hard road is almost certain to cause the rot. It is sometimes produced in a sheep-house, where a number of suckling ewes are kept, from the heating nature of the dung, if not frequently cleaned out. Sheep which are afflicted with this disease frequently feed as well as when in good health; but notwithstanding this, they seldom thrive, and often rapidly lose flesh, and more especially their fat, which no doubt is caused by the copiousness of the discharge.

REMEDIEs.-As soon as sheep are observed to go lame, the feet should be inspected, and if affected with this disease, the horn should be pared away, so as to permit the matter to escape. Caustic remedies are found the most effectual in foot-rot. 
Three recipes have been recommended as efficacious in this disorder; these are as follows:-

$$
\text { NO. I.-MILD RECIPE. }
$$

Sulphate of copper . . 2 ounces;

dissolved in twelve ounces of water.

Strong sulphuric acid . . 2 drachms;

to be applied with a sponge or feather to the proudflesh.

NO. II. --STRONGER RECIPE.

Verdigris, powdered . . I ounce,

Nitrous acid . . . . 2 ounces,

Water. . . . 6 ounces.

NO. III.-STRONGEST RECIPE.

Red nitrate of mercury . . . I ounce,

Nitrous acid . . . 2 ounces,

Spirit of wine . . . 4 ounces.

The nitrated mercury should be first dissolved in the acid, and then the spirit of wine should be added; to facilitate the decomposition of the nitrate, add a teaspoonful or two of pure water, as frequently no action and decomposition will take place without water being added.

After these appliances have been repeated twice or thrice, it will be found that the flesh assumes a more healthy aspect; the parts should then be healed by an application of tincture of myrrh, or Friar's balsam. But should a discharge continue to flow from the parts, they should be carefully washed with a weak solution of soda, or Goulard's extract. The animals must be kept in a dry situation until the feet are perfectly healed.

The treatment pursued by that enterprising 
agriculturist, Sir George S. Mackenzie, is first to administer a dose of Glauber's salts, from one to two ounces, according to the size of the animal. Then the ulcer is laid open, and cleaned and washed with a weak caustic ley of potash or soda, and then filled up with scraped linen, steeped in Goulard cerate. This dressing to be continued every evening, until the granulations of the healing process become apparent. If ulceration continues, then the part may be again washed with caustic ley. When the hollow part caused by ulceration is tolerably well filled up, a dressing of cerate may be applied, after which it will soon heal, in most instances. Sometimes, however, the flesh grows too rapidly and in too great a quantity; which must be checked by a mixture of red precipitate and burnt alum dusted upon it. When the discharge has changed from a green hue to a yellowish-white, it may then be assumed that the parts are healing; and they should be brought together at the edges by gentle pressure, and kept so by adhesive plaster; but care should be taken to leave free egress for the matter if it still continues to flow. To keep down the swelling, the lower portion of the limbs may be washed with vinegar.

Where the flock is a large one and the cases are frequent and numerous, the method of treatment recommended by Mr Buttar, of Corston, is the most effective and least expensive. Mr Buttar's directions are as follows: "Pass the whole flock twice during the year through a solution of arsenic, which is thus prepared: Boil 2 lbs. of arsenic with 2 lbs. of potash (pearl ash) in one gallon of water over a slow fire for half-an-hour. Keep stirring, and at any signs of boiling over, pour in a little cold water, then add five gallons of cold water. Put this solution to the depth 
of one inch to one and a half inch (just sufficient to cover the hoofs of the sheep), in a strong, well-made, water-tight trough, twelve feet long by eighteen inches wide, and about six inches deep, with narrow strips of wood nailed across the bottom to prevent the sheep from slipping. The trough must be set and fixed perfectly level alongside a wall or other fence in some out-of-the-way place. It should be provided with a good waterproof lid, secured by a padlock, so as to prevent the possibility of danger from any poison which might be left in the trough.

"There should also be a wooden fence on the other side of the trough, extended somewhat at the entrance end to guide the sheep into it.

"Before the sheep are driven through the trough, their feet should be well pared: then walk them quietly through, and let them remain in Pen No. 2 for half-an-hour or so before taking them back to their pasture.

"If sheep are badly attacked, I would recommend drawing out all the affected ones and passing them through the trough a second time, after remaining for half-an-hour in the pen. Should this not cure them, repeat the process in a fortnight or three weeks time. Having got free from foot-rot, the passing the flock through the solution twice a year, will completely prevent any new attack."

\section{VERMIN.}

Sheep are liable to be infested with various kinds of vermin, especially those that are lean and out of condition, as well as the young animals. Various kinds of insects deposit their eggs in the skin of sheep, which produce little tumours. These are the re- 
ceptacles of the grub or maggot, after the eggs are hatched. The insects which lay their eggs in the skin of animals are provided with an instrument called an ovipositor, with which they penetrate the skin, and at the same instant they deposit their eggs. The tumours produced by the grub after it is hatched create considerable uneasiness to the sheep ; and when a single sheep has several of these in his hide, he will seldom feed well, from the constant irritation produced by the motion of the grub. The shepherd, when he sees this uneasiness manifested, should search for the tumours, and pick out the grubs with a pin, or squeeze them out with his thumb nails, as there is always a small aperture on the surface of the tumour through which the grub escapes in due season.

The sheep-tick, called hippobosca ovina, is also very troublesome to sheep and lambs, especially before they are smeared.

REMEDIES. - When the former of these vermin infest sheep, the flowers of sulphur mixed with hogs' lard, and rubbed over the fleece, generally has the effect of preventing insects from alighting on them for the purpose of depositing their egg. A solution of aloes, spirit of turpentine, and black soap have also been successfully used for this purpose. Strong, colourless spirit of tar or carbolic acid, has likewise the effect of preventing flies from alighting on sheep. It also destroys the tick. A solution of corrosivesublimate has the property of extirpating flies.

\section{WOUNDS.}

We have already given directions concerning the treatment of wounds, which is alike in all quadrupeds. But we must remind our readers that the application of 
greasy substances to a fresh wound makes it suppurate, and become a sore, and consequently Friar's balsam, or tying or bringing the sides of the wound together by means of adhesive plaster, will in most cases prove the best remedy. We would therefore recommend the shepherd to have always at hand a piece of adhesive plaster in case of need; and he should have a bottle of Friar's balsam, or the following preparation in his possession, which will answer equally well in fresh wounds :-

Saltpetre, finely ground Spirit of turpentine . Opodeldoc .
I ounce,

2 ounces,

4 ounces.

When sheep are bitten by that species of snake called the viper, or adder, the wound being so small, it is seldom known until considerable swelling has taken place, in consequence of the poison entering the system. The only specific which we know in such cases is to administer doses of castor oil to the extent of from three-quarters of an ounce to an ounce and a quarter. Half-an-ounce of hartshorn, mixed with water, has been also found effectual in allaying swelling produced from the bite of the viper.

\section{FRACTURES.}

When bones get fractured in sheep, if in the limbs. the ends of the bones should be placed in contact, and splints of wood placed round the part, firmly bound together by means of a plastic bandage an inch and a half in breadth; and where this cannot be conveniently procured, broad tape may be substituted, which should be replaced as soon as a proper bandage can be had. A piece of pasteboard will also answer the purpose of splints. The bandage should be wound round in a 
spiral direction. The splints will require to be worn for about twenty-one days, when they may be removed, and the bandage alone worn, until the leg has acquired sufficient strength. If the limb swells considerably, the bandage must be slackened, and again tightened when the swelling has subsided. If the fracture is comminuted, that is, in more than one piece, the safest thing is to destroy the animal immediately, as there is but little chance of a recovery under such circumstances.

\section{OF BLEEDING.}

The best and easiest mode of bleeding a sheep is to open a large vein which passes over the cheek. This vein can be readily felt, by pressing the finger on the edge of the lower jaw-bone, where its trunk passes over, and from thence its greater branches emanate, and spread over the cheek about two inches from the corner or angle of the jaw, nearly opposite to the third grinding or molar tooth. When blood-letting is to be performed, the operator must hold the sheep between his limbs, with the croup placed against a wall to prevent him from getting backwards. Place the left side of the jaws undermost, and seize the lower jaw in such a way that the fingers come upon its right side, so as to press above the trunk of the vein a small way below where the lancet is to be inserted. Make an incision obliquely across the vein, where the trunk is largest, which we have marked by letter $a$, Plate xrv, fig. I. This will be distinctly felt through the skin. The pressure prevents the flow of blood beyond the spot where the finger is applied, and consequently must pass through the opening, as it can find no other course. The oblique incision is found to answer better than one made along the course of the vein, or 
directly across it. To prevent the vein from shifting while the lancet is inserted, it should be pierced as near to the point of the finger as possible.

In our figure above referred to it will be seen that the trunk of the vein passes from under the lower jaw at $b$, and that its branches go along the soft portion of the cheek. At $c$ a small nerve takes an opposite course, passing over the vein, and in bleeding care should be taken not to divide it. Under this nerve there is a thick, fleshy muscle, which is called the zigomaticus major, whose office is to take a principal part in moving the jaw during mastication. The letter $d$ marks the situation of another much thinner muscle, beneath which the branches of the cheek-vein pass, and lead to the corner of the mouth, and assist in giving motion to the lips.

\section{ON THE AGE OF SHEEP AS INDICATED BY THE TEETH.}

The sheep, as well as all the other ruminating animals, is destitute of the upper fore, or cuttingteeth, which are also termed the incisors, but is furnished with eight cutting-teeth in the lower jaw.

At two years of age two of these drop out, and are replaced by other two. At three years old four of them are renewed, and the others at the age of four years.

The teeth undergo considerable changes between the first and eighth years, which we have delineated in Plate xiv, so as to enable persons to judge of the age of sheep by their dentition.

Fig. 2 exhibits the appearance of the teeth at one year old. Their cutting, or outer edges, form a crescent-shaped line, the teeth being nearly all of a 
similar breadtn, almost flat, and very smooth on their outer surface, and considerably concave internally.

Fig. 3 shows their aspect at two years of age, in which the two central teeth are considerably broader than the others, with the cutting edge not nearly so much arcuated as at two years.

Fig. 4 represents the structure of the teeth at three years, from which it will be observed that the four central ones are considerably broader and longer than the other two on each side, which rather suddenly shorten, the external teeth being shortest and narrowest : the cutting edge of the four central ones is but slightly curved.

Fig. 5 characterises the teeth at four years, and shows that the six central ones are nearly of uniform breadth and length; their cutting edge being but very slightly curved, the external tooth on each side being materially shorter than the others. The whole of the teeth are much stronger than in any of the preceding years.

Fig. 6 shows the structure of the teeth on the fifth year. It will be observed that the whole eight teeth are nearly straight on their cutting margin, and that there is but a small difference in their breadth, and they almost gradually diminish from the centre to the sides. The basal range above the gums is also nearly parallel.

Fig. 7 shows the character of the teeth on the sixth year, when the general length and breadth continue nearly as in the fourth and fifth years; but the two central ones are generally considerably worn down, or broken, as exhibited in our figure, and frequently dark-green, or brown, at their base above the gums, and their hue rather yellow.

As in cattle, so in sheep, selection and feeding 
causes the teeth to appear earlier or later in the mouth, and in a flock of well-bred and well-fed sheep, the teeth generally appear in the following order of time :-

At birth, or by the seventh day, the lamb has 4 incisors,

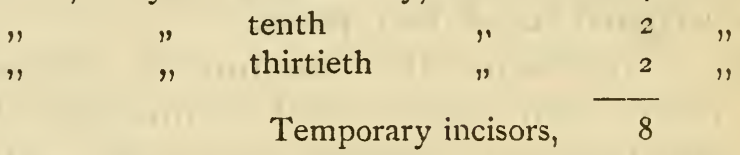

At three weeks the rst, 2nd, and 3rd temporary molars on each side and in both jawbones appear.

At three months the $4^{\text {th }}$ molars appear, but they are permanent and not sucking teeth.

At nine months the $5^{\text {th }}$ molars (also permanent) appear.

At one year to one and a quarter the 2 central temporary incisor teeth are thrown off and replaced by the 2 central permanent.

At one and a half year to one and three-quarters, the 2 middle temporary incisors are thrown off and replaced by the 2 middle permanent.

At one and a half year the 6th molar (permanent) appears.

At two years the Ist, 2nd, and 3rd temporary molars are thrown off and replaced by the Ist, 2 nd, and 3 rd permanent molars.

At two and a quarter years to two and a half years the $z$ lateral temporary incisors are thrown off and replaced by the 2 lateral permanent incisors.

At three years to three and a quarter the 2 corner temporary incisors are thrown off and replaced by the 2 corner permanent incisors. The permanent teeth of sheep are easily distinguished from the temporary or sucking, by being double their size. See Plate xIV, figs. 3,4 , and 5 . 


\section{CHAPTER V.}

Gestation, Parturition, etc.

THE RAM.

OF late years much attention has been paid to the form of the ram from which to breed, as well as the quality of his wool, fleeces, and other properties which experience has shown to be the most appropriate.

The head should be fine, and rather small than otherwise; his eyes large and prominent, ears thin, his nostrils wide and expanded, his collar full from the breast and shoulders, but gradually tapering from the junction of the head and neck, and the latter of a graceful and gentle curve, without any appearance of hanging skin underneath; the shoulders broad and full, and joining smoothly to the collar forward, and to the chine backward, without permitting a hollow in either situation; the muscle upon his arm or forethigh must swell boldly from its superior part to the knee; the legs straight with a clean and fine bone; the knee must not have any loose skin about it, and the hair from thence, as well as from the hough downwards, without long or coarse hair; the breast broad and well-formed, and his fore-legs placed at a proper distance from each other; his chest of considerable girth, full and deep, free from any hollow between the shoulders, and the fore-flank quite full: 
the back and loins broad, flat, and straight, with the ribs finely arched; his belly straight, and the quarter long and full, furnished with ample muscle down to the hough, which should be nearly straight; the junction of his thighs deep, wide, and full ; the entire body should be covered with a thin pelt, and thick, with fine, bright, soft wool.

There can be little doubt but the above is an excellent and graphic description of a well-formed ram, although some individuals differ with respect to some of the points. But it is hardly to be expected that all will agree on any given form, however symmetrical it may be, and at the same time well adapted for taking on flesh.

Considerable differences of opinion exist among sheep-farmers, as to whether a large or small ram is the best. This, however, can only be determined by local circumstances, and other considerations, which may strike the fancy of the breeder. Much will also depend upon the nature of the pastureground, as there can be no doubt that large rams require more food than small ones. Therefore, on lighter pastures a middling size should be preferred to those which require a greater proportion of food.

The ram lives to the age of about fifteen years, and is fit for procreation at one year old. When castrated, they are termed wedders; and they soon fatten, and the flesh becomes better flavoured, and finer in the fibre.

\section{LEAPING.}

In former times it was the practice to turn rams loose among the ewes; but they are now kept apart from them during the rutting season. A separate 
paddock, or small enclosure, is appropriated for the purpose, and to keep them quiet a couple of ewes are permitted to remain beside them. The ewes of the flock are brought to them, and they are only permitted to leap them once. The consequence of this improved method is, a ram is enabled to impregnate nearly double the number of ewes he would do, if allowed to go amongst them without any restraint, more especially a young ram. Formerly from sixty to eighty ewes were considered as many as a ram could serve; whereas, now, from one hundred to one hundred and twenty are impregnated by a single ram. Instances have occurred where one ram has served seven score in a season, but this is decidedly injudicious.

Much attention is required by the shepherd during the rutting season, to see that rams leap those ewes which are in season, as the ram will sometimes take a fancy for a particular sheep, which probably is not in sufficient heat to receive him, and will remain with her a whole day inactive, and will drive off such other ewes as come near him. When the shepherd notices a circumstance of this kind, he should remove the favourite ewe to a great distance from the ram; that is, of course, in cases where he is not kept apart from the flock.

To ascertain when the ewes have been served, it is a good practice to rub the breast of the ram with red keel, or other dry paint, which will leave a mark on those which he has leaped. But this is not a sure criterion, as tups frequently leap without accomplishing their end.

It is of great consequence that both rams and ewes should be in good condition before the rutting season, which of course must be regulated by climate, 
and the necessary quantity of spring food. It is likewise important that lambs should be dropped as early as possible, so that they may be well nursed, and able to provide for themselves before the winter commences.

\section{THE EWE.}

The ewe generally produces one lamb at a birth, sometimes two, and rarely three or four. She goes with young five months, and brings forth in spring. Some, however, produce their lambs at Christmas.

\section{LAMBING.}

When ewes are far gone in parturition, they should be moved about as seldom as possible, and feed upon dry ground, and in a quiet, retired situation, as much annoyance is apt to make them miscarry. When they happen to part with their lambs prematurely, they should be taken into the house if the weather be cold; but if the weather be mild and dry, they may be allowed to remain on the pasturage, if it is in a favourable locality. When ewes are about to yean, they should be located on as dry and smooth ground as possible, which will be of much benefit to them, as well as to their lambs.

When lambs are dropped on a place where they have difficulty in rising, they ought to be lifted, placed on their feet, and carried close to their dams. But when they can rise without aid, it is better to let them alone. It is usual to dock lambs when two or three days old, but if the weather is cold it should be delayed, and the remaining portion should not exceed two or three inches in length. It is of much consequence for ewes to have short tails, as it not 
unfrequently happens that lambs at birth are entangled by the tails of the ewes, and in this way get strangled or killed. The tails of the males need not be docked until they are about to be castrated. Short tails are of much consequence when they are attacked by the disorder called pinding.

While ewes are nursing, the pasture should be good, and they should be kept on the same feeding ground until their lambs are weaned.

It is of importance that the shepherd should be almost constantly with his flock during the lambing season, so as to assist such ewes as require it in cases of difficult parturition. In this office he should act with great gentleness, and watch the times when nature is acting her part to discharge the lamb.

When lambs are weakly, the shepherd should hold them up to the teats of their dam to suck. And in cases where the dam dies, he should endeavour to get another ewe to adopt the orphan lamb; and when he cannot succeed in this, should carry it home, as well as any weakly ones, so that they may receive his fostering care, or that of his family. This may be done by drawing off the milk from the udders of ewes that have an abundance of it, or from ewes which have lost their lambs. Lambs seldom attempt to eat grass for fourteen days, and during this time their whole sustenance consists of milk.

It not unfrequently happens that lambs die from hunger, in consequence of their dams refusing to suckle them. When this is the case, the poor lamb will be seen running about, and following any sheep it may come near, while it utters a mournful bleat. When it attempts to suck its dam, she will run off from it to a distance. When this is noticed the shepherd should 
examine the ewe, when he will find that sore nipples, or some other disease, is frequently the cause of her want of maternal care. If he discovers that sore nipples is the reason she deserts her young, they should be anointed with some emollient ointment to heal them : such as common cerate, or palm-oil. If the want of a sufficiency of milk is the cause, then the ewe should be supplied plentifully with green, rich food, and the lamb fed in the meantime with cow's milk, or from the milk of a ewe which has lost her lamb.

To make a ewe which has lost her lamb take to another, the following device has been adopted. The dead one is skinned, and the skin fixed on the other lamb, and both ewe and lamb confined together in a particular place, when the ewe will take to it, and then the false skin may be removed. In those cases where the ewe refuses to allow the lamb to suck, tie her by the horns or head and place her in a small corner pen, where she can neither kick the lamb, nor turn round upon it, when the little creature desires to suck. Give the ewe hand-feeding, and allow her to remain tied up for a day or two. As a rule she now takes kindly to the lamb. If not, introduce within the vagina a small quantity of common salt, and this by acting on the mucous membrane of the vagina and womb excites the maternal feeling and desire to give milk.

\section{WEANING LAMBS.}

It is rather a difficult task to wean lambs. They should be allowed to suck for three months and a half; after which time they should be removed to a distance from their dams, and kept apart for two or three weeks, when they may again be returned to the pasturage along with the ewes, and will give no further trouble. 
They should be so far off that their bleating cannot be heard by the ewes, otherwise they are sure to be extremely restless and anxious, and will hardly settle to feed.

Ewes will generally cease to have milk in about ten or twelve days, if they are not milked regularly, which some farmers are in the habit of doing for the purpose of making cheese. If, however, this is long continued, it cannot fail to weaken them, and reduce them to too low a condition before the rutting season. It will be proper to milk them every second day at first, when the lambs are removed from them, and allow a longer interval at each milking, until they cease to secrete milk, or at least in small quantities, which will be carried off by absorption.

\section{CASTRATING LAMBS.}

This operation may be performed at any time, from fourteen days old, to that of a month or six weeks. In some districts it is deferred to a much later period. We would, however, recommend it to be done early, as there is much less danger of inflammation occurring at an early period than there is at a later one. Attention must be paid to the state of health of the animal at the time, as if weakly, or labouring under any other disease, constitutional irritation may ensue, and death is most likely to follow.

This operation is usually performed by the shepherd. It is executed by the excision of a portion of the scrotum or cod with a sharp scalpel, and pushing out the testicles with the spermatic cord attached to them. Some shepherds perform the operation of dividing the cord with their teeth.

It is evident that this must be a painful and 
dangerous operation, when not judiciously performed; and some lambs die on the day they are castrated. The second and third days, however, are those on which it may be judged of the probability of safely surviving it.

Strict attention should be paid to the state of the weather when castration is performed : the atmosphere should be dry, but not too warm, and, if possible, the lambs which have been gelded kept in a dry, quiet, and sheltered situation, until the inflammation has subsided. If wet weather comes on, it would be prudent to have them removed to a shed, or barn, but not too many crowded into one place, as they should have room to move about freely. The operation should not be performed when the nights are frosty.

\section{INFLAMED UDDER, ETC.}

When the ewe is suckling; she is liable to inflammation as well as to hard tumours, in the udder. These are caused by obstructions in the lacteals, or milkvessels; and unless they are speedily removed, mortification will ensue in two or three days. When tumours are discovered, the ewe should be taken into the house, the wool must be cut closely off, and the hard parts frequently rubbed with camphorated spirit of wine and goulard water, in the proportion of three parts of the former to one of the latter. Should the parts not get softened by the means recommended, and suppuration appear likely to follow, in that case the tumours should be opened with a lancet, and the wound carefully dressed with Friar's balsam. It sometimes happens that the loss of one or both teats follow this complaint; when this is the case, then the best way is to fatten the ewe, and dispose of her, as she will never afterwards be adapted for breeding from. 


\section{CHAPTER VI.}

Management, Feeding, etc., of Sheep.

SHELTER.

THE natural habit of sheep is to range at liberty, and that, too, over a wide extent of country; therefore, confinement is ever to be avoided, except under peculiar circumstances. At the same time, all sheepfarmers must be aware of the positive necessity of their being sheltered sometimes; and it will be universally acknowledged that those which are protected from storms and much wet always thrive the best. Therefore, on all sheep-farms, sheds or other shelter should be provided for the animals in case of need.

\section{DRIFTING SNOW.}

In all mountainous countries sheep are liable to be overtaken by sudden falls of snow, and few situations afford natural shelter. Therefore large circular enclosures should be constructed of turf or stone, into which the sheep should be driven on the approach of snow. These should generally be on the south or west sides of the hill, as snow-storms usually proceed from the north or east. This should always be situated on dry ground, and advantage taken of any natural shelter, such as an elevated rock, or side of a ravine. The walls of turf enclosures should be four 
feet thick at the base, and two feet at top, and the height not less than six feet. There should be two or three openings in each, and these always on the south side. The spot on which they are situated should be on a slightly inclined plane; and drains should be properly constructed, so as to carry off the rain or melted snow; these should pass through holes in the walls, and be received in a large drain outside.

When sheep have been driven a few times into these enclosures, they will go into them of their own accord, on the approach of a storm or even during indifferent weather. Consequently, such enclosures are of much advantage to a shepherd, as he will be certain that his sheep will be safe in them, when a snow-storm has taken place, without the danger to which he is liable, in attending to them personally.

Besides these enclosures, it has been found that strips and clumps of Scotch fir-trees are of much utility as shelter for sheep; and where such exist, sheep will always retire to the lee side of them on the approach of boisterous or rainy weather.

Where gentlemen feed sheep in their parks, there is generally sufficient shelter; but in the formation of new ones, we would especially recommend the introduction of a considerable proportion of sprucefirs, as better adapted for shelter both during snow and rain, owing to the thickness of their foliage. Dry knolls or rocky ground should always be preferred for planting clumps.

In localities where foxes are plentiful, it becomes necessary to have folds or cots constructed for the protection of lambs during the night, where they should be driven with their dams by the shepherd at nightfall. There are various methods of constructing these, which, however, comes more within the 
province of a work devoted to agriculture, than one on farriery. There is one, however, of a very simple and cheap construction, invented by $\mathrm{Mr}$ Plowman, of Broome, Norfolk, which we may notice. This is twenty-one feet long, and three feet eleven inches high, composed of a top rail, with bars below passed through uprights. These have low cast-iron wheels below, on which they move strongly, but so lightly constructed that a boy may remove them from one place to another. It saves much time besides, for it can be changed from one locality to another in a few minutes; that is a fold which can contain at least three hundred sheep.

\section{EFFECTS OF RAIN.}

It is almost impossible to shelter large flocks during rainy weather, unless very large sheds were erected, which would cost too much money, besides occupying too great a space of ground. In the summer months, however, little danger is to be apprehended from sheep being long exposed to rain. But in winter, weak sheep are very liable to be affected by their fleece getting drenched with rain, without an opportunity of drying themselves. When such, however, is the case, they must be taken into a barn, or shed, to enable them to get dry, otherwise disease is sure to be induced.

\section{HEAT AND FLIES.}

In low and flat localities without shelter, sheep are frequently subjected to the oppressive heat of the sun, and likewise to the attacks of flies and various other tormenting insects, and even when they have shelter in flats, still they are not free from these pests. It is very common for these insects to deposit 
their eggs at the roots of the horns, or other parts about the head, as well as under the tail, which in the course of time will change into the grub or larva state. It is the duty of the shepherd to see that sheep are free from these, and to destroy their eggs when he finds them. This is most effectually done by the following ointment, which he must apply on the places where eggs are deposited:-

$\begin{array}{llll}\text { Mercurial ointment } & \cdot & \cdot & \text { I ounce, } \\ \text { Resin, finely ground } & \cdot & 5 & \text { I ounce, } \\ \text { Hogs' lard } & \cdot & \cdot & 3 \text { ounces. }\end{array}$

Let the lard be melted in an earthen or other pot, and the powdered resin added, and when properly incorporated, let the ointment be put in, and the whole stirred until cold, to prevent the mercury from falling to the bottom. A very small portion of the above, well rubbed on the parts, will have the desired effect.

As a preventive, the head and tail may be rubbed with the following composition :-

$$
\begin{aligned}
& \text { Tar } \\
& \text { Train oil }
\end{aligned} \quad \cdot \quad \cdot \quad \cdot \quad \cdot \quad \cdot \quad \begin{aligned}
& \text { I pint, } \\
& \frac{1}{2} \text { gallon. }
\end{aligned}
$$

The time when flies are most troublesome to sheep is after shearing. Sheep that are fed on mountainpasture are free from the attacks of insects.

\section{WASHING.}

Preparatory to shearing, it is necessary to cleanse the fleece. The best method of performing this operation is to rail off a portion of the river, or pond, with a sloping opening at one end, by which the sheep are driven into the water, at a part so deep that they will be unable to cross without swimming. A cask loaded should be fixed so that 
the men may stand in it dry, with a plank or bridge leading to it, to wash the sheep, before allowing each individual to pass to the opposite side, where they can walk out by means of an inclined plane. Before they reach the man who washes them they should be well soaked in the water, so as to save time. When washing is over, the flock should be driven to a dry, clean pasture. Of course, dusty roads must be carefully avoided, until the animals have become fairly dried. The lambs do not require more than simply swimming through the water.

\section{SHEARING.}

June is the time when shearing commences. It requires considerable care and nicety to perform this operation without mangling the fleece, and leaving large patches of wool adhering to the backs of the animals. After sheep are shorn, they are liable to the attacks of different insects, such as ticks and gad-flies, etc. The former of these should be carefully picked off, and the skin smeared all over with the following composition:-

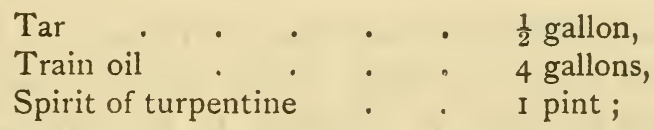

but oil of tar may be substituted for the turpentine, being considerably cheaper.

The method employed in sheep-shearing is so well known that a detailed account of it is unnecessary.

\section{FOOD.}

It is considered prejudicial to change the food of sheep frequently, as it has a tendency to derange the 
regular action of the bowels, and to induce disease. Sudden change from one kind of pasture to another is certain to affect sheep, more particularly in the spring and autumn. It is, however, more dangerous to change suddenly from poor to rich diet than the reverse.

Sheep thrive best where there is a tolerable uniformity of pasture all the year round. If the ground is wet, with the water standing on it here and there, that is quite unfit for pasturing sheep.

There is no better spring food for sheep than ryegrass and red clover; the former comes in most appropriately after turnip feeding. Cole, tares, and rye are likewise of much consequence as spring food.

For winter feeding, nothing is better than turnips, which should be accompanied by hay, or the straw of peas, beans, or wheat, chopped or cut. Swedish turnips are most approved of in most parts of England. Cabbages come in well late in the season, and sheep fatten quickly upon them.

\section{FATTENING.}

Various methods have been adopted to force the feeding of sheep; among these may be particularly mentioned oil-cake, in addition to the ordinary food, half a cake being given daily. But hay, bran, chaff, and corn have all a tendency to fatten, and of the latter two pounds may be given daily with advantage. The cake, corn, and chaff should be given in troughs, and the hay in racks, which may be placed over the troughs as a roof to keep the cake from being soaked with rain. From eighteen to twenty pounds of turnips are required daily for each sheep. Barley-meal has also been successfully used in feeding, as well as pease 
and beans and meal made from them. These last two are the most nutritious of all food; and it will be found advantageous to have a piece of rich land for the final stages of feeding.

On the subject of fattening there has been considerable diversity of opinion, but much depends upon the particular locality; for what may answer well in one place, may not prove effectual in another. 


\section{CHAPTER VII.}

\section{Different Breeds of Sheep.}

THE introduction of the Merino sheep has done much to improve the different breeds of British sheep. And in almost every county, which formerly had their distinct and recognisable breeds, the Merino blood has, to a certain extent, been introduced. In our description of the ram, page 565 , we have detailed the points which are now generally admitted to be the best in the form of sheep. So many crosses have taken place of late years, that there is hardly a breed which possesses characters by which they can be distinguished, if we except those which feed on the mountainous tracts of Wales and Scotland, which are for the most part small animals, and many of the flocks with black faces, and both males and females provided with horns. These sheep are remarkable for their fine, close-grained, muscular fibre. Nearly allied to these are those of the Westmoreland, Yorkshire, and Northumberland mountain sheep, with white faces. Those which are now most propagated are long-woolled, and also such as is of a fine texture. These were first successfully improved in the following counties, viz., Cumberland, Dorset, Hereford, Norfolk, and Sussex; and these may be considered as the parent stocks of all our modern improved varieties. To attempt a specific description of these is now almost impossible, as the distinguishing characteristics of each are mere shades of difference. 


\section{SECTION IV.}

\section{DISEASES OF SWINE.}

\section{INTRODUCTION.}

THE most erroneous opinions prevailed for a long time respecting the nature of swine, as also their mode of treatment. It was fancied that this animal would thrive equally well, whether kept clean or dirty, as it was supposed its natural habits were to wallow in the dirt and mire. Modern experience, however, has proved these fancies to be most incorrect, and it is well known that pigs always prefer a clear sty and clean litter to those that are dirty; and it is an equally well-ascertained fact, that they never thrive when these important points are neglected.

If it is possible, swine-sties should be constructed in localities through which a running stream passes, and their sleeping-houses on a sloping bank, so that they may be dry and comfortable for the animals, and the inclined plane will carry off any urine from the enclosure appropriated to them. Those who keep a number of pigs should have separate sties for the young and the old, as well as for the accommodation of those which are in pig; and where they should be kept until they farrow.

Swine are liable to various diseases; but their treatment has not yet been sufficiently ascertained, in 
consequence of the difficulty of administering medical treatment to them. They are, besides, not robust in their constitutions, being exceedingly liable to complaints of the lungs, if kept in cold situations, from the effects of which they are frequently afflicted with a hard, dry, and husky cough. As with almost all our domestic animals, it is of infinite consequence that their food should be regularly given to them. 


\section{CHAPTER I.}

\section{Internal Complaints.}

\section{INFLAMMATION OF THE LUNGS.}

THIS complaint makes its appearance in animals predisposed to it, when subjected to wet and cold sties. It is also induced by their being too hastily changed from poor feeding to that which is rich.

REMEDIES.-In the highly inflammable state, with much cough, cooling aperients may be given. The following has been found effectual in affording relief :-

$$
\begin{aligned}
& \text { Cream of tartar . . . . } \quad \mathrm{I} \frac{1}{4} \text { ounce, } \\
& \text { Saltpetre . . . . . } \frac{1}{2} \text { ounce, } \\
& \text { Tartarised antimony . . . I drachm; }
\end{aligned}
$$

to be dissolved in warm water, and mixed with the food. The same quantity to be repeated every twelve hours, until an abatement of the complaint is apparent.

\section{QUINSY.}

This complaint manifests itself by a quickness of the breathing, and is often accompanied by a wheezing sound.

Remedies.-Clean, plentiful, and dry bedding is of the first importance; and give a spoonful of tartarised antimony in the food, every twelve hours. If swell- 
ings in the glands follow, and matter collects under the jaws, the gathering should be let out with a lancet, after which dress it with tar-ointment.

\section{BLOOD-STRIKING.}

Pigs labouring under this complaint will frequently drop suddenly down and die almost immediately. Sometimes, however, there are premonitory symptoms, indicating the approach of the complaint, by staggering, or the fore-legs giving way under the animal. This malady is caused by an overflow of blood to the head, and is analogous to the staggers in horses, cattle, sheep, etc. When pigs die of this complaint, putrefaction almost instantly ensues, which renders the carcase entirely useless.

Remedies.-Whenever the above symptoms are noticed, aperient medicines must be freely given; the following will act more rapidly than any other :-

$$
\begin{aligned}
& \text { Tartarised antimony. . . } \mathrm{r} \frac{1}{2} \mathrm{drachm} \text {, } \\
& \text { Epsom salts . . . . } 4 \text { ounces ; }
\end{aligned}
$$

to be given in gruel of oatmeal, or linseed-meal, and repeated every twelve or fifteen hours, if necessary. 


\section{CHAPTER II.}

\section{GARGUT, OR DISTENDED UDDER.}

THis complaint is occasioned by the lacteal vessels becoming obstructed, and the udders being distended by coagulated milk, and internal inflammation of the parts is induced. In some instances, this disease is caused by too nutritious food previous to the time of farrowing; and when it occurs afterwards, it is attributed to the teats not being sucked or drawn down sufficiently soon after having farrowed.

The first remedy is to milk the animal with the hand, as young pigs will not suck their dams, when the milk is corrupted, nor would it be proper to allow them to do so, if they were so inclined. And when this has been accomplished, the following ointment must be gently applied, and slight friction used :-

Camphorated spirit of wine . . r ounce, Florence oil . . . . . $\frac{1}{2}$ ounce, Goulard's extract . . . $\frac{1}{2}$ ounce.

Two grains of calomel for two or three nights may also be given with advantage. 


\section{CHAPTER III.}

\section{Breeding, Gestation, Parturition.}

BREEDING, ETC.

Like all our other domestic animals, the hog has, of late years, been much improved, and nearly a uniform shaped animal is now generally diffused throughout Great Britain and Ireland. In the latter country, fifty years ago, the prevailing character was more that of a greyhound than of a hog: long-bodied and legged, high-backed, and leanness, being their ordinary characteristics.

The body should be of moderate length; the head broad and rather flat; the carcase well rounded and compact; the legs of moderate length, and rather short than otherwise, and fine in the bone; the shoulders broad, breast wide, and the quarters full; the hide must be fine and thin, and the ears rather small; the feet should be rather small, with the claws upright and even. The form which we have particularised holds good, whether the animals be of a large or small size.

The sow goes four months with young, and brings forth from six to twelve, and even so many as twenty at a litter, and that twice a year. At the time of farrowing, the sow must be carefully watched, as it is not uncommon for her to devour her young.

It has always been found that the best pigs are 
produced from a full-grown boar; but when they become aged, the progeny seldom proves good. The sow should be wide in the chest, and capacious in the belly; and if well nursed before weaning, she may procreate at the age of seven or eight months, and produce an excellent litter of young. But we think ten or twelve months is soon enough to commence breeding, as the animal will by that time have gained sufficient strength to bear the exertion of gestation and parturition. And no boar should be put to a sow until he is at least a twelvemonth old.

It is of great importance that sows should farrow in warm weather, as when they produce in the winter season, the pigs are very liable to die with cold. When they are farrowed late in August, the chances are much against many of them getting through the winter; and those which do are seldom strong and healthy. It is, therefore, advisable to part with these as soon as practicable.

During pregnancy sows should be kept separate from others which are not in that condition, as by keeping many together, the pigs are liable to be injured in the womb, and even killed, which will have a very prejudicial influence on the rest of the progeny, as well as on the sow.

It is of great importance to keep the sow and her young in a dry and warm situation, with plenty of good litter; and neither should be permitted to go abroad in bad weather. When sows have the unnatural propensity of devouring their young, they should be carefully watched for some days after their birth; and such as have this desire, should be parted with, as useless for breeding from.

During the time of pregnancy, sows should be well fed, and have a meal of a rich mash twice a 
day, at least, and, besides, a good supply of vegetable substances, such as cabbages, carrots, or potatoes; and when they are nursing their young, they should have a still larger supply of food, to keep up that secretion of milk so essentially necessary for the supply of such a numerous progeny.

Pigs may be weaned when about eight weeks old, and separated from the sow. She should be shut up by herself for a week or so, and well fed, to restore what she has lost in suckling her young. She will very soon after this manifest a desire to take the boar.

The times at which hogs are fattened, are twice a year, namely, beginning in October, for the winter, and February or March for the spring-time. Where skimmed-milk can be spared, it will be found the best liquid, and mixed with ground oats, barley-meal, peameal, or bean-meal, as also pollard; these may be given combined, with great advantage. Indeed, it is better to neutralise the heating effects of pea and bean-meal, by a mixture of some of the other farinaceous bodies. Malted barley has also been given to pigs while fattening, with considerable success. Potatoes and carrots boiled, mixed with skimmed or butter-milk, and even whey, prove an excellent variety for inducing an increase of substance in the animals. The refuse of the brewhouse and distillery are also found to be beneficial in fattening swine, with other farinaceous substances mixed in them.

From the contumacious and unruly nature of hogs, there is great difficulty, if not an utter impossibility, of performing operations on them, as with other animals, and consequently little progress has been made in the application of the veterinary art to these animals. The ordinary methods, of cutting off part 
of the ears and tail, can clo but little good; and equally ineffective is the plan of opening a vein in the roof of the mouth, about an inch from the front in a line between the second and third tooth, an operation that it is almost impossible to perform on a pig, and the bleeding is never of sufficient extent to relieve inflammation. Purgatives will consequently be the best remedies to be adopted. Epsom salts, varying in quantity, from one to two ounces, according to the age of the animal, and sulphur, in doses of from one to three drachms, may be administered. Attention should, therefore, be given chiefly to preventive, rather than remedial, means. It is of great consequence to let the animals have occasionally a quantity of green meat, as they will always be found to eat it with avidity. Grass, tares, or clover will be used with advantage. 



\section{SECTION V.}

\section{DISEASES OF DOGS.}

\section{INTRODUCTION.}

THE dog seems to have been destined by the Creator to be the friend and assistant of man. Throughout the dangers and difficulties which beset the human being, particularly in an inartificial condition of society, the dog has ever proved himself the vigorous and faithful defender of his life and property, as well as a powerful and essential auxiliary in subduing other animals to his purpose, and of securing them as his food. Without the assistance of the dog, man would not even yet have obtained a beneficial dominion over the various races of wild animals of the earth, or been able to watch with sufficient care those creatures destined for his support.

Of all animals known to mankind, the dog is the most diversified in form, size, proportions, properties, and intellect, agreeing only in one striking and admirable peculiarity - his constant attachment and fidelity to his master. Our domestic dog, with all the varied and striking varieties of form, is, however, but one species; changed and modified by local circumstances, domestication, and breeding; consequently, it is improper to consider and call pointers, terriers, mastiffs, and greyhounds species, as they are 
mere varieties. The parent stock from which all our domesticated races have sprung, has long been a dispute among naturalists, and is at this time an unsettled point. Buffon was of opinion that the shepherd's dog was the progenitor of them all.

Dogs in a domesticated condition are liable to a variety of diseases; but, above all others, what has been termed canine madness, or hydrophobia, is the worst, and most to be dreaded by mankind. They are, besides, subject to many complaints, in some respects similar to those of the human body. We believe there is none more common with them than lung disease and worm complaints, diseases to which mankind in this country are very liable. Our friend, $\mathrm{Mr}$ Mackenzie, keeper of the anatomical museum in the University of Edinburgh, an expert anatomist and physiologist, concurs with me in this opinion, and informs me that he has made many dissections of dogs, and found diseased lungs very prevalent in them. This is chiefly to be attributed to sleeping in damp situations, and in the open air, at times, when they are for the most part accustomed to be kept comfortable and dry in a house. Some families of dogs are constitutionally liable to this disorder; and sportsmen would do well to avoid breeding from a stock predisposed to lung disease. 


\section{CHAPTER I.}

\section{Diseases of the Head and Chest.}

\section{SWELLING IN THE THROAT.}

Symptons.-This is a common complaint, and consists of an enlargement of the glands of the throat. It is a disease to which the smaller breeds of dogs are more particularly subject, and comparatively of rare occurrence with the larger kinds. Young dogs are most liable to it ; and if not attended to early, it may become a fixed swelling, not reducible at any after period. It produces a stiffness of the neck; and if swelling is on one side, the animal sometimes has his head inclining to the opposite side. There is seldom much pain, nor does it often terminate fatally. It, however, occasions pain if pressed upon.

REMEdies. - The hair should be clipped off the swelling, and the part well rubbed with the following ointment :-

$$
\begin{aligned}
& \text { Calomel } \quad \cdot \quad \cdot \quad \cdot \quad \text { I drachm, } \\
& \text { White cerate }
\end{aligned}
$$

This to be repeated daily, until a cure is effected; but if no diminution is visible in a week, add half-an-ounce of blistering-ointment to the above proportions. Let the animal have a quarter of an ounce of nitre, in his drink, daily.

If the swelling is noticed early, fomentations will 
often perform a cure. Sew up a quantity of camomile flowers in a bag, immerse them in hot water, and apply it to the parts affected. Let it be applied as hot as the animal can bear it. The application should be taken off several times during the day, and heated again.

\section{INTERNAL ULCERATION OF THE EAR.}

Symptoms. - This disease is manifested by the dog continually shaking his head; and a discharge of matter will take place after the complaint is matured; but before the matter is seen, a dry, scurfy, red scab is formed on the inside of the ear. It is occasioned by too high feeding, and by the animal taking the water when heated.

REMEDIES.-When the case is not severe, all that will be necessary is to use the following wash, slightly warmed:-

$$
\begin{aligned}
& \text { Sugar of lead } \quad \cdot \quad \cdot \quad \text { I drachm, } \\
& \text { Rose, or rain-water } \quad \cdot \quad 4 \text { ounces. }
\end{aligned}
$$

About a teaspoonful should be poured in night and morning.

When the disease is of a more severe kind, the following remedy must be applied :-

$$
\begin{aligned}
& \text { White vitriol } \quad \cdot \quad \cdot \quad \text { I8 grains, } \\
& \text { Decoction of oak-bark } \quad \cdot \quad 4 \text { ounces. }
\end{aligned}
$$

When the above fails to prove effectual, recourse must be had to mercury. Let the ears be well washed with a solution of soda, and then rubbed with the following :-

Strong mercurial ointment $\quad$ - $\quad$ I ounce,
Hogs' lard . $\quad . \quad \frac{1}{2}$ ounce.

Besides rubbing internally, the ointment should be 
well applied at the base of the ear externally, close to the head.

\section{COUGHS.}

It frequently happens that cough precedes the distemper; and if the dog is young, and no probable reason can be assigned for the cold he has taken, it may reasonably be inferred that it is a premonitory symptom of that disease; and precautionary measures ought to be adopted. The cough which precedes distemper is always accompanied by dullness, and cold shiverings, with wasting of the flesh; and it is always dry and short in its character. Still there is little perceptible diminution in his appetite. For this, the following may be given with great advantage :-

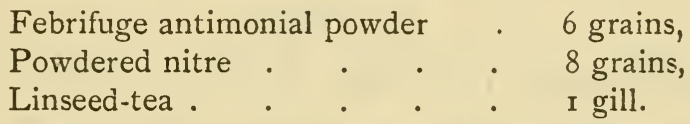

A cough arising from a cold is distinguished from that connected with the distemper by its shortness and frequency.

Sometimes cough is occasioned by worms; when this is the case, the coat stares, and the breath is very fetid. When this is suspected, it must be treated as recommended for worms, under its proper head.

\section{INFLAMMATION OF THE LUNGS.}

This is occasioned by colds, and many dogs die of it. It is a complaint which, with the canine species, is very rapid in its effects; and, therefore, must be speedily attended to.

Symproms. - An animal labouring under this malady holds up his head, as if to give him more freedom in breathing, the pulse is much accelerated, with an oppressed beating of the heart. 
Remenies.-Apply a copious mustard poultice to the chest over the region of the lungs. Then give the following mixture:-

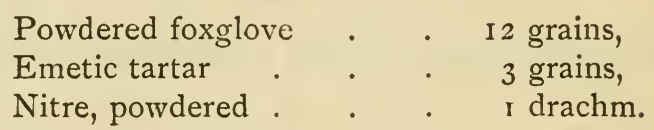

If for a large dog, let it be made into six powders; for one of a middling size, nine; and for a small one twelve. One to be given every three hours, until the symptoms are abated, and then they may be given every six hours, for a day afterwards. The animal must be kept warm. If the powders produce vomiting, they must be divided into two parts each, and given less frequently.

\section{ASTHMA.}

Confinement and high feeding are the causes of asthma; a complaint to which dogs are very liable, and are attacked with it at any period of life, but more especially at three or four years, where they are over-fed; but with those who are less pampered, it is seldom known until they are six or seven. It may be brought on at an earlier period by severe colds, and inflammatory complaints in the lungs. When it proceeds from over-feeding, it is caused by an accumulation of fat in the regions of the heart and lungs. It manifests itself by a short, harsh, and dry cough, and difficulty in breathing, more particularly when the animal has been much exercised, or by catching cold. This malady generally comes on gradually. Remedies should be adopted as soon as any indications of the complaint are noticed; otherwise, if allowed to attain a height, it is likely to prove fatal.

REMEDIES.-If there appear to be inflammation 
at an early stage of the complaint, bleeding will be necessary, which should be followed by gentle alteratives. The following purgative must be administered :-

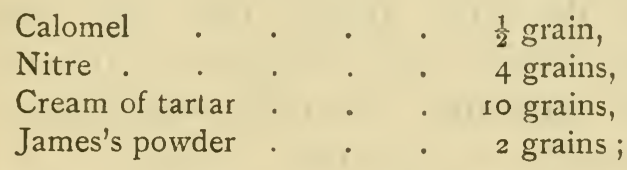

to be made into a ball with linseed meal and treacle, and put down the animal's throat. To be repeated every morning, and, if the complaint is severe, each evening likewise. The above is for an ordinary sized dog, and is rather too much for a small one; but for a large animal it must be increased.

If the calomel sickens the dog, the following preparation may be given instead of the above :-

Emetic tartar $\quad \cdot \quad \cdot \quad \frac{1}{2}$ grain,
Nitre $\cdot \quad \cdot \quad \begin{aligned} & 3 \text { grains, } \\ & \text { Powdered foxglove }\end{aligned} \quad \cdot \quad \frac{1}{2}$ grain;

prepared as a ball, with the linseed meal and treacle.

\section{THE DISTEMPER.}

This universal and fatal disorder made its first appearance among the dogs of Great Britain about I 50 years ago, at which period its ravages were widely diffused over the country. At that time about seven out of every ten fell victims to its malignity. The virulence of this disease and its baneful effects have now, however, greatly abated, either from the preventive remedies which are had recourse to at its commencement, or from the operation of certain medicines applied in the actual progress of the disease. I, however, find that this 
malady has been known for a much longer time on the Continent than in this country. It is as infectious among dogs as the small-pox, measles, and scarlet fever among the human species; and the contagious miasmata, like those arising from the diseases just mentioned, retain their destructive properties a long time after separation from the distempered animal. Young hounds, for example, brought in a state of health into a kennel where others have gone through the distemper seldom escape it. Kennels have been carefully washed with water, then whitewashed, and even repeatedly fumigated with muriatic acid, without any good results. The dogs generally sicken the second week after exposure to the contagion. It commences with inflammation of the substance of the lungs, and generally of the mucous membrane of the bronchia. The inflammation at the same time seizes on the membranes of the nostrils, and those lining the bones of the nose, particularly the nasal portion of the ethmoid bone. These membranes are often inflamed to such a degree as to occasion extravasation of blood.

Dr Jenner mentions a case which came under his observation, of a dog dying within twenty-four hours after infection, and in that short space of time the greater portion of the lungs was, from exudation, converted into a substance nearly as solid as the liver of a sound animal. When inflammation of the lungs is very severe, the dog frequently dies on the third day.

By judicious treatment, the distemper might be, in all probability, entirely banished, or at least its features be very much mitigated.

Colonel Hawker, in his Instructions to Young Sportsmen, mentions a case or a dog belonging to 
himself, on which he performed inoculation, by vaccine virus, or the matter of cow-pox, had the effect of preventing the distemper completely; and this was found an effectual preventive by James Drearden, Esq., of Rochdale, Lancashire, confirmed by an extensive and successful practice. It would certainly be worth while to try this expedient, as being exceedingly simple; and we have ascertained that in the instances where dogs have had the distemper after vaccination, it has been very mild.

The manner in which this operation is performed on a dog, is to make a puncture or scratch inside of the fore-leg, within the shoulder pit: the abrasion is then rubbed with a small quill with the virus. A better method, however, is to puncture with a lancet, charged with the virus, the inside of the ear. The virus acts with more certainty on the sensible skin devoid of hair, and the animal cannot remove it by licking with the tongue.

Although the distemper is a disease which, for the most part, attacks puppies from four to twelve months old, yet it is said there are instances where old dogs have been affected by it, which had not the disease when young. Without entering into a more lengthened detail of this disorder, I shall only notice the prevailing symptoms, and mode of treatment.

Symptoms. - There are some symptoms in the distemper which predominate, although the general ones are not invariably the same. In the first stages of the disease, the dog has a hard, dry cough, a want of nervous energy, depression of spirits, a swelling of the glands of the throat, and almost total loss of appetite; after which he is seized with a running at the nose and eyes, when emaciation and great weakness ensue, more especially in the hinder extremities. These 
symptoms are universally followed by convulsive twitchings of the head, and sometimes in other parts of the body, indicating considerable irritation in the brain and spinal marrow. The bowels are violently affected, either by being costive or extremely loose; and in either case the dog suffers great pain. When these acute symptoms continue, they are soon followed by vertigo, or giddiness, which produces dimness of the sight; in which case the dog runs round, foams at the mouth, and makes a piteous moaning or howling. In truth, inflammation and effusion take place in the brain, producing water in the head, a disease to which young children are liable, from long-continued irritation in the stomach and bowels.

These affections are accompanied by great irritability of the stomach, which discharges everything as soon as taken; and it not unfrequently happens that the poor animal expires in one of these spasmodic affections. When the distemper reaches this degree of virulence, few dogs recover: but there have been instances where Blaine's medicine has proved effectual, even in the worst stages of the disease. Dr James's powders have also, in extreme cases, produced a favourable change. Even in the worst cases the dog always retains its sanity, and will drink water, though certainly not very freely, two circumstances which will readily distinguish the disease in question from canine madness.

Remedies.-If this disease is attended to at its first appearance, the after-symptoms may be greatly mitigated, by administering opening medicines, in small quantities, and persisting in their use; and afterwards in larger doses, as the disease makes progress. This will be found of the greatest consequence in all complaints where the nucous membrane 
is likely to be affected, which is almost invariably the case where there is inflammation of the lungs; and was satisfactorily proved, by my late talented friend, Dr Macintosh, Lecturer on the Practice of Physic, Edinburgh, to be a general accompaniment of all pulmonary diseases in the human species. For this purpose I would recommend, so soon as the symptoms appear, to administer an ounce of castor oil, and, after its operation has abated, the following bolus should be given :-

Crocus metallorum, finely levigated
White antimonial powder
Diaphoretic calx of antimony . $\quad \begin{gathered}6 \text { grains, } \\ \text {. }\end{gathered}$

to be mixed up with treacle and linseed meal into a ball and given every two hours; the dog should be kept very warm during its operation, and should be supplied frequently with new milk or water gruel. If this medicine occasions sickness, or brings on great laxity in the bowels, the doses must be temporarily stopped, or greatly diminished.

The above dose is sufficient for a pointer, foxhound, harrier, or other large dog of ten months old. If younger, or a smaller dog, the quantities must be proportionally diminished.

$\mathrm{Mr}$ Shaw, principal gamekeeper to His Grace the Duke of Buccleuch, at Dalkeith, has generally found the distemper easily disposed of by attending to the first symptoms of the disease, and immediately administering a dose of calomel and jalap every second day, and paying strict attention to the food of the patient. He seldom found it necessary to give more than three doses. He has, however, remarked that when the disease begins with a flux, it generally proves fatal. This discharge is brought on by cold, and by sleeping in damp quarters. He mentions 
having lost several brace of greyhounds from one night's bad lodging.

Mr Daniel is of opinion that Blaine's medicine is an effectual remedy: it is made up in packets, marked with different numbers, I, 2, and 3. For a Newfoundland dog, mastiff, pointer, and setter, No. I should be used, for fox-hounds, harriers, and other dogs of a middling. size, use No. 2; and for cockers, and all other varieties, No. 3 will prove a sufficient dose. $\mathrm{He}$ found that soon after administering Blaine's powders, even although the disease had got to a height, the violence of the symptoms abated, the spasms became less frequent, and generally within twenty-four hours they completely subsided, leaving only a slight discharge from the nose. Our own experience completely coincides with that of $\mathrm{Mr}$ Daniel, although in some instances it will last for weeks; in the event of which I would recommend strict attention to the state of the bowels, and that the nose be frequently fomented with pieces of flannel dipped in hot milk and water.

Colonel Hawker recommends, in the case of a discharge from the nose, the use of a lotion, made by mixing half-an-ounce of sugar of lead, and the same quantity of alum, with a pint of water, and that the nose should be syringed with it. However effectual such applications may be in stopping the discharge, yet I cannot too strongly condemn the use of them, as having a tendency to bring on other diseases in the mucous membrane of the nose, and thereby affect, if not totally destroy, the olfactory nerves. But the truth is, the discharge from the nose is by no means an unfavourable symptom: the main risk the dog runs in this disease is from internal inflammation, and not from any affection of the 
organ; and it is a very generally received opinion in medical practice, that it is not safe to check discharges suddenly.

When the irritability of the stomach continues, and it refuses to retain the medicine, the latter should be mixed up with a small piece of butter, and from thirty to fifty drops of laudanum added, according to the age, size, and strength of the dog. Should this not remain in the stomach, an hour and a half afterwards the same quantity of laudanum should be given in a little broth. The powder must also be administered, in twenty minutes or half-an-hour, made into a kind of paste with treacle and flour, or linseed meal, which will certainly have the effect of allaying the vomiting. But if the bowels are obstructed, which generally follows the use of much laudanum, and the retching still continues, in this case some active purgative should be had recourse to, such as twenty grains of jalap, or fifteen grains of calomel, accompanied with from five to eight drops of laudanum, to allay the irritation; either of these should be made up into a ball, and put down the dog's throat ; or two tablespoonfuls of castor oil may be given in their stead. Should these prescriptions fail, a clyster should next be tried, composed of oatmeal gruel, salt, and oil; and when the bowels are moved by this, the medicine may then be given, accompanied with a few drops of laudanum.

When there is great alvine flux attending this disease, from thirty to forty drops of laudanum must be administered, mixed with an ounce of sweet or almond oil. It will be of no use to give the powder until the irritation has somewhat abated, as its effect will be nearly lost in passing too rapidly through the intestines. 
The following remedies have been found effectual :-

One ounce of Peruvian bark, in a glass of port wine, to be given twice a day. Norris's drops, to the extent of a tablespoonful, in the same quantity of port wine, given three times a day; the quantity to be diminished as the dog grows better.

Colonel Hawker recommends the following :-

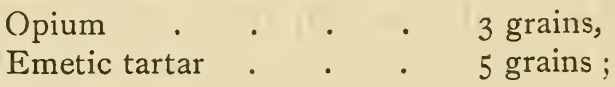

to be administered at night; and the same dose to be repeated every third night, until the dog recovers, taking care to keep him warm, and always to feed him with warm liquid diet, such as broth, gruel, etc.

Dr Taylor, of East Yarmouth, gave :-

$\begin{array}{ll}\text { Gamboge } & \quad \cdot \quad \cdot \quad 20 \text { grains, } \\ \text { White hellebore powder } & \text {. } \quad 30 \text { grains; }\end{array}$

made into six balls, one to a full-grown dog, six successive mornings, or half the quantity to a puppy, or a small dog, such as a cocker, terrier, etc.

For a half-grown pointer, or other young dog :-

$$
\begin{aligned}
& \text { Jalap, in powder . . } 20 \text { grains, } \\
& \text { Calomel . . . . } 4 \text { grains; }
\end{aligned}
$$

made into a ball with treacle.

For a full-grown pointer, or other large dog :-

$$
\begin{aligned}
& \text { Jalap, in powder . . } 25 \text { grains, } \\
& \text { Calomel . . . . } 7 \text { grains ; }
\end{aligned}
$$

mixed as above, and one of the doses given every second morning, mixed with a little butter ; and if the dog will not take it in this form, it must be made into a small ball, and forced down his throat. The food should always be light and easy of digestion.

I was favoured with the following recipe from a friend, who has had a great deal of experience in the 
rearing of dogs, for upwards of twenty-five years. $\mathrm{He}$ says it has always proved a most effectual remedy, in all cases in which he has applied it :-

$\begin{array}{lllll}\text { Calomel } & \cdot & \cdot & \cdot & \text { I drachm, } \\ \text { Tartar emetic } & \cdot & \cdot & \cdot & 20 \text { grains, } \\ \text { Jalap . } & \cdot & \cdot & \cdot & \text { I drachm, } \\ \text { Gamboge } & \cdot & \cdot & \text {. } & \text { I drachm; }\end{array}$

to be made into six balls, with conserve of roses, and one to be given every morning for a week.

Care should be taken to support the strength of the animal by light, nourishing diet, after the inflammatory stage is over, and the discharge from the nostrils fully established. Various authors recommend the too general use of emetics in the distemper, which, I conceive, must frequently be attended with evil consequences, as they are, for the most part, inadmissible in inflammatory diseases.

As the distemper is infectious, those dogs labouring under this malady should be kept apart from others; and exposure to the air, when not under the effects of medicine, will be found beneficial. This disorder has an affinity to some human diseases, and rarely attacks a dog a second time. Fortunately for humanity, the distemper is not communicable to man. Neither the effluvia from the diseased dog, nor the bite, has proved in any instance infectious.

As this disease is a parasitic one, and due to a specific bacillus which breeds in the lung tissue, producing structural changes therein, and is attended with great bodily debility, and as apparently the disease cannot be cut short, but must, like other specific diseases, run its course, the treatment prescribed should be backed up by good nursing, frequent change of diet, soups, milk, beef-tea, a uniform temperature (near to a fire), and flannel rugs and bandages. 


\section{RABIES, OR HYDROPHOBIA.}

In the dog this disease is termed "Rabies Canina," canine madness: in the human subject, Hydrophobia, from the dread of drinking water, the sense of suffocation felt in the act being awful. The dog as a rule laps liquids with facility, but the pouring of water from one tumbler into another is sufficient in some persons rabid to bring on powerful spasms of the muscles of deglutition.

This is a specific disease which develops primarily (if such an expression or opinion is allowable) in dogs, wolves, jackals, foxes, and cats, but the specific poison or organism on which it depends once having declared itself present in these creatures, may by inoculation (bite or abrasion) produce the disease in men, cattle, sheep, deer, rabbits, horses, swine, etc.

The virus is contained in the saliva, salivary glands, the tissues of the brain, medulla oblongata, medulla spinalis, cerebro-spinal fluid, and portions of the pneumogastric nerves.

Symptoms.-Like all other specific diseases, there is a period of incubation from the reception of the virus until symptoms of indisposition are exhibited, and this period varies widely, from eight days to several months. The symptoms also vary according to the form it takes, or, in other words, according to the structures invaded. In the dog we have two well-marked forms of the disease-(I) Raging madness : here the so-called blood poison acts powerfully on the brain, and the animal barks and bites and furiously attacks all objects which come within its range of action. (2) Dumb madness: here the dog neither barks nor bites, but sits gazing at objects, his hind limbs being paralysed, as also his lower jaw. 
The face presents a strange idiotic look, with the lower jaw hanging down, and the saliva in large quantity flowing from the mouth. In these cases the blood poison apparently principally affects the spinal cord and certain of its nerves.

A very marked symptom of rabies canina is the strange craving the animal has for seizing and swallowing all kinds of rubbish, e.g., paper, straw, hair, wool, wood, stones, nails, etc. etc.

Post-mortem.-There is no specific lesion which indicates after death the absolute certainty that the dog was rabid. Generally the tongue is relaxed and swollen, and the mucous membrane lining the fauces, pharynx, and larynx, congested or inflamed. The stomach is congested, and in some cases acutely inflamed (gastritis), and if the dog has not been confined, it invariably contains many curious articles. The membranes of the brain and the spinal cord may also be congested.

But all these post-mortem appearances, and the foreign bodies in the stomach, may be found when no such disease as rabies has existed. Indigestion and gastric affections are common to the dog, from the irregularity in feeding and the variety of food of which the dog partakes when he has to forage for himself, and, as every one knows, it is stray dogs which are so frequently seized by the police as rabid animals.

When a dog has suffered from indigestion and gastric irritation for a time, just like the cow eating bed-clothes, old leather shoes, etc., and the horse swallowing sand, stones, and other rubbish, so the dog commences to swallow rubbish, and sooner or later becomes raging mad, although not rabid. On this point Prof. Crookshank, in his standard work on Bacteriology, makes the following remarks:- "Dogs 
suffer from symptoms simulating those of rabies, and formerly when human beings were bitten, it was impossible to determine whether the dog had been suffering from rabies or not. We are indebted to Pasteur for the only reliable test which can be applied, and we are now in a position, when a human being is bitten by a dog supposed to be, but not really, rabid, to remove all cause for the anxiety which would otherwise remain for months and even years.

"Pasteur found that a dog inoculated under the dura-mater (covering of brain) with virus from the spinal cord of a rabid animal will develop rabies as a rule, within eighteen days. By trephining rabbits and inoculating the virus, and by, in the same way, transmitting the virus from rabbit to rabbit, the incubation period gradually shortens, until it is reduced to six or seven days. The virus has then reached its maximum virulence in the rabbit, and is 'fixed.' Pasteur then studied the possibility of producing immunity. The medulla of a rabbit containing the virulent virus was suspended in a glass bottle over caustic potash at a temperature of $25^{\circ} \mathrm{C}$. If a number of spinal cords were thus treated, and examined from day to day, it was found that they gradually lost their virulence, becoming completely inert in from sixteen to twenty days. A series of cords were thus obtained with diminishing virulence; by injecting subcutaneously an infusion of rabid spinal cord crushed in broth, and beginning with an inert cord on the first day, and using the next in the series on the second day, and so on till a fresh spinal cord could be injected, it was found that dogs were rendered insusceptible to the strongest virus administered by inoculation or by exposing them to the bites of rabid dogs. Dogs have usually an incubation period of 
several weeks, and Pasteur conceived that it would be possible to anticipate the symptoms which would naturally follow in a dog which had been bitten or inoculated, by giving them a mild form of hydrophobia by the injection of attenuated virus of short incubation period. These experiments showed that it was possible to do this, and the outcome was the introduction of a system of protective inoculation in the human subject. Pasteur succeeded in giving immunity from hydrophobia to about fifty dogs of every age and breed.

"In I 885, Joseph Meister, a boy nine years of age, bitten badly by a mad dog upon the hands, legs, and thighs, was brought to Pasteur. At a post-mortem examination of the dog, its stomach was found full of bits of hay, straw, and wood, and it had been unquestionably rabid. On 6th July, sixty hours after Meister had been bitten, a syringe full of marrow from a rabbit which had died on 2 Ist June, and therefore fifteen days old, was injected beneath the skin over the right hypochondriac region. The next morning Meister was inoculated with a spinal cord fourteen days old, and so on every day, till on the sixteenth a cord only one day old was used. So many injections, however, need not have been given, as it was subsequently found that the spinal marrows injected during the first five days were inert when tested on rabbits. The marrows of the next five days showed an ascending scale of virulency, until, on the last two days of the treatment, Meister had been inoculated with a virus so virulent that it was capable of causing hydrophobia in dogs after ten days' incubation. Meister remained completely free from hydrophobia. From that time to the present day, many thousands of patients have been treated in 
Paris by slightly modified methods, and it is very generally believed that a real prophylactic agent has been discovered."

In dealing with rabies in the dog, or rather a dog suspected of being rabid (for our experience would lead us to assert that not one per cent. of the dogs alleged to be rabid are rabid), the usual practice is to kill the animal where found. Instead of doing so the dog should be secured with a lasso and placed in confinement. If the dog be really rabid, hourly he will become worse and will be dead in a few days, whereas, if not rabid and allowed rest and food and plenty of water to drink, in all probability, the symptoms which similate rabies so exactly, will gradually disappear and the animal be restored to health.

This has been our experience of the disease in many instances, and if the dog belongs to a person who values him, it is pleasing, and to say the least, highly satisfactory and reassuring to the individual who has been bitten by a dog believed to be rabid.

Remedies.-Treatment is not allowed in the lower animals. The rabies order makes it imperative on reporting to the Local Authority; and their veterinary inspector is required to see the animal destroyed.

Precaution.-When a person is bitten by a mad dog, the wound should be immediately burnt with lunar caustic; but the most effectual way to prevent the poison from getting into the system, is to cut out the piece of muscle which has been bitten.

\section{BITES OF VIPERS.}

If the part is rubbed immediately with sweet oil, 
the effect of the poison is destroyed; and the following composition may be afterwards used :-

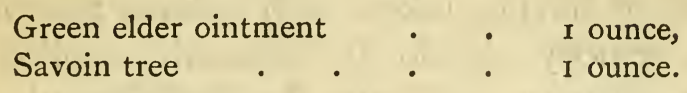

It not unfrequently happens that considerable swelling of the head takes place after the bite of a viper; when this occurs, recourse should be had to purgative medicines. The following must be given, once a day, until the swelling subsides:-

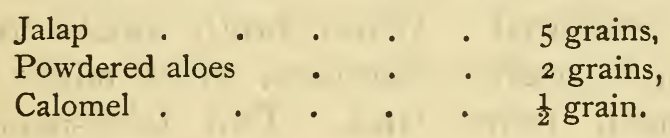

\section{POISON.}

When it has been discovered that a dog has taken poison, give the following specific as soon as possible :-

Emetic tartar, dissolved in water . 15 grains, which will have the effect of producing vomiting; and after the retching has subsided, give him two ounces of castor oil, or ten grains of turpeth mineral, if a large dog, mixed up in butter, and rubbed on his nose. If the dog is middle-sized, eight grains; and if very small, five grains will have the effect.

Turpeth mineral is useful as an emetic for a dog upon any occasion; eight grains of it may be mixed with a little butter, and rubbed on his nose; he will lick it off, and it will operate in about five minutes.

Warreners very often make use of nux vomica as a poison, which is not unfrequently concealed in a piece of meat, to entice foumarts and weasels to eat it. Hounds and other sporting dogs pick it up, 
and if no remedy is administered, convulsive fits, and death itself, will shortly ensue. When dogs are suspected of having taken such poison, the following effectual remedy should be instantly applied:-Put as much common salt into the dog's mouth as can be got down, hold the head upwards, and force open the mouth, and by fixing a stick across, prevent the mouth from shutting, whilst the throat is filled with salt; a sufficient quantity, both to prove an emetic and a laxative, will soon dissolve with the saliva, and be swallowed. Warm broth should frequently be given, to prevent faintness, which might, without nourishment, prove fatal. Two tablespoonfuls of castor oil would accelerate its action downwards. When the effects of the salt, etc., have ceased, give the dog fifteen drops of laudanum.

\section{BLINDNESS.}

During the time that dogs are afflicted with the distemper, they are sometimes subject to diseases of the eyes; the most frequent of which is an abscess in the transparent cornea, which, on its first appearance, is of a blue tinge; in some instances there is a darkness of the part affected, in the middle of which a speck is visible, which gradually assumes the form of an abscess. This suppurates, leaving an ulcer, which not unfrequently extends over the entire pupil, often entirely preventing the animal from distinguishing objects, and having such an appearance, that no hope of recovery can be entertained. But, however intense the affection may be, whenever the distemper ceases, the ulcer dries up, and the animal gradually acquires its wonted vision. Let the 
eye be washed with the following, twice or thrice

a day :--

$$
\begin{aligned}
& \text { White vitriol - . . } 8 \text { grains, } \\
& \text { Burnt alum, powdered . . Io grains, } \\
& \text { Litharge . . . . I drachm, } \\
& \text { Distilled water . . . } 3 \text { ounces. }
\end{aligned}
$$

The same wash may be used for other complaints of the eye. 


\section{CHAPTER II.}

Diseases of the Abdomen, etc.

\section{INFLAMMATION OF THE BOWELS.}

Sympтомs.-This complaint is indicated by extreme restlessness in the dog, getting up and lying down, frequently changing places, and groaning. Sometimes he will lose the use of his hinder extremities; and if his back, over the loins, be touched, he will exhibit indications of suffering; if he is moved from one place to another, he will utter loud screams. Costiveness frequently accompanies this disease.

REMEDIES.-Bathing the animal in a tub of warm water frequently has a salutary effect. $\mathrm{He}$ should be kept in the water for a considerable time, and well rubbed while he is in it. When taken out let him be wiped quite dry, and rolled up in a warm blanket, and kept near a fire, if the weather be cold. Spring is the time dogs are most liable to attacks of this kind.

If the animal does not exhibit signs of being better from this treatment, let him have the following mixture, which is for a dog of middle size :-

$\begin{array}{lllll}\text { Laudanum } & \cdot & \cdot & \cdot & 20 \text { drops, } \\ \text { Sulphuric } x \text { ther } & \cdot & \cdot & \cdot & 25 \text { drops, } \\ \text { Castor oil } & \text {. } & \text {. } & \text {. } & \text { I ounce. }\end{array}$

This may be facilitated in its operation by the use 
of a clyster of gruel, butter, and salt. If the above fails to move the bowels in six or seven hours, then the following must be given :-

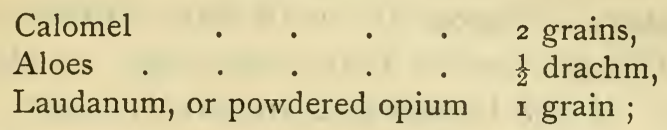

to be made into a ball with treacle and linseed meal, or simply with butter. Should it not operate in six hours, a like quantity may be given; and when the bowels have been moved, they must be kept open for two or three days.

Should the hinder parts still continue semiparalysed, the following stimulant should be used for rubbing them, which may be done three or four times daily :-

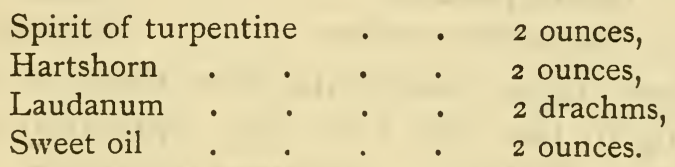

It is not likely that the animal will suddenly recover the use of the limbs; it will, therefore, be necessary to persevere in the use of the warm bath every day or two. The animal must, in the meantime, be sparingly fed with thin oatmeal-porridge and milk or other farinaceous food.

Sometimes costiveness is mistaken for the animal's bowels being in a lax state. This is when the animal passes its fæces in small balls, accompanied by a quantity of mucous matter; but this is a sure sign of the bowels being really bound up.

When inflammation has its origin in cold, accompanied by panting, heat, and thirst, and the dog is very restless, and the stomach will not retain the food, accompanied by heat in the belly, redness of the eyeballs, and there is an alternation of hotness and cold 
ness of the nose, and the dog groans, it may be inferred that the inflammation is considerable; bleeding must, therefore, be had recourse to immediately, and clysters of castor oil, with from fifteen to twenty drops of laudanum in them, according to the size of the dog. If the bowels become costive, they must be kept open with the following purgative, until the dog recovers.

$$
\begin{aligned}
& \text { Jalap } \\
& \text { Aloes, powdered } \quad \cdot \quad \cdot \quad \cdot \quad \cdot \quad 5 \text { grains, }
\end{aligned}
$$

If the evacuations are accompanied with blood, and the bowels in a naturally loose condition, laxatives

\begin{tabular}{|c|}
\hline $\begin{array}{l}\text { Powdered colombo } \\
\text { Prepared chalk } \\
\text { Opium, powdered } \\
\text { Gum-arabic, powdered }\end{array}$ \\
\hline
\end{tabular}
must not be had recourse to, but the following used :-

The above to be made into from three to six balls, according to the size of the dog, and given every six hours, until the bloody symptoms disappear.

\section{INFLAMMATION OF THE BLADDER.}

Symptoms. - Panting and extreme restlessness, heat in the belly, with swelling and tenderness, are the indications of this complaint, accompanied by the urine being voided frequently, and in small quantities, and in some cases a total suppression of it ; at other times, it is evacuated by drops, and mixed with blood.

REMEDIES. - As the bowels are generally constipated, the following opening medicine may be given :-
Calomel
2 grains,
Extract of colocynth
2 grains.

In his drink should be given, twice or thrice a day,

Nitre . . . . I drachm,

Powdered gum-arabic . . 2 drachms. 
If the animal exhibits pain when he voids his urine, then it will be necessary to give half-a-grain of powdered opium, put in a little meal and butter, and forced down his throat. Fomentations applied over the region of the bladder will have a good effect in alleviating pain. The food should be bread or biscuits soaked in gravy, and unimited supply of milk and water to drink.

WORMS-INTESTINAL PARASITES.

Of all the domesticated animals the dog is most frequently affected with intestinal parasites.

Worms are generally classified into tape and cystic worms, sucking worms, and round worms. Time will only permit us to mention a few of the more important of these creatures, which may be found in the intestines of the dog.

TAPE-WORMS.

So called because they are broad and expanded like various sizes of tape.

(I) Tania Serrata.-This tape in its cystic form is the Cysticercus Pisiformis, and is found in the rabbit.

(2) Tania Conurus.-Cystic form is tlie Cœnurus Cerebralis, found in the brain of sheep and cattle.

(3) Tania Echinococcus. - In its cystic form it is found in various animals, and in man.

(4) Tania Cucumerina.-Cystic form, also found in the rabbit.

(5) Tania Marginata.-Cystic form Cysticercus Tennicollis in the sheep, pig, etc. 
ROUND-WORMS.

(I) Ascaris Marginata. This perfectly developed worm is found in the small intestines, and sometimes in considerable numbers.

(2) Spiroptera Sanguinolenta. Found in tumours of the stomach and œesophagus.

(3) Trichosoma Plica. Found in the bladder.

(4) Filaria Immitis. Found in the heart.

(5) Eustrongylus Gigas. Found in the kidneys, bladder, and under the peritoneum.

SUCKING-WORMS.

(I) Holostomum Alatum. Found in the intestines.

(2) Pentastomum Tanioides. Found in the frontal and ethmoidal sinuses.

Symp'oms.-The dog, under the influence of worms, becomes thin and even emaciated; is dull and stupid; his eyes are heavy, and his nose swelled and dry. He continually rubs his nose with his paws. In the earlier stages of the disease he eats voraciously, but afterwards loathes his food. He has an inclination to move his tongue about in his mouth, and has a tendency to hang his under jaw. He is often afflicted with severe pains in the abdomen, which cause him to scream violently; during sleep he has spasmodic twitchings, and frequently draws all his limbs together : the belly becomes tumid and hard, and saliva runs from his mouth while asleep. Another symptom is sitting down on his hind-quarters, and in this position dragging himself forward with his fore-paws, while his hind ones continue in a sitting posture; and it often happens that his hair stands on end, which is termed staring by sportsmen.

If a dog has had the distemper and is seized with 
vertigo or giddiness, there is strong reason to suspect that worms are the cause, and that they have either become very numerous, or have taken their lodgment in the upper region of the alimentary canal, or in the stomach. In the latter case, the disease is generally attended with sickness, and even violent vomiting. Worms also produce convulsions and death, from the extreme irritation of the stomach and bowels. If the freces passed by the dog are examined carefully, a portion of the worm may be seen.

REMEDIES.-If the dog is infested with tape-worms allow it to fast for twelve hours, and on the empty stomach administer :-

Oil of the male shield-fern . . Io to 20 minims, Spirits of turpentine . . . 10 to 20 minims, Spirits of nitric ether . . . 20 to 60 minims.

This may be repeated, but care should be exercised as to its frequency. A dose of castor oil following a single administration of the above medicine frequently effects an expulsion of tape-worm.

For round-worms, a combination of oil of the male shield-fern and powdered areca-nut, or santonin, repeated twice a week, and followed by a purgative, may be prescribed with advantage.

Turpentine is apt to produce an irritation in the neck of the bladder; and when the animal urinates, if a painful straining is noticed, or the urine is voided frequently, and in small quantities, it will be necessary to give linseed-tea, boiled up with a little butchers' meat, to form a kind of soup, and let the animal drink plentifully of this. When all traces of parts of these worms disappear, it will be necessary to give tonic medicines, so as to strengthen the digestive organs, which will have the effect of preventing more effectually 
the recurrence of these worms. The following will be found beneficial :-

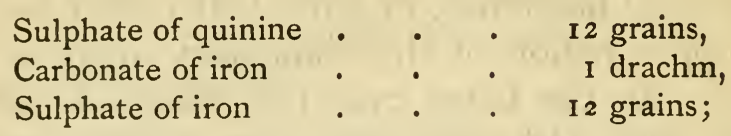

to be divided into from six to twelve powders, according to the size of the animal, and one given daily, for a week or ten days, mixed with linseed meal and treacle, in the form of small balls.

Castor oil and turpentine will be found a valuable remedy for all worm complaints.

\section{DROPSY.}

This disease consists of an accumulation of water in the abdomen, and is not uncommon in dogs, and often proves fatal. It generally has its origin in some disease of the chest or kidneys. It sometimes manifests itself by a strong, hard cough; the belly becomes swelled and tense, and a want of appetite follows, with quick and difficult breathing, accompanied by great thirst, and in the more advanced stages, a considerable tendency to suffocation. A good way of testing the existence of dropsy, is to place one hand on the near side of the body, and strike the off side with the other hand, when an undulating motion will be perceived. The following will be found beneficial :-

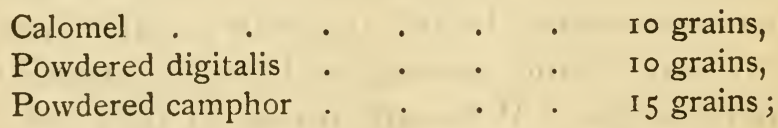

to be divided into six powders, and one to be given daily; but if for a small dog, it must be made into eight powders. Should the camphor lead to the 
animal refusing his food, it should be discontinued at once.

In extreme cases tapping will afford relief, and may be performed several times, but ought not to be attempted by any one but an experienced veterinarian.

\section{COSTIVENESS.}

Dogs are very liable to costiveness, and the freces which they void is sometimes extremely hard, and not unfrequently of a limy consistence.

REMEDIES.-Occasional doses of castor oil, varying from halt-an-ounce to two ounces, will be found the most effectual remedy for this ; and its operation will be facilitated by giving the animal frequent draughts of warm oatmeal gruel or weak broth.

\section{COLIC.}

Symptoms.- The animals labouring under this complaint are dull, restless, with inflamed eyeballs, dry and warm nose, loss of appetite; and the dog suffers considerable pain.

Remedies.-The application of stimulating embrocations applied to the belly are useful; and the warm bath, brought to a considerably high temperature, often affords relief. Half-an-ounce of castor oil should be given immediately, and six drops of laudanum. This is given when the habit is costive; but when the bowels are lax, give two tablespoonfuls of the following mixture every four hours:-

Compound tincture of senna . . 3 drachms,

Sweet spirit of nitre . . $\frac{1}{2}$ ounce,

Compound tincture of rhubarb . 3 drachms,

Laudanum

2 drachms,

Water

I pint. 


\section{DIARRHCEA, OR LOOSENESS.}

During distemper, this is a common malady; and when it happens with this complaint every means must be adopted to check its progress, as it is apt to produce great weakness, which the animal is ill able to bear, as want of appetite is too common an attendant upon distemper; and when this is the case there is a great difference in the colour of the excrement, it being sometimes very dark, at others yellow, and sometimes of an albuminous character.

The acrimonious state of the fæces produces inflammation of the anus, and a constant desire to stool, which some persons erroneously take for constipation; and under that idea give the animal cathartic medicines, which have an injurious tendency, the bowels being already too much relaxed, and not unfrequently cause the death of the animal.

REMEdiEs.-When dogs are labouring under this complaint give the following twice or thrice daily, if the complaint is at all severe ; but when not so, once a day will suffice.

$\begin{array}{lll}\text { Peruvian bark } & \text {. } & \text { I scruple, } \\ \text { Powdered ginger } & \cdot & \text { I drachm, } \\ \text { Powdered camphor } & \text {. } & 4 \text { grains, } \\ \text { Laudanum . } & \text {. } & \text { I } 2 \text { drops. }\end{array}$

Rhubarb and magnesia have an excellent effect in absorbing and carrying downwards the acrid irritating state of the bowels.

\section{BLEND WATER, OR BLOODY URINE.}

Dogs are frequently afflicted with this malady, which has its origin in a diseased condition of the neck of the bladder, or a rupture in the vessels of the urethra. It is also caused by fungiform growths on the penis, in 
which case the blood issues in almost single drops at a time. The irritation during the course of urinating causes the animal to strain, which presses on the fungi, and causes blood to issue from them.

Remedies. - When the malady proceeds from any of the two former causes, the following should be administered every day until the animal recovers :-

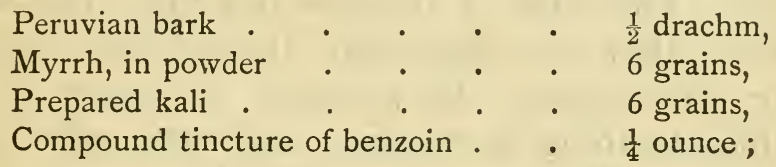

the whole to be formed into a ball.

If the complaint arises from fungi, the best plan is to have recourse to excision of them with a scalpel or sharp knife; or they may be reduced by means of caustic applications. Burnt alum may be first tried, and if it does not prove effectual, about an eighth of the quantity of red oxide of mercury should be mixed with it.

\section{PILES.}

CAUSE.-Confinement, heat, and dry, heating food, as well as frequent costiveness, produce piles.

Sympтoms.-The anus is generally protruded, and red, which is frequently much aggravated by the dog dragging his hinder parts on the ground, from the itching and sharp pain of the parts. When they are habitual, the best appliance is the following ointment :-

$$
\begin{aligned}
& \text { Sugar of lead . . . . . } 6 \text { grains, } \\
& \text { Tar . . . . . . } \frac{1}{2} \mathrm{drachm} \text {, } \\
& \text { Elder ointment, or hog's lard . . } 3 \text { drachms ; }
\end{aligned}
$$

the part to be anointed with it three or four times a day. 


\section{FITS.}

Dogs are very liable to be afflicted with fits of several kinds, and arising from a variety of causes.

Epileptic fits.-As in the human being, these are accompanied by severe convulsions, and they occur at all ages. The cause of these is not well understood. However, they are frequently the followers of distemper, costiveness, the presence of worms in the intestines, teething in puppies, and some affection of the brain. These are most difficult to cure, if not incurable in many instances. Bleeding often has the effect of removing fits, but aperients should always be administered ; and the following will be found of much use :-

$\begin{array}{llll}\text { Calomel } & \cdot & \cdot & \text { I } 2 \text { grains, } \\ \text { Powdered foxglove } & \cdot & \cdot & \text {. } 12 \text { grains, } \\ \text { Powdered miseltoe } & \cdot & \cdot & \text {. } 2 \text { drachms }\end{array}$

to be divided into nine, twelve, or fifteen powders, according to the size of the dog, and one given every morning. Let them be made up in the form of pills, with treacle and a little linseed meal, and put down the animal's throat.

The natural ardour of dogs, more particularly those used in field-sports, frequently causes them to have fits, from the great excitement produced by the sight or smell of game; more especially at the commencement of the season; and particularly in such dogs as have not been sufficiently exercised. When they are attacked with epileptic fits in the field, the best and easiest remedy is to plunge them into water immediately, which will have the effect of rousing them. Setters are more liable to these attacks than pointers, from their more ardent temperament; and 
especially if they have been subjected to severe chastisement for flushing game, which they are very apt to do at the commencement of the shooting season. Costiveness also may produce fits, and when this is the cause, we need hardly say that active purgatives are the best and speediest remedies.

It is very imprudent to allow bitches to suckle many puppies at a time, as they are frequently attacked with fits in consequence. Two or three at most are sufficient for an animal to rear. Puppies are liable to fits from the effects of teething. Worms also produce fits in them; and they are sometimes afflicted with convulsions when the distemper is approaching. 


\section{CHAPTER III.}

\section{Diseases of the External Parts, etc.}

\section{DISEASES OF THE EYE.}

Young dogs which are recovering from the distemper are liable to be afflicted with sore eyes; but this is only part of the disease, and he generally recovers when the disease is fairly eradicated. But should the eyes continue sore afterwards, then a seton in the neck, and washes with Goulard's extract, will facilitate the recovery.

Weak and watery eyes are not of uncommon occurrence with dogs, which when carefully examined in a strong light, will be found to be red at the bottom, as well as on the inside of the eyelids. When the animals labouring under this complaint are exposed to a strong light, they exhibit painful symptoms, as well as a great desire to be freed from its glare.

The following wash should be used :-

$\begin{array}{llllll}\text { Sugar of lead . } & \cdot & \cdot & \cdot & \cdot & \frac{1}{2} \text { drachm, } \\ \text { Rose-water } & \cdot & \cdot & \text {. } & \text { 10 ounces. }\end{array}$

After the inflammation has somewhat subsided, let the following be applied:-

$$
\begin{aligned}
& \text { White vitriol . . . Io grains, } \\
& \text { Rose-water . . . . ro ounces. }
\end{aligned}
$$

The above may also be used for other eye complaints, 
as well as for injuries inflicted from blows, scratches, or punctures.

Cataract.-This is a disease of the crystalline lens of the eye, which becomes white and opaque, and generally of a hard, horny consistence, instead of being clear and transparent, as in its natural state. When complete, this disease causes total blindness. It may be brought on by an injury, and generally only affects the injured eye. Aged dogs are very subject to the complaint.

The only remedy for this malady is an operation which is termed couching; which should only be attempted by an experienced veterinary surgeon. The operation is performed in two different ways. In the first of these, a needle is introduced, and the covering of the lens torn, and its contents depressed into the posterior chamber of the eye, where it is gradually absorbed by the vessels of the eye, called the absorbents. The second is called extraction, by which the lens is entirely taken out, and a small incision made with a knife in front of the eye. The latter mode is most perfect, and when neatly performed, is done without much hazard to the eye.

Washes are recommended for this complaint, but they are very unlikely to produce a cure, although they may keep down inflammation.

\section{EXTERNAL ULCER OF THE EAR.}

This disease has by some been mistaken for canker, but it is very dissimilar. This complaint is known by a foul ulcer, which is situated on the lower edge of the flap, or one on both ears, in the form of a slit. The pain of this sore induces the dog almost continually to shake his head, which irritates the sore, and increases the discharge of matter. 
The most frequent remedy is burning out the sore, by means of a cauteriser, or with some caustic substances. But the following is more effectual :-

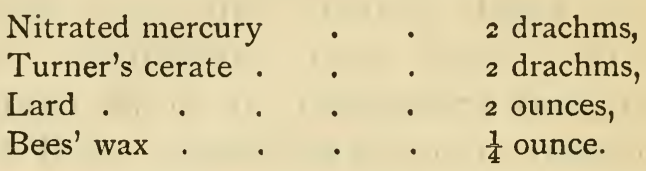

It must be well mixed together, and applied twice a day. The part must be covered with a cloth, well secured, otherwise the dog will remove all the ointment by scratching.

The following has also been found effectual :-

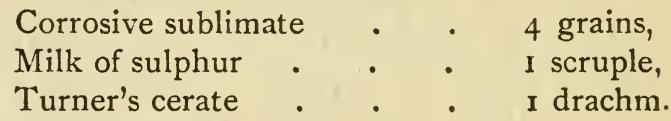

The following wash may be applied when the case is not severe, and will generally prove effectual :-

$\begin{array}{llll}\text { Corrosive sublimate } & \text {. } & \text { - } & 6 \text { grains, } \\ \text { Rose-water } & \text {. } & \text {. } & 5 \text { ounces. }\end{array}$

CANKER IN THE EAR.

This consists of an ulcerating sore, which spreads widely if not stopped in time.

The treatment consists in syringing the affected ear or ears out, thereby removing all wax, etc., and thereafter injecting a mild astringent lotion. If this does not affect a cure the animal had better be handed over to the veterinary surgeon for further treatment.

THE COMMON MANGE.

This is a common disease among dogs, and is attributed to filth, want of proper exercise, and foul feeding. It is a chronic inflammation of the skin, 
sometimes the effect of a morbid constitutional action, and in others dependent upon a parasite.

Some authors affirm that there are four distinct kinds of mange, but we know that there are two which can easily be distinguished, namely, the common, and red-mange. The latter is more difficult to cure than the former. We shall first point out that for the more prevalent form of the disease.

Remedies.-Cleanliness is the first remedy; and, whenever it is perceived that the dog is attacked by the complaint, which will be indicated by his constantly scratching himself, recourse should be immediately had to the following mixture :-

$\begin{array}{llll}\text { Train oil } & \cdot & \text { I pint, } \\ \text { Spirit of turpentine } & \cdot & \cdot & \frac{1}{2} \text { pint, } \\ \text { Powdered sulphur } & \cdot & \cdot & \frac{1}{4} \text { pound. }\end{array}$

The following is another remedy :-

First let the dog be well washed with lime-water, and when perfectly dry, anoint the parts affected with the following ointment, which is said to be effectual in both the common and red-mange:-

$\begin{array}{llll}\text { Sulphur vivum } & \cdot & \cdot & 4 \text { ounces, } \\ \text { Hellebore powder } & \text {. } & \cdot & 2 \text { ounces, } \\ \text { Bayberry powder } & \text {. } & \text {. } & 2 \text { ounces, } \\ \text { Spirit of turpentine } & \cdot & \cdot & \text { I ounce, } \\ \text { Hogs' lard . } & \text {. } & \text {. } & \frac{1}{2} \text { pound. }\end{array}$

The washing and anointing must be repeated every second day until a cure is effected. But to remove the ointment effectually, it will be necessary to use a strong solution of soda, which of itself will have a powerful tendency to remove the complaint. Unless the state of the dog's system is bad, three or four applications generally have the effect of performing a 
cure. The bowels of the animal should be kept gently open with the following alterative, viz:-

Nitre · • . . $\frac{1}{2}$ drachm,

Sulphur . . . I $\mathrm{I}$ drachm.

$\mathrm{Mr}$ Mackenzie, of Edinburgh, recommends the dog to have a powerful laxative every second day, and to be rubbed all over with soft soap, and allowed to remain untouched, and then to be well washed with a scrubbing-brush and hot water; this two or three times repeated will generally effect a cure, unless the disease is of a bad kind. If this fails, an application of common flour of sulphur, in the proportion of one ounce to two ounces of hogs' lard, four drachms of carbolic acid, applied three or four times, will generally prevail.

We have, however, in obstinate cases, found that they resisted all the above remedies, which rendered it necessary to have recourse to mercurial preparations, which it is always desirable to avoid, if possible. In such cases the animal must be kept very warm.

\begin{tabular}{|c|c|}
\hline White precipitate & 2 drachms, \\
\hline Sulphur & I $\frac{1}{2}$ ounce, \\
\hline Hogs' lard. & 4 ounces; \\
\hline
\end{tabular}

to be rubbed with the above for three or four times, every second day, first washing the dog with a strong solution of soda, after the ointment has been applied for twelve or fourteen hours.

The following ointment has also been successfully used for mange :-

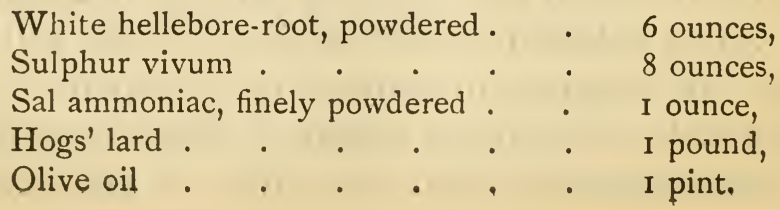


Rub the parts affected with this, night and morning, for six or seven days, and the following purgative ball should be given, and repeated twice after it has operated, allowing an interval of three days:-

\begin{tabular}{|c|c|c|}
\hline Powdered jalap & & 20 grains, \\
\hline Calomel & & 3 grains, \\
\hline Ginger, powdered & & 3 grains, \\
\hline Syrup of buckthorn & & I drachm \\
\hline
\end{tabular}

to be made into a ball, and concealed in a small bit of butter. The above doses are for a fox-hound, greyhound, setter, and pointer. For larger dogs the quantity to be increased, and for smaller ones diminished.

\section{THE RED-MANGE.}

As already stated, this is a more obstinate disease than the common. It is known by a red inflammatory state of the skin over the whole body, and is accompanied by great itching, and the skin feels hot to the touch. It affects the roots of the hair, so that it falls off, frequently in large patches, in some instances leaving the body almost naked. The hair frequently becomes discoloured. In obstinate cases, ulceration often takes place; and when this is observed, after washing with soft soap, or a very strong solution of soda, as recommended in the common complaint, the scabs should be picked off, and the fresh sore rubbed with the following solution:-

Corrosive sublimate . . $\frac{1}{4}$ ounce,

Spirit of wine, or strong whisky $\frac{1}{2}$ pint ;

which should be applied with a small piece of sponge.

The ordinary remedy is the following:-

Strong mercurial ointment . . 4 ounces,

Venice turpentine . . . 2 ounces,

Hogs' lard . . . . . I pound; 
the turpentine and mercurial ointment to be well rubbed together until properly incorporated. An ounce of this to be rubbed on the parts affected daily for three days. It should be well washed off on the fourth day; but if the redness continues on the fifth, another application will be indispensable.

\section{FOUL COATS.}

Sometimes the coats of dogs stare, that is, the hair stands on end, and assumes an irregular disposition, which condition is termed foul by sportsmen. To obviate this the following ointment has been found effectual :-

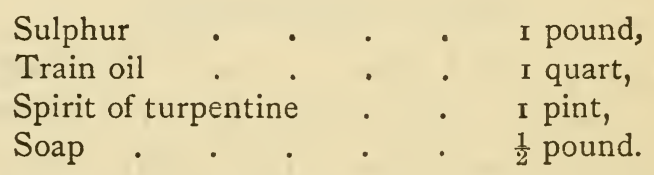

Two or three applications of the above will have the desired effect. It proceeds from an irritability of the skin. Some sportsmen use the above, as a preventive, two or three times during the season, and before the sporting-time particularly.

\section{SORE FEET.}

During the hunting and shooting seasons, dogs are very liable to be affected with sore feet : they should be frequently washed with strong brine, pot-liquor, or salt and vinegar, a handful of the former to a pint of the latter. But as it will be found easier to prevent than cure the affection, we would recommend that the feet of dogs should be washed every day, on returning from the field, with pot-liquor or brine, which will have the effect of hardening the skin.

When the feet have become sore and continue raw, 
let them be well washed with a solution of soda, and then the following mixture applied three or four times a day with a feather :-

Sulphuric acid $\quad \cdot \quad$. 6 drops,
Tincture of myrrh . $\quad$ I ounce.

\section{WOUNDS.}

Dogs are very liable to be wounded when in pursuit of game, or in their ardour in ranging to find it.

When these wounds are of a superficial kind, they will only require to be washed, and afterwards, by the dog constantly licking them, they soon heal. But where they are of a more serious character, other means must be adopted. The mode of cure will depend upon the situation and nature of the wound, and other circumstances.

We would, however, generally recommend that they should be washed with Friar's balsam, or tincture of myrrh. But when they are severe, it would be prudent to have the advice of a veterinary surgeon.

\section{SPRAINS AND BRUISES.}

Like wounds, it would be difficult to recommend a specific which may be generally useful, as these may vary so much in situation and degree; therefore veterinary aid will be necessary; but when that cannot be easily obtained, we would recommend the following applications, to be well rubbed on the parts affected :-

Spirit of turpentine

Spirit of wine
I ounce,

2 ounces.

Or where there is heat and swelling accompanying the 
sprain, the following lotion will be found the best, if diligently applied :-

$$
\begin{aligned}
& \text { Sugar of lead . . . } 2 \text { ounces, } \\
& \text { Vinegar . . . . } \quad \text { r pint; }
\end{aligned}
$$

to be kept on the part with rags well wetted in the lotion; and when the inflammation is completely removed, use the following embrocation:-

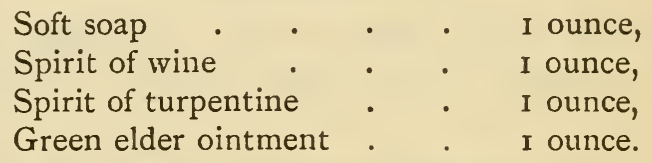

For a strained leg or foot, the following embrocation will be found effectual in removing it :-

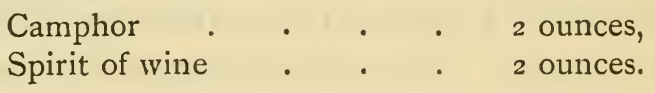

When the camphor is dissolved, add to it Goulard's extract, one ounce, and the gall of an ox, and rub the part affected twice or thrice a day. Fomentations of hot water or camomile flowers will also be of much service. For swelling of the stifle, an ounce of tincture of cantharides added to the above, will be found useful to rub the part with.

\section{SCALDS.}

The ordinary application of lime and oil is the best, to be used immediately after the dog has been scalded, taking care to cover up the part, and secure it in such a way that the dog cannot lick it off. Next day, when the heat has subsided, the animal may be permitted to lick the part, and if the blister be broken, all that will be necessary is to allow nature to work her own cure, which will be facilitated by 
the dog licking the part. When the scalded part has healed, it will be found denuded of hair.

\section{RICKETS.}

This is an affection of the joints of the legs, which it enlarges, and the limbs become crooked, and the animals are in consequence called wrylegged. This complaint is hereditary in some dogs, and those should not be bred from. It is more common in the smaller kinds of dogs, such as terriers, lap-dogs, etc. It attacks them when very young, soon after they begin to walk. Whenever puppies can walk properly, they ought to be exposed as much as possible to the open air, and have plenty of exercise, good, plain food, and be kept clean and dry, which will generally have the effect of preventing rickets.

\section{THORNS.}

During the sporting season, dogs are very liable to get thorns in their feet; and when these cannot be picked out, a poultice of ground linseed or boiled oatmeal may be applied, the surface of which must be rubbed with butter or lard, to keep it clean. This will have the effect of sucking out the thorn. But if it fails to do so on the first application, a second must be applied.

\section{FLEAS AND TICKS, ETC.}

Dogs are very liable to be molested by these insects, which are very troublesome to them. The most effectual remedy is to rub the whole animal all over with train oil, allow it to remain from half-an- 
hour to three-quarters, and then wash it off with salt of tartar, or soda and warm water. Soft soap made into a thick paste and rubbed over the body will have the same effect; it must be allowed to remain on an hour before washing it off. Warm water is all that is required. 


\section{CHAPTER IV.}

\section{Operations.}

\section{BLOOD-LETTING.}

THE best way to perform this operation on the dog is to take the blood from the jugular vein. Tie a ligature pretty tight round the animal's neck, close to the shoulders, and when the vein has risen enough to be seen distinctly, take a common lancet and make an oblique puncture in it, when it will bleed copiously. When a sufficient quantity has been taken away, remove the ligature, and the bleeding will instantly cease; the incision will heal afterwards without any application whatever.

\section{METHOD OF GIVING A BOLUS OR PILL.}

The method of administering a bolus, pill, or other medicine to a dog, is to pull the tongue pretty far out of his mouth, then put the medicine as close to the ront as possible, and when the animal draws in his tongue, the medicine will descend into his stomach.

\section{WORMING.}

Worming dogs has been considered a preventive of hydrophobia since the time of Pliny; but how such an absurd idea should have prevailed for 1760 years, is quite unaccountable. $\operatorname{Dr}$ Blain very 
properly says, it is a custom founded on ignorance, and should not be perpetuated by publishing written directions how to perform the operation. Concurring, as we do, in the opinion of Dr Blain, we abstain from any description of the operation.

\section{FRACTURES.}

Fracture of the thigh-bone is extremely common, but is by no means so difficult of cure as might be supposed. If the flesh has been injured by the fracture, the first thing to be attended to is to get quit of the swelling and inflammation, which must be accomplished by means of fomentations of vinegar and water, until allayed. Then apply a long piece of wood, in the form of a lath, which must reach from the foot to a little way above the fracture. But before applying this, a pitch-plaster sufficiently large to surround the thigh must be applied, and then the slip of wood firmly bound over it by means of a roller of cotton cloth, an inch and a half broad. But care must be taken not to make it too tight, otherwise the circulation may be retarded, or entirely stopped, which will in all probability terminate in mortification.

If the leg-bone is broken, then several splints of wood must surround the limb, carefully wrapped round with a bandage.

When fractures of the limbs are comminuted, that is, broken in two or more places, then the cure becomes more difficult and uncertain in its issue, as it is difficult for an inexperienced person to place the pieces of broken bone in their proper position. The safest plan is to have compound fractures set and attended to by veterinary surgeons. 
CROPPING.

This is at least a barbarous custom, and extremely presumptuous in man to attempt to improve nature. We believe this outrageous practice originated in the barbarous ages, when dog-fighting formed an amusement amongst the fashionable. Happily this is now of rare occurrence; and we hope the time is not far distant when this, as well as the brutal practice of cock-fighting, will be prevented by an act of the legislature. 


\section{CHAPTER V.}

Gestation, Parturition, etc.

\section{OF BREEDING GENERALLY.}

From the earliest times great diversity of opinion has existed respecting the proper age at which dogs should be allowed to breed, with the view of producing strong and healthy puppies.

We are informed by Aristotle, who flourished in the fourth century before the Christian era, that ancient sportsmen, for the ennobling of their race of dogs, did not suffer the male to engender till he was four years of age, and the female three; and that the former was only allowed to propagate for eight years thereafter, and the latter for six years. They conceived that the progeny of such would be much stronger than those bred at an earlier age. This opinion does not, however, hold good, although it is found that the puppies produced by a female under twelve months, are generally weak. It is now ascertained, from patient investigation and experience, that a female should be fully twelve months old before she is allowed to engender, and that whelps produced by a dam about this age, are as good as those at any later period of the animal's life. The male ought to be at his full strength and symmetry, but not aged, as the offspring of such are generally dull and deficient in spirit.

The usual time at which females are first seized 
with the furor uterinus, is at the age of twelve months; but there are many instances where it is felt at eight, and it generally lasts about eighteen days. For the first four or five days she will not receive the embraces of the male, but keeps up a sportive dalliance with him; after which unbounded vent is given to her desires, and even with a variety of males; from the termination of which period, she is gravid nine weeks or sixty-three days, and produces from five to twelve puppies, according to her size.

Breeders are in the habit of restraining the desire of the female; and in general only allow her to be warded two or three times, which proves as effectual as the most unrestrained commerce. Indeed, it has been found, that from six to eight puppies have been the fruits of one coitus, and, in some rare instances, so many as eleven.

Albertus mentions that he saw a mastiff bitch, which brought forth at three litters fifty whelps, viz.: nineteen at the first, eighteen at the second, and thirteen at the third litter. Some females have been known to produce puppies three times a year; but this generally has the effect of destroying their constitution. Those, therefore, who have a regard for their dogs, and wish to preserve a healthy race, will never allow the female to produce oftener than once a year.

It need hardly be remarked, that if it is wished to preserve a breed of dogs uncontaminated, all intercourse with those of different races must be strictly guarded against. The animals from which a breed is to be taken should be as perfect in their form as possible, as it would be in vain to expect handsome puppies from an ill-made sire and dam; although it not unfrequently happens, that in the case of one of the parents being cross in its make, an elegant progeny 
are produced, by the other possessing a more perfect symmetry, more especially when the female is beautifully formed. But this ought never to be depended upon, unless the sire or dam have very pre-eminent qualities, which it is desirable to possess. Crosses should be carefully guarded against.

\section{"Consider well}

His lineage ; what his father did of old, Chiefs of the pack, and first to climb the rock, Or plunge into the deep."

SOMERVILLE.

In dogs of the chase, care should be taken that the dog is stout, his shape good, and colour of the right kind, his nose fine, and that he has a proper method of hunting. Be sure that he is no babbler or skirter. The former is the worst fault a hound can have, and is apt to be followed by others. Those that skeit are always unsteady and changing, and lose more foxes than they kill. If the dogs are otherwise good, this imperfection may be rectified by a more steady parent of the opposite sex.

Breeding from the same stock is to be strictly avoided, as it is found that all animals (and even man himself) soon degenerate by too close a union in blood. If, therefore, a perfect race is wished, every possible attention should be paid to obtain alliances betwixt the sexes by animals not connected by consanguinity, or at least, not very near.

The time of producing in most animals lies with the female. In the dog species the spring is the usual season of desire, commencing generally in February or March; and this is certainly the best time of the year; for puppies whelped in summer are always stronger and more likely to be straight and firm about the joints than those of a winter litter, 
which often suffer materially from the cold, and become rickety.

Thus far as regards the breeding of dogs generally; but in sporting dogs there are various opinions as to the time at which they should be bred. The strictest attention, however, should be observed in regard of both male and female being in good health, and as perfect as possible in their points of form ; their faculty of smell should also be in high perfection, although it sometimes happens that cross, ill-made dogs possess excellent qualities in this respect; and when this is the case, and a breed is desired from them, a good male or female of the opposite sex should be selected, as the case may be.

Much vigilance is necessary on the part of the feeder, to watch the females going to heat, and whenever the slightest indication of this becomes apparent, they should immediately be separated from the rest of the pack, if dogs of chase, which will prevent quarrelling among the males. This should also be attended to with other sporting dogs. From neglect of this kind, it has not unfrequently happened that the best dogs of the pack, or the finest pointers and setters, have fallen victims to these broils.

While in pup the female should not be hunted more than the first month, as it often proves injurious to her as well as her litter. During gestation, however, moderate exercise will be found of great service in preserving her health, and is in consequence likely to give strength to her offspring.

$\mathrm{Mr}$ Daniel is of opinion that winter whelps, if they survive, come in well the following season, and that they are generally hardy and handsome; and he recommends to have no puppies later than April, as they seldom thrive in very hot weather; and that of the 
early puppies, five or six should be kept, and of the later ones, not more than half that number. We, however, beg to dissent from this conclusion, for the reasons stated in a former paragraph.

When the female has littered, and the puppies have been cleaned by her tongue, it will be proper to select such as are to be kept, while those that are deemed superfluous should be immediately drowned. In the chase a preference should be given to those having a resemblance to dogs of the pack of established worth, and possessing at the same time the strongest make, as the smaller puppies are likely to turn out weak. If a whole litter is wished to be preserved, and if it is larger than can be nursed with ease and safety by the dam, a few should be taken from her and given to a foster mother. Sometimes, however, it is difficult to get another bitch to nurse strange puppies. A method, which has been successfully practised, is to rub the puppies so selected with a little of the fostermother's milk, when, in general, she will carefully lick them and adopt them as her own.

While nursing, the bitches should be well fed with flesh, broth, milk, porridge, etc., several times a day. When puppies are a few weeks old, milk should be offered them, and they will soon learn to lap it, which will greatly rellieve the dams. By the end of six weeks they will be able to feed themselves, and may then be removed from the nursing quarters. These observations apply to dogs generally.

Many of the most experienced sportsmen, and also writers on this subject, conceived that hounds may be hunted while nursing; but this is an opinion with which no physiologist or medical man can concur; for violent exercise of any kind has a strong tendency 
to injure the quality of the milk, and must, consequently, have a bad effect on the young progeny.

When the puppies of dogs of the chase are three or four days old, the points of their tails should be twisted off. This operation is performed by placing the tail between the fore-finger and thumb; press the nail of the latter on the point, and twist the tip of the tail gently round, and a slight pull will separate it (but this operation should never be performed on a setter, springer, or cocker); after which the dew claws must be taken off with scissors.

It is necessary to give physic to females when their young ones are removed from them. Three of the following laxative balls, given every alternate day, will have the desired effect :-

Jalap, in powders Calomel
20 grains,

4 grains ;

to be made into a small ball or pill with treacle. But during the process of drying up the milk, as little liquid as possible must be given to her, which should consist entirely of water. Whey is recommended by some veterinarians, as being a less abrupt transition from their former diet, and containing but little nutriment.

In the event of this not proving effectual, the laxative balls must be continued, and the teats of the animal rubbed twice a day with a composition of goose grease and rum, in equal proportions ; or brandy and salid oil, used in the same way, will have a similar effect. This treatment, continued for three or four days, generally answers the purpose. But if, in spite of strict attention to the above appliances for dispersing the milk, it accumulates in large quantity, which will sometimes be the case, it will be necessary to draw off 
some of the milk by means of a nipple-glass or suckingpipe, to relieve the animal from the pain of her udder being distended. A mixture of warm vinegar and brandy may be also successfully applied to dry up the milk.

In breeding pointers and setters, some sportsmen are anxious to have the puppies produced at such a time as to become fit for breaking in the succeeding spring, so as to be ready for hunting in the autumn ; and also that the bitch may not be in case, so as to prevent her from running along with the males, or to be so heavy with young that she cannot work by herself during the last part of the sporting season. This may certainly be a saving knowledge, but the opportunity of saving a breed of a good kind ought not to be sacrificed for so paltry an object.

\section{PARTURITION, OR PUPPING.}

It is no uncommon occurrence for bitches to lose their lives in pupping. Protracted labour will cause the death of the young in the womb, in which case they frequently are not discharged at the time, but come away piecemeal some days afterwards. Sometimes fits are brought on by tedious labours, in which case give the following specific :-

$\begin{array}{llll}\text { Ether } & \cdot & . & \text { I drachm, } \\ \text { Laudanum . } & \cdot & \text {. } & \text { I drachm, } \\ \text { Strong ale . } & . & \text {. } & 2 \text { ounces. }\end{array}$




\section{CHAPTER VI.}

FEEDING, MANAGEMENT, TRAINING, ETC.

The natural food of the dog is flesh, and it is found that those in a wild state prefer it to any other kind of nutriment. It is this desire that gives to him the instinctive property of pursuing other animals; and without this craving of nature he never would hunt. Many have been of opinion that to feed a dog on flesh destroys the acuteness of his olfactory sense. This doctrine we most positively deny, and that, too, upon the most common principles of physiology; for it is difficult to conceive how any animal should be formed with a natural desire for a particular sort of food, the use of which would prove destructive to some of his faculties.

Although, however, the dog is strictly a carnivorous animal, yet he can subsist on many kinds of food; which is the case, also, with various other animals more highly carnivorous in their nature. It has been said of man himself, that feeding on flesh destroys his sense of smell in some degree; and in support of this statement it is alleged, that certain natives of India who feed entirely on grain, have the olfactory sense in such a degree of perfection, that they can distinguish the smell of the water of one spring from that of another. But such accuracy of discrimination has been satisfactorily ascertained to be entirely the result of practice. 
The nutriment best adapted for sporting dogs, so as to enable them to perform their work well, should consist of at least two-thirds of flesh, with a judicious mixture of farinaceous vegetables. It is an established fact, that dogs in a domesticated state invariably become lean if fed entirely upon flesh.

Good water is of great consequence to the health of dogs, as they drink frequently and copiously, and particularly setters; but the idea that dogs being kept long without water produces canine madness, is a vulgar prejudice.

The dog is naturally a voracious animal; and yet he can endure hunger for a very great length of time, and be brought by habit to subsist on a very scanty meal. In the Memoirs of the Academy of Sciences, it is mentioned, that a bitch which was forgot in a country house, where she had access to no other nourishment, lived forty days on the wool of an old mattress, which she had torn to pieces.

An extraordinary instance of a similar kind occurred with a terrier bitch belonging to a friend of my own. One day, when following her master through a grass park near Gilmerton, it happened that she started a hare. During the pursuit her master suddenly lost sight of her, and in a few days she was considered either killed or lost. Six weeks afterwards, a person happening to look down the shaft of an old coal-pit, was surprised by hearing a dog howling. He immediately returned to the village, and having procured a hand-basket, let it down by a rope into the shaft; the dog instantly leapt into it, and on being brought to the surface, it turned out to be Gipsy, the lost terrier bitch of my friend, worn to perfect skin and bone. How she had existed in this subterranean abode it is impossible to tell. 
Staghounds, foxhounds, harriers, and beagles, are generally fed on oatmeal; and the older it is the better, so that it is not fusty. Store sufficient for twelve or eighteen months' consumption ought, therefore, always to be kept by those who have a pack. The meal should be well dried and broken into grits, but not too fine. It is best kept in bins in a granary, well trodden down. Some persons are in the habit of using barleymeal, but it is not nearly so nutritious as the former. Others are of opinion that oatmeal and barleymeal, in equal proportions, form a preferable food. But nothing is better than oatmealporridge, with the addition of a little milk, and occasionally the kitchen-offal, such as remnants of butchers' meat, broth, and soups, the raspings and refuse of bakers' shops, or hard, coarse, sea-biscuit, well soaked and boiled with bullocks' liver or horse-flesh. Wellboiled greens are an excellent addition to the food of all dogs, and may be given twice a week; but this ought to be discontinued during the shooting season with pointers, setters, cockers, and greyhounds ; and also during the hunting-season with foxhounds, harriers, and beagles, as they are apt to render the bowels too open for hard work. The flesh should be first thoroughly boiled, and then taken out before the oatmeal is added to the broth.

Dogs which are regularly worked are better for having two meals a day; the principal one, of course, should be given in the evening. Nothing is better than good, wholesome horse-flesh (avoiding by all means such as have died full of drugs; but let it be those which have been killed, and in a healthy state), boiled, and the liquor mixed well with the oatmealporridge, the quantity of each about equal. If horseflesh is not to be had, cracklings or greaves are a 
proper substitute, if they are good. They are generally broken small, and mixed with about half the quantity of oatmeal, and boiling-water poured on them, and well stirred together ; or they may be boiled together like porridge. Dogs, like men, tire of the same kind of food; therefore, a judicious feeder, like a good cook, will contrive to vary his bill of fare. Porridge and milk, the offal of the kitchen, the offal of bullocks or sheep, which should be well boiled, make an excellent variety; but we would by no means recommend a too frequent repetition of the latter food. Potatoes make also a good variety, and although not so nutritious as oatmeal, they are less heating. Care should be taken never to present more to a dog than he will eat with a good appetite; and when oatmeal and barleymeal are used, mixed, the former should be first boiled for twenty minutes, and then the other added, and boiled only for about eight or ten minutes. The latter meal should, however, never be given in the hunting season, as it is too heating, and occasions the dogs to be perpetually drinking. Their food should be given to them pretty thick, as thin porridge does not stay in the stomach so well; and it should be well cooled before being presented to them. The feeding-troughs for hounds should be wide at the bottom, and not exceeding three feet in length. They should be thoroughly cleaned out, and scalded with hot water every second day. Dogs should always have plenty of fresh water.

During the hunting season, hounds should have sulphur mixed up with their meals once a week, in the proportion of three drachms to each. At the end of the season, the same quantity of sulphur should be given, with the addition of one and a half drachm of antimony. After a hard day's work an entire meal of 
horse-flesh should be given them, and as newly killed as possible; and when this cannot be had, bullocks' paunches or sheep's trotters, both of which ought to be well boiled.

\section{GREYHOUNDS}

Should be fed principally on animal food, such as sheep's trotters or neats' feet, boiled or stewed down, and mixed with bread or oatmeal, and given moderately in the morning and afternoon (the dog never being allowed, on any occasion, to eat a great quantity at a time), or on other hard meat, as it will enlarge and strengthen the muscular fibre without increasing the cellular tissue and adipose substance, which has an invariable tendency to affect their breathing. The butcher's meat should be of the best quality. Within a few days of a coursing-match, some sportsmen give each dog two or three ounces of beefsteak, moderately fried in a little brandy, with two or three teaspoonfuls of assafœtida dropped into it. This braces their stomachs, and produces other stimulating effects. After they have been coursed, they should be well brushed all over, a little oil being used in the operation.

The kennels of greyhounds should be kept particularly warm and dry, and at the same time they ought to be properly ventilated. Indeed, pure air is an essential requisite to the health and vigour of all animals.

Nothing is of greater consequence to the health and efficiency of dogs than cleanliness. Their kennels ought to be frequently replenished with dry and clean straw, and their apartments well aired. Their beds should, if possible, be placed on a wooden bench, or, at least, on some dry place. On attention to cleanli- 
ness also depends the perfection of their olfactory nerves, and sense of smelling; for if accustomed to disagreeable effluvia, a dog will be ill-adapted to trace the fail of a deer, or scent of a fox, through greasy fallows or ground tainted by the grazing of sheep.

\section{OF TRAINING FOXHOUNDS.}

The first thing to be attended to in young dogs of all kinds is to make them understand their names well, and answer to them, before training, for which they should be rewarded and caressed. After foxhounds have learned to follow freely, they should be coupled, and led out amongst sheep, deer, etc., and made to understand that such is not their game. But when they have arrived at the locality where sheep and deer are, they must be let loose, and only a few at a time; and if they attempt to run after them, they must be severely chastised, and the cry of 'ware sheep be often repeated to them, which cry will generally, on future occasions, have the effect of stopping them from sheep-running, without the necessity of using the whip. Great care is necessary at the offset to prevent them from worrying a sheep, which they will sometimes do under the management of careless trainers. If they are allowed to taste the blood, it will be very clifficult afterwards to break them off this bad habit.

Young hounds should be often walked about the courts of the kennel, followed by the whipper-in, who ought to rate them after the huntsmen. They should also be frequently taken out with people on foot, which teaches them to be more tractable, attentive, and much more manageable. It is usual to take them out to their walks in a country where they are to be hunted, 
as the sooner they acquire a knowledge of it the better; and when they are thrown out, or left behind, are more likely to find their way home.

In entering foxhounds, it should always be at their own game, although some are foolish enough to begin them with hares, which just requires a second training to break them from that pursuit. Nothing is so good for rendering young hounds obedient as walking them frequently out amongst sheep, hares, and deer, and restraining their pursuits of these animals. This probation will teach them to be steady. A fox-cub should then be turned out before them, in company of some old hounds, as leaders, which has the effect of training them in a short time instinctively, as it were, to hunt themselves. After they have tasted blood, it will be more difficult to repress their ardour than to excite it. Every means of encouraging them should be used in the earlier stages of their training, and punishment only administered after they have made some progress. In flogging a hound for a fault, the whipper-in should use his voice at the same time; this teaches him to know for what he is beaten. It has been recommended, and successfully adopted, that a live hare should be introduced into the kennel, and the dogs soundly flogged whenever they attempt to approach her.

Hounds at their first entering cannot be encouraged too much. When they are become hardy, love a scent, and begin to know what is right, it will be soon enough to chastise them for doing wrong; in which case one severe beating will save a deal of trouble. It should be recommended to the whipperin, when he flogs a hound, to make use of his voice as well as his whip; and let him remember that a smack of the whip is often of as much use as the lash, 
to one that has felt it. If any are very unsteady, it will not be amiss to send them out by themselves, when the men go out to exercise their horses. If hares are plentiful in the neighbourhood, let some be found sitting, and turned out before them, and you will soon find that the most riotous will not run after them. If they are to be made steady from deer, they should see them often, and they will not regard them. Flogging hounds frequently in kennel is a practice with many huntsmen, but which ought on no account to be permitted; it is unreasonable, unjust, and cruel. Hounds that are old offenders, that are very riotous, and at the same time very cunning, may be difficult to catch-such hounds may be excepted-they deserve punishment, whenever it happens, and huntsmen of course should not fail to give it them when they can. This we will allow is a particular case, and necessity may excuse it; but let not the peace and quiet of the kennel be often thus disturbed. When hounds offend, punish them-when caught in the act, then let them suffer-and if severity be exercised, let it be just.

\section{TRAINING OF POINTERS AND SETTERS.}

One of the earliest lessons which a dog should be taught is obedience; this can be accomplished by walking him out near home, and when he ranges to too great a distance he must be called back, using the words here, or come in. To teach him to go behind, the words back or behind, should be used.

At this period it will be proper to accustom the dog to be tied up in a stable, but not for too long a time at the outset, as a dog's growth is apt to be cramped by confining him too much at an early age. Where the situation is favourable, dogs under twelve months 
should always be allowed to go at liberty through the day, taking care to tie them up at night, because in strange quarters, where this restraint may be necessary, if not used to it they may howl, and disturb a whole neighbourhood.

Dogs should, if possible, receive their education in an open moorland country, where there are neither pheasants nor rabbits. They will then have full scope for their faculties, acquire a gallant range, and learn to quarter their ground properly. In this process the words used should be few and simple, and accompanied by signs, which ought ultimately to become their substitutes; as we hold, that when a dog is thoroughly made, a whistle or a signal will be sufficient for his guidance in the field. To the young sportsman, the Vocabulary and Maxims which follow may be useful, and tend to impress him with a proper notion of the business in hand.

\section{VOCABULARY.}

Hey away, hey on, good dog!-is a general encouragement to general beat.

Have a care. - A caution when you perceive him pressing rashly upon his birds, and in danger of springing them.

Hold up, good dog.-When he is diffident of following up his game, or dwelling upon a false haunt.

Take heed, or to-ho! - A caution to him when he fixes to his point, and warning to other dogs to back, accompanied by holding up the right hand, the same as to down.

Down charge. - The word of command, instantly after firing, which must be imperative.

Seek-dead.-A notice for him to go in search of dead or wounded birds. 
Ware bird, or Ware dead.-A check when he attempts to mouth or snap a dead or dying bird.

Ware chase!-A challenge (loud and sharp) for chasing birds upon the wing.

Ware hare!-A check of decisive recall from an unlawful pursuit.

Ware lark!-A summons to cease from puzzling on a worthless haunt.

Gone, gone :-A notice that birds are away; and signal to go on.

Come in, or Come in here.-Or the whistle; a summons he should promptly obey, and come in to heel.

The following should be most strictly attended to :-

\section{MAXIMS.}

Never let your dog have a will of his own; but impress upon him, from the first, that your command is to be the rule of his actions; and

Never allow him to ramble about the neighbourhood alone, or at the risk of falling into bad company. "Evil communications," etc.

Never take the field without your whip: it is the only legitimate weapon of punishment; and the sight of it may, in many instances, save the skin of your pupil.

Never pass a blunder unnoticed, nor a fault unpunished: nevertheless, "love mercy." Keep your pupil down under lecture till you are friends again, then hey-on!

Never administer punishment without endeavouring to make him understand the nature of his offence -ware chase! take heed! or down charge! as the case may be-duly impressed upon him.

Never avenge upon your dog your own errors in 
shooting; neither let your anxiety to bag a bird induce you to join him in a race for the possession thereof. Footing out-winged birds is the finest opportunity you have of developing his powers, and rather lose the bird than the advantage of such a lesson.

Never permit a race after a hare; therefore never be tempted to shoot at one which rises before your dog. In a case of necessity shoot her in her seat.

Never head your dog, nor let him trifle his time behind you; but keep him ahead in his beat, and go hand-in-hand with him up to his point.

Never allow your dog to break field, that is, make off into another field before his master, or to hunt out of your sight at any time. Neither let him take another dog's point out of his teeth, but make him back up at a respectful distance; or, which is better, lie close till desired to go on.

Never hunt a dog when tired down, lest he become a dealer in false points, and lose his gallantry of range.

Do not suffer your dog to ramble when you are going to or returning from the field, but keep him strictly to your heel. It is not in the way of business.

By way of preparation for taking the field (which may be as soon after six months old as convenient), we trust the master or keeper of young pointers or setters has betimes accustomed them to know their names distinctly, and answer to them, and to prostrate themselves to the imperious down and uplifted hand. Down charge should also be taught at home, as blinking shot on the field is one of the worst evils which can befall us. This can be accomplished at small expense of gunpowder-reward and caresses with puppies. Sheep and poultry should likewise be made 
familiar to them, and respected. If a young dog makes a mistake in the flock or poultry-yard, the whip will cure him ; but if an old one takes to such practices, to the gallows with him! he is never to be trusted.* Every sportsman, we presume, will understand the advantage of giving a young dog the wind, against which he should take his range, making regular tacks, like a vessel beating up to windward. If he stretches too far to the one hand, a whistle, a wave, and inclination of the body in another direction should bring him about. In this manner continue to work him till you see him on the haunt of game; then caution him by have a care-take heed! as he is fixing to his point, and fix him with a stern, authoritative down! Keep him to his point, and if the birds lie, make a circuit round them, keeping your eye strictly upon the dog, checking every attempt to move with the uplifted hand and down! When you see him reconciled to lie close, give the word, hold up! to your birds. If he is diffident, pat and encourage him with kind words; if too forward, check him. When the birds spring, should he pursue them, ware chase! must be thundered in his ears, which may perhaps stop him; if not, have patience, keep your station, and he will return after his rioting is over. Beware of severity now, lest you check him; but lead him again to the haunt of the birds, and there lecture him -take heed, sir! down! At this stage of the business a check-cord, a few yards in length, may be of advan-

* The following specimen of the "tender mercies" of a reverend sportsman may amuse, if not instruct.--"To break a sheep-biting dog, take some wool off a sheep's rump, steep it in train oil, put it in the dog's jaws, and sew up his mouth. For killing poultry, boil a chicken in its feathers, take it hot from the boiler, squeeze the water from it, and put it into his jaws, and tie them together."-DaniEL, iii. p. 337. 
tage to prevent him from bolting in or escaping under punishment. When you have got him steady to his point, you may proceed to down charge! to which we have supposed the pupil ready drilled; nevertheless, when a bird drops, he may break in, and this should be instantly and invariably resisted with down charge! ware dead! and the application of the whip when persisted in.

We now come to the important act of bagging the birds which are down. Seek dead is an easy process if they are dead; but, if birds are winged, and run, your dog will require great indulgence and encouragement in footing them out, till experience teaches him to distinguish the fail of a wounded bird from that of one which is not. If he make it out, caress him with seek dead! good dog! etc., laying the bird before his nose and feet; but never permitting him to mouth or pull the feathers off it. The perfecting a dog in this branch of education, which enables him to road out the scattered birds of a covey through all their doublings, adds more to the pleasure of a really scientific votary of the trigger than perhaps any other, and, therefore, should be patiently and particularly attended to. When we have got two or more so far in their course, we may proceed to hunt them in company, and learn them to back, which will not be difficult, if they are decidedly subject to the take heed -down! Some are in the practice of making their dogs back up the dog which has the point: we prefer having them to drop and lie till he makes out his birds, which he is entitled to do, and ought to show the same respect in turn. Dogs are not exempt from jealousy, and often break through the rules when a rival is at hand. When in company they should hunt in an independent manner, crossing and quartering their 
ground in opposite courses, and not following at the tail of one another. When a dog is found to be slowish in this respect, he should be hunted with a strange one, or alone; he may thus be made to act for himself.

If in following up this system, we have brought them to have a gallant range, quarter their ground properly, point, back, lie-charge, and foot out their birds correctly, we are in the high road to perfection; this, however, is not to be attained in a few weeks, or months, but is the reward of continued care and perseverance. But whatever rules may be laid down, much must be left to the common-sense and discernment of the trainer, as a passionate or stupid man can never make a first-rate dog.

Spring is the best time for training dogs, as the birds are then paired, and lie better, and are not so easily flushed as after the breeding-season. Besides being less numerous at that season, the game are not so likely to excite the dog. But a dog cannot be expected to be rendered quite staunch until a few birds have been killed before him. After this, and about the beginning of July, the young dogs ought to be taken for a few weeks to the moors, to be hunted with steady dogs, and blank shots fired over them, so as to prepare them for their work when the season commences. Even old dogs are the better for this occasionally.

It is of great importance to choose a proper day for training-such a one as the scent lies well with. The air should be at its mean gravity, rather moist, but inclining to grow drier, with a mild and gentle breeze. The moderate gravity buoys up the scent, and enables the dog more readily to find his game without lowering his head, or inclining to rake.

A good property in a dog, is to hunt with his nose 
high, as in this way he will find more birds than when he rakes, that is, runs with his nose close to the ground and follows the bird by tracking. Whenever this vice is observed, he should be called to in an angry tone of voice to hold up! or up head! 'This may often flurry him, but after a little practice he will soon learn to take the wind of his own accord, and hunt high. If a dog obstinately persists in raking, there is little hopes of his ever turning out a good ranger, and he should be thrown aside. If anything will break him of this very bad fault, it will be the puzzle. This instrument is made of a piece of hard-wood or fir, one foot in length, and an inch and a half in breadth, tapering a little at one end; at the broader end there are two holes running longitudinally, through which the collar of the dog is put, and the whole is buckled round his neck: the piece of wood being projected beyond his nose is then fastened with a piece of leather thong round his jaw ; by this means the peg protruding seven or eight inches beyond his muzzle, effectually prevents him from putting his nose to the ground.

The following is a representation of an iron puzzle, recommended by Colonel Hawker, which is more convenient than a wooden one.

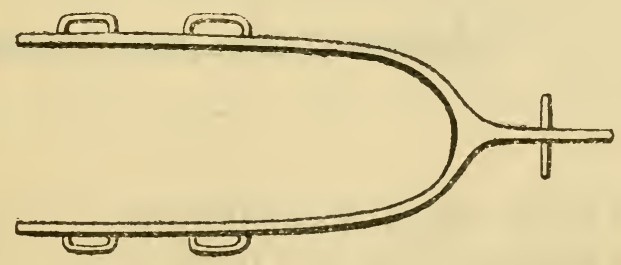

When a dog perfectly knows his game, it is time to bring him under complete discipline and obedience. He should be taught, before being brought to the field, 
to lie down the instant he is desired, by repeating to him the word down! If he is unruly, it will be necessary to use the trash-cord, which is a rope or strong cord, about twenty yards in length, attached to his collar, which he is allowed to drag after him while ranging. Endeavour always to keep within reach of the cord, and when it is necessary to call him, check him smartly with it, if he fails to obey. This, repeated several times, in general has the effect of teaching him to come at call. When expedient, he should be caressed and rewarded with a bit of bread, and this should be done as often as he yields his due obedience.

The check-collar is an excellent instrument for this purpose, and has been found more effectual in curing dogs of these bad habits than the trash-cord. The following representation of one will be easily understood. The whole is made of iron.

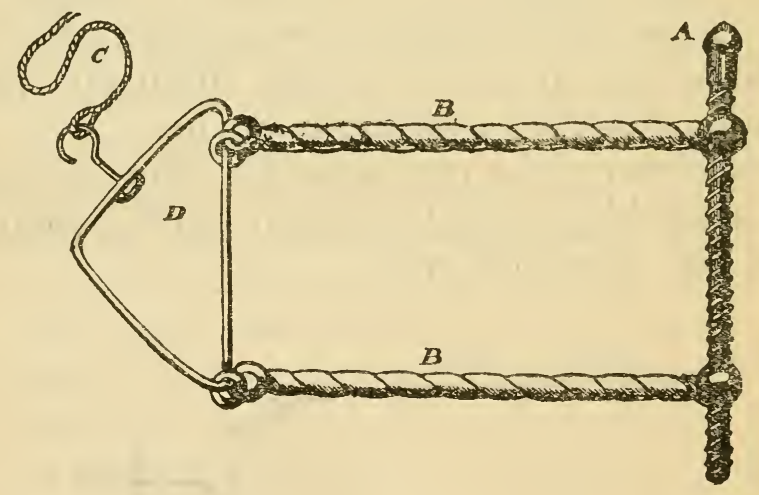

$A$ is a screw which keeps the sides of the collar at the proper distance from each other, and by which they are adjusted to the thickness of the dog's neck; it rests upon the back of his neck, above the collar. It is necessary to unscrew this, to admit the dog's head. $B B$, the sides of the collar. $D$, a triangle 
which the rope $C$ is fastened to, and which, being pulled, draws the sides together, and presses severely upon the wind-pipe of the dog, by the external pressure. It will be observed that this triangle is attached to the sides of the collar by small rings, passing through a hole at the end of each side. The harder the rope is pulled the more it presses on the dog's throat.

\section{RETRIEVERS.}

Gentlemen who keep large establishments of sporting dogs, generally keep one or two retrievers for the express purpose of finding lost and wounded game, and hence their name retrievers. These consist of the Newfoundland dog, the greater and lesser water-dogs, and the large water-spaniel. The last is decidedly the best adapted for general use, from the qualities which he possesses, and which will be more particularly described when treating of the specific character of sporting dogs. Genuine dogs of this last kind are now very rare in Great Britain. The mode of training is similar to that employed in teaching a water-dog to search for, and fetch and carry his game. They ought to know well the distinction betwixt fur and feather, otherwise they can never be adepts in retrieving. They should be carefully instructed and encouraged to carry kindly, that is, without mouthing their game in a rough manner, and they should invariably be instructed to lie charge.

\section{WATER-DOGS.}

In the shooting of wild-fowl, various dogs are used to fetch the birds out of the water as soon as shot. The above-mentioned dogs, or retrievers, are all fitted for this sport; and sometimes the poodle, 
or small water-dog, will be found extremely useful, particularly if he is tolerably large, as he is a rapid and expert swimmer and diver, and very ardent in his temperament. There will be no difficulty in teaching any of the above varieties to take the water, as they are impelled by a natural instinct to do so.

When puppies are five or six months old, they should be taught to fetch and carry, as a preliminary to breaking them. This is easily done by throwing a glove or other article to them in the house, or during a walk, and desiring them to fetch it. With young dogs, the most gentle means are certainly the best; and when any animal proves obstinate, his correction should be of a moderate kind; and if he seems much disheartened by beating, it will be best to suspend teaching for a time, and in the interim he may be propitiated with gentle caresses.

A method which has been successfully employed in training the dogs under consideration is, to get a rabbit's skin, stuffed, and begin by tossing it about a room. When the dog, which should have a small line attached to his collar, takes up the skin, bring him to you by a gentle pull, with the skin in his mouth; encourage him three or four times, and then take the line off. When the dog begins to enjoy this sport, take a small line and run it through a pulley fixed to the ceiling; then tie the rabbit's skin to one end of the line, and keep the other in your hand; after this, fire a pistol, and let the skin drop. The dog will soon become fond of the sport, and will thereafter readily bring every head of game and wild-fowl that is shot. After some proficiency is made, take two or three together into a room, fire the pistol, and order first one dog, and then another 
to bring the skin: with a little practice, they will soon be perfect.

Should all these means prove unavailing, the task ought to be abandoned until he is old enough to be broken in; and he will then be better able to bear correction, and to understand for what cause it is inflicted.

Recourse must next be had to throwing a piece of wood into the water, and desiring the dog to fetch it out, which he will soon do by a little practice.

\section{GREYHOUNDS.}

The varieties and qualities of these dogs will be pointed out in our specific account of them.

Greyhound puppies should be kept extremely warm, being very tender dogs. They never grow straight if exposed to much cold; and where gentlemen have not appropriate establishments for this purpose, we would recommend that they be allowed to run about the kitchen fireside; although in this situation they are liable to accidents.

The time for trying and training greyhounds is at the age of twelve months, although fifteen months is soon enough. Some sportsmen enter and try them at ten months; but this is by no means to be recommended, as they are apt to get strained, if the course should happen to be long and severe, and, in many cases, they never get the better of an exertion of this kind at so early an age. They require but little training, as they are endowed with an instinctive propensity to course; and in some greyhounds their ardour is so great, that it is almost impossible to restrain them. They ought always to be entered at first with a trained dog of known 
abilities; and they should never be allowed to suppose, after having tasted blood, that a hare can escape them: and, on this account, ought never to be slipped at a hare when jaded and exhausted, or when too far ahead to be overtaken.

When they have been taught properly to know their game, the next thing to be attended to is to accustom them to the slips, and when a hare is found, a distinct stand should be made by their keepers, and the words so-ho! several times repeated in a firm manner; and the young dogs should never be slipped until the hare is at some distance, lest, being over anxious to possess it, they strain their limbs too much.

The training of a greyhound requires from three to six weeks, the time, however, depending much upon the condition and constitution at the commencement. If ton fat, it is necessary to begin by administering laxatives. The exercise ought to be on turf, and occasionally on the road, with a horse, and by hard galloping, to strengthen their wind, as well as to keep their claws short; also in the morning and afternoon, before feeding, at first gently, and for an hour and a half at each time. As the training advances, and the condition of the dog improves, it should be more severe and of shorter duration, till the greyhound is capable of accompanying a horse at speed for a couple of miles, without showing any signs of distress. After severe exercise dogs should be walked for a quarter of an hour, and then returned to the kennel, and brushed, cleaned, and fed. The brushing and cleaning removes all scurf, etc., from the skin, and renders the animal more cheerful and active.

No remedy has yet been found to prevent a 
greyhound from running false or cunning; that is, in place of following close after the hare, and capturing it by superior speed, to endeavour to cut off its retreat, by making towards a vacant space in a wall or hole, through which hares are in the habit of escaping. It is a propensity which greyhounds are apt to indulge in when they have run frequently over the same ground; and as soon as they take to it, their previous excellence, let it have been ever so great, becomes a dead letter, as they are no longer to be depended on in running matches.

\section{THE KENNEL.}

We cannot do better than give Mr Beckford's directions respecting the management of the kennel, who was one of the most experienced sportsmen of his day.

"Cleanliness is not only absolutely necessary to the nose of the hound, but also to the preservation of his health. Dogs are naturally clean animals; they seldom, when they can help it, dung where they lie; air and fresh straw are necessary to keep them healthy. They are subject to the mange, a disorder to which poverty and nastiness do very much contribute. This, though easily stopped at its first appearance, if suffered to continue long, greatly lessens the power of the animal; and the remedies which must then be used, being in themselves violent, often injure his constitution: it had better be prevented. Let the kennel, therefore, be your particular care.

'Upon some little eminence erect, And fronting to the ruddy dawn; its courts

On either hand wide op'ning, to receive

The sun's all-cheering beams, when mild he shines, And gilds the mountain tops.' 
Such as Somerville directs may be the situation; its size must be suited to the number of its inhabitants; the architecture of it may be conformable to your own taste.

"However deserving your confidence, still it should be the care of the master to see the state of the kennel frequently. Two kennels are absolutely necessary to the well-being of the hounds: when there is but one, it is seldom sweet; and when cleaned out, the hounds, particularly in winter, suffer both whilst it is cleaning, and as long as it remains wet afterwards. To be more clearly understood, I shall call one of these the hunting-kennel, by which I mean, that kennel into which the hounds are drafted which are to hunt the next day. Used always to the same kennel, they will be drafted with little trouble; they will answer to their names more readily, and you may count your hounds into the kennel with as much ease as a shepherd counts his sheep out of the fold.

"When the feeder comes to the kennel in the morning, he should let out the hounds into the outer court, at the same time opening the door of the hunting-kennel, as want of rest, or bad weather, may incline them to go into it. The lodging-room should then be cleaned out, the doors and windows of it opened, the litter shaken up, and the whole kennel made sweet and clean before the hounds return to it again. The great court and the other kennels are not less to be attended to, nor should any omission that is hurtful to the hounds be passed over in silence.

"The floor of each lodging-room should be bricked, and sloped on both sides, to run to the centre, with a gutter left to carry off the water, that when they are washed they may be soon dry. If water should stand through any fault of the floor, it should 
be carefully mopped up, for as warmth is in the greatest degree necessary after work, so damps are equally prejudicial. Orders given without skill are seldom well obeyed; and when the master is either ignorant or inattentive, the servants will be idle.

"Contrary to the usual practice in building kennels, there should be three doors, two in the front and one in the back; the last to have a lattice window in it with a wooden shutter, which is constantly to be kept close when the hounds are in, except in summer, when it should be left open all day. This door answers two very necessary purposes: it gives an opportunity of carrying out the straw when the lodging-room is cleaned, and as it is opposite to the window, will be the means to let in a through air, which will greatly contribute to the keeping it sweet and wholesome. The other doors will be of use in drying the rooms, when the hounds are out; and as one is to be kept shut and the others hooked back (allowing just room for a dog to pass), they are not liable to any objection. The great window, in the centre, should have a folding shutter; half or the whole of which may be closed at nights, according to the weather; and the kennels by that means may be kept warm or cool."

The two great lodging-rooms are exactly similar, and having a court belonging to each, are distinct kennels, situate at opposite ends of the building. In the centre is the boiling-house and feeding-yard; a lesser kennel, either for hounds that are drafted off, and those that are sick and lame, or for any other required purpose, is on each side; at the back of which, as they are but half the depth of the two larger kennels, are places for coals, etc., for the use of the kennel. There is also a small building in the rear for the accommodation of bitches in heat. The 
inner court floors should be bricked or flagged, and sloping towards the centre like those of the lodgingrooms ; and water, brought in by a leaden pipe, should run through the channel in the middle. In the centre of each court is a well sufficiently large to dip a bucket in to clean the kennels : to keep it from wanting repair it should be faced with stone. A wooden cover should be fixed over the well of the feeding-yard.

The benches, which must be open to let the urine through, should have hinges and hooks in the wall, that they may fold up when the kennel is washed; let them be made as low as possible, that when a hound is tired he may have no difficulty in jumping up, and at no time should the space under it be so great that he will be able to creep under.

A large bricked court in front, having a grass court adjoining, and a brook running through the middle of it, completes the kennel. This court should be planted round, and also have some lime and horsechestnut trees near the centre for shade; some posts, bound round with galbanum, may be placed to prevent the hounds urining against the trees. The brook may be used as a cold bath for hounds lamed in the stifle and strained. A high pale should enclose the whole, which, to the height of four feet, should be close; the other open, two inches wide. At the back of the kennel should be a thatched house, and fenced up at the sides, to contain at least a load of straw; a pit for receiving the dung, and a gallows for the flesh. If a piece of ground adjoining the kennel can be enclosed for such dog-horses as may be brought alive, it will be of great service, as the disorders of condemned horses are not always to be discovered, and may be dangerous to others; the hounds may also be brought into this field to empty themselves after 
feeding; and the draft for the next day's hunting can be here made with greater accuracy than when confined in the kennel.

Should ticks at any time be troublesome, the walls of the kennel should be well washed; if that does not destroy them, white-washing should then be adopted.

When the hunting season is over, one kennel will suffice; the other, with the grass-yard adjoining to it, may be allowed to the young hounds. This separation, which should continue till the season commences, is necessary, as it prevents many accidents which might happen at this time of the year. Should there be conveniences, the dogs being kept separate from the bitches during the summer months, will be all the better. When hounds are very riotous, the feeder may sleep in a cot in the adjoining kennel: if well chastised at the first quarrel, his voice will be sufficient afterwards. 


\section{CHAPTER VII.}

Different Dogs, used in Field Sports and for Domestic Purposes, Watch-Dogs, etc.

I HAVE already said that dogs, in all their varieties of form, are but one species, modified and altered by adventitious circumstances ; climate, feeding, crossing one breed with another, accident, and education, all combining to give that almost infinite variety of form and intelligence now to be met with amongst dogs of every country and climate. How the distinct races which have characters and propensities peculiar to themselves were originally produced it is impossible now to tell. If care is taken to prevent these from commingling with spurious blood, it is certain that by the result of education, the habits to which they have been trained have become hereditary. Many instances have occurred where pointer pups have lost their parent, and been suckled by a bitch of a totally different breed; and upon being taken into a field, for the first time, have pointed game.

The subjugation and domestication of the dog by man may be esteemed as one of the most important conquests which he has made in the animal kingdom.

Without the vices of man, nature has formed the dog with an ardour and purity of attachment, which when once matured, appears unsullied and inviolable. His whole ambition seems a desire to execute the 
commands of his master, and to yield implicit obedience to his every wish; while he, at the same time, has a great dread of giving offence. His whole actions are marked by zeal, vigour, and gratitude, for the little kindnesses he receives at the hand of his master. He seems perfectly sensible of favours bestowed on him. Under correction, whether deserved or not, he in general displays a degree of firmness, by submitting to, and not avoiding chastisement; while with conciliating and impressive looks and supplications he endeavours to allay the wrath of his chastiser, and will lick the hand by which the blows are inflicted. Every kindness he receives from his master is remembered, while his too often undeserved punishment is speedily forgotten. But, on the other hand, he will act quite differently with strangers, as he will boldly protect himself against an unmerited injury, and will seldom forget it.

To illustrate the services of this faithful animal, we shall quote the reflections and remarks of $\mathrm{Mr}$ Burchell, given in his Travels in Africa.

" Our pack of dogs," says he, "consisted of about five and twenty, of various sorts and sizes. This variety, though not altogether intentional, as I was obliged to take any that could be procured, was of the greatest service on such an expedition, as I observed that some gave notice of service in one way, and some in another. Some were more disposed to watch against men, and others against wild beasts; some discovered an enemy by their quickness of hearing, others by that of scent; some were useful for speed in pursuing game; some for their vigilance and barking ; and others for their courage in holding ferocious animals at bay. So large a pack was not, indeed, maintained without adding greatly to our care 
and trouble, in supplying them with meat and water, for it was sometimes difficult to procure them enough of the latter; but their services were invaluable, often contributing to our safety, and always to our ease, by their constant vigilance; as we felt a confidence that no danger could approach us at night, without being announced by their barking. No circumstance could render the value and fidelity of these animals so conspicuous and sensible as a journey through regions which, abounding in wild beasts of almost every class, gave continual opportunities of witnessing the strong contrast in their habits, between the ferocious beasts of prey, which fly at the approach of man, and those kind, but too often injured companions of the human race. Many times, when we have been travelling over plains where those have fled the moment we appeared in sight, have I turned my eyes towards my dogs to admire their attachment, and have felt a grateful affection towards them for preferring our society to the wild liberty of other quadrupeds. Often, in the middle of the night, when all my people have been asleep around a fire, have I stood to contemplate these faithful animals lying by their side, and have learnt to esteem them for their social inclination to mankind. When wandering over pathless deserts, oppressed with vexation and distress, at the conduct of my own men, I have turned to these as my only friends, and felt how much inferior to them was man, when actuated only by selfish views. It is impossible for the naturalist, when taking a survey of the whole animal creation, not to feel the conviction, that this friendship between two creatures so different from each other, must be the result of the laws of nature; nor can the humane and feeling mind avoid the belief, that kindness to those animals, from which he derives con- 
tinued and essential assistance, is part of his moral duty."

\section{SECTION I.-DOGS OF THE CHASE.}

THE STAGHOUND.

The staghound is now the largest and most powerful of all the dogs which go under the general denomination of hound. $\mathrm{He}$ is held in higher estimation than any other dog of the chase, and has a most commanding and dignified aspect.

The staghounds, exclusively devoted to that sport in the royal establishment in this country, it is well known, are an improved breed, produced by a cross between the old English southern-hound, and the fleeter foxhound, grafted upon the bloodhound.

It has been asserted by the most celebrated naturalists, that the hound, harrier, turnspit, water$\mathrm{dog}$, and spaniel, are all sprung from the same race; and there seems to be strong reasons for believing this to be the case, as their forms and instinctive properties are nearly allied in all these kinds: the principal difference consisting in the length of their legs and the size of their ears, which are in all of them soft in their texture and pendulous. The head is large, broad, and shorter in proportion than that of the foxhound, with wider nostrils, and the hams more muscular, and he carries his tail high, whether at rest or in the chase. The hound and harrier are supposed to be natives of Britain, France, and Germany; an opinion which is attended with some degree of reason, for when transported to warmer climates they degenerate.

It seems extremely probable that this large, strong, and bony hound was the primeval stock from which 
all the collateral branches of this race have descended; and that all deviations from the original stem have been the result of crosses and improvements during many centuries, by those skilled in breeding and rearing dogs of the chase, and varied in size and strength, according to the particular sport for which they were intended. At the present day, it is well known that the practical breeder, by judicious crosses, can either enlarge or diminish the stature and strength of his pack in the course of three or four generations.

The following fact affords a striking proof of the wonderful spirit of the staghound in supporting a continuance of exertion. Many years since, a very large and powerful stag was turned out at Whinfield Park, in the county of Westmoreland, and was pursued by the hounds till, by fatigue or accident, the whole pack was thrown out, except two staunch and favourite dogs, which continued the chase the greatest part of the day. The stag returned to the park from whence he set out, and, as his last effort, leapt the wall, and immediately expired. One of the hounds pursued him to the wall, but being unable to get over, lay down and almost instantly died; the other was found dead at a little distance.

The length of the chase is uncertain; but, as they were seen at Red-kirk, near Annan, in Dumfriesshire (distant by the post-road forty-six miles), it is conjectured that the circuitous and uneven course they might be supposed to take would not be less than one hundred and twenty miles. To commemorate this fact, the horns of the stag, which were the largest ever seen in that part of the country, were placed on a tree of enormous size in the Park (afterwards called " Hart'shorn Tree"), accompanied with this inscription :-

"Hercules kill'd Hart o' Greece ;

And Hart o' Greece kill'd Hercules!" 
The horns have been since removed, and are now at Julian's Bower, in the same county.

\section{THE FOXHOUND.}

The muzzle of the foxhound is rather long, and his head small in proportion to his body; his ears long and pendulous, though not so much so as those of the bloodhound or staghound, and a little lower at the shoulders, and more slender in his make. His limbs are very straight, his feet round, and not too large; his chest is very deep, and breast wide; his back broad, his neck thin, with the shoulders lying well back, and his tail thick and bushy, which he carries high while in the chase. The ground-colour of his whole skin is white, variously patched with black and tan in different parts of the body, as well as the head, and generally with one at the root of his tail.

No country in Europe can boast of foxhounds equal in swiftness, strength, and agility to those of Britain, where the utmost attention is paid to their breeding, education, and food. The climate also seems congenial to their nature; for when taken to France or Spain, and other southern countries of Europe, they quickly degenerate, and lose all the admirable qualities for which they are remarkable in this country.

"In thee alone, fair land of liberty,

Is bred the perfect hound, in scent and speed

As yet unrivall'd; while in other climes

Their virtue fails-a weak, degenerate race."

Somerville.

The chief excellence of a pack of foxhounds is the head they carry, taken collectively; and on this and the fineness of their noses depend their speed. $\mathrm{Mr}$ Beckford says, "that hounds go, like the horses of the 
sun, all abreast." Five and twenty couples are a sufficient force at any time to be taken into the field; they are a match for any fox, supposing them steady and their speed nearly equal; too heavy dogs always do more mischief than service. Hounds that are meant to run well together should never have too many old ones amongst them. Five or six seasons are sufficient to destroy the speed of most dogs.

Fox-hunting at the present day is not carried to that extreme which was the custom twenty years ago, as it then killed many of the finest horses. When George IV. was Prince of Wales, he hunted with a pack of hounds which were silent in the chase.

\section{THE HARRIER.}

The harrier is considerably less than the foxhound, and was originally generated in a double cross, between the beagle and the southern hound and the beagle. Sportsmen, however, have different sizes of harriers adapted for the kind of country in which they hunt, as well as the fancy of the owner of the pack. The colour and markings are similar to those of the foxhound, but frequently the dark colours occupying a greater extent of surface. It is this dog which is now almost universally used in Britain and Ireland for hare-hunting.

The harrier pursues the hare with much eagerness and swiftness, allowing her but little time to breathe or double. The keenest sportsman, and well-mounted, frequently find it difficult to keep up with the pack; and when following a strong hare, becomes rather fatiguing work. The mingled voices of a pack of harriers produce a cheerful and agreeable harmony.

$\mathrm{Mr}$ Beckford justly remarks, that "harriers, to be 
good, like all other hounds, must be kept to their own game: if they are permitted to run a fox, they are afterwards spoiled, and no reliance can thereafter be placed upon them. Hounds of all kinds cannot be perfect, unless used to one scent and one style of hunting." Somerville says,

"A different hound for every different chase

Select with judgment; nor the timorous hare

O'ermatch'd destroy, but leave that vile offence

To the mean, murderous, coursing crew, intent

On blood and spoil."

\section{THE BEAGLE.}

The beagle is the smallest of the dogs of the chase. In form he bears considerable resemblance to the harrier, but with his limbs proportionally much shorter, and on that account much inferior to that dog in point of speed. His sense of smell, however, is equally acute, and he pursues the hare with indefatigable vigilance and energy. Every winding, and all her mazes are traced by him with a matchless degree of exactness, whilst the soft and melodious tones of the pack afford ecstatic pleasure to the lovers of the chase, and is thus beautifully described by the poet of "The Chase":-

"Hark! from yon covert, where those towering oaks Above the humble copse aspiring rise, What glorious triumphs burst in every gale Upon our ravish'd ears! The hunter's shout, The clanging horns swell their sweet winding notes, The pack wide opening load the trembling air

With various melody; from tree to tree The propagated cry redoubling bounds, And wingèd zephyrs waft the floating joy Through all the regions near..... . The puzzling pack unravel, mile by mile, Maze within maze." 
Colonel Smith is of opinion that the beagle is the same as the brachet of the Middle Ages; and thinks it the only species of long-eared dog known in the West during the Roman empire, and noticed by Oppian under the name of Agassus.

THE TERRIER.

It is now impossible to trace from what stock the terrier has emanated; but possessing as he does so many peculiar characteristics, one would be tempted to consider him a primitive race. There are several distinct varieties of terriers, but all of them possessing the same inherent properties and propensities. For many ages distinct breeds of this kind have been maintained in purity, by the assiduity of careful breeders.

Although the pure terrier is a dog of small stature, he is possessed of wonderful strength and courage. The rough-haired, or Scotch-terrier of the pure breed, seldom exceeds twelve or fourteen inches in height. His body is strong and muscular, his legs short and stout, his ears small and half-pricked; his head rather large in proportion to his body; and the muzzle considerably pointed; and possessing a most exquisite sense of smell, which enables him to trace the footsteps of other animals with unerring certainty. His colour for the most part sandy, and in some black. If white or pied, it is a sure mark of impurity of the breed. The hair is rather long, matted, and hard, over almost every part of his body.

There are three distinct varieties of the Scotch terrier: viz., the ordinary kind above described; that of the Isle of Sky, with long, somewhat flowing hair, the body long, and the legs short, and bent inwards at the knees. This kind is somewhat larger than the 
common breed. The third is a larger dog, standing from fifteen to eighteen inches in height, and with the hair very strong and stiff, and hence termed wirehaired.

The name terrier seems to be derived from the Latin word terra, "the earth," from the avidity with which he takes the earth in pursuit of all those animals which burrow.

The terrier, amongst the higher orders of sportsmen, is preserved in his greatest purity, and with the most assiduous attention; and it seems of the utmost importance not to increase its size, which would render him unsuitable for the purposes in which he is employed, namely, that of entering to drive out other animals from their burrows, for which his make and strength, and invincible ardour, peculiarly fit him. On this account he is the universal attendant upon a pack of foxhounds; and though last in the pursuit, he is not the least in value. It is when the fox is supposed to have earthed that the services of the terrier are more essentially required: he enters with the utmost eagerness, and soon informs the ear of the sportsman whether or not the fox is in, and at what distance from the mouth, when he is speedily dug out.

\section{THE OTTER-HOUND.}

This animal is a mixed breed between the hardy southern hound, and the larger rough, wire-haired terrier. His head is large and broad, and his ears long and pendulous; his size being about that of a harrier; his shoulders and quarters are thick; his hair strong and wiry, and somewhat shaggy. His colour is, for the most part, of a sandy hue, although we have met with good dogs which were white and with large patches of black. $\mathrm{He}$ is a bold and fierce 
dog, with a full and harmonious voice; he carries his tail similar to the foxhound.

Otter-hunting is now not a very common sport in Great Britain, as the animal is now very scarce, from the population becoming more dense, and cultivation more widely extended; and besides, gamekeepers use all kinds of traps to catch and destroy them.

\section{THE GREYHOUND.}

The greyhound is supposed to have had its origin from the Irish greyhound, but to have acquired its thinner and more delicate and elegant form by the influence of climate and culture, and brought to his present state of high perfection by the persevering attention of zealous breeders. The strong similitude of these dogs in shape and general character holds out good grounds for the adoption of such an idea; the smallness of the muzzle, length of neck, and depth of chest, and the light and graceful airiness of his whole figure, and especially the length and elegance of his legs, all contribute to render this the most elegant of the canine tribe.

We owe much of the superiority of the present breed of greyhounds to the perseverance and judgment of the late Earl of Orford, of Houghton, in Norfolk; and it is said he obtained the great depth of chest and strength of his breed from crossing with the bull-dog. At his death, his greyhounds were sold by auction, and some of the best were purchased by Colonel Thornton; from one of them, Claret, which was put to a favourite bitch of Major Topham's, was produced Snowball, the best greyhound that ever appeared; although he was nearly equalled by his brothers, Major and Sylvia, who were all of the same litter. They were never beaten; and may 
be considered as examples of the most perfect greyhound.

The shape, make, elegant structure, and other characteristics of high blood, were equally distinguished in all the three; the colour of Snowball was a jet black, and, when in good running condition, was as fine in the skin as black satin. Major and Sylvia were singularly, but beautifully brindled. Snowball won ten large pieces of silver plate, and upwards of forty matches, his master having accepted every challenge, whatever might be the dogs of different counties which might be brought against him. His descendants have generally been also very successful.

The last match run by this celebrated dog, was against the famous greyhound, Speed, the property of Hall Plumber, Esq., of Bilton Park, Yorkshire. $\mathrm{He}$ gained the match; and so severe was the run, that Speed died soon after it. This terminated the career of Snowball's public coursing, as the owner, in consideration of his age, then declared he should never run another match.

Snowball was perhaps the fleetest of his race that ever ran; and like the Flying Childers, which was the swiftest of horses, may never be outstripped in rapidity of movements.

Wynken de Worde, who wrote a work on sports in 1496 , describes what then constituted the most perfect form of the greyhound in his day, which holds good still :-

"Headed lyke a snake,

Necked lyke a drake,

Footed lyke a catte,

Taylled lyke a ratte,

Syded lyke a teme,

And chyned lyke a beame." 
The greyhound is the fleetest of all dogs, and is the only one who can cope with the hare in speed. The keenness with which he pursues his game is thus beautifully described by Somerville:-

"With emulation fired,

They strain to leave the field, top the barred gate,

O'er the deep ditch exulting bound, and brush

The thorny-twining hedge : the ridges bend

O'er their arched necks; with steady bounds by turns

Indulge their speed, or moderate their pace."

The greyhound never gives tongue, like other dogs of the chase, when in pursuit of his game.

Greyhounds have been held in much estimation for many centuries in Great Britain. In the time of King John they were accepted by him as payment in lieu of money for the renewal of grants, fines, and forfeitures due to the crown. There is one fine upon record, paid to that monarch in $\mathrm{I}_{20} \mathrm{O}$, which specifies "five hundred merks, ten horses, and ten leashes of greyhounds;" and we find another mentioned in I 2 IO, of "one swift horse, and six greyhounds."

The following curious circumstance is related by Froissart, of King Richard the Second, when confined in the castle of Flint; which we shall give in the author's words.

"And it was informed me, Kynge Richarde had a greyhounde called Mathe, who always waited upon the kynge, and would know no one else. For whensoever the kynge did ride, he that kept the greyhounde did let him lose, and he wolde streyght runne to the kynge, and fawne upon him, and leap with his fore-fete upon the kynge's shoulders. And as the kynge and the Erle of Derby talked togyder in the courte, the greyhounde, who was wont to leap upon the kynge, left the kynge, and came to the Erle of 
Derby, Duke Lancaster, and made to hym the same friendly continuance and chere as he was wont to do to the kynge. The duke, who knew not the greyhounde, demanded of the kynge what the greyhounde would do? 'Cosyn,' quod the kynge, 'it is a great good token to you, and an evil sygne to me.' 'Sir, how know ye that?' quod the duke. 'I know it well,' quod the kynge; 'the greyhounde maketh you chere this daye as King of England, as ye shall be, and I shall be deposed; the greyhounde hath this knowledge naturally, therefore, take him to you: he will follow you, and forsake me.' The duke understood well those words, and cheryshed the greyhounde, who would never after followe Kynge Richarde, but followed the Duke of Lancaster."

We have no information why the name "greyhound" was given to this dog. It is, in all probability, a corruption of "gazehound"; as a variety, or probably the same kind, was known in ancient times by this name, which name it no doubt acquired from hunting by the eye instead of the scent.

THE SCOTTISH HIGHLAND GREYHOUND, OR WOLF-DOG.

The Scottish Highland greyhound differs from the common greyhound in one essential particular, as he will either hunt in packs or singly. In size this dog is equal to the Irish greyhound, and very powerful, with great depth of chest, small loins, and his back slightly arched, and long legs. His general aspect is commanding and fierce; his head is long, and muzzle rather sharp; his ears pendulous, but not long; his eyes large, keen, and penetrating, half concealed among the long, stiff, bristly hair, with which his face is covered. His hair is shaggy and 
wiry, and his general colour reddish sand-colour, mixed with white; his tail is rough, which he carries somewhat in the manner of a staghound, but not quite so erect.

It was this noble dog which the Highland chieftains of Scotland used in former times in their grand hunting parties. The breed is now exceedingly rare, and in all likelihood will become extinct. One of the largest and finest which we have ever seen was possessed by the late Sir Walter Scott, Bart., and was an appropriate guardian for his unique seat of Abbotsford. He was the gift of the late spirited and high-minded chieftain Macdonnel, of Glengarry.

This fine specimen of the dog probably brought on himself premature old age by the excessive fatigue and exercise to which his natural ardour and love of sport inclined him, for he had the greatest pleasure in accompanying the common greyhounds; and although, from his great size and strength, he was not adapted for coursing, yet he not unfrequently turned and even ran down hares.

The name of this dog was Maida; and he lies buried at the gate of Abbotsford, which he long protected, as he had more the habits of a watchdog than those of the common greyhound. A gravestone is placed over him, with the figure of a dog cut on it, by $\mathrm{Mr}$ John Smith, of Melrose, and is thus inscribed :-

"Maida, tu marmorea dormis sub imagine, Maidæ Ad januam domini. Sit tibi terra levis!"

The Scottish Highland greyhound is endowed with an exquisite sense of smell. One of Glengarry's dogs, named Bran, when held on the leash, followed 
the track of a wounded stag; and that, too, in most unfavourable, rainy weather, for three successive days, at the end of which time the stag was shot. The present Glengarry wrote me an account of it, in which he mentions, "he," the stag, "was wounded just within nine miles of Invergarry-house, and was traced that night to the estate of Glenmoriston. At dusk in the evening the deer-stalkers placed a stone on each side of the last fresh print of his hoof, and another over it; and this they did each night following. On the succeeding morning they removed the upper stone, when the dog recovered the scent, and the stag was that day traced over a great part of Glenmoriston's grounds. On the third day he was re-traced to the lands of Glengarry, and there shot."

Glengarry also furnished me with the following gallant achievement of one of his dogs. He says, "My present dog, Comhstri, to great courage unites the quality of a gentle disposition, with much fidelity and attachment. Though not so large as some of his kindred, he is nevertheless as high-spirited and determined as any of his race, which the following circumstance will testify:-

"About three years ago, a deer from the wood of Derrygarbh, whose previous wounds had been healed, came out of Glengarry's pass, who wounded it in the body with a rifle bullet. The deerhounds were inmediately laid on the blood-track. The stag was started in the course of a few minutes; the dogs were instantly slipped, and the fine animal ran to bay in a deep pool of water, below a cascade, on the Garyquulach stream. Comhstri immediately plunged in and seized the stag by the throat, both went under water, surrounded with the white foam, slightly tinged with the deer's blood. The dog soon came to the surface to 
recover his breath, and before the stag could do so, Comhstri dived, and again seized him by the throat. The stag was soon after taken out of the pool dead."

We think it very probable that the Irish greyhound is only a variety of the same stock as the Highland greyhound. The former has much smoother hair than the Scottish breed.

\section{SECTION II.-DOGS USED IN SHOOTING.}

\section{THE ENGIISH POINTER.}

The Spanish pointer is the stock from which our English pointer was derived. $\mathrm{He}$ is much lighter in form, and much more rapid in his movements. $\mathrm{He}$ was originally produced by a cross of the Spanish pointer and the foxhound, and afterwards re-crossed with the harrier. $\mathrm{He}$ is now to be met with of a variety of sizes; but it has been found that when pointers are too large, they are far less active than those which are smaller; but in this particular there is a variety of tastes.

The pointer is possessed of a beautiful symmetry of frame; and in this respect is perhaps the most proportionably formed of all the canine race. His docility and pliability of temper are truly astonishing. $\mathrm{He}$ enjoys the sense of smelling in an exquisite degree.

In proportion as the breed of pointers diverge in blood from their Spanish progenitor, the difficulty of training them is experienced, and also that steadiness of the original, which renders them staunch for the field.

Pointers are never considered complete in training unless they are perfectly staunch to bird, dog, and gun ; which implies, first, standing singly to a bird or covey; secondly, to backing or pointing the moment he per- 
ceives another dog to stand at game; and thirdly, not to stir from his own point at the rising of any bird, or the firing of any gun in the field, provided the game is neither sprung nor started at which he himself originally pointed. Whenever a pointer is conscious of his own powers and education, he makes it his whole business to serve and amuse his master. At the same time he will also perform his work to others to whom he may be lent, and is sensible of the duty required of him the moment he enters the field.

Pointers are seldom used for any other kind of shooting than that of grouse, partridge, and snipe.

We are informed of Mr Daniel, that he possessed a pointer which would always go round close to the hedges of a field before he would quarter his ground; the dog being sensible that he most frequently found his game in the course of this circuit, and therefore very naturally took the middle road to discover it.

The following interesting fact was communicated to me by an eye-witness. The circumstance happened in Forfarshire, and is a strong proof of the reasoning powers of a dog. "Two or three weeks ago, an acquaintance and I were out shooting in this neighbourhood, when we saw the most beautiful thing we ever witnessed in the way of a point: one of our pointers, a bitch, was going over a stone dyke, about four feet high, and just as she made the leap, got the scent of some birds on the other side of the wall. She hung by her fore-legs until we came up. It appeared at the distance we were from her, as if her fore-legs had got fastened amongst the stones in the wall, and that she could not extricate herself. You may judge of our delight when coming up to find that it was her caution, for fear of flushing the birds, that prevented her from taking the leap. It is impossible adequately 
to convey to you in writing the beauty of this point!"

Was this mere instinct, or was it reason?

Sir Charles Stewart Menteath, Bart. of Closeburn, Dumfriesshire, had an extremely small and beautifully formed pointer. His length, from the point of the nose to the tip of the tail, was only two feet and half an inch. From one fore-foot to the other, across the shoulders, two feet; length of the head, six inches; girth of the chest, one foot three inches. He was a most perfect miniature of the best formed English pointer. His colour was white, with dark livercoloured patches on each side of the head, extending half-way down the neck; the ears, with some patches down the back, were also of the same colour; and numerous dark liver-coloured spots sprinkled his entire body. His olfactory sense exhibited itself in a high degree.

The late Earl of Lauderdale had some of the same dogs, which were broke in and pointed most admirably. Sir James Colquhoun, Bart. of Luss, had one of the same kind, which was considerably smaller than Sir C. Menteath's.

THE ENGLISH SETTER.

This celebrated breed was produced between the Spanish pointer, the English water-spaniel, and springer, which, by careful and assiduous cultivation, has attained a very high degree of perfection as a sporting dog. His figure is elegant, and his fur presents a very pleasing diversity of colour, with beautifully flowing hair, extremely villous on the lower margin of the tail, and in the middle of the belly.

The setter possesses all the high qualities of the pointer, with a greater degree of speed and natural 
vivacity of temper; he, however, is not so easily broken in as the pointer, and requires a certain degree of training every season to make him continue staunch. There have been, however, various instances of setters being self-taught.

The setter ranges with great speed, and is a very hardy dog. Many prefer him to the pointer; and if water is plentiful he is certainly more useful ; for his feet are much better defended against the sharp cutting of the heath than those of the pointer, as a great deal of hair grows between the toes, and round the ball of the foot, of which the pointer is almost destitute. Besides, he ranges much faster, and can endure much more fatigue. $\mathrm{He}$ can serve in thick coverts, where a pointer will not enter, and on this account is useful in woodcock-shooting, where springers or cockers are not kept.

Formerly the setter was used for the purpose of taking partridges with the draw-net; and was generally taught to squat down when the game was within a proper distance-hence the name setter.

The setter, besides his uses in the field, is much employed in duck-shooting, as he is an excellent swimmer, and takes the water very readily. $\mathrm{He}$ is naturally of a timid disposition, and much afraid of correction, which, if inflicted with too much severity, never fails to render him ever afterwards unfit for field-sports. He becomes what is termed blinked, and in this case is so overpowered with fear, when threatened with correction, that he will sink at his master's feet, and will steal away, after which it will be impossible to rouse him to further exertion in finding game; consequently it is of much consequence that both breaker and master should cautiously ascertain the dog's natural character. 
The following beautiful stanzas of Somerville finely depicts the style of ranging by the setter :-

"When Autumn smiles, all beauteous in decay, And paints each chequer'd grove with various hues, My setter ranges in the new-shorn fields, His nose in air erect; from ridge to ridge Panting he bounds, his quarter'd ground divides In equal intervals, nor careless leaves

One inch untried. At length the tainted gales His nostrils wide inhale ; quick joy elates His beating heart, which, awed by discipline Severe, he dares not own, but cautious creeps," etc.

\section{THE SPRINGER.}

The springer is supposed to have had its origin in Great Britain, although it is now to be met with in almost all countries. $\mathrm{He}$ is much used, and eagerly sought after in the wild sports of the East.

In form, the true English-bred springer differs but little from the setter, except in size, the former being about two-fifths less in height and strength, with a more delicate form, the ears longer in proportion, very soft and pliable, covered with long, silky, waving hair; the head is larger in proportion to the bulk of his body, and the nose red or black, the latter, however, being the surest mark of high-breeding; the tail is bushy and pendulous, and is always in motion when the dog is employed in pursuit of game.

Differently from other dogs used in shooting, both the springer and cocker give tongue the moment they either see or smell game, and this is an indication to the sportsmen, who generally station themselves on the skirts of the wood or covert, to which pheasants, woodcocks, and snipes are known to fly, when started.

It will thus be seen how admirably adapted the different varieties of dogs are for the particular kind 
of sport in which they are to be employed. The dogs of the chase give tongue whenever their game is started, and continue their cry until the object of their pursuit is run down, while their mingled and varied voices produce the most delightful, exhilarating, and harmonious sounds, so beautifully apostrophised by the poet of the Chase, as already quoted at page 679. The greyhound alone pursues his game in silence, which could not be otherwise, as every nerve is strained to outstrip his prey. And here we find in the springer and cocker, which are required for driving game, that shelter in thick coverts and brushwood, from their retreats, where a sportsman cannot penetrate, barking whenever they find either of those for which they are trained, and thus affording a premonitory warning to look out.

From the time the springer is thrown off in the field, he gives evident proofs of the pleasure he experiences in being thus employed, by the perpetual motion of his tail, which is termed feathering amongst sportsmen; and upon the increasing vibration of which, the experienced fowler well knows he is getting nearer to the object of his attraction. The more closely he approaches the game, the more energetic the dog becomes in his endeavour to succeed; tremulous whispers escape him as a symptom of doubt; but the moment this doubt is dispelled, and the game is found, his clamorous raptures break forth in full force. He expresses his gratification by loud and quick barking, which may be relied on as a proof that he has not sought in vain, leaving the happy owner exultingly to boast, that "he is in possession of at least one faithful domestic who never tells a lie."

The following anecdoie, related by $\mathrm{Mr}$ Blain, is 
one proof among many, showing that dogs are endowed with some wonderful instinctive knowledge, by which they can traverse with unerring certainty a country with which they are unacquainted, in pursuit of their master or former home. He says- "I took a spaniel, bred in London, forty-eight miles in the close rumble-tumble of a chaise into Essex, where she remained with me some months. - During the journey she was only once taken out of this close confinement for a few minutes in an inn yard. She proved useless as a sporting dog, and I gave her to a friend to breed from, who was on a visit with me. I accompanied him on his return from Essex, and she was brought back with us exactly in a similar manner to that in which she had been before taken; and it is most certain, that neither in going nor coming did she ever see twenty yards of the road. On our arrival in London she was removed to my friend's kennel, from whence she contrived to escape during the night, by digging her way out in a most extraordinary manner, and travelled the whole forty-eight miles back into Essex so expeditiously, that a servant found her at the door of my residence in the country, in the morning when she arose. The bitch remained at large during the day; but finding I was not in the country, she again set off in the evening, and returned to London; and in the morning once more presented herself at my friend's house in search of me."

\section{THE COCKER.}

This dog is considerably smaller than the springer, and is generally used for woodcock and snipe shooting. His diminutive size peculiarly fits him for ranging in low, thick coverts, for which purpose nature seems particularly to have adapted him. 
The form of the cocker is shorter and more compact than that of the springer: his head is rounder and his muzzle shorter; his ears are very long, and the limbs short and strong. The entire ears, neck, and body are covered with longer and more curled hair than the springer. He varies also from liver and white, red, red and white, or entirely livercoloured, with tanned legs and muzzle.

This beautiful and lively dog seems to have been produced originally by a cross between the small water-spaniel and the springer; as he not only resembles the figure of the latter, but also possesses many of his habits, combined with the lively and active disposition of them both. From the beauty and temper of the cocker, he has been very generally diffused throughout the kingdom, and on these accounts he is more frequently a companion of the parlour than used for the sports of the field.

It is the unalterable nature of the cocker to spring, flush, or start all the game before them, and they pursue without distinction, hare, pheasant, partridge, woodcock, snipe, quail, and plover. It consequently becomes necessary to hunt them within gun-shot of covert, and they never should be allowed, if possible, to go beyond call of the sportsman or his whistle. The following beautiful passage, from Somerville, depicts the cocker in field-sports :--

"But if the shady woods my cares employ,

In quest of feathered game my spaniels beat,

Puzzling th' entangled copse ; and from the brake

Push forth the whirling pheasant; higher in air

He waves his varied plumes, stretching away

With hasty wing. Soon from the uplifted tube

The mimic thunder bursts, the leaden death

O'ertakes him, and, with many a giddy whirl,

To earth he falls, and at my feet expires." 
THE SMALLER SPANIEL, OR KING CHARLES'S COCKER.

This beautiful little dog is considerably less than the cocker, to which he is closely allied in character and form; he has, however, much longer ears, and the tail is also longer in proportion to the size of his body. Like the springer and cocker, he is extremely fond of pursuing birds of all kinds, and like them, also, he gives tongue in the pursuit. His diminutive size unfits him for field sports. He is easily tired, and his legs too short to get through swampy ground.

King Charles the First was much attached to these dogs, and was always followed by from eight to a dozen of them. In all the portraits of that monarch or his family they were introduced; and in particular we may allude to the celebrated portrait by Vandyke.

The Dukes of Marlborough have preserved in the utmost purity this race of dogs; and the late General Maxwell, of Parkhill, Stirlingshire, had a beautiful breed of them. They were black, with the insides of their ears and legs tan-coloured: their ears were extremely long.

\section{THE LARGE WATER-SPANIEL.}

This dog is somewhat near the size of an ordinary setter but considerably stronger in the bone, and shorter in the legs. His head is long, and his muzzle moderately acute, his face quite smooth as well as his legs in front; his ears are long, which, together with his whole body, is covered with long, curled, crisped hair. His ordinary hue is dark liver-colour, with white legs, neck, and belly; and sometimes, although rarely met with, entirely black.

His sense of smell is very acute, and he swims and dives with very great dexterity; on which account 
he is very valuable as a retriever in wild-fowl shooting. He watches with minute observation the actions of his master, and whenever a bird is killed, he plunges instantly into the water and fetches it out, and lays it at the foot of his owner.

This dog is very acute at finding the haunts of wildfowl, and is easily taught to fetch and carry articles, and also to seek articles which have been lost; which latter quality obtained for him in former times the appellation of "the finder."

The native country of this dog is Spain; but we conceive that the variety we possess, which is a very distinct one, is not the pure breed as originally imported into this country, but that it is the produce of the large water-dog and the English setter, as it appears to be intermediate between these, not only in figure, but also in their united qualities.

THE SMALL WATER-SPANIEL, OR POODLE.

The small water-spaniel is supposed to be the offspring of the large water-dog and the cocker: he has all the appearance of the former in shape, and also in the thickly-coloured silky hairs. No dog whatever possesses more activity and buoyancy of spirit than this; and he is highly susceptible of instruction of almost any kind. He is a most dexterous diver; and so exquisite in his olfactory sense that he will find at the bottom of a river or pool, a stone which is thrown in by his master, even where there are many other stones. The poodle has been taught to perform many domestic offices, and those, too, with the utmost accuracy, such as to go errands, shut a door, ring a bell, fetch his master or mistress's shoes or gloves, etc. The general colour of this dog is white. He is 
very useful as a retriever, and will fetch anything out of the water.

\section{SECTION III.--WATCH-DOGS.}

\section{THE MASTIFF.}

The mastiff is a large and powerful dog, with a large flat head, and rather long and pendulous ears; his forehead large and broad; his muzzle wide and obtuse, his lips are full, loose, the upper one hanging considerably over the lower, at the two extremities of the mouth; his aspect is grave and somewhat sullen, and his bark loud and deep-toned; his chest is very wide and deep; his limbs strong, and the whole muscles of his frame full and largely developed.

There can be but little doubt but that the mastiff is a truly British dog, and had his origin in this country. We are borne out in this opinion by finding that, at so early a period as the time of the Roman empire, this country was celebrated on account of its dogs of this kind; and at the period when Great Britain was under the Roman yoke, an officer was appointed to live here, whose sole business was to breed, select, and send to Rome such as promised, by their size and strength, to become fit for the combats of the amphitheatre. Dr Caius informs us that three mastiffs were reckoned a match for a bear, and four for a lion.

From the large size and commanding aspect of the mastiff, he is naturally calculated to intimidate strangers; consequently is peculiarly well suited for the protection of large and extensive premises; and he watches them with the most scrupulous care and assiduity. $\mathrm{He}$ is so instinctively impressed with the importance of his charge, that he will lose his lite 
rather than abandon it. But with this commanding aspect, he possesses qualities of the best kind. $\mathrm{He}$ is remarkable for the great mildness of his disposition, when disencumbered of his charges, and is as solicitous to gain attention, and as faithfully grateful for favours, as the most diminutive of the canine race.

The mastiff has one peculiarity in his character which seems inherent:-his ferocity is always increased by the degree of restraint under which he is kept. If kept constantly on the chain, his temper is irritable and ferocious, and it is consequently dangerous for a stranger to approach him; from whence it evidently appears, that what may be considered a friendly kindness on one side, is always productive of confidence on the other. He seems conscious of his own great strength, power, and authority, and will seldom condescend to lower his dignity by servile fawning, while he appears to consider his services as only befitting a trust of the highest importance. This dog is naturally possessed of strong instinctive sensibility, speedily obtains a knowledge of all the duties required of him, and discharges them too with the most punctual assiduity. In the protection of houses, woodyards, gardens, and widely-extended manufactories, his vigilance is very striking: he makes regular rounds of the whole premises like a watchman, examines every part of them with a careful eye: his penetration reaches even the remotest corner, and not a spot is passed by until he is satisfied that all is in a state of perfect security. During the night he gives a signal of his presence by repeated and vociferous barkings, which are increased upon the least cause of alarm; and, contrary to the spirit of the bull-dog, whose invariable practice is to bite before he barks, the mastiff always warns before he attacks. 
This breed is now exceedingly difficult to be obtained in purity, from the various admixtures and experimental crosses which have lately taken place. The genuine English mastiff is now rarely to be seen, although we have dogs of various sizes and colours which go under that name. At Lyme Hall, in Cheshire, there is a pretty pure breed of these dogs. The true mastiff has very rarely been known to attack the human being, except under the most palpable provocation.

The mastiff usually shows a remarkable and peculiar warmth in his attachments, and, on the other hand, he is equally distinguished for inveteracy in his dislike. If he is once severely corrected or insulted, it is almost impossible to eradicate the feeling from his memory, and it is no less difficult to obtain a reconciliation with him. He seems conscious of his own strength, power, and authority, and will seldom condescend to lower his dignity by servile fawning; nor will he easily be induced to combat an inferior enemy, as will be illustrated by the following anecdote :-

A farmer in the neighbourhood of Falkirk had a large mastiff dog, which used to go regularly to church, and was always attended by a very small mongrel. In their way to and from church, in passing through the principal street, where there were always a number of butchers' dogs, who used to be very clamorous, although they never ventured actually to attack the church-going couple, probably having had proof of the mastiff's powers. One Sunday, however, the diminutive cur was induced to give battle to one of those dogs, which, the mastiff discovering, turned back, and the butcher's dog scampered off. The mastiff did not condescend to give chase, but taking up his little friend with his mouth, by the neck, carried him 
to the extreme end of the town and set him down, after which they quietly walked home together.

\section{THE BULL-DOG.}

There can be little doubt respecting the original country of this dog, as all authors are agreed in opinion that it is of British origin. Some writers affirm that it was the bull-dog and not the mastiff which was held in such high estimation when the Romans invaded Britain. But if it was the bull-dog it must have been a very different animal in point of size from what it is at the present day, for the best breed is now low in stature, very deep-chested, and strongly made about the shoulders and thighs; the muscles of both of which are extremely developed. His head is broad, his nose short and flat, with the under jaw projecting beyond the upper one, producing a fierce and disagreeable aspect. His eyes are distant, and prominent, appearing as if forced out of their sockets, and giving him a suspicious-like leer, which, with the distention of his nostrils, produce a contemptuous look; and in conjunction with these, the teeth being always exposed, he has the constant appearance of grinning, while he is perfectly placid. $\mathrm{He}$ is the most ferocious and unrelenting of the canine tribe, and is, doubtless, courageous beyond every other animal in the world; for no animal, however great in magnitude, will be exempt from his attack. He is hardly capable of any education, and seems fitted only for combat and ferocity. He gives no warning by barking when he attacks, and holds with such determined pertinacity the part that he seizes upon, that it is with the utmost difficulty he can be disengaged. He generally lays hold of the lip, tongue, or side of the cheek or eye, which he maintains in spite of the most desperate 
efforts of the animal to free himself from his antagonist ; and no command of his master will induce him to desist, which can only be effected by squeezing the windpipe till he is nearly choked; and instances have occurred where he has been mutilated and still maintained his grip. The thorough-bred bull-dog always attacks animals in front, seizing either on the head or throat, and when they lay hold of any of the extremities, it is reckoned a degeneracy from the original purity of blood.

This animal derived its name from his having been employed in former times in assaulting the bull; and he is used for the same brutal purpose at the present day, where such amusements are still practised; but these are, like cock-fighting, happily on the decline.

The first bull-baiting, of which we have a wellauthenticated account, took place in the reign of King John, in I 209, at Stamford, in Lincolnshire, and had its origin in the following circumstance:-William, Earl Warren, Lord Stamford, standing upon the walls of his castle, saw two bulls fighting for a cow in the castle-meadow, till all the butchers' dogs pursued one of the bulls (which was maddened by the noise and multitude) through the town. This sight so pleased the Earl, that he gave the castle-meadow, where the bulls' combat began, for a common to the butchers of the town, after the first grass was mowed, on condition that they should find "a mad bull" on a day six weeks before Christmas, for the continuance of that sport for ever. At Tutbury, in Staffordshire, about the year I374, an establishment of a similar nature was instituted, which was abolished by the praiseworthy and humane exertions of the Dule of Devonshire, in 1778 , as steward of Tutbury. 
There is a singular will, bearing date May i8th, I66I, by which a person named George Staverton, gave the whole rent of his dwelling-house, situated at Staines, in Middlesex (after two lives) to buy a bull annually for ever, which bull he gave to the poor of Workingham, in Berkshire, to be there baited, then killed and equitably divided; the offal, hide, and giftmoney (collected from the spectators) to be laid out in shoes and stockings, to be distributed among the children of the poor. These seem to be the principal donations mentioned in history upon which the practice of bull-baiting was unhappily founded originally, and has been since continued in this country under the plausible pretext of charity. To give it a still greater degree of singularity in the town of Workingham, St Thomas's day is annually dedicated to this sublime sport.

Many strenuous efforts have been made for the abolition of this barbarous and inhuman amusement; among these, the Rev. Dr Barry preached a sermon in the church of Workingham, at the request of the Rev. Mr Bremner, then resident clergyman of the parish, on Sunday, the 20th December I80I, being the day previous to the festival of St Thomas, which was afterwards published, and from which we extract the following excellent admonition :-

"Gracious God! benevolent Parent of the universe! what prodigy must he be in a Christian land, who could thus disgrace his nature by such gigantic infamy, at which the blood of a heathen, of a very Hottentot, might curdle! Two useful animals, the bull, which propagates our food, and the faithful dog, which protects our property, to be thus tormented! and for what purpose? Does it tend, as some have said,* to keep alive the spirit or the * Mr Windham, in the House of Commons. 
English character? In answer to this we must remark that the barbarous sport (if sport it can be called) was totally unknown to the ancient bravery of our ancestors ; was introduced into this country during the reign of a bad king; and earnestly do I pray to Almighty God that in the reign of a most pious and benevolent prince it may be for ever set aside. Cowards, of all men the least unmoved, can both inflict and witness cruelties. The heroes of a bull-bait, the patrons of mercenary pugilists, and the champions of a cock-fight, can produce, I should think, but few, if any, disciples brought up under their tuition, who have done service to their country, either as warriors or citizens; but abundant are the testimonies which have been registered at the gallows, of her devoted victims, trained up by these pursuits of bull-baiting!"

\section{THE BULL-TERRIER.}

The bull-terrier has now become a fixed variety of the dog: it was originally propagated by a cross between the bull-dog and terrier, and in point of form is handsomer than either of its progenitors. $\mathrm{He}$ is a sprightly and showy animal, and even better adapted for mischievous sport than either of those dogs from which he has sprung. $\mathrm{He}$ is airy and pleasant tempered, but possesses great fierceness when his energies are called into action. His head is rather square and large, his neck short and thick, his chest deep, and legs very strong and cleanly formed, his hair is stiff and hard. This dog has great strength of jaw, and he has risen into great reputation with gentlemen of the "fancy!" but it is to be lamented that the services of this excellent dog are too often misapplied, and his prowess turned to the most wanton mischief, such as fighting with other dogs, 
tearing to pieces the domestic cat, worrying and maiming unfortunate and starving stray dogs-sports which cannot fail to be viewed by the humane with horror, and cannot be too severely reprobated.

Some time ago, no fashionable young man could appear without a bull-terrier at his heels, which $\mathrm{Mr}$ Egan, a popular and amusing author of the day, depicted in the following graphic caricature:- "The new breed," says he, "which has become so truly the go that no rum or queer kiddy, or man of cash, from Tothil Street in the west, to north-eastern Holloway, far less any swell rising sixteen, with a black, purple, or green Indiaman round his squeeze, the corner of his variegated $d a b$ hanging from his pocket, and his pantaloons well creased and puckered, but must have a tike of the new cut at the heels of himself or his prad. The swells of Brunswick and the adjoining squares have dropped even the Newfoundland and the poodle, to be followed by one of a new edition of the dog."

The late Sir Walter Scott had a very intelligent dog of this breed, and kindly furnished me with the following account of him :- " The wisest dog I ever had was what is called the bull-terrier. I taught him to understand a great many words, insomuch that I am positive that the communication betwixt the canine species and ourselves might be greatly enlarged. Camp once bit the baker who was bringing bread to the family. I beat him, and explained the enormity of his offence; after which, to the last moment of his life, he never heard the least allusion to the story, in whatever voice or tone it was mentioned, without getting up and retiring into the darkest corner of the room, with a great appearance of distress. Then if you said, 'The baker was well paid,' or 'The 
baker was not hurt after all,' Camp came forth from his hiding-place, capered and barked, and rejoiced. When he was unable, towards the end of his life, to attend me when on horseback, he used to watch my return, and the servant used to tell him 'his master was coming down the hill, or through the moor,' and although he did not use any gesture to explain his meaning, Camp was never known to mistake him, but either went out at the front to go up the hill, or at the back to get down to the moor-side. He certainly had a singular knowledge of spoken language."

\section{THE NEWFOUNDLAND DOG.}

The powerful and beautiful dog known by this name, now plentifully distributed throughout Great Britain, is not the pure breed, but a race procured by a mixture with others. The pure Newfoundland dog, however, differs from the Esquimaux and other wild races, in his muzzle being much broader, his lips more hanging, his ears partially pendulous, his back longer, and more loose in his general structure.

The Newfoundland dog, as now improved in this country, is unquestionably one of the noblest of the canine race, both in regard to his majestic appearance, large size and strength, and surprising intelligence. The full-sized animal measures from the nose to the end of the tail about six and a half feet, from the one fore-foot to the other, over the shoulders, five feet eight inches; girth behind the shoulders, three feet four inches; round the head, across the ears, two feet ; round the upper part of the fore-leg, ten inches; length of the head, fourteen inches; his feet are webbed, or with the membrane connecting the toes extending to their tips, which enables him to swim with great ease and dexterity. $\mathrm{He}$ is covered with 
long, shaggy, flowing hair, and his fore-legs are feathered behind, as well as the hinder ones, as far as the hock-joint. The fur in many is white, with black or dark brown patches and spots, but they are also met with plentifully of almost all colours, as chocolate, fawn-colour, cream colour, and black.

This dog is not remarkable for symmetry of form, or in the setting on of his legs, and consequently his motion is somewhat awkward and loose, and therefore he is not distinguished for speed-a defect which might be remedied by breeding, were an improvement in that particular desirable.

The Newfoundland dog is docile to a very great degree, and nothing can exceed his affection. Naturally athletic and active, he is ever eager to be employed, and seems delighted to perform any little office required of him. Nature has given him a great share of emulation, and hence to be surpassed or overcome is to him the occasion of great pain. Active on every emergency, he is the friend of all, and is naturally without the least disposition to quarrel with other animals. He seldom or never offers offence, but will not receive an insult or injury with impunity. Such is the capacity of his understanding, that he can be taught almost everything which man can inculcate, and of which his own strength and frame are capable. His sagacity can only be exceeded by his energies, and he perseveres with unabated ardour in whatever shape he is employed, and while he has a hope of success he will never slacken in his efforts to attain it. The amazing pliability of his temper peculiarly fits him for the use of man, and he never shrinks from any service which may be required of him, but undertakes it with an ardour proportionate to the difficulty of the execution. 
Sagacity, and a peculiarly faithful attachment to the human species, are characteristics inseparable from this dog; and hence he is ever on the alert to ward off from his master every impending danger, and to free him from every peril to which he may be exposed. $\mathrm{He}$ is endowed with an astonishing degree of courage, whether to resent an insult or to defend his friends, even at the risk of his own life.

As a proof of the intellectual superiority of the Newfoundland dog, we may mention, that innumerable instances have occurred of their having saved persons from drowning, of their own accord, which the following facts will prove :-

A farmer's servant man, passing a deep water on horseback, with a woman behind him, the latter slipped off, and after a few struggles sank to the bottom, unperceived by the clown, who rode on. Some spectators at a distance hastened to the waterside, and beheld the efforts of a Newfoundland dog which attended the careless servant. The friendly animal had perceived the woman fall, and instantly swam to the place and dived after her. At first he brought her cap to the shore, but looking at it he dived again, and brought up her cloak: when he had brought it to the water side, he looked at it for some moments as if with the anger of disappointment, and rushed back to the place the third time, and, to the wonder of the people present, he brought up the woman, over whom he expressed every demonstration of joy. She soon recovered, and was afterwards housekeeper to a clergyman in Norfolk.

In the summer of 1792 a gentleman went to Portsmouth for the benefit of sea-bathing. He was conveyed in one of the machines into the water; but being unacquainted with the steepness of the shore, 
and no swimmer, he found himself, the instant he quitted the vehicle, out of his depth. The state of alarm into which he was thrown increased his danger, and, unnoticed by the person who attended the machine, he would inevitably have been drowned, had not a large Newfoundland dog, which by accident was standing on the shore and observed his distress, plunged in to his assistance: the animal seized him by the hair, and conducted him safely to the land; but it was some time before he recovered. The gentleman afterwards purchased the dog at a high price, and preserved him as a treasure of equal value with his whole fortune.

As a proof that dogs understand, in many instances, spoken language, we have seen a Newfoundland $\operatorname{dog}$ fetch any particular thing which his master asked him. A large assemblage of various articles were promiscuously piled up in the middle of the floor of an adjoining room, such as shoes, boots, gloves, sticks, umbrellas, horse-whips, spurs, hats, slippers, etc. We were asked what we wished him to bring, and instantly on it being named, the dog went to the next room and fetched it. We first requested a pair of slippers to be brought, when Cæsar brought one, laid it down, and returned for the other. He was next required to fetch a stick; he instantly obeyed, and returned with the one his master invariably used, although there were several others in the heap.

\section{THE SHEPHERD'S DOG.}

The shepherd's dog is characterised by his upright ears, sometimes slightly drooping at the tips, sharp muzzle, his long and rather woolly-like hair, with a great villosity on the under part of the tail, 
as well as on the back of the fore-legs. The body is somewhat elongated, and the legs rather short. There is a character which pervades most of the individuals of this race, namely, that their feet are provided with one, and frequently with two superfluous toes, which are destitute of bony adhesion or muscular action, and hang dangling at the hind part of the leg more like an unnatural excrescence than a necessary appendage to the animal. But as "Nature has made nothing in vain," these must certainly be destined for some useful purpose with which we are yet unacquainted. These dew-claws, as they are termed, are sometimes found in the spaniel, pointer, and cur-dogs; in the two former they are generally cut off at an early age, as they are impediments in covers, and frequently get torn, thereby creating sores, and sometimes rendering the dog lame for days together. The shepherd's dog seldom reaches two feet in height.

This truly useful and intelligent animal is exceeded by no other member of the canine race. $\mathrm{He}$ is remarkable for his placid, obedient, serene, and grateful character. $\mathrm{He}$ is ever alive to the slightest indication of his master's wishes, prompt and gratified to execute them, and he seems to enjoy the highest delight when employed in any kind of useful service. Formed by nature with an instinctive propensity to industry, he is never more pleased than in exerting his talents for the benefit of man, and in affording constant proofs of his inviolable attachment.

The inherent calmness, patience, and devoted faithfulness of the shepherd's dog render him insensible to all attractions beyond the arduous duties connected with the flock under his care. When once properly trained, he not only becomes perfectly ac- 
quainted with the extent of his beat, but also with every individual in the flock; he will most carefully select his own, and drive off such as encroach on his limits. This appears the more extraordinary, when we consider the vast extent of mountain country (especially in Scotland), and the numerous flocks committed to the charge of a single shepherd, a duty which he could not possibly perform but for the invaluable services of this sagacious animal. A word or signal from the shepherd will direct the dog so as to conduct the flock to any point required, and that signal he will obey with energy and unerring certainty.

Some have imagined the shepherd's dog destitute of the social characters for which most dogs are remarkable, and that his nature is sullen and sleepy; these, however, originate in mistaking the true virtues of the animal. Accustomed to the company of his master alone in those dreary and unfrequented wilds, he naturally acquires a thoughtful and expressive gravity; and, like man himself, when unaccustomed to society, he becomes habitually taciturn and shy. This is his character in remote situations; for where he is accustomed to see strangers, he exhibits all the amiable qualities of other dogs. His sagacity, affection, fidelity, comprehensive penetration and activity are exceeded by no other dogs; the frequent excursions he necessarily makes during the day afford him sufficient exercise. This is his character all over Europe.

Of the wonderful susceptibility for training, the following anecdotes will sufficiently illustrate the intelligence of the shepherd's dog.

Few instances of greater sagacity in a dog can be adduced than the following: - The owner himself 
having been hanged some years before for sheepstealing, this fact, among others respecting his dog, was fully authenticated by evidence on the trial. When the man intended to steal a sheep, he did not do it himself, but directed his dog to perform the business. With this view, under pretence of looking at the sheep with an intention to purchase them, he went over the grounds with the dog at his feet, to whom he secretly gave a signal, so as to let him know the individuals he wanted, to the number of perhaps from ten to twenty, out of a flock of some hundreds; he then went away, and from a distance of several miles sent back the dog by himself in the night-time, who picked out the individual sheep that had been pointed out to him, separated them from the rest, and drove them before him the distance of ten or twelve miles, till he came up with his master, to whom he delivered his charge.

"My dog Sirrah," says the late celebrated author, Mr James Hogg, the Ettrick Shepherd, "was, beyond all comparison, the best dog I ever saw: he was of a surly and unsociable temper-disdaining all flattery, he refused to be caressed; but his attention to my commands and interests will never again, perhaps, be equalled by any of the canine race. When I first saw him, a drover was leading him in a rope; he was both lean and hungry, and far from being a beautiful animal, for he was almost all black, and had a grim face, striped with dark brown. The man had bought him of a boy somewhere on the border for three shillings, and had fed him very ill on his journey. I thought I discovered a sullen intelligence in his countenance, notwithstanding his dejected and forlorn appearance: I gave the drover a guinea for him, and I believe there never was a guinea so well laid out, 
at least I am satisfied that I never laid out one to so good a purpose. He was scarcely a year old, and knew so little of herding that he had never turned a sheep in his life; but as soon as he discovered that it was his duty to do so, and that it obliged me, I can never forget with what anxiety and eagerness he learned his different evolutions. He would try every way deliberately until he found out what I wanted him to do, and when I once made him understand a direction, he never forgot or mistook it again. Well as I knew him, he often astonished me; for, when hard pressed in accomplishing the task that he was put to, he had expedients of the moment that bespoke a great share of the reasoning faculty."

Amongst other remarkable exploits of Sirrah's as illustrative of his sagacity, $\mathrm{Mr}$ Hogg relates that, upon one occasion, about seven hundred lambs, which were under his care at weaning-time, broke up at midnight, and scampered off in three divisions, across the neighbouring hills, in spite of all that he and an assistant could do to keep them together. "Sirrah," cried the shepherd, in great affliction, "my man, they're a' awa'." The night was so dark that he could not see Sirrah; but the faithful animal heard and knew the import of his master's words, - words such as, of all others, were sure to set him on the alert; and without more ado, he silently set off in quest of the recreant flock. Meanwhile the shepherd and his companion did not fail to do all in their power to recover their lost charge; they spent the whole night in scouring the hills for miles round, but of neither the lambs nor Sirrah could they obtain the slightest trace. It was the most extraordinary circumstance that had ever occurred in the annals of pastoral life. They had nothing for it, day having 
dawned, but to return to their master, and inform him that they had lost his whole flock of lambs, and knew not what was become of them. "On our way home, however," says Hogg, "we discovered a lot of lambs at the bottom of a deep ravine, called the "Flesh Cleuch,' and the indefatigable Sirrah standing in front of them, looking round for some relief, but still true to his charge. The sun was then up, and when we first came in view, we concluded it was one of the divisions which Sirrah had been unable to manage, until he came to that commanding situation. But what was our astonishment, when we discovered that not one lamb of the whole flock was wanting! How he had got all the divisions collected in the dark is beyond my comprehension. The charge was left entirely to himself, from midnight until the rising sun; and if all the shepherds in the forest had been there to have assisted him, they could not have effected it with greater propriety. All that I can further say is, that I never felt so grateful to any creature under the sun as I did to my honest Sirrah that morning."

\section{THE COACH-DOG, OR DALMATIAN.}

This dog, once so common an attendant upon gentlemen's carriages, has now become exceedingly scarce. Some authors have confounded him with the Danish dog. Buffon and others imagine him to be the harrier of Bengal; but his native country is Dalmatia, a mountainous district of European Turkey.

In Britain the Dalmatian has only been used for ornament, while in Italy he was long the harrier of that country, and used for upwards of two centuries as a dog of the chase. He has also been used as a pointer. for which he has been found even more adapted than for hunting; and many instances have occurred where 
he has turned out very staunch. His form is handsome, as if a medium between the foxhound and pointer; his head, however, is more acute than that of the latter, and his ears fully longer; his general colour is white, and his entire skin covered with small black or reddish-brown spots. The pure breed has tanned cheeks and black ears. In size he is considerably smaller than the Danish dog. A barbarous opinion prevailed at one time in this country, that the Dalmatian looked better with his ears cropped; and we remember the time when hardly one that we met with but had been denuded of those elegant appendages, but happily this depraved taste has now become nearly extinct.

Lord Maynard lost a dog of this kind in France, which he in vain endeavoured to recover while in that country. He returned to England, where he had not long arrived before the dog appeared at his residence; but the mode of his return has remained for ever unexplained, though it is probable that the dog's sagacity, when he had made his escape from confinement, prompted him to go to the sea-coast, where he must have found means to get on board some vessel bound for the opposite shore. 



\section{A P P E N D IX.}

\section{RULES OF RACING.}

These Rules apply to all meetings held under the sanction of the Jockey Club, and to all races run at such meetings.

\section{Interpretation of Words and Phrases.}

3. A "recognised meeting" is a meeting held under the sanction of the Jockey Club, or other Turf authority, of the country in which it is held, or (where no such authority exists) under these rules.

The Turf authorities of the United Kingdom are :-

The Jockey Club.

The National Hunt Committee.

The Turf Club of Ireland.

The Irish National Hunt Steeple Chase Committee.

The Channel Islands Racing and Hunt Club.

"Stewards."-Wherever the word "Steward" or "Stewards" is used, it means Steward or Stewards of the meeting, or their duly appointed deputy or deputies.

Ireland, the Channel Islands, and the Isle of Man are not included in the expression "Great Britain," but they are in the expression "the United Kingdom."

The "Registry Office" is the office for the time being appointed as the registry office by the Jockey Club.

"Registered" and "Registration" mean "Registered" and "Registration" at such office.

N.B.-The Present Registry Office is Messrs Weatherby's Office, 6 Old Burlington Street, London, W., and at Newmarket.

The "Racing Calendar," comprising the "Sheet Calendar," the "Monthly Calendar," the "Book Calendars," of "Races Past" and of "Races to Come," and the "Stud Book" are the works published under those names respectively by authority of the Jockey Club.

A "horse" includes mare, gelding, colt, and filly.

A " maiden" is a horse which has never won a race (other than a match or private sweepstakes) at any recognised meeting in the United Kingdom, or in any other country; A maiden means a maiden at the time of the start.

A "race" means plate, cup, sweepstakes, private sweepstakes, or match, but not steeple chase, hurdle race, or hunters' race on the flat.

A "plate" is a race to be run for money or other prize without any stake being made by the owners of the horses engaged.

A "sweepstakes" is a race in which stakes are made by the owners of three $\mathrm{cr}$ more horses engaged, to be paid to the winner or other horses placed, and any such race is still a sweepstakes when money or other prize is added.

A "private sweepstakes" is one to which no money is added, and which has not been advertised previous to closing.

A match or "catchweights" means one for which the riders need not weigh before or after the race. 
A "cup" is any prize not given in money.

The "Forfeit List" is a record of arrears published under the sanction of the Turf a uthorities of the United Kingdom.

A "handicap" is a race in which the weights to be carried by the horses are adjusted by the handicapper for the purpose of equalising their chances of winning.

A "free handicap" is one in which no liability for stake or forfeit is incurred until acceptance.

A "Nursery handicap" is one confined to two years old horses.

A "post race" is a race for which a person may, under one subscription, enter two or more horses, and run any one or more of them as the conditions prescribe.

A "produce race" is one to be run for by the produce of the horses named or described at the time of entry.

A "selling race" is one, the conditions of which require that every horse running, if a loser, may be claimed, and if the winner, must be offered for sale by auction, or be liable to be claimed.

A "weight-for-age" race is any race which is not a handicap or a selling race.

The "nominator" is the person in whose name a horse is entered for a race.

"Owner" includes "part owner" or "lessee."

"Authorised agent" means an agent appointed by a document signed by the owner and lodged at the Registry Office, or, if for a single meeting only, with the Clerk of the Course. "Authorised agent" includes sub-agent, if authority to appoint a sub-agent, is provided by the document.

"Arrears" are any sums unpaid in respect of fines, compensation, fees, entrance money, stakes, subscriptions, forfeits, and purchase money in races with selling conditions.

The "time of entry" means the time fixed for closing.

"Started"-Every horse shall be considered as having started which is under the Starter's orders when the advance flag has been raised.

\section{Calculation of Time.}

2. When the last day for doing anything under these rules falls on a Sunday, it may be done on the following Monday, unless a race to which such act relates is appointed for that day, in which case it must be done on the previous Saturday.

3. "A Month" means a calendar month: "a Day" means twenty-four hours.

4. Racing Season. - No race shall be run earlier than in the week which includes the 25 th of March unless that be the one next before Easter Sunday, in which case races may be run in the week preceding, nor later than in the week which includes the 22 nd of November, but in the interval between the last and first named weeks the Jockey Club will sanction weight-for-age races for four years old and upwards over a distance of two miles and upwards, with a minimum weight of 9 st $7 \mathrm{lb}$, and of not less value than $£^{200}$, such races not to be selling races, and to be under the authority of the National Hunt Committee.

\section{Stewards of Meetings.}

5. There must be at least two Stewards for every meeting.

6 . Each Steward may appoint a deputy at any time, or if there be only one Steward present he shall, in case of necessity, appoint one or more persons to act with him. If none of the Stewards are present, the Clerk of the Course shall request two or more persons to act during the absence of such Stewards.

7. Powers of the Stewards. - The Stewards of a meeting have full power to make (and if necessary to vary) all such arrangements for the conduct of the meeting as they think fit, and, under special circumstances, to put off any races from day to day until a Sunday intervene.

8. The Stewards have control over, and they and the Stewards of the Jockey Club have free access to, all stands, rooms, enclosures, and other places used for the purposes of the meeting.

9. They shall exclude from all places under their control :-

(i.) Every person who is warned off Newmarket Heath. 
(ii.) Every person whose name has been published in the Unpaid Forfeit List, until the default is cleared.

(iii.) Every person who has been reported as a defaulter under Corrupt Practices, etc., until it has been officially notified that his default is cleared.

(iv.) Every person who has been declared by the Turf authorities of, or by the Stewards of, any recognised meeting in this or any other country, to have been guilty of any corrupt or fraudulent practice on the turf.

(v.) They have power to exclude at their discretion any person from all or any places under their control.

I0. The Stewards have power to regulate and control the conduct of all officials, and of all trainers, jockeys, grooms, and persons attendant on horses.

II. They have power to punish at their discretion any person subject to their control with a fine not exceeding $£ 50$, and with suspension from acting or riding at the same meeting, and to report to the Stewards of the Jockey Club should they consider any further fine or punishment necessary.

12. The Stewards have power to determine all questions arising in reference to racing at the meeting subject to appeal under Disputes, Objections, etc., and should no decision have been arrived at by the Stewards, within seven days of an objection being lodged, the Clerk of the Course shall then report the case to the Stewards of the Jockey Club, who may at their discretion decide the matter, and, if they consider there has been any negligence, may order any additional expense arising therefrom to be defrayed out of the funds of the meeting at which the case occurred.

13. The Stewards have power to call for proof that a horse is neither itself dis. qualified in any respect ; nor nominated by, nor the property, wholly or in part, of a disqualified person; and in default of such proof being given to their satisfaction they may declare the horse disqualified.

14. The Stewards have power at any time to order an examination by such person or persons as they think fit, of any horse entered for a race, or which has run in a race.

15. The Stewards, as such, shall not entertain any disputes relating to bets.

16. Powers of the Stewards of the Jockey Club. - The Stewards of the Jockey Club may impose any fine not exceeding 6 roo.

17. The Stewards of the Jockey Club have power, at their discretion, to grant, and to withdraw, licences to Officials, Jockeys, and Race Courses, to fix the dates on which all meetings shall be held, to make inquiry into, and deal with, any matters relating to racing, and to warn any person off Newmarket Heath.

18. The Stewards of the Jockey Club take no cognisance of any disputes or claims with respect to bets, but they will give effect to an official report of default made to them by the Committee of the Subscription Rooms, at Newmarket, or at Tattersall's.

\section{Officials.}

19. The following officials shall be appointed for every meeting, subject to the approval of the Stewards, viz., Clerk of the Course, Handicapper, Stakeholder, Clerk of the Scales, Starter, and Judge, each of whom, as a qualification for his office, requires a licence to be granted annually by the Stewards of the Jockey Club. No two offices, except those of Clerk of the Course and Stakeholder, shall be held by one person unless by special permission.

20. In case of emergency, the Stewards may, during a meeting, appoint an unlicensed substitute to fill any of the above-named offices for that meeting only.

21. Every complaint against an official shall be made to the Stewards in writing, signed by the complainant.

22. Clerk of the Course.-The Clerk of the Course, or his authorised substitute, is the sole person responsible to the Stewards for the general arrangements of the meeting, and shall see that all courses are properly measured and marked.

23. The Clerk of the Course shall arrange for the publication, under the sanction of the Stewards, of a daily official card of the races, containing the conditions of each race, as published in the Racing Calendar, the names, or other description, of the horses engaged with a number attached to each and such further particulars as the Stewards may require. A horse may appear on the card 
in the name of the owner instead of that of the nominator, and the Stewards may, under special circumstances, grant permission for a horse to run in the name of the trainer. The Clerk of the Course shall, in such latter case, make a report to the Registry Office, stating the grounds upon which the permission was granted.

24. The Clerk of the Course shall make a return to the Registry Office of any Deputy Steward or official appointed, of all complaints to and decisions of the Stewards, of all fines inflicted, and of all horses sold or claimed.

25. The Clerk of the Course shall have in his possession, for the information of the Stewards, a list of persons warned off Newmarket Heath, and of suspended jockeys, and also a copy of the latest Monthly Forfeit List, and he shall not allow any horse which, or the owner or nominator of which, is in the Forfeit List to start for any race.

26. Handicapper. - The Handicapper shall append to the weights for every handicap the day and hour from which winners will be liable to a penalty, and no alteration shall be made after publication, except that, by express permission of the Stewards, a weight may be affixed to a horse duly entered whose name or weight has been omitted from the handicap.

27. No handicapper shall handicap at any other meeting held during the same week, except by express permission of the Stewards of the Jockey Club. He must attend the meeting either personally or by licensed deputy. The name of the handicapper attending the meeting shall appear in the Calendar containing the report.

28. Stakeholder.-The Stakeholder shall not allow a jockey to be weighed out for any horse until such horse's stake for that race, and the forfeits for every horse belonging to the same owner, or standing in his name, the jockey's fee, and any arrears claimed under these rules, have been paid, or the Stakeholder shall himself be liable: Provided that he shall not be liable for arrears in respect of a meeting elsewhere than the place at which the race is run, unless notice of such arrears being overdue has been published in the Forfeit List, or delivered in writing, signed by the party claiming the arrears, to him, and to the person indebted, before ten in the evening preceding the race.

29. The Stakeholder shall at the expiration of fifteen days after the meeting render an account and pay over all stakes and added money to the persons entitled, and at the same time notify at the Registry Office all arrears, and one month before the publication of the next available Forfeit List he shall further notify at the Registry Office all arrears then remaining unpaid, for publication therein.

30. Clerk of the Scales. - The Clerk of the Scales shall exhibit the number (as allotted on the official card) of each horse for which a jockey has been weighed out, and shall forthwith furnish the starter with a list of such numbers, and the numbers shall not be taken down till the horses are started.

31. If extra, or special, weight be declared for any horse, such weight shall be exhibited with the number, also any declaration to win, or alteration of colours.

32. He shall in all cases weigh in the riders of the horses placed by the Judge and report to the Stewards any jockey not presenting himself to be weighed in.

33. The Clerk of the Scales shall always put $2 \mathrm{lb}$ extra into the scale to prove that the horse has not carried too much weight.

34. He shall at the close of each day's racing send a return to the Registry Office of the weights carried in every race and the names of the jockeys, specifying over-weight if any.

35. The Starter.-The Starter shall give all orders necessary for securing a fair start. The horses must be started from a walk, and also, as far as possible, in a line, but they may be started at such reasonable distance behind the starting post as the Starter thinks necessary.

36. Any jockey presuming to start, or even to put his horse into a trot or canter, with a view to take any advantage, before the flags are dropped, wilfully turning his horse round, not starting when the flags are dropped, hanging behind, or refusing to obey the commands of the starter in any respect whatever, shall be reported to the Stewards.

37. A start in front of the starting-post, or on a wrong course, or before the appointed time, is void, and the horses must be started again as soon as practicable.

38. The Starter shall report to the Stewards the time at which each race was 
started; and shall also report by whom, or by what cause, any delay was occasioned.

39. The Judge.-The judge, or his authorised substitute, must occupy the judge's box at the time the horses pass the winning post. He must announce his decision immediately, and such decision shall be final, unless an objection to the winner, or any placed horses, is made and sustained : Provided that this rule shall not prevent a judge from correcting any mistake, such correction being subject to confirmation by the Stewards.

40. The judge shall, at the close of each day's racing, sign and send a report of the result of each race to the Registry Office.

\section{Regulations for Race Meetings.}

41. All Race-courses must be licensed, and all meetings sanctioned, by the Stewards of the Jockey Club.

42. The conditions of every race before closing, and the full programme of every meeting, before it takes place, must be advertised in the "Racing Calendar," and no alteration can be made in the conditions of any race after the last advertisement.

43. The last rule shall not preclude the addition of more money to a race before the time of closing, or of a new race to the programme of a meeting subsequent to the publication of the last Calendar issued previous to the meeting being held.

44. The Stewards of the Jockey Club may at their discretion prohibit the advertisement of any race or meeting in the "Racing Calendar," or call upon the Stewards to alter or expunge any conditions, even after advertisement.

45. At every meeting advertised in the "Racing Calendar," one-half at least of the total amount guaranteed for prizes shall be apportioned to races of a mile or over for three-years-old or upwards, and of this half not less than a moiety shall be for races of a mile and a quarter or upwards; but where more than one Meeting is held at the same place during the current racing season the apportionment may be calculated over each Meeting, or over all the Meetings; provided that in the case of Meetings where races are already closed, the Stewards of the Jockey Club shall have power to relax the above Rule as to the apportionment of the money. No two-years-old shall run in any race with more than 200 sov. added before the Ist of June.

46. There shall be no race of less distance than five furlongs.

47. The programme of each day shall be subject to the following regulations :-

(i) There shall be at least two races of a mile or upwards of the minimum aggregate distance of two miles and a half. These races must differ at least one furlong in distance, and they shall fill with five entries. Neither of these races shall be open to two-years-old ; and one of them shall be neither a handicap nor a race with selling conditions.

(ii) There shall not be more than two races, to which three-years-old or upwards are admitted, of less than seven furlongs, and of these two races only one may be less than six furlongs.

(iii) Of the races confined to two-years-old, there shall not be more than two races a day before September Ist, nor more than three a day at any time.

(iv) Matches and private sweepstakes may be sanctioned by the Stewards, independent of the above regulations.

48. The advertisements must state the days on which the meeting is to be held, the dates for closing the stakes, the names of the persons to receive nominations and declarations of forfeit, and a place or places for the entry for every race, the dates when weights for the handicaps will be published and when forfeits will be due, and, before the time fixed for closing (races for future years excepted), the names of two or more persons as Stewards, and of the Judge, Starter, Clerk of the Course, Handicanper, Stakeholder, and Clerk of the Scales. 


\section{Omitted Conditions.}

49. When the weights are omitted from the advertised conditions of any race, the colts shall carry 9st, and fillies 8st IIlb, subject to penalties and allowances. If the horses be of different age, the weight shall be fixed by the sanctioned scale appended to these rules.

50. When no course is mentioned, it shall be as follows :-

If two years old, six furlongs.

If three years old, one mile.

If four years old, two miles.

If five years old, or upwards, four miles.

And if the horses be of different age, the course shall be fixed by the age of the youngest.

At any meeting where there are two or more courses of the same distance, the special course shall be selected by the Stewards.

51. When no day is fixed for a race, it shall be run on the last day of the meeting, unless otherwise agreed by all the parties engaged, and sanctioned by the Stewards.

\section{Special Rules applicable to Handicaps, Produce Races, and Selling Races. - Handicaps.}

52. The top weight to be allotted in a handicap shall not be less than 9st.

If in a handicap for which there is a minor forfeit declared by a fixed time, the highest weight accepting was originally less than 9 st, it shall be raised to that weight, and the other acceptances equally.

It shall be in the power of Stewards, by notice in the programme, to extend this last-mentioned Rule to the highest weight left in at ten o'clock the preceding evening in handicaps for plates and stakes, where there is no declaration of forfeit, provided the weights are fixed the night before running.

53. In handicaps there shall be no clause permitting an alteration of the weights after publication by the claim of a selling allowance.

54. Produce Races.-If the produce of a mare is dropped before the Ist of January, or if there is no produce, or if the produce is dead when dropped, a declaration to that effect by the time prescribed in the conditions renders the entry of such mare void; if twins are dropped, the nominator shall at the same time declare to which of them the engagement attaches.

55. Selling Races.-All lads who, while under age, have, of their own free will, and with the consent of their parents or guardians, bound themselves to a trainer for a term of not less than three years, are permitted during their apprenticeship to claim $51 \mathrm{lb}$ allowance in all selling races which are not handicaps, provided no horse carry less than $6 \mathrm{st}$. They will be entitled to this $5 \mathrm{lb}$ allowance for one year (or 365 days) after winning their first race in any country.

56 . In no selling race shall the lowest selling price be less than $50 \mathrm{sov}$.

Every horse running shall, if the winner be liable to be claimed for the selling price by the owner of any other horse engaged in the race, or his authorised agent, but if it is a condition of the race that the winner is to be sold by auction, the sale shall take place immediately after the race, and the surplus over the selling price shall be divided between the owner of the second horse and the race fund.

If sold, or bought in, the lorse shall not leave the place of sale without permission of the auctioneer, and a written order given for his delivery; and if the horse be not paid for, or the price secured to the satisfaction of the auctioneer within a quarter of an hour, he may put the horse up a second time, and the purchaser at the first sale shall be responsible for any deficiency arising from the second, and shall be treated as a defaulter until it is paid.

Whoever issues the delivery order for a horse sold, bought in, or claimed, is responsible for the money, and shall, if required, pay the same over to the person entitled the day after the horse is delivered.

57. All other horses starting may be claimed for the selling price plus the 
value of the stakes or plate by the owners of horses running in the race, or their authorised agents.

(i) Owners of horses placed shall have priority of claim in the order of their places, and if the owners of two or more horses having equal rights claim, they are to draw lots. The owner of the winner has the last claim.

(ii) No person can claim more than one horse.

(iii) Every claim must be made in writing to the Clerk of the Scales within a quarter of an hour after the winner has passed the scale, but subject to the Rules in cases of objection.

58. The price of every horse claimed must be paid to the Stakeholder, and an order given by him for the delivery of the horse.

In the case of a horse being claimed, if the price be not paid before seven o'clock in the evening of the day of the race, the claimant forfeits his right. But the owner may insist on the claimant taking and paying for the horse, and if he refuse or neglect to do so, he shall be treated as a defaulter in respect of the price.

59. If a horse walk over (or there be no second horse placed) for a selling race, the winner is still liable to be sold, but any surplus above the selling price will go to the fund.

60 . The foregoing Rules relating to claiming and selling races, in cases where the horse placed first is objected to, are subject, when practicable, to the following provisions :-

(i) If an objection be made before the horse lias been sold, the time for selling and claiming shall be fixed by the Stewards.

(ii) Where the objection is made and sustained after the horse has been sold or bought in, the sale or buying in, and any claim in respect of the horse placed second, shall be annulled, and all moneys paid thereunder returned. The horse disqualified shall be liable to be claimed as a beaten horse, and the Stewards shall fix a time for the exercise of such right of claiming, and a time and place for the sale of the horse adjudged to be the winner.

Should the Stewards find the above provisions impracticable they shall make a report to the Registry Office.

(iii) In the case of a dead heat, the time for claiming or selling is postponed until the dead heat is run off. In case of a division, each of the horses dividing is a winner for the purposes of the Rules relating to claiming and selling, and if an auction race, both shall be put up to auction, and any surplus shall be divided, half to go between those horses, and half to the Race Fund.

$6 \mathrm{r}$. In all other races with selling conditions only such horses as run to be sold shall be liable to be sold or claimed; but, with this exception, and the allowance to apprentices, the foregoing rules relating to selling races shall apply.

\section{Race-Horses.-Age.}

62. The age of a horse shall be reckoned as beginning on the Ist of January, in the year in which he is foaled.

63. Yearlings shall not run for any race.

64. Two-years-old shall not run more than six furlongs before the rst of July, nor shall they run for handicaps before the Ist of September, nor in handicaps with older horses.

65. Names of Horses.-(i) A name can only be claimed for a horse by application at the Registry Office in London, with the description according to rule, when, if there is no other horse of the same name, the name will be registered and published in the first Sheet Calendar after it has been claimed, and will, from the date of publication, be the horse's name under these rules.

(ii) In the case of a horse which has been entered elsewhere than in Great Britain, under the same name as one already registered, the name may be claimed for him if accompanied by a numeral, and the name with the numeral will then be registered and published as his name.

(iii) If the same name be simultaneously claimed for two or more horses, the order of priority shall be determined by lot at the Registry Office. 
66. Disqualifications of Horses.-If a horse run at any unrecognised meeting, he is disqualified for all races to which these Rules apply.

67 . No horse is qualified to be entered or run which is wholly or partly the property of, or in any way under the care or superintendence of, a disqualified person, or to run in any race so long as any disqualified person has any interest in the horse's winnings in that race.

68. Any horse which has been the subject of fraudulent practice may, at the discretion of the Stewards of the Jockey Club, be disqualified for such time and for such races as they shall determine.

69. Foreign Horses. - A horse foaled out of the United Kingdom shall not be qualified to start for any race until there have been deposited at the Registry Office, and a fee of 5 s paid on each certificate (I) such a foreign certificate, and (2) such a certificate of age as are next mentioned, that is to say :-

The foreign certificate must state the age, sex, pedigree, and colour of the horse, and any mark by which it may be distinguished, and must be signed by the secretary or other officer of some approved racing club of the country in which the horse was foaled, or by some magistrate, mayor, or public officer of that country.

The Stewards of the Jockey Club may from time to time approve any racing club for the purposes of this Rule, and prescribe the magistrate, mayor, or public officer, by whom a foreign certificate must be signed. They may also require any further proof or confirmation in any particular case, and may declare any horse disqualified in default thereof.

The certificate of age must be signed by a veterinary surgeon in the United Kingdom, approved for this purpose by the Stewards of the Jockey Club, either by general order or in the particular case.

70. A horse which has been out of the United Kingdom (otherwise than as a foal at the foot of his dam) before having run in Great Britain, shall not be qualified to start for any race until a certificate of age, signed as in the last Rule mentioned, has been deposited at the Registry Office.

NOTE. - The Stewards of the Jockey Club have approved generally of certificates given by the Members of the Royal College of Veterinary Surgeons and by persons holding the Highland and Agricultural Society's diploma.

\section{Entries, Subscriptions, Declarations of Forfeit, and Acceptances for} Races.

7I. Every entry shall close, and every declaration of forfeit or of acceptance shall be fixed to be made at IO P.M., and upon Tuesdays only, except in the case of races closing within four days of their being run.

All entries for handicaps for which the weights are to appear before the week in which a Meeting is held, must be in the Sheet Calendar at least one week before the weights are published, and for those handicaps for which the weights are to appear during the week of the Meeting, the entries shall close at least twenty-four hours before the publication of the weights.

No declaration of forfeit shall be fixed to be made for races for two-years-old only, between the second Tuesday in October, when they are yearlings, and the last Tuesday in March in the following year.

72. A horse is not qualified to run for any race unless he is duly entered for the same.

73. All entries made elsewhere than at the Registry Office (except entries made during the week of, or on the Saturday preceding, the Meeting) shall be lodged at that office within forty-eight hours, or, if intended for publication in the next Calendar, within twelve hours after the day of closing.

In default of observance of this rule, the receiver of nominations shall be reported to the Stewards of the Jockey Club, and unless the nominator can prove to their satisfaction that the entry was made in due time, it shall be void.

74. The list of entries shall be closed at the advertised time, and no entry shall be admitted on any ground after that time.

75. An entry or subscription may, before the time of closing, be altered or withdrawn. 
76. Allowances, when practicable under the conditions of the race, must be claimed at the time of entry, or they shall not be allowed.

77. Form of Entry or Nomination.-Entries shall be made in writing, signed by the owner of the horse, or his authorised agent, and declarations of forfeit in like manner by the owner of the engagement or his authorised agent, subject to the rules relating to sales with engagements. Entries made by telegram shall be equally binding, but must be confirmed in writing. Declarations of forfeits made by telegram shall be confirmed in writing, or the forfeit shall not be saved. The above confirmations must be made as soon as possible, and, if the Stewards consider that there has been any unnecessary delay, they may inflict a fine.

78. A horse cannot be entered in the real or assumed name of any person as his owner unless that person's interest or property in the horse is at least equal to that of any other one person, and has been so registered.

79. The entry shall be in the name, or assumed name of one person, and shall state the name, or assumed name, of the owner, the name of the horse, if registered, or his description, according to rule, if name not registered.

8o. In entering a horse whose name has not been registered, it shall be described by stating the age, the colour (when possible), and whether a horse, mare, or gelding, and the registered or Stud-book names or description of its sire and dam. If the dam was covered by more than one stallion the names of all must be stated. In all cases such pedigree and description must be given as will clearly distinguish the horse entered from all other horses, and if the pedigree of the sire or dam be unknown, such further particulars must be given as to where, when, and from whom it was purchased, or obtained, as will identify it.

$8 \mathrm{r}$. Whenever the name under which a horse has run at any recognised Meeting in any country is changed, or abandoned, his old name, as well as his new rame or description, must be given in every entry until the change has been published in two Monthly Calendars or the Book Calendar, and a fee of $£ 5$ paid.

82. No alteration or addition shall be made in any entry after the time fixed for closing, except in cases expressly sanctioned by these rules.

83. In the event of a horse being entered for a race with an incorrect or imperfect description according to the rules of entry, such error or omission may, it accidental, be corrected on payment of a fine of 65 at any time before the horse's number is exhibited for that race, provided that the identity of the horse be satisfactorily proved.

If the above correction be not made, or the horse struck out, within seven days after the error has been officially brought to the knowledge of the owner, or his agent, or if any horse runs without the prescribed correction having been made, the Stewards of the Jockey Club may inflict fines upon, or otherwise deal with any persons responsible for such errors.

NOTE.-The omission, under fine, of horses from races for which they are not qualified, is now dealt with under Rule ro8.

84. When, subsequently to the time fixed for closing, a registered name has been published for any horse entered, the entry may be amended by inserting such name in addition to the name or description given in the original entry.

85. A subscriber to a race may transfer the right of entry under his subscription to any other person.

86. Subscriptions and all entries or rights of entry under them become void on the death of the subscriber, and entries (except such as are made under another person's subscription) become void on the death of the person in whose name they are made.

87. If either party to a match die the match is off.

88. An acceptance of the weight for a free handicap shall be considered as equivalent to an entry, but if the horse be wrongly described the acceptance shall be void.

89. In making an entry for a produce race, the produce is entered by specifying the dam and sire, or sires.

90. No horse shall be considered as struck out of any of his engagements until the owner, or some duly authorised person, shall have given notice in writing, or by telegraph to be confirmed in writing, at the Registry Office or to the Clerk of the Course where the horse is engaged. 
The notification of the death of a horse shall be equivalent to a declaration of forfeit.

\section{Sale with Engagements.}

91. When a horse is sold with his engagements, or any part of them, the seller cannot strike the horse out of any of such engagements, and, on default of the purchaser, remains liable for the amount of the forfeits in each, but should the horse, or his owner, appear in the Forfeit List, the seller may, at any time before their names are removed from the list, strike the horse out of any engagement, if he can by this means reduce his liability.

92. Sales, with engagements, by private treaty must be registered to entitle either seller or purchaser to a vail himself of the conditions of the preceding rule; but when the horse is sold by public auction or claimed out of a selling race the advertised conditions of either the race or sale are sufficient evidence. If certain engagements only be specified those only are sold with the horse.

NOTE.-The following are known and recognised as "Lord Exeter's Conditions":-

"The horses are sold without their engagements, but the purchaser has the right of running for any of them by paying half the stake, and in the event of the horse winning, or being entitled to second or third money, one-third shall be paid to the vendor, but the vendor reserves to himself the right of striking the horse out of any race, in time to save a minor forfeit or discount, unless the purchaser shall give notice that he wishes to run for any particular race, when he will become liable for half the stake or forfeit. Horses purchased under Lord Exeter's conditions cannot be re-sold under the same conditions without the written consent of the original vendor."

\section{Assumed Names and Partnerships.}

93. An assumed name cannot be used unless annually registered, and the fee paid. A registration continues effectual only during the current year.

(i) A person cannot register more than one assumed name at the same time, nor can he use his real name so long as he has a registered one.

(ii) An assumed name may be changed at any time by registering a new assumed name.

(iii) A person cannot register as his assumed name one which has been already registered by any other person, or the real name of any owner of race-horses.

(iv) Any person who has registered an assumed name may at any time abandon it by giving written notice at the Registry Office; such notice of cancellation shall be published in the next Racing Calendar, after which all entries which have been made in the assumed name, shall be altered to the real name of the owner.

94. Partnerships, etc.-All partnerships and the name and address of every person having any interest in a horse, the relative proportions of such interest, and the terms of any sale with contingencies, lease, or arrangement, must be signed by all the parties, or their authorised agents, and lodged at the Registry Office, or with the Clerk of the Course for transmission to the Registry Office, before a horse sold with contingencies, or leased, or which is a joint property, can be entered or start for any race, and the document shall state with whom the power of entry or declaration of forfeit rests, and all partners shall be jointly and severally liable for every stake or forfeit. No part owner shall assign his share or any part thereof in a horse without the consent of his partners. A fee of I sov. shall be paid on each horse, and all partnerships, sales with contingencies, or leases, shall be published in the real or assumed names in the next available Racing Calendar. Any termination or severance must be notified within fouteen days to the Registry Office for publication, or a second fee will be incurred.

\section{Jockeys' Licenses, etc.}

95. (i) No jockey or apprentice shall, after the last day of the week in which he shall have first ridden, ride in any race until he shall have obtained a license from the Sicwards of the Jockey Club. Such license must be applied for annually: 
with full name and address, at the Registry Office, and will only be granted on the condition that a jockey is not an owner of any race-horse. Under exceptional circumstances, leave may be granted to jockeys, who are also trainers, to own one or more horses, provided that such horse or horses are trained in their own stables.

(ii) Any person who shall employ a jockey, in contravention of this rule, shall be liable to be fined by the Stewards of the Jockey Club.

(iii) A list of the licensed jockeys shall be published annually in the Racing Calendar.

(iv) Every jockey shall pay I sov., and every apprentice Ios, for his license, to be applied as his subscription to the Bentinck Benevolent and Provident Fund.

(v) No jockey whose license has been withdrawn, or refused on the ground of misconduct, will be eligible to ride trials, or be allowed in any weighing-room, stand, or enclosure, during the time his suspension from riding continues.

96. Any gentleman wishing to ride in races on even terms with jockeys shall obtain a permission, current till revoked, from the Stewards of the Jockey Club, and make a donation of $£ 5$ to the Bentinck Benevolent Fund.

97. Jockeys' Retainers. - In the absence of special agreement, a jockey's retainer terminates at the end of the racing season. Half the agreed retaining fee must be paid in advance, and the remainder at the termination of the retainer.

98. Every jockey at the termination of his apprenticeship is free to form engagements for himself, irrespective of any which have been made for him during such apprenticeship.

The terms of all agreements shall be registered, and a fee of $5 \mathrm{~s}$ paid.

99. Employers retaining the same jockey have precedence according to the priority of their retainers.

100. The Stewards of the Jockey Club may adjudicate between persons claiming the services of any jockey, and on disputes between jockeys and their employers, and have power to cancel any agreement between them.

IOr. Jockeys' Fees.-In the absence of special agreement to ride for a lower sum, the fee to a winning jockey shall be $£ 5,5 \mathrm{~s}$, and to a losing jockey $£ 3,3 \mathrm{~s}$, and no further charge shall be made, except when requested to leave home for the purpose of riding, in which case the cost of travelling expenses, and $\oint_{\mathrm{I}} \mathrm{a}$ day for living, shall be charged to the owner, or divided between the owners, at whose request he left home.

(a) In the case of a dead heat, if the owners divide, each jockey shall be paid a winning fee. In case of a run off, no additional fee shall be paid; but when either horse is ridden by a fresh jockey, the rider on each occasion is entitled to the prescribed fee. stakes.

All jockeys' fees shall be paid to the Stakeholder at the same time as the

Every jockey sending in a charge for expenses at a meeting shall state at the same time what charge has been made to other owners for attending the same meeting.

I02. Stable Lads.-(i) No trainer shall engage any stable servant who has previously been in a training stable without referring to his last employer, and receiving a satisfactory reply in writing.

(ii) Any trainer infringing this rule and continuing to employ such servant after notice has been served on him by the late employer or through the Registry Office shall be reported to the Stewards of the Jockey Club.

(iii) Any servant prevented by this rule from obtaining or retaining employment shall have the right of appeal to the Stewards of the Jockey Club.

\section{Racing Colours.}

I03. Racing colours shall be registered either annually on payment of $5 \mathrm{~s}$, or for the life of the person registering on payment of 5 sov. Colours so registered shall not be taken by any other person.

All disputes as to the right to particular colours shall be settled by the Stewards of the Jockey Club. 
104. Any person running a horse in colours other than those registered in his own or assumed name, without a special declaration over-night to the Clerk o1 the Course (at a time to be prescribed), shall be fined not less than I sov., nor more than Io sov.

A special declaration is also required where the owner is not the nominator.

\section{Entrance Money, Forfeits, and Stakes.}

105. Entrance money, forfeits, stakes, and arrears, must be paid in cash (if so required) to the Clerk of the Course, or authorised stakeholder, and entrance money must (if so required) be paid at the time of entry.

106. Entrance money shall go to the race fund of the meeting, unless otherwise specified in the conditions of the race and subject to the application of surplus under Prizes (see p. 73I).

107. Entrance fees, subscriptions, stakes, and forfeits shall be in pounds, not guineas.

108. The nominator is liable, as well as every partner in the horse at the time of nomination, for the entrance money and stake or forfeit. A person making a wrong nomination is equally liable, but where a horse has been accidentally entered for a race for which he is not qualified, he may be withdrawn on payment of a fine, in lieu of forfeit, of 5 sov. at any time before the race, the fines under this rule to go as the forfeits.

A subscriber to a sweepstakes is liable for the stake or forfeit; but if he transfer the right of entry to any other person he is liable only in case of default by the transferee.

I09. A person taking an entry under another person's subscription, where forfeit must be declared by a particular time shall, if he do not declare forfeit by that time, be considered to have taken the engagement upon himself, and it shall be transferred to his name.

I IO. A jockey shall not be weighed out for any horse for a race unless there have been previously paid (I) any stake, forfeit, entrance money or fee payable by the owner or nominator, in respect of that race; (2) all arrears due from any person for such horse, or due for the same or any other horse from any person by whom such horse is wholly or partly owned, or in whose name or under whose subscription he is entered; and (3) the jockey's fee.

\section{The Unpaid Forfeit List.}

III. An Unpaid Forfeit List shall be kept at the Registry Office, and shall be published in the Sheet Calendar after the Newmarket July Meeting, and again at the conclusion of the Racing season in every year. It shall include all arrears which have been notified by the Stakeholder of any recognised meeting in the United Kingdom, or as otherwise provided under these rules, and shall state the real name or names, and also the assumed name or names (if any) of the persons from whom, and the horses in respect of which, the same are due. "Arrears" which have been so published must be paid directly into the Registry Office, with fees according to rule, and until so paid shall not be removed from the list.

II2. Where a person is prevented by these rules from entering or starting a horse for any race without paying arrears for which he would not otherwise be liable, he may, by paying the same, enter or start the horse and place the arrears on the Forfeit List as due to himself, and in like manner the seller of a horse with engagements may, if compelled to pay them by the purchaser's default, place the amount on the Forfeit List, as due from the purchaser to himself.

II3. So long as the name of a person is in the Forfeit List, no horse can be entered by him or under his subscription for any race, whether acting as an agent or otherwise, and no horse which has been entered by him, or in his name, or under his subscription, or of which he is, or was at the time of entry, wholly or partly the owner, can run for any race; and no horse which shall be proved to the satisfaction of the Stewards to be directly or indirectly under the care, training, management, or superintendence of a person whose default has been twice published in the Racing Calendar, shall be qualified to be entered or run for any race. 
So long as any horse is in the Forfeit List, such horse shall not be qualified to be entered or run for any race.

This rule shall not prevent the entry for a Produce stake of mares and stallions that are in the Forfeit List.

The disabilities under this rule extend to all arrears officially notified by a recognised Turf authority of any country.

II4. A corrected alphabetical Index of the horses and owners in the last Forfeit List and Irish Forfeit List shall be published in the first Calendar of every month during the racing season. Such monthly list shall commence not less than three years before the time at which it is published, and shall be carried down to and include the latest Forfeit List which has been published in the Sheet Calendar as above nentioned.

I I5. If a horse which, or the owner of which, is in the Forfeit List be entered for any race in contravention of these rules, the nominator of such horse may be fined $t 50$.

\section{Weights, Penalties, and Allowances.}

II6. No horse shall carry less than 6 st in any race.

II7. Penalties. - In estimating the amount a horse has won in any one or more races, account shall be taken of all cups or moneys, whether derived from stakes, bonus, or any other source, gained by him for his owner or of any other person, deducting only his own stake and interest.

I18. Winnings during the year shall include all prizes to the time appointed for the start, and apply to all races in any country, and include dividing, walking over, or receiving forfeit.

Penalties for winning a fixed sum shall be understood to mean for winning it in one race unless specified to the contrary.

I19. No horse shall carry extra weight for having run second, or in any lower place in a race.

I20. Extra weights shall not be incurred in respect of matches or private sweepstakes.

I2I. Penalties are not cumulative unless so declared by the conditions of the race.

I22. When any race is in dispute both the horse that came in first and any horse claiming the race shall be liable to all the penalties attaching to the winner of that race till the matter be decided.

For penalties on dividing after dead heats (see Dead Heats, p. 730).

123. All penalties and allowances shall be calculated according to the amount of the value of each race as reported in the Racing Calendar.

I24. Allowances.-Allowances must be claimed at the time of entry where practicable (see Entries, Subscriptions, etc., p. 724), but omission to claim is not a source of disqualification, and a claim for allowance to which a horse is not entitled does not disqualify unless carried out at scale.

I25. No horse shall receive allowance of weight, or be relieved from extra weight, for having been beaten in one or more races; provided that this rule shall not prohibit maiden allowances, or allowances to horses that have not won within a specified time, or races of a specified value.

I26. Allowances to the produce of untried horses or mares are for the produce of horses whose produce never won a race in the United Kingdom, but these allowances can only be claimed for horses bred in the United Kingdom.

127. Allowances are cumulative unless otherwise specified.

\section{Weighing out, Exhibiting Numbers, etc.}

I28. The Weighing-Room.-No person shall, without special leave from the Stewards, be admitted to the weighing-room except the owner, trainer, and jockey, or other person having the care of a horse engaged in the race, and any person refusing to leave shall be reported to the Stewards.

129. Weighing out. - Every jockey must be weighed for a specified horse by the Clerk of the Scales, at the appointed place, not less than a quarter of an hour before the time fixed for the race, and the numbers shall then be exhibited as soon 
as possible. In exceptional cases, or where the delay of a previous race has rendered punctuality impossible, the Stewards may extend the time allowed for weighing, declaring weight, and for exhibiting the numbers.

I 30. When the numbers have been exhibited, no alteration or addition can be made without the leave of the Stewards, whose reasons for such permission shall be reported at the Registry Office. Should any horse not start whose number has been exhibited, the owner and any other person responsible may be fined, or otherwise dealt with, at the discretion of the Stewards.

I 3 I. If a jockey intend to carry over-weight, he must declare the amount thereof at the time of weighing out, or, if in doubt as to his proper weight he may declare the weight he intends to carry.

132. It is optional for the jockey to weigh out or in with his bridle, and the Clerk of the Scales shall, if requested, allow rlb for a curb or double bridle, but if a horse run in a hood, muzzle, martingale, breastplate, or clothing, it must be put into the scale, and included in the jockey's weight.

133. No whip or substitute for a whip shall be allowed in the scales.

\section{Starting.}

I34. The horses must be started by the official Starter or his authorised substitute.

135. Every horse shall be at the post, ready to start, at the appointed time.

I 36. All jockeys, on arrival at the starting post, must immediately place themselves under the Starter's orders.

I37. No horse, when once under the Starter's hands, shall go back, except in the case of an accident.

138. The horses shall (so far as is practicable) be drawn up before the start in an order to be determined by lots to be drawn by the jockeys at the time of weighing out.

\section{Running and Walking Over.}

139. An owner running two or more horses in a race may declare to win with one of them, and such declaration must be made at scale. A jockey riding a horse with which the owner was not declared to win, must on no account stop such horse, except in favour of the stable companion on whose behalf declaration to win has been made.

140. (i) A horse which crosses another in any part of the race so as to interfere with that or any other horse's chance is liable to disqualification, unless it be proved that he was two clear lengths in front when he crossed.

(ii) If a horse or his jockey jostle another horse, or jockey, the aggressor is disqualified unless it be proved that the jostle was wholly caused by the fault of some other jockey, or that the jostled horse or his jockey was partly in fault.

(iii) If a horse run the wrong side of a post he must turn back and run the course from such post.

I4I. If a race has been run by all the horses at wrong weights, or over a wrong course, or distance, or before the appointed time, or if the Judge is not in the box at the time the horses pass the winning post, the Stewards shall order the race to be run again the same day if practicable, but if otherwise, it shall be void.

I42. Walking Over.-When one horse pays forfeit for a match, the other need not walk-over, but for a sweepstakes even if all the horses but one, have declared forfeit, that horse must walk-over, except by the written consent of the persons who pay forfeit ; in the case of a plate, the consent of the Stewards is necessary.

I 43. On a division after a dead heat it shall not be necessary for a horse to walkover.

\section{Dead Heats.}

144. Every horse running a dead heat for first place shall be deemed the winner of the race until the dead heat is run off or the owners agree to divide, and, if the owners agree to divide, each horse which divides shall be deemed a winner 
of the race, and liable to any penalty for the full amount he would have received if he had won.

I 45 (i) A dead heat for the first place shall be run off on the same day at a time to be appointed by the Stewards, unless the owners agree to divide. The other horses shall be deemed to have been beaten, but they shall be entitled to their places (if any) as if the race had been finally determined the first time.

(ii) In running off a dead heat, the rules as to declaration of over-weight, weighing out, and weighing in, shall apply, but the same jockeys need not ride.

(iii) If, in running a dead heat off, either horse should be disqualified, it shall be decided by the Stewards whether the disqualification shall extend to the loss of the second place, and if so, the horse that originally ran third shall be entitied to the second place.

I 46 . When a dead heat is run for second place, and an objection is made to the winner of the race, if such objection be declared valid in time for the dead heat to be run off on the day of the race, the Stewards may direct it to be run off accord. ingly: otherwise the horses which ran the dead heat shall divide.

147. If a dead heat be run by two or more horses for second or any lower place in a race, the orvners shall divide, subject to the provisions of the last preceding rule.

I48. When owners divide they shall divide equally all the moneys or other prizes which any of them could take if the dead heat were run off.

149. If the dividing owners cannot agree as to which of them is to have a cup or other prize which cannot be divided, the question shall be determined by lot by the Stewards who shall decide what sum of money shall be paid by the owner who takes such cup or other indivisible prize to the other owners or owner.

I50. On a dead heat for a match the match is off.

\section{Weighing In.}

I51. Every jockey must, immediately after pulling up, ride his horse to the place of weighing, dismount, and present himself to be weighed by the clerk of the scales: Provided that, if a jockey be prevented from riding to the place of weighing by reason of accident or illness, by which he or his horse is disabled, he may walk or be carried to the scales.

I52. If a horse carry more than two pounds over his proper or declared weight he is disqualified, unless the Stewards be satisfied that such excess of weight has been caused by wet.

I53. If a jockey do not present himself to weigh in, or be short of weight, or be guilty of any fraudulent practice with respect to weight or weighing, or dismount before reaching the scales, or touch (except accidently) any person or thing other than his own equipments before weighing in, his horse is disqualified, unless he can satisfy the Stewards that he was justified by extraordinary circumstances.

\section{Prizes.}

154. The value of prizes not in money must be advertised.

I55. Prizes, stakes and forfeits in a race belong to the winner, except as otherwise declared in the conditions.

155. No plate or sweepstakes shall be run for unless the clear value to the winner (calculated as in Weights, Penalties, etc., p. 729), in case the race be run by two or more horses, will amount to $£$ Ioo. But if the value would amount to $£ \mathrm{IoO}$, if the race were so run, a horse may walk over, a though he thereby receives less than $£$ roo.

I57. In all races with not less than 5 entries, the second horse shall at least save his stake and entrance.

I58. When a sweepstakes has been so reduced by the death of subscribers that the payments to second or other horses, according to conditions, would reduce the value, if run for, to less than $£ 100$, the winner shall receive a clear $£ 100$, and only the balance, if any, be divided proportionally between the other horses entitled to participate in the stakes.

I j9. In all races, should there be iny surplus from entrance or subscription 
over the advertised value it shall be paid to the winner, unless provided by the conditions to go to other horses in the race. A race may be declared void provided the number of entries required by advertisement be not obtained, but the value of a prize once advertised shall not be reduced.

I6o. When a cup or plate or any added money is advertised to be run for, it shall be given in the event of a walk-over.

When a walk-over (except after a dead heat) is the result of arrangement by the owners of horses engaged, neither a cup nor any portion of the advertised money need be given.

I6I. Any money or prize which by the conditions is to go to the horse placed second, or in any lower place, shall, if the winner have walked over or no horse has been placed second, be dealt with as follows, namely :-

(i) If part of the stakes or plate it shall go to the winner ; or

(ii) If a separate donation from the race fund, or any other source, it shall not be given at all; or

(iii) If entrance money for the race, it shall go to the race fund of the meeting.

I62. No prize shall be given to any but to the first four horses placed.

I63. No deductions shall be made from the value of any race except such as are gained by other horses in the race, and except Clerks, stakeholding, and weighing fees, as fixed by rule, or any sums assigned, under the conditions of a race to the Bentinck Benevolent or Rous Memorial Funds.

164. If a race be never run or be void, stakes, forfeits, and entrance money shall be returned.

I65. A race may be declared void if no qualified horse cover the course according to rule.

\section{Disputes, Objections, Appeals, etc.}

166. Every objection shall be decided by the Stewards, but their decision shall (if they are not the Stewards of the Jockey Club) be subject to appeal to the Stewards of the Jockey Club, so far as relates to points involving the interpretation of these rules, or to any question, other than a question of fact; on which there shall be no appeal unless by leave of the Stewards and with the consent of the Stewards of the Jockey Club. Notice of appeal must be given in writing to the Clerk of the Course within forty-eight hours of the decision being made known.

I67. All disputes, objections, and appeals referred to or brought before the Stewards of the Jockey Club for their decision, shall be decided by the three Stewards; if only two Stewards be present they shall fix upon a third person, being a member of the Club, in lieu of the absent Steward, but the Stewards may call in any other members of the Jockey Club to their assistance, or may, if they think the importance or difficulty of the case requires such a course, refer it to a general meeting.

168. Objections, when and how made.-(i) If an objection to a horse engaged in a race be made not later than half-past ten in the morning of the day of the race, the Stewards may require his qualification to be proved before the race; and in default of such proof being given to their satisfaction, they may declare him disqualified.

(ii) An objection to the distance of a course officially designated must be made before a race. once.

(iii) An objection to any decision of the Clerk of the Scales must be made at

(iv) An objection to a horse, on the ground of a cross, jostle, or any act on the part of his jockey, or of his not having run the proper course, or of the race having been run on a wrong course, or of any other matter occurring in the race, must be made within a quarter of an hour after the winner has weighed in, unless, under special circumstances, the Stewards are satisfied that it could not have been made within that time.

(v) An objection on the ground,

(a) That the horse which ran was not the horse which he was represented 
to be at the time of entry, or that he was not qualified under the conditions of the race, or

(b) That he has run in contravention of the rules of partnership, may be received within fourteen days of the conclusion of a meeting.

(vi) An objection on the ground that a horse is disqualified by reason of any default entered in the Forfeit List, may be received within six months after the race.

(vii) In any other case an objection must be made within twenty-four hours of the race being run, exclusive of Sunday, save in the case of any fraud, or wilful mis-statement, when there shall be no limit to the time for objecting, provided the Stewards are satisfied there has been no unnecessary delay on the part of the objector.

169. Every objection shall be in writing, and must be signed by the owner of some horse engaged in the race, or by his authorised agent, trainer, jockey, or groom, and must be made to one of the Stewards, to the Clerk of the Course, or Clerk of the Scales; the objector shall, at the time he makes the objection, deposit in the hands of the Stakeholder the sum of 5 sov., and on an appeal, an additional sum of Io sov. shall be lodged by the appellant. If the case be decided against the depositor, his deposit shall be forfeited to the Bentinck Benevolent or Rous Memorial Funds, unless the Stewards who heard the case, or the Stewards of the Jockey Club on the appeal, shall certify that there was good and reasonable ground for the objection of the appeal.

I70. An objection may also be made without deposit by a Steward or licensed official of a meeting in his official capacity.

17I. An objection cannot be withdrawn without leave of the Stewards.

172. No horse shall be disqualified on account of any error or violation of rule in the entry, which might have been corrected on payment of a fine, but the Stewards may inflict fines upon, or otherwise deal with, any persons responsible for such errors.

I73. If an objection to a horse which has won, or been placed in a race, be declared valid, the horse shall be regarded as having been last in the race, and the other horses shall take positions accordingly.

174. All costs and expenses in relation to determining an objection or conducting an enquiry, and any reasonable compensation for outlay incurred, shall be paid by such person, or persons, and in such proportions, as the Stewards shall direct.

I75. Pending the determination of an objection, any prize which the horse objected to may have won or may win in the race, shall be withheld until the objection is determined, and any forfeit payable by the owner of any other horse shall be paid to and held by the Stakeholder for the person who may be entitled to it.

\section{Corrupt Practices and Disqualifications of Persons.}

I76. (i) If any person corruptly give or offer, or promise directly or indirectly, any bribe in any form to any person having official duties in relation to a race or race-horse, or to any trainer, jockey, or agent, or to any other person having charge of, or access to, any race-horse ; or

(ii) If any person having official duties in relation to a race, or if any trainer, jockey, agent, or other person, having charge of, or access to, any race-horse corruptly accept or offer to accept any bribe, in any form; or

(iii) Wilfully enter or cause to be entered or to start for any race a horse which he knows or believes to be disqualified; or

(iv) If any person be proved to the satisfaction of the Stewards of the Jockey Club to have surreptitiously obtained information respecting a trial from any person or persons engaged in it, or in the service of the owner or trainer of the horses tried, or respecting any horse in training from any person in such service ; or

(v) If any person be guilty of or shall conspire with any other person for the commission of, or shall connive at any other person being guilty of any other corrupt or fraudulent practice in relation to racing in this or any other country ; 
Every person so offending shall be warned off Newmarket Heath and other places where these Rules are in force.

I77. If any person be reported by the Committee of the Subscription Rooms at Newmarket or at Tattersall's as being a defaulter in bets, he shall be warned off as in the last rule mentioned so long as his default continues.

I78. When a person is warned off Newmarket Heath and so long as his exclusion continues, he shall not be qualified, whether acting as an agent or otherwise, to subscribe for or to enter or run any horse for any race either in his own name or in that of any other person, and any horse of which he is the nominator, or is (or was at the time of entry) wholly or partly the owner, or which (after the fact of his being warned off has been twice published in the Racing Calendar) shall be proved to the satisfaction of the Stewards to be, or to have been, directly or indirectly, under his care, training, management, or superintendence, shall be disqualified.

\section{Fees and Fines.}

I79. The Stakeholder shall be allowed to retain, out of the stakes in his hands, the following fees, viz.:-

For every match, one pound.

For every subscription or sweepstakes where the lowest forfeit amounts to 20 sov. $\frac{1}{2}$ per cent. on the whole stake, and on all other races I per cent.

180. There shall also be payable the following maximum fees :-

For every entry, a Clerk's fee of $2 \mathrm{~s}$. $6 \mathrm{~d}$.

For weighing, a Clerk's fee of $2 \mathrm{~s}$. 6 d.

For registration of a horse's name, a Clerk's fee of $2 \mathrm{~s}$. $6 \mathrm{~d}$.

Registration Fees. The following fees are also payable:-

I8I. (i) For registration of authority to act generally on behalf of an owner, five shillings.

(ii) For every registration or change of an assumed name, $£ 30$.

(iii) For every registration of partnership, and on every change thereof one sovereign for each horse.

(iv) For every annual registration of colours, five shillings.

(v) For every registration of colours for life, five sovereigns.

(vi) For registration of foreign and veterinary certificates, five shillings.

(vii) For registration of an agreement with a jockey, five shillings.

(b) On fixtures being granted to a meeting, the following fees shall be paid (unless the Stewards of the Jockey Club shall see fit in any case to remit the same):-All meetings having four days consecutively, or six days at any time, IOO sov.; all others, 5 sov. a day.

182. Except where otherwise provided, all fees and fines shall be paid to the credit of the Jockey Club, all fines to be afterwards transferred, either wholly or in such proportions as the Stewards may direct, either to the Bentinck Benevolent Fund or the Rous Memorial Fund.

\section{New Rules.}

183. Every new rule (in which term is included the repeal or alteration of an existing rule) must be advertised three times in the Sheet Calendar, with the date of the meeting of the Jockey Club at which it is to be proposed, and be submitted to the Rules Committee, before it is brought up for discussion.

I84. Every new rule must be confirmed at a meeting subsequent to that at which it is passed, and be then twice advertised in the Sheet Calendar, but shall not come into operation until the Ist of January following, unless urgency he declared by the Stewards on confirmation. 


\section{NATIONAL HUNT RULES.}

\section{Definitions and Application of these Rules.}

I. (i) A "recognised meeting" is a meeting held under the sanction of the National Hunt Committee, or other Turf authority, of the country in which it is held; or (where no such authority exists) under these rules.

The Turf authorities of the United Kingdom are-

The National Hunt Committee.

The Jockey Club.

The Turf Club of Ireland.

The Irish National Hunt Steeple Chase Committee.

The Channel Islands Racing and Hunt Club.

"Stewards."-Wherever the word "Steward" or "Stewards" only is used, it means Steward or Stewards of the meeting, or their duly appointed deputy or deputies.

The expression "United Kingdom" includes Great Britain, Ireland, the Channel Islands, and the Isle of Man.

The "Registry Office" is the office for the time being appointed as the registry office by the National Hunt Committee.

"Registered" and "Registration" mean "Registered" and "Registration" at such office.

N.B.-The present Registry Office is Messrs Weatherby's Office, 6 Old Burlington Street, London, W., and Newmarket.

The "Racing Calendar," comprising the "Sheet Calendar" and the "Book Calendar" of "Steeple Chases Past," and the "Stud Book" are the works published under those names respectively by authority of the National Hunt Committee or the Jockey Club.

The "Forfeit List" is a record of "arrears," published under the sanction of the Turf authorities of the United Kingdom.

A "horse" includes mare, gelding, colt, and filly, save when the context requires otherwise.

A "welter flat race" is a weight-for-age race, of not less value than 200 sov., for four-years-old and upwards, sanctioned by the Jockey Club to be run under these Rules, during the close time of Flat Racing only, at a minimum weight of $9 \mathrm{st} 7 \mathrm{lb}$, and over a distance of not less than two miles.

A "maiden" is a horse which has never won a steeple chase, hurdle race, or National Hunt flat race (other than a match or private sweepstakes) at any recognised meeting in the United Kingdom, or in any other country. In welter flat races the word "maiden" signifies a horse which has never won a race of that description or any race to which the Rules of Racing apply. A maiden means a maiden at the time of the start.

A horse which has "never started" is one that has never started for a steeple chase, hurdle race, or National Hunt flat race (other than a match or private sweepstakes) in any country.

A "race" means plate, sweepstakes, cup or match, but refers only, under these rules, to steeple chases, hurdle races, National Hunt flat races, or welter flat races.

A "plate" is a race to be run for money or other prize without any stake being made by the owners of the horses engaged.

A "sweepstake" is a race in which stakes are made, or a fixed sum subscribed for, by the owners of three or more horses engaged, and any such race is still a sweepstakes, when money or other prize is added.

A "private sweepstakes" is one to which no money is added, and which has not been publicly advertised previous to closing.

A "cup" is any prize not given in money.

A "handicap" is a race in which the weights to be carried by the horses are adjusted by the handicapper for the purpose of equalising their chances of winning.

A "free handicap" is one in which no liability for stake or forfeit is incurred until acceptance.

A "post race" is a race for which a person may, under one subscription, enter 
two or more horses, and run any one or more of them, as the conditions prescribe.

A "produce race" is one to be run for by the produce of the horses named at the time of entry.

A "selling race" is one in which any horse running "to be sold," if a loser, may be claimed, and if the winner, must be offered for sale by auction, or be liable to be claimed.

A "weight-for-age" race is a race in which weights are apportioned to horses according to their ages, and remains a weight-for-age race, even if there are penalties and allowances.

A match at "catch weights" means one for which the riders need not weigh before or after the race.

The "nominator" is the person in whose name a horse is entered for a race.

"Owner" includes "part-owner" or "lessee."

"Arrears" are any sums unpaid in respect of fines, compensation, fees, entrance money, stakes, subscriptions, forfeits, and purchase money in races with selling conditions.

The expression "disqualified person" includes-

(a) Every person who is warned off New market Heath or other courses.

(b) Every person whose name is in the For'eit List or list of arrears officially notified by a recognised Turf authority of any country until the default is cleared.

(c) Every person who has been reported as a defaulter in bets under Corrupt Practices, etc., until it has been officially notified that such default is cleared.

(d) Every person who has been declared ty the Turf authorities of, or by the Stewards of, any recognised meeting in the United Kingdom or any other country to have been guilty of any corrupt or fraudulent practice on the lurf.

(ii) These Rules apply to all meetings held under the sanction of the National Hunt Committee, and to all races run at such meetings, but they do not apply to

(a) Yeomanry races confined to chargers and troop horses, and held during the time of the annual training, under the Stewardship of the Commanding Officer.

(b) Point to point steeple chases held under the Stewardship of the Master of Foxhounds or Staghounds, or of a Master of Harriers (being a member of the Association of Masters of Harriers), of the country in which they are run, of which notice shall have been given at the Registry Office (on a form to be obtained therefrom) not less than seven days before the holding thereof, with a fee of ros., and for which a certificate in form I of Appendix C to these Rules, and signed by such Master as aforesaid, shall be lodged at the Registry Office within fourteen days after the holding thereof.

All other meetings, to which the public are admitted, not held under the sanction of the National Hunt Committee or other Turf authority of the country in which they are held, are " unrecognised meetings," and every horse which has run at such meeting is disqualified for all races under these rules.

\section{Calculation of Time.}

2. A meeting shall be deemed to commence at ten in the morning of the day for which the first race is advertised, and to conclude at ten in the evening of the last day of the races.

3. When the last day for doing anything under these rules falls on a Sunday, it may be done on the following Monday, unless a race to which such act relates is appointed for that day, in which case it must be done on the previous Saturday.

4. A " month" means a calendar month; a "day" means twenty-four hours.

\section{Stewards of the National Hunt Committee and their Powers.}

5. (i) The Stewards of the National Hunt Committee for the time being have full power to sanction or prohibit any meeting to which these Rules apply, or any 
alteration in the date of holding such a meeting, and to supervise and make such alterations as they may think advisable in the programme of, or the conditions of, any race at a meeting.

(ii) They have also the following powers:-

(a) To grant, withdraw, or suspend jockey's' licenses ;

(b) To prohibit any person from acting in any official capacity in connection with a meeting ;

(c) To investigate any case which appears to them to require their interference (whether or not referred to them by the Stewards of a meeting), and to give a final decision thereon;

(d) To entertain and determine appeals from the Stewards of a meeting, as provided by Disputes, Objections, etc. ;

(e) To receive reports from the Stewards of a meeting and other persons, as provided by these Rules, and to act thereon;

$(f)$ To impose any fine not exceeding 50 sov., and to warn off any person from all Courses where these Rules are in force for such period as they think fit;

and generally to exercise any of the powers specifically conferred upon them by, or which they think necessary for the carrying out of, any of these Rules.

6. Stewards of Meetings. - There must be at least two Stewards for every meeting.

7. If only one Steward is present on any day of a meeting, the Clerk of the Course shall, before the running of the second race, request such Steward to appoint some person to act as a deputy Steward, and if no Steward is present, the Clerk of the Course shall appoint two or more deputy Stewards, and shall notify conspicuously such appointment.

8. Powers of the Stewards.-The Stewards of a meeting have full power to make all such arrangements for the conduct of the meeting as they think fit, and, subject to the provisions of Regulations for Race Meetings, to postpone any races for any time not later than the week following that for which they were originally fixed, and all nominations and subscriptions shall stand good as if the race had taken place on the day originally fixed.

9. The Stewards have control over, and they and the Members of the National Hunt Committee have free access to, all stands, rooms, enclosures, and other places used for the purposes of the meeting.

IO. The Stewards shall exclude from all places under their control any "disqualified person," and they have power in like manner at their discretion to exclude any other person or persons.

II. The Stewards have power to regulate and control the conduct of all officials, and of all trainers, riders, grooms, and persons attendant on horses.

12. They have power to punish at their discretion any person subject to their control with fine not exceeding 50 sov., and with suspension from acting or riding at the same meeting, and to report to the Stewards of the National Hunt Committee should they consider any further fine or punishment necessary.

I3. The Stewards have power to determine all questions arising in reference to racing at the meeting, subject to appeal under Disputes, Objections, etc.

14. Betting.-The Stewards of the National Hunt Committee take no cognisance of any disputes or claims with respect to bets, nor shall the Stewards of a meeting, as such, entertain any disputes relating thereto. But the Stewards of the National Hunt Committee will give effect to an official report of default made to them by the Committee of the Subscription Rooms at Newmarket, or at Tattersall's, by warning persons so reported off all Courses where these Rules are in force.

Officials.

I5. One or more Inspectors of Steeple Chase Courses shall be annually appointed by, and will receive their instructions from the Stewards of the National Hunt Committee.

I6. The following officials shall be appointed for every meeting, subject to the approval of the Stewards, viz., Clerk of the Course, Handicapper, Stakeholder, Clerk of the Scales, Starter, and Judge. 
I7. In case of emergency, the Stewards may, during a meeting, appoint a substitute to fill any of the above-named offices for that meeting only.

I8. Every complaint against an official shall be made to the Stewards in writing, signed by the complainant.

19. Clerk of the Course. - The Clerk of the Course, or his authorised substitute, is the sole person responsible to the Stewards for the general arrangements of the meeting.

20. The Clerk of the Course shall arrange for the publication, under the sanction of the Stewards, of a daily official card of the races, containing the conditions of each race, as published in the Racing Calendar, the names of the horses engaged, with a number attached to each, and such further particulars as the Stewards may require. A horse may appear on the card in the name of the owner, instead of that of the nominator, and the Stewards may, under special circumstances, grant permission for a horse to run in the name of some other person. The Clerk of the Course shall, in such latter case, make a report to the Registry Office, stating the grounds upon which the permission was granted.

2I. The Clerk of the Course shall, at once, make a return to the Registry Otfice of any Deputy Steward, or official appointed, of all complaints to, and decisions of, the Stewards, of all fines inflicted, and of all horses sold or claimed.

22. The Clerk of the Course shall have in his possession, for the information of the Stewards, a list of persons "warned off," and of suspended jockeys, and also a copy of the latest Monthly Forfeit List, and he shall not, under a penalty not excéeding ro sov., allow any horse which, or the owner or nominator of which, is in the Forfeit List to start for any race.

23. Handicapper. - The Handicapper shall append to every handicap the day and hour from which winners will be liable to a penalty.

24. The top weight to be allotted in a handicap shall not be less than I2st $7 \mathrm{lb}$, nor the lowest weight less than Iost, except in handicap steeple chases of three miles and a half or upwards, when the lowest weight may be 9 st 7 lb.

25. Stakeholder.-The Stakeholder shall not allow a rider to be weighed out for any horse until such horse's entrance money and stake for that race, and the forfeits for every horse belonging to the same owner, or standing in his name, the jockey's fee, and any arrears claimed under rule, have been paid, or the Stakeholder shall himself be liable: Provided that the Stakeholder shall not be liable for arrears in respect of a meeting elsewhere than at the place at which the race is run, unless notice of such arrears being overdue has been published in the Forfeit List, or delivered in writing, signed by the person claiming the arrears to the Stakeholder and to the person indebted, before ten in the evening preceding the race.

26. The Stakeholder shall, at the expiration of fifteen days after the meeting, render an account and pay over all stakes and added money to the persons entitled, and at the same time notify at the Registry Office all arrears then unpaid, and one month before the publication of the next available Forfeit List he shall further notify at the Registry Office all arrears then remaining unpaid, for publication therein.

27. Clerk of the Scales.-The Clerk of the Scales shall exhibit the number (as allotted on the official card) of each horse for which a rider has been weighed out, and shall forthwith furnish the starter with a list of such numbers, and the numbers shall not be taken down until the horses are started.

28. If extra, or special, weight be declared for any horse, such weight shall be exhibited with the number, also any declaration to win, or alteration of colours.

29. The Clerk of the Scales shall in all cases weigh in the riders of the horses placed by the Judge, and report to the Stewards any rider not presenting himself to be weighed in.

30. He shall always put $4 \mathrm{lb}$ extra into the scale for steeple chases and hurdle races, and $2 \mathrm{lb}$ extra for National Hunt flat races and welter flat races, to prove that the horse has not carried too much weight.

3I. He shall at the close of each day's racing send a return to the Registry Office of the weights carried in every race and the names of the riders, specifying over-weight, if any.

32. The Starter.-The Starter shall give all orders necessary for securing a fair 
start. The horses must be started from a walk, and as far as possible in a line, but they may be started at such reasonable distance behind the starting post as the Starter thinks necessary.

33. Any rider misconducting himself at the post, or refusing to obey the commands of the Starter in any respect whatever, shall be reported to the Stewards.

34. A start in front of the starting post, or on a wrong course, is void, and the horses must be started again.

35. The Judge.-The Judge, or his authorised substitute, or a Steward, must occupy the Judge's box at the time the horses pass the winning post, or the race must be run again. He must announce his decision immediately, and such decision shall be final, unless an objection to the winner, or any placed horses, is sustained: Provided that this Rule shall not prevent a Judge from correcting any mistake, such correction being subject to confirmation by the Stewards.

36. The Judge shall, at the close of each day's racing, sign and send a report of the result of each race to the Registry Office.

\section{Regulations for Race Meetings.}

37. All meetings under these Rules must be sanctioned by the Stewards of the National Hunt Committee.

38. The conditions of every race before closing, and the full programme of every meeting, before it takes place, must be advertised in the Racing Calendar, and no alteration can be made in the conditions of any race after the last advertisement.

39. The preceding rule shall not preclude the addition of more money to a race, or of a new race to the programme of a meeting subsequent to the publication of the last Calendar issued previous to the meeting being held.

40. The advertisement must state that the meeting is to be subject to the National Hunt Rules, the days on which it is to be held, the dates for closing the stakes, the names of the persons to receive nominations and declarations of forfeit, and a place or places for the entry of every race, and, before the time fixed for closing (races for future years excepted), the names of two or more persons as Stewards, and of the Judge, Starter, Clerk of the Course, Handicapper, Stakeholder, and Clerk of the Scales.

4I. The Stewards of the National Hunt Committee may at their discretion prohibit the advertisement of any meeting or race in the Racing Calendar, or call upon the Stewards to alter or expunge any conditions, even after advertisement.

42. At every meeting advertised to take place solely under these Rules, there shall be in each day's programme at least two steeple chases, one of which must be of three miles or upwards; and, of the total amount guaranteed for prizes, one half at least shall be apportioned to steeple chases.

But this rule shall not apply in the case of Yeomanry races held during the annual training, and conjointly under Regimental and National Hunt Rules, the programmes of which have been submitted to, and approved by, the Stewards of the National Hunt Committee.

43. There shall be no race less than two miles.

44. In all Steeple Chase Courses there shall be at least twelve fences (exclusive of hurdles) in the first two miles, and at least six fences in each succeeding mile. There shall be a water jump at least twelve feet wide and 2 feet deep, to be left open, or guarded only by a perpendicular fence not exceeding 2 feet in height. There shall be in each mile at least one ditch 6 feet wide and 3 feet deep on the taking off side of the fence, which ditch may be guarded by a single rail, or left open, and which fence must be 4 feet 6 inches in height, and, if of dead brushwood or gorse, 2 feet in width.

45. In all hurdle race courses there shall be not less than eight flights of hurdles in the first two miles, with an additional flight of hurdles for every quarter of a mile or part of one beyond that distance, the height of the hurdles being not less than 3 feet 6 inches from the bottom bar to the top bar. rules.

46. No pony or Galloway race shall take place at any meeting held under these 
47. There shall be no restrictions with regard to training stables in the conditions of any race.

48. Alterations of Dates and Postponements.-The following provisions apply to any alterations in the dates of holding a meeting, and to postponements-

(i) The date for holding a meeting cannot be altered without the sanction of the Stewards of the National Hunt Committee, unless the weather or ground be in an unfit state for running, when a meeting or race may be postponed by the Stewards of the meeting for any time not later than the end of the following week.

(ii) In the latter case a certificate must be drawn up containing the reason for postponement, and stating the day and hour when such decision was arrived at, which shall not be before noon on the day preceding that on which the meeting or race has been advertised to take place, or to which it may have been previously adjourned; such certificate must be signed by two of the Stewards, or by one Steward and the Clerk of the Course, and at once dispatched to the Registry Office.

(iii) The meetings set out in Appendix A, shall not interfere with each other by postponement except with the sanction of the Stewards of the National Hunt Committee, who have the power to vary the list.

\section{Omitted Conditions.}

49. When the weights are omitted from the conditions of any race, the horses shall carry I $2 \mathrm{st} 7 \mathrm{lb}$ each, subject to penalties and allowances. If the horses be of different age, the weights shall be fixed by the sanctioned scale given in Appendix B

50. When no course is mentioned, it shall be as follows :-

If for three-years-old, two miles.

If for four-years-old, two miles and a half.

If for five-years-old, three miles.

If for six-years-old or upwards, four miles.

If for horses of different ages, the course shall be fixed by the age of the youngest.

5I. When no day is fixed for a race, it shall be run on the last day of the meeting, unless otherwise agreed by all the parties engaged, and sanctioned by the Stewards.

\section{Special Conditions applicable to Handicaps, Produce Races, and Selling Races.}

52. Handicaps. - If in a handicap for which there is a minor forfeit declared by a fixed time, the highest weight accepting was originally less than I 2 st $7 \mathrm{lb}$, it shall be raised to that weight, and the other acceptances equally.

53. In handicaps there shall be no clause permitting an alteration of the weights after publication by the claim of a selling allowance.

54. Produce Races. - If the produce of a mare is dropped before the Ist of January, or if there is no produce, or if the produce is dead when dropped, the entry of such mare is void if it is declared by the time prescribed in the conditions; if twins are dropped the nominator shall at the same prescribed time declare to which of them the engagement attaches.

55. Selling Races. - In a selling race, the lowest selling price shall in no case be less than $£ 50$.

56. (i) Any horse running for any race "to be sold," shall, if the winner, be liable to be claimed for the entered selling price by the owner of any other horse starting in the race.

(ii) If it is a condition of the race that the winner is to be sold by auction, the sale shall take place immediately after the race, and the surplus, if any, over the entered selling price shall be equally divided between the owner of the second horse and the Race Fund. Any such sale by auction shall be subject to a right to bid for, or to buy in such horse, on behalf of the seller.

(iii) If sold, or bought in, the horse shall not leave the place of sale without the written permission of the Clerk of the Course, and if the horse be not paid for, or the price secured to the satisfaction of the Clerk of the Course within a quarter of 
an hour, he may order the horse to be put up a second time, and the purchaser at the first sale shall be responsible for any deficiency arising from the second, and shall be treated as a defaulter until it is paid.

57. (i) All other horses starting "to be sold" may be claimed for the entered selling price, plus the value of the stakes or plate by owners of horses running in the race.

(ii) Owners of horses placed, other than the winner, have priority of claim in order of their places, and if the owners of two or more horses having equal rights, claim, they must draw lots ; the owner of the winner has the last claim.

(iii) No person can claim more than one horse.

(iv) Every claim must be made in writing to the Clerk of the Scales within a quarter of an hour after the winner has weighed in, but subject to the rules in cases of objection.

58. The price of every horse claimed must be paid to the Clerk of the Course, and a written order given by him for delivery of the horse.

In the case of a horse being claimed, if the price be not paid before seven o'clock in the evening of the day of the race, the claimant forfeits his right. But the owner may insist on the claimant taking and paying for the horse, and if he refuse or neglect to do so, he shall be treated as a defaulter in respect of the price.

59. In selling races where a horse walks over, or there is no second horse placed, the winner is liable to be sold, and any surplus above the entered selling price will go to the fund; but where a horse walks over and the whole of the added money is not given, he shall not be liable to be sold or to be penalised for such walk-over in cases where selling race winners are exempted from penalties.

6o. In cases where there are not the required number of starters, and the added money is reduced according to the conditions, the race ceases to be a selling race, the winner is exempt from sale, and the remaining horses cannot be claimed.

6I. In the cases hereafter mentioned, the following provisions as to claiming and selling shall apply-

(i) Where a horse is objected to-

(a) If such objection is made before the horse has been sold or delivered, the time for selling or delivering him shall be postponed until such time after the determination of the objection as the Stewards appoint.

(b) Where the objection is made and sustained after the horse has been sold or bought in, the sale or buying in, and any claim in respect of the horse placed second, shall be annulled, and all moneys paid thereunder returned. The horse disqualified shall be liable to be claimed as a beaten horse, and the Stewards shall fix a time for the exercise of such right of claiming, and a time and place for the sale of the horse adjudged to be the winner.

Should the Stewards find the above provisions impracticable, they shall make a report to the Registry Office.

(ii) In the case of a dead heat, the time for claiming or selling is postponed until the dead heat is run off. In case of a division, each of the horses dividing is a winner for the purposes of the Rules relating to claiming and selling, and if an auction race, both shall be put up to auction, and any surplus shall be divided, half to go between those horses and half to the Race Fund.

\section{Race Horses.}

62. Age.-The age of a horse shall be reckoned as beginning on the Ist of January in the year in which he is foaled.

63. No horse shall run under four years of age for a steeple chase or National Hunt flat race, or for a hurdle race until the Ist of September of the year in which he is three years old.

64. Disqualifications of Horses.-

(i) No horse $(a)$ which has run at an unrecognised meeting in the United Kingdom or any other country, or

(b) which is nominated by, or is wholly or partly the property of a "disqualified person," or is in any way under the care, training, management, or superintendence of any person whose name has been twice published in the Racing Calendar as being in default, or as being warned off, or 
(c) which is in the Forfeit List or list of arrears officially notified by a recognised Turf authority of any country, or

(d) which is the property of a partnership, sold with contingencies, leased, or the subject of any other joint arrangement, unless such partnership, sale with contingencies, lease or other joint arrangement has been registered in accordance with the Rules relating to partnerships and other joint interests

is qualified to be entered to start for any race under these Rules-

(ii) (a) No horse not duly entered or in whose entry any mis-statement, omission, or error has occurred and has not been corrected on payment of a fine as hereinafter provided, or which is not qualified according to the conditions of the race.

(b) No foreign horse, until the certificates required by these Rules have been deposited at the Registry Office, and

- (c) No horse in whose winnings in such race any disqualified person has any interest,

is qualified to start for any race under these Rules.

Provided always that if in any of the cases occurring under sub-sections (i) or (ii), any such horse has been allowed to be entered and start for any race, it may be declared disqualified after such a race as hereinafter provided.

(iii) The following horses shall also be disqualified under these Rules-

(a) Any horse ridden by a "disqualified person," or by a person not qualified according to the conditions of the race.

(b) Any horse whose owner or rider has run or ridden a horse at any unrecognised meeting within the preceding twelve months.

(c) Any horse with regard to which any infringement of these Rules as to weighing, riding, or running, has occurred, entailing disqualification.

65. Any horse which has been the subject of fraudulent practice may at the discretion of the Stewards of the National Hunt Committee be disqualified for such time and for such races as they shall determine.

66. The Stewards may either-

(i) Themselves call for proof that any horse is not disqualified, and at any time order an examination by such person or persons as they think fit of any horse entered, or which has run in a race, or

(ii). On objection made to them in accordance with the provisions of Disputes, Objections, etc., decide whether any horse is disqualified.

If the Stewards come to the conclusion that any grounds of disqualification are proved, or that any objection made as aforesaid is valid, they may declare the horse to be disqualified; such horse will then either be unable to start, or, if the race has been run, and he has won, or been placed, he will be regarded as having finished last, and the other horses shall take positions accordingly.

67. Foreign Horses.-(i) A horse foaled out of the United Kingdom shall not be qualified to start for any race until there has been deposited at the Registry Office, and a fee of $5 s$. paid on each certificate.

(a) A foreign certificate stating the name (if any), age, sex, pedigree, and colour of the horse, and any mark by which it may be distinguished, signed by the secretary or other officer of a racing club of the country in which the horse was foaled, approved by the Stewards of the National Hunt Committee, or by some magistrate, mayor, or public officer of that country prescribed by them.

(b) A certificate of age, signed by a Veterinary Surgeon in the United Kingdom, approved for this purpose by the Stewards of the National Hunt Committee either by general order or in the particular case.

(ii) The Stewards of the National Hunt Committee may also require any further proof or confirmation in any particular case, and may declare any horse lisqualified in default thereof.

(iii) A horse which has been out of the United Kingdom (otherwise than as a foal at the foot of his dam) before having run in Great Britain, shall not be qualified to start for any race under these Rules until such a certificate of age has been deposited at the Registry Office.

NOTE-The Stewards of the National Hunt Committee have approved generally of certificates given by the Members of the Royal College of Veterinary 
Surgeons, and by persons holding the Highland and Agricultural Society's diploma.

\section{Entries, Subscriptions, Declarations of Forfeit, and Acceptances for Races.}

68. Every entry shall close, and every declaration of forfeit or acceptance shall be fixed to be made at IO P.M., and upon Tuesday only, except in the case of races closing within four days of their being run.

69. All entries made elsewhere than at the Registry Office (except entries made during the week of, or on the Saturday preceding, the meeting) shall be lodged at that office within forty-eight hours, or, if intended for publication in the next Calendar, within twelve hours, after the day of closing.

In default of observance of this rule, the receiver of nominations shall be reported to the Stewards of the National Hunt Committee, and unless the nominator can prove to their satisfaction that the entry was made in due time, it shall be void.

70. The list of entries shall be closed at the advertised time, and no entry shall be admitted on any ground after that time.

71. An entry or subscription may, before the time of closing, be altered or withdrawn.

72. In any race where there shall be any particular conditions required as a qualification to start, such conditions shall extend to the time of starting.

73. Form of Entry or Nomination. - (i) Entries and declarations of forfeit shall be made in writing, or by telegraph (which shall be equally binding), to be confirmed in writing, and shall be signed by the owner of the horse, or of the engagement, or by some person authorised by him.

(ii) (a) An entry by telegraph must be confirmed in writing before the time of weighing, or the horse shall not be qualified to start.

(b) A declaration of forfeit by telegraph must be confirmed in writing at the earliest opportunity, or the forfeit shall not be saved.

74. A horse cannot be entered in the real or assumed name of any person as his owner unless that person's interest or property in the horse is at least equal to that of any other one person, and has been so registered.

75. In entering a horse for the first time for any race under these rules he shall be described by stating a name, the colour (when possible), and whether a horse, mare, or gelding, and the Calendar or Stud-book names of his sire and dam. If the dam was covered by more than one stallion the names of all must be stated. If the sire or dam has no name in the Calendar or Stud-book, such further pedigree and description must be added as will clearly distinguish the horse entered from all other horses, and if the pedigree of the sire or dam be unknown, such further particulars as to where, when, and from whom the horse was purchased or obtained must be given as will identify him.

NOTE. - Any horse for which a name has been registered under the Rules of Racing shall be run under the same name under these Rules, subject to provisions of Rule 79.

76. The entry shall be in the name or assumed name of one person, and shall state the name, or assumed name, of the owner, the name of the horse, and the age he will be at the time of the race.

77. The name and description of the horse must be repeated in every entry until he has run under these rules, and a report of the race has been published in the "Racing Calendar." In any entry after such publication it will be sufficient to enter him by his name and with his age only.

78. When entering under a new ownership, a horse which has run previously under these rules, the name of the person who last ran the horse must be attached to every entry until the horse has run again unier these rules.

79. Whenever the name of a horse which has run at any recognised meeting in any country is changed, his old name as well as his new name and his description, must be given in every entry until the change has been published in the Book Calendar of Steeple Chases Past.

80. An entry of a horse for a race with the wrong age, or an incorrect or 
imperfect description according to the rules of entry, may be corrected on payment of a fine of 5 sov., provided that it be proved to the satisfaction of the Stewards, or the Stewards of the National Hunt Committee, that the error was accidental, and provided also that the correction be made within seven days of the publication of the Calendar in which the wrong entry appeared. The correction, if it relate to a race, the entries for which have not been published in the Racing Calendar, must be made before the numbers are exhibited for that race.

On payment of the fine, an entry may, within the like time, be omitted from a race for which the horse was not qualified, but no other horse may be substituted.

$8 \mathrm{I}$. No other alteration or addition shall be made in any entry after the time fixed for closing, except when the name of the horse has been changed, or an assumed name of the nominator has been abandoned.

82. A subscriber to a race may transfer the right of entry under his subscription to any other person.

83. Subscriptions and all entries or rights of entry under them become void on the death of the subscriber, and entries (except such as are made under another person's subscription) become void on the death of the persons in whose names they are made.

84. If either party to a match die the match is off.

85. An acceptance for a free handicap shall be considered as equivalent to an entry, but if the horse be wrongly described the acceptance shall be void.

86. In making an entry for a produce race, the produce is entered by speciiying the dam and sire, or sires.

87. No horse shall be considered as struck out of any of his engagements, until the owner or some authorised person, shall have given notice in writing, or by telegraph, to be confirmed in writing at the earliest possible opportunity, at the Registry Office or to the Clerk of the Course where the horse is engaged. forfeit.

The notification of the death of a horse shall be equivalent to a declaration of

\section{Sale with Engagements.}

88. When a horse is sold with his engagements, or any part of them, the seller cannot strike the horse out of any of such engagements, but, on default of the purchaser, remains liable for the amount of the forfeits in each.

89. In all cases of sale by private treaty, the written acknowledgment of both parties that the horse was sold with the engagement is necessary to prove the fact, but when the horse is sold by public auction or claimed out of a selling race, the advertised conditions of either the race or the sale are sufficient evidence. If certain engagements only be specified, those only are sold with the horse.

NOTE.-The following are known and recognised as "Lord Exeter's Conditions":-

"The horses are sold without their engagements, but the purchaser has the right of running for any of them by paying half the stake, and in the event of the horse winning, or being entitled to second or third money, one-third shall be paid to the vendor, but the vendor reserves to himself the right of striking the horse out of any race, in time to save a minor forfeit or discount, unless the purchaser shall give notice that he wishes to run for any particular race, when he will become liable for half the stake or forfeit. Horses purchased under Lord Exeter's conditions cannot be re-sold under the same conditions without the written consent of the original vendor."

\section{Assumed Names, Partnerships, and other Joint Interests.}

90. Assumed Names.-Assumed names can only be used subject to the following provisions-

(i) An assumed name must be annually registered, and a fee of Io sov. paid. A registration continues effectual only during the current year.

(ii) A person cannot register more than one assumed name at the same time, nor can he use his real name so long as he has a registered one.

(iii) A person cannot register as his assumed name one which has been already registered by any other person, or the real name of any owner of race horses. 
(iv) An assumed name may be changed at any time by registering a new assumed name.

(v) Any person who has registered an assumed name may at any time abandon it, by giving written notice at the Registry Office; such notice of cancellation shall be published in the next Racing Calendar, after which all entries which have been made in the assumed, shall be altered to the real, name of the owner.

91. Partnerships, Sales with Contingencies, Leases, etc.-(i) In the case of partnerships, a document stating (a) the name and address of every person having an interest in a horse; (b) the relative proportions of such interest; and (c) with whom the power of entry and declaration of forfeit rests, must be signed by all the parties, and lodged at the Registry Office, or with the Clerk of the Course, for transmission to the Registry Office, before a horse which is partnership property can be entered or start for any race. All partners shall be jointly and severally liable for every stake or forfeit, and no part owner shall assign his share or any part thereof, in a horse without the consent of his partners.

(ii) In the case of a horse sold with contingencies, leased, or the subject of any other joint arrangement, a document stating the names and addresses of all the parties interested, shall be signed by them and lodged as above, and shall state fully the terms of such sale with contingencies, lease, or other joint arrangement before any such horse shall be entered or start for a race.

(iii) In all the above cases a registration fee of I sov. shall be paid on each horse.

(iv) If partnerships, sales with contingencies, leases, or other joint arrangements existing either at entry or at the time of the race, have not been registered as above, a horse shall be disqualified.

(v) All partnerships, sales with contingencies, leases, and other joint arrangements, shall be published, in the real or assumed names in the next available Racing Calendar, and any termination or severance must be notified at once to the Registry Office for publication.

\section{Riders, Gentlemen Riders, Qualified Riders, Jockeys, etc.}

92. (i) Persons who have never ridden for hire, and who are not otherwise disqualified under these rules, need no qualification to ride in Steeple Chases or Hurdle Races unless the conditions of any such steeple chase or hurdle race require a particular qualification, but for welter flat races riders must be "Qualified Riders" or Jockeys holding licences under these Rules.

(ii) "Qualified Riders" under these Rules are persons who have never ridden for hire, and who are qualified either
(a) as Gentlemen,
(b) as Farmers,
(c) by Election,
(d) Yeomen when riding at their own regimental meeting.

(a) Riders qualified as Gentlemen must be members of the National Hunt Committee, the Irish National Hunt Steeple Chase Committee, or of one of the following Clubs-The Jockey Club, Turf Club of Ireland, Croxton Park, Bibury, Southdown and Ludlow Race Clubs, the New Rooms at Newmarket, the Jockey Club of Paris, Berlin and Vienna, the Army and Navy, Junior Army and Navy, Arthur's, Turf, Boodle's, Brooks, Carlton, Junior Carlton, Guards', Cavalry, Pratt's, Travellers', United Service, Junior United Service, East India United Service, the Union, White's, the Conservative, the Oxford and Cambridge, the Naval and Military, the Oriental, the Badminton, the Devonshire, the New University, the Windham, the St. James' or the United University Club; the Kildare-street, Sackville-street, Hibernian United Service, or Stephen's Green Clubs in Dublin; the Western Meeting (Ayr), or the New Club, Edinboro'; or that they be Officers on full pay in the Army or Navy, or persons holding Commissions under the Crown, or bearing titles in their own right or by courtesy.

(b) Riders qualified as farmers must be now farming at least 100 acres of land, and their Sons if following the same occupation, and for the purposes of this rule a "Farmer" shall be understood to mean one who resides permanently on his farm, working it himself, and deriving therefrom his principal and ostensible means of subsistence. 
(c) Persons not qualified as "Gentlemen Riders" or "Farmers," who are desirous of becoming "Qualified Riders," must send their names in for election, with the names of their proposer and seconder, who must be members of the National Hunt Committee, to the Registry Office for publication in at least one Calendar before the day of election. The names of persons elected must be submitted annually to the Committee, at the General Meeting on the second Monday in December, for re-election. The fee to be paid on election, or reelection, is I sov.

93. Should any Qualified Rider subsequently ride for hire, or appear in the Forfeit List, or be reported by the Committee of the Subscription Rooms at New. market, or at Tattersall's, as being a defaulter for bets lost on horse racing, he will lose his qualification, and if a qualified rider by election, his name will be erased from the list of Qualified Riders.

94. Jockeys' Licences, etc.-(i) No jockey shall, after the last day of the week in which he has first ridden, ride in any race until he shall have obtained a licence from the Stewards of the National Hunt Committee. Such licence must be obtained annually, on application with full name and address, at the Registry Office, and will only be granted on the condition that a jockey is not an owner, or part owner, of any race horse. Under exceptional circumstances, leave may be granted to Jockeys who are also trainers, to own one or more horses, provided that such horse, or horses, are trained in their own stables.

(ii) Any person who shall employ a jockey in contravention of this rule shall be liable to be fined by the Stewards of the National Hunt Committee.

(iii) A list of the licenced jockeys shall be published annually in the Racing Calendar.

(iv) Every jockey shall pay I sov. for his licence, to be applied as his subscription to the Bentinck Benevolent and Provident Fund.

(v) No jockey whose licence has been withdrawn, or refused on the ground of misconduct, will be eligible to ride trials, or be allowed in any weighing room, stand, or enclosure, during the time his suspension from riding continues.

95. Jockeys' Retainers. - In the absence of special agreement, a jockey's retainer terminates at the end of the current year. The agreed retaining fee must be paid in advance in respect of each year.

The terms of all agreements shall be registered, and a fee of $5 \mathrm{~s}$. paid.

96. Employers retaining the same jockey have precedence according to the priority of their retainers.

97. The Stewards of the National Hunt Committee may adjudicate between persons claiming the services of any jockey, and upon disputes between jockeys and their employers, and have power to cancel any agreement between them.

98. Jockeys' Fees. - In the absence of special agreement to ride for a lower sum, the fee to a winning jockey shall be Io sov. in steeple chases and hurdle races, and 5 sov. in welter flat races, and to a losing jockey, 5 sov., and 3 sov. respectively, and no further charge shall be made, except when requested to leave home for the purpose of riding, in which case the cost of travelling expenses, and I sov. a day for living may be charged to the owner, or divided between the owners, at whose request he left home.

All jockeys' fees shall be paid to the Stakeholder at the same time as the stakes except under a private arrangement with the jockey, in which case notification must have been previously made at the Registry Office, or to the Stakeholder, for transmission thereto.

99. Stable Lads.-(i) No trainer shall engage any stable servart who has previously been in a training stable, without referring to his last employer, and receiving a satisfactory reply in writing.

(ii) Any trainer infringing this rule and continuing to employ such servant after notice has been served on him by his late employer, or through the Registry Office, shall be reported to the Stewards of the National Hunt Committee.

(iii) Any servant prevented by this rule trom obtaining or retaining employment shall have the right of appeal to the Stewards of the National Hunt Committee. 


\section{Racing Colours.}

100. Racing colours may be registered either annually, on payment of 5s., or for the life of the person registering, on payment of 2 sov.

All disputes as to the right to particular colours shall be settled by the Stewards of the National Hunt Committee.

IOI. Any person running a horse in colours other than those registered in his own or assumed name without a special declaration over-night to the Clerk of the Course (at a time to be prescribed) shall be fined not less than I sov., nor more than Io sov.

A special declaration is also required where the owner is not the nominator.

\section{Entrance Money, Stakes, Forfeits, and Arrears.}

102. Entrance money, stakes, forfeits, and arrears, must be paid in cash (if so required) to the Clerk of the Course or authorised stakeholder, and entrance money must (if so required) be paid at the time of entry.

No entrance money or forfeit to any fund but that devoted to horses running in the race shall exceed 5 per cent. on the added money, or value of other prize. Entrance fees, subscriptions, stakes, and forfeits shall be in pounds, not guineas.

103. A person entering is liable, as well as the nominator, and every partner in the horse at the time of nomination, for the entrance money and stake or forfeit. A person making a wrong nomination is equally liable.

A subscriber to a sweepstakes is liable for the stake or forfeit; but if he transfer the right of entry to any other person he is liable only in case of default by the transferee.

I04. A person taking an entry under another person's subscription, where forfeit must be declared by a particular time shall, if he do not declare forfeit by that time, be considered to have taken the engagement upon himself, and it shall be transferred to his name.

105. Every horse whose number has been exhibited shall be liable for his whole stake.

I06. A rider shall not be weighed out for a race unless there have been previously paid (i) any stake, forfeit, entrance money, or fee payable by the owner or nominator in respect of that race; (ii) all arrears due for the horse, or from his rider, or due for the same or any other horse from any person by whom such horse is wholly or partly owned, or in whose name or under whose subscription he is entel $€ d$; and (iii) the jockey's fee.

\section{The Unpaid Forfeit List.}

107. An Unpaid Forfeit List shall be kept at the Registry Office, and shall be published annually in the Sheet Calendar. It shall include all arrears which have been notified by the Stakeholder of any recognised meeting in the United Kingdom, or as otherwise provided under these rules, and shall state the real name or names, and also the assumed name or names (if any), of the persons from whom, and the horses (if any) in respect of which the same are due. "Arrears" which have been so published must be paid direct to the Registry Office, and until so paid shall not be removed from the list.

IOS. Where a person is prevented by these rules from entering or starting a horse for any race without paying arrears, for which he would not otherwise be liable, he may, by paying the same, enter or start the horse, and place the arrears on the Forfeit List as due to himself, and in like manner the seller of a horse with engagements may, if compelled to pay them by the purchaser's default, place the amount on the Forfeit List as due from the purchaser to himself.

109. So long as the name of a person is in the Forfeit List, no horse can be entered by him, or under his subscription, for any race, whether acting as an agent or otherwise, nor can any horse be ridden by him for any race, and no horse which has been entered by him, or in his name, or under his subscription, or of which he is, or was at the time of entry, wholly or partly the owner, can run for any race; and no horse which shall be proved to the satisfaction of the 
Stewards to be directly or indirectly under the care, training, management, or superintendence of a person whose default has been twice published in the Racing Calendar, shall be qualified to be entered or run for any race, and so long as any horse is in the Forfeit List, such horse shall not be qualified to be entered or run for any race. The disabilities under this rule extend to all arrears officially notified by a recognised Turf authority of any country.

IIO. A corrected Alphabetical Index of the horses and owners in the last Forfeit List shall be published in the first Calendar of every month. Such monthly list shall commence not less than three years before the time at which it is published, and shall be carried down to and include the latest Forfeit List which has been published in the Sheet Calendar as above mentioned.

II I. If a horse which, or the owner of which, is in the Forfeit List be entered for any race, the nominator of such horse shall be liable to a fine not exceeding 50 sov.

\section{Weights, Penalties, and Allowances.}

II2. Weights.-(i) No horse shall carry less than Iost in a Hurdle Race or Steeple Chase (except in a Handicap Steeple Chase of three miles and a half, or upwards, when the lowest weight may be 9st $7 \mathrm{lb}$ ); nor less than IIst in a National Hunt flat race.

(ii) Catch weights are only permissible for matches or private sweepstakes.

II 3. Penalties. - In estimating the amount a horse has won in any one or more races, account shall be taken of cups or moneys, whether derived from stakes, bonus, or any other source, gained by him in steeple chases, hurdle races, and National Hunt flat races, in any country, of the value of 20 sov. or upwards, but in the case of welter flat races, only of winnings in such races and in others to which the "Rules of Racing" apply, deducting only his own stake and entrance. Winnings during the year shall include all prizes from the Ist of January preceding, to the time appointed for the start, and winning shall include dividing, walking-over, or receiving forfeit.

A Challenge Cup is not estimated in the value of a race until it is finally won, provided the sweepstakes or added money amount to 20 sov. or upwards, but when won outright the winner must carry a penalty in respect of the full value of the Cup and money prize.

Penalties for winning a fixed sum shall be understood to mean for winning it in one race unless specified to the contrary.

II4. No horse shall carry extra weight for having run second, or in any lower place in a race.

II5. Extra weight shall not be incurred in respect of matches or private sweepstakes.

I 6. Penalties are not cumulative unless so declared by the conditions of the race.

II7. Where winners of selling races are exempted from penalties, only such horses as have run to be sold, shall be entitled to the exemption.

II8. When any race is in dispute, both the horse that came in first and any horse claiming the race, shall be liable to all the penalties attaching to the winner of that race until the matter be decided.

For penalties on dividing after dead heats, see Dead Heats.

II9. All penalties and allowances shall be calculated according to the amount of the value of each race as reported in the Racing Calendar.

120. Allowances. - No horse shall receive allowance of weight or be relieved from extra weight, for having been beaten in one or more races; Provided that this rule shall not prohibit maiden allowances, or allowances to horses that have not won within a specified time, or races of a specified value.

I2I. Allowances to the produce of untried horses are for the produce of horses whose produce never won a race-in any country.

122. Allowances are cumulative unless otherwise specified.

I23. No horse shall be entitled to allowance of weight for being half-bred.

Weighing Out, Exhibiting Numbers, etc.

124. The Weighing Room.-No person shall, without special leave from the 
Stewards, be admitted to the Weighing-room, except Members of the National Hunt Committee, the owner, trainer, and rider, or other person having the care of a horse engaged in the race, and any person refusing to leave shall be reported to the Stewards.

125. Weighing Out. - Each rider must be weighed for a specified horse by the Clerk of the Scales, at the appointed place, not less than a quarter of an hour before the time fixed for the race, and the numbers shall then be exhibited as soon as possible. In exceptional cases, or where the delay of a previous race has rendered punctuality impossible, the Stewards may extend the time allowed for weighing, declaring weight, and for exhibiting the numbers.

I26. When the numbers have been exhibited, no alteration or addition can be made without the leave of the Stewards, whose reasons for such permission shall be reported at the Registry Office.

Should any horse not start whose number has been exhibited, the owner and any other person responsible may be fined, or otherwise dealt with, at the discretion of the Stewards.

127. If a rider intends to carry over-weight, he must declare the amount thereof at the time of weighing out, or, if in doubt as to his proper weight, the weight he intends to carry.

I28. It is optional for a rider to weigh out or in with his bridle, and the Clerk of the Scales shall, if requested, allow Ilb for a curb or double bridle, but if a horse run in a hood, martingale, breastplate, or clothing, it must be put into the scale and included in the riders' weight.

I29. No whip or substitute for a whip shall be allowed in the scales.

\section{Starting.}

I30. The horses must be started by the official starter or his authorised substitute.

I3I. Every horse must be at the post ready to start at the appointed time; any owner, trainer, or rider making default herein may be fined at the discretion of the Stewards a sum not exceeding 5 sov.

132. All riders, on arrival at the starting post, must immediately place themselves under the Starter's orders, and no horse, when once under his orders, shall go back, except in the case of an accident.

\section{Running and Walking Over.}

133. Running.-An owner running two or more horses in a race may declare to win with one of them, and such declaration must be made at scale. The rider of a horse with which the owner has not declared to win, must on no account stop such horse except in favour of the horse on whose behalf declaration to win has been made.

I34. (i) In a National Hunt flat race or welter flat race a horse which crosses another in any part of the race is disqualified, unless it be proved that he was two clear lengths ahead of the other when he crossed.

(ii) In a steeple chase or hurdle race a horse shall be disqualified if his rider, by foul riding, jeopardised the chance of success of any other horse in the race, and in the run home from the last hurdle or fence, section (i) of this rule shall apply; the Stewards have power to fine a rider for the above offence any sum not exceeding 50 sov. In all cases the Stewards have power of suspending a rider until the expiration of the meeting, or, should they consider such punishment insufficient, until the case can be heard and decided by the Stewards of the National Hunt Committee.

(iii) If a horse or his rider jostle another horse or rider, the aggressor is disqualified, unless it be proved that the jostle was wholly caused by the fault of some other rider, or that the jostled horse or his rider was partly in fault.

(iv) If a horse run the wrong side of a post he must turn back and run the course from such post.

I35. If a race has been run by all the horses at wrong weights, or over a wrong course or distance, or if the Judge, or his authorised substitute or a Steward is 
not in the box at the time the horses pass the winning post, the Stewards shall order it to be run again on the same day if practicable, if otherwise the race shall be void.

136. Walking Over.-A walk-over shall in no case be deemed necessary. It shall be sufficient if a horse be weighed out for, mounted, and proceed to the starting post, when, if no competitor appear in due time, he shall be the winner.

\section{Dead Heats.}

137. Every horse running a dead heat for the first place shall be deemed the winner of the race until the dead heat is run off or the owners agree to divide, and, if the owners agree to divide, each horse which divides shall be deemed a winner of the race, and liable to the penalty for the full amount he would have received if he had won.

I38. A dead heat for the first place shall be run off on the same day at a time to be appointed by the Stewards, unless the owners agree to divide. The other horses shall be deemed to have been beaten, but they shall be entitled to their places (if any) as if the race had been finally determined the first time.

If, in running a dead heat off, either horse should be disqualified, it shall be decided by the Stewards whether the disqualification shall extend to the loss of the second place, and, if so, the horse that was originally placed third shall be entitled to the second place.

139. When a dead heat is run for second place, and an objection is made to the winner of the race, if such objection be declared valid in time for the dead heat to be run off on the day of the race, the Stewards may direct it to be run off accordingly; otherwise the horses which ran the dead heat shall divide.

I40. If a dead heat be run by two or more horses for second or any lower place in a race, the owners shall divide, subject to the provisions of the last preceding rule.

I4I. When owners divide they shall divide equally all the moneys or other prizes which any of them could take if the dead heat were run off.

I42. If the dividing owners cannot agree as to which of them is to have a cup or other prize which cannot be divided, the question shall be determined by lot by the Stewards, who shall decide what sum of money shall be paid by the owner who takes such cup or other indivisible prize, to the other owner or owners.

143. On a dead heat for a match the match is off.

\section{Weighing In.}

I44. Every rider must, immediately after pulling up, ride his horse to the place of weighing, dismount and present himself to be weighed by the Clerk of the Scales: Provided that, if a rider be prevented from riding to the place of weighing by reason of accident or illness, by which he, or his horse is disabled, he may walk or be carried to the scales.

I45. If a rider do not present himself to be weighed in, or be short of weignt, or be guilty of any fraudulent practice with respect to weight or weighing, or dismount before reaching the place of weighing, or touch (except accidentally) any person or thing other than his own equipments before weighing in, his horse is disqualified, unless he can satisfy the Stewards that he was justified by extraordinary circumstances.

I46. If a horse carry more than four pounds over his declared weight for a steeple chase or hurdle race, or two pounds for a National Hunt flat race or welter flat race, he is disqualified unless the Stewards be satisfied that such excess of weight has been caused by wet or mud.

I 47. If a rider be unable to draw his weight with the one pound allowed for the curb or double bridle, he shall have the option of weighing in with his bridle ; but no weight shall be allowed for a snaffle bridle unless it is put into the scales before the horse is led away.

\section{Prizes.}

148. The value of prizes not in money must be advertised.

149. Prizes, stakes, and forfeits in a race belong to the winner, except as otherwise declared in the conditions. 
150. No plate or sweepstakes shall be run for unless the clear value to the winner (calculated as in Weights, Penalties, etc.), in case the race be run by two or more horses, will amount to 20 sov. But if the value would amount to 20 sov., if the race were so run, a horse may walk over, although he thereby receives less than 20 sov.

I5I. When a sweepstakes has been so reduced by the death of subscribers that the payments to second or other horses, according to the conditions, would reduce the value, if run for, to less than 20 sov., the winner shall receive a clear 20 sov., and the balance only, if any, shall then be divided proportionately between the other horses entitled to participate in the stakes.

I52. In all plates or sweepstakes (private sweepstakes excepted) the second horse shall at least save his stake and entrance.

I53. When a cup (other than a Challenge Cup) is advertised to be run for it shall be given, even in the event of a walk-over.

I54. When a walk-over (except after a dead heat) is the result of arrangement by the owners of horses engaged, neither a cup nor any portion of the advertised money need be given.

I55. Any money or prize which by the conditions is to go to the horse placed second, or in any lower place, shall, if the winner has walked over or no horse has been placed second, etc., be dealt with as follows, namely :-

(i) If part of the stakes or plate, it shall go to the winner.

(ii) If a separate donation from the Race-fund, or any other source, it shall not be given at all.

(iii) If entrance money for the race, it shall go to the Race-fund of the meeting.

156. No deductions shall be made from the value of any race except such as are gained by other horses in the race, and except Clerks', stakeholding, and weighing fees, as fixed by rule.

157. If a race be never run or be void, stakes, forfeits, and entrance money shall be returned.

\section{Disputes, Objections, Appeals, etc.}

158. All disputes, objections, and appeals referred to or brought before the Stewards of the National Hunt Committee, shall be determined by them (three to form a quorum); if only two Stewards are present, they shall appoint a third person, being a member of the Committee, in lieu of the absent Steward, but they may call in any other members of the Committee to their assistance, or may, if they think the importance or difficulty of the case requires such a course, refer it to a general meeting.

I59. Objections. - (i) Every objection shall be determined by the Stewards, but should no decision be given by them within seven days of an objection being lodged, the Clerk of the Course shall report the case to the Stewards of the National Hunt Committee, who may at their discretion decide the matter, and if they consider there has been any negligence order any additional expense arising therefrom to be defrayed out of the funds of the meeting at which the case occurred.

(ii) The decision of the Stewards on any objection shall be subject to appeal to the Stewards of the National Hunt Committee through the Stewards or the meeting, and with their consent, and that of the Stewards of the National Hunt Committee, and not otherwise.

(iii) Every objection shall be in writing, and must be signed by the owner of some horse engaged in the race, or by his deputed agent, trainer, or rider, and must be made to one of the Stewards, to the Clerk of the Course, or Clerk of the Scales; the objector shall, at the time he makes the objection deposit in the hands of the Stakeholder the sum of 5 sov., which, if the case be decided against him, shall be forfeited to the National Hunt Fund, unless the Stewards shall certify that there was good and reasonable grounds for the objection; and in the event of the objection being decided to be frivolous or vexatious, it shall be in the power of the Stewards to find the objector any additional sum not exceeding 20 sov.

(iv) An objection may also be made without deposit by a Steward or official of a meeting in his official espacity.

(v) An objection cannot be withdrawn without leave of the Stewards. 
160. (i) If an objection to a horse engaged in a race be made not later than half-past ten in the morning of the day of the race, the Stewards may require his qualification to be proved before the race; and in default of such proof being given to their satisfaction, they may declare him disqualified.

(ii) An objection to any decision of the Clerk of the Scales must be made at once.

(iii) An objection to a horse, on the ground of a cross, jostle or foul riding, or of his not having run the proper course, or of any other matter occurring in the race, or on the ground of any irregularity on the part of a rider at the scales or before weighing in, must be made within a quarter of an hour after the winner has weighed in, unless, under special circumstances, the Stewards are satisfied that it could not have been made within that time.

(iv) An objection on the ground,

(a) Of mis-statement, omission, or error in the entry under which a horse has run, or

(b) That the horse which ran was not the horse, or of the age, which he was represented to be at the time of entry, or that he was not qualified under the conditions of the race, or

(c) That he has run in contravention of the rules as to registration of partnerships, or other joint interests, or

(d) That he has run at any unrecognised meeting, or that his owner or rider has run or ridden a horse at any unrecognised meeting during the preceding twelve months ;

may be received within fourteen days of the conclusion of a meeting.

(v) An objection on the ground that a horse is disqualified by reason of any default entered in the Forfeit List, may be received within six months after the race.

(vi) An oojection on the ground of carrying wrong weight, or on any other ground not specified in the foregoing sections of this rule, must be made within twenty-four hours of the race being run, exclusive of Sunday, save in the case of fraud, or wilful mis-statement, when there shall be no limit to the time for objecting.

I6I. All costs and expenses in relation to determining an objection or conducting an enquiry, and any reasonable compensation for outlay incurred, shall be paid by such person, or persons, and in such proportions, as the Stewards, or the Stewards of the National Hunt Committee, shall direct.

I62. Pending the determination of an objection, any prize which the horse objected to may have won, or may win in the race, shall be withheld until the objection be determined, and any forfeit payable by the owner of any other horse shall be paid to and held by the Clerk of the Course for the person who may be entitled to it.

163. Appeals. - When leave of appeal has been granted the appellant shall forthwith notify in writing to the Clerk of the Course, for transmission to the Registry Office, his intention of appealing, and at the same time deposit the sum of Io sov.; in all cases, except where fraud or wilful mis-statement is alleged, such notice and deposit must be received at the Registry Office within seven days of the decision of the Stewards having been given or the a ppellant shall forfeit his right.

Should an appeal be decided to be frivolous or vexatious, the deposit shall be forfeited to the National Hunt Fund, or if otherwise, returned to the appellant.

\section{Disqualifications of Persons and Corrupt Practices.}

164. No "disqualified person" shall act as a Steward or in any other official capacity in relation to a meeting, nor nominate, enter, run, or ride any horse in any race under these rules, and any horse so nominated, entered, run, or ridden by a "disqualified person" shall be disqualified.

165. Any person riding or running a horse for any race whatever in Great Britain, not advertised to be run under the Rules of Racing, or the National Hunt Rules, and not specially exempted from the operation of these rules under Definitions and Application of these Rules, shall be disqualified from riding or running 
a horse where the National Hunt Rules are in force, for twelve months from the date of such offence, but this rule shall not apply to

(a) Races at Meetings confined to Resident Members of the Universities of Oxford and Cambridge.

(b) Pony or Galloway races at Meetings confined to Pony or Galloway racing.

166. (i) If any person corruptly give or offer, or promise directly or indirectly, any bribe in any form to any person having official duties in relation to a race or race-horse, or to any trainer, rider, or agent, or to any other person having charge of, or access to, any race-horse; or

(ii) If any person having official duties in relation to a race, or if a trainer, rider, agent, or other person, having charge of, or access to, any race-horse, corruptly accept or offer to accept any bribe in any form ; or

(iii) Wilfully enter or cause to be entered or to start for any race a horse which he knows or believes to be disqualified; or

(iv) If any person be proved to the satisfaction of the Stewards of the National Hunt Committee to have surreptitiously obtained information respecting a trial from any person or persons engaged in it, or in the service of the owner or trainer of the horses tried, or respecting any horse in training from any person in such service; or

(v) If any person be guilty of, or shall conspire with any other person for the commission of, or shall connive at any other person being guilty of, any other corrupt or fraudulent practice in relation to racing in this or any other country.

Every person so offending shall be warned off all courses where these Rules are in force.

167. If any person be reported by the Committee of the Subscription Rooms at Newmarket or at Tattersall's as being a defaulter in bets he shall be warned off as in the last Rule mentioned, so long as his default continues.

I68. When a person is warned off all courses where these Rules are in force, and so long as his exclusion continues, he shall not be qualified, whether acting as an agent or otherwise, to subscribe for, enter, run, or ride any horse for any race either in his own name or in that of any other person, and any horse of which he is the nominator, or is (or was at the time of entry) wholly or partly the owner, or which after the fact of his being warned off has been twice published in the Racing Calendar, shall be proved to the satisfaction of the Stewards to be, or to have been, directly or indirectly, under his care, training, management, or superintendence, shall be disqualified.

\section{Qualification for National Hunt Flat Races.}

169. The following provisions apply to National Hunt Flat Races-

(I) A horse to qualify to run for a National Hunt Flat Race must have been placed by the Judge first, second, or third in a steeple chase in Great Britain or Ireland, after having jumped all the fences, and completed the whole distance of the race to the satisfaction of at least two of the Stewards, to whom previous notice shall have been given in writing through the Clerk of the Course.

(2) Such Stewards shall give a certificate to the above effect in the form 2 of Appendix C.

(3) Such certificate, or in the case of a horse qualified in Ireland, a copy of such certificate, signed by the Secretary, and countersigned by a Steward of the Irish National Hunt Steeple Chase Committee, must be lodged at the Registry Office one clear week before running, with a fee of $5 \mathrm{~s}$. for registration.

(4) Any certificate is invalidated by the disqualification of the horse for the race in which it was obtained.

(5) Horses for National Hunt Flat Races must be ridden by Qualified Riders. They cannot be handicapped, but shall not carry less than I Ist.

(6) The provisions of Running and Walking Over as to running shall be in force in National Hunt Flat Races so far as applicable. 


\section{Special Rules relating to Steeple Chases and Hurdle Races.}

I70. (i) In steeple chases and hurdle races, any horse getting away from his rider may be remounted in any part of the field or enclosure in which the occurrence took place, but should such horse not be caught until he shall have entered another field, then he shall be ridden or brought back to the one in which he parted from his rider. Any rider so losing his horse may be assisted in catching him and remounting him without risk of disqualification; and in the event of a rider being disabled, his horse may be ridden home by any person of sufficient weight, provided he be qualified according to the conditions of the race. No penalty shall be exacted for carrying overweight in this instance.

NOTE.-In artificially constructed steeple chase courses, and in hurdle races, the spaces between the fences or hurdles are considered as fields or enclosures for the purposes of this rule.

(ii) If any flag, post, or boundary mark be placed in the course or altered after the riders have been shown over the ground, or had the course pointed out to them, it shall not be considered binding or of any effect unless such addition or alteration shall have been particularly named, previous to starting, to all the riders in the race, by one of the Stewards, the Clerk of the Course, or by their representatives.

(iii) If a horse refuse any fence, or hurdle, in a race, and it can be proved to the satisfaction of the Stewards that he has been led over it by any of the bystanders, or has been given a lead over by any horseman not riding in the race, the horse shall be disqualified.

\section{Fees and Fines.}

I7I. Fees.-The Stakeholder shall be allowed to retain, out of the stakes in his hands, the following fees, viz.-

For every match, I sov.

For every subscription or sweepstakes where the lowest forfeit amounts to 20 sov. $\frac{1}{2}$ per cent. on the whole stake, and on all other races I per cent., the minimum fee to be I sov.

I72. There shall also be payable the following fees-

(i) For every entry a maximum Clerk's fee of $5 \mathrm{~s}$., to include weighing.

(ii) On election or re-election as Qualified Rider, I sov.

(iii) For every registration or change of an assumed name, ro sov.

(iv) For every registration of partnership, and on every change thereof $I$ sov. for each horse.

(v) For every annual registration of colours, $5 \mathrm{~s}$.

(vi) For every registration of colours for life, 2 sov.

(vii) For registration of foreign and veterinary certificates, 5s. on each certificate.

(viii) For registration of National Hunt Flat Race Certificates, 5s.

(ix) For registration of Point to Point Steeple Chase Certificates, Ios.

(x) For registration of an agreement with a jockey, $5 \mathrm{~s}$.

I73. Fines. - All fines shall be paid to the funds of the National Hunt Committee.

\section{New Rules.}

174. No new rule can be passed, nor can any existing rule be rescinded or altered, without being previously advertised three times in the "Sheet Calendar," and without notice being given of the meeting of the National Hunt Committee at which it is to be proposed; and no new rule, or repeal or alteration of a rule, shall take effect until it has been twice subsequently published in the "Sheet Calendar." Any owner of race-horses, or persons affected by such proposed new rule, recission, or alteration, may, before it is made, petition the National Hunt Committee through the Secretaries. All such petitions shall be laid before the meeting before the question is put. 


\section{APPENDIX A.-List of Meetings Referred to in Regulations for Race Meetings.}

[The Stewards of the National Hunt Committee have power from time to time to alter or extend this list.]

Birmingham, Cardiff, Derby, Folkestone, Gatwick, Hawthorn Hill, Hurst Park, Keele Park, Kempton Park, Leicester, Lingfield, Manchester, Newmarket, Nottingham, Plumpton, Sandown Park, Sheffield and Rotherham, Southweil, Warwick, Windsor, Wolverhampton (Dunstall Park), Wye.

\section{APPENDIX B.-Scale of Weight-for-Age (see Omitted Conditions).}

The Committee recommend the following scale of weight-for-age :-

For Steeple Chases of three miles and upwards.

From Ist Jan. to 30 th June, inclusive :

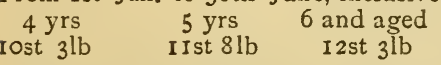

From Ist July to 3 Ist Dec, inclusive :
4 yrs
5 yrs
6 and aged
I Ist
I Ist I $2 \mathrm{lb}$
I 2 st $31 \mathrm{~b}$

For Steeple Chases of less than three miles.

From Ist Jan. to 30 th June, inclusive :

4 yrs
Iost Iolb yrs 6 and aged

From Ist July to 3 Ist Dec., inclusive :

$\begin{array}{ccc}4 \mathrm{yrs} & 5 \mathrm{yrs} & 6 \text { and aged } \\ \text { IIst } 6 \mathrm{lb} & \text { I2st } & \text { I2st } 3 \text { lb }\end{array}$

\section{For Hurdle Races.}

From Ist Jan. to 3 Ist Aug., inclusive :

$\begin{array}{ccc}4 \mathrm{yrs} & 5 \mathrm{yrs} & 6 \text { and aged } \\ \text { IIst } & \text { I Ist Iolb } & \text { I } 2 \mathrm{st}\end{array}$

From Ist Sept. to 3 Ist Dec., inclusive : 3 yrs $\quad 4$ yrs 5, 6 and aged Iost $7 \mathrm{lb}$ I Ist I $2 \mathrm{lb}$ I $2 \mathrm{st} 3 \mathrm{lb}$

For National Hunt Flat Races.

From Ist Jan. to 30 th June, inclusive :

\begin{tabular}{|c|c|}
\hline $4 \mathrm{j} \cdot \mathrm{rs}$ & $5 \mathrm{yrs}$ \\
\hline
\end{tabular}

From Ist July to 3 Ist Dec., inclusive :

$\begin{array}{ccc}4 \mathrm{yrs} & 5 \mathrm{yrs} & 6 \text { and aged } \\ \text { I } 2 \mathrm{st} & \text { I2st } 5 \mathrm{lb} & \text { I2st } 7 \mathrm{lb}\end{array}$

\section{APPENDIX C. -Fcrm I.}

Point to Point Steeple Chase Certificate (see Definitions, Application, etc.).

$I$ hereby certify-

I. That bona fide Point to Point Steeple Chases took place at on

2. That not more than three steeple chases, and no other races of any description, took place there on that day.

3. That the course was unflagged, except at the winning post, and at* other places where, in the opinion of the Stewards, flags were absolutely necessary for the general guidance of the riders.

\section{* Number specified, not exceeding three.}

4. That no money was taken at any gate, or at any stand or enclosure in connection with the races.

5. That the winning flag was placed within the limits of the country hunted over by my hounds.

Date Master of the 189

Hounds and Stewards.

** This Certificate must be signed by a Master of Foxhounds or Staghounds or by a Master of Harriers (being a Member of the Association of Masters of Harriers), and must be lodged with Messrs Weatherby and Sons, 6 Old Burlington Street, London, W., within fourteen days of the day of the races. 


\section{Form 2.}

Certificate of Qualification for National Hunt Flat Races (see Qualification for National Hunt Flat Races).

We, the undersigned, Stewards of the that $\mathrm{Mr}$ 's

Meeting, hereby certify has this day been placed in the

Steeple Chase, and has jumped all the fences and completed the whole distance of the race to our satisfaction.

Date

I8

Signed,

Address,

Signed,

Address,

** This certificate must be lodged with Messrs Weatherby and Sons, 6 Old Burlington Street, London, IV., one clear week before the horse is to run, with a fee of 5 s. for registration.

\section{RULES ON BE'TTING.}

Although the Stewards of the Jockey Club take no cognisance of betting, yet, for the convenience of such persons as are interested in the subject, we subjoin a copy of the Rules as re-arranged by the Committee of the Subscription Rooms, at Tattersall's, on February 8, I886.

The Committee of Tattersall's and the Committee of the Newmarket Rooms have authority to settle all questions relating to bets, to adjudicate on all cases of default, and, at their discretion, to report defaulters and persons guilty of any malpractice to the Jockey Club. In the following rules the words "the Committee" refer to either of those bodies.

2. In all bets there must be a possibility to win when the bet is made: "you cannot win when you cannot lose." No betting first past the post will be recognised by either of the Committees.

3. All bets are P.P.-play or pay-with the following exceptions-I. When the nominator dies before the decision of the race. 2. When the race is postponed to a future week, or the conditions are altered after the bets are made. 3 . Bets on matches. 4. Bets made after the running numbers are telegraphed about a horse that is not subsequently under the starter's orders.

4. If no objection is lodged within seven days of the race, exclusive of the day on which the race was run, bets go to the horse placed first by the Judge, and the settling, except in cases of fraud, shall not be disturbed. If an objection is made within the said time, bets go with stakes.

5. Bets made on one horse against another, or that one horse beats another, are determined if either of them should win: unless agreed by the parties, it is not indispensable that both horses should start. Bets made between horses I, 2, 3 are determined by the places assigned by the Judge-it is not necessary to say the best of $I, 2,3$.

6. If odds are laid in running or immediately after the horses pass the post, and a dead heat is the result: and in "double events," if either is decided in the backer's favour, and the other results in a dead heat, the money betted must be put together and equally divided. As, according to racing custom, matches which result in a dead heat are void, bets are void also.

7. If a bet is made on one of the horses that runs a dead heat against a beaten horse, and the owners agree to divide, he who backed the horse that ran the dead heat wins half his bet. If odds are laid on one horse against another I, 2, 3, and they run a dead heat for either place, the money betted must be put together and equally divided.

8. The person who lays the odds has the right to choose a horse or the field; when a person has chosen a horse, the field is what starts against him. If odds are laid without mentioning the horse before the race is over, the bet must be determined by the state of the odds at the time of making it. 
9. Bets made after a race that the winner will be disqualified, stand, even if no objection be made.

Io. Any bet made from signal or indication when the race has been determined, shall be considered fraudulent and void.

II. All bets on matches and private sweepstakes depending between any two horses shall be void if those horses subsequently become the property of the same person, or of his avowed confederate.

I2. Double event bets are determined when the first event is lost.

I3. Bets made on horses winning any number of races within the year shall be understood to mean between the Ist of January and the 3 Ist of December.

I4. Money given to have a bet laid shall not be returned, though the race be not run.

I5. In the event of a race being ordered to be run over again, starting price bets shall be regulated by the price current at the time the race was first run. Bets in favour of any horse which started on the first but did not go to the post on the second occasion are lost.

I6. Confirmed bets cannot be declared off except by mutual consent, but on any allegation of fraud or corrupt practice, the Committee will investigate the case and may declare the bet void. Either of the bettors may demand stakes to be made on proving to the satisfaction of the Committee that he has just cause for doing so. If ordered, the bets must be covered or sufficient security offered, and a person refusing to cover shall be expelled the Subscription Room at Newmarket and at Tattersall's.

I7. The Committee will not necessarily enforce the settlement of a compromised account. Before giving a decision they may require the books of the debtor and a statement of his accounts to be submitted to them; but they have authority, in all such cases, to order the account to be settled if they think a reasonable offer is made.

18. If a debtor does not satisfy the claims of his creditors within twelve months he shall not be entitled to receive any debts which may be due to him; but if he does so within the prescribed time, viz., "one year," i.e., 365 days, inclusive of the day when tine money was won, the Committee will support his just claims to receive payment from his debtors.

I9. If any extraordinary occasion should arise, or in cases of notorious and palpable fraud, any of the before-mentioned rules may be suspended by the Committee.

20. The Stewards of races have no authority ex cficio to take cognisance of any disputes or claims with respect to bets. 


\section{IN DEX}

A

Abdomen, diseases of-

\begin{tabular}{|c|c|c|c|c|c|c|}
\hline ttle & & & • & • & . & 449 \\
\hline $\begin{array}{l}\text { dogs } \\
\text { horses }\end{array}$ & - & • & • & - & . & 614 \\
\hline $\begin{array}{l}\text { horses } \\
\text { sheep }\end{array}$ & - & • & - & - & - & 449 \\
\hline $\begin{array}{l}\text { heep } \\
\text { domen, }\end{array}$ & & . & & . & . & 542 \\
\hline $\begin{array}{l}\text { lomen, d } \\
\text { lomen of }\end{array}$ & dise & ses 0 & , dog & s. & . & 614 \\
\hline en of & of th & hors & & • & . & 239 \\
\hline ragr & & . & • & . & $24 \mathrm{I}$ & 249 \\
\hline & & . & • & . & . & 240 \\
\hline & & & & & & \\
\hline & • & • & • & • & • & \\
\hline hor: & rses & . & 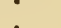 & • & • & \\
\hline & & . & . & & & 50 \\
\hline & & 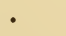 & • & 0.4 & 85 & \\
\hline & & & • & . & • & \\
\hline
\end{tabular}

American horses . . . 412

Anatomical structure of the horse $16 \mathrm{I}$

Anthrax . . . . 467

cleansing and disinfection $\quad .472$

disposal of carcases . . . $47 \mathrm{I}$

procedure . • . . 470

symptoms of . . . . . 468

Arabian horses . . . . 403

breeding from. . . . 304

Arm of the horse . . . 87

Asiatic horses . . . . 403

Ass, the : $: \quad \therefore 416$

Asthma, dogs . . . 596

Attachments in front of the pastern bones, horse

\section{B}

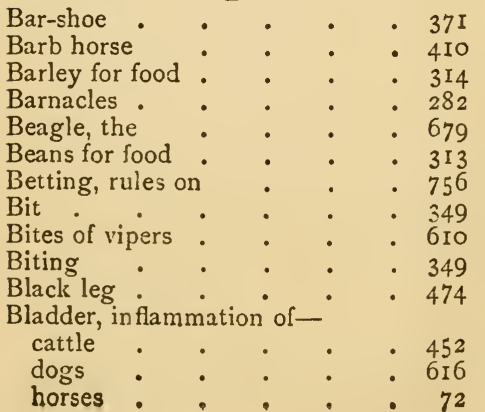

Bladder of the horse - • 252 stone in . . . . 73

Bleeding, horses : $\quad \cdot \quad 284$ sheep . . . . $56 \mathrm{I}$

Blend-water, dogs : . 622

Blindness and imperfect visiondogs . . . . 6r2 horses . . . . 37, 180 sheep * . . .535

Blistering, horses • • . 288

Blood-letting, dogs . . . 637

Blood-striking, swine . • . 584

Bloody water, dogs . . . 622

Bog-spavin . . . II9

Bolus, method of giving : . 637

Bone-sparin . . . . II5

Boots, water $\quad . \quad$. $\quad 374$ poultice . . . . $\quad .374$

Bots, the . . . . 81

Bowels, entanglement of : • 79

Bowels, inflammation ofcattle . . . . . 449 dogs : . . . $\quad .6$ I4 horses . . . . 75

Brain, inflammation of, cattle $\quad 426$

Braxy or sickness, sheep . . 544

Breeding dogs . . . . 640 horses . . . . . 304 swine . . . . 586

Breeds of the horse . . . 388 African horse . . . . 410 American horse . . . 4I2 Arabian horse . . . 403 Asiatic horse . . . . 403 Barb . . . . 410 British horse . $\quad . \quad$ * 395 charger, the : . . . 399 Cleveland bays . . . 400 coach horse, the . . . 400 draught horse. . . . 40I Egyptian horse $\cdot \quad \cdot \quad \cdot 4$ II hackney, the - . . $\quad$ - 397 hunter, the . . . . 396 Nubian or Dongola horse - 4 II Persian horse . . . 408 Suffolk punch-horse . . 402 Tartar horse . . . . 408 Toorkoman horse . . . 409 Turkish horse . . . 409 
Breeds of horned cattle Galloway breed Kyloes, or Highland long-horned cattle . milch cows short-horned, or Holsteir

Breeds of sheep

British horses

Broken wind

Bronchitis

Bruises, dogs

Bull-dog, the terrier, the

C

Calf-bed, falling down of

Calves, rearing of

Calving

Canker, dogs horses

Capped hocks

Carrots for food

Cartilages of the foot

Castrating lambs

Cataract dogs

Catarrhal mammitis

Catarrh, or common cold, cattle . 442 horses . . . . 56 sheep • • . 536

Cattle, breeds of . . . . $52 \mathrm{I}$ dentition of . . . . 5 54 diseases of . . . . 42 I feeding of . . . 516

Cavesson . . . . 320

Chaps . . . . 500

Charger, the : • • • 399

Chest, diseases of-

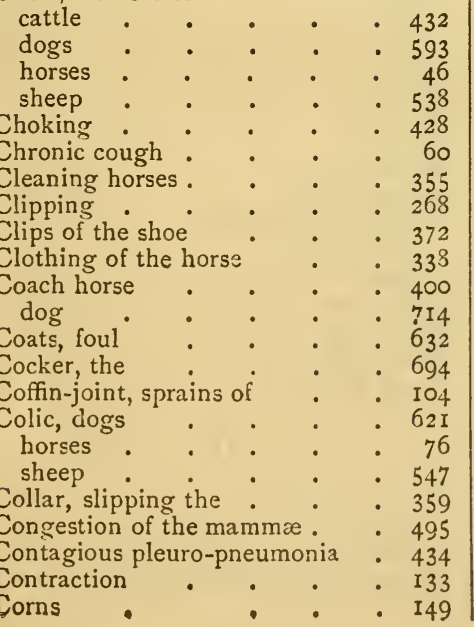

Costiveness, cattle . . . 463 dogs . . . . 621 Cough, cattle, - . . . 444 dogs . . . . 595

Cow-houses $\cdot \quad \cdot \quad \cdot 516$

Cow-pox . . . . 480

Cramp of the legs . $\quad$. 554

Cranium of the horse . . . 166

Crib-biting . . • . 357

Cropping . . . . $\quad 639$

Crupper • • • • $\quad$. 324

Curb • • • •

Cuticle, the . . . . 261

Cutis, the . . . . 262

\section{D}

Dalmatian . • • • • 7I4

Defects of the fore-leg . . 217

Dentition of cattle . . . 5I4

Diabetes . • • . 74

Diaphragm of the horse . . 241

Diarrhœa or looseness-
cattle . 458

dogs : . . . 622

sheep . . . 546

Diseases of the abdomen-

cattle . . . 4 $42 \mathrm{I}, 449$

dogs . . . 6 I4

horses . • . $\quad . \quad 68$

sheep . . 542

Diseases of the chest-
cattle . . 432

dogs . . . . 593

horses . . . • • 46

sheep • • . . 538

Diseases of dogs . . 591

Diseases of the external parts-

cattle . . . . . 479

dogs . . . . 626

horses . . . . 84

sheep . . . . 549

Diseases of the eye-
dogs . . $62 \epsilon$

horses . . . 32

Diseases of the head-
cattle . . 426

dogs . . . . . 593

horses . . . . . 5

sheep . . . . 533

Diseases of the
hind legs . . . 218

horse . . . . . 5

lips . . . $\quad 32$

mammary gland or udder $\quad 494$

neck . . . . 40

sheep . . . . . $53 \mathrm{I}$

skin of the horse . . . 26I

swine . . . 581

teeth . . . . . 30

tongue . . . . 29 
Distemper-

\begin{tabular}{|c|c|c|c|c|}
\hline dogs & & - & - & 597 \\
\hline horses & & - & - & $6 I$ \\
\hline Diuretic medicin & & - & - & 251 \\
\hline Docking & . & . & - & - 299 \\
\hline ogs- & & & & \\
\hline breeding & - & - & - & - 640 \\
\hline diseases of & • & - & - & $59 I$ \\
\hline feeding . & . & - & - & - 647 \\
\hline of the chase & & & - & - 675 \\
\hline used in shooti & & & & - 688 \\
\hline watch- & & & . & - 698 \\
\hline Domestication o & f th & hor & & - 389 \\
\hline Draught horse & - & . & . & - 401 \\
\hline Drifting snow & . & . & . & 573 \\
\hline Drink for horses & & - & - & 3 I 6 \\
\hline Dropsy, dogs & - & - & - & 620 \\
\hline $\begin{array}{l}\text { Dysentery- } \\
\text { cattle }\end{array}$ & & & & \\
\hline sheep & . & $\dot{\bullet}$ & • & 47 \\
\hline & & & & \\
\hline
\end{tabular}

E

Earcanker in, dogs ulceration of .

Egyptian horse .

Elbow, enlargement of fracture of

Epilepsy, horses.

Epileptic fits, dogs

Erysipelas, sheep

Ewe, the .

Exercise of the horse

Eye, diseases of-

dogs horses

Eye, its visual structure muscles of, etc.

Eyelashes of the horse

Eyelids, eruption of

\section{F}

Falling down of the calf-bed

False quarter : : :

Farcy

Fardel-bound

Fattening of sheep

Febrile diseases .

Feeding cattle

cow-house

dogs

horses

sheep

Feet of the horse, management of

Feet, sore, dogs .

Fetlock sprain of the .

Fever cutting of the.

Firing
Fistulous wither. . . $\quad$. 64

Fits, dogs . . . . . 624

horses . . . . 14

Fleas. . . . . 635

Fluke, or liver rui . $\quad . \quad$. 538

Fog sickness . . . . 440

Food for cattle . . . . 517

dogs . . . . 647

horses . . . . 310

sheep . . . . 577

Foot, bones of the horse's . 215, 218 cartilages of the . . . 228

diseases of . . . . 129

external parts of . . . 223

integuments of . . . 218

nerve, vein, and artery of . 216

rot, sheep . . . . 555

Fore legs . . . . . 84

Foul coats, dogs . . . 632

Fouls, the . . . . . 482

Founder, acute . . . . I29

chronic . . . . . I33

Foxhound . . . . . 677

training the . . 652

Fracture of the skull, horses . 39

tuberosities of the haunch 107

Fractures, dogs . . . . 638

sheep . . . . 560

G

Gadflies, cattle . • • 4483

horses . . . . . 82

Galloway breed . . . . 523

Gargut, or distended udder . $\quad 585$

Gauchos . . . . . 413

Gestation, cattle. $\quad$. $\quad .492$ dogs . . . . 640

sheep . . . . . 565

swine . . . . 586

Gibbing • • • • • 345

Glanders . . . . . 16

Grass-ill • • • • 540

Grease • • • • .123

Greyhounds . . . 651,682

Scottish Highland . • . 685

training . . . . 665

Gripes • • • • • 455

Grogginess . . . . 100

Grooming . • • • 337

Gullet, obstruction of . • • 43

Gutta serena . . . . 36

\section{$\mathrm{H}$}

Hair of the horse. • . 266 colour of the • • • . 270

Harrier, the . . 678

Haunch and hind legs, diseases of 107

Haw of the eye . . $\quad 35,178$ 
Hay for food

Head of the horse, external anatomy internal anatomy

Head, diseases of cattle dogs horses. sheep

Heart of the horse . . $\quad 233$

Heat and flies . . . . 575

Hernia

Hide-bound

Hind legs . . . . . 107 diseases of . . . 218

Hinder-shoe . . . . 372

History of the horse . . . 388

Hocks, capped . . . . IIO enlargement of $\cdot$. $\quad$ II2

lameness in . . . . 214

Hock-joint, structure of : 2 II

Hoof, preparation for shoeing of 362

Hoose or husk . . . . 445

Horses-

abdomen of . . . . 239

age of : - 164, 185, 187

anatomical structure of . . I6I

bladder. . . . 252

bleeding . . . . 284

blistering . . . . 288

breeding of . . . . 304

chest of . . . . . 23I

dental indications of age. $\quad$ I87

diaphragm, or midriff . . 249

docking . . . . . 299

drink for . . . . 316

exercise of . . . . 336

eye and its visual structure . I78

feeding of . . . 3 IO

feet, management of . $\quad$. 334

firing . $\cdot$., 292

grooming $\quad$ • $\quad$. 337

hair . . . . 266

heart of . . . . 233

how to buy . • . $\quad 376$

internal organisation of . 230

intestines . . . . 253

kidneys . . . . . 250

lady's • . . . . 386

liver of . . . . . 247

lungs of . . . . 230

muscles and eye . . . I74

neurotomy . . . . 294

nicking • • • . 300

not lying down . . . 358

omentum or caul of. . . 248

palate and teeth . . . I7I

pancreas of . . . . 249

pulse $\mathrm{cf}$.

\section{Horses-continued}

skin . . . . 26I

spleen of : : : $\quad: 248$

stable • • • . $\quad 328$

stomach of . . . . 243

teeth . . . . I84

training..$\quad \cdot \quad \cdot \quad \cdot 319$

vices of . . . . . 340

Hoven . . . . 440

How to buy a horse - • . 376

Hunter, the . • • . 396

Hunting shoe . . . . 370

Husk • . • . • 445

Hydrophobia . . . . 606

Inflamed udder . $\quad . \quad$ - 572

Inflammation of the

bladder, cattle. $\quad$. $\quad 452$

dogs. . . . $6 \mathrm{I} 6$

horses. . . . 72

bowels, cattle . . . . 449

dogs . . . . 6I4

horses. . . . . 75

brain, cattle . . . . 426

eye, horses . • • . 32

kidneys, cattle. . . . 45I

horses. . . . $7 \mathrm{I}$

liver, cattle $\quad \cdot \quad$. $\quad . \quad \cdot 439$

horses . . . 68

lungs, cattle : . . . 432

dogs . . . . 595

horses . . . 46, 203

sheep . . . . 540

swine . . . . 583

mucous membrane, horses . 75

stomach, cattle . . . $43^{8}$

womb, cattle - . . . 452

Influenza . . . . 6 I

Intestinal worms . $\quad . \quad 80$

parasites . . . . 6I 7

Intestines of the horse. . . 253

intussusception of . . . 79

stones in $\cdot \quad \cdot \quad \cdot \quad \cdot 79$

Interstitial mammitis, cattle 496,498

Intussusception of the intestines . 79

J

Jaundice, or yellows-

cattle • • • • • 447

horses : $: \quad: \quad 69$

sheep . . . . 54I

Jockey's licenses, etc. . . . 726

Kennel, the . . . . 667

Kicking • . • • • 347

Kidneys, inflammation of, cattle . $45 \mathrm{I}$ of the horse . . . 250 inflammation of . . . 7 I 
Knees, complaints and injuries of Knee-tied . 95 Kyloes, or Highland cattle. $\quad 523$

\section{L}

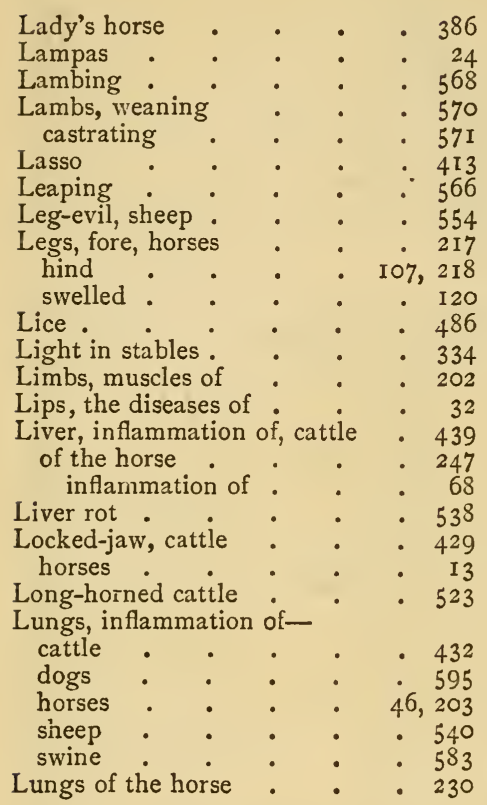

Muscles of the limbs . . . 202

thigh . . . . 208 Musty grain : $\quad \cdot \quad \cdot \quad \cdot 3 \mathrm{I} 2$ Mucous membrane : . 75

\section{$\mathrm{N}$}

Nasal gleet $\quad$ • $\quad$. 22

National Hunt Rules. $\quad . \quad 735$

Navicular-joint disease . . I 39

Neck, diseases of . . . 40

Nerve, cutting of a . . . 294

Neurotomy . . . 216, 294

Newfoundland dog . . . 706

Nicking . . . . 300

Nostrils of the horse . . . 169

Nubian and Dongola horses :4II

\section{$\mathrm{O}$}

Oats for food . . 310

Omentum or caul of the horse 248

Operations-

dogs . . . . 637

horses . . . . . 282

Ophthalmia . . . . 33

Ossification of the back sinew . IO5 of the cartilages . . . 157

Otter-hound, the $\quad . \quad$ 68I

$\mathrm{P}$

Palate of the horse . . . I7I

Palsy. . . . . . I5

Pancreas of the horse. . . 249

Parturient apoplexy . • . 476

Parturition-

cattle . . . . 492, 505

dogs . . . . 640

horses . . . . 308

sheep . . . . 565

swine . . . 586

Pastern and foot, their bones and integuments . . 218

nerve vein and artery of . $\quad 26 \mathrm{I}$

Pasteur, Dr . . . . 608

Pawing • • • • . 359

Pease for food . . . . 313

Pelt-rot, sheep • • . . 553

Pelvis . . . . . IO7

Peritoneum of the horse - . 240

Persian horse . . . . 408

Piles, dogs. . . . . 623

Pill, method of giving : . 637

Pinding, sheep . . . . 548

Pin-toed horses . . . $\quad$. 378

Pleurisy . . . . 50

Pleuro-pneumonia : . . 434

Pneumonia $\therefore$. . $\quad 432$

Prick or wound in the sole. $\quad$ I4 6

Pointer, the English . . . 688

how to train. . . 652 
Poison

Poisonous plants

Poll evil

Polypus

Poodle

Post-mortem, dogs . . . 607

Poultice boot . . . . 374

Puffing the glims . . . 165

Pulse of the horse . . . 236

Pupping . . . . 646

\section{Q}

Quinsy • • . $\quad 583$

Quittor • • • • $\mathbf{I} 44,225$

\begin{tabular}{|c|c|c|c|c|c|c|}
\hline \multicolumn{7}{|l|}{ Rabies- } \\
\hline dogs & - & - & . & - & - & 606 \\
\hline horses & & . & - & - & $\dot{v}$ & I 2 \\
\hline Race-horse & es, ag & & $\theta^{\circ}$ & • & . & 723 \\
\hline Racing col & lours & . & . & . & . & 727 \\
\hline rules of & . & . & . & . & 717 & 735 \\
\hline shoe & . & . & . & . & . & $37 \mathrm{I}$ \\
\hline Ram, the & . & . & . & . & . & 565 \\
\hline Rearing & & . & - & . & . & 344 \\
\hline of calves & & & & . & - & 510 \\
\hline Red-water, & catt & & . & . & • & 453 \\
\hline Red-water & blibe & s, she & & . & . & 543 \\
\hline Regulation & is for & race & meeti & ings & . & 721 \\
\hline Resp or rec & d-wat & er, sh & heep & . & • & 542 \\
\hline Restivenes: & $\mathrm{s}$ in $\mathrm{h}$ & 1orses & & - & 340 , & 353 \\
\hline while sh & oeing & & . & 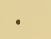 & . & 356 \\
\hline Rete muco & sum, & the & • & • & • & 265 \\
\hline Retrievers & . & - & . & 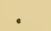 & . & 663 \\
\hline Rickets & . & . & . & . & . & 635 \\
\hline Ringbone & . & . & . & . & 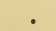 & 104 \\
\hline Roaring & & 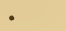 & . & . & . & $4^{2}$ \\
\hline Rolling & & - & • & . & . & 360 \\
\hline Rules of ra & acing & . & • & . & 7 I 7, & 735 \\
\hline on bettin & & & . & . & . & 756 \\
\hline Running a & way & horse & & 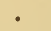 & • & 350 \\
\hline Rupture, o & or her & & & . & • & 70 \\
\hline f h & & & & & & 100 \\
\hline
\end{tabular}

\section{S}

\begin{tabular}{|c|c|}
\hline Saddle-galls & 66 \\
\hline Safety-shoe & - 373 \\
\hline Sallenders. & - III \\
\hline Sand-crack & I 35 \\
\hline Scab or itch, cattle & 479 \\
\hline sheep & . 549 \\
\hline Scalds. & - 634 \\
\hline Scurf, scab & . 479 \\
\hline Setter, the Englis & - 690 \\
\hline how to train. & . 654 \\
\hline Setons & - 298 \\
\hline Shearing . & . 577 \\
\hline
\end{tabular}

Sheep, age of, as indicated by the teeth. . . . 562 breeds of . . . . 580 diseases of . . . . 531 fattening of . . . $\quad 578$ feeding of . . . . 573 food for . • • • • . 577 shelter for . . . . 573 washing . . . . 576

Shelter for sheep . . $\quad 573$ drifting snow . • • . 573 effects of rain . . . . 575 heat and flies. . . . 575

Shepherd's dog . . . . 709

Shoeing . . . . 36r bar shoe. • • • $\cdot 37 \mathrm{r}$ clips of the shoe $\quad$. $\quad . \quad 372$ concave-seated shoe • • 366 expanding shoe • • • 370 hinder shoe . . . . 372 hunting shoe . • . 370 preparation of the hoof for $\quad 362$ putting on of the shoe - . 364 racing shoe . • • $\quad 37 \mathrm{I}$ restiveness while : $\quad . \quad 356$ safety-shoe . . . . 373 seated shoe $\quad \cdot \quad$ • $\quad$ - 369 tips . . . 373 Short-horned or Holstein breed of cattle . $\quad . \quad 52 \mathrm{I}$ Shoulder, muscles of . $\quad . \quad 202$ Shying . . . . 350 Side line . . . . 283 Sight of the horse, testing the, . $\quad \mathbf{8} \mathbf{2}$ Sinew, ossification of the kack . I05 sprain of the back . . . 95 Singeing . $\quad \cdot \quad \cdot 269$ Sinuses, frontal . $\quad . \quad$. 167 Skeleton of the horse . . . r6I Skin of the horse . . . 26I Skull, fracture of . . . 39 Snores . . . . 430 Soles, felt or leather . . $\quad$ - 375 Sore feet, dogs . . . . 632 Spaniel, water . . . . 696 Spavin, bone . . . . II5 bog . . . . . II9

Speedy cut . . . 994

Sprains and bruises, dogs . . 633 of the back sinews, horses $\quad$. 95 of the coffin-joint . . . IO4 of the fetlock . . . . rOI of the round bone . . . 107 of the shoulder $\quad \cdot \quad \cdot 85$ of the stifle-joint . . . IOS Spleen of the horse • • 248 Splenic apoplexy $\quad . \quad \cdot 467$ Splent, or splint $\quad$ • $\quad$ • 92 Springer, the . . . . 692 Staggers or apoplexy, horses $\quad 8$ 
Staggers or apoplexy, sheep - 533

Stables light in : $\quad: \quad * 334$

Staling of blood. . . . 74

Stomach of the horse . . . 243

Stomach or grass staggers, cattle 446 horses

Stomach, inflammation of the $\quad 438$

Stones in the bladder. . . 73

Stones in the intestines . . 79

Strains and bruises . . . 487

Strangles . . . . 24

Strangury • • • • 4493

String halt . . . . II4

Suffolk punch-liorse . • • 402

Surfeit . . • • . 276

Swelled legs . . . . I 20

Swelling of the idder. • . 494

Swine, diseases of $\quad . \quad$. $58 \mathrm{I}$

$\mathrm{T}$

Tares • • . • 314

Tartar horse . . . . 408

Teats, ulcer ted. . . . 500

Teeth, sheep . . . $\quad 562$ horses . . . 30, I84, I7I

Terrier, the . . . . 680

Tetanus . . . . . I3

Thick wind $\cdot \quad \cdot \quad 57$

Thigh, muscl s of the . . 208

Thorns . . . . 635

Thorough-pin . . . . 109

Throat, sore $\quad$. . . 430

Thrush • . . . . I53

Ticks, etc. . . . . . 635

Tips . . . . . . 373

Tongue . $\quad$ • $\quad$ • 29

Toorkoman horse . . . 409

Training dogs . . . . 647

foxhounds . . . . 652

horses . . . . . 3I9

pointers . . . . 654

setters . . . . . 654

Travis . . . . 282

Tread, or overiench . . . I43

Tripping . . . . 360

Tuberculosis • • • 524

Turkish horse . . . . 409

Turnips for food . . . 314

$\mathrm{U}$

Udder, diseases of
Udder, swelling of - • 494 swine . $\quad . \quad 585$ Ulceration of the ear, dogs . 594, 627 Ulcerated teats . . . 500

\section{V}

Vein, inflammation of the jugular 44

Vermin . . . 558

Vices of the horse . . . 340

Vipers, bites of, in dogs . . 610

\section{W}

Warbles • • . . . 66

Warts : $: \quad: \quad 36,28$ r

Washing sheep . . . . 576

Watch-dogs . . . . 698

Water-boot . . . . 374

IVater-dogs : . . . 663

Water-dropwort . . . . 3I5

Water-parsley . . . . 3I5

Water-spaniel, large . . . . 696

small . . . . 697

Weakness of the foot . . . 158

Weaning lambs . . . 570

Weaving . . . . 360

Wheat for food . . . . 313

Wildfire • $\quad \cdot \quad \cdot \quad \cdot 553$

Wind, broken . . . 58

thick . . . . . 57

Wind-galls $\quad . \quad$. $\quad .99$

Wind-sucking . . . . 358

Wintering cattle . . . 518

Withers, fistulous . . . 64

Wolf-dog . . . . . 685

Womb, inflammation of . 452

Wood-evil . . . . 554

Worming . . . . . 637

Worms : . . 6I7

Wounds, cattle . . . . 487

dogs . . . . 633

horses . . . . . 27

sheep • . • . . 559

Wurbles, or wormals . • . 483

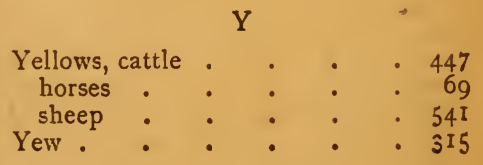


Webster Cumm vulicine at

Ti:"

200 vinuaver

North Graton MA U1536 


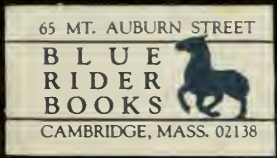


